\title{
Estimação e teste de hipótese baseados em verossimilhanças perfiladas
}

\author{
Michel Ferreira da Silva
}

Tese apresentada

$\mathrm{aO}$

Instituto de Matemática e Estatística

da

Universidade de São Paulo

para

obtenção do grau

de

Doutor em Estatística

Área de concentração: Estatística

Orientadora: Profa. Dra. Silvia Lopes de Paula Ferrari

Co-orientador: Prof. Dr. Francisco Cribari Neto

Durante a elaboração deste trabalho, o autor recebeu apoio financeiro da FAPESP.

São Paulo, junho de 2005 


\section{Estimação e teste de hipótese baseados em verossimilhanças perfiladas}

Este exemplar corresponde à redação final da tese devidamente corrigida e defendida

por Michel Ferreira da Silva e aprovada pela comissão julgadora.

São Paulo, junho de 2005.

Banca examinadora:

Profa. Dra. Silvia Lopes de Paula Ferrari (orientadora) (IME-USP)

Prof. Dr. Francisco Cribari Neto (co-orientador) (UFPE)

Prof. Dr. Enrico Antônio Colosimo (UFMG)

Profa. Dra. Reiko Aoki (ICMC-USP)

Prof. Dr. Filidor Edilfonso Vilca Labra (UNICAMP) 
Aos meus pais, José Ferreira e Ranucia, aos meus avós maternos, José Correia (in memorian) e Maria, e aos meus irmãos, Micheline e Jean, minha homenagem e gratidão. 


\section{AGRADECIMENTOS}

Aqui cabe minha gratidão por meio da menção a algumas pessoas e instituições que contribuíram para a realização deste trabalho:

- os professores Silvia Lopes de Paula Ferrari e Francisco Cribari Neto pela orientação, amizade, paciência e confiança transmitida ao longo do meu doutoramento;

- os amigos que conviveram comigo durante os últimos cinco anos, com os quais compartilhei momentos difíceis, mas também comemorei vitórias. Entre estes, vale mencionar: Laélia, Viviana e Héctor, Dione e Pledson, Isaura, Marilia, Alberto, Roger e Lorena, Pedro, Tito e Carla, Terêncio, Guillermo, Flávio;

- os professores do Departamento de Estatística da Universidade de São Paulo, que enriqueceram minha formação estatística;

- a Fundação de Amparo à Pesquisa do Estado de São Paulo, pelos recursos financeiros disponibilizados para meu doutoramento, sem os quais este trabalho não seria concretizado;

- os participantes da banca examinadora pelas sugestões. 


\section{RESUMO}

Tratar a função de verossimilhança perfilada como uma verossimilhança genuína pode levar a alguns problemas, como, por exemplo, inconsistência e ineficiência dos estimadores de máxima verossimilhança. Outro problema comum refere-se à aproximação usual da distribuição da estatística da razão de verossimilhanças pela distribuição qui-quadrado, que, dependendo da quantidade de parâmetros de perturbação, pode ser muito pobre. Desta forma, torna-se importante obter ajustes para tal função. Vários pesquisadores, incluindo Barndorff-Nielsen (1983,1994), Cox e Reid (1987,1992), McCullagh e Tibshirani (1990) e Stern (1997), propuseram modificações à função de verossimilhança perfilada. Tais ajustes consistem na incorporação de um termo à verossimilhança perfilada anteriormente à estimação e têm o efeito de diminuir os vieses da função escore e da informação.

Este trabalho faz uma revisão desses ajustes e das aproximações para o ajuste de Barndorff-Nielsen $(1983,1994)$ descritas em Severini (2000a). São apresentadas suas derivações, bem como suas propriedades. Para ilustrar suas aplicações, são derivados tais ajustes no contexto da família exponencial biparamétrica. Resultados de simulações de Monte Carlo são apresentados a fim de avaliar os desempenhos dos estimadores de máxima verossimilhança e dos testes da razão de verossimilhanças baseados em tais funções.

Também são apresentadas aplicações dessas funções de verossimilhança em modelos não pertencentes à família exponencial biparamétrica, mais precisamente, na família de distribuições $\mathcal{G}_{A}^{0}(\alpha, \gamma, \mathcal{L})$, usada para modelar dados de imagens de radar, e no modelo de Weibull, muito usado em aplicações da área da engenharia denominada confiabilidade, considerando dados completos e censurados. Aqui também foram obtidos resultados numéricos a fim de avaliar a qualidade dos ajustes sobre a verossimilhança perfilada, analogamente às simulações realizadas para a família exponencial biparamétrica. Vale mencionar que, no caso da família de distribuições $\mathcal{G}_{A}^{0}(\alpha, \gamma, \mathcal{L})$, foi avaliada a aproximação da distribuição da estatística da razão de verossimilhanças sinalizada pela distribuição normal padrão. Além disso, no caso do modelo de Weibull, vale destacar que foram derivados resultados distribucionais relativos aos estimadores de máxima verossimilhança e às estatísticas da razão de verossimilhanças para dados completos e censurados, apresentados em apêndice. 


\begin{abstract}
The profile likelihood function is not genuine likelihood function, and profile maximum likelihood estimators are typically inefficient and inconsistent. Additionally, the null distribution of the likelihood ratio test statistic can be poorly approximated by the asymptotic chi-squared distribution in finite samples when there are nuisance parameters. It is thus important to obtain adjustments to the likelihood function. Several authors, including Barndorff-Nielsen (1983,1994), Cox and Reid (1987, 1992), McCullagh and Tibshirani (1990) and Stern (1997), have proposed modifications to the profile likelihood function. They are defined in a such a way to reduce the score and information biases.

In this dissertation, we review several profile likelihood adjustments and also approximations to the adjustments proposed by Barndorff-Nielsen (1983, 1994), also described in Severini (2000a). We present derivations and the main properties of the different adjustments. We also obtain adjustments for likelihood-based inference in the two-parameter exponential family. Numerical results on estimation and testing are provided.

We also consider models that do not belong to the two-parameter exponential family: the $\mathcal{G}_{A}^{0}(\alpha, \gamma, \mathcal{L})$ family, which is commonly used to model image radar data, and the Weibull model, which is useful for reliability studies, the latter under both noncensored and censored data. Again, extensive numerical results are provided. It is noteworthy that, in the context of the $\mathcal{G}_{A}^{0}(\alpha, \gamma, \mathcal{L})$ model, we have evaluated the approximation of the null distribution of the signalized likelihood ratio statistic by the standard normal distribution. Additionally, we have obtained distributional results for the Weibull case concerning the maximum likelihood estimators and the likelihood ratio statistic both for noncensored and censored data.
\end{abstract}




\section{ÍNDICE}

LISTA DE SÍMBOLOS - x

1. INTRODUÇÃO 1

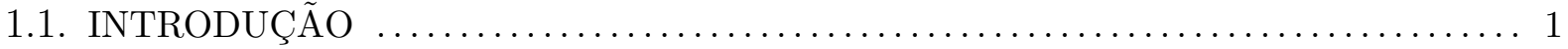

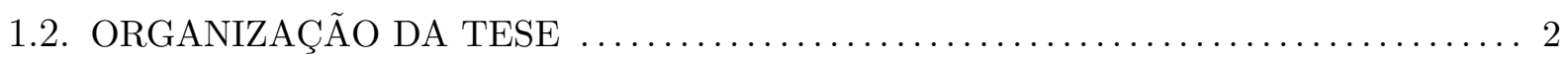

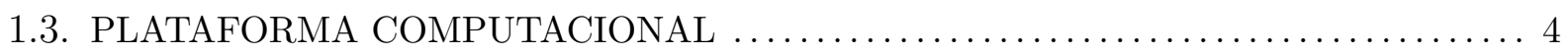

2. AJUSTES PARA VEROSSIMILHANÇAS PERFILADAS 6

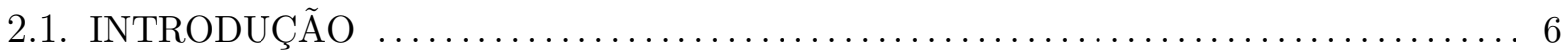

2.2. FUNÇÃO DE VEROSSIMILHANÇA PERFILADA $\ldots \ldots \ldots \ldots \ldots \ldots \ldots \ldots \ldots \ldots$

2.3. VEROSSIMILHANÇA PERFILADA MODIFICADA PROPOSTA POR

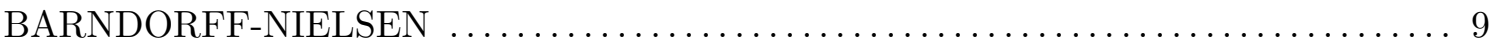

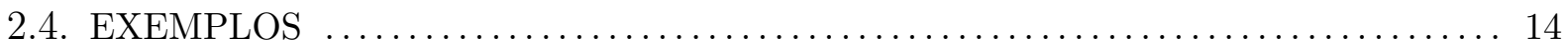

2.5. VEROSSIMILHANÇA PERFILADA MODIFICADA PROPOSTA POR

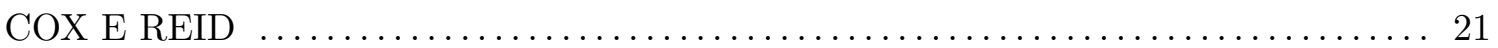

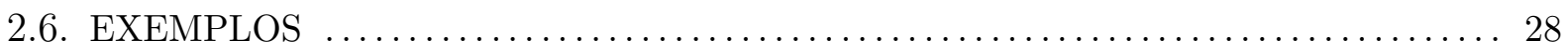

2.7. VEROSSIMILHANÇA PERFILADA MODIFICADA PROPOSTA POR

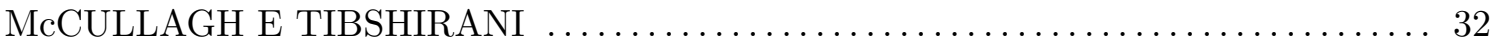

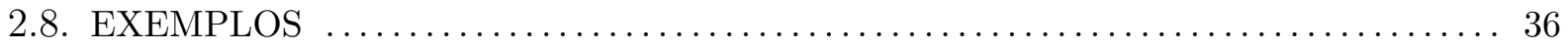

2.9. VEROSSIMILHANÇA PERFILADA MODIFICADA PROPOSTA POR

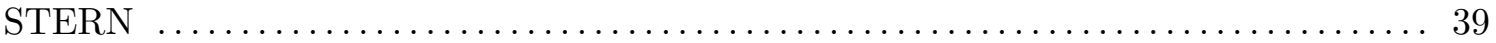

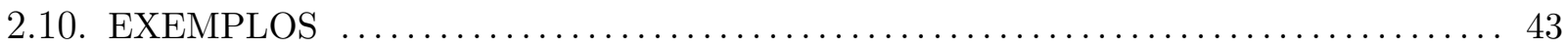

2.11. APROXIMAÇÕES PARA A VEROSSIMILHANÇA PROPOSTA POR

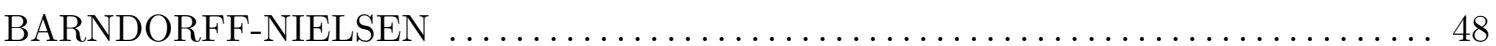

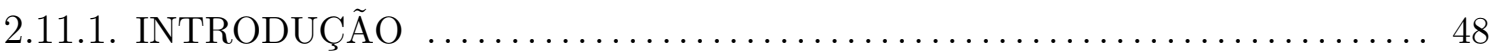

2.11.2. UMA APROXIMAÇÃO BASEADA NAS COVARIÂNCIAS DE DERIVADAS DO LOGARITMO DA FUNÇÃO DE VEROSSIMILHANÇA ........ 49 2.11.3. UMA APROXIMAÇÃO BASEADA EM COVARIÂNCIAS EMPÍRICAS . . . 52 2.11.4. UMA APROXIMAÇÃO BASEADA NUMA ESTATÍSTICA

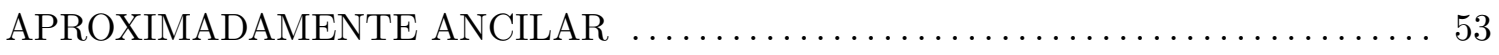

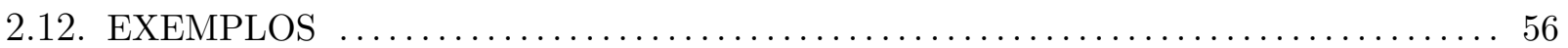

3. FAMÍLIA EXPONENCIAL BIPARAMÉTRICA: RESULTADOS NUMÉRICOS

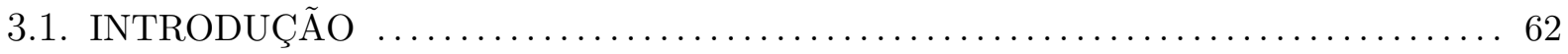

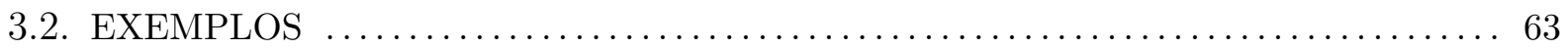

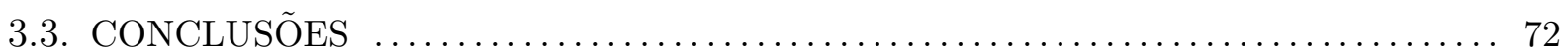


4. PARÂMETRO DE HETEROGENEIDAdE DA $\mathcal{G}_{A}^{0}(\alpha, \gamma, \mathcal{L})$ : TESTES UNICAUDAIS E ESTIMAÇÃO $\quad \mathbf{7 4}$

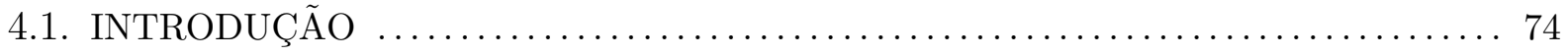

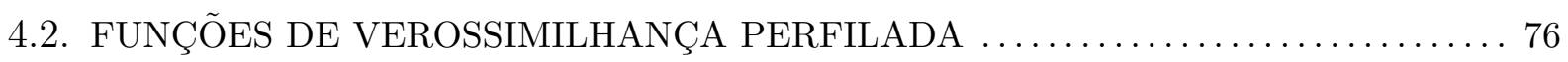

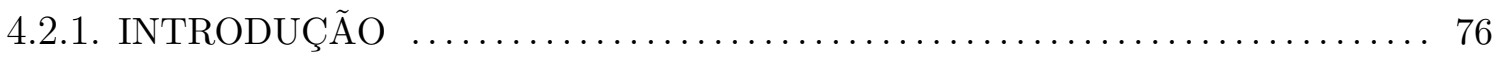

4.2.2. APROXIMAÇÕES PARA A VEROSSIMILHANÇA PROPOSTA POR

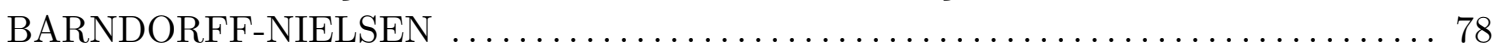

4.2.3. VEROSSIMILHANÇA PROPOSTA POR COX E REID $\ldots \ldots \ldots \ldots \ldots \ldots . \ldots 79$

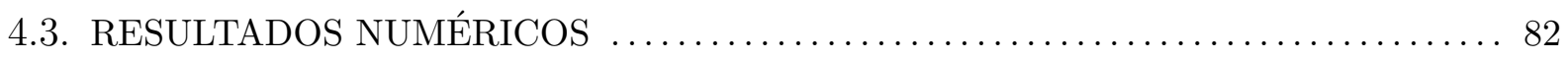

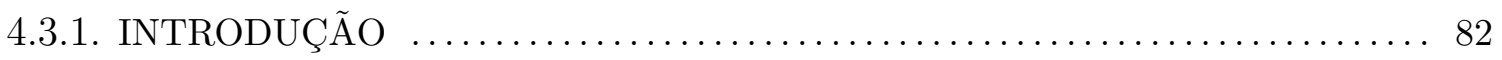

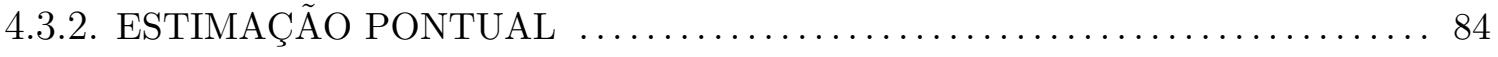

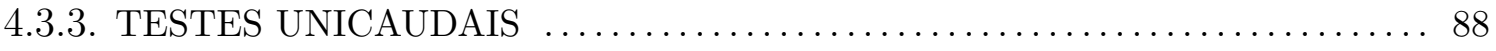

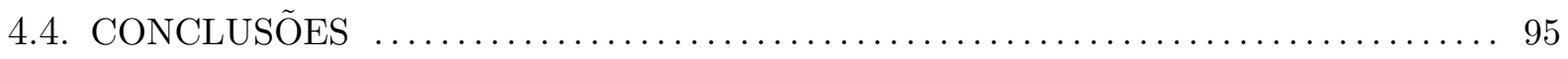

5. ESTIMADORES DE MÁXIMA VEROSSIMILHANÇA E TESTES DA RAZÃO DE VEROSSIMILHANÇAS NO MODELO DE WEIBULL 97

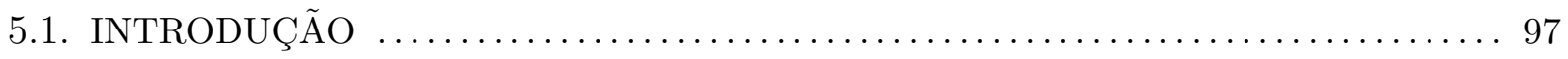

5.2. TESTE DE HIPÓTESE E ESTIMAÇÃO DO PARÂMETRO DE FORMA ........ 99

5.2.1. OBSERVAÇÕES INDEPENDENTES E IDENTICAMENTE DISTRIBUÍDAS 99

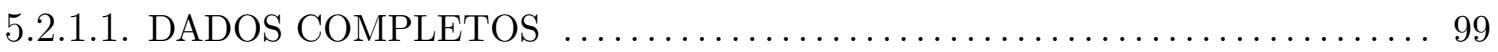

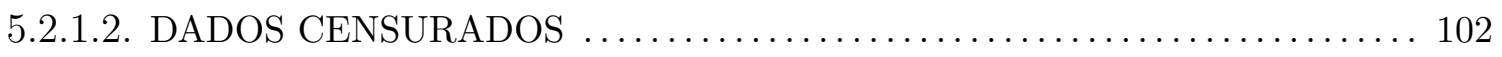

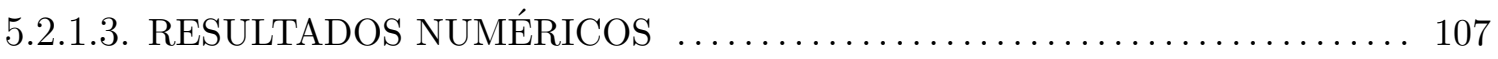

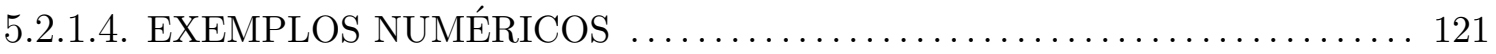

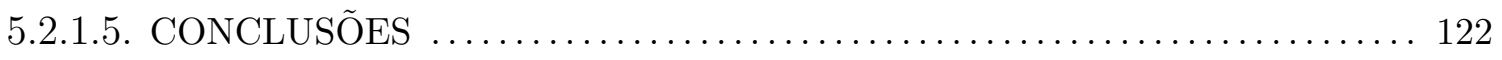

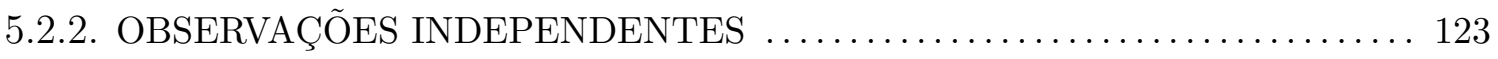

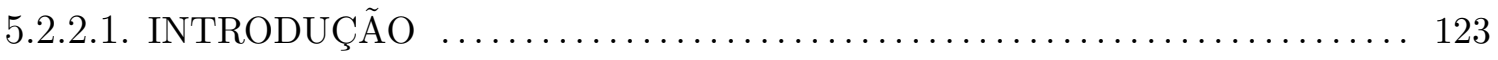

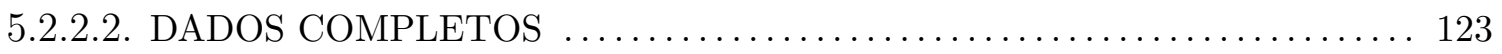

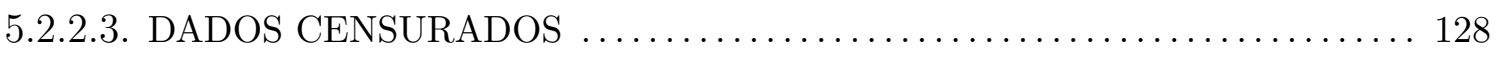

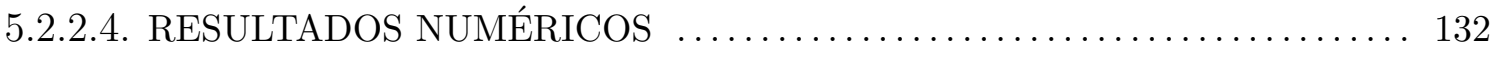

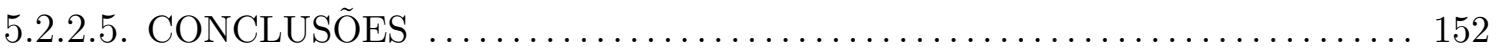

5.3. TESTES SOBRE OS COEFICIENTES DA REGRESSÃO $\ldots \ldots \ldots \ldots \ldots \ldots \ldots \ldots \ldots$

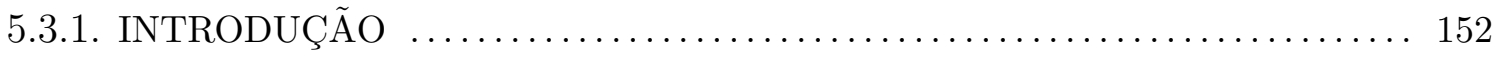

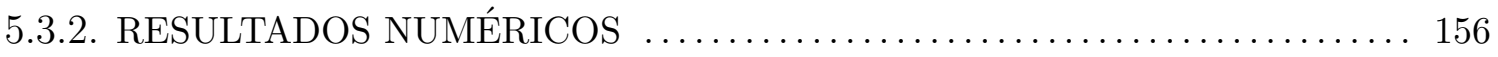

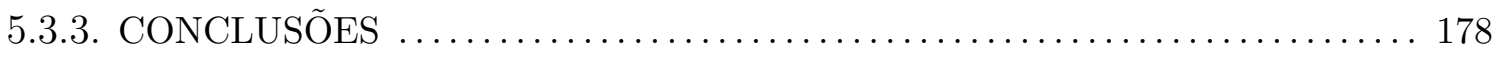

A. DETALHES COMPUTACIONAIS $\mathbf{1 7 9}$

A.1. GERADORES DE NÚMEROS ALEATÓRIOS $\ldots \ldots \ldots \ldots \ldots \ldots \ldots \ldots \ldots \ldots \ldots \ldots \ldots$

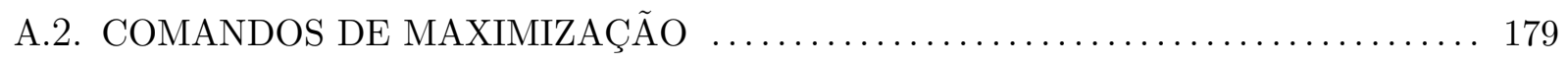

B. OTIMIZAÇÃO ALTERNADA 183 
C. PROGRAMA COMPUTACIONAL $\mathbf{1 8 5}$

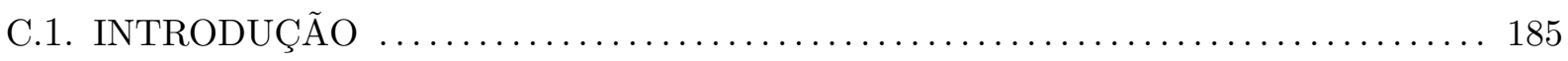

C.2. EXEMPLO 2.4: DISTRIBUIÇÃO GAUSSIANA INVERSA $\ldots \ldots \ldots \ldots \ldots \ldots \ldots \ldots$

D. ESTIMADORES DE MÁXIMA VEROSSIMILHANÇA E ESTATÍSTICAS DA RAZÃO DE VEROSSIMILHANÇAS NO MODELO DE WEIBULL: RESULTADOS DISTRIBUCIONAIS $\quad 207$

D.1. OBSERVAÇÕES INDEPENDENTES E IDENTICAMENTE DISTRIBUÍDAS .... 207

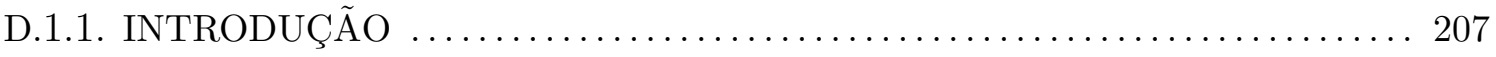

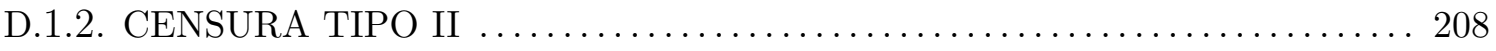

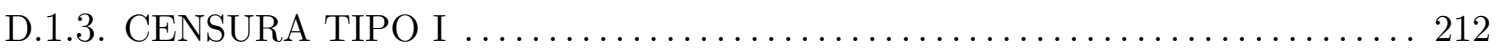

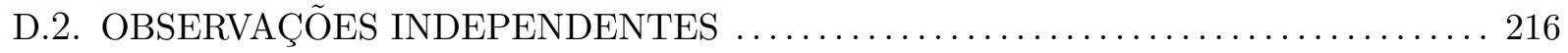

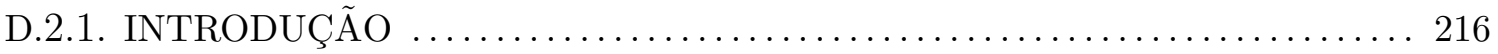

D.2.2. ESTIMADORES E ESTATÍSTICAS DE TESTES $\ldots \ldots \ldots \ldots \ldots \ldots \ldots \ldots 217$

D.3. TESTES SOBRE OS COEFICIENTES DA REGRESSÃO $\ldots \ldots \ldots \ldots \ldots \ldots \ldots \ldots \ldots \ldots \ldots \ldots$

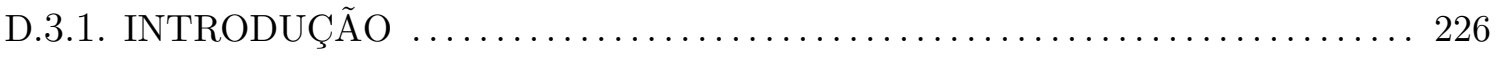

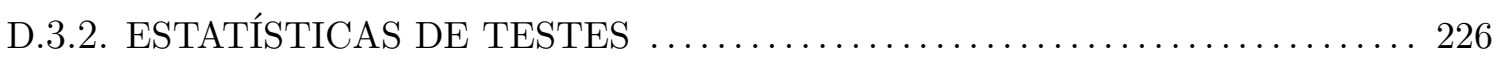

REFERÊNCIAS 231 


\section{LISTA DE SÍMBOLOS}

$y$

$p(y)$ ou $p(y ; \theta)$

$p(y \mid x)$ ou $p(y \mid x ; \theta)$

$F(y)$ ou $F(y ; \theta)$

$\operatorname{dim} \theta$

$E\{y\}$ ou $E_{\theta}\{y\}$

$\operatorname{Var}\{y\}$ ou $\operatorname{Var}_{\theta}\{y\}$

$R V$

$|A|$

$N\left(\mu, \sigma^{2}\right)$

$\Gamma(\cdot)$

$G(\alpha, \beta)$

$B(a, b)$

$\doteq \mathrm{ou} \approx$

$W$

$S_{R}$

$\operatorname{tr}\{A\}$

$\left[a^{r}\right]$

$\left[a_{r s}\right]$

$\sim$

$A^{-}$

$0_{n \times m}$

$\chi_{n}^{2}$

$\operatorname{Pr}(E)$

$\psi(\cdot)$
Variável (vetor) aleatória(o) ou valor observado desta(e)

Função densidade ou de probabilidade de $y$

Função densidade ou de probabilidade condicional de $y$ dado $x$

Função de distribuição de $y$

Dimensão do vetor paramétrico $\theta$

Esperança da(o) variável (vetor) aleatória(o) y

Variância (matriz de covariância) da(o) variável (vetor) aleatória(o) y

Estatística da razão de verossimilhanças

Determinante da matriz $A$

Distribuição normal com média $\mu$ e variância $\sigma^{2}$

Função gama

Distribuição gama com parâmetro de escala $1 / \beta$ e parâmetro de configuração (forma) $\alpha$

Distribuição beta com parâmetros $a$ e $b$

Aproximadamente igual

Estatística de Wald

Estatística escore de Rao

Traço da matriz $A$

Vetor com elemento genérico $a^{r}$

Matriz com elemento genérico $a_{r s}$

Distribuído como

Inversa generalizada da matriz $A$

Matriz nula de dimensão $n \times m$

Distribuição qui-quadrado com $n$ graus de liberdade

Probabilidade do evento $E$

Função digama 


$\begin{array}{ll}W B(\alpha, \beta) & \text { Distribuição de Weibull com parâmetro de escala } \alpha \text { e parâmetro de } \\ & \text { configuração (forma) } \beta \\ N^{-}\left(\mu, \mu \sigma^{2}\right) & \text { Distribuição gaussiana inversa com média } \mu \text { e coeficiente de } \\ & \text { variação } \sigma \\ V E(\eta, \sigma) & \text { Distribuição valor extremo com parâmetros de locação } \eta \text { e de } \\ & \text { escala } \sigma \\ \csc (\cdot) & \text { Função cossecante } \\ \min (u, w) & \text { Menor valor entre } u \text { e } w \\ \mathcal{E} x p(\lambda) & \text { Distribuição exponencial de média } \lambda \\ \operatorname{Ber}(p) & \text { Distribuição de Bernoulli de média } p \text { (probabilidade de sucesso) } \\ \operatorname{Bin}(n, p) & \text { Distribuição binomial, onde } n \text { é o número de ensaios de Bernoulli e } \\ & p, \text { a probabilidadede de sucesso } \\ \# \mathrm{E} & \text { Cardinalidade do conjunto E } \\ t_{n} & \text { Distribuição } t \text {-Student com } n \text { graus de liberdade } \\ U(a, b) & \text { Distribuição uniforme contínua no intervalo }(a, b)\end{array}$




\section{ESTIMAÇÃO E TESTE DE HIPÓTESE BASEADOS EM VEROSSIMILHANÇAS PERFILADAS}


"I have no objection to the study of likelihood as such."

Sir Harold Jeffreys 


\section{CAPÍTULO 1 INTRODUÇÃO}

\subsection{INTRODUÇÃO}

Um modelo estatístico é uma coleção de possíveis distribuições para os dados observados. Os problemas de inferência estatística suscitados por tal modelo freqüentemente envolvem aspectos da distribuição de probabilidade de uma variável populacional.

Suponha que aspectos de interesse primário sejam descritos por um vetor de parâmetros $\mu$ e aqueles de interesse secundário, necessários para a adequada descrição da variabilidade da população, sejam expressos por um vetor de parâmetros $\nu$. Ao longo do texto, por convenção, vetor é vetor-coluna.

Em alguns casos, a inferência sobre $\mu$ (parâmetro de interesse) pode ser baseada numa função de verossimilhança marginal ou de verossimilhança condicional. Entretanto, essas funções podem ser obtidas apenas quando o modelo tem uma estrutura particular.

Neste trabalho, considera-se uma pseudo-verossimilhança que não requer a existência de tais verossimilhanças e tem sido utilizada quando o modelo a ser estimado envolve parâmetros de perturbação, $\nu$. Aqui, a idéia é substituir este vetor paramétrico por uma estimativa consistente na verossimilhança original, comumente, a estimativa de máxima verossimilhança para valores fixados de $\mu$.

A função resultante, chamada de função de verossimilhança perfilada, depende, portanto, somente dos parâmetros de interesse. Evidentemente, essa função de verossimilhança não é uma verossimilhança genuína. De fato, algumas propriedades básicas das funções de verossimilhança não são válidas para as verossimilhanças perfiladas. Por exemplo, a função escore não tem necessariamente média zero e a informação pode apresentar vício. Tratar a função de verossimilhança perfilada como uma verossimilhança genuína pode levar a alguns problemas, como, por exemplo, inconsistência e ineficiência de estimadores. Estimadores inconsistentes podem ocorrer quando o número de parâmetros de perturbação não é fixo mas depende do tamanho da amostra. Outro problema comum refere-se à aproximação usual da distribuição da estatística da razão de verossimilhanças pela distribuição qui-quadrado. Tal estatística é proporcional à diferença entre o valor máximo do logaritmo da função de verossimilhança perfilada e o valor deste logaritmo sob a hipótese nula. Se o número de parâmetros de perturbação não for muito pequeno quando comparado com o tamanho da amostra, a aproximação por qui-quadrado pode ser bastante pobre. Desta forma, torna-se importante obter ajustes para as verossimilhanças perfiladas que reduzam esses problemas. Vários pesquisadores, incluindo Barndorff-Nielsen (1983, 1994), Cox e Reid (1987, 1992), Mc-

Cullagh e Tibshirani (1990) e Stern (1997), propuseram ajustes à função de verossimilhança 
perfilada.

A função de verossimilhança perfilada tipicamente induz "excesso de precisão", dado que trata o parâmetro de incômodo como conhecido, uma função dos dados e do parâmetro de interesse, e, assim, despreza a incerteza inerente a estimação de $\nu$. Por isso, em geral, a curvatura do logaritmo desta verossimilhança é bem acentuada em torno do ponto de máximo. Os ajustes sobre tal função reduzem este grau de curvatura, corrigindo o "excesso de precisão", e consistem na incorporação de um termo à verossimilhança perfilada anteriormente à estimação de $\mu$. Além disso, têm o efeito de diminuir os vieses da função escore e da informação. Uma avaliação numérica dos efeitos destes ajustes pode ser encontrada no trabalho de Stern (1997).

Paralelamente a esses trabalhos, DiCiccio e Stern (1994) mostraram que é possível obter correção de Bartlett para a estatística da razão de verossimilhanças proveniente de uma verossimilhança perfilada ajustada. O objetivo desta correção é a redução na distorção de tamanho do teste em amostras de tamanho típico através de uma aceleração da taxa de convergência do tamanho verdadeiro para o tamanho nominal (assintótico).

Uma estatística da razão de verossimilhanças sinalizada pode ser baseada numa verossimilhança perfilada modificada. Este enfoque é considerado por Sartori et al. (1999), usando o ajuste de Barndorff-Nielsen $(1983,1994)$. Neste caso, eles mostraram que as propriedades de tal estatística são freqüentemente superiores àquelas da estatística da razão de verossimilhanças sinalizada usual.

A verossimilhança perfilada modificada proposta por Barndorff-Nielsen $(1983,1994)$ é freqüentemente difícil de ser calculada, por isso várias propostas de aproximação desta função foram desenvolvidas e algumas destas estão descritas em Severini (2000a).

Em geral, as verossimilhanças perfiladas modificadas têm propriedades mais próximas daquelas de uma verossimilhança genuína. Fergunson et al. (1991) mostraram que a função escore baseada na proposta de Barndorff-Nielsen $(1983,1994)$ tem aproximadamente média zero. Mukerjee (1992) mostrou que o teste da razão de verossimilhanças baseado neste ajuste tem, até segunda ordem, o mesmo poder que o teste da razão de verossimilhanças do caso em que $\nu$ é conhecido. Esta propriedade também é observada em algumas das aproximações para a modificação devida a Barndorff-Nielsen $(1983,1994)$. Uma teoria geral de pseudoverossimilhança é dada em Severini (1998b).

\subsection{ORGANIZAÇÃO DA TESE}

Esta tese de doutorado está dividida em cinco capítulos e quatro apêndices. No segundo capítulo, é definida, matematicamente, a verossimilhança perfilada e são apresentadas diversas modificações desta com suas respectivas propriedades. Para exemplificar a obtenção de tais verossimilhanças, é considerada uma amostra aleatória de uma variável com densidade ou 
função de probabilidade na família exponencial biparamétrica. Assim, obtêm-se expressões gerais para as verossimilhanças derivadas desta família, que são aplicadas, posteriormente, em algumas distribuições desta classe (normal, gama, beta e gaussiana inversa).

O terceiro capítulo consiste de resultados numéricos provenientes de simulações de Monte Carlo. São apresentadas medidas amostrais de posição e de dispersão de alguns estimadores de máxima verossimilhança que foram derivados das várias verossimilhanças perfiladas obtidas nos quatro exemplos estudados ao longo do Capítulo 2 (normal, gama, beta e gaussiana inversa). Também são mostradas taxas de rejeições de hipóteses testadas sobre os parâmetros de interesse, usando as estatísticas da razão de verossimilhanças obtidas das verossimilhanças perfiladas, bem como média e variância amostrais destas. Além disso, gráficos contendo as discrepâncias entre os quantis amostrais dessas estatísticas e os quantis assintóticos. Assim, é possível comparar o desempenho do teste original e dos testes baseados em funções de verossimilhanças perfiladas ajustadas.

Os Capítulos 4 e 5 tratam de aplicações de tais funções de verossimilhanças em modelos não pertencentes à família exponencial biparamétrica.

Mais precisamente, no quarto capítulo, o interesse é pelo parâmetro de heterogeneidade da distribuição proposta por Frery et al. (1997) para modelar dados provenientes de radares do tipo SAR (Synthetic Aperture Radar). Sua estimação é realizada através da maximização de uma aproximação para a verossimilhança perfilada modificada proposta por Barndorff-Nielsen (1983), descrita em Severini (2000a), da maximização da verossimilhança perfilada proposta por Cox e Reid $(1987,1989)$ e da maximização da verossimilhança original. A estimação desse parâmetro é de grande importância para produzir uma imagem da região monitorada pelo radar e esta pode ser utilizada, por exemplo, nos diversos campos de estudo das ciências ambientais. Resultados numéricos comparando tais estimativas são apresentados. Além disso, são realizados testes unicaudais sobre este parâmetro, utilizando as raízes quadradas sinalizadas das estatísticas da razão de verossimilhanças. O objetivo é comparar os desempenhos das verossimilhanças a partir das distorções de tamanho dos testes associados e do poder destes.

O quinto capítulo consiste numa aplicação das verossimilhanças perfiladas modificadas no ajuste de modelos da área de análise de sobrevivência, mais especificamente, em modelos paramétricos utilizando a distribuição de Weibull, que é freqüentemente usada para descrever o tempo de vida de produtos industriais. O interesse é pelo parâmetro de forma desta distribuição, que é determinante na inclinação da função de taxa de falha. A estimação deste é realizada através das maximizações de aproximações para a verossimilhança perfilada proposta por Barndorff-Nielsen (1983), descritas em Severini (2000a), e da maximização da verossimilhança perfilada proposta por Cox e Reid (1987, 1989). Resultados numéricos comparando tais estimativas são apresentados. Além disso, são realizados testes bicaudais sobre este parâmetro, utilizando as estatísticas da razão de verossimilhanças perfiladas. O objetivo é comparar os desempenhos das verossimilhanças perfiladas a partir das taxas de rejeições dos testes sob as hipóteses nulas. Os resultados numéricos são obtidos a partir de 
dados completos e dados censurados (tipos I e II). Este capítulo está estruturado em duas partes: na primeira, as observações dos tempos de falhas são independentes e identicamente distribuídas; na segunda, as observações são apenas independentes, não são identicamente distribuídas. Neste caso, supõe-se um modelo de regressão, ou seja, diferentemente do trabalho de Yang e Xie (2003), aqui é estudado e simulado o caso em que se modela o parâmetro $\alpha$ através de algumas covariáveis. O objetivo dessa parte é observar os desempenhos das verossimilhanças perfiladas modificadas na presença de mais de um parâmetro de incômodo. Aqui também são realizados testes da razão de verossimilhanças sobre os coeficientes da regressão. Mais uma vez, o objetivo é comparar os desempenhos das verossimilhanças a partir das distorções de tamanho dos testes associados e do poder destes.

O Apêndice A desta tese trata dos geradores de números aleatórios e de algumas funções de maximização disponíveis na linguagem de programação 0x, sumariamente apresentada a seguir e utilizada para realizar as simulações computacionais deste trabalho. O Apêndice B apresenta o algoritmo de otimização proposto por Frery et al. (2004) e utilizado para as simulações computacionais do Capítulo 4. O Apêndice C consiste na apresentação de um programa implementado na linguagem de programação Ox que foi utilizado para algumas simulações computacionais realizadas neste trabalho. O Apêndice D apresenta alguns resultados relativos às distribuições dos estimadores de máxima verossimilhança do parâmetro de forma da distribuição de Weibull, que foram usados para contestar resultados numéricos produzidos por Yang e Xie (2003).

\subsection{PLATAFORMA COMPUTACIONAL}

As linguagens de programação 0x, $\mathrm{R}$ e (Plain) TEX constituem a plataforma computacional usada na elaboração deste trabalho.

As simulações computacionais foram realizadas usando a versão 3.30 da linguagem de programação matricial Ox em um computador Intel e sob o sistema operacional Windows XP. Esta linguagem de programação foi criada por Jurgen A. Doornik em 1994, durante o desenvolvimento de sua tese de doutorado na Universidade de Oxford (Inglaterra). Foi desenvolvida com base na linguagem de programação C, é bastante flexível e tem se mostrado muito útil em computação numérica. Suporta também programação orientada a objeto. A versão mais atual desta linguagem de programação está disponível gratuitamente para uso acadêmico no endereço http://www.nuff.ox.ac.uk/Users/Doornik. Apesar do Ox ser uma linguagem de programação de alto nível, não apresenta os problemas de eficiência inerentes a outras linguagens de alto nível (e.g., S-PLUS) e permite a implementação de novas técnicas

estatísticas com facilidade. Portanto, seu uso é aconselhável em tarefas computacionalmente intensivas, como bootstrap, métodos de amostragem de Gibbs e integração de Monte Carlo, onde são exigidas precisão numérica e eficiência computacional. Maiores detalhes sobre 
esta linguagem de programação podem ser encontrados em Doornik (2001), em CribariNeto (1997) e em Cribari-Neto e Zarkos (2003).

Para a manipulação gráfica de resultados, adotou-se a linguagem de programação R. Esta linguagem caracteriza-se pelo compromisso entre a flexibilidade oferecida por algumas linguagens compiladas (como C, C++ e FORTRAN) e a conveniência dos tradicionais pacotes estatísticos. Foi criada por Ross Ihaka e Robert Gentleman na Universidade de Auckland (Nova Zelândia) com o objetivo de produzir um ambiente de programação parecido com o S, uma linguagem desenvolvida no AT\&T Bell Laboratories, mas sem sofrer dos mesmos problemas de demanda de memória e desempenho. A versão 1.7.1 foi a utilizada neste trabalho e a versão mais atual está disponínel gratuitamente (inclusive o código fonte) no endereço http://www.r-project.org/. Maiores detalhes sobre esta linguagem de programação podem ser encontrados em Cribari-Neto e Zarkos (1999) e em Ihaka e Gentleman (1996).

Para datilografar esta tese, foi usado o programa $\mathrm{T}_{\mathrm{E}} \mathrm{X}$, desenvolvido por Donald Knuth. Por ser uma linguagem de programação, TEXpermite a definição de novos comandos (macros) por parte do usuário para obtenção do efeito desejado no texto. É um programa poderoso e flexível, por isto muitas vezes utilizado para a confecção de livros. Para maiores detalhes sobre $\mathrm{T}_{\mathrm{E} X}$, ver Knuth (1986). MiKTeX é uma implementação do $\mathrm{T}_{\mathrm{EX}}$ para o sistema operacional windows e está disponível gratuitamente no endereço http: //www .miktex .org/. 


\section{CAPÍTULO 2 \\ AJUSTES PARA VEROSSIMILHANÇAS PERFILADAS}

\subsection{INTRODUÇÃO}

Este capítulo consiste de um estudo de vários métodos de ajustes para verossimilhanças perfiladas, exemplificados na classe da família exponencial biparamétrica. Assim, $y_{1}, \ldots, y_{n}$ são variáveis aleatórias independentes e identicamente distribuídas com função densidade ou de probabilidade da forma

$$
p(y ; \mu, \nu)=\exp \left[\alpha_{1}(\mu, \nu) d_{1}(y)+\alpha_{2}(\mu, \nu) d_{2}(y)-\rho(\mu, \nu)+v(y)\right],
$$

onde $(\mu, \nu)$ representa o vetor de parâmetros desconhecidos, $\alpha_{1}(\cdot, \cdot), \alpha_{2}(\cdot, \cdot), \rho(\cdot, \cdot), d_{1}(\cdot)$, $d_{2}(\cdot)$ e $v(\cdot)$ são funções conhecidas e considera-se que o suporte de $p(y ; \mu, \nu)$ não depende de $(\mu, \nu)$. Também assume-se que $\alpha_{1}(\cdot, \cdot), \alpha_{2}(\cdot, \cdot)$ e $\rho(\cdot, \cdot)$ têm as quatro primeiras derivadas contínuas em relação aos componentes do vetor de parâmetros $(\mu, \nu)$. Aqui, a estatística suficiente minimal é $\left(\sum_{j=1}^{n} d_{1}\left(y_{j}\right), \sum_{j=1}^{n} d_{2}\left(y_{j}\right)\right)$. *

A motivação é a de se trabalhar com uma família de distribuições relativamente simples mas que compreende um amplo leque de distribuições como casos particulares. Dentro deste contexto, são derivadas modificações da verossimilhança perfilada propostas por BarndorffNielsen (1983), Cox e Reid (1987, 1993), McCullagh e Tibshirani (1990) e Stern (1997), além de aproximações para o ajuste de Barndorff-Nielsen (1983), descritas em Severini (2000a). Esta é a contribuição deste capítulo. Para isto, são apresentados e estudados os diversos ajustes sobre a verossimilhança perfilada.

\subsection{FUNÇÃO DE VEROSSIMILHANÇA PERFILADA}

Seja $\theta$ o vetor paramétrico de um modelo estatístico hipotético com função de verossimilhança $L(\theta)$ para o qual $\mu=\mu(\theta)$ é o vetor de parâmetros de interesse. A função de verossimilhança perfilada para $\mu$ é definida por

$$
L_{p}(\mu)=\sup _{\theta \mid \mu} L(\theta)
$$

onde $\theta \mid \mu$ denota todos os valores de $\theta$ tais que $\mu(\theta)=\mu$. O logaritmo da função de verossimilhança perfilada é indicado por $\ell_{p}(\mu)$. E, por fim, a função escore perfilada, $u_{p}(\mu)=$ $\partial \ell_{p}(\mu) / \partial \mu$. O ponto de máximo de $L_{p}(\mu)$ é denotado por $\widehat{\mu}_{p}$.

* Neste texto, $y$ denota tanto a variável aleatória quanto seu valor observado. 
Agora, suponha que $\theta=(\mu, \nu)$. Assim, pela definição, a verossimilhança perfilada é dada por

$$
L_{p}(\mu)=L\left(\mu, \widehat{\nu}_{\mu}\right)
$$

em que $\widehat{\nu}_{\mu}$ é a estimativa de máxima verossimilhança de $\nu$ para $\mu$ fixado. Ou seja, $\widehat{\nu}_{\mu}$ é a raiz da equação $\partial \ell(\theta) / \partial \nu=0$, onde $\ell(\theta)=\log L(\theta)$.

Essa função de verossimilhança não é uma verossimilhança genuína, pois é derivada da substituição de $\nu$ por $\widehat{\nu}_{\mu}$ na verossimilhança original. Conseqüentemente, propriedades como

$$
E\{u(\theta)\}=0 \quad \text { e } E\left\{u(\theta) u^{\top}(\theta)\right\}+E\left\{\frac{\partial u(\theta)}{\partial \theta^{\top}}\right\}=0,
$$

em que $u(\theta)=\partial \ell(\theta) / \partial \theta$ é a função escore associada a $L(\theta)$, não são satisfeitas por $u_{p}(\mu)$ geralmente. Assim, diz-se que $u_{p}(\mu)$ é viciada, pois sua esperança matemática não é nula, e que a informação apresenta viés, pois a segunda igualdade acima não é obedecida pela função escore perfilada.

Entretanto, essa função tem algumas propriedades interessantes (Pace e Salvan, 1997, Capítulo 4), de validade geral, não apenas observadas na família exponencial biparamétrica:

(i) $\widehat{\mu}_{p}=\widehat{\mu}$, onde $\widehat{\mu}$ é a estimativa de máxima verossimilhança de $\mu$ e $\widehat{\mu}_{p}$, a estimativa de máxima verossimilhança perfilada;

(ii) ao testar hipóteses sobre $\mu$, a estatística da razão de verossimilhanças baseada em $\ell_{p}(\mu)$ é igual à baseada em $\ell(\mu, \nu)$, ou seja,

$$
R V=2\left[\ell(\widehat{\mu}, \widehat{\nu})-\ell\left(\mu, \widehat{\nu}_{\mu}\right)\right]=2\left[\ell_{p}(\widehat{\mu})-\ell_{p}(\mu)\right]
$$

onde $\widehat{\nu}$ é a estimativa de máxima verossimilhança de $\nu$;

(iii)

$$
\ell_{\mu}\left(\mu, \widehat{\nu}_{\mu}\right)=\left.\frac{\partial \ell(\mu, \nu)}{\partial \mu}\right|_{(\mu, \nu)=\left(\mu, \widehat{\nu}_{\mu}\right)}=u_{p}(\mu) \quad \mathrm{e} \quad\left(j_{p}(\mu)\right)^{-1}=j^{\mu \mu}\left(\mu, \widehat{\nu}_{\mu}\right)
$$

onde $j_{p}(\mu)=-\partial^{2} \ell_{p}(\mu) / \partial \mu^{\top} \partial \mu$ é a informação perfilada observada e $j^{\mu \mu}(\mu, \nu)$ é o bloco superior-esquerdo do inverso da matriz de informação observada $j(\theta)=-\partial^{2} \ell(\theta) / \partial \theta^{\top} \partial \theta$;

(iv) as estatísticas de Wald e escore de Rao, relativamente a um teste de hipótese sobre $\mu$, são, respectivamente,

$$
W=(\widehat{\mu}-\mu)^{\top}\left(i^{\mu \mu}(\widehat{\mu}, \widehat{\nu})\right)^{-1}(\widehat{\mu}-\mu) \quad \text { e } \quad S_{R}=\ell_{\mu}^{\top}\left(\mu, \widehat{\nu}_{\mu}\right) i^{\mu \mu}\left(\mu, \widehat{\nu}_{\mu}\right) \ell_{\mu}\left(\mu, \widehat{\nu}_{\mu}\right),
$$

em que $i^{\mu \mu}(\mu, \nu)=E\left\{j^{\mu \mu}(\mu, \nu)\right\}$. Assintoticamente, estas estatísticas têm distribuição qui-quadrado com número de graus de liberdade igual à dimensão do vetor $\mu$. Em tais expressões, a informação esperada pode ser substituída pela informação observada sem modificação da distribuição assintótica. Conseqüentemente, por (iii),

$$
W=(\widehat{\mu}-\mu)^{\top} j_{p}(\widehat{\mu})(\widehat{\mu}-\mu) \quad \text { e } \quad S_{R}=u_{p}^{\top}(\mu)\left(j_{p}(\mu)\right)^{-1} u_{p}(\mu) .
$$


Na classe da família exponencial biparamétrica, $\mu$ e $\nu$ são unidimensionais. Dada a observação de uma amostra aleatória $\left(y_{1}, \ldots, y_{n}\right)$ de $n$ variáveis com função densidade ou de probabilidade $p(y ; \mu, \nu)$, dada em $(2.1)$, o logaritmo da função de verossimilhança para estes parâmetros, representado por $\ell=\ell(\mu, \nu)$, é expresso por

$$
\ell=\ell(\mu, \nu)=\sum_{j=1}^{n}\left[\alpha_{1}(\mu, \nu) d_{1}\left(y_{j}\right)+\alpha_{2}(\mu, \nu) d_{2}\left(y_{j}\right)-\rho(\mu, \nu)\right]
$$

Observe que foi desprezada a parcela $\sum_{j=1}^{n} v\left(y_{j}\right)$. Por convenção, as expressões de todas as funções de verossimilhança apresentadas neste texto contêm apenas parcelas que dependem dos parâmetros.

$\operatorname{Sejam} \alpha_{i}^{(s, t)}(\mu, \nu)=\partial^{s+t} \alpha_{i}(\mu, \nu) / \partial \mu^{s} \partial \nu^{t}(i=1,2)$ e $\rho^{(s, t)}(\mu, \nu)=\partial^{s+t} \rho(\mu, \nu) / \partial \mu^{s} \partial \nu^{t}$. Assim,

$$
\frac{\partial \ell}{\partial \mu}=\sum_{j=1}^{n} \sum_{i=1}^{2}\left[\alpha_{i}^{(1,0)}(\mu, \nu) d_{i}\left(y_{j}\right)\right]-n \rho^{(1,0)}(\mu, \nu)
$$

e

$$
\frac{\partial \ell}{\partial \nu}=\sum_{j=1}^{n} \sum_{i=1}^{2}\left[\alpha_{i}^{(0,1)}(\mu, \nu) d_{i}\left(y_{j}\right)\right]-n \rho^{(0,1)}(\mu, \nu)
$$

Do fato de que $E\{\partial \ell / \partial \mu\}=E\{\partial \ell / \partial \nu\}=0$, vem

$$
\rho^{(1,0)}(\mu, \nu)=\sum_{i=1}^{2} \alpha_{i}^{(1,0)}(\mu, \nu) \beta_{i}(\mu, \nu) \quad \text { e } \quad \rho^{(0,1)}(\mu, \nu)=\sum_{i=1}^{2} \alpha_{i}^{(0,1)}(\mu, \nu) \beta_{i}(\mu, \nu)
$$

com

$$
\beta_{i}(\mu, \nu)=E\left\{d_{i}(y)\right\} \text { para } i=1,2 .
$$

Considerando estas duas equações e resolvendo o sistema com relação a $\beta_{1}(\mu, \nu)$ e $\beta_{2}(\mu, \nu)$, a solução é dada por

$$
\beta_{1}=\frac{\rho^{(1,0)} \alpha_{2}^{(0,1)}-\rho^{(0,1)} \alpha_{2}^{(1,0)}}{\alpha_{1}^{(1,0)} \alpha_{2}^{(0,1)}-\alpha_{1}^{(0,1)} \alpha_{2}^{(1,0)}} \quad \text { e } \quad \beta_{2}=\frac{\rho^{(0,1)} \alpha_{1}^{(1,0)}-\rho^{(1,0)} \alpha_{1}^{(0,1)}}{\alpha_{1}^{(1,0)} \alpha_{2}^{(0,1)}-\alpha_{1}^{(0,1)} \alpha_{2}^{(1,0)}} .
$$

Aqui, por simplicidade, o argumento $(\mu, \nu)$ das funções é omitido e assume-se que

$$
\alpha_{1}^{(1,0)} \alpha_{2}^{(0,1)}-\alpha_{1}^{(0,1)} \alpha_{2}^{(1,0)} \neq 0
$$

Os estimadores de máxima verossimilhança satisfazem simultaneamente:

$$
\sum_{j=1}^{n} \sum_{i=1}^{2}\left[\alpha_{i}^{(1,0)}(\widehat{\mu}, \widehat{\nu}) d_{i}\left(y_{j}\right)\right]-n \rho^{(1,0)}(\widehat{\mu}, \widehat{\nu})=0
$$

e

$$
\sum_{j=1}^{n} \sum_{i=1}^{2}\left[\alpha_{i}^{(0,1)}(\widehat{\mu}, \widehat{\nu}) d_{i}\left(y_{j}\right)\right]-n \rho^{(0,1)}(\widehat{\mu}, \widehat{\nu})=0
$$


Considerando estas duas equações, resolvendo o sistema com relação a $\sum_{j=1}^{n} d_{1}\left(y_{j}\right)$ e $\sum_{j=1}^{n} d_{2}\left(y_{j}\right)$ e usando (2.2), a solução é dada por

$$
\sum_{j=1}^{n} d_{1}\left(y_{j}\right)=n \beta_{1}(\widehat{\mu}, \widehat{\nu}) \quad \text { e } \quad \sum_{j=1}^{n} d_{2}\left(y_{j}\right)=n \beta_{2}(\widehat{\mu}, \widehat{\nu}) .
$$

Utilizando a expressão obtida para $\rho^{(0,1)}(\mu, \nu)$, a derivada $\partial \ell / \partial \nu$ pode ser escrita como

$$
\frac{\partial \ell}{\partial \nu}=n \sum_{i=1}^{2} \alpha_{i}^{(0,1)}(\mu, \nu)\left[\beta_{i}(\widehat{\mu}, \widehat{\nu})-\beta_{i}(\mu, \nu)\right]
$$

Dessa forma, considerando $\mu$ fixado, o estimador de máxima verossimilhança de $\nu\left(\widehat{\nu}_{\mu}\right)$ satisfaz

$$
\sum_{i=1}^{2} \alpha_{i}^{(0,1)}\left(\mu, \widehat{\nu}_{\mu}\right)\left[\beta_{i}(\widehat{\mu}, \widehat{\nu})-\beta_{i}\left(\mu, \widehat{\nu}_{\mu}\right)\right]=0
$$

Portanto, o logaritmo da função de verossimilhança perfilada para $\mu$ é dado por

$$
\ell_{p}(\mu)=\ell\left(\mu, \widehat{\nu}_{\mu}\right)=n\left\{\sum_{i=1}^{2}\left[\alpha_{i}\left(\mu, \widehat{\nu}_{\mu}\right) \beta_{i}(\widehat{\mu}, \widehat{\nu})\right]-\rho\left(\mu, \widehat{\nu}_{\mu}\right)\right\}
$$

onde $\widehat{\nu}_{\mu}$ é a raiz da equação $(2.3)$.

Usar $\ell_{p}(\mu)$ para realizar inferências sobre um modelo estatístico qualquer assemelha-se a tratar $\nu$ como se fosse um parâmetro conhecido e igual a $\widehat{\nu}_{\mu}$. Isto pode não ser razoável caso os dados não contenham muita informação sobre $\nu$, algo que geralmente ocorre se a dimensão deste parâmetro é grande quando comparada ao tamanho da amostra. Para compensar esta carência de informação, diminuindo os vieses da função escore perfilada e da correspondente informação, várias versões modificadas da verossimilhança perfilada foram propostas e são apresentadas a seguir. Algumas dessas versões são descritas em Severini (2000a, Capítulo 9); ver também Pace e Salvan (1997, Capítulo 11). As reduções destes vieses são importantes para estabelecer resultados em grandes amostras para estimadores de máxima verossimilhança (consistência) e para testes assintóticos $\left(R V, W\right.$ e $\left.S_{R}\right)$.

\subsection{VEROSSIMILHANÇA PERFILADA MODIFICADA PROPOSTA POR BARNDORFF-NIELSEN}

O fraco desempenho de $\ell_{p}(\mu)$ em alguns casos pode ser parcialmente explicado pelo fato desta função não constituir uma tentativa de aproximação para verossimilhanças marginal e condicional genuínas. 
A verossimilhança perfilada modificada proposta por Barndorff-Nielsen (1983) pode ser derivada como uma aproximação de uma verossimilhança marginal ou de uma verossimilhança condicional para $\mu$, se uma destas funções existir. Nos dois casos, utiliza-se a fórmula $p^{*}$ (Barndorff-Nielsen, 1980, 1983), que é uma aproximação para a função densidade do estimador de máxima verossimilhança condicionalmente a uma estatística ancilar. Considerando novamente um modelo estatístico hipotético com vetor paramétrico $\theta$, estatística suficiente minimal $s$ e função de verossimilhança $L(\theta ; s)$, onde $s$ ressalta a dependência desta função das observações da variável modelada, sua expressão é

$$
p^{*}(\widehat{\theta} \mid a ; \theta)=c(\theta, a)|j(\widehat{\theta} ; \widehat{\theta}, a)|^{1 / 2} \frac{L(\theta ; \widehat{\theta}, a)}{L(\widehat{\theta} ; \widehat{\theta}, a)},
$$

onde $a$ é a estatística ancilar, ou seja, sua distribuição não depende de $\theta, \widehat{\theta}$ é a estimativa de máxima verossimilhança de $\theta,(\widehat{\theta}, a)$ é uma função um-a-um de $s$ e $j(\theta ; \widehat{\theta}, a)=$ $-\partial^{2} \log L(\theta ; \widehat{\theta}, a) / \partial \theta^{2}$ é a informação observada. Aqui, $L(\theta ; \widehat{\theta}, a)=L(\theta ; s)$, bem como, $j(\theta ; \widehat{\theta}, a)=j(\theta ; s)$.

Esta aproximação tem um erro relativo de ordem $O\left(n^{-3 / 2}\right)$ para $\widehat{\theta}=\theta+O_{p}\left(n^{-1 / 2}\right)$ (Skovgaard, 1990). Mais precisamente,

$$
p(\widehat{\theta} \mid a ; \theta)=p^{*}(\widehat{\theta} \mid a ; \theta)\left(1+O\left(n^{-3 / 2}\right)\right) .
$$

Uma propriedade importante desta expansão é que reparametrizar o modelo e calcular $p^{*}$ a partir da nova função de verossimilhança equivale a calcular $p^{*}$ inicialmente e transformá-la segundo as regras de mudanças de variáveis.

A constante de proporcionalidade $c(\theta, a)$ garante uma propriedade básica de funções densidade,

$$
\int p^{*}(\widehat{\theta} \mid a ; \theta) \widehat{d \theta}=1
$$

e é invariante sob reparametrizações. Pode-se mostrar que $c(\theta, a)=(2 \pi)^{-\operatorname{dim} \theta / 2}\left(1+O\left(n^{-1}\right)\right)$ (Pace e Salvan, 1997, Capítulo 11); assim, tal constante é muitas vezes tomada como sendo $(2 \pi)^{-\operatorname{dim} \theta / 2}$. Neste caso, obtém-se uma aproximação para $p(\widehat{\theta} \mid a ; \theta)$ com erro relativo de ordem $O\left(n^{-1}\right)$, ou seja,

$$
p(\widehat{\theta} \mid a ; \theta)=p^{\dagger}(\widehat{\theta} \mid a ; \theta)\left(1+O\left(n^{-1}\right)\right),
$$

com

$$
p^{\dagger}(\widehat{\theta} \mid a ; \theta)=(2 \pi)^{-\operatorname{dim} \theta / 2}|j(\widehat{\theta} ; \widehat{\theta}, a)|^{1 / 2} \frac{L(\theta ; \widehat{\theta}, a)}{L(\widehat{\theta} ; \widehat{\theta}, a)}
$$

Observe que, ao calcular $p^{*}$, pode-se extrair qualquer fator da verossimilhança original que dependa apenas de elementos da amostra observada. Como conseqüência, esta fórmula é invariante sob transformações um-a-um dos dados. Aqui, as observações não precisam ser independentes ou identicamente distribuídas, mas podem, por exemplo, ser realizações de uma série temporal estacionária. Vale salientar também que a maioria dos resultados aqui 
discutidos mantêm-se quando empregamos a informação esperada, $i(\theta ; \widehat{\theta}, a)=E_{\theta}\{j(\theta ; \widehat{\theta}, a)\}$, avaliada em $\widehat{\theta}$, ao invés da informação observada, $j(\widehat{\theta} ; \widehat{\theta}, a)$, em (2.4).

A exatidão da fórmula $p^{*}$ é observada, por exemplo, em famílias de locação e escala. A função densidade de $\widehat{\theta}=(\widehat{\kappa}, \widehat{\sigma})$ condicionalmente em $a=\left(a_{1}, \ldots, a_{n}\right)$, onde $a$ é a estatística de configuração com $a_{i}=\left(y_{i}-\widehat{\kappa}\right) / \widehat{\sigma}, i=1, \ldots, n$, é igual à fórmula $p^{*}$ calculada. Aqui, $y_{1}, \ldots, y_{n}$ são realizações independentes de uma variável aleatória com função densidade $p(y ; \kappa, \sigma)=$ $\sigma^{-1} p_{0}((y-\kappa) / \sigma)$, onde $-\infty<\kappa<\infty, \sigma>0$ e $p_{0}(\cdot)$ é uma função densidade conhecida. Este resultado estende-se a todos os modelos de transformação (Barndorff-Nielsen, 1980, 1983). Para tais modelos, $c(\theta, a)=c(a)$.

A derivação da verossimilhança proposta por Barndorff-Nielsen $(1983,1994)$ nos dois casos possíveis mencionados anteriormente é feita sob a seguinte suposição em relação ao modelo: a existência de uma estatística $a_{0}$ tal que, quando $\mu$ é considerado fixo, $\left(\widehat{\nu}_{\mu}, a_{0}\right)$ é suficiente minimal e $a_{0}$ é ancilar. Isto é, a distribuição de $a_{0}$ pode depender de $\mu$, mas não de $\nu$.

Para derivar tal função como uma aproximação de uma verossimilhança marginal, suponha que $(\widehat{\mu}, \widehat{\nu}, a)$ seja uma transformação um-a-um da estatística suficiente minimal do modelo. Aqui, $\widehat{\mu}$ e $\widehat{\nu}$ são as estimativas de máxima verossimilhança dos respectivos parâmetros e $a$, uma estatística ancilar. Assuma também que, fixado $\mu, \widehat{\mu}$ seja ancilar, portanto, sua distribuição não depende de $\nu$. Sabe-se que

$$
p(\widehat{\mu}, \widehat{\nu} \mid a ; \mu, \nu)=\frac{p(\widehat{\mu}, \widehat{\nu}, a ; \mu, \nu)}{p(a)} \quad \text { e } \quad p(\widehat{\nu} \mid \widehat{\mu}, a ; \mu, \nu)=\frac{p(\widehat{\mu}, \widehat{\nu}, a ; \mu, \nu)}{p(\widehat{\mu}, a ; \mu)} .
$$

Logo, a distribuição condicional de $\widehat{\mu}$ dado $a$ pode ser escrita como

$$
\frac{p(\widehat{\mu}, \widehat{\nu} \mid a ; \mu, \nu)}{p(\widehat{\nu} \mid \widehat{\mu}, a ; \mu, \nu)}=\frac{p(\widehat{\mu}, a ; \mu)}{p(a)}=p(\widehat{\mu} \mid a ; \mu) .
$$

Uma aproximação tipo- $p^{*}$ para $p(\widehat{\mu} \mid a ; \mu)$ é obtida usando a fórmula $p^{*}$ para aproximar as funções densidade $p(\widehat{\mu}, \widehat{\nu} \mid a ; \mu, \nu)$ e $p(\widehat{\nu} \mid \widehat{\mu}, a ; \mu, \nu)$. Dessa forma,

$$
p^{*}(\widehat{\mu}, \widehat{\nu} \mid a ; \mu, \nu)=c_{1}(\mu, \nu, a)|j(\widehat{\mu}, \widehat{\nu} ; \widehat{\mu}, \widehat{\nu}, a)|^{1 / 2} \frac{L(\mu, \nu ; \widehat{\mu}, \widehat{\nu}, a)}{L(\widehat{\mu}, \widehat{\nu} ; \widehat{\mu}, \widehat{\nu}, a)}
$$

e, considerando o modelo com $\mu$ fixado $\left(a_{0}=(\widehat{\mu}, a)\right)$,

$$
p^{*}\left(\widehat{\nu}_{\mu} \mid \widehat{\mu}, a ; \mu, \nu\right)=c_{2}(\mu, \nu, a)\left|j_{\nu \nu}\left(\mu, \widehat{\nu}_{\mu} ; \widehat{\mu}, \widehat{\nu}_{\mu}, a\right)\right|^{1 / 2} \frac{L\left(\mu, \nu ; \widehat{\mu}, \widehat{\nu}_{\mu}, a\right)}{L\left(\mu, \widehat{\nu}_{\mu} ; \widehat{\mu}^{\prime}, \widehat{\nu}_{\mu}, a\right)},
$$

onde $j_{\nu \nu}\left(\mu, \nu ; \widehat{\mu}, \widehat{\nu}_{\mu}, a\right)=-\partial^{2} \log L\left(\mu, \nu ; \widehat{\mu}, \widehat{\nu}_{\mu}, a\right) / \partial \nu^{2}$. Como $\widehat{\nu}_{\mu}$ é uma função de $\widehat{\nu}$, fazendo uma mudança de variável na expressão anterior, obtém-se

$$
p^{*}(\widehat{\nu} \mid \widehat{\mu}, a ; \mu, \nu)=c_{2}(\mu, \nu, a)\left|j_{\nu \nu}\left(\mu, \widehat{\nu}_{\mu} ; \widehat{\mu}, \widehat{\nu}, a\right)\right|^{1 / 2} \frac{L(\mu, \nu ; \widehat{\mu}, \widehat{\nu}, a)}{L\left(\mu, \widehat{\nu}_{\mu} ; \widehat{\mu}, \widehat{\nu}, a\right)}\left|\frac{\partial \widehat{\nu}_{\mu}}{\partial \widehat{\nu}}\right| .
$$


Agora, substituindo $p(\widehat{\mu}, \widehat{\nu} \mid a ; \mu, \nu)$ e $p(\widehat{\nu} \mid \widehat{\mu}, a ; \mu, \nu)$ em (2.5) pelas expansões construídas usando (2.6) e (2.7), respectivamente, e omitindo termos que dependem apenas das observações, obtém-se uma verossimilhança marginal aproximada para $\mu$ baseada em $\widehat{\mu}$. Tal aproximação é a verossimilhança perfilada modificada para $\mu$ proposta por Barndorff-Nielsen:

$$
L_{B N}(\mu)=\left|\frac{\partial \widehat{\nu}_{\mu}}{\partial \widehat{\nu}}\right|^{-1}\left|j_{\nu \nu}\left(\mu, \widehat{\nu}_{\mu} ; \widehat{\mu}, \widehat{\nu}, a\right)\right|^{-1 / 2} L_{p}(\mu)
$$

Esta função apresenta um erro relativo de ordem $O\left(n^{-3 / 2}\right)$ ao ser comparada com a verossimilhança marginal exata obtida de $p(\widehat{\mu} \mid a ; \mu)$, caso esta exista, e, tomando logaritmo, obtém-se

$$
\log L_{B N}(\mu)=\ell_{B N}(\mu)=\ell_{p}(\mu)-\log \left|\frac{\partial \widehat{\nu}_{\mu}}{\partial \widehat{\nu}}\right|-\frac{1}{2} \log \left|j_{\nu \nu}(\mu, \widehat{\nu} \mu \widehat{\mu}, \widehat{\nu}, a)\right| .
$$

A correspondente função escore é denotada por $u_{B N}(\mu)$ e é igual a $\partial \ell_{B N}(\mu) / \partial \mu$. Os vieses desta função e da informação são de ordem $O\left(n^{-1}\right)$, ou seja,

$$
E\left\{u_{B N}(\mu)\right\}=O\left(n^{-1}\right) \quad \text { e } \quad E\left\{u_{B N}(\mu) u_{B N}^{\top}(\mu)\right\}+E\left\{\frac{\partial u_{B N}(\mu)}{\partial \mu^{\top}}\right\}=O\left(n^{-1}\right)
$$

ver Ferguson et al. (1991) e DiCiccio et al. (1996). O ponto de máximo de $\ell_{B N}(\mu)$ é indicado por $\widehat{\mu}_{B N}$.

$L_{B N}(\mu)$, como $L_{p}(\mu)$, é invariante sob reparametrizações da forma $(\mu, \nu) \rightarrow(\xi, \lambda)$, onde $\xi=\xi(\mu)$ e $\lambda=\lambda(\mu, \nu)$. Entretanto, em geral, para seu cálculo, é requerida a especificação de uma estatística ancilar, $a$, a fim de que $(\widehat{\mu}, \widehat{\nu}, a)$ seja uma estatística suficiente minimal do modelo.

Essa verossimilhança pode ser derivada também como uma aproximação para uma verossimilhança condicional. Neste caso, supõe-se que, fixado $\mu,\left(\widehat{\nu}_{\mu}, a\right)$ seja a estatística suficiente minimal do modelo. Assim, dado que

$$
p(\widehat{\mu} \mid \widehat{\nu}, a ; \mu, \nu)=\frac{p(\widehat{\mu}, \widehat{\nu}, a)}{p(\widehat{\nu}, a)}=\frac{p(\widehat{\mu}, \widehat{\nu} \mid a ; \mu, \nu)}{p(\widehat{\nu} \mid a ; \mu, \nu)},
$$

é possível, por passos análogos ao procedimento anterior aplicados às funções densidade $p(\widehat{\mu}, \widehat{\nu} \mid a ; \mu, \nu)$ e $p\left(\widehat{\nu}_{\mu} \mid a ; \mu, \nu\right)$, obter $L_{B N}(\mu)$.

A expressão da função de verossimilhança perfilada modificada proposta por BarndorffNielsen pode ser apresentada de outra forma, conforme a demonstração a seguir.

A quantidade $\widehat{\nu}_{\mu}$ satisfaz

$$
\left.\frac{\partial \ell(\mu, \nu ; \widehat{\mu}, \widehat{\nu}, a)}{\partial \nu}\right|_{(\mu, \nu)=\left(\mu, \widehat{\nu}_{\mu}\right)}=0
$$

Derivando esta expressão com relação a $\widehat{\nu}$, produz-se a igualdade

$$
\left(\left.\frac{\partial^{2} \ell(\mu, \nu ; \widehat{\mu}, \widehat{\nu}, a)}{\partial \nu^{2}}\right|_{(\mu, \nu)=\left(\mu, \widehat{\nu}_{\mu}\right)}\right) \frac{\partial \widehat{\nu}_{\mu}}{\partial \widehat{\nu}}+\frac{\partial}{\partial \widehat{\nu}}\left(\left.\frac{\partial \ell(\mu, \nu ; \widehat{\mu}, \widehat{\nu}, a)}{\partial \nu}\right|_{(\mu, \nu)=\left(\mu, \widehat{\nu}_{\mu}\right)}\right)=0 .
$$


A parcela

$$
\frac{\partial}{\partial \widehat{\nu}}\left(\frac{\partial \ell(\mu, \nu ; \widehat{\mu}, \widehat{\nu}, a)}{\partial \nu}\right)
$$

é uma derivada com respeito ao espaço amostral, comumente denotada por $\ell_{\nu ; \widehat{\nu}}(\mu, \nu ; \widehat{\mu}, \widehat{\nu}, a)$. Logo,

$$
\frac{\partial \widehat{\nu}_{\mu}}{\partial \widehat{\nu}}=j_{\nu \nu}\left(\mu, \widehat{\nu}_{\mu} ; \widehat{\mu}, \widehat{\nu}, a\right)^{-1} \ell_{\nu ; \widehat{\nu}}\left(\mu, \widehat{\nu}_{\mu} ; \widehat{\mu}, \widehat{\nu}, a\right) .
$$

Portanto, $L_{B N}(\mu)$ pode ser escrita como

$$
L_{B N}(\mu)=\left|\ell_{\nu ; \widehat{\nu}}\left(\mu, \widehat{\nu}_{\mu} ; \widehat{\mu}, \widehat{\nu}, a\right)\right|^{-1}\left|j_{\nu \nu}(\mu, \widehat{\nu} \mu ; \widehat{\mu}, \widehat{\nu}, a)\right|^{1 / 2} L_{p}(\mu) .
$$

Mais adiante, algumas aproximações para esta função serão derivadas a partir de aproximações para $\ell_{\nu ; \widehat{\nu}}(\mu, \nu ; \widehat{\mu}, \widehat{\nu}, a)$. Para o cálculo desta quantidade, é indispensável a especificação da estatística ancilar $a$.

Nos casos em que os estimadores de máxima verossimilhança dos parâmetros são estatísticas suficientes minimais, não se faz necessária a estatística ancilar $a$. Isto ocorre com a família exponencial biparamétrica, aqui considerada mais uma vez.

Da equação (2.3), vem

$$
\alpha_{1}^{(0,1)}\left(\mu, \widehat{\nu}_{\mu}\right)\left[\beta_{1}(\widehat{\mu}, \widehat{\nu})-\beta_{1}\left(\mu, \widehat{\nu}_{\mu}\right)\right]+\alpha_{2}^{(0,1)}\left(\mu, \widehat{\nu}_{\mu}\right)\left[\beta_{2}(\widehat{\mu}, \widehat{\nu})-\beta_{2}\left(\mu, \widehat{\nu}_{\mu}\right)\right]=0 .
$$

Derivando ambos os lados desta igualdade com relação a $\widehat{\nu}$, após manipulações algébricas, obtém-se

$$
\frac{\partial \widehat{\nu}_{\mu}}{\partial \widehat{\nu}}=\frac{\sum_{i=1}^{2} \alpha_{i}^{(0,1)}\left(\mu, \widehat{\nu}_{\mu}\right) \beta_{i}^{(0,1)}(\widehat{\mu}, \widehat{\nu})}{\sum_{i=1}^{2} \alpha_{i}^{(0,1)}\left(\mu, \widehat{\nu}_{\mu}\right) \beta_{i}^{(0,1)}\left(\widehat{\mu}, \widehat{\nu}_{\mu}\right)-\alpha_{i}^{(0,2)}\left(\mu, \widehat{\nu}_{\mu}\right)\left[\beta_{i}(\widehat{\mu}, \widehat{\nu})-\beta_{i}\left(\mu, \widehat{\nu}_{\mu}\right)\right]},
$$

onde $\beta_{i}^{(s, t)}(\mu, \nu)=\partial^{s+t} \beta_{i}(\mu, \nu) / \partial \mu^{s} \partial \nu^{t}$.

A quantidade $j_{\nu \nu}(\mu, \nu ; \widehat{\mu}, \widehat{\nu})$ avaliada em $\left(\mu, \widehat{\nu}_{\mu}\right)$ tem expressão

$$
j_{\nu \nu}\left(\mu, \widehat{\nu}_{\mu} ; \widehat{\mu}, \widehat{\nu}\right)=n \sum_{i=1}^{2} \alpha_{i}^{(0,1)}\left(\mu, \widehat{\nu}_{\mu}\right) \beta_{i}^{(0,1)}\left(\widehat{\mu}, \widehat{\nu}_{\mu}\right)-\alpha_{i}^{(0,2)}\left(\mu, \widehat{\nu}_{\mu}\right)\left[\beta_{i}(\widehat{\mu}, \widehat{\nu})-\beta_{i}\left(\mu, \widehat{\nu}_{\mu}\right)\right] .
$$

Conseqüentemente, desprezando termos que não dependem do parâmetro de interesse, o logaritmo da função de verossimilhança perfilada modificada para $\mu$ proposta por BarndorffNielsen é dado por

$$
\begin{gathered}
\ell_{B N}(\mu)=\ell_{p}(\mu)-\log \left\{\sum_{i=1}^{2} \alpha_{i}^{(0,1)}\left(\mu, \widehat{\nu}_{\mu}\right) \beta_{i}^{(0,1)}(\widehat{\mu}, \widehat{\nu})\right\}+ \\
+\frac{1}{2} \log \left\{\sum_{i=1}^{2} \alpha_{i}^{(0,1)}\left(\mu, \widehat{\nu}_{\mu}\right) \beta_{i}^{(0,1)}\left(\mu, \widehat{\nu}_{\mu}\right)-\alpha_{i}^{(0,2)}\left(\mu, \widehat{\nu}_{\mu}\right)\left[\beta_{i}(\widehat{\mu}, \widehat{\nu})-\beta_{i}\left(\mu, \widehat{\nu}_{\mu}\right)\right]\right\} .
\end{gathered}
$$




\subsection{EXEMPLOS}

Aqui, são apresentados resultados relativos aos parâmetros de algumas distribuições pertencentes à família exponencial biparamétrica. Mais especificamente, são exemplificadas as obtenções de $\ell_{p}(\mu)$ e de $\ell_{B N}(\mu)$. Nas expressões destas funções, são consideradas apenas parcelas que dependem dos parâmetros.

EXEMPLO 2.1: distribuição normal com média $\tau$ e variância $\phi(-\infty<\tau<+\infty, \phi>0)$.

Os parâmetros $\tau$ e $\phi$, que indexam a distribuição deste exemplo, são ortogonais. Isto significa que os elementos da diagonal secundária da matriz de informação de Fisher são nulos e, portanto, os estimadores de máxima verossimilhança são assintoticamente independentes.

A função densidade de uma variável aleatória y com distribuição $N(\tau, \phi)$ é dada por

$$
p(y ; \tau, \phi)=\frac{1}{\sqrt{2 \pi \phi}} \exp \left\{-\frac{1}{2}\left(\frac{y-\tau}{\sqrt{\phi}}\right)^{2}\right\}, \quad-\infty<y<+\infty .
$$

Conseqüentemente, as quantidades $\alpha_{1}(\cdot, \cdot), \alpha_{2}(\cdot, \cdot), \rho(\cdot, \cdot), d_{1}(\cdot), d_{2}(\cdot)$ e $v(\cdot)$ que definem $p(y ; \tau, \phi)$ segundo $(2.1)$ são

$$
\begin{array}{ccc}
\alpha_{1}(\tau, \phi)=\frac{\tau}{\phi}, & \alpha_{2}(\tau, \phi)=-\frac{1}{2 \phi}, \quad \rho(\tau, \phi)=\frac{\tau^{2}}{2 \phi}+\frac{\log \phi}{2}, \\
d_{1}(y)=y, & d_{2}(y)=y^{2} \quad \text { e } & v(y)=-\frac{1}{2} \log 2 \pi .
\end{array}
$$

As funções $\beta_{1}(\tau, \phi)=E\left\{d_{1}(y)\right\}$ e $\beta_{2}(\tau, \phi)=E\left\{d_{2}(y)\right\}$ são dadas por

$$
\beta_{1}(\tau, \phi)=\tau \quad \text { e } \quad \beta_{2}(\tau, \phi)=\phi+\tau^{2}
$$

Por (2.2), estes mesmos resultados podem ser obtidos.

Seja $\left(y_{1}, \ldots, y_{n}\right)$ uma realização de uma amostra aleatória de uma variável com distribuição $N(\tau, \phi)$. O logaritmo da função de verossimilhança para $(\tau, \phi)$ é

$$
\ell(\tau, \phi)=\frac{\tau}{\phi} \sum_{j=1}^{n} y_{j}-\frac{1}{2 \phi} \sum_{j=1}^{n} y_{j}^{2}-n\left(\frac{\tau^{2}}{2 \phi}+\frac{\log \phi}{2}\right) .
$$

As estimativas de máxima verossimilhança dos parâmetros são

$$
\widehat{\tau}=\bar{y} \quad \text { e } \quad \widehat{\phi}=\frac{1}{n} \sum_{j=1}^{n}\left(y_{j}-\bar{y}\right)^{2}
$$


A fim de exemplificar as obtenções dos logaritmos das verossimilhanças perfilada e perfilada modificada até aqui estudadas, são considerados dois casos. No primeiro, $\tau$ é o parâmetro de interesse e $\phi$, o de incômodo. No segundo, inverte-se esta situação.

Assim, inicialmente, $\mu=\tau$ e $\nu=\phi$. Fixando $\tau$ e resolvendo a equação $\partial l(\tau, \phi) / \partial \phi=0$ com relação a $\phi$ ou usando a equação (2.3), a estimativa de máxima verossimilhança para $\phi$ é

$$
\widehat{\phi}_{\tau}=\frac{1}{n} \sum_{j=1}^{n}\left(y_{j}-\tau\right)^{2} .
$$

Usando a definição do logaritmo da verossimilhança perfilada, obtém-se

$$
\ell_{p}(\tau)=\ell\left(\tau, \widehat{\phi}_{\tau}\right)=-\frac{n}{2} \log \sum_{j=1}^{n}\left(y_{j}-\tau\right)^{2} .
$$

A função escore perfilada é dada por

$$
u_{p}(\tau)=\frac{\partial \ell_{p}(\tau)}{\partial \tau}=n \frac{\sum_{j=1}^{n}\left(y_{j}-\tau\right)}{\sum_{j=1}^{n}\left(y_{j}-\tau\right)^{2}}
$$

e, conseqüentemente, a estimativa de máxima verossimilhança perfilada para $\tau$, $\widehat{\tau}_{p}$, que satisfaz $u_{p}\left(\widehat{\tau}_{p}\right)=0$, é a média amostral.

Agora, calculando os termos que compõem a expressão geral do logaritmo da verossimilhança perfilada modificada, obtêm-se

$$
\begin{array}{cl}
\alpha_{1}^{(0,1)}(\tau, \phi)=-\frac{\tau}{\phi^{2}}, \quad \beta_{1}^{(0,1)}(\tau, \phi)=0, & \alpha_{2}^{(0,1)}(\tau, \phi)=\frac{1}{2 \phi^{2}}, \quad \beta_{2}^{(0,1)}(\tau, \phi)=1, \\
\alpha_{1}^{(0,2)}(\tau, \phi)=\frac{2 \tau}{\phi^{3}} \quad \text { e } \quad \alpha_{2}^{(0,2)}(\tau, \phi)=-\frac{1}{\phi^{3}} .
\end{array}
$$

As funções $\beta_{1}(\tau, \phi)$ e $\beta_{2}(\tau, \phi)$ foram determinadas anteriormente.

Avaliando essas funções em $\left(\tau, \widehat{\phi}_{\tau}\right)$ e em $(\widehat{\tau}, \widehat{\phi})$ segundo a expressão de $\ell_{B N}(\mu)$, vem

$$
\ell_{B N}(\tau)=\left(1-\frac{n}{2}\right) \log \sum_{j=1}^{n}\left(y_{j}-\tau\right)^{2},
$$

cuja primeira derivada com relação a $\tau$ é

$$
u_{B N}(\tau)=\frac{\partial \ell_{B N}(\tau)}{\partial \tau}=(n-2) \frac{\sum_{j=1}^{n}\left(y_{j}-\tau\right)}{\sum_{j=1}^{n}\left(y_{j}-\tau\right)^{2}} .
$$

Dessa forma, a estimativa de máxima verossimilhança perfilada modificada é, também, a média amostral, ou seja, $\widehat{\tau}_{B N}=\bar{y}$.

A esperança da expansão de Taylor em torno da média amostral da razão

$$
\sum_{j=1}^{n}\left(y_{j}-\tau\right) / \sum_{j=1}^{n}\left(y_{j}-\tau\right)^{2}
$$


considerada como uma função de $\tau$, é nula. Portanto,

$$
E\left\{u_{p}(\tau)\right\}=E\left\{u_{B N}(\tau)\right\}=0,
$$

satisfazendo uma propriedade das funções de verossimilhança genuínas.

Note que

$$
\ell_{B N}(\tau)=\frac{n-2}{n} \ell_{p}(\tau)=\frac{\ell_{p}(\tau)}{n /(n-2)} .
$$

Esta transformação em escala sobre a verossimilhança perfilada, que define sua versão modificada proposta por Barndorff-Nielsen, corresponde a mesma transformação em $u_{p}(\tau)$ que torna o viés da informação associado a $u_{B N}(\tau)$ nulo, conforme a modificação proposta por McCullagh e Tibshirani (1990) para a função escore perfilada (ver seções 2.7 e 2.8), que, aqui, apresenta tal viés de ordem $O(1)$.

Denotando as estatísticas da razão de verossimilhanças derivadas de $\ell_{B N}(\tau)$ e de $\ell_{p}(\tau)$ por $R V_{B N}$ e por $R V_{p}$, respectivamente, tem-se

$$
R V_{B N}=2 \frac{n-2}{n}\left[\ell_{p}\left(\widehat{\tau}_{B N}\right)-\ell_{p}(\tau)\right]=\frac{2(n-2)}{n}\left[\ell_{p}\left(\widehat{\tau}_{B N}\right)-\ell_{p}\left(\widehat{\tau}_{p}\right)\right]+\frac{n-2}{n} R V_{p}
$$

e, portanto,

$$
R V_{B N}<R V_{p}
$$

com probabilidade um. Conseqüentemente, neste caso, usando $R V_{B N}$ como estatística de teste, pode-se observar menor rejeição da hipótese nula do que fazendo uso de $R V_{p}$.

O segundo caso considera $\phi$, a variância, como parâmetro de interesse e $\tau$ como parâmetro de incômodo. Dessa forma, fixando $\phi$ e resolvendo a equação $\partial l(\tau, \phi) / \partial \tau=0$, obtém-se

$$
\widehat{\tau}_{\phi}=\bar{y}
$$

Pela definição, o logaritmo da função de verossimilhança perfilada para $\phi$ é

$$
\ell_{p}(\phi)=\ell\left(\widehat{\tau}_{\phi}, \phi\right)=-\frac{n}{2} \log \phi-\frac{1}{2 \phi} \sum_{j=1}^{n}\left(y_{j}-\bar{y}\right)^{2}
$$

A primeira derivada desta função, denominada função escore perfilada, é

$$
u_{p}(\phi)=\frac{1}{2 \phi^{2}} \sum_{j=1}^{n}\left(y_{j}-\bar{y}\right)^{2}-\frac{n}{2 \phi} .
$$

Como se trata da verossimilhança perfilada, a raiz da equação $u_{p}(\phi)=0$ é a estimativa de máxima verossimilhança de $\phi$. Ou seja, $\widehat{\phi}_{p}=\widehat{\phi}$. Observa-se que $E\left\{u_{p}(\phi)\right\}=-1 /(2 \phi) \neq 0$.

Aqui, $(\mu, \nu)=(\phi, \tau)$. Permutando os argumentos das funções $\alpha_{1}(\cdot, \cdot), \alpha_{2}(\cdot, \cdot), \beta_{1}(\cdot, \cdot)$ e $\beta_{2}(\cdot, \cdot)$ a fim de utilizar a expressão geral do logaritmo da verossimilhança perfilada modificada proposta por Barndorff-Nielsen, os termos desta expressão são dados por

$$
\alpha_{1}^{(0,1)}(\phi, \tau)=\frac{1}{\phi}, \quad \beta_{1}^{(0,1)}(\phi, \tau)=1, \quad \alpha_{2}^{(0,1)}(\phi, \tau)=0, \quad \beta_{2}^{(0,1)}(\phi, \tau)=2 \tau
$$




$$
\alpha_{1}^{(0,2)}(\phi, \tau)=0 \quad \text { e } \quad \alpha_{2}^{(0,2)}(\phi, \tau)=0 .
$$

As funções $\beta_{1}(\phi, \tau)$ e $\beta_{2}(\phi, \tau)$ foram determinadas anteriormente.

Avaliando essas funções em $\left(\phi, \widehat{\tau}_{\phi}\right)$ e em $(\widehat{\phi}, \widehat{\tau})$ segundo $\ell_{B N}(\mu)$, vem

$$
\ell_{B N}(\phi)=-\frac{n-1}{2} \log \phi-\frac{1}{2 \phi} \sum_{j=1}^{n}\left(y_{j}-\bar{y}\right)^{2} .
$$

Esta função é igual ao logaritmo da verossimilhança marginal para $s^{2}$ e, também, ao logaritmo da verossimilhança condicional dado $\bar{y}$, em que $s^{2}=\sum_{j=1}^{n}\left(y_{j}-\bar{y}\right)^{2}$. Note que $\widehat{\tau}_{\phi}=$ $\widehat{\tau}=\bar{y}, \log \mathrm{o}, \partial \widehat{\tau}_{\phi} / \partial \widehat{\tau}=1$.

Derivando essa função com relação a $\phi$, encontra-se a função escore perfilada modificada, a saber

$$
u_{B N}(\phi)=\frac{1}{2 \phi^{2}} \sum_{j=1}^{n}\left(y_{j}-\bar{y}\right)^{2}-\frac{n-1}{2 \phi} .
$$

Diferentemente de $u_{p}(\phi)$, a esperança de $u_{B N}(\phi)$ é nula. Aqui,

$$
u_{B N}(\phi)=u_{p}(\phi)+\frac{1}{2 \phi} \text {. }
$$

Esta transformação sobre a função escore perfilada torna o viés da informação associado a $u_{B N}(\phi)$ nulo, conforme a modificação proposta por McCullagh e Tibshirani (1990) para $u_{p}(\phi)$ (ver seções 2.7 e 2.8).

Além disso, observa-se também que

$$
\widehat{\phi}_{B N}=\frac{1}{n-1} \sum_{j=1}^{n}\left(y_{j}-\bar{y}\right)^{2},
$$

portanto, um estimador não viciado de $\phi$. Vale ressaltar que o estimador de máxima verossimilhança deste parâmetro não tem esta propriedade.

EXEMPLO 2.2: distribuição gama com parâmetros $\omega$ e $\vartheta(\omega>0, \vartheta>0)$.

A função densidade de uma variável aleatória $y$ com distribuição $G(\omega, \vartheta)$ é

$$
p(y ; \omega, \vartheta)=\frac{\vartheta^{\omega} y^{\omega-1} \exp (-\vartheta y)}{\Gamma(\omega)}, \quad y>0 .
$$

De acordo com a parametrização desta distribuição, $\omega$ é o parâmetro de configuração ou de forma e $1 / \vartheta$ é o parâmetro de escala.

Diferentemente do exemplo anterior, aqui são apresentados apenas as expressões dos logaritmos das verossimilhanças perfilada e perfilada modificada proposta por BarndorffNielsen e quantidades derivadas destas. São considerados dois casos: no primeiro, o interesse 
é pelo parâmetro $\omega$; no segundo, pelo parâmetro $\vartheta$. Nestas situações, os parâmetros de incômodo são, respectivamente, $\vartheta$ e $\omega$.

$$
\mu=\omega \quad \nu=\vartheta
$$

- $\ell_{p}(\omega)$ e $u_{p}(\omega)$

$$
\begin{gathered}
\ell_{p}(\omega)=n \omega \log \left(\frac{\omega}{\bar{y}}\right)+\omega \sum_{j=1}^{n} \log y_{j}-n \omega-n \log \Gamma(\omega) . \\
u_{p}(\omega)=n \log \left(\frac{\omega}{\bar{y}}\right)-n \psi(\omega)+\sum_{j=1}^{n} \log y_{j} \quad \text { e } \quad \widehat{\omega}_{p} \text { não tem forma fechada. }
\end{gathered}
$$

- $\ell_{B N}(\omega)$ e $u_{B N}(\omega)$

$$
\begin{gathered}
\ell_{B N}(\omega)=-\frac{1}{2} \log \omega+\ell_{p}(\omega) \quad \text { e } \quad u_{B N}(\omega)=-\frac{1}{2 \omega}+u_{p}(\omega) . \\
\widehat{\omega}_{B N} \text { não tem forma fechada. }
\end{gathered}
$$

- viés da função escore

$$
\begin{gathered}
E\left\{u_{p}(\omega)\right\}=n[\log (n \omega)-\psi(n \omega)] \quad \text { e } \quad E\left\{u_{B N}(\omega)\right\}=n\left[\left(\log (n \omega)-\frac{1}{2 n \omega}\right)-\psi(n \omega)\right] . \\
\psi(z)=\log (z)-\frac{1}{2 z}-\frac{1}{12 z^{2}}\left(1+O\left(\frac{1}{z^{2}}\right)\right), \quad|\operatorname{Arg}(z)|<\pi,|z| \rightarrow \infty .
\end{gathered}
$$

(Abramowitz e Stegun, 1965)

Fazendo $z=n \omega$,

$$
\begin{gathered}
E\left\{u_{p}(\omega)\right\}=O(1) \quad \text { e } \quad E\left\{u_{B N}(\omega)\right\}=O\left(n^{-1}\right) . \\
\mu=\vartheta \quad \nu=\omega
\end{gathered}
$$

Resolvendo a equação $\partial \ell(\omega, \vartheta) / \partial \omega=0$, obtém-se

$$
n \log \vartheta+\sum_{j=1}^{n} \log y_{j}=n \psi\left(\widehat{\omega}_{\vartheta}\right) .
$$

- $\ell_{p}(\vartheta)$ e $u_{p}(\vartheta)$

$$
\ell_{p}(\vartheta)=n \widehat{\omega}_{\vartheta} \psi\left(\widehat{\omega}_{\vartheta}\right)-\vartheta \sum_{j=1}^{n} y_{j}-n \log \Gamma\left(\widehat{\omega}_{\vartheta}\right) \quad \text { e } \quad u_{p}(\vartheta)=n \frac{\widehat{\omega}_{\vartheta}}{\vartheta}-\sum_{j=1}^{n} y_{j}
$$




$$
\widehat{\vartheta}_{p} \text { não tem forma fechada. }
$$

- $\ell_{B N}(\vartheta)$ e $u_{B N}(\vartheta)$

$$
\begin{gathered}
\ell_{B N}(\vartheta)=\ell_{p}(\vartheta)+\frac{1}{2} \log \left(\psi^{\prime}\left(\widehat{\omega}_{\vartheta}\right)\right) \quad \text { e } \quad u_{B N}(\vartheta)=u_{p}(\vartheta)+\frac{\psi^{\prime \prime}\left(\widehat{\omega}_{\vartheta}\right)}{2 \vartheta \psi^{\prime 2}\left(\widehat{\omega}_{\vartheta}\right)} .{ }^{*} \\
\widehat{\vartheta}_{B N} \text { não tem forma fechada. }
\end{gathered}
$$

- viés da função escore

Não foi possível determinar as esperanças $E\left\{u_{p}(\vartheta)\right\}$ e $E\left\{u_{B N}(\vartheta)\right\}$.

EXEMPLO 2.3: distribuição beta com parâmetros $a$ e $b(a>0, b>0)$.

A função densidade de uma variável aleatória $y$ com distribuição $B(a, b)$ é

$$
p(y ; a, b)=\frac{\Gamma(a+b)}{\Gamma(a) \Gamma(b)} y^{a-1}(1-y)^{b-1}, \quad 0<y<1 .
$$

Para esta variável aleatória,

$$
E\{y\}=\frac{a}{a+b}, \quad \operatorname{Var}\{y\}=\frac{a b}{(a+b)^{2}(a+b+1)}
$$

e o coeficiente de assimetria é igual a

$$
\frac{2(b-a)(a+b+1)^{1 / 2}}{(a+b+2)(a b)^{1 / 2}}
$$

Portanto, quanto maior o valor absoluto da diferença entre $b$ e $a$, maior a assimetria da distribuição.

Considerando apenas o caso $\mu=a$ e $\nu=b$, tem-se

$$
\frac{\partial \ell\left(a, \widehat{b}_{a}\right)}{\partial b}=\sum_{j=1}^{n} \log \left(1-y_{j}\right)+n\left[\psi\left(a+\widehat{b}_{a}\right)-\psi\left(\widehat{b}_{a}\right)\right]=0
$$

- $\ell_{p}(a)$ e $u_{p}(a)$

$$
\ell_{p}(a)=\ell\left(a, \widehat{b}_{a}\right)=a \sum_{j=1}^{n} \log y_{j}+\widehat{b}_{a} \sum_{j=1}^{n} \log \left(1-y_{j}\right)-n \log \left[\frac{\Gamma(a) \Gamma\left(\widehat{b}_{a}\right)}{\Gamma\left(a+\widehat{b}_{a}\right)}\right] .
$$

$\star \psi^{\prime}(x)=d \psi(x) / d x \quad$ e $\quad \psi^{\prime \prime}(x)=d \psi^{\prime}(x) / d x$. 


$$
\begin{gathered}
u_{p}(a)=\sum_{j=1}^{n}\left[\log y_{j}-\psi(a)\right]+\frac{\psi^{\prime}\left(a+\widehat{b}_{a}\right)}{\psi^{\prime}\left(\widehat{b}_{a}\right)-\psi^{\prime}\left(a+\widehat{b}_{a}\right)} \sum_{j=1}^{n}\left[\log \left(1-y_{j}\right)-\psi\left(\widehat{b}_{a}\right)\right] \\
-n \psi\left(a+\widehat{b}_{a}\right) \frac{\psi^{\prime}\left(\widehat{b}_{a}\right)}{\psi^{\prime}\left(\widehat{b}_{a}\right)-\psi^{\prime}\left(a+\widehat{b}_{a}\right)} . \\
\widehat{a}_{p} \text { não tem forma fechada. }
\end{gathered}
$$

- $\ell_{B N}(a)$ e $u_{B N}(a)$

$$
\begin{gathered}
\ell_{B N}(a)=\ell_{p}(a)+\frac{1}{2} \log \left\{\psi^{\prime}\left(\widehat{b}_{a}\right)-\psi^{\prime}\left(a+\widehat{b}_{a}\right)\right\} \\
u_{B N}(a)=u_{p}(a)+\frac{1}{2} \frac{\psi^{\prime}\left(a+\widehat{b}_{a}\right)}{\left[\psi^{\prime}\left(\widehat{b}_{a}\right)-\psi^{\prime}\left(a+\widehat{b}_{a}\right)\right]^{2}}\left[\psi^{\prime \prime}\left(\widehat{b}_{a}\right)-\psi^{\prime \prime}\left(a+\widehat{b}_{a}\right)\right]-\frac{1}{2} \frac{\psi^{\prime \prime}\left(a+\widehat{b}_{a}\right)}{\psi^{\prime}\left(\widehat{b}_{a}\right)-\psi^{\prime}\left(a+\widehat{b}_{a}\right)} . \\
\widehat{a}_{B N} \text { não tem forma fechada. }
\end{gathered}
$$

- viés da função escore

Não foi possível determinar as esperanças $E\left\{u_{p}(a)\right\}$ e $E\left\{u_{B N}(a)\right\}$.

EXEMPLO 2.4: distribuição gaussiana inversa com média $\gamma$ e coeficiente de variação $\zeta$ $(\gamma>0, \zeta>0)$.

A função densidade de uma variável aleatória $y$ com distribuição $N^{-}\left(\gamma, \gamma \zeta^{2}\right)$ é

$$
p(y ; \gamma, \zeta)=\left[\frac{\gamma}{2 \pi y^{3} \zeta^{2}}\right]^{1 / 2} \exp \left[-\frac{(y-\gamma)^{2}}{2 y \gamma \zeta^{2}}\right], \quad y>0
$$

A razão do nome gaussiana inversa deve-se a uma relação inversa entre as funções geratrizes de cumulantes desta distribuição e da normal $N\left(\mu, \sigma^{2}\right)$.

Os coeficientes de assimetria e de curtose são $3 \zeta$ e $\left(15 \zeta^{2}+3\right)$, respectivamente. Portanto, tal distribuição aproxima-se da normal à medida que $\zeta$ decresce (Chhikara e Folks, 1989).

Dada uma amostra aleatória, o estimador de máxima verossimilhança de $\gamma$ é a média amostral, que tem distribuição $N^{-}\left(\gamma, \gamma(\zeta / \sqrt{n})^{2}\right)$. Neste caso, o coeficiente de variação é $(\zeta / \sqrt{n})$. Considerando o caso em que o parâmetro de interesse é $\zeta(\nu=\gamma)$, seu estimador de máxima verossimilhança é

$$
\widehat{\zeta}=\left(\frac{\bar{y} \sum_{j=1}^{n}\left(1 / y_{j}\right)}{n}-1\right)^{1 / 2}
$$


- $\ell_{p}(\zeta)$ e $u_{p}(\zeta)$

$$
\begin{gathered}
\ell_{p}(\zeta)=\frac{n}{2} \log \left(\frac{n \zeta^{2}+\sqrt{\Delta_{\zeta}}}{\zeta^{2}}\right)+\frac{2 n-\sqrt{\Delta_{\zeta}}}{2 \zeta^{2}} \quad \text { e } \quad u_{p}(\zeta)=\frac{\sqrt{\Delta_{\zeta}}-n\left(\zeta^{2}+2\right)}{\zeta^{3}} . \\
\Delta_{\zeta}=n^{2} \zeta^{4}+4 n \bar{y} \sum_{j=1}^{n}\left(1 / y_{j}\right) \quad \text { e } \quad \widehat{\zeta}_{p}=\widehat{\zeta} .
\end{gathered}
$$

- $\ell_{B N}(\zeta)$ e $u_{B N}(\zeta)$

$$
\begin{gathered}
\ell_{B N}(\zeta)=\ell_{p}(\zeta)-\frac{1}{4} \log \Delta_{\zeta}+\log \zeta \quad \text { e } \quad u_{B N}(\zeta)=u_{p}(\zeta)+\frac{4 n \bar{y} \sum_{j=1}^{n}\left(1 / y_{j}\right)}{\zeta \Delta_{\zeta}} . \\
\widehat{\zeta}_{B N} \text { não tem forma fechada. }
\end{gathered}
$$

- viés da função escore

Não foi possível determinar as esperanças $E\left\{u_{p}(\zeta)\right\}$ e $E\left\{u_{B N}(\zeta)\right\}$.

\subsection{VEROSSIMILHANÇA PERFILADA MODIFICADA PROPOSTA POR COX E REID}

Suponha, mais uma vez, um modelo estatístico hipotético com vetor de parâmetros $(\mu, \nu)$ e função de verossimilhança $L(\mu, \nu)$. Quando a fatoração

$$
L=L(\mu, \nu)=L_{1}(\mu) L_{2}(\nu)
$$

é possível, diz-se que $L$ tem parâmetros separáveis. Conseqüentemente, o logaritmo da função de verossimilhança é dado por

$$
\ell=\ell(\mu, \nu)=\ell_{1}(\mu)+\ell_{2}(\nu)
$$

e, assim, $\widehat{\nu}_{\mu}=\widehat{\nu}$ e $\ell_{p}(\mu)=\ell_{1}(\mu)$. Ou seja, o cálculo da verossimilhança perfilada e de quantidades relacionadas tornam-se mais simples.

Uma forma de tentar reproduzir esta situação consiste em utilizar uma parametrização ortogonal. Neste caso, os componentes do vetor escore, $\partial \ell / \partial \mu$ e $\partial \ell / \partial \nu$, são não correlacionados. As seguintes implicações, devido à ortogonalidade de $\mu$ e $\nu$, são enumeradas: 
(i) os estimadores de máxima verossimilhança destes parâmetros são assintoticamente independentes,

(ii) a matriz de covariância de $\widehat{\mu}$ pode ser calculada como se $\nu$ fosse conhecido,

(iii) há diminuição do custo computacional na determinação numérica das estimativas de máxima verossimilhança destes parâmetros,

(iv) $\widehat{\nu}_{\mu}-\widehat{\nu}=O_{p}\left(n^{-1}\right)$, enquanto que, em geral, observa-se $\widehat{\nu}_{\mu}-\widehat{\nu}=O_{p}\left(n^{-1 / 2}\right)$.

A aproximação do logaritmo da função de verossimilhança com parâmetros ortogonais para aquele com parâmetros separáveis pode também ser constatada. Suponha $\mu$ e $\nu$ unidimensionais e, por enquanto, não necessariamente ortogonais. A expansão de $\ell(\mu, \nu)$ em série de Taylor em torno de $(\widehat{\mu}, \widehat{\nu})$ é

$\ell(\mu, \nu)=\ell(\widehat{\mu}, \widehat{\nu})-\frac{1}{2}(\mu-\widehat{\mu})^{2} j_{\mu \mu}(\widehat{\mu}, \widehat{\nu})-\frac{1}{2}(\nu-\widehat{\nu})^{2} j_{\nu \nu}(\widehat{\mu}, \widehat{\nu})-(\mu-\widehat{\mu})(\nu-\widehat{\nu}) j_{\mu \nu}(\widehat{\mu}, \widehat{\nu})+O_{p}\left(n^{-1 / 2}\right)$,

onde

$$
j_{\mu \mu}(\mu, \nu)=-\partial^{2} \ell(\mu, \nu) / \partial \mu^{2}, j_{\nu \nu}(\mu, \nu)=-\partial^{2} \ell(\mu, \nu) / \partial \nu^{2} \text { e } j_{\mu \nu}(\mu, \nu)=-\partial^{2} \ell(\mu, \nu) / \partial \mu \partial \nu .
$$

Se $\mu$ e $\nu$ são ortogonais, $j_{\mu \nu}(\mu, \nu)$ e $j_{\mu \nu}(\widehat{\mu}, \widehat{\nu})$ são de ordem $O_{p}\left(n^{1 / 2}\right)$. Logo,

$$
\ell(\mu, \nu)=\ell(\widehat{\mu}, \widehat{\nu})-\frac{1}{2}(\mu-\widehat{\mu})^{2} j_{\mu \mu}(\widehat{\mu}, \widehat{\nu})-\frac{1}{2}(\nu-\widehat{\nu})^{2} j_{\nu \nu}(\widehat{\mu}, \widehat{\nu})+O_{p}\left(n^{-1 / 2}\right),
$$

ou seja,

$$
\ell(\mu, \nu)=\ell_{1}(\mu)+\ell_{2}(\nu)+O_{p}\left(n^{-1 / 2}\right),
$$

$\operatorname{para}(\mu, \nu)=(\widehat{\mu}, \widehat{\nu})+O\left(n^{-1 / 2}\right)$.

Entretanto, nem sempre é possível encontrar uma parametrização ortogonal. Somente há garantia da existência de tal parametrização quando o parâmetro de interesse é escalar.

Assim, considere o caso no qual $\mu$ é escalar e $\nu=\left(\nu_{1}, \ldots, \nu_{q}\right)$ é o vetor de parâmetros de perturbação, com $\mu$ e $\nu$ não ortogonais. Deseja-se, então, encontrar uma nova parametrização $(\mu, \lambda)$, com $\lambda=\lambda(\mu, \nu)=\left(\lambda_{1}(\mu, \nu), \ldots, \lambda_{q}(\mu, \nu)\right)$, tal que $\mu$ e $\lambda$ sejam ortogonais. Observe que

$$
\ell^{\perp}(\mu, \lambda)=\ell(\mu, \nu(\mu, \lambda)),
$$

onde $\nu(\mu, \lambda)=\left(\nu_{1}(\mu, \lambda), \ldots, \nu_{q}(\mu, \lambda)\right)$ e $\ell^{\perp}(\mu, \lambda)$ denota o logaritmo da função de verossimilhança na nova parametrização.

Derivando ambos os lados dessa igualdade com relação a $\mu$,

$$
\frac{\partial \ell^{\perp}}{\partial \mu}=\frac{\partial \ell}{\partial \mu}+\sum_{r=1}^{q} \frac{\partial \ell}{\partial \nu_{r}} \frac{\partial \nu_{r}}{\partial \mu}
$$

e, pela segunda vez, com relação a $\lambda_{m}$, obtém-se

$$
\frac{\partial^{2} \ell^{\perp}}{\partial \mu \partial \lambda_{m}}=\sum_{h=1}^{q} \frac{\partial^{2} \ell}{\partial \mu \partial \nu_{h}} \frac{\partial \nu_{h}}{\partial \lambda_{m}}+\sum_{r=1}^{q} \sum_{h=1}^{q} \frac{\partial^{2} \ell}{\partial \nu_{r} \partial \nu_{h}} \frac{\partial \nu_{h}}{\partial \lambda_{m}} \frac{\partial \nu_{r}}{\partial \mu}+\sum_{r=1}^{q} \frac{\partial \ell}{\partial \nu_{r}} \frac{\partial^{2} \nu_{r}}{\partial \mu \partial \lambda_{m}}
$$


para $m=1, \ldots, q$.

Como

$$
E\left\{\frac{\partial^{2} \ell^{\perp}}{\partial \mu \partial \lambda_{m}}\right\}=0 \quad \text { e } \quad E\left\{\frac{\partial \ell}{\partial \nu_{r}}\right\}=0,
$$

para $r, m=1, \ldots, q$, então, para obter a ortogonalidade entre $\mu$ e $\lambda$, é necessário resolver um conjunto de $q$ equações diferenciais parciais, a saber

$$
\sum_{h=1}^{q} \frac{\partial \nu_{h}}{\partial \lambda_{m}}\left(i_{\mu \nu_{h}}+\sum_{r=1}^{q} i_{\nu_{r} \nu_{h}} \frac{\partial \nu_{r}}{\partial \mu}\right)=0, \quad m=1, \ldots, q .
$$

Aqui,

$$
i_{\mu \nu_{h}}=-E\left\{\frac{\partial^{2} \ell}{\partial \mu \partial \nu_{h}}\right\} \quad \text { e } \quad i_{\nu_{r} \nu_{h}}=-E\left\{\frac{\partial^{2} \ell}{\partial \nu_{r} \partial \nu_{h}}\right\}
$$

O jacobiano da transformação de $(\mu, \nu)$ para $(\mu, \lambda)$ não pode ser nulo. Assim, o sistema de equações passa a ser

$$
\sum_{r=1}^{q} i_{\nu_{r} \nu_{h}} \frac{\partial \nu_{r}}{\partial \mu}=-i_{\mu \nu_{h}}, \quad h=1, \ldots, q .
$$

Estas equações diferenciais parciais determinam a dependência de $\nu$ sobre $\mu$, mas permitem uma certa liberdade na escolha da relação de dependência entre $\nu$ e $\lambda$. Além disso, se $\mu=\left(\mu_{1}, \mu_{2}\right)$, é possível calcular independentemente $\partial \nu_{r} / \partial \mu_{1}$ e $\partial \nu_{r} / \partial \mu_{2}$, entretanto, não se garante que $\partial^{2} \nu_{r} / \partial \mu_{1} \partial \mu_{2}=\partial^{2} \nu_{r} / \partial \mu_{2} \partial \mu_{1}$. Por isso, em geral, quando o parâmetro de interesse não é um escalar, não é possível obter a ortogonalidade entre este parâmetro e o parâmetro de perturbação.

A fim de conseguir uma parametrização ortogonal na família exponencial biparamétrica (2.1), é preciso resolver a equação diferencial parcial

$$
i_{\mu \nu}+i_{\nu \nu} \frac{\partial \nu(\mu, \lambda)}{\partial \mu}=0
$$

onde

$$
i_{\mu \nu}=\alpha_{1}^{(1,0)}(\mu, \nu) \beta_{1}^{(0,1)}(\mu, \nu)+\alpha_{2}^{(1,0)}(\mu, \nu) \beta_{2}^{(0,1)}(\mu, \nu)
$$

e

$$
i_{\nu \nu}=\alpha_{1}^{(0,1)}(\mu, \nu) \beta_{1}^{(0,1)}(\mu, \nu)+\alpha_{2}^{(0,1)}(\mu, \nu) \beta_{2}^{(0,1)}(\mu, \nu)
$$

Explorando as conseqüências da ortogonalidade, Cox e Reid (1987) propuseram uma versão modificada da verossimilhança perfilada. Assim, considerando $\mu$ e $\nu$ ortogonais, sendo $\mu$ o parâmetro de interesse, escalar, e $\nu$, um vetor de parâmetros de incômodo, a expansão (2.8) é dada por

$$
\ell(\mu, \nu)=\ell(\widehat{\mu}, \widehat{\nu})-\frac{1}{2}\left\{(\mu-\widehat{\mu})^{2} j_{\mu \mu}(\widehat{\mu}, \widehat{\nu})+(\nu-\widehat{\nu})^{\top} j_{\nu \nu}(\widehat{\mu}, \widehat{\nu})(\nu-\widehat{\nu})\right\}+O_{p}\left(n^{-1 / 2}\right) .
$$


Conseqüentemente,

$$
\begin{gathered}
p(y ; \mu, \nu)=\exp \{\ell(\mu, \nu)\}=\exp \{\ell(\widehat{\mu}, \widehat{\nu})\} \exp \left\{-\frac{1}{2}(\mu-\widehat{\mu})^{2} j_{\mu \mu}(\widehat{\mu}, \widehat{\nu})\right\}(2 \pi)^{q / 2}\left|j_{\nu \nu}(\widehat{\mu}, \widehat{\nu})\right|^{-1 / 2} \\
\times(2 \pi)^{-q / 2}\left|j_{\nu \nu}(\widehat{\mu}, \widehat{\nu})\right|^{1 / 2} \exp \left\{-\frac{1}{2}(\nu-\widehat{\nu})^{\top} j_{\nu \nu}(\widehat{\mu}, \widehat{\nu})(\nu-\widehat{\nu})\right\} \exp \left\{O_{p}\left(n^{-1 / 2}\right)\right\},
\end{gathered}
$$

onde $q$ é a dimensão do vetor $\nu$.

Geralmente, a distribuição assintótica de $\widehat{\nu}$ pode ser aproximada por uma distribuição normal de média $\nu$ e matriz de covariância $j^{\nu \nu}$, onde $j^{\nu \nu}$ é o bloco inferior-direito da matriz

$$
j(\mu, \nu)^{-1}=\left(\begin{array}{cc}
j_{\mu \mu} & j_{\mu \nu} \\
j_{\nu \mu} & j_{\nu \nu}
\end{array}\right)^{-1}=\left(\begin{array}{ll}
j^{\mu \mu} & j^{\mu \nu} \\
j^{\nu \mu} & j^{\nu \nu}
\end{array}\right) .
$$

Devido à ortogonalidade dos parâmetros, $j_{\mu \nu}(\mu, \nu), j_{\nu \mu}(\mu, \nu), j_{\mu \nu}(\widehat{\mu}, \widehat{\nu})$ e $j_{\nu \mu}(\widehat{\mu}, \widehat{\nu})$ são de ordem $O_{p}\left(n^{1 / 2}\right)$. Logo, $j^{\nu \nu} \doteq\left(j_{\nu \nu}\right)^{-1}$, ou ainda, $j_{\nu \nu} \doteq\left(j^{\nu \nu}\right)^{-1}$. Dessa forma, a aproximação para a função densidade de $\widehat{\nu}$ é dada por

$$
p(\widehat{\nu} ; \mu, \nu) \doteq(2 \pi)^{-q / 2}\left|j_{\nu \nu}(\widehat{\mu}, \widehat{\nu})\right|^{1 / 2} \exp \left\{-\frac{1}{2}(\nu-\widehat{\nu})^{\top} j_{\nu \nu}(\widehat{\mu}, \widehat{\nu})(\nu-\widehat{\nu})\right\} .
$$

Agora, usando essa aproximação e supondo que $(\widehat{\nu}, w)$ seja uma transformação um-a-um de $y$, onde $w$ é uma função qualquer conveniente das observações, constata-se que $p(y \mid \widehat{\nu} ; \mu)$ não depende de $\nu$, ou seja, ocorre o que se define como suficiência parcial. Assintoticamente, $\widehat{\nu}$ é parcialmente suficiente para $\nu$, pois

$p(y \mid \widehat{\nu} ; \mu)=\exp \{\ell(\widehat{\mu}, \widehat{\nu})\} \exp \left\{-\frac{1}{2}(\mu-\widehat{\mu})^{2} j_{\mu \mu}(\widehat{\mu}, \widehat{\nu})\right\}(2 \pi)^{q / 2}\left|j_{\nu \nu}(\widehat{\mu}, \widehat{\nu})\right|^{-1 / 2} \exp \left\{O_{p}\left(n^{-1 / 2}\right)\right\} \widehat{J}$,

onde $\widehat{J}$ é o jacobiano da transformação e é função apenas dos dados. Dessa forma, justifica-se o uso deste modelo condicional a fim de realizar inferências sobre $\mu$.

De (2.11), vem

$$
\ell(\widehat{\mu}, \widehat{\nu})=\ell(\mu, \nu)+\frac{1}{2}\left\{(\mu-\widehat{\mu})^{2} j_{\mu \mu}(\widehat{\mu}, \widehat{\nu})+(\nu-\widehat{\nu})^{\top} j_{\nu \nu}(\widehat{\mu}, \widehat{\nu})(\nu-\widehat{\nu})\right\}+O_{p}\left(n^{-1 / 2}\right) .
$$

Portanto, o logaritmo da função de verossimilhança condicional, omitindo termos que não dependem dos parâmetros, pode ser aproximado por

$$
\log p(y \mid \widehat{\nu} ; \mu) \doteq \ell(\mu, \nu)-\frac{1}{2} \log \left|j_{\nu \nu}(\mu, \nu)\right|+\frac{1}{2}(\nu-\widehat{\nu})^{\top} j_{\nu \nu}(\mu, \nu)(\nu-\widehat{\nu}) .
$$

Aqui, utilizou-se o fato que $j_{\nu \nu}(\widehat{\mu}, \widehat{\nu}) \doteq j_{\nu \nu}(\mu, \nu)$ quando $n \rightarrow \infty$.

Note que esta função não é independente de $\nu$, pois a suficiência parcial de $\widehat{\nu}$ para $\nu$ é somente observada assintoticamente, além disso foi utilizada uma aproximação para a distribuição deste estimador de máxima verossimilhança. Uma solução natural é substituir $\nu$ por $\widehat{\nu}_{\mu}$. Como já foi mencionado, quando $\mu$ e $\nu$ são ortogonais, observa-se $\widehat{\nu}_{\mu}-\widehat{\nu}=O_{p}\left(n^{-1}\right)$. 
Por conseguinte, o termo $\left(\widehat{\nu}_{\mu}-\widehat{\nu}\right)^{\top} j_{\nu \nu}\left(\mu, \widehat{\nu}_{\mu}\right)\left(\widehat{\nu}_{\mu}-\widehat{\nu}\right)$ torna-se de ordem $O_{p}\left(n^{-1}\right)$ e pode ser desprezado.

Assim, o logaritmo da função de verossimilhança perfilada modificada proposta por Cox e Reid (1987) é definido por

$$
\ell_{C R}(\mu)=\ell\left(\mu, \widehat{\nu}_{\mu}\right)-\frac{1}{2} \log \left|j_{\nu \nu}\left(\mu, \widehat{\nu}_{\mu}\right)\right|=\ell_{p}(\mu)-\frac{1}{2} \log \left|j_{\nu \nu}\left(\mu, \widehat{\nu}_{\mu}\right)\right|
$$

Note que, assim como $\ell_{B N}(\mu), \ell_{C R}(\mu)$ é uma versão penalizada do logaritmo da função de verossimilhança perfilada. Aplicando a função inversa da função logarítmica em ambos os membros dessa igualdade, obtém-se

$$
L_{C R}(\mu)=\left|j_{\nu \nu}\left(\mu, \widehat{\nu}_{\mu}\right)\right|^{-1 / 2} L_{p}(\mu)
$$

onde $L_{C R}(\mu)$ é a função de verossimilhança perfilada modificada proposta por Cox e Reid (1987). A correspondente função escore é denotada por $u_{C R}(\mu)$ e é igual a $\partial \ell_{C R}(\mu) / \partial \mu$. Aqui, $E\left\{u_{C R}(\mu)\right\}=O\left(n^{-1}\right)$ (Liang, 1987), mas, em geral, o viés da informação não é de ordem $O\left(n^{-1}\right)$; ver DiCiccio et al. (1996). O ponto de máximo de $\ell_{C R}(\mu)$ é indicado por $\widehat{\mu}_{C R}$.

A utilização dessa função requer a ortogonalidade dos parâmetros $\mu$ e $\nu$. Além disso, a mesma não é invariante sob reparametrizações da forma $(\mu, \nu) \rightarrow(\xi, \lambda)$, em que $\xi=\xi(\mu)$ e $\lambda=\lambda(\mu, \nu)$, diferentemente de $L_{B N}(\mu)$. Para esta função, essa propriedade é garantida pela parcela $\left|\partial \widehat{\nu}_{\mu} / \partial \widehat{\nu}\right|$ presente na sua expressão.

Note que se $\widehat{\nu}_{\mu}=\widehat{\nu}$ para qualquer valor de $\mu$, então

$$
L_{B N}(\mu)=\left|j_{\nu \nu}\left(\mu, \widehat{\nu}_{\mu}\right)\right|^{-1 / 2} L_{p}(\mu)=L_{C R}(\mu),
$$

pois $\mu$ e $\nu$ são parâmetros ortogonais neste caso (Cox e Reid, 1987) e, assim, o viés da informação associado a $u_{C R}(\mu)$ é de ordem $O\left(n^{-1}\right)$. Infelizmente, em geral, não é possível parametrizar um modelo de tal forma que $\widehat{\nu}_{\mu}=\widehat{\nu}$. Contudo, quando $\mu$ e $\nu$ são ortogonais, para $\mu=\widehat{\mu}+O_{p}\left(n^{-1 / 2}\right)$, observa-se $\widehat{\nu}_{\mu}-\widehat{\nu}=O_{p}\left(n^{-1}\right)$. Logo, aplicando a aproximação

$$
\left|\frac{\partial \widehat{\nu}_{\mu}}{\partial \widehat{\nu}}\right|=1+O_{p}\left(n^{-1}\right)
$$

em $L_{B N}(\mu)$, obtém-se $L_{C R}(\mu)$.

A expansão de $\log (1+x)$ em série de Taylor em torno de zero avaliada em $x=O_{p}\left(n^{-1}\right)$ fornece

$$
\log \left|\frac{\partial \widehat{\nu}_{\mu}}{\partial \widehat{\nu}}\right|=O_{p}\left(n^{-1}\right)
$$

Como $\widehat{\nu} \widehat{\mu}=\widehat{\nu}$, então

$$
\ell_{B N}(\mu)-\ell_{B N}(\widehat{\mu})=\ell_{C R}(\mu)-\ell_{C R}(\widehat{\mu})+O_{p}\left(n^{-1}\right) .
$$


Conseqüentemente, ao testar hipóteses sobre $\mu$, as estatísticas da razão de verossimilhança derivadas de $\ell_{B N}(\mu)$ e de $\ell_{C R}(\mu)$ diferem de um termo de ordem $O_{p}\left(n^{-1}\right)$.

Em algumas aplicações pode ser relativamente fácil obter a ortogonalidade entre os parâmetros de interesse e de perturbação resolvendo o sistema de equações diferenciais parciais (2.9). Contudo, em problemas mais complexos, a determinação explícita do novo parâmetro $\lambda=\lambda(\mu, \nu)$, em que $\mu$ e $\nu$ não são ortogonais, dificulta ainda mais os procedimentos inferenciais. Sabe-se que a verossimilhança perfilada é invariante sob reparametrizações da forma $(\mu, \nu) \rightarrow(\xi, \lambda)$, em que $\xi=\mu$ e $\lambda=\lambda(\mu, \nu)$, o que não ocorre com a parcela de penalização de $\ell_{p}(\mu)$ na expressão de $\ell_{C R}(\mu),(1 / 2) \log \left|j_{\lambda \lambda}\left(\mu, \widehat{\lambda}_{\mu}\right)\right|$. Assim, seria desejável aproximar esse termo de correção por uma função de $\left(\mu, \widehat{\nu}_{\mu}\right)$ independentemente da parametrização ortogonal produzida segundo (2.9).

Utilizando notação já definida nesta seção,

$$
\ell^{\perp}(\mu, \lambda)=\ell(\mu, \nu(\mu, \lambda))
$$

Lembre que $\nu$ e $\lambda$ são vetores de dimensão $q$ e que $\mu$ é um escalar. Derivando ambos os lados dessa igualdade com relação ao componente $\lambda_{m}$ de $\lambda$, tem-se

$$
\frac{\partial \ell^{\perp}(\mu, \lambda)}{\partial \lambda_{m}}=\sum_{r=1}^{q} \frac{\partial \ell(\mu, \nu(\mu, \lambda))}{\partial \nu_{r}} \frac{\partial \nu_{r}(\mu, \lambda)}{\partial \lambda_{m}}
$$

e, pela segunda vez, com relação a $\lambda_{t}$, obtém-se

$$
\frac{\partial^{2} \ell^{\perp}(\mu, \lambda)}{\partial \lambda_{m} \partial \lambda_{t}}=\sum_{r=1}^{q}\left[\left(\sum_{s=1}^{q} \frac{\partial^{2} \ell(\mu, \nu(\mu, \lambda))}{\partial \nu_{r} \partial \nu_{s}} \frac{\partial \nu_{s}(\mu, \lambda)}{\partial \lambda_{t}}\right) \frac{\partial \nu_{r}(\mu, \lambda)}{\partial \lambda_{m}}+\frac{\partial \ell(\mu, \nu(\mu, \lambda))}{\partial \nu_{r}} \frac{\partial^{2} \nu_{r}(\mu, \lambda)}{\partial \lambda_{m} \partial \lambda_{t}}\right] .
$$

Note que

$$
\frac{\partial \ell\left(\mu, \nu\left(\mu, \widehat{\lambda}_{\mu}\right)\right)}{\partial \nu_{r}}=\frac{\partial \ell\left(\mu, \widehat{\nu}_{\mu}\right)}{\partial \nu_{r}}=0
$$

Conseqüentemente,

$$
\frac{\partial^{2} \ell^{\perp}\left(\mu, \widehat{\lambda}_{\mu}\right)}{\partial \lambda_{m} \partial \lambda_{t}}=\sum_{r=1}^{q} \sum_{s=1}^{q} \frac{\partial^{2} \ell\left(\mu, \widehat{\nu}_{\mu}\right)}{\partial \nu_{r} \partial \nu_{s}} \frac{\partial \nu_{s}\left(\mu, \widehat{\lambda}_{\mu}\right)}{\partial \lambda_{t}} \frac{\partial \nu_{r}\left(\mu, \widehat{\lambda}_{\mu}\right)}{\partial \lambda_{m}}
$$

e, escrevendo na forma matricial,

$$
j_{\lambda \lambda}\left(\mu, \widehat{\lambda}_{\mu}\right)=\frac{\partial \nu^{\top}\left(\mu, \widehat{\lambda}_{\mu}\right)}{\partial \lambda} j_{\nu \nu}\left(\mu, \widehat{\nu}_{\mu}\right) \frac{\partial \nu\left(\mu, \widehat{\lambda}_{\mu}\right)}{\partial \lambda^{\top}}
$$

onde

$$
\frac{\partial \nu^{\top}\left(\mu, \widehat{\lambda}_{\mu}\right)}{\partial \lambda}=\left(\frac{\partial \nu\left(\mu, \widehat{\lambda}_{\mu}\right)}{\partial \lambda^{\top}}\right)^{\top}=\frac{\partial(\nu)}{\partial(\lambda)}
$$


Assim,

$$
\left|j_{\lambda \lambda}\left(\mu, \widehat{\lambda}_{\mu}\right)\right|=\left|\frac{\partial(\nu)}{\partial(\lambda)}\right|\left|j_{\nu \nu}\left(\mu, \widehat{\nu}_{\mu}\right)\right|\left|\left(\frac{\partial(\nu)}{\partial(\lambda)}\right)^{\top}\right|
$$

e, portanto,

$$
\log \left|j_{\lambda \lambda}\left(\mu, \widehat{\lambda}_{\mu}\right)\right|=\log \left|j_{\nu \nu}\left(\mu, \widehat{\nu}_{\mu}\right)\right|+2 \log \left|\frac{\partial(\nu)}{\partial(\lambda)}\right|,
$$

em que $|\partial(\nu) / \partial(\lambda)|$ é o jacobiano da transformação entre $\nu$ e $\lambda$ avaliado em $\left(\mu, \widehat{\lambda}_{\mu}\right)$.

Usando este resultado, o logaritmo da verossimilhança proposta por Cox e Reid (1987) pode ser expresso como

$$
\ell_{C R}(\mu)=\ell_{p}(\mu)-\frac{1}{2} \log \left|j_{\lambda \lambda}\left(\mu, \widehat{\lambda}_{\mu}\right)\right|=\ell_{p}(\mu)-\frac{1}{2} \log \left|j_{\nu \nu}\left(\mu, \widehat{\nu}_{\mu}\right)\right|-\log \left|\frac{\partial(\nu)}{\partial(\lambda)}\right| .
$$

Expandindo em série de Taylor o último termo desta expressão em torno de um ponto arbitrário $\left(\mu_{0}, \lambda_{0}\right)$, obtém-se

$$
\ell_{C R}(\mu)=\ell_{p}(\mu)-\frac{1}{2} \log \left|j_{\nu \nu}\left(\mu, \widehat{\nu}_{\mu}\right)\right|-\left.\left(\mu-\mu_{0}\right) \frac{\partial}{\partial \mu}\left[\log \left|\frac{\partial(\nu)}{\partial(\lambda)}\right|\right]\right|_{\left(\mu_{0}, \lambda_{0}\right)}+\ldots
$$

Utilizando propriedades de diferenciação de matrizes, encontradas, por exemplo, em Magnus e Neudecker (1988), $\ell_{C R}(\mu)$ pode ser escrita como

$$
\ell_{C R}(\mu)=\ell_{p}(\mu)-\frac{1}{2} \log \left|j_{\nu \nu}\left(\mu, \widehat{\nu}_{\mu}\right)\right|-\left.\left(\mu-\mu_{0}\right) \operatorname{tr}\left\{\left(\frac{\partial(\nu)}{\partial(\lambda)}\right)^{-1} \frac{\partial}{\partial \mu}\left(\frac{\partial(\nu)}{\partial(\lambda)}\right)\right\}\right|_{\left(\mu_{0}, \lambda_{0}\right)}+\ldots
$$

O sistema de equações (2.9) pode ser expresso da seguinte forma

$$
i_{\nu \nu} \frac{\partial \nu}{\partial \mu}=-i_{\nu \mu}
$$

na qual $i_{\nu \nu}$ é uma matriz $q \times q$ com elementos $i_{\nu_{r} \nu_{s}}=-E\left\{\partial^{2} l / \partial \nu_{r} \partial \nu_{s}\right\}, \partial \nu / \partial \mu$ é um vetor $q \times 1$ com componentes $\partial \nu_{r} / \partial \mu$ e $i_{\nu \mu}$ é um vetor de mesma dimensão com elementos $i_{\nu_{r} \mu}=-E\left\{\partial^{2} l / \partial \nu_{r} \partial \mu\right\}$, com $r, s=1, \ldots, q$. Conseqüentemente,

$$
\frac{\partial \nu}{\partial \mu}=-i^{\nu \nu} i_{\nu \mu}=-m
$$

em que $i^{\nu \nu}$ é a inversa da matriz $i_{\nu \nu}$ e $m$ é um vetor $q \times 1$ cujos componentes dependem de $(\mu, \nu(\mu, \lambda))$. Derivando os dois lados desta igualdade com relação a $\lambda^{\top}$, obtém-se

$$
\frac{\partial^{2} \nu}{\partial \lambda^{\top} \partial \mu}=-m_{\nu} \frac{\partial \nu}{\partial \lambda^{\top}}
$$

onde $m_{\nu}=\partial m / \partial \nu^{\top}$ é uma matriz $q \times q$. Logo,

$$
\ell_{C R}(\mu) \doteq \ell_{p}(\mu)-\frac{1}{2} \log \left|j_{\nu \nu}\left(\mu, \widehat{\nu}_{\mu}\right)\right|+\left.\left(\mu-\mu_{0}\right) \operatorname{tr}\left\{m_{\nu}\right\}\right|_{\left(\mu_{0}, \nu_{0}\right)} .
$$


Se $\nu$ é unidimensional, então

$$
\ell_{C R}(\mu) \doteq \ell_{p}(\mu)-\frac{1}{2} \log \left|j_{\nu \nu}\left(\mu, \widehat{\nu}_{\mu}\right)\right|+\left.\left(\mu-\mu_{0}\right) \frac{\partial m(\mu, \nu)}{\partial \nu}\right|_{\left(\mu_{0}, \nu_{0}\right)} .
$$

Fazendo $\left(\mu_{0}, \nu_{0}\right)=(\widehat{\mu}, \widehat{\nu})$, obtém-se a seguinte aproximação para $\ell_{C R}(\mu)$ (Cox e Reid, 1993)

$$
\ell_{A C R}(\mu)=\ell_{p}(\mu)-\frac{1}{2} \log \left|j_{\nu \nu}\left(\mu, \widehat{\nu}_{\mu}\right)\right|+\mu \operatorname{tr}\left\{\widehat{m}_{\nu}\right\},
$$

em que $\widehat{m}_{\nu}$ é igual a $m_{\nu}$ avaliado em $(\widehat{\mu}, \widehat{\nu})$. Usando resultados de Ferguson (1992) e McCullagh e Tibshirani (1990), para $\mu=\widehat{\mu}+O\left(n^{-1 / 2}\right)$, tem-se

$$
E\left\{u_{A C R}(\mu)\right\}=O\left(n^{-1}\right),
$$

em que $u_{A C R}(\mu)=\partial \ell_{A C R}(\mu) / \partial \mu$.

Essa aproximação para o logaritmo da verossimilhança proposta por Cox e Reid (1987)

não requer ortogonalidade dos parâmetros de interesse e de perturbação, mas continua não sendo invariante sob reparametrizações da forma $(\mu, \nu) \rightarrow(\xi, \lambda)$, em que $\xi=\mu$ e $\lambda=\lambda(\mu, \nu)$.

Observe que se os parâmetros $\mu$ e $\nu$ são ortogonais, então $i_{\nu \mu}=0$. Assim, $m=0$ e, por conseguinte, $\ell_{A C R}(\mu)=\ell_{C R}(\mu)$.

Para a família exponencial biparamétrica, tal aproximação é dada por

$$
\begin{gathered}
\ell_{A C R}(\mu)=\ell_{p}(\mu)+\mu m^{(0,1)}(\widehat{\mu}, \widehat{\nu}) \\
-\frac{1}{2} \log \left\{\sum_{i=1}^{2} \alpha_{i}^{(0,1)}\left(\mu, \widehat{\nu}_{\mu}\right) \beta_{i}^{(0,1)}\left(\mu, \widehat{\nu}_{\mu}\right)-\alpha_{i}^{(0,2)}\left(\mu, \widehat{\nu}_{\mu}\right)\left[\beta_{i}(\widehat{\mu}, \widehat{\nu})-\beta_{i}\left(\mu, \widehat{\nu}_{\mu}\right)\right]\right\},
\end{gathered}
$$

na qual

$$
m=\frac{\left(\alpha_{1}^{(1,0)} \beta_{1}^{(1,0)}+\alpha_{2}^{(1,0)} \beta_{2}^{(1,0)}\right)\left(\alpha_{1}^{(1,0)} \beta_{1}^{(0,1)}+\alpha_{2}^{(1,0)} \beta_{2}^{(0,1)}\right)}{\left(\alpha_{1}^{(1,0)} \alpha_{2}^{(0,1)}-\alpha_{1}^{(0,1)} \alpha_{2}^{(1,0)}\right)\left(\beta_{1}^{(1,0)} \beta_{2}^{(0,1)}-\beta_{1}^{(0,1)} \beta_{2}^{(1,0)}\right)} \quad \text { e } \quad m^{(0,1)}=\frac{\partial m}{\partial \nu} .
$$

Aqui, por simplicidade, o argumento $(\mu, \nu)$ das funções é omitido e assume-se que

$$
\alpha_{1}^{(1,0)} \alpha_{2}^{(0,1)}-\alpha_{1}^{(0,1)} \alpha_{2}^{(1,0)} \neq 0 \quad \text { e } \quad \beta_{1}^{(1,0)} \beta_{2}^{(0,1)}-\beta_{1}^{(0,1)} \beta_{2}^{(1,0)} \neq 0
$$

Se $\mu$ e $\nu$ são ortogonais, $\alpha_{1}^{(1,0)} \beta_{1}^{(0,1)}+\alpha_{2}^{(1,0)} \beta_{2}^{(0,1)}=0$. Neste caso, $m=0$.

\subsection{EXEMPLOS}

A seguir, é dada continuidade aos exemplos da seção 2.4, derivando as verossimilhanças $\ell_{C R}(\mu)$ e $\ell_{A C R}(\mu)$ para algumas distribuições pertencentes a família exponencial biparamétrica. 
EXEMPLO 2.1 (continuação): distribuição normal com média $\tau$ e variância $\phi$.

Os parâmetros $\tau$ e $\phi$, que indexam a distribuição deste exemplo, são ortogonais. Assim, não é necessário obter a ortogonalidade entre os parâmetros de interessere e de perturbação em qualquer uma das duas situações: $\mu=\tau$ e $\nu=\phi, \mu=\phi$ e $\nu=\tau$.

Considerando o primeiro caso,

$$
\widehat{\phi}_{\tau}=\frac{1}{n} \sum_{j=1}^{n}\left(y_{j}-\tau\right)^{2}=\widehat{\phi}+(\bar{y}-\tau)^{2}=\widehat{\phi}+O_{p}\left(n^{-1}\right),
$$

pois $\bar{y}-\tau=\widehat{\tau}-\tau=O_{p}\left(n^{-1 / 2}\right)$.

Da seção 2.4,

$$
\ell_{p}(\tau)=-\frac{n}{2} \log \sum_{j=1}^{n}\left(y_{j}-\tau\right)^{2}
$$

Calculando a informação observada $j_{\phi \phi}(\tau, \phi)$, obtém-se

$$
j_{\phi \phi}(\tau, \phi)=\frac{n^{3}}{2\left[\sum_{j=1}^{n}\left(y_{j}-\tau\right)^{2}\right]^{2}} .
$$

Conseqüentemente,

$$
\ell_{C R}(\tau)=\ell_{p}(\tau)-\frac{1}{2} \log j_{\phi \phi}\left(\tau, \widehat{\phi}_{\tau}\right)=\left(1-\frac{n}{2}\right) \log \sum_{j=1}^{n}\left(y_{j}-\tau\right)^{2}=\ell_{B N}(\tau) .
$$

Assim, $\ell_{C R}(\tau)$ herda todas as propriedades de $\ell_{B N}(\tau)$.

Agora, considere a variância $\phi$ como parâmetro de interesse. Sabe-se que $\widehat{\tau}_{\phi}=\bar{y}$. Como $\widehat{\tau}=\bar{y}$, então $\partial \widehat{\tau}_{\phi} / \partial \widehat{\tau}=1$. Logo,

$$
\ell_{C R}(\phi)=\ell_{B N}(\phi)=-\frac{n-1}{2} \log \phi-\frac{1}{2 \phi} \sum_{j=1}^{n}\left(y_{j}-\bar{y}\right)^{2}
$$

Aqui, também, $\ell_{C R}(\phi)$ herda todas as propriedades de $\ell_{B N}(\phi)$.

EXEMPLO 2.2 (continuação): distribuição gama com parâmetros $\omega$ e $\vartheta$.

Os parâmetros $\omega$ e $\vartheta$ que indexam a distribuição $G(\omega, \vartheta)$ não são ortogonais. Assim, como essa distribuição pertence à família exponencial biparamétrica, uma parametrização ortogonal é obtida resolvendo a equação diferencial (2.10). 


$$
\mu=\omega \quad \nu=\vartheta
$$

Aplicando (2.10), duas possíveis soluções para $\vartheta(\omega, \lambda)$ são

$$
\vartheta_{1}\left(\omega, \lambda_{1}\right)=\frac{\omega}{\lambda_{1}} \quad \text { e } \quad \vartheta_{2}\left(\omega, \lambda_{2}\right)=\omega \exp \left(\lambda_{2}\right)
$$

Desprezando os termos que dependem apenas da amostra, os logaritmos das verossimilhanças propostas por Cox e Reid $(1987), \ell_{C R 1}(\omega)$ e $\ell_{C R 2}(\omega)$, são iguais:

$$
\ell_{C R 1}(\omega)=\ell_{C R 2}(\omega)=\ell_{p}(\omega)-\frac{1}{2} \log \omega .
$$

Observe que $\ell_{C R 1}(\omega)=\ell_{C R 2}(\omega)=\ell_{B N}(\omega)$ e, assim, tais verossimilhanças herdam as propriedades de $\ell_{B N}(\omega)$, como, por exemplo, viés da informação de ordem $O\left(n^{-1}\right)$.

$$
\mu=\vartheta \quad \nu=\omega
$$

Pela equação (2.10),

$$
\frac{\partial \omega(\delta, \vartheta)}{\partial \vartheta}=\frac{1}{\vartheta \psi^{\prime}(\omega(\delta, \vartheta))} \quad \Longleftrightarrow \quad \frac{\partial \psi(\omega(\delta, \vartheta))}{\partial \vartheta}=\frac{1}{\vartheta}
$$

Assim, $\psi(\omega(\delta, \vartheta))=\log \vartheta+K(\delta)$, em que $K(\delta)$ é uma função qualquer de $\delta$. Observe que não é possível obter explicitamente $\omega=\omega(\delta, \vartheta)$ tal que $\delta$ e $\vartheta$ sejam ortogonais. Conseqüentemente, não é possível obter o logaritmo da função de verossimilhança proposta por Cox e Reid (1987) quando o parâmetro de interesse é $\vartheta$. Esta situação é conveniente para utilização da aproximação $\ell_{A C R}(\mu)$ proposta por Cox e Reid (1993).

Usando (2.12), tem-se

$$
\ell_{A C R}(\vartheta)=\ell_{p}(\vartheta)+\vartheta m^{(0,1)}(\widehat{\vartheta}, \widehat{\omega})-\frac{1}{2} \log \left(\psi^{\prime}\left(\widehat{\omega}_{\vartheta}\right)\right),
$$

em que $\widehat{\omega}_{\vartheta}$ satisfaz a equação

$$
n \log \vartheta+\sum_{j=1}^{n} \log y_{j}=n \psi\left(\widehat{\omega}_{\vartheta}\right)
$$

Aqui,

$$
m(\vartheta, \omega)=-\frac{\omega}{\vartheta\left(1+\omega \psi^{\prime}(\omega)\right)} \quad \text { e } \quad m^{(0,1)}(\vartheta, \omega)=\frac{\omega^{2} \psi^{\prime \prime}(\omega)-1}{\vartheta\left(1+\omega \psi^{\prime}(\omega)\right)^{2}} .
$$

A correspondente função escore é dada por

$$
u_{A C R}(\vartheta)=n \frac{\widehat{\omega}_{\vartheta}}{\vartheta}-\sum_{j=1}^{n} y_{j}+m^{(0,1)}(\widehat{\vartheta}, \widehat{\omega})-\frac{\psi^{\prime \prime}\left(\widehat{\omega}_{\vartheta}\right)}{2 \vartheta\left(\psi^{\prime}\left(\widehat{\omega}_{\vartheta}\right)\right)^{2}} .
$$


EXEMPLO 2.3 (continuação): distribuição beta com parâmetros $a$ e $b$.

Para calcular a verossimilhança proposta por Cox e Reid (1987), é necessário que os parâmetros de interesse e de incômodo sejam ortogonais. Esta propriedade não é observada entre os parâmetros da distribuição $B(a, b)$. Entretanto, para $\mu=a$ e $\nu=b$, não é possível obter explicitamente um parâmetro de incômodo ortogonal. Logo, é conveniente o uso da aproximação proposta por Cox e Reid (1993).

- $\ell_{A C R}(a)$ e $u_{A C R}(a)$

$$
\ell_{A C R}(a)=\ell_{p}(a)+a m^{(0,1)}(\widehat{a}, \widehat{b})-\frac{1}{2} \log \left(\psi^{\prime}\left(\widehat{b}_{a}\right)-\psi^{\prime}\left(a+\widehat{b}_{a}\right)\right),
$$

em que $\widehat{b}_{a}$ satisfaz a equação

$$
\sum_{j=1}^{n} \log \left(1-y_{j}\right)+n\left[\psi\left(a+\widehat{b}_{a}\right)-\psi\left(\widehat{b}_{a}\right)\right]=0
$$

e $m^{(0,1)}(a, b)$ é igual a

$$
\begin{gathered}
\frac{\left[\psi^{\prime \prime}(b) \psi^{\prime}(a+b)-\psi^{\prime}(b) \psi^{\prime \prime}(a+b)\right]\left[\psi^{\prime}(a+b)-\psi^{\prime}(a)\right]^{2}-\psi^{\prime}(a) \psi^{\prime \prime}(a+b)\left(\psi^{\prime}(a+b)\right)^{2}}{\left[\psi^{\prime}(a+b) \psi^{\prime}(a)+\psi^{\prime}(b)\left(\psi^{\prime}(a+b)-\psi^{\prime}(a)\right)\right]^{2}} . \\
u_{A C R}(a)=u_{p}(a)+m^{(0,1)}(\widehat{a}, \widehat{b})+\frac{\psi^{\prime \prime}\left(a+\widehat{b}_{a}\right) \psi^{\prime}\left(\widehat{b}_{a}\right)-\psi^{\prime}\left(a+\widehat{b}_{a}\right) \psi^{\prime \prime}\left(\widehat{b}_{a}\right)}{2\left[\psi^{\prime}\left(\widehat{b}_{a}\right)-\psi^{\prime}\left(a+\widehat{b}_{a}\right)\right]^{2}} \\
\widehat{a}_{A C R} \text { não tem forma fechada. }
\end{gathered}
$$

- viés da função escore

Não foi possível determinar a esperança $E\left\{u_{A C R}(a)\right\}$.

EXEMPLO 2.4 (continuação): distribuição gaussiana inversa com média $\gamma$ e coeficiente de variação $\zeta$.

$$
\begin{gathered}
\mu=\zeta \quad \nu=\gamma \\
\Delta_{\zeta}=n^{2} \zeta^{4}+4 n \bar{y} \sum_{j=1}^{n}\left(1 / y_{j}\right)
\end{gathered}
$$

- parametrização ortogonal $(\zeta, \lambda)$

$$
\gamma_{1}\left(\zeta, \lambda_{1}\right)=\lambda_{1}\left(\zeta^{2}+2\right) \quad \text { e } \quad \gamma_{2}\left(\zeta, \lambda_{2}\right)=\exp \left(\lambda_{2}\right)\left(\zeta^{2}+2\right)
$$


- $\ell_{C R 1}(\zeta)$ e $u_{C R 1}(\zeta)$

$$
\ell_{C R 1}(\zeta)=\ell_{p}(\zeta)+\log \left(\frac{\zeta}{\zeta^{2}+2}\right)+\log \left(n \zeta^{2}+\sqrt{\Delta_{\zeta}}\right)-\frac{1}{4} \log \Delta_{\zeta}
$$

$u_{C R 1}(\zeta)=u_{p}(\zeta)+\frac{4 n \bar{y} \sum_{j=1}^{n}\left(1 / y_{j}\right)}{\zeta \Delta_{\zeta}}+\frac{2 n \zeta}{\sqrt{\Delta_{\zeta}}}-\frac{2 \zeta}{\zeta^{2}+2} \quad$ e $\quad \widehat{\zeta}_{C R 1}$ não tem forma fechada.

- $\ell_{C R 2}(\zeta)$ e $u_{C R 2}(\zeta)$

$$
\begin{gathered}
\ell_{C R 2}(\zeta)=\ell_{p}(\zeta)-\frac{1}{4} \log \Delta_{\zeta}+\log \zeta \quad \text { e } \quad u_{C R 2}(\zeta)=u_{p}(\zeta)+\frac{4 n \bar{y} \sum_{j=1}^{n}\left(1 / y_{j}\right)}{\zeta \Delta_{\zeta}} \\
\widehat{\zeta}_{C R 2} \text { não tem forma fechada. }
\end{gathered}
$$

- viés da função escore

Não foi possível determinar as esperanças $E\left\{u_{C R 1}(\zeta)\right\}$ e $E\left\{u_{C R 2}(\zeta)\right\}$. Note que $\ell_{C R 2}(\zeta)=$ $\ell_{B N}(\zeta)$, conseqüentemente, os vieses da função escore e da informação associados a $u_{C R 2}(\zeta)$ são de ordem $O\left(n^{-1}\right)$.

\subsection{VEROSSIMILHANÇA PERFILADA MODIFICADA PROPOSTA POR McCULLAGH E TIBSHIRANI}

A modificação da verossimilhança perfilada apresentada por Barndorff-Nielsen (1983) reduz os vieses da informação e da função escore, enquanto, a proposta de Cox e Reid (1987), apenas este último. Entretanto, as obtenções de tais verossimilhanças requerem, respectivamente, uma estatística ancilar e a ortogonalidade dos parâmetros de interesse e de perturbação.

Se $u(\theta)$ é a função escore associada a uma função de verossimilhança genuína, então

$$
E\{u(\theta)\}=0 \quad \text { e } \quad E\left\{u(\theta) u^{\top}(\theta)\right\}+E\left\{\frac{\partial u(\theta)}{\partial \theta^{\top}}\right\}=0 .
$$

A fim de ajustar a verossimilhança perfilada para que a função escore derivada obedeça as igualdades acima, McCullagh e Tibshirani (1990) propuseram uma modificação na função escore perfilada e definiram a seguinte função escore, denotada por $u_{M T}(\mu)$ :

$$
u_{M T}(\mu)=w(\mu)\left\{u_{p}(\mu)-m(\mu)\right\}
$$

em que as funções $m(\mu)$ e $w(\mu)$ são obtidas do sistema de equações

$$
E_{\left(\mu, \widehat{\nu}_{\mu}\right)}\left\{u_{M T}(\mu)\right\}=0 \quad \text { e } \quad E_{\left(\mu, \widehat{\nu}_{\mu}\right)}\left\{u_{M T}(\mu) u_{M T}^{\top}(\mu)\right\}+E_{\left(\mu, \widehat{\nu}_{\mu}\right)}\left\{\frac{\partial u_{M T}(\mu)}{\partial \mu^{\top}}\right\}=0 .
$$


Observe que as esperanças são calculadas sob $\left(\mu, \widehat{\nu}_{\mu}\right)$ e para todo $\mu$.

Dessa forma, o logaritmo da verossimilhança perfilada modificada proposta por McCullagh e Tibshirani (1990) é dado por

$$
\ell_{M T}(\mu)=\int^{\mu} u_{M T}(t) d t
$$

Aqui, mais uma vez, não se trata de uma verossimilhança genuína. Espera-se que, ao fazer esses ajustes em $u_{p}(\mu)$, o comportamento assintótico de quantidades derivadas da nova verossimilhança melhore. O respectivo estimador de máxima verossimilhança é denotado por $\widehat{\mu}_{M T}$.

Quando $\mu$ é unidimensional, a solução do sistema de equações para $m(\mu)$ e $w(\mu)$ é

$$
m(\mu)=E_{\left(\mu, \widehat{\nu}_{\mu}\right)}\left\{u_{p}(\mu)\right\} \quad \text { e } \quad w(\mu)=\frac{-E_{\left(\mu, \widehat{\nu}_{\mu}\right)}\left\{u_{p}^{\prime}(\mu)\right\}+m^{\prime}(\mu)}{\operatorname{Var}_{\left(\mu, \widehat{\nu}_{\mu}\right)}\left\{u_{p}(\mu)\right\}},
$$

em que $u_{p}^{\prime}(\mu)=\partial u_{p}(\mu) / \partial \mu$ e $m^{\prime}(\mu)=\partial m(\mu) / \partial \mu$. Quando $\mu$ é multidimensional, $u_{p}(\mu)$ é um vetor de mesma dimensão. Conseqüentemente,

$$
m(\mu)=E_{\left(\mu, \widehat{\nu}_{\mu}\right)}\left\{u_{p}(\mu)\right\}
$$

é um vetor e

$$
w(\mu)=\operatorname{Var}_{\left(\mu, \widehat{\nu}_{\mu}\right)}^{-1}\left\{u_{p}(\mu)\right\}\left\{-E_{\left(\mu, \widehat{\nu}_{\mu}\right)}\left\{\frac{\partial u_{p}(\mu)}{\partial \mu^{\top}}\right\}+\frac{\partial m(\mu)}{\partial \mu^{\top}}\right\}^{\top}
$$

é uma matriz quadrada. Neste caso, em geral, $\partial u_{M T}^{a}(\mu) / \mu^{b} \neq \partial u_{M T}^{b}(\mu) / \mu^{a}$, onde $u_{M T}^{a}(\mu)$ e $\mu^{a}$ denotam o $a$-ésimo componente dos vetores $u_{M T}(\mu)$ e $\mu$, respectivamente. Portanto, quando $\mu$ é multidimensional, geralmente não existe uma função com vetor gradiente $u_{M T}(\mu)$.

Como $\ell_{p}(\mu)$, essa verossimilhança é invariante sob reparametrizações que preservam $\mu$. Observe que se $m(\mu)=0$, então os estimadores de máxima verossimilhança obtidos da $\exp \left(\ell_{M T}(\mu)\right)$ e da verossimilhança original são iguais, pois $w(\mu) \neq 0$. Agora, se $m(\mu) \neq 0$, então, mesmo que $w(\mu)$ seja igual a 1 , tal igualdade não é necessariamente observada.

O cálculo de $\ell_{M T}(\mu)$ requer a obtenção das seguintes quantidades:

$$
m(\mu)=E_{\left(\mu, \widehat{\nu}_{\mu}\right)}\left\{u_{p}(\mu)\right\}, \quad \operatorname{Var}_{\left(\mu, \widehat{\nu}_{\mu}\right)}\left\{u_{p}(\mu)\right\} \quad \text { e } \quad E_{\left(\mu, \widehat{\nu}_{\mu}\right)}\left\{\frac{\partial u_{p}(\mu)}{\partial \mu^{\top}}\right\} .
$$

Todas estas envolvem esperanças com respeito a $p\left(y ;\left(\mu, \widehat{\nu}_{\mu}\right)\right)$ que, às vezes, podem ser calculadas analiticamente. Porém, em geral, recorre-se a simulações de Monte Carlo para estimá-las. Assim, quando $\mu$ é unidimensional, o algoritmo para o cálculo do logaritmo da verossimilhança proposta por McCullagh e Tibshirani (1990) é dado por:

(i) obtenha $\widehat{\nu}_{\mu}$ e calcule $\ell_{p}(\mu)$;

(ii) defina um conjunto de valores para $\mu$ (em geral, 40 valores igualmente espaçados); 
(iii) para cada um dos valores de $\mu$, gere $B$ amostras (em geral, 25) de $p\left(y ;\left(\mu, \widehat{\nu}_{\mu}\right)\right)$ do mesmo tamanho da amostra original e obtenha $k$ valores para $\ell_{p}(\mu)$ e para $u_{p}(\mu)$, denotados, genericamente, por $\ell_{p}^{k}(\mu)$ e $u_{p}^{k}(\mu)$;

(iv) para cada um dos valores de $\mu$, estime as quantidades acima por:

$$
\widehat{m}(\mu)=\frac{1}{B} \sum_{k=1}^{B} u_{p}^{k}(\mu), \quad \widehat{E}_{\left(\mu, \widehat{\nu}_{\mu}\right)}\left\{u_{p}^{\prime}(\mu)\right\}=\frac{1}{B} \sum_{k=1}^{B} u_{p}^{\prime k}(\mu)
$$

e

$$
\widehat{w}(\mu)=\left\{-\widehat{E}_{\left(\mu, \widehat{\nu}_{\mu}\right)}\left\{u_{p}^{\prime}(\mu)\right\}+m^{\prime}(\mu)\right\} / \frac{1}{B} \sum_{k=1}^{B}\left(u_{p}^{k}(\mu)-\widehat{m}(\mu)\right)^{2} ;
$$

(v) a partir do conjunto de valores obtidos para $m(\mu)$ e $w(\mu)$, calcule numericamente a integral abaixo

$$
\ell_{M T}(\mu)=\int^{\mu}\left\{u_{p}(t)-m(t)\right\} w(t) d t
$$

Para estimar esta integral, pode ser usada a regra dos trapézios. Já para estimar $m^{\prime}(\mu)$, uma possibilidade é o método de diferenças finitas. Para maiores detalhes, ver Ruggiero e Lopes (1997).

Ainda, diante da dificuldade de obtenção de $\ell_{M T}(\mu)$, podem ser usadas as aproximações de primeira ordem, derivadas em McCullagh e Tibshirani (1990), para a esperança dos componentes do vetor escore $u_{p}(\mu)$ e para os elementos da matriz de covariância destes componentes calculados sob o valor verdadeiro do vetor paramétrico. Assim, tais expressões são funções de $(\mu, \nu)$ e não apenas de $\mu$. Na prática, $\nu$ é substituído por $\widehat{\nu}_{\mu}$, introduzindo assim uma dependência dos dados em $m(\mu)$ e $w(\mu)$. * Como conseqüência, a nulidade dos vieses da função escore e da informação pode não ser observada, como exemplificado em Macaskill (1993).

Para apresentar estas aproximações, são necessárias algumas convenções e notações. Para denotar cada um dos componentes do vetor $\mu$ são utilizados os índices $a, b, c, \ldots$ e, no caso do vetor $\nu, i, j, k, \ldots$ Quando um destes índices aparece duas ou mais vezes em um produto de elementos de matrizes ou de vetores, então subentende-se que há uma soma sobre tal índice. Por exemplo, o produto interno de dois vetores de mesma dimensão, $v$ e $z$, pode ser expresso, segundo esta convenção, por

$$
<v, z>=v^{r} z^{r} .
$$

Já a forma quadrática $v^{\top} A v$, em que $A=\left[a_{i j}\right]$ é uma matriz quadrada, pode ser apresentada como

$$
v^{\top} A v=a_{i j} v^{i} v^{j} .
$$

* Da equação (7.10) de McCullagh (1987), vem $\widehat{\nu}_{\mu}^{i}-\nu^{i}=O_{p}\left(n^{-1 / 2}\right)$. 
Além disso, são utilizadas as seguintes notações para derivadas do logaritmo da verossimilhança original $\ell=\ell(\mu, \nu)$ :

$$
\begin{aligned}
& \ell_{a}=\frac{\partial \ell}{\partial \mu^{a}}, \quad \ell_{a b}=\frac{\partial^{2} \ell}{\partial \mu^{a} \partial \mu^{b}}, \quad \ell_{i}=\frac{\partial \ell}{\partial \nu^{i}}, \quad \ell_{i j}=\frac{\partial^{2} \ell}{\partial \nu^{i} \partial \nu^{j}}, \\
& \ell_{a, b}=\ell_{a} \ell_{b}, \quad \ell_{a i}=\frac{\partial^{2} \ell}{\partial \mu^{a} \partial \nu^{i}} \\
& \ell_{i, j}=\ell_{i} \ell_{j}, \quad \ell_{a, i}=\ell_{a} \ell_{i}, \quad \ell_{a, i, j}=\ell_{a} \ell_{i} \ell_{j}, \quad \ell_{i, j, k}=\ell_{i} \ell_{j} \ell_{k} .
\end{aligned}
$$

Os respectivos cumulantes são denotados por:

$$
\begin{gathered}
\kappa_{a}=E\left\{\ell_{a}\right\}=0, \quad \kappa_{i}=E\left\{\ell_{i}\right\}=0, \quad \kappa_{a b}=E\left\{\ell_{a b}\right\}=-\kappa_{a, b}, \quad \kappa_{i j}=E\left\{\ell_{i j}\right\}=-\kappa_{i, j}, \\
\kappa_{a i}=E\left\{\ell_{a i}\right\}=-E\left\{\ell_{a} \ell_{i}\right\}, \kappa_{a, i, j}=E\left\{\ell_{a} \ell_{i} \ell_{j}\right\}, \kappa_{i, j, k}=E\left\{\ell_{i} \ell_{j} \ell_{k}\right\}, \kappa_{a, i j}=E\left\{\ell_{a} \ell_{i j}\right\} .
\end{gathered}
$$

Assim, McCullagh e Tibshirani (1990) obtiveram

$$
E\left\{\frac{\partial \ell_{p}(\mu)}{\partial \mu^{a}}\right\}=-\frac{1}{2}\left(\kappa_{a, i j}-\kappa_{a, k} \kappa^{k, l} \kappa_{l, i j}\right) \kappa^{i, j}-\frac{1}{2}\left(\kappa_{a, i, j}-\kappa_{a, k} \kappa^{k, l} \kappa_{l, i, j}\right) \kappa^{i, j}+O\left(n^{-1}\right),
$$

em que $\left[\kappa^{i, j}\right]$ denota a matriz inversa de $\left[\kappa_{i, j}\right]$, que é a matriz de informação de Fisher para $\nu$ com $\mu$ fixado. Este resultado foi utilizado por Stern (1997) para derivar uma verossimilhança perfilada modificada com vieses da função escore e da informação de ordem $O\left(n^{-1}\right)$.

A obtenção de uma expansão para a covariância de elementos do vetor $u_{p}(\mu)$ é mais complicada no caso geral. Assim, McCullagh e Tibshirani (1990) derivaram tal expansão para a situação em que, fixado $\mu$, o logaritmo da verossimilhança para $\nu$ é um modelo da família exponencial com a dimensão da estatística suficiente igual a dimensão de $\nu$. Não é necessário aqui que $\ell(\mu, \nu)$ seja do tipo da família exponencial. Pode-se reparametrizar $\nu$ para que $\ell_{i j}$ seja uma constante: parametrização canônica. Sob tais condições, segue que

$$
\begin{gathered}
E\left\{\frac{\partial \ell_{p}(\mu)}{\partial \mu^{a}}\right\}=-\frac{1}{2} \kappa_{a, i, j} \kappa^{i, j}+O\left(n^{-1}\right) \\
\operatorname{Cov}\left\{\frac{\partial \ell_{p}(\mu)}{\partial \mu^{a}}, \frac{\partial \ell_{p}(\mu)}{\partial \mu^{b}}\right\}=\kappa_{a, b}-\frac{1}{2} \kappa_{a, i, j} \kappa_{b, k, l} \kappa^{i, k} \kappa^{j, l}+O\left(n^{-1}\right)
\end{gathered}
$$

$\mathrm{e}$

$$
-E\left\{\frac{\partial}{\partial \mu^{b}}\left[\frac{\partial \ell_{p}(\mu)}{\partial \mu^{a}}-M_{a}\right]\right\}=\kappa_{a, b}-\frac{1}{2} \kappa_{a, b, i} \kappa_{j, k, l} \kappa^{i, j} \kappa^{k, l}-\frac{1}{2} \kappa_{a, i, j} \kappa_{b, k, l} \kappa^{i, k} \kappa^{j, l}+O\left(n^{-1}\right),
$$

onde $M_{a}=-(1 / 2) \kappa_{a, i, j} \kappa^{i, j}$ é o viés de primeira ordem do a-ésimo componente do vetor $u_{p}(\mu)$.

Apesar da originalidade da proposta de modificação da verossimilhança perfilada apresentada por McCullagh e Tibshirani (1990), ainda assim, tal verossimilhança, em geral, parece não produzir os resultados esperados, conforme Macaskill (1993), que utilizou tal proposta em um problema de regressão não-linear. Além disso, se $\widehat{\nu}_{\mu}$ não tiver forma fechada, 
então possivelmente $u_{M T}(\mu)$ também não terá e, assim, não será possível determinar $\ell_{M T}(\mu)$, mesmo numericamente (ver seção 2.8 , exemplos 2.2 e 2.3).

\subsection{EXEMPLOS}

A seguir, é exemplificada a obtenção de $\ell_{M T}(\mu)$ a partir de algumas distribuições da família exponencial biparamétrica. Como já foi salientado, a derivação exata de $\ell_{M T}(\mu)$ é por vezes difícil, inviabilizando seu uso em algumas situações. Assim, aqui, os cálculos das esperanças matemáticas necessárias para obter $m(\mu)$ e $w(\mu)$ são realizados sob $(\mu, \nu)$ e, quando necessário, $\nu$ é substituído por $\widehat{\nu}_{\mu}$ na expressão final.

EXEMPLO 2.1 (continuação): distribuição normal com média $\tau$ e variância $\phi$.

$$
\begin{gathered}
\mu=\tau \quad \nu=\phi \\
u_{p}(\tau)=n \frac{\sum_{j=1}^{n}\left(y_{j}-\tau\right)}{\sum_{j=1}^{n}\left(y_{j}-\tau\right)^{2}} \quad \text { e } \quad E\left\{u_{p}(\tau)\right\}=0 .
\end{gathered}
$$

Conseqüentemente,

$$
m(\tau)=0 \quad \text { e } \quad \operatorname{Var}\left\{u_{p}(\tau)\right\}=E\left\{u_{p}^{2}(\tau)\right\} .
$$

Aqui,

$$
\frac{\partial u_{p}(\tau)}{\partial \tau}=\frac{2 u_{p}^{2}(\tau)}{n}-\frac{n^{2}}{\sum_{j=1}^{n}\left(y_{j}-\tau\right)^{2}} .
$$

Sabe-se que

$$
z=\sum_{j=1}^{n}\left(\frac{y_{j}-\tau}{\sqrt{\phi}}\right)^{2} \sim \chi_{n}^{2} \quad \text { e } \quad E\left\{\frac{1}{z}\right\}=\frac{1}{n-2} .
$$

Calculando $E\left\{u_{p}^{2}(\gamma)\right\}$, obtém-se

$$
E\left\{u_{p}^{2}(\tau)\right\}=\frac{n^{2}}{2 \phi} \frac{\Gamma((n-2) / 2)}{\Gamma(n / 2)}=\frac{n^{2}}{2 \phi} \frac{1}{(n / 2)-1}=\frac{n^{2}}{\phi(n-2)} .
$$

Logo,

$$
-E\left\{\frac{\partial u_{p}(\tau)}{\partial \tau}\right\}=-\frac{2}{n} \frac{n^{2}}{\phi(n-2)}+n^{2} \frac{1}{\phi(n-2)}=\frac{n}{\phi} .
$$

Pela definição de $w(\mu)$, vem

$$
w(\tau)=\frac{n / \phi}{n^{2} / \phi(n-2)}=\frac{n-2}{n} .
$$


Assim,

e, portanto,

$$
u_{M T}(\tau)=\frac{n-2}{n} u_{p}(\tau)
$$

$$
\begin{gathered}
\ell_{M T}(\tau)=\frac{n-2}{n} \ell_{p}(\tau)=\ell_{B N}(\tau)=\ell_{C R}(\tau) . \\
\mu=\phi \quad \nu=\tau \\
u_{p}(\phi)=\frac{1}{2 \phi^{2}} \sum_{j=1}^{n}\left(y_{j}-\bar{y}\right)^{2}-\frac{n}{2 \phi} \quad \text { e } \quad E\left\{u_{p}(\phi)\right\}=m(\phi)=-\frac{1}{2 \phi} .
\end{gathered}
$$

Por conseguinte,

$$
\frac{\partial u_{p}(\phi)}{\partial \phi}=-\frac{1}{\phi^{3}} \sum_{j=1}^{n}\left(y_{j}-\bar{y}\right)^{2}+\frac{n}{2 \phi^{2}}, \quad \operatorname{Var}\left\{u_{p}(\phi)\right\}=\frac{1}{4 \phi^{4}} \operatorname{Var}\left\{\sum_{j=1}^{n}\left(y_{j}-\bar{y}\right)^{2}\right\}
$$

e

$$
m^{\prime}(\phi)=1 /\left(2 \phi^{2}\right) .
$$

Como

$$
\frac{\sum_{j=1}^{n}\left(y_{j}-\bar{y}\right)^{2}}{\phi} \sim \chi_{n-1}^{2},
$$

então

$$
-E\left\{\frac{\partial u_{p}(\phi)}{\partial \phi}\right\}=\frac{1}{\phi^{3}} \phi(n-1)-\frac{n}{2 \phi^{2}}=\frac{n}{2 \phi^{2}}-\frac{1}{\phi^{2}}
$$

e

$$
\operatorname{Var}\left\{u_{p}(\phi)\right\}=\frac{1}{4 \phi^{4}} 2 \phi^{2}(n-1)=\frac{n-1}{2 \phi^{2}} .
$$

Usando estas quantidades, $w(\phi)=1 \mathrm{e}$, pela definição,

$$
u_{M T}(\phi)=u_{p}(\phi)+\frac{1}{2 \phi} .
$$

Observe que $u_{M T}(\phi)=u_{B N}(\phi)=u_{C R}(\phi)$. Dessa forma,

$$
\ell_{M T}(\phi)=\ell_{B N}(\phi)=\ell_{C R}(\phi)=-\frac{n-1}{2} \log \phi-\frac{1}{2 \phi} \sum_{j=1}^{n}\left(y_{j}-\bar{y}\right)^{2} .
$$

EXEMPLO 2.2 (continuação): distribuição gama com parâmetros $\omega$ e $\vartheta$.

Nos dois casos considerados ao longo deste capítulo, $(\mu, \nu)=(\omega, \vartheta)$ e $(\mu, \nu)=(\vartheta, \omega)$, não é possível obter a expressão exata de $\ell_{M T}(\mu)$ analiticamente. 


$$
\begin{gathered}
\mu=\omega \quad \nu=\vartheta \\
u_{p}(\omega)=n \log \left(\frac{\omega}{\bar{y}}\right)-n \psi(\omega)+\sum_{j=1}^{n} \log y_{j} \quad \text { e } \quad E\left\{u_{p}^{\prime}(\omega)\right\}=\frac{n}{\omega}-n \psi^{\prime}(\omega) .
\end{gathered}
$$

Usando o fato que $\bar{y} \sim G(n \omega, n \vartheta)$, obtém-se

$$
m(\omega)=E\left\{u_{p}(\omega)\right\}=n[\log (n \omega)-\psi(n \omega)],
$$

cuja derivada é

$$
m^{\prime}(\omega)=n\left[\frac{1}{\omega}-n \psi^{\prime}(n \omega)\right] .
$$

O cálculo da variância $\operatorname{Var}\left\{u_{p}(\omega)\right\}$, necessária para derivar $w(\omega)$, requer a esperança $E\left\{\log (\bar{y}) \times \log \left(\prod_{j=1}^{n} y_{j}\right)\right\}$. A integral envolvida nesta esperança matemática é de difícil resolução. Assim, aqui pode ser utilizada a aproximação (2.14), pois, fixado $\omega$ em $l(\omega, \vartheta)$, o logaritmo da verossimilhança para $\vartheta$ é um modelo da família exponencial com a dimensão da estatística suficiente igual a um e com parâmetro canônico $\vartheta$.

Segundo McCullagh e Tibshirani (1990), considerando a parametrização ortogonal $(\omega, \lambda)$, em que $\lambda=\omega / \vartheta$ (seção 2.6), pode ser mostrado que $m(\omega) \doteq 1 /(2 \omega)$ e $w(\omega) \doteq 1$. Dessa forma, $\ell_{M T}(\omega) \doteq \ell_{B N}(\omega)$.

$$
\begin{gathered}
\mu=\vartheta \quad \nu=\omega \\
u_{p}(\vartheta)=n \frac{\widehat{\omega}_{\vartheta}}{\vartheta}-\sum_{j=1}^{n} y_{j},
\end{gathered}
$$

em que $\widehat{\omega}_{\vartheta}$ satisfaz

$$
n \log \vartheta+\sum_{j=1}^{n} \log y_{j}=n \psi\left(\widehat{\omega}_{\vartheta}\right) .
$$

Como $u_{p}(\vartheta)$ não é definida unicamente (explicitamente) em função de $\vartheta$, torna-se impossível derivar analiticamente uma expressão para a verossimilhança perfilada proposta por McCullagh e Tibshirani (1990), mesmo usando as aproximações (2.13), (2.14) e (2.15), pois $\ell_{M T}(\vartheta)$ é a primitiva da integral indefinida de $u_{M T}(\vartheta)$.

Diante da dificuldade de derivação de uma expressão para $u_{M T}(\mu)$ analiticamente, podese usar simulações de Monte Carlo para estimar $m(\mu)$ e $w(\mu)$ e, em seguida, utilizar um método de integração numérica para obter $\ell_{M T}(\mu)$. Mas, aqui, observe que não possível obter valores para $u_{p}(\vartheta)$ e, assim, mesmo numericamente, não é possível obter $\ell_{M T}(\vartheta)$. 
EXEMPLO 2.3 (continuação): distribuição beta com parâmetros $a$ e $b$.

Como no exemplo 2.2 (distribuição gama com parâmetros $\omega$ e $\vartheta$ ), e, mais precisamente, como no caso em que $(\mu, \nu)=(\vartheta, \omega)$, não é possível obter $\ell_{M T}(a)$, tanto analiticamente quanto numericamente $(\nu=b)$.

EXEMPLO 2.4 (continuação): distribuição gaussiana inversa com média $\gamma$ e coeficiente de variação $\zeta$.

$$
\begin{gathered}
\mu=\zeta \quad \nu=\gamma \\
u_{p}(\zeta)=\frac{\sqrt{\Delta_{\zeta}}-n\left(\zeta^{2}+2\right)}{\zeta^{3}},
\end{gathered}
$$

em que $\Delta_{\zeta}=n^{2} \zeta^{4}+4 n \bar{y} \sum_{j=1}^{n}\left(1 / y_{j}\right)$

Aqui, não é possível obter a expressão exata de $\ell_{M T}(\zeta)$ analiticamente e, nem mesmo, utilizando as aproximações $(2.13),(2.14)$ e (2.15), pois, fixando o parâmetro de interesse $\zeta$, o logaritmo da verossimilhança para $\gamma$ não é um modelo da família exponencial completa (dimensão da estatística suficiente é dois). Entretanto, é possível obter $\ell_{M T}(\zeta)$ através de simulação computacional, usando o método "bootstrap paramétrico" para estimar os momentos da função escore perfilada numericamente, conforme o algoritmo apresentado na seção 2.7 .

\subsection{VEROSSIMILHANÇA PERFILADA MODIFICADA PROPOSTA POR STERN}

A fim de melhorar as propriedades assintóticas de quantidades derivadas da função de verossimilhança perfilada, Barndorff-Nielsen (1983) e Cox e Reid (1987) propuseram ajustes aditivos ao logaritmo desta função. Este mesmo enfoque foi seguido por Stern (1997). Sua proposta de modificação deste logaritmo tornou os vieses da função escore e da informação de ordem $O\left(n^{-1}\right)$, quando, em geral, os vieses apresentados por estas quantidades derivadas de uma verossimilhança perfilada são de ordem $O(1)$. Diferentemente das verossimilhanças propostas por McCullagh e Tibshirani (1990) e Cox e Reid (1987), tal verossimilhança sempre pode ser obtida no caso de um parâmetro de interesse multidimensional. Além disso, não requer uma estatística ancilar e nem a ortogonalidade dos parâmetros de interesse e 
de perturbação. Enfim, não apresenta as especificidades das verossimilhanças anteriores e, portanto, é aplicável a uma estrutura paramétrica mais geral.

Além das convenções e notações adotadas na seção 2.7, são necessárias mais algumas considerações para apresentar a proposta de modificação da verossimilhança perfilada devida a Stern (1997). Como $\theta=(\mu, \nu)$, para denotar um componente de $\theta$, são usados os índices $r, s, t, \ldots$ Assim, se estes variam de 1 a $(p+q)$, então $a, b, c, \ldots$ assumem valores entre $1 \mathrm{e}$ $p$ e $i, j, k, \ldots$, entre $(p+1)$ e $(p+q)$. Aqui, $\ell_{r}=\partial \ell / \partial \theta^{r}, \ell_{r s}=\partial^{2} \ell / \partial \theta^{r} \partial \theta^{s}, \kappa_{r}=E\left\{\ell_{r}\right\}$, $\kappa_{r s}=E\left\{\ell_{r s}\right\}, \kappa_{r, s}=E\left\{\ell_{r} \ell_{s}\right\}, \kappa_{r s t}=E\left\{\ell_{r s t}\right\}, \kappa_{r s / t}=\partial \kappa_{r s} / \partial \theta^{t}, \kappa_{r s / t u}=\partial^{2} \kappa_{r s} / \partial \theta^{t} \partial \theta^{u}$, etc.. A matriz inversa de $\left[\kappa_{r s}\right]$ é denotada por $\left[\kappa^{r s}\right]$ e $\eta^{r s}=\kappa^{r s}-\tau^{r s}$, onde $\tau^{r s}=\kappa^{r a} \kappa^{s b} \sigma_{a b}$ com $\left[\sigma_{a b}\right]$ sendo a inversa de $\left[\kappa^{a b}\right]$, o bloco superior-esquerdo de dimensão $p \times p$ da matriz $\left[\kappa^{r s}\right]$. Note que todos os elementos da matriz $\left[\eta^{r s}\right]$ são nulos, exceto o bloco inferior-direito de dimensão $q \times q$, que é a inversa da matriz $\left[\kappa_{i j}\right]$.

Stern (1997) propôs o seguinte ajuste aditivo ao logaritmo da função de verossimilhança perfilada

$$
\ell_{S T}(\mu)=\ell_{p}(\mu)+\Lambda(\tilde{\theta}(\mu))+\Omega(\tilde{\theta}(\mu))
$$

em que

- $\tilde{\theta}(\mu)=\left(\mu, \widehat{\nu}_{\mu}\right)$

- $\Lambda(\theta)=A^{a}(\theta) \ell_{a}(\theta), \mathrm{com}$

$$
\ell_{a}(\theta)=\partial \ell(\theta) / \partial \mu^{a} \quad \text { e } \quad A^{a}(\theta)=\kappa^{a r} \eta^{s t}\left(\kappa_{r s / t}-\frac{1}{2} \kappa_{r s t}\right) ;
$$

- $\Omega(\theta)=\frac{1}{2} B^{a b}(\theta) \ell_{a}(\theta) \ell_{b}(\theta), \mathrm{com}$

$$
\begin{aligned}
B^{a b}(\theta) & =\kappa^{a r} \kappa^{b s} \kappa^{t v} \eta^{u w}\left(\frac{1}{2} \kappa_{r s t} \kappa_{u v w}-\kappa_{r s t} \kappa_{u v / w}+\kappa_{r t / s} \kappa_{u v / w}+\kappa_{s t / r} \kappa_{u v / w}\right. \\
& \left.-\frac{1}{2} \kappa_{r t / s} \kappa_{u v w}-\frac{1}{2} \kappa_{s t / r} \kappa_{u v w}\right) \\
& +\kappa^{a r} \kappa^{b s} \eta^{t v} \eta^{u w}\left(\frac{1}{2} \kappa_{r t u} \kappa_{s v w}-\kappa_{r t u} \kappa_{s v / w}-\kappa_{r t / u} \kappa_{s v w}+\kappa_{r t / u} \kappa_{s w / v}\right. \\
& \left.+\kappa_{r t / u} \kappa_{v w / s}+\kappa_{t u / r} \kappa_{s v / w}-\frac{1}{2} \kappa_{r t u} \kappa_{v w / s}-\frac{1}{2} \kappa_{t u / r} \kappa_{s v w}\right) \\
& -\kappa^{a r} \kappa^{b s} \eta^{t u}\left(\frac{1}{2} \kappa_{r s t u}-\kappa_{r s t / u}+\kappa_{r t / s u}+\kappa_{s t / r u}-\frac{1}{2} \kappa_{r t u / s}-\frac{1}{2} \kappa_{s t u / r}\right)
\end{aligned}
$$

A derivação desta verossimilhança é baseada em resultados obtidos por McCullagh e Tibshirani (1990) e DiCiccio et al. (1996). Vale salientar que a expressão para $B^{a b}(\theta)$ originalmente proposta por Stern apresentava alguns erros e foi corrigida por Ferrari et al. (2004). A correspondente função escore é denotada por $u_{S T}(\mu)$ e é igual a $\partial \ell_{S T}(\mu) / \partial \mu$. O ponto de máximo de $\ell_{S T}(\mu)$ é indicado por $\widehat{\mu}_{S T}$. Esta verossimilhança não é invariante sob reparametrizações que preservam o parâmetro de interesse. 
McCullagh e Tibshirani (1990) mostraram que

$$
E\left\{u_{p}^{a}(\mu)\right\}=\sigma_{a b} \kappa^{b r} \eta^{s t}\left(\frac{1}{2} \kappa_{r s t}-\kappa_{r s / t}\right)+O\left(n^{-1}\right)
$$

em que $u_{p}^{a}(\mu)=\partial \ell_{p}(\mu) / \partial \mu^{a}$.

Stern (1997) mostrou que

$$
E\left\{\Lambda_{a}(\tilde{\theta}(\mu))\right\}=\sigma_{a b} \kappa^{b r} \eta^{s t}\left(\kappa_{r s / t}-\frac{1}{2} \kappa_{r s t}\right)+O\left(n^{-1}\right)
$$

em que $\Lambda_{a}(\tilde{\theta}(\mu))=\partial \Lambda(\tilde{\theta}(\mu)) / \partial \mu^{a}$. Conseqüentemente,

$$
E\left\{u_{p}(\mu)+\frac{\Lambda(\tilde{\theta}(\mu))}{\partial \mu}\right\}=O\left(n^{-1}\right)
$$

Mesmo com o acréscimo do termo $\Lambda(\tilde{\theta}(\mu))$ ao logaritmo da verossimilhança perfilada, o viés da informação é, tipicamente, de ordem $O(1)$. Para torná-lo de ordem $O\left(n^{-1}\right)$, Stern propõe a adição de um segundo termo, $\Omega(\tilde{\theta}(\mu))$, baseado em um resultado obtido por DiCiccio et al. (1996).

Denotando o logaritmo de uma verossimilhança perfilada modificada por $\ell_{p m}(\mu)$ e supondo que a correspondente função escore apresenta viés de ordem $O\left(n^{-1}\right)$ - conforme o ajuste sob a função escore perfilada proporcionado pela adição de $\Lambda_{a}(\tilde{\theta}(\mu))$, ou seja, $\ell_{p m}(\mu)=$ $\ell_{p}(\mu)+M(\mu)$, onde $M(\mu)$, como $\Lambda(\tilde{\theta}(\mu))$, satisfaz (2.16) —, DiCiccio et al. (1996) mostraram que $\ell_{p m}(\mu)$ pode ser usado para construir uma nova verossimilhança, cujo logaritmo é aqui denotado por $\bar{\ell}_{p m}(\mu)$, tal que sua função escore, definida por

$$
\bar{u}_{p m}^{a}(\mu)=u_{p m}^{a}(\mu)+C_{a}^{b}(\mu) u_{p m}^{b}(\mu)
$$

apresenta viés da informação menor que aquele derivado de $\ell_{p m}(\mu)$. Aqui,

$$
\bar{u}_{p m}^{a}(\mu)=\frac{\partial \bar{\ell}_{p m}(\mu)}{\mu^{a}}, \quad u_{p m}^{a}(\mu)=\frac{\partial \ell_{p m}(\mu)}{\mu^{a}}, \quad C_{a}^{b}(\mu)=\varsigma_{a}^{b}(\theta)+O_{p}\left(n^{-3 / 2}\right)
$$

e $\varsigma_{a}^{b}(\theta)$ é uma quantidade não-aletória de ordem $O\left(n^{-1}\right)$. Note que $E\left\{\bar{u}_{p m}^{a}(\mu)\right\}=O\left(n^{-1}\right)$.

Assim, DiCiccio et al. (1996) obtiveram a seguinte expansão para o viés da informação de $\bar{u}_{p m}(\mu)$

$$
\begin{gathered}
E_{\theta}\left\{\bar{u}_{p m}^{a}(\mu) \bar{u}_{p m}^{b}(\mu)\right\}+E_{\theta}\left\{\partial \bar{u}_{p m}^{a}(\mu) / \partial \mu^{b}\right\}= \\
\Delta_{a b}-\rho_{a} \rho_{b}+\rho_{a / r} \sigma_{b c} \kappa^{r c}+\sigma_{a c} \rho_{b / r} \kappa^{c r}-\Psi_{b a}-\sigma_{a c} \varsigma_{b}^{c}+O\left(n^{-1}\right),
\end{gathered}
$$

em que

$$
\begin{gathered}
\Delta_{a c}=E_{\theta}\left\{u_{p}^{a}(\mu) u_{p}^{c}(\mu)\right\}+E_{\theta}\left\{\partial u_{p}^{a}(\mu) / \partial \mu^{c}\right\}, \\
\rho_{a}(\theta)=\sigma_{a b} \kappa^{b r} \eta^{s t}\left(\kappa_{r s / t}-\frac{1}{2} \kappa_{r s t}\right),
\end{gathered}
$$




$$
\rho_{a / r}=\partial \rho_{a}(\theta) / \partial \theta^{r}
$$

e

$$
\Psi_{a c}=E_{\theta}\left\{\frac{\partial^{2} M(\mu)}{\partial \mu^{a} \partial \mu^{c}}\right\} .
$$

Logo, o viés da informação é de ordem $O\left(n^{-1}\right)$ quando

$$
\begin{aligned}
\varsigma_{a}^{b}(\theta) & =\left(\Delta_{a c}-\rho_{a} \rho_{c}+\rho_{a / r} \sigma_{c d} \kappa^{r d}+\sigma_{a d} \rho_{c / r} \kappa^{d r}-\Psi_{a c}\right) \kappa^{b c}+O\left(n^{-2}\right)= \\
& =\Delta_{a c} \kappa^{b c}-\rho_{a} \rho_{c} \kappa^{b c}+\rho_{a / r} \kappa^{b r}+\sigma_{a d} \rho_{c / r} \kappa^{b c} \kappa^{d r}-\Psi_{a c} \kappa^{b c}+O\left(n^{-2}\right) .
\end{aligned}
$$

Para obter tal redução no viés da informação, é necessário que $\varsigma_{a}^{b}(\theta)$ seja avaliado em $\check{\theta}(\mu)$ tal que $\check{\theta}(\mu)-\theta=O_{p}\left(n^{-1 / 2}\right)$.

Utilizando este resultado, Stern (1997) acrescentou ao primeiro ajuste do logaritmo da verossimilhança perfilada, $\ell_{p}(\mu)+\Lambda(\tilde{\theta}(\mu))$, cuja função escore tem viés de ordem $O\left(n^{-1}\right)$ e é invariante sob reparametrizações que preservam $\mu$, uma segunda parcela, para reduzir o viés da informação.

Fazendo

$$
\ell_{p m}(\mu)=\ell_{p}(\mu)+A^{a}(\tilde{\theta}(\mu)) \ell_{a}(\tilde{\theta}(\mu))+\frac{1}{2} B^{a b}(\tilde{\theta}(\mu)) \ell_{a}(\tilde{\theta}(\mu)) \ell_{b}(\tilde{\theta}(\mu)),
$$

em que $B^{a b}(\theta)$ é uma função de ordem $O\left(n^{-2}\right)$, note que $u_{p m}(\mu)$ tem viés de ordem $O\left(n^{-1}\right)$. Aqui,

$$
M(\mu)=A^{a}(\tilde{\theta}(\mu)) \ell_{a}(\tilde{\theta}(\mu))+\frac{1}{2} B^{a b}(\tilde{\theta}(\mu)) \ell_{a}(\tilde{\theta}(\mu)) \ell_{b}(\tilde{\theta}(\mu)) .
$$

Usando (2.17), é possível construir um nova verossimilhança cujo viés da informação é reduzido a ordem $O\left(n^{-1}\right)$ quando $\varsigma_{a}^{b}(\theta)$ satisfaz (2.19). Calculando $\Psi_{a c}$, Stern (1997) mostrou que $\varsigma_{a}^{b}(\theta)=O\left(n^{-2}\right)$. Assim, por (2.18), o viés da informação de $\bar{u}_{p m}(\mu)$ é $O\left(n^{-1}\right)$ quando

$$
\Delta_{a b}-\rho_{a} \rho_{b}+\rho_{a / r} \sigma_{b c} \kappa^{r c}+\sigma_{a c} \rho_{b / r} \kappa^{c r}-\Psi_{b a}=0
$$

pois $\sigma_{a c} \varsigma_{b}^{c}=O\left(n^{-1}\right)$. Por (2.20), Stern (1997) obteve a expressão de $B^{a b}$ que torna o viés da informação de ordem $O\left(n^{-1}\right)$. *

Os cálculos das quantidades $\Lambda(\theta)$ e $\Omega(\theta)$ são bastante simplificados quando os parâmetros de interesse e de perturbação são ortogonais. Dessa forma, considerando $\mu$ e $\nu$ unidimensionais e ortogonais, obtém-se

e

$$
\Lambda(\theta)=-\frac{1}{2} \frac{\ell_{\mu} \kappa_{\mu \nu \nu}}{\kappa_{\mu \mu} \kappa_{\nu \nu}}
$$

$$
\begin{aligned}
\Omega(\theta) & =\frac{1}{2} \ell_{\mu}^{2}\left\{\frac{\kappa_{\nu \mu \nu}}{\kappa_{\mu \mu}^{3} \kappa_{\nu \nu}}\left[\frac{1}{2} \kappa_{\mu \mu \mu}-\kappa_{\mu \mu / \mu}\right]+\frac{\kappa_{\mu \mu \nu}}{\kappa_{\mu \mu}^{2} \kappa_{\nu \nu}^{2}}\left[\frac{1}{2} \kappa_{\nu \nu \nu}-\kappa_{\nu \nu / \nu}\right]\right. \\
& \left.+\frac{\kappa_{\mu \nu \nu}}{\kappa_{\mu \mu}^{2} \kappa_{\nu \nu}^{2}}\left[\frac{1}{2} \kappa_{\mu \nu \nu}-\kappa_{\nu \nu / \mu}\right]-\frac{1}{\kappa_{\mu \mu}^{2} \kappa_{\nu \nu}}\left[\frac{1}{2} \kappa_{\mu \mu \nu \nu}-\kappa_{\mu \mu \nu / \nu}-\kappa_{\mu \nu \nu / \mu}\right]\right\} .
\end{aligned}
$$

* Na verdade, as funções $A^{a}(\theta)$ e $B^{a b}(\theta)$ não precisam ser avaliadas em $\tilde{\theta}(\mu)$, mas em $\check{\theta}(\mu)$ tal que $\check{\theta}(\mu)-\theta=O_{p}\left(n^{-1 / 2}\right)$. Entretanto, a forma de $B^{a b}(\theta)$ depende da escolha de $\check{\theta}(\mu)$. 
Como os parâmetros de interesse e de perturbação têm dimensão um, preferiu-se usar $\ell_{\mu}$ no lugar de $\ell_{a}$ e $\kappa_{\nu \nu}$ ao invés de $\kappa_{i j}$, trocar $\kappa_{a b i}$ por $\kappa_{\mu \mu \nu}$ e $\kappa_{a b i / j}$ por $\kappa_{\mu \mu \nu / \nu}$, etc..

Supondo ainda $\mu$ e $\nu$ ortogonais e unidimensionais, mas, agora, como parâmetros da família exponencial biparamétrica, tais funções são expressas por

$$
\Lambda(\theta)=\frac{1}{2 n} \frac{s_{11,01}}{s_{10,10 s_{01,01}}} \sum_{j=1}^{n} \sum_{i=1}^{2} \alpha_{i}^{(1,0)}(\theta)\left[d_{i}\left(y_{j}\right)-\beta_{i}(\theta)\right]
$$

e

$$
\begin{aligned}
\Omega(\theta)= & \frac{1}{2 n^{2}}\left\{-\frac{s_{10,20} s_{11,01}}{2 s_{10,10}^{3} s_{01,01}}-\frac{s_{11,10} s_{01,02}+2 s_{11,01} s_{01,11}-s_{11,01} s_{10,02}}{2 s_{10,10}^{2} s_{01,01}^{2}}\right. \\
& \left.+\frac{s_{12,10}+3 s_{11,11}+s_{21,01}}{2 s_{10,10}^{2} s_{01,01}}\right\}\left\{\sum_{j=1}^{n} \sum_{i=1}^{2} \alpha_{i}^{(1,0)}(\theta)\left[d_{i}\left(y_{j}\right)-\beta_{i}(\theta)\right]\right\}^{2},
\end{aligned}
$$

em que $s_{j k, l m}=\sum_{i=1}^{2} \alpha_{i}^{(j, k)}(\theta) \beta_{i}^{(l, m)}(\theta)$.

\subsection{EXEMPLOS}

Aqui, é exemplificada a obtenção de $\ell_{S T}(\mu)$ para algumas distribuições pertencentes a família exponencial biparamétrica. Como já foi salientado, a derivação da parcela $\Omega(\theta)$ é simplificada quando há ortogonalidade entre os parâmetros de interesse e de perturbação. Assim, os exemplos são apresentados sob tal condição e apenas termos que dependem dos parâmetros nas expressões dos logaritmos das funções de verossimilhança são considerados.

EXEMPLO 2.1 (continuação): distribuição normal com média $\tau$ e variância $\phi$.

Aqui, os parâmetros $\tau$ e $\phi$ são ortogonais. Logo, não é necessário obter a ortogonalidade entre os parâmetros de interesse e de perturbação em qualquer uma das duas situações: $(\mu, \nu)=(\tau, \phi)$ e $(\mu, \nu)=(\phi, \tau)$. Lembre que

$$
\begin{gathered}
\alpha_{1}(\tau, \phi)=\frac{\tau}{\phi}, \quad \alpha_{2}(\tau, \phi)=-\frac{1}{2 \phi}, \quad d_{1}(y)=y, \quad d_{2}(y)=y^{2} \\
\beta_{1}(\tau, \phi)=\tau \quad \text { e } \quad \beta_{2}(\tau, \phi)=\phi+\tau^{2} .
\end{gathered}
$$

Considerando o primeiro caso, $\theta=(\mu, \nu)=(\tau, \phi)$, tem-se

$$
\widehat{\phi}_{\tau}=\frac{1}{n} \sum_{j=1}^{n}\left(y_{j}-\tau\right)^{2} \quad \text { e } \quad \ell_{p}(\tau)=-\frac{n}{2} \log \sum_{j=1}^{n}\left(y_{j}-\tau\right)^{2} .
$$


Além disso,

$$
\begin{gathered}
\sum_{j=1}^{n} \sum_{i=1}^{2} \alpha_{i}^{(1,0)}(\theta)\left[d_{i}\left(y_{j}\right)-\beta_{i}(\theta)\right]=\frac{n}{\phi}(\bar{y}-\tau), \\
s_{10,10}=\sum_{i=1}^{2} \alpha_{i}^{(1,0)}(\theta) \beta_{i}^{(1,0)}(\theta)=\frac{1}{\phi}, \quad s_{01,01}=\sum_{i=1}^{2} \alpha_{i}^{(0,1)}(\theta) \beta_{i}^{(0,1)}(\theta)=\frac{1}{2 \phi^{2}}
\end{gathered}
$$

e

$$
s_{12,10}=\sum_{i=1}^{2} \alpha_{i}^{(1,2)}(\theta) \beta_{i}^{(1,0)}(\theta)=\frac{2}{\phi^{3}}
$$

Observe que $\forall \theta$,

$$
\begin{array}{ll}
s_{11,01}=\sum_{i=1}^{2} \alpha_{i}^{(1,1)}(\theta) \beta_{i}^{(0,1)}(\theta)=0, & s_{21,01}=\sum_{i=1}^{2} \alpha_{i}^{(2,1)}(\theta) \beta_{i}^{(0,1)}(\theta)=0, \\
s_{11,11}=\sum_{i=1}^{2} \alpha_{i}^{(1,1)}(\theta) \beta_{i}^{(1,1)}(\theta)=0, & s_{01,02}=\sum_{i=1}^{2} \alpha_{i}^{(0,1)}(\theta) \beta_{i}^{(0,2)}(\theta)=0 .
\end{array}
$$

Portanto,

$$
\Lambda(\theta)=0
$$

e

$$
\Omega(\theta)=\frac{1}{2 n^{2}} \frac{s_{12,10}}{2 s_{10,10}^{2} s_{01,01}}\left\{\sum_{j=1}^{n} \sum_{i=1}^{2} \alpha_{i}^{(1,0)}(\theta)\left[d_{i}\left(y_{j}\right)-\beta_{i}(\theta)\right]\right\}^{2}=\frac{(\bar{y}-\tau)^{2}}{\phi} .
$$

Dessa forma,

$$
\Omega\left(\tau, \widehat{\phi}_{\tau}\right)=n \frac{(\bar{y}-\tau)^{2}}{\sum_{j=1}^{n}\left(y_{j}-\tau\right)^{2}}
$$

e

$$
\ell_{S T}(\tau)=-\frac{n}{2} \log \sum_{j=1}^{n}\left(y_{j}-\tau\right)^{2}+n \frac{(\bar{y}-\tau)^{2}}{\sum_{j=1}^{n}\left(y_{j}-\tau\right)^{2}}
$$

Observe que

$$
n \frac{(\bar{y}-\tau)^{2}}{\sum_{j=1}^{n}\left(y_{j}-\tau\right)^{2}}=\log \sum_{j=1}^{n}\left(y_{j}-\tau\right)^{2}-\log \sum_{j=1}^{n}\left(y_{j}-\bar{y}\right)^{2}+O_{p}\left(n^{-2}\right) .
$$

Como

$$
\ell_{C R}(\tau)=\ell_{B N}(\tau)=\left(1-\frac{n}{2}\right) \log \sum_{j=1}^{n}\left(y_{j}-\tau\right)^{2}
$$


então

$$
\ell_{S T}(\tau)=\ell_{B N}(\tau)-\log \sum_{j=1}^{n}\left(y_{j}-\bar{y}\right)^{2}+O_{p}\left(n^{-2}\right)
$$

Aqui,

$$
u_{S T}(\tau)=\left(n^{2}-2 n\right) \frac{\bar{y}-\tau}{\sum_{j=1}^{n}\left(y_{j}-\tau\right)^{2}}+2 n^{2} \frac{(\bar{y}-\tau)^{3}}{\left[\sum_{j=1}^{n}\left(y_{j}-\tau\right)^{2}\right]^{2}} .
$$

Assim, $\widehat{\tau}_{S T}=\bar{y}$

Agora, considere a variância $\phi$ como parâmetro de interesse, $\theta=(\mu, \nu)=(\phi, \tau)$. Sabe-se que

$$
\widehat{\tau}_{\phi}=\bar{y} \quad \text { e } \quad \ell_{p}(\phi)=-\frac{n}{2} \log \phi-\frac{1}{2 \phi} \sum_{j=1}^{n}\left(y_{j}-\bar{y}\right)^{2} .
$$

A derivada do logaritmo da verossimilhança original com respeito a $\phi, \partial \ell(\theta) / \partial \phi$, vale

$$
\sum_{j=1}^{n} \sum_{i=1}^{2} \alpha_{i}^{(1,0)}(\theta)\left[d_{i}\left(y_{j}\right)-\beta_{i}(\theta)\right]=-\frac{n}{2 \phi}+\frac{1}{2 \phi^{2}} \sum_{j=1}^{n}\left(y_{j}-\tau\right)^{2}
$$

Os outros termos constituintes das expressões de $\Lambda(\theta)$ e $\Omega(\theta)$ valem

$$
\begin{gathered}
s_{11,01}=\sum_{i=1}^{2} \alpha_{i}^{(1,1)}(\theta) \beta_{i}^{(0,1)}(\theta)=-\frac{1}{\phi^{2}}, \quad s_{01,01}=\sum_{i=1}^{2} \alpha_{i}^{(0,1)}(\theta) \beta_{i}^{(0,1)}(\theta)=\frac{1}{\phi}, \\
s_{10,10}=\sum_{i=1}^{2} \alpha_{i}^{(1,0)}(\theta) \beta_{i}^{(1,0)}(\theta)=\frac{1}{2 \phi^{2}}, \\
s_{10,02}=\sum_{i=1}^{2} \alpha_{i}^{(1,0)}(\theta) \beta_{i}^{(0,2)}(\theta)=\frac{1}{\phi^{2}}, \quad s_{21,01}=\sum_{i=1}^{2} \alpha_{i}^{(2,1)}(\theta) \beta_{i}^{(0,1)}(\theta)=\frac{2}{\phi^{3}} .
\end{gathered}
$$

Note que $\forall \theta$,

$$
\begin{gathered}
s_{10,20}=\sum_{i=1}^{2} \alpha_{i}^{(1,0)}(\theta) \beta_{i}^{(2,0)}(\theta)=0, \quad s_{11,10}=\sum_{i=1}^{2} \alpha_{i}^{(1,1)}(\theta) \beta_{i}^{(1,0)}(\theta)=0 \\
s_{11,11}=\sum_{i=1}^{2} \alpha_{i}^{(1,1)}(\theta) \beta_{i}^{(1,1)}(\theta)=0 \\
s_{01,11}=\sum_{i=1}^{2} \alpha_{i}^{(0,1)}(\theta) \beta_{i}^{(1,1)}(\theta)=0, \quad s_{12,10}=\sum_{i=1}^{2} \alpha_{i}^{(1,2)}(\theta) \beta_{i}^{(1,0)}(\theta)=0 .
\end{gathered}
$$


Por conseguinte,

$$
\Lambda(\theta)=\frac{1}{2}-\frac{1}{2 n \phi} \sum_{j=1}^{n}\left(y_{j}-\tau\right)^{2}
$$

e

$$
\Omega(\theta)=\frac{\phi^{2}}{n^{2}}\left(-\frac{n}{2 \phi}+\frac{1}{2 \phi^{2}} \sum_{j=1}^{n}\left(y_{j}-\tau\right)^{2}\right)^{2} .
$$

Como $\widehat{\tau}_{\phi}=\bar{y}$, então

$$
\ell_{S T}(\phi)=-\frac{n}{2} \log \phi-\frac{n+2}{2 n \phi} \sum_{j=1}^{n}\left(y_{j}-\bar{y}\right)^{2}+\frac{1}{4 n^{2} \phi^{2}}\left[\sum_{j=1}^{n}\left(y_{j}-\bar{y}\right)^{2}\right]^{2} .
$$

A correspondente função escore é dada por

$$
u_{S T}(\phi)=-\frac{n}{2 \phi}+\frac{n+2}{2 n \phi^{2}} \sum_{j=1}^{n}\left(y_{j}-\bar{y}\right)^{2}-\frac{1}{2 n^{2} \phi^{3}}\left[\sum_{j=1}^{n}\left(y_{j}-\bar{y}\right)^{2}\right]^{2},
$$

em que

$$
\frac{\sum_{j=1}^{n}\left(y_{j}-\bar{y}\right)^{2}}{\phi} \sim \chi_{n-1}^{2}
$$

Logo,

$$
\begin{aligned}
E\left\{u_{S T}(\phi)\right\} & =-\frac{n}{2 \phi}+\frac{n+2}{2 n \phi}(n-1)-\frac{1}{2 n^{2} \phi}\left[2(n-1)+(n-1)^{2}\right]= \\
& =\frac{1}{2 n^{2} \phi}-\frac{1}{n \phi}=O\left(n^{-1}\right) .
\end{aligned}
$$

Pode-se mostrar também que

$$
E\left\{\frac{\partial u_{S T}(\phi)}{\partial \phi}\right\}=-\frac{n}{2 \phi^{2}}+\frac{1}{2 \phi^{2}}+\frac{2}{n \phi^{2}}-\frac{3}{2 n^{2} \phi^{2}}
$$

e

$$
E\left\{u_{S T}^{2}(\phi)\right\}=\frac{n}{2 \phi^{2}}-\frac{1}{2 \phi^{2}}-\frac{2}{n \phi^{2}}+\frac{17}{2 n^{2} \phi^{2}}+\frac{1}{n^{3} \phi^{2}}-\frac{15}{4 n^{4} \phi^{2}} .
$$

Assim,

$$
E\left\{\frac{\partial u_{S T}(\phi)}{\partial \phi}\right\}+E\left\{u_{S T}^{2}(\phi)\right\}=O\left(n^{-2}\right)
$$

EXEMPLO 2.2 (continuação): distribuição gama com parâmetros $\omega$ e $\vartheta$.

Os parâmetros $\omega$ e $\vartheta$ que indexam a distribuição $G(\omega, \vartheta)$ não são ortogonais. Aqui são consideradas as parametrizações ortogonais obtidas na Seção 2.6 deste capítulo para este 
exemplo. A fim de exemplificar a obtenção da verossimilhança proposta por Stern, é considerado apenas o caso $(\mu, \nu)=(\omega, \vartheta)$, pois quando $(\mu, \nu)=(\vartheta, \omega)$, não foi possível obter explicitamente $\omega=\omega(\delta, \vartheta)$ tal que $\delta$ e $\vartheta$ sejam ortogonais.

- parametrização ortogonal $(\omega, \lambda)$

$$
\vartheta_{1}\left(\omega, \lambda_{1}\right)=\frac{\omega}{\lambda_{1}} \quad \text { e } \quad \vartheta_{2}\left(\omega, \lambda_{2}\right)=\omega \exp \left(\lambda_{2}\right)
$$

- $\ell_{S T 1}(\omega)$ e $\ell_{S T 2}(\omega)$

$$
\begin{aligned}
\ell_{S T 1}(\omega)= & \ell_{S T 2}(\omega)=\ell_{p}(\omega)+\frac{1}{2 n\left(\omega \psi^{\prime}(\omega)-1\right)}\left[\sum_{j=1}^{n} \log y_{j}-n \psi(\omega)+n \log (\omega / \bar{y})\right] \\
& -\frac{1}{4 n^{2}} \frac{\omega^{2} \psi^{\prime \prime}(\omega)+\omega \psi^{\prime}(\omega)}{\left(\omega \psi^{\prime}(\omega)-1\right)^{3}}\left[\sum_{j=1}^{n} \log y_{j}-n \psi(\omega)+n \log (\omega / \bar{y})\right]^{2} .
\end{aligned}
$$

Para as duas parametrizações ortogonais consideradas aqui,

$$
\ell_{C R 1}(\omega)=\ell_{C R 2}(\omega)=\ell_{B N}(\omega)=\ell_{p}(\omega)-\frac{1}{2} \log \omega .
$$

Lembre que os vieses da função escore e da informação relativos à verossimilhança proposta por Barndorff-Nielsen são de ordem $O\left(n^{-1}\right)$. Observe a simplicidade deste ajuste comparativamente àquele obtido em $\ell_{S T 1}(\omega)$.

EXEMPLO 2.3 (continuação): distribuição beta com parâmetros $a$ e $b$.

Para este exemplo, não foi possível obter o logaritmo da função de verossimilhança proposta por Cox e Reid (1987) quando o parâmetro de interesse era $a$, pois não foi possível a determinação explícita de um parâmetro de incômodo ortogonal. Assim, aqui, não será exemplificada a obtenção da verossimilhança proposta por Stern (1997).

EXEMPLO 2.4 (continuação): distribuição gaussiana inversa com média $\gamma$ e coeficiente de variação $\zeta$.

$$
\begin{gathered}
\mu=\zeta \quad \nu=\gamma \\
\Delta_{\zeta}=n^{2} \zeta^{4}+4 n \bar{y} \sum_{j=1}^{n}\left(1 / y_{j}\right)
\end{gathered}
$$


- parametrização ortogonal $(\zeta, \lambda)$

$$
\gamma_{1}\left(\zeta, \lambda_{1}\right)=\lambda_{1}\left(\zeta^{2}+2\right) \quad \text { e } \quad \gamma_{2}\left(\zeta, \lambda_{2}\right)=\exp \left(\lambda_{2}\right)\left(\zeta^{2}+2\right)
$$

- $\ell_{S T 1}(\zeta)$ e $u_{S T 1}(\zeta)$

$$
\begin{gathered}
\ell_{S T 1}(\zeta)=\ell_{p}(\zeta)+\left[\frac{1}{\zeta^{4}}+\frac{5}{2 \zeta^{2}}+\frac{\zeta^{2}}{4}\right]-\frac{\sqrt{\Delta_{\zeta}}}{n}\left[\frac{1}{2}+\frac{5}{2 \zeta^{2}}+\frac{2-\zeta^{2}}{\zeta^{4}\left(\zeta^{2}+2\right)}\right] \\
+\frac{\Delta_{\zeta}}{n^{2}}\left[\frac{\zeta^{2}+3}{4 \zeta^{2}\left(\zeta^{2}+2\right)}+\frac{1}{\zeta^{4}\left(\zeta^{2}+2\right)^{2}}\right] \cdot \\
u_{S T 1}(\zeta)=u_{p}(\zeta)+\left[\frac{3 \zeta}{2}+\frac{1}{\zeta}-\frac{2 \zeta}{\left(\zeta^{2}+2\right)^{2}}-\frac{5}{\zeta^{3}}-\frac{4}{\zeta^{5}}\right]-\frac{2 n \zeta^{3}}{\sqrt{\Delta_{\zeta}}}\left[\frac{1}{2}+\frac{5}{2 \zeta^{2}}+\frac{2-\zeta^{2}}{\zeta^{4}\left(\zeta^{2}+2\right)}\right] \\
-\frac{\sqrt{\Delta_{\zeta}}}{n}\left[-\frac{5}{\zeta^{3}}+\frac{4 \zeta^{4}-8 \zeta^{2}-16}{\zeta^{5}\left(\zeta^{2}+2\right)^{2}}\right]-\frac{\Delta_{\zeta}}{n^{2}}\left[\frac{\zeta^{2}+3}{2 \zeta^{3}\left(\zeta^{2}+2\right)}+\frac{\zeta^{4}+8}{2 \zeta^{5}\left(\zeta^{2}+2\right)^{2}}+\frac{4}{\zeta^{3}\left(\zeta^{2}+2\right)^{3}}\right] . \\
\widehat{\zeta}_{S T 1} \text { não tem forma fechada. }
\end{gathered}
$$

- $\ell_{S T 2}(\zeta)$ e $u_{S T 2}(\zeta)$

$$
\ell_{S T 2}(\zeta)=\ell_{S T 1}(\zeta) \quad \text { e } \quad u_{S T 2}(\zeta)=u_{S T 1}(\zeta)
$$

\subsection{APROXIMAÇÕES PARA A VEROSSIMILHANÇA PROPOSTA POR BARNDORFF-NIELSEN}

\subsubsection{INTRODUÇÃO}

Conforme foi mostrado na seção 2.3, a verossimilhança proposta por Barndorff-Nielsen (1983) pode ser expressa na forma

$$
L_{B N}(\mu)=\left|\ell_{\nu ; \widehat{\nu}}\left(\mu, \widehat{\nu}_{\mu} ; \widehat{\mu}, \widehat{\nu}, a\right)\right|^{-1}\left|j_{\nu \nu}\left(\mu, \widehat{\nu}_{\mu} ; \widehat{\mu}, \widehat{\nu}, a\right)\right|^{1 / 2} L_{p}(\mu)
$$

em que

$$
\ell_{\nu ; \widehat{\nu}}(\mu, \nu ; \widehat{\mu}, \widehat{\nu}, a)=\frac{\partial}{\partial \widehat{\nu}}\left(\frac{\partial \ell(\mu, \nu ; \widehat{\mu}, \widehat{\nu}, a)}{\partial \nu}\right)
$$


é uma derivada com respeito ao espaço amostral.

Observe que a estatística ancilar $a$ não é necessária para o cálculo de $j_{\nu \nu}(\mu, \widehat{\nu}, \widehat{\mu}, \widehat{\nu}, a)$, mas é indispensável para o cálculo da derivada do espaço amostral. Embora, por exemplo, para modelos da família exponencial de posto completo, a especificação de tal estatística seja desnecessária, para modelos em geral, o cálculo de derivadas como $\ell_{\nu ; \widehat{\nu}}(\mu, \nu ; \widehat{\mu}, \widehat{\nu}, a)$ pode ser difícil ou impossível.

Motivado por tal dificuldade, nesta seção são apresentadas algumas aproximações para $\ell_{B N}(\mu)$ obtidas a partir de aproximações para $\ell_{\nu ; \widehat{\nu}}(\mu, \nu ; \widehat{\mu}, \widehat{\nu}, a)$, que estão descritas em Severini (2000a, Capítulos 6 e 9). Tais aproximações caracterizam-se por não dependerem de a. Uma revisão e comparação dos vários métodos de aproximação para derivadas do espaço amostral é dada em Severini (2000b).

\subsubsection{UMA APROXIMAÇÃO BASEADA NAS COVARIÂNCIAS DE DERIVADAS DO LOGARITMO DA FUNÇÃO DE VEROSSIMILHANÇA}

Para $\theta=\theta_{0}+\delta / \sqrt{n}$, as expansões em séries de Taylor de $\ell_{\theta}(\theta ; \widehat{\theta}, a)$ e de $\ell_{\theta ; \widehat{\theta}}(\theta ; \widehat{\theta}, a)$ em torno de $\theta=\widehat{\theta}$ e avaliadas em $\widehat{\theta}=\theta_{0}$ são dadas por

$$
\ell_{\theta}\left(\theta ; \theta_{0}, a\right)=\ell_{\theta}\left(\theta_{0} ; \theta_{0}, a\right)+\ell_{\theta \theta}\left(\theta_{0} ; \theta_{0}, a\right)\left(\theta-\theta_{0}\right)+O(1)
$$

$\mathrm{e}$

$$
\ell_{\theta ; \widehat{\theta}}\left(\theta ; \theta_{0}, a\right)=\ell_{\theta ; \widehat{\theta}}\left(\theta_{0} ; \theta_{0}, a\right)+\ell_{\theta \theta ; \widehat{\theta}}\left(\theta_{0} ; \theta_{0}, a\right)\left(\theta-\theta_{0}\right)+O(1) .
$$

Ao longo da seção 2.11,

$$
\ell_{\theta}(\theta)=\ell_{\theta}(\theta ; \widehat{\theta}, a)=\frac{\partial \ell(\theta ; \widehat{\theta}, a)}{\partial \theta} \quad \text { e } \quad \ell_{\theta ; \widehat{\theta}}(\theta)=\ell_{\theta ; \widehat{\theta}}(\theta ; \widehat{\theta}, a)=\frac{\partial \ell_{\theta}(\theta ; \widehat{\theta}, a)}{\partial \widehat{\theta}}
$$

Lembre que $(\widehat{\theta}, a)$ é suficiente e minimal. Seja $I\left(\theta ; \theta_{0}\right)=E_{\theta_{0}}\left\{\ell_{\theta}(\theta) \ell_{\theta}\left(\theta_{0}\right)^{\top}\right\}$. Usando o fato que

$$
\frac{1}{n} E_{\theta}\left\{\ell_{\theta \theta}(\theta) \ell_{\theta}(\theta)\right\}=\frac{1}{n} \ell_{\theta \theta ; \widehat{\theta}}(\theta ; \theta, a)+O\left(n^{-1 / 2}\right)
$$

obtém-se

$$
I\left(\theta ; \theta_{0}\right)-I\left(\theta_{0} ; \theta_{0}\right)=\ell_{\theta ; \widehat{\theta}}\left(\theta ; \theta_{0}, a\right)-\ell_{\theta ; \widehat{\theta}}\left(\theta_{0} ; \theta_{0}, a\right)+O(1)
$$

Ou seja,

$$
\ell_{\theta ; \widehat{\theta}}\left(\theta ; \theta_{0}, a\right)=I\left(\theta ; \theta_{0}\right)+\ell_{\theta ; \widehat{\theta}}\left(\theta_{0} ; \theta_{0}, a\right)-I\left(\theta_{0} ; \theta_{0}\right)+O(1)
$$

Derivando a igualdade $\ell_{\theta}(\widehat{\theta} ; \widehat{\theta}, a)=0$ com relação a $\widehat{\theta}$ e avaliando em $\widehat{\theta}=\theta$, tem-se

$$
\ell_{\theta \theta}(\theta ; \theta, a)+\ell_{\theta ; \widehat{\theta}}(\theta ; \theta, a)=0 \Rightarrow \ell_{\theta ; \widehat{\theta}}(\theta ; \theta, a)=-\ell_{\theta \theta}(\theta ; \theta, a)=j(\theta)
$$


onde $j(\theta)$ é a informação observada.

Logo,

$$
\begin{gathered}
I\left(\theta ; \theta_{0}\right)+\ell_{\theta ; \widehat{\theta}}\left(\theta_{0} ; \theta_{0}, a\right)-I\left(\theta_{0} ; \theta_{0}\right)=I\left(\theta ; \theta_{0}\right)+j\left(\theta_{0}\right)-i\left(\theta_{0}\right)= \\
=I\left(\theta ; \theta_{0}\right)+i\left(\theta_{0}\right)\left\{i\left(\theta_{0}\right)^{-1} j\left(\theta_{0}\right)-D\right\}= \\
=I\left(\theta ; \theta_{0}\right)+\left\{I\left(\theta ; \theta_{0}\right)+O\left(n^{1 / 2}\right)\right\}\left\{i\left(\theta_{0}\right)^{-1} j\left(\theta_{0}\right)-D\right\}= \\
=I\left(\theta ; \theta_{0}\right) i\left(\theta_{0}\right)^{-1} j\left(\theta_{0}\right)+O(1),
\end{gathered}
$$

onde $D$ denota a matriz identidade e $i(\theta)$ é a matriz de informação de Fisher. Observe que são utilizadas as seguintes expansões:

$$
i\left(\theta_{0}\right)=I\left(\theta ; \theta_{0}\right)+O\left(n^{1 / 2}\right) \quad \text { e } \quad i\left(\theta_{0}\right)^{-1} j\left(\theta_{0}\right)=D+O\left(n^{-1 / 2}\right) .
$$

Por conseguinte,

$$
\ell_{\theta ; \widehat{\theta}}\left(\theta ; \theta_{0}, a\right) \doteq I\left(\theta ; \theta_{0}\right) i\left(\theta_{0}\right)^{-1} j\left(\theta_{0}\right)
$$

Assim, fazendo $\theta_{0}=\widehat{\theta}$, obtém-se uma aproximação para $\ell_{\theta: \widehat{\theta}}(\theta ; \widehat{\theta}, a)$ com erro relativo de ordem $O\left(n^{-1 / 2}\right)$, a saber

$$
\bar{\ell}_{\theta ; \widehat{\theta}}(\theta ; \widehat{\theta}, a)=I(\theta ; \widehat{\theta}) i(\widehat{\theta})^{-1} j(\widehat{\theta}) .
$$

Como $i(\widehat{\theta})^{-1} j(\widehat{\theta})=D+O_{p}\left(n^{-1 / 2}\right)$, costuma-se fazer

$$
\bar{\ell}_{\theta ; \widehat{\theta}}(\theta ; \widehat{\theta}, a) \doteq I(\theta ; \widehat{\theta}) .
$$

Este método de aproximação da derivada do espaço amostral foi originalmente proposto por Barndorff-Nielsen (1995) para modelos da família exponencial curvada e foi estendido para modelos em geral por Severini (1998a). Ver também Skovgaard (1996) e DiCiccio e Stern (1993).

Utilizando um argumento análogo, $\bar{\ell}_{\nu ; \widehat{\nu}}\left(\mu, \widehat{\nu}_{\mu}\right)$ pode ser aproximado por $I_{\nu}(\mu, \widehat{\nu} \mu ; \widehat{\mu}, \widehat{\nu})$, onde

$$
I_{\nu}\left(\mu, \nu ; \mu_{0}, \nu_{0}\right)=E_{\left(\mu_{0}, \nu_{0}\right)}\left\{\ell_{\nu}(\mu, \nu) \ell_{\nu}\left(\mu_{0}, \nu_{0}\right)^{\top}\right\} .
$$

Note que $I_{\nu}(\mu, \widehat{\nu} \mu \widehat{\mu}, \widehat{\nu})$ não depende da estatística ancilar $a$ e que $I_{\nu}\left(\mu, \nu ; \mu_{0}, \nu_{0}\right)$ é, por definição, a covariância entre $\ell_{\nu}(\mu, \nu)$ e $\ell_{\nu}\left(\mu_{0}, \nu_{0}\right)$.

Portanto, a correspondente aproximação para a verossimilhança proposta por BarndorffNielsen, $\bar{\ell}_{B N}(\mu)$, é dada por

$$
\bar{\ell}_{B N}(\mu)=\ell_{p}(\mu)+\frac{1}{2} \log \left|j_{\nu \nu}\left(\mu, \widehat{\nu}_{\mu}\right)\right|-\log \left|I_{\nu}\left(\mu, \widehat{\nu}_{\mu} ; \widehat{\mu}, \widehat{\nu}\right)\right| .
$$

Esta aproximação também tem erro relativo de ordem $O\left(n^{-1 / 2}\right)$. O respectivo estimador de máxima verossimilhança é denotado por $\widehat{\bar{\mu}}_{B N}$ e a função escore, por $\bar{u}_{B N}(\mu)=\partial \bar{\ell}_{B N}(\mu) / \partial \mu$. 
Para a família exponencial biparamétrica,

$$
\ell_{\nu}(\mu, \nu)=\frac{\partial \ell}{\partial \nu}=\sum_{j=1}^{n} \sum_{i=1}^{2} \alpha_{i}^{(0,1)}(\mu, \nu)\left[d_{i}\left(y_{j}\right)-\beta_{i}(\mu, \nu)\right]
$$

Conseqüentemente,

$$
\begin{gathered}
I_{\nu}\left(\mu, \nu ; \mu_{0}, \nu_{0}\right)=n\left[\alpha_{1}^{(0,1)}(\mu, \nu) \alpha_{2}^{(0,1)}\left(\mu_{0}, \nu_{0}\right)+\alpha_{2}^{(0,1)}(\mu, \nu) \alpha_{1}^{(0,1)}\left(\mu_{0}, \nu_{0}\right)\right] \operatorname{Cov}\left\{d_{1}(y), d_{2}(y)\right\} \\
+n \alpha_{1}^{(0,1)}(\mu, \nu) \alpha_{1}^{(0,1)}\left(\mu_{0}, \nu_{0}\right) \operatorname{Var}\left\{d_{1}(y)\right\}+n \alpha_{2}^{(0,1)}(\mu, \nu) \alpha_{2}^{(0,1)}\left(\mu_{0}, \nu_{0}\right) \operatorname{Var}\left\{d_{2}(y)\right\}
\end{gathered}
$$

onde $\operatorname{Cov}\left\{d_{1}(y), d_{2}(y)\right\}$ denota a covariância entre $d_{1}(y)$ e $d_{2}(y)$. Lembre que esta quantidade e as variâncias são calculadas sob $\left(\mu_{0}, \nu_{0}\right)$ e que $y_{1}, \ldots, y_{n}$ são variáveis aleatórias independentes e identicamente distribuídas.

Considerando a parametrização canônica da família exponencial biparamétrica,

$$
\ell\left(\alpha_{1}, \alpha_{2}\right)=\sum_{j=1}^{n}\left[\alpha_{1} d_{1}\left(y_{j}\right)+\alpha_{2} d_{2}\left(y_{j}\right)-\rho^{*}\left(\alpha_{1}, \alpha_{2}\right)+v\left(y_{j}\right)\right]
$$

tem-se

$$
\operatorname{Var}\left\{d_{i}(y)\right\}=\frac{\partial^{2} \rho^{*}\left(\alpha_{1}, \alpha_{2}\right)}{\partial \alpha_{i}^{2}} \quad \text { e } \operatorname{Cov}\left\{d_{1}(y), d_{2}(y)\right\}=\frac{\partial^{2} \rho^{*}\left(\alpha_{1}, \alpha_{2}\right)}{\partial \alpha_{1} \partial \alpha_{2}},
$$

em que $i=1,2$. Após o cálculo destas derivadas, como $\alpha_{1}=\alpha_{1}(\mu, \nu)$ e $\alpha_{2}=\alpha_{2}(\mu, \nu)$, tais quantidades serão funções de $\left(\mu_{0}, \nu_{0}\right)$.

Uma outra forma de obtenção dos elementos da matriz de covariância de $\left(d_{1}(y), d_{2}(y)\right)$ consiste em construir um sistema de equações cujas incógnitas são $\operatorname{Var}\left\{d_{1}(y)\right\}, \operatorname{Var}\left\{d_{2}(y)\right\}$ e $\operatorname{Cov}\left\{d_{1}(y), d_{2}(y)\right\}$, usando as igualdades

$$
E\left\{\left(\frac{\partial \ell}{\partial \mu}\right)^{2}\right\}=-E\left\{\frac{\partial^{2} \ell}{\partial \mu^{2}}\right\}, E\left\{\left(\frac{\partial \ell}{\partial \nu}\right)^{2}\right\}=-E\left\{\frac{\partial^{2} \ell}{\partial \nu^{2}}\right\}, E\left\{\frac{\partial \ell}{\partial \mu} \frac{\partial \ell}{\partial \nu}\right\}=-E\left\{\frac{\partial^{2} \ell}{\partial \mu \partial \nu}\right\} .
$$

Assim, omitindo o argumento $(\mu, \nu)$ das funções, em forma matricial, tem-se

$$
\left(\begin{array}{ccc}
{\left[\alpha_{1}^{(1,0)}\right]^{2}} & {\left[\alpha_{2}^{(1,0)}\right]^{2}} & 2 \alpha_{1}^{(1,0)} \alpha_{2}^{(1,0)} \\
{\left[\alpha_{1}^{(0,1)}\right]^{2}} & {\left[\alpha_{2}^{(0,1)}\right]^{2}} & 2 \alpha_{1}^{(0,1)} \alpha_{2}^{(0,1)} \\
\alpha_{1}^{(1,0)} \alpha_{1}^{(0,1)} & \alpha_{2}^{(1,0)} \alpha_{2}^{(0,1)} & \left(\alpha_{1}^{(1,0)} \alpha_{2}^{(0,1)}+\alpha_{1}^{(0,1)} \alpha_{2}^{(1,0)}\right)
\end{array}\right)\left(\begin{array}{c}
\operatorname{Var}_{1} \\
\operatorname{Var}_{2} \\
\operatorname{Cov}_{1,2}
\end{array}\right)=\left(\begin{array}{c}
s_{10,10} \\
s_{01,01} \\
s_{10,01}
\end{array}\right)
$$

onde $\operatorname{Var}_{1}=\operatorname{Var}\left\{d_{1}(y)\right\}, \operatorname{Var}_{2}=\operatorname{Var}\left\{d_{2}(y)\right\}$ e $\operatorname{Cov}_{1,2}=\operatorname{Cov}\left\{d_{1}(y), d_{2}(y)\right\}$. Note que $s_{10,01}=s_{01,10}$.

A solução deste sistema de equações é

$$
\operatorname{Var}_{1}=\frac{s_{10,10}\left[\alpha_{2}^{(0,1)}\right]^{2}+s_{01,01}\left[\alpha_{2}^{(1,0)}\right]^{2}-s_{10,01} 2 \alpha_{2}^{(0,1)} \alpha_{2}^{(1,0)}}{\left(\alpha_{1}^{(1,0)} \alpha_{2}^{(0,1)}-\alpha_{1}^{(0,1)} \alpha_{2}^{(1,0)}\right)^{2}}
$$




$$
\operatorname{Var}_{2}=\frac{s_{10,10}\left[\alpha_{1}^{(0,1)}\right]^{2}+s_{01,01}\left[\alpha_{1}^{(1,0)}\right]^{2}-s_{10,01} 2 \alpha_{1}^{(0,1)} \alpha_{1}^{(1,0)}}{\left(\alpha_{1}^{(1,0)} \alpha_{2}^{(0,1)}-\alpha_{1}^{(0,1)} \alpha_{2}^{(1,0)}\right)^{2}}
$$

e

$$
\operatorname{Cov}_{1,2}=-\frac{s_{10,10} \alpha_{1}^{(0,1)} \alpha_{2}^{(0,1)}+s_{01,01} \alpha_{1}^{(1,0)} \alpha_{2}^{(1,0)}-s_{10,01}\left(\alpha_{1}^{(1,0)} \alpha_{2}^{(0,1)}+\alpha_{1}^{(0,1)} \alpha_{2}^{(1,0)}\right)}{\left(\alpha_{1}^{(1,0)} \alpha_{2}^{(0,1)}-\alpha_{1}^{(0,1)} \alpha_{2}^{(1,0)}\right)^{2}}
$$

Aqui, assume-se que $\alpha_{1}^{(1,0)} \alpha_{2}^{(0,1)}-\alpha_{1}^{(0,1)} \alpha_{2}^{(1,0)} \neq 0$. Avaliando estas quantidades em $\left(\mu_{0}, \nu_{0}\right)$, obtém-se $I_{\nu}\left(\mu, \nu ; \mu_{0}, \nu_{0}\right)$.

Vale salientar que caso $\alpha_{1}=\alpha_{1}(\mu)$ e $\alpha_{2}=\alpha_{2}(\nu)$ (e vice-versa), ou seja, se cada uma destas funções depender exclusivamente de cada um dos parâmetros, a aproximação $\bar{\ell}_{B N}(\mu)$ é exata.

\subsubsection{UMA APROXIMAÇÃO BASEADA EM COVARIÂNCIAS EMPÍRICAS}

Supondo que os dados consistem de $n$ observações independentes, as covariâncias usadas ao construir $\bar{\ell}_{\theta ; \widehat{\theta}}(\theta)$ podem ser trocadas pelas covariâncias empíricas sem mudança da ordem do erro de aproximação.

Dessa forma, $I\left(\theta ; \theta_{0}\right)$ pode ser aproximado por

$$
\breve{I}\left(\theta ; \theta_{0}\right)=\sum_{j=1}^{n} \ell_{\theta}^{(j)}(\theta) \ell_{\theta}^{(j)}\left(\theta_{0}\right)^{\top}
$$

onde $\ell_{\theta}^{(j)}(\theta)$ é a função escore baseada na $j$-ésima observação.

Assim, uma aproximação com erro relativo de ordem $O\left(n^{-1 / 2}\right)$ para $\ell_{\theta ; \widehat{\theta}}(\theta)$ é dada por

$$
\breve{\ell}_{\theta ; \widehat{\theta}}(\theta)=\breve{I}(\theta ; \widehat{\theta}) \widehat{i}(\widehat{\theta})^{-1} j(\widehat{\theta})
$$

onde $\widehat{i}(\widehat{\theta})=\breve{I}(\widehat{\theta} ; \widehat{\theta})$. Esta aproximação é baseada em Severini (1999).

Analogamente, $\ell_{\nu ; \widehat{\nu}}\left(\mu, \widehat{\nu}_{\mu}\right)$ pode ser aproximado por $\breve{\ell}_{\nu ; \widehat{\nu}}\left(\mu, \widehat{\nu}_{\mu}\right) \mathrm{e}$

$$
\breve{\ell}_{\nu ; \widehat{\nu}}\left(\mu, \widehat{\nu}_{\mu}\right) \doteq \breve{I}_{\nu}\left(\mu, \widehat{\nu}_{\mu} ; \widehat{\mu}, \widehat{\nu}\right)
$$

em que

$$
\breve{I}_{\nu}\left(\mu, \nu ; \mu_{0}, \nu_{0}\right)=\sum_{j=1}^{n} \ell_{\nu}^{(j)}(\mu, \nu) \ell_{\nu}^{(j)}\left(\mu_{0}, \nu_{0}\right)^{\top}
$$


Logo, uma aproximação para a verossimilhança proposta por Barndorff-Nielsen é expressa por

$$
\breve{\ell}_{B N}(\mu)=\ell_{p}(\mu)+\frac{1}{2} \log \left|j_{\nu \nu}\left(\mu, \widehat{\nu}_{\mu}\right)\right|-\log \left|\breve{I}_{\nu}(\mu, \widehat{\nu} \mu \widehat{\mu}, \widehat{\nu})\right| .
$$

Esta aproximação também tem erro relativo de ordem $O\left(n^{-1 / 2}\right)$. O respectivo estimador de máxima verossimilhança é denotado por $\widehat{\breve{\mu}}_{B N}$ e a função escore, por $\breve{u}_{B N}(\mu)$.

Para a família exponencial biparamétrica,

$$
\ell_{\nu}^{(j)}(\mu, \nu)=\sum_{i=1}^{2} \alpha_{i}^{(0,1)}(\mu, \nu)\left[d_{i}\left(y_{j}\right)-\beta_{i}(\mu, \nu)\right] .
$$

Conseqüentemente, pela definição, tem-se

$$
\begin{gathered}
\breve{I}_{\nu}\left(\mu, \widehat{\nu}_{\mu} ; \widehat{\mu}, \widehat{\nu}\right)=\sum_{j=1}^{n} \sum_{i=1}^{2} \alpha_{i}^{(0,1)}\left(\mu, \widehat{\nu}_{\mu}\right) \alpha_{i}^{(0,1)}(\widehat{\mu}, \widehat{\nu})\left[d_{i}^{2}\left(y_{j}\right)-\beta_{i}^{2}(\widehat{\mu}, \widehat{\nu})\right]+ \\
+\left[\alpha_{1}^{(0,1)}(\widehat{\mu}, \widehat{\nu}) \alpha_{2}^{(0,1)}\left(\mu, \widehat{\nu}_{\mu}\right)+\alpha_{2}^{(0,1)}(\widehat{\mu}, \widehat{\nu}) \alpha_{1}^{(0,1)}\left(\mu, \widehat{\nu}_{\mu}\right)\right] \times \sum_{j=1}^{n}\left[d_{1}\left(y_{j}\right) d_{2}\left(y_{j}\right)-\beta_{1}(\widehat{\mu}, \widehat{\nu}) \beta_{2}(\widehat{\mu}, \widehat{\nu})\right] .
\end{gathered}
$$

Observe que não há dependência da estatística ancilar $a$.

Aqui, também, se cada uma das funções $\alpha_{1}(\mu, \nu)$ e $\alpha_{2}(\mu, \nu)$ depender exclusivamente de cada um dos parâmetros, então $\breve{\ell}_{B N}(\mu)=\ell_{B N}(\mu)$.

Esta aproximação é conveniente para situações em que há dificuldade no cálculo da esperança do produto de derivadas do logaritmo da verossimilhança $\left(I_{\nu}(\mu, \widehat{\nu} \mu \widehat{\mu}, \widehat{\nu})\right)$, pois é fácil de ser obtida numericamente.

\subsubsection{UMA APROXIMAÇÃO BASEADA NUMA ESTATÍSTICA APROXIMADAMENTE ANCILAR}

Seja $f(\theta)$ uma função qualquer que depende dos dados somente através da estatística suficiente $(\widehat{\theta}, a)$. Logo, é indiferente escrever $f(\theta ; y)$ ou $f(\theta ; \widehat{\theta}, a)$. Suponha que as dimensões de $\widehat{\theta}$ e $a$ sejam, respectivamente, $d$ e $m$, onde $m \leq n-d$ ( $n$ é o tamanho da amostra). Aqui, $y=\left(y_{1}, \ldots, y_{n}\right)$. Assim,

$$
f_{; y}(\theta)=\frac{\partial f(\theta ; y)}{\partial y}=\left(f_{\widehat{;}}(\theta) \quad f_{; a}(\theta)\right)\left(\begin{array}{c}
\partial \widehat{\theta} / \partial y \\
\partial a / \partial y
\end{array}\right) .
$$

Seja $A^{-}$a inversa generalizada de uma matriz $A .^{\star} \operatorname{Logo}$,

$$
f_{; y}(\theta)\left(\begin{array}{c}
\partial \widehat{\theta} / \partial y \\
\partial a / \partial y
\end{array}\right)^{-}\left(\begin{array}{c}
\partial \widehat{\theta} / \partial y \\
\partial a / \partial y
\end{array}\right)=\left(\begin{array}{ll}
f_{\widehat{\theta}}(\theta) & f_{; a}(\theta)
\end{array}\right)\left(\begin{array}{c}
\partial \widehat{\theta} / \partial y \\
\partial a / \partial y
\end{array}\right) .
$$

* A inversa generalizada de uma matriz $A$ satisfaz $A A^{-} A=A$. 
Desta igualdade vem

Fazendo

$$
\left(\begin{array}{ll}
f_{\widehat{\theta}}(\theta) & f_{; a}(\theta)
\end{array}\right)=f_{; y}(\theta)\left(\begin{array}{c}
\partial \widehat{\theta} / \partial y \\
\partial a / \partial y
\end{array}\right)^{-}
$$

$$
\left(\begin{array}{c}
\partial \widehat{\theta} / \partial y \\
\partial a / \partial y
\end{array}\right)^{-}=\left(\begin{array}{ll}
V_{1} & V_{2}
\end{array}\right)
$$

em que $V_{1}$ é uma matriz de dimensão $n \times d$ e $V_{2}$, de dimensão $n \times m$, tem-se

$$
f_{; \widehat{\theta}}(\theta)=f_{; y}(\theta) V_{1}
$$

e

$$
\left(\begin{array}{l}
\partial \widehat{\theta} / \partial y \\
\partial a / \partial y
\end{array}\right)\left(\begin{array}{ll}
V_{1} & V_{2}
\end{array}\right)\left(\begin{array}{l}
\partial \widehat{\theta} / \partial y \\
\partial a / \partial y
\end{array}\right)=\left(\begin{array}{l}
\partial \widehat{\theta} / \partial y \\
\partial a / \partial y
\end{array}\right) .
$$

Por esta segunda igualdade, as matrizes $V_{1}$ e $V_{2}$ satisfazem

$$
\frac{\partial \widehat{\theta}}{\partial y} V_{1} \frac{\partial \widehat{\theta}}{\partial y}+\frac{\partial \widehat{\theta}}{\partial y} V_{2} \frac{\partial a}{\partial y}=\frac{\partial \widehat{\theta}}{\partial y}
$$

$\mathrm{e}$

$$
\frac{\partial a}{\partial y} V_{1} \frac{\partial \widehat{\theta}}{\partial y}+\frac{\partial a}{\partial y} V_{2} \frac{\partial a}{\partial y}=\frac{\partial a}{\partial y} .
$$

Observe que se a matriz $V_{1}$ for conhecida, para $f(\theta)=\ell_{\theta}(\theta)$, obtém-se a derivada com respeito ao espaço amostral $\ell_{\theta ; \widehat{\theta}}(\theta)=\ell_{\theta ; y}(\theta) V_{1}$.

Suponha uma matriz $V$ de dimensão $n \times d$ satisfazendo

$$
\frac{\partial a}{\partial y} V=0_{m \times d} \quad \text { e } \quad\left|\frac{\partial \widehat{\theta}}{\partial y} V\right| \neq 0,
$$

em que $0_{m \times d}$ denota uma matriz nula de dimensão $m \times d$.

Multiplicando ambos os lados da igualdade (2.20) à direita pela matriz $V$, obtém-se

$$
\frac{\partial \widehat{\theta}}{\partial y} V_{1} \frac{\partial \widehat{\theta}}{\partial y} V=\frac{\partial \widehat{\theta}}{\partial y} V
$$

Dessa forma, $V_{1}$ pode ser expressa como

$$
V_{1}=V\left(\frac{\partial \widehat{\theta}}{\partial y} V\right)^{-1} .
$$

Substituindo esta proposta para $V_{1}$ em (2.20) e (2.21), vem

$$
\frac{\partial \widehat{\theta}}{\partial y} V_{2} \frac{\partial a}{\partial y}=0_{d \times n} \quad \text { e } \quad \frac{\partial a}{\partial y} V_{2} \frac{\partial a}{\partial y}=\frac{\partial a}{\partial y} .
$$


Note que $V_{2}$ pode ser uma inversa generalizada da matriz $\partial a / \partial y(m \times n)$, satisfazendo $(\partial \widehat{\theta} / \partial y) V_{2}=0_{d \times m}$.

Portanto,

$$
f_{\widehat{;} \widehat{\theta}}(\theta)=f_{; y}(\theta) V\left(\frac{\partial \widehat{\theta}}{\partial y} V\right)^{-1}
$$

em que a matriz $V$ satisfaz (2.22). Se é possível expressar explicitamente $y$ em termos de $(\widehat{\theta}, a)$, pode-se fazer $V=\partial y / \partial \widehat{\theta}$.

Derivando ambos os lados da igualdade $\ell_{\theta}(\widehat{\theta})=0$ com respeito a $y$, obtém-se

$$
\ell_{\theta \theta}(\widehat{\theta}) \frac{\partial \widehat{\theta}}{\partial y}+\ell_{\theta ; y}(\widehat{\theta})=0
$$

Por conseguinte,

$$
\frac{\partial \widehat{\theta}}{\partial y}=j(\widehat{\theta})^{-1} \ell_{\theta ; y}(\widehat{\theta})
$$

Usando esta igualdade, pode-se re-escrever $f_{; \widehat{\theta}}(\theta)$ como

$$
f_{\widehat{;} \widehat{\theta}}(\theta)=f_{; y}(\theta) V\left(\ell_{\theta ; y}(\widehat{\theta}) V\right)^{-1} j(\widehat{\theta}) .
$$

Observe que esta expressão é exata e não requer que $a$ seja uma estatística ancilar, apenas que $(\widehat{\theta}, a)$ seja suficiente. Portanto, fazendo $f(\theta)=\ell_{\theta}(\theta)$, tem-se

$$
\ell_{\theta ; \widehat{\theta}}(\theta)=\ell_{\theta ; y}(\theta) V\left(\ell_{\theta ; y}(\widehat{\theta}) V\right)^{-1} j(\widehat{\theta}) .
$$

Agora, considere a determinação da matriz $V$ baseada numa estatística aproximadamente ancilar. Suponha que $y_{1}, \ldots, y_{n}$ sejam variáveis aleatórias independentes e que $y_{j}$ tenha função distribuição $F_{j}(\cdot ; \theta) . \quad F_{j}\left(y_{j} ; \theta\right)$ tem uma distribuição uniforme no intervalo $[0,1]$. Seja $c_{j}=F_{j}\left(y_{j} ; \widehat{\theta}\right), j=1, \ldots, n$. Dessa forma, $c=\left(c_{1}, \ldots, c_{n}\right)$ pode ser considerada uma estatística aproximadamente ancilar e $y$, como uma função de $(\widehat{\theta}, c)$. Logo, usando

$$
\widehat{V}=\left(\begin{array}{lll}
\partial y_{1} / \partial \widehat{\theta} & \cdots & \partial y_{n} / \partial \widehat{\theta}
\end{array}\right)^{\top},
$$

obtém-se uma aproximação para $\ell_{\theta ; \widehat{\theta}}(\theta)$, a saber

$$
\tilde{l}_{\theta ; \widehat{\theta}}(\theta)=\ell_{\theta ; y}(\theta) \widehat{V}\left(\ell_{\theta ; y}(\widehat{\theta}) \widehat{V}\right)^{-1} j(\widehat{\theta}) .
$$

Tal aproximação tem erro relativo de ordem $O\left(n^{-1}\right)$. Aqui,

$$
\frac{\partial y_{j}}{\partial \widehat{\theta}}=-\frac{\partial F_{j}\left(y_{j} ; \widehat{\theta}\right) / \partial \widehat{\theta}}{p_{j}\left(y_{j} ; \widehat{\theta}\right)},
$$


em que $p_{j}\left(y_{j} ; \widehat{\theta}\right)$ denota a função densidade de $y_{j}$ para $\theta=\widehat{\theta}$. Este método de aproximação é devido a Fraser e Reid $(1995,1999)$ e Fraser, Reid e Wu (1999).

Por um procedimento semelhante, obtém-se a seguinte expressão para a derivada com respeito ao espaço amostral $\ell_{\nu ; \widehat{\nu}}(\mu, \nu ; \widehat{\mu}, \widehat{\nu}, a)$,

$$
\ell_{\nu ; \widehat{\nu}}(\mu, \nu ; \widehat{\mu}, \widehat{\nu}, a)=\ell_{\nu ; y}(\mu, \nu) V_{\nu}\left(\frac{\partial \widehat{\nu}}{\partial y} V_{\nu}\right)^{-1}
$$

em que $\mu$ tem dimensão $p(p<d)$ e $V_{\nu}$ é uma matriz $n \times(d-p)$ satisfazendo

$$
\frac{\partial(\widehat{\mu}, a)}{\partial y} V_{\nu}=0_{(m+1) \times(d-p)} \quad \text { e } \quad\left|\frac{\partial \widehat{\nu}}{\partial y} V_{\nu}\right| \neq 0 .
$$

Analogamente, uma aproximação para tal derivada é obtida ao fazer $V_{\nu}=\widehat{V}_{\nu}$, onde

$$
\widehat{V}_{\nu}=\left(\begin{array}{lll}
-\frac{\partial F_{1}\left(y_{1} ; \widehat{\theta}\right) / \partial \widehat{\nu}}{p_{1}\left(y_{1} ; \widehat{\theta}\right)} & \cdots & -\frac{\partial F_{n}\left(y_{n} ; \widehat{\theta}\right) / \partial \widehat{\nu}}{p_{n}\left(y_{n} ; \widehat{\theta}\right)}
\end{array}\right)^{\top}
$$

Dessa forma, desprezando termos que não dependem de $\mu$, uma aproximação para $\ell_{B N}(\mu)$ é dada por

$$
\tilde{\ell}_{B N}(\mu)=\ell_{p}(\mu)+\frac{1}{2} \log \left|j_{\nu \nu}\left(\mu, \widehat{\nu}_{\mu}\right)\right|-\log \left|\ell_{\nu ; y}\left(\mu, \widehat{v}_{\mu}\right) \widehat{V}_{\nu}\right| .
$$

O respectivo estimador de máxima verossimilhança é denotado por $\widehat{\widetilde{\mu}}_{B N}$ e a função escore, por $\tilde{u}_{B N}(\mu)$.

Para a família exponencial biparamétrica, tem-se

$$
\ell_{\nu ; y}(\mu, \nu)=\left(\sum_{i=1}^{2} \alpha_{i}^{(0,1)}(\mu, \nu) d_{i}^{\prime}\left(y_{1}\right) \quad \cdots \quad \sum_{i=1}^{2} \alpha_{i}^{(0,1)}(\mu, \nu) d_{i}^{\prime}\left(y_{n}\right)\right),
$$

onde $d_{i}^{\prime}(y)=d d_{i}(y) / d y$. Além disso, como $y_{1}, \ldots, y_{n}$ são variáveis identicamente distribuídas, então $p_{j}\left(y_{j} ; \theta\right)=p\left(y_{j} ; \theta\right)$ e $F_{j}\left(y_{j} ; \theta\right)=F\left(y_{j} ; \theta\right)$, em que $p(y ; \theta)$ é dado por $(2.1)$ e $F(y ; \theta)$ é a correspondente função de distribuição.

\subsection{EXEMPLOS}

A seguir, dando continuidade aos exemplos apresentados neste capítulo, são derivadas algumas dessas aproximações para $\ell_{B N}(\mu)$. São consideradas apenas aquelas cujas expressões não coincidem com a forma exata.

EXEMPLO 2.1 (continuação): distribuição normal com média $\tau$ e variância $\phi$.

Neste exemplo são considerados dois casos: $\mu=\tau$ e $\mu=\phi$. 
Quando $\phi$ é o parâmetro de interesse, todas as aproximações apresentadas na seção 2.11 são exatas, ou seja, $\bar{\ell}_{B N}(\phi)=\breve{\ell}_{B N}(\phi)=\tilde{\ell}_{B N}(\phi)=\ell_{B N}(\phi)$.

Quando $\tau$ é o parâmetro de interesse, observa-se que $\bar{\ell}_{B N}(\tau)=\ell_{B N}(\tau)$. Entretanto, $\breve{\ell}_{B N}(\tau) \neq \ell_{B N}(\tau)$ e $\tilde{\ell}_{B N}(\tau) \neq \ell_{B N}(\tau)$.

De seções anteriores, sabe-se que $(\nu=\phi)$

$$
\ell_{p}(\tau)=-\frac{n}{2} \log \sum_{j=1}^{n}\left(y_{j}-\tau\right)^{2}
$$

Calculando $j_{\phi \phi}\left(\tau, \widehat{\phi}_{\tau}\right)$,

$$
\frac{1}{2} \log \left|j_{\phi \phi}\left(\tau, \widehat{\phi}_{\tau}\right)\right|=-\log \sum_{j=1}^{n}\left(y_{j}-\tau\right)^{2} .
$$

Aqui, $\breve{I}_{\phi}\left(\tau, \widehat{\phi}_{\tau} ; \widehat{\tau}, \widehat{\phi}\right)$ é igual a

$$
\begin{aligned}
& \left\{n^{4} \tau \bar{y} \sum_{j=1}^{n}\left(y_{j}-\bar{y}\right)^{2}+\frac{n^{4}}{4}\left[\sum_{j=1}^{n} y_{j}^{4}-\frac{1}{n}\left(\sum_{j=1}^{n} y_{j}^{2}\right)^{2}\right]-\frac{n^{4}(\tau+\bar{y})}{2}\left(\sum_{j=1}^{n} y_{j}^{3}-\bar{y} \sum_{j=1}^{n} y_{j}^{2}\right)\right\} \\
& \times\left[\sum_{j=1}^{n}\left(y_{j}-\tau\right)^{2}\right]^{-2}\left[\sum_{j=1}^{n}\left(y_{j}-\bar{y}\right)^{2}\right]^{-2},
\end{aligned}
$$

enquanto que

$$
\ell_{\phi ; y}\left(\tau, \widehat{\phi}_{\tau}\right) \widehat{V}_{\phi}=\frac{n^{2}}{2 \widehat{\phi}\left[\sum_{j=1}^{n}\left(y_{j}-\tau\right)^{2}\right]^{2}} \sum_{j=1}^{n}\left(y_{j}-\tau\right)\left(y_{j}-\widehat{\tau}\right)
$$

Assim,

$$
\begin{gathered}
\breve{\ell}_{B N}(\tau)=\ell_{p}(\tau)+\frac{1}{2} \log \left|j_{\phi \phi}\left(\tau, \widehat{\phi}_{\tau}\right)\right|-\log \left|\breve{I}_{\phi}(\tau, \widehat{\phi} \tau ; \widehat{\tau}, \widehat{\phi})\right|= \\
=\ell_{B N}(\tau)-\log \left\{-\tau \sum_{j=1}^{n}\left(y_{j}-\bar{y}\right)^{3}-\left[\bar{y} \sum_{j=1}^{n} y_{j}^{3}-\bar{y}^{2} \sum_{j=1}^{n} y_{j}^{2}-\frac{1}{2} \sum_{j=1}^{n} y_{j}^{4}+\frac{1}{2 n}\left(\sum_{j=1}^{n} y_{j}^{2}\right)^{2}\right]\right\}
\end{gathered}
$$

e

$$
\begin{aligned}
\tilde{\ell}_{B N}(\tau)= & \ell_{p}(\tau)+\frac{1}{2} \log \left|j_{\phi \phi}\left(\tau, \widehat{\phi}_{\tau}\right)\right|-\log \left|\ell_{\phi ; y}\left(\tau, \widehat{\phi}_{\tau}\right) \widehat{V}_{\phi}\right|= \\
& =\ell_{B N}(\tau)-\log \sum_{j=1}^{n}\left(y_{j}-\tau\right)\left(y_{j}-\bar{y}\right)
\end{aligned}
$$


em que

$$
\ell_{B N}(\tau)=\left(1-\frac{n}{2}\right) \log \sum_{j=1}^{n}\left(y_{j}-\tau\right)^{2}
$$

Derivando $\tilde{\ell}_{B N}(\tau)$ com respeito a $\tau$,

$$
\frac{\partial \tilde{\ell}_{B N}(\tau)}{\partial \tau}=\tilde{u}_{B N}(\tau)=u_{B N}(\tau)=(n-2) \frac{\sum_{j=1}^{n}\left(y_{j}-\tau\right)}{\sum_{j=1}^{n}\left(y_{j}-\tau\right)^{2}} .
$$

Logo, a estimativa de máxima verossimilhança obtida de $\tilde{\ell}_{B N}(\tau)$ é $\bar{y}$ e $\tilde{u}_{B N}(\tau)$ herda todas as propriedades de $u_{B N}(\tau)$ (por exemplo, esperança nula).

EXEMPLO 2.2 (continuação): distribuição gama com parâmetros $\omega$ e $\vartheta$.

A função densidade de uma variável aleatória $y$ com distribuição $G(\omega, \vartheta)$ é

$$
p(y ; \omega, \vartheta)=\frac{\vartheta^{\omega} y^{\omega-1} \exp (-\vartheta y)}{\Gamma(\omega)}, \quad y>0 .
$$

Expressando essa função na forma (2.1), obtém-se

$$
\begin{gathered}
\alpha_{1}(\omega, \vartheta)=\omega, \quad \alpha_{2}(\omega, \vartheta)=-\vartheta, \quad \rho(\omega, \vartheta)=\log \Gamma(\omega)-\omega \log \vartheta \\
d_{1}(y)=\log y, \quad d_{2}(y)=y \quad \text { e } \quad v(y)=-\log y .
\end{gathered}
$$

Portanto, como as funções $\alpha_{1}(\omega, \vartheta)$ e $\alpha_{2}(\omega, \vartheta)$ dependem exclusivamente de cada um dos parâmetros, então $\bar{\ell}_{B N}(\omega)=\breve{\ell}_{B N}(\omega)=\ell_{B N}(\omega)$ e $\bar{\ell}_{B N}(\vartheta)=\breve{\ell}_{B N}(\vartheta)=\ell_{B N}(\vartheta)$.

Agora, considere as determinações das aproximações baseadas numa estatística aproximadamente ancilar $\tilde{\ell}_{B N}(\omega)$ e $\tilde{\ell}_{B N}(\vartheta)$. Para isto, é feita a suposição que $\omega$ seja inteiro. Assim, $y$ tem uma distribuição Erlang e sua função distribuição é dada por

$$
F(y ; \omega, \vartheta)=1-\exp (-\vartheta y) \sum_{k=0}^{\omega-1} \frac{(\vartheta y)^{k}}{k !}, \quad \text { onde } y, \omega, \vartheta>0 \text { e } \omega \text { é inteiro. }
$$

Fazendo inicialmente $\mu=\omega$, tem-se

$$
\ell_{\vartheta ; y}\left(\omega, \widehat{\vartheta}_{\omega}\right) \widehat{V}_{\vartheta}=\frac{n \bar{y}}{\widehat{\vartheta}}
$$

em que $\widehat{\vartheta}$ denota a estimativa de máxima verossimilhança de $\vartheta$ (não tem forma fechada). Note que este produto só depende dos dados.

Calculando $j_{\vartheta \vartheta}\left(\omega, \widehat{\vartheta}_{\omega}\right)$, obtém-se

$$
\frac{1}{2} \log \left|j_{\vartheta \vartheta}\left(\omega, \widehat{\vartheta}_{\omega}\right)\right|=\frac{1}{2} \log \left(\frac{n \bar{y}}{\omega}\right)
$$


Como

$$
\ell_{p}(\omega)=n \omega \log \left(\frac{\omega}{\bar{y}}\right)+\omega \sum_{j=1}^{n} \log y_{j}-n \omega-n \log \Gamma(\omega),
$$

então

$$
\tilde{\ell}_{B N}(\omega)=\ell_{p}(\omega)+\frac{1}{2} \log \left|j_{\vartheta \vartheta}\left(\omega, \widehat{\vartheta}_{\omega}\right)\right|-\log \left|\ell_{\vartheta ; y}\left(\omega, \widehat{\vartheta}_{\omega}\right) \widehat{V}_{\vartheta}\right|=\ell_{B N}(\omega) .
$$

Agora, considere $\vartheta$ como parâmetro de interesse e $\omega$ como parâmetro de perturbação. Neste caso,

$$
\ell_{\omega ; y}\left(\vartheta, \widehat{\omega}_{\vartheta}\right) \widehat{V}_{\omega}=\sum_{j=1}^{n} \frac{\psi(\widehat{\omega})-\log \left(\widehat{\vartheta} y_{j}\right)}{\widehat{\vartheta}_{y_{j}}} \quad \text { e } \quad j_{\omega \omega}\left(\vartheta, \widehat{\omega}_{\vartheta}\right)=n \psi^{\prime}\left(\widehat{\omega}_{\vartheta}\right),
$$

onde $\widehat{\omega}_{\vartheta}$ satisfaz a equação

$$
n \log \vartheta+\sum_{j=1}^{n} \log y_{j}=n \psi\left(\widehat{\omega}_{\vartheta}\right) .
$$

Portanto,

$$
\tilde{\ell}_{B N}(\vartheta)=\ell_{p}(\vartheta)+\frac{1}{2} \log \left(\psi^{\prime}\left(\widehat{\omega}_{\vartheta}\right)\right)
$$

em que

$$
\ell_{p}(\vartheta)=n \widehat{\omega}_{\vartheta} \psi\left(\widehat{\omega}_{\vartheta}\right)-\vartheta \sum_{j=1}^{n} y_{j}-n \log \Gamma\left(\widehat{\omega}_{\vartheta}\right) .
$$

Aqui, também, $\tilde{\ell}_{B N}(\vartheta)=\ell_{B N}(\vartheta)$.

EXEMPLO 2.3 (continuação): distribuição beta com parâmetros $a$ e $b$.

A função densidade de uma variável aleatória $y$ com distribuição $B(a, b)$ é

$$
p(y ; a, b)=\frac{\Gamma(a+b)}{\Gamma(a) \Gamma(b)} y^{a-1}(1-y)^{b-1}, \quad 0<y<1 .
$$

Escrevendo $p(y ; a, b)$ na forma (2.1), obtém-se

$$
\begin{gathered}
\alpha_{1}(a, b)=a, \quad \alpha_{2}(a, b)=b, \quad \rho(a, b)=-\log \Gamma(a+b)+\log \Gamma(a)+\log \Gamma(b), \\
d_{1}(y)=\log y, \quad d_{2}(y)=\log (1-y) \quad \text { e } \quad v(y)=-\log [y(1-y)] .
\end{gathered}
$$

Observe que as funções $\alpha_{1}(a, b)$ e $\alpha_{2}(a, b)$ dependem exclusivamente de cada um dos parâmetros. Logo, as aproximações $\bar{\ell}_{B N}(a), \breve{\ell}_{B N}(a), \bar{\ell}_{B N}(b)$ e $\breve{\ell}_{B N}(b)$ são exatas.

Aqui, a função de distribuição da variável aleatória $y$ é expressa em função de uma integral cuja primitiva não é conhecida e, além disso, $p(y ; a, b)$ não pertence a uma família 
de locação e/ou escala, como observado numa distribuição normal. Assim, não é possível obter as aproximações baseadas numa estatística aproximadamente ancilar $\tilde{\ell}_{B N}(a)$ e $\tilde{\ell}_{B N}(b)$.

EXEMPLO 2.4 (continuação): distribuição gaussiana inversa com média $\gamma$ e coeficiente de variação $\zeta$.

$$
\mu=\zeta \quad \nu=\gamma
$$

- $\bar{\ell}_{B N}(\zeta)$ e $\bar{u}_{B N}(\zeta)$

$$
\begin{gathered}
\bar{\ell}_{B N}(\zeta)=\ell_{p}(\zeta)+\log \zeta+\log \left(n \zeta^{2}+\sqrt{\Delta_{\zeta}}\right)+\frac{1}{4} \log \Delta_{\zeta} \\
-\log \left\{2 n^{3} \bar{y} \sum_{j=1}^{n}\left(1 / y_{j}\right)+\left[2 n \bar{y} \sum_{j=1}^{n}\left(1 / y_{j}\right)+\left(n \zeta^{2}+\sqrt{\Delta_{\zeta}}\right)^{2}\right]\left[\bar{y} \sum_{j=1}^{n}\left(1 / y_{j}\right)-n\right]^{2}\right\} . \\
\bar{u}_{B N}(\zeta)=u_{p}(\zeta)+\frac{1}{\zeta}+\frac{2 n \zeta}{\sqrt{\Delta_{\zeta}}}+\frac{n^{2} \zeta^{3}}{\Delta_{\zeta}} \\
-\frac{4 n \zeta\left(n \zeta^{2}+\sqrt{\Delta_{\zeta}}\right)^{2}\left(\bar{y} \sum_{j=1}^{n}\left(1 / y_{j}\right)-n\right)^{2}}{\left\{2 n^{3} \bar{y} \sum_{j=1}^{n}\left(1 / y_{j}\right)+\left[2 n \bar{y} \sum_{j=1}^{n}\left(1 / y_{j}\right)+\left(n \zeta^{2}+\sqrt{\Delta_{\zeta}}\right)^{2}\right]\left[\bar{y} \sum_{j=1}^{n}\left(1 / y_{j}\right)-n\right]^{2}\right\} \sqrt{\Delta_{\zeta}}} . \\
\widehat{\bar{\zeta}}_{B N} \text { não tem forma fechada. }
\end{gathered}
$$

- $\breve{\ell}_{B N}(\zeta)$ e $\breve{u}_{B N}(\zeta)$

$$
\begin{gathered}
\breve{\ell}_{B N}(\zeta)=\ell_{p}(\zeta)+\log \zeta+\log \left(n \zeta^{2}+\sqrt{\Delta_{\zeta}}\right)+\frac{1}{4} \log \Delta_{\zeta} \\
-\log \left\{4 n\left(\sum_{j=1}^{n}\left(1 / y_{j}\right)\right)^{2}\left[\sum_{j=1}^{n}\left(y_{j}-\bar{y}\right)^{2}+\bar{y}^{2}\left(\bar{y} \sum_{j=1}^{n}\left(1 / y_{j}\right)-n\right)\right]+\left(n \zeta^{2}+\sqrt{\Delta_{\zeta}}\right)^{2} \times\right. \\
\left.\times\left[n\left(\bar{y} \sum_{j=1}^{n}\left(1 / y_{j}\right)-n\right)+\bar{y}^{2}\left(n \sum_{j=1}^{n}\left(1 / y_{j}\right)^{2}-\left(\sum_{j=1}^{n}\left(1 / y_{j}\right)\right)^{2}\right)\right]\right\} . \\
-\left\{4 n \zeta\left(n \zeta^{2}+\sqrt{\Delta_{\zeta}}\right)^{2}\left[n\left(\bar{y} \sum_{j=1}^{n}\left(1 / y_{j}\right)-n\right)+\bar{y}^{2}\left(n \sum_{j=1}^{n}\left(1 / y_{j}\right)^{2}-\left(\sum_{j=1}^{n}\left(1 / y_{j}\right)\right)^{2}\right)\right]\right\} \div
\end{gathered}
$$




$$
\begin{gathered}
\div\left\{\left\{n\left(\sum_{j=1}^{n}\left(1 / y_{j}\right)\right)^{2}\left[\sum_{j=1}^{n}\left(y_{j}-\bar{y}\right)^{2}+\bar{y}^{2}\left(\bar{y} \sum_{j=1}^{n}\left(1 / y_{j}\right)-n\right)\right]+\left(n \zeta^{2}+\sqrt{\Delta_{\zeta}}\right)^{2} \times\right.\right. \\
\left.\left.\times\left[n\left(\bar{y} \sum_{j=1}^{n}\left(1 / y_{j}\right)-n\right)+\bar{y}^{2}\left(n \sum_{j=1}^{n}\left(1 / y_{j}\right)^{2}-\left(\sum_{j=1}^{n}\left(1 / y_{j}\right)\right)^{2}\right)\right]\right\} \sqrt{\Delta_{\zeta}}\right\} . \\
\widehat{\breve{\zeta}}_{B N} \text { não tem forma fechada. }
\end{gathered}
$$

Aqui, $\tilde{\ell}_{B N}(\zeta)=\ell_{B N}(\zeta)$. Lembre que $\Delta_{\zeta}=n^{2} \zeta^{4}+4 n \bar{y} \sum_{j=1}^{n}\left(1 / y_{j}\right)$. 


\section{CAPÍTULO 3 \\ FAMÍLIA EXPONENCIAL BIPARAMÉTRICA: RESULTADOS NUMÉRICOS}

\subsection{INTRODUÇÃO}

Este capítulo consiste de resultados numéricos provenientes de simulações de Monte Carlo. Aqui, são apresentadas medidas amostrais de posição e de dispersão de alguns estimadores de máxima verossimilhança que foram derivados das várias verossimilhanças perfiladas obtidas nos quatro exemplos estudados ao longo do Capítulo 2. Também são mostradas taxas de rejeições de hipóteses testadas sobre os parâmetros de interesse, usando as estatísticas da razão de verossimilhanças obtidas das verossimilhanças perfiladas, bem como média e variância amostrais destas. Além disso, gráficos contendo as discrepâncias relativas entre os quantis amostrais e os quantis assintóticos dessas estatísticas versus quantis assintóticos. Mais precisamente, supondo qualquer uma das verossimilhanças e denotando o quantil amostral de ordem $q$ do conjunto de valores da respectiva estatística de teste por $R V(q)$ e o correspondente quantil da distribuição qui-quadrado com um grau de liberdade por $\chi_{1}^{2}(q)$, a discrepância relativa supracitada é definida como

$$
\frac{R V(q)-\chi_{1}^{2}(q)}{\chi_{1}^{2}(q)}
$$

Assim, é possível comparar o desempenho do teste original e dos testes baseados em verossimilhanças perfiladas ajustadas.

Em geral, a verossimilhança perfilada é considerada "muito precisa", pois trata o parâmetro de perturbação como função conhecida dos dados e do parâmetro de interesse $\mu$. Ou seja, espera-se que a curvatura do logaritmo desta verossimilhança seja bem acentuada em torno do ponto de máximo. A fim de investigar este fato, foi construído, no caso em que o parâmetro de interesse é a média de uma distribuição normal, um gráfico com as curvas das várias funções de verossimilhanças perfiladas calculadas no Exemplo 2.1. Assim, foram comparadas as curvaturas de tais curvas alinhando-as a partir de um ponto comum. Fixando a curva do logaritmo da verossimilhança perfilada, aplicaram-se transformações (translações) aos pontos das curvas dos logaritmos das verossimilhanças perfiladas modificadas de tal forma que o ponto e o valor de máximo destas fossem $\left(\widehat{\mu}_{p}, \ell_{p}\left(\widehat{\mu}_{p}\right)\right)$.

Sobre a organização dos resultados da estimação pontual do parâmetro de interesse, os seguintes valores são apresentados em tabelas: valor verdadeiro, média, viés, estimativa da variância, estimativa do erro quadrático médio (EQM) e estimativas dos coeficientes de assimetria e de curtose. 
Quanto à organização dos resultados do teste de hipótese sobre o parâmetro de interesse, os seguintes valores são apresentados em tabelas: taxa de rejeição observada sob a hipótese nula, considerando níveis nominais de $15 \%, 10 \%, 5 \%, 3 \%, 1 \%, 0.5 \%, 0.1 \%, 0.05 \%$ e 0.01\%; média e variância amostrais das estatísticas de teste, bem como os valores assintóticos destes momentos.

Para denotar os estimadores de máxima verossimilhança derivados das várias verossimilhanças perfiladas, assim como o logaritmo destas verossimilhanças, é utilizada a notação definida no capítulo anterior e resumida na Tabela 3.1 .

Tabela 3.1. Notação para estimadores de máxima verossimilhança perfilada e logaritmos das verossimilhanças perfiladas.

\begin{tabular}{lc}
\hline estimador & log-verossimilhança \\
\hline$\widehat{\mu}_{p}$ & perfilada: $\ell_{p}$ \\
$\widehat{\mu}_{B N}$ & Barndorff-Nielsen: $\ell_{B N}$ \\
$\widehat{\mu}_{C R}$ & Cox e Reid: $\ell_{C R}$ \\
$\widehat{\mu}_{A C R}$ & Aprox. para Cox e Reid: $\ell_{A C R}$ \\
$\widehat{\mu}_{M T}$ & McCullagh e Tibshirani: $\ell_{M T}$ \\
$\widehat{\mu}_{S T}$ & Stern: $\ell_{S T}$ \\
$\widehat{\bar{\mu}}_{B N}$ & $\mathrm{ABN}{ }^{*}-$ covariância: $\bar{\ell}_{B N}$ \\
$\widehat{\breve{\mu}}_{B N}$ & $\mathrm{ABN}-$ covar. empír.: $\breve{\ell}_{B N}$ \\
$\widehat{\tilde{\mu}}_{B N}$ & $\mathrm{ABN}-$ est. aprox. anc.: $\tilde{\ell}_{B N}$ \\
\hline
\end{tabular}

* $\mathrm{ABN}=$ Aproximação para Barndorff-Nielsen.

\subsection{EXEMPLOS}

Nesta seção, para alguns dos exemplos estudados ao longo do Capítulo 2, são apresentados resultados numéricos provenientes de simulações de Monte Carlo.

EXEMPLO 2.1 (continuação): distribuição normal com média $\tau$ e variância $\phi$.

Como pode ser observado no capítulo anterior, para este exemplo, foram explorados dois casos: $(\mu, \nu)=(\tau, \phi)$ e $(\mu, \nu)=(\phi, \tau)$.

Considere inicialmente a estimação da média (parâmetro de interesse) de uma distribuição normal com variância desconhecida. Como pode ser observado ao longo do Capítulo 2, os estimadores de máxima verossimilhança são iguais à média amostral, que é o estimador 
não viesado de variância uniformemente mínima; a exceção é o estimador obtido de $\breve{\ell}_{B N}(\tau)$, que não foi considerado devido a dificuldades numéricas encontradas na maximização desta verossimilhança. Aqui, os dois parâmetros são ortogonais e as seguintes verossimilhanças coincidem: Barndorff-Nielsen (1983), Cox e Reid (1987), McCullagh e Tibshirani (1990) e a aproximação para Barndorff-Nielsen baseada na covariância de derivadas do logaritmo da verossimilhança.

Os valores verdadeiros dos parâmetros são $\tau=-3$ e $\phi=25$ e todos os resultados são baseados em 500000 réplicas.

A Tabela 3.2 apresenta taxas de rejeição sob a hipótese nula $\left(\mathcal{H}_{0}: \tau=-3\right)$ de diferentes testes da razão de verossimilhanças com vários níveis de significância. Considere, por exemplo, o nível de significância de 5\%. A taxa de rejeição da hipótese nula dos testes baseados em $\ell_{p}(\tau), \ell_{B N}(\tau), \tilde{\ell}_{B N}(\tau)$ e $\ell_{S T}(\tau)$ são, respectivamente, $9.777 \%, 3.241 \%, 3.241 \%$ e $5.581 \%$. Para os níveis nominais usados na prática, o teste baseado no ajuste de Stern (1997) teve o melhor desempenho. Este ajuste mostrou-se liberal, enquanto o teste baseado na modificação proposta por Barndorff-Nielsen (1983) mostrou-se conservador. Observe que os testes baseados em $\ell_{B N}(\tau)$ e $\tilde{\ell}_{B N}(\tau)$ apresentaram as mesmas taxas de rejeição. Finalmente, note que todas as modificações sobre a verossimilhança perfilada produziram inferências mais precisas relativamente aos resultados obtidos pelo teste da razão de verossimilhanças original.

Tabela 3.2. Taxas de rejeição da hipótese nula $\left(\mathcal{H}_{0}: \tau=-3\right)$ de diferentes testes da razão de verossimilhanças, distribuição normal, $n=5$.

\begin{tabular}{crrrr}
\hline nível nominal & \multicolumn{1}{c}{$\ell_{p}$} & \multicolumn{1}{c}{$\ell_{B N}$} & \multicolumn{1}{c}{$\tilde{\ell}_{B N}$} & \multicolumn{1}{c}{$\ell_{S T}$} \\
\hline $15 \%$ & 22.519 & 11.639 & 11.639 & 14.552 \\
$10 \%$ & 16.489 & 7.284 & 7.284 & 10.117 \\
$5 \%$ & 9.777 & 3.241 & 3.241 & 5.581 \\
$3 \%$ & 6.674 & 1.793 & 1.793 & 3.655 \\
$1 \%$ & 2.948 & 0.498 & 0.498 & 1.522 \\
$0.5 \%$ & 1.771 & 0.212 & 0.212 & 0.889 \\
$0.1 \%$ & 0.542 & 0.029 & 0.029 & 0.256 \\
$0.05 \%$ & 0.323 & 0.012 & 0.012 & 0.149 \\
$0.01 \%$ & 0.095 & 0.002 & 0.002 & 0.043 \\
\hline
\end{tabular}

Na Tabela 3.3, são mostradas a média e a variância amostrais de diferentes estatísticas de teste.

Tabela 3.3. Médias e variâncias amostrais de diferentes estatísticas de teste e os valores assintóticos destas, distribuição normal, $n=5$.

\begin{tabular}{cccccc}
\hline momentos & $\chi_{1}^{2}$ & $\ell_{p}$ & $\ell_{B N}$ & $\tilde{\ell}_{B N}$ & $\ell_{S T}$ \\
\hline média & 1.000 & 1.405 & 0.843 & 0.843 & 1.005 \\
variância & 2.000 & 3.903 & 1.405 & 1.405 & 2.479 \\
\hline
\end{tabular}


Observe na Tabela 3.3 que as estatísticas obtidas das verossimilhanças ajustadas têm médias e variâncias mais próximas dos valores assintóticos comparativamente aos valores apresentados pela estatística de teste obtida da verossimilhança original. Por exemplo, enquanto a estatística de teste derivada de $\ell_{B N}(\tau)$ tem média e variância iguais a 0.843 e 1.405 , tais valores, relativos ao uso de $\ell_{S T}(\tau)$, são 1.005 e 2.479 , respectivamente. Note também que a média e a variância da estatística da razão de verossimilhanças original (1.405 e 3.903) são consideravelmente maiores do que os valores assintóticos (1 e 2).

A Figura 3.1 apresenta o gráfico das discrepâncias relativas de quantis versus os correspondentes quantis assintóticos. Quanto mais próxima da ordenada zero a curva estiver, melhor a aproximação assintótica utilizada no teste. Note que as discrepâncias de quantis das estatísticas de teste baseadas em $\ell_{B N}(\tau)(\mathrm{BN})$ e $\tilde{\ell}_{B N}(\tau)$ (aprox. BN) são sempre negativas, enquanto as discrepâncias de quantis da estatística de teste derivada da verossimilhança perfilada (perfilada) são sempre positivas. Observe também que a estatística de teste cuja distribuição nula é melhor aproximada pela distribuição assintótica $\chi_{1}^{2}$ é a estatística derivada do ajuste de Stern (1997).

Figura 3.1. Gráfico das discrepâncias relativas de quantis, $n=5$.

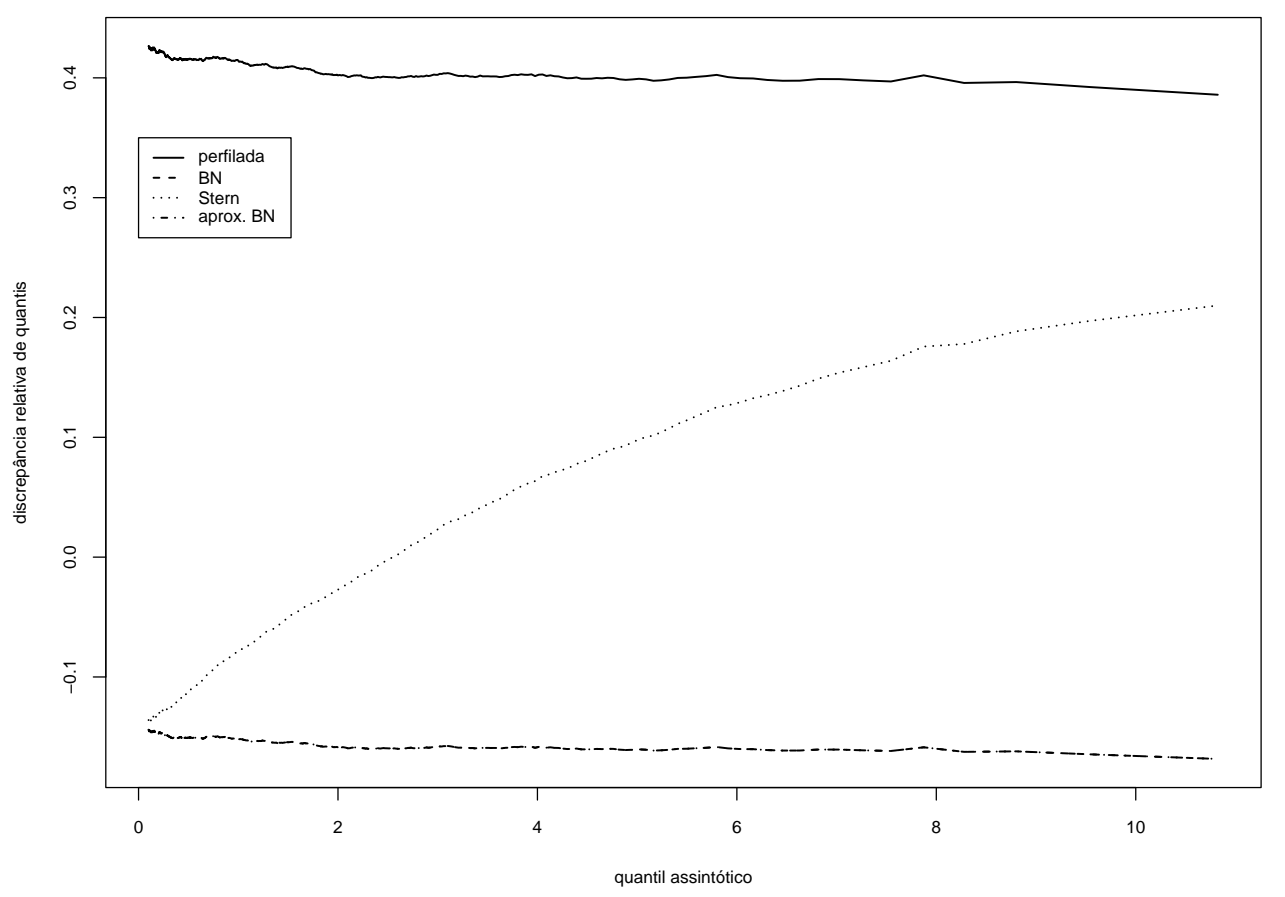

A função de verossimilhança perfilada tipicamente induz "excesso de precisão", dado que trata o parâmetro de incômodo como conhecido e, assim, despreza a incerteza inerente a estimação deste parâmetro. A Figura 3.2 apresenta as curvas dos logaritmos das verossimilhanças perfiladas como uma função de $\tau$ (média). As curvas foram ajustadas de tal forma que tivessem um ponto comum no valor de máximo destas. A linha vertical tracejada indica o valor verdadeiro do parâmetro (-3). A amostra usada para construir esta figura 
foi $(-6.4557,-1.4973,1.0981,-6.5075,4.1651)$. Note que a curva do logaritmo da função de verossimilhança perfilada está completamente abaixo das demais, e, portanto, sua curvatura é mais acentuada em torno do ponto de máximo. Ou seja, neste caso, aparentemente, a informação contida nos dados para a estimação da média de uma distribuição normal é maior comparativamente ao desempenho das demais verossimilhanças. Em valores, a segunda derivada de $\ell_{p}(\tau)$ avaliada no seu ponto de máximo, neste caso, $\widehat{\tau}_{p}=\bar{y}$, é maior, em módulo, do que os respectivos valores desta derivada associados às verossimilhanças perfiladas modificadas.

Figura 3.2. Gráfico dos logaritmos das verossimilhanças perfiladas, $n=5$.

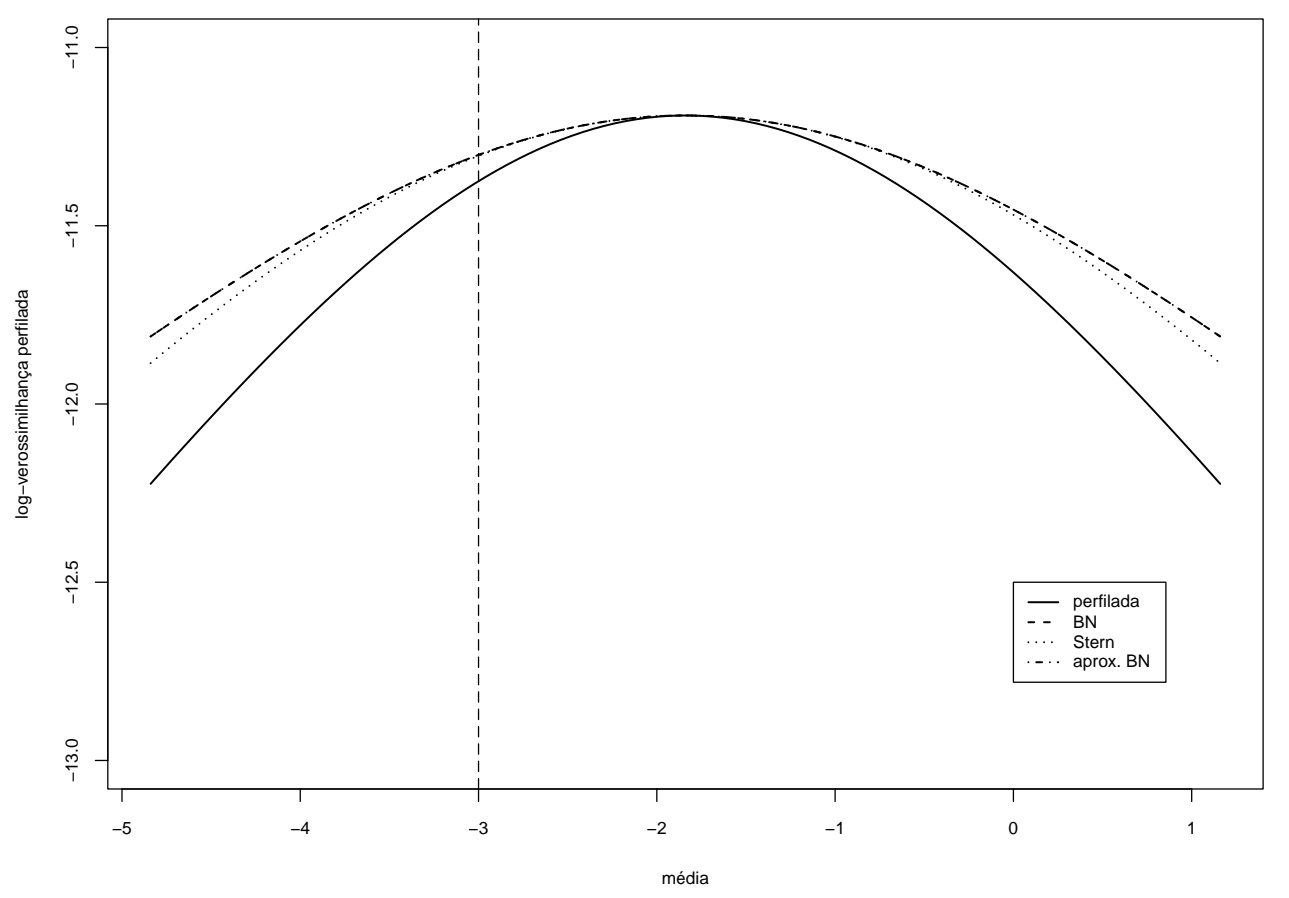

Agora, considere a estimação da variância de uma distribuição normal com média desconhecida. As aproximações da verossimilhança proposta por Barndorff-Nielsen foram exatas. Além disso, $\ell_{M T}(\phi)=\ell_{C R}(\phi)=\ell_{B N}(\phi)$.

Aqui, foram geradas 1000001 de amostras de Monte Carlo de tamanho 5 de uma distribuição $N(3,36)$, porém uma destas amostras foi descartada, pois o ponto de máximo encontrado para a verossimilhança perfilada modificada proposta por Stern (1997) não era global. Assim, os valores verdadeiros dos parâmetros são $\tau=3$ e $\phi=36$, e todos os resultados são baseados em 1000000 de réplicas.

Observando os resultados do Capítulo 2, note que*

$$
\frac{5 \widehat{\phi}_{p}}{36} \sim \chi_{4}^{2} \quad \text { e } \quad \frac{\widehat{\phi}_{B N}}{36} \sim \frac{1}{4} \chi_{4}^{2} .
$$

$\star s^{2} / \phi \sim \chi_{n-1}^{2}$, em que $s^{2}=\sum_{j=1}^{n}\left(y_{j}-\bar{y}\right)^{2}$. 
Assim, os valores verdadeiros das medidas apresentadas na Tabela 3.4 referentes a estes estimadores são conhecidos e, portanto, estimativas obtidas numericamente seriam desnecessárias. Entretanto, o estimador $\widehat{\phi}_{S T}$ não tem forma fechada e, por isto, são mostrados resultados numéricos na tabela a seguir.

Tabela 3.4. Medidas descritivas de diferentes estimadores de $\phi$, distribuição normal, $n=5$.

\begin{tabular}{cccccccc}
\hline parâmetro & estimador & média & variância & \multicolumn{1}{c}{ viés } & EQM & assimetria & curtose \\
\hline \multirow{2}{*}{$\phi=36$} & $\widehat{\phi}_{p}$ & 28.804 & 414.924 & -7.196 & 466.703 & 1.418 & 6.073 \\
& $\widehat{\phi}_{B N}$ & 36.005 & 648.318 & 0.005 & 648.318 & 1.418 & 6.073 \\
& $\widehat{\phi}_{S T}$ & 35.674 & 636.449 & -0.326 & 636.556 & 1.418 & 6.073 \\
\hline
\end{tabular}

Na Tabela 3.4, note que as estimativas dos vieses dos estimadores de máxima verossimilhança derivados de modificações na verossimilhança perfilada, $\widehat{\phi}_{B N}(0.005)$ e $\widehat{\phi}_{S T}(-0.326)$, foram, em módulo, bem menores que o correspondente valor para $\widehat{\phi}_{p}$. Os valores verdadeiros relativos a $\widehat{\phi}_{p}$ e $\widehat{\phi}_{B N}$ são, respectivamente, -7.2 e zero. Entretanto, estas diminuições foram acompanhadas de aumentos das estimativas das variâncias comparativamente àquela obtida para $\widehat{\phi}_{p}$ : $648.318\left(\widehat{\phi}_{B N}\right)$ e $636.449\left(\widehat{\phi}_{S T}\right)$. Aqui, os valores verdadeiros das variâncias são $414.72\left(\widehat{\phi}_{p}\right)$ e $648\left(\widehat{\phi}_{B N}\right)$.

Um critério de avaliação de estimadores é o valor do erro quadrático médio: quanto menor, melhor. Assim, segundo o erro quadrático médio estimado (EQM), o estimador de máxima verossimilhança perfilada teve o melhor desempenho, pois apresentou 466.703 como estimativa para tal medida, contra 648.318 de $\widehat{\phi}_{B N}$ e 636.556 de $\widehat{\phi}_{S T}$, concordando, em parte, com a comparação entre os valores verdadeiros desta quantidade, 466.56 para $\widehat{\phi}_{p}$ e 648 para $\widehat{\phi}_{B N}$.

Observe que as estimativas dos coeficientes de assimetria e de curtose dos três estimadores foram iguais a 1.418 e 6.073. Para uma distribuição qui-quadrado com 4 graus de liberdade, tais coeficientes são $\sqrt{2}$ e 6 , respectivamente. Portanto, tais estimativas estão próximas dos seus verdadeiros valores.

A Tabela 3.5 apresenta taxas de rejeição sob a hipótese nula $\left(\mathcal{H}_{0}: \phi=36\right)$ de diferentes testes da razão de verossimilhanças com vários níveis de significância. Considere, por exemplo, o nível de significância de 10\%. As taxas de rejeição da hipótese nula dos testes baseados em $\ell_{p}(\phi), \ell_{B N}(\phi)$ e $\ell_{S T}(\phi)$ são, respectivamente, $17.661 \%, 11.356 \%$ e $12.998 \%$. Para todos os níveis nominais mostrados na tabela, o teste baseado no ajuste de Barndorff-Nielsen (1983) teve o melhor desempenho. Aqui, todos os testes da razão de verossimilhanças rejeitaram a hipótese nula além do esperado (nível nominal), entretanto as duas modificações sobre a verossimilhança perfilada produziram inferências mais precisas relativamente aos resultados obtidos pelo teste da razão de verossimilhanças original.

Na Tabela 3.6, são mostradas a média e a variância amostrais de diferentes estatísticas de teste. Observe que as estatísticas obtidas das verossimilhanças ajustadas têm médias e variâncias mais próximas dos valores assintóticos comparativamente aos valores apresentados 
Tabela 3.5. Taxas de rejeição da hipótese nula $\left(\mathcal{H}_{0}\right.$ : $\phi=36)$ de diferentes testes da razão de verossimilhanças, distribuição normal, $n=5$.

\begin{tabular}{crrr}
\hline nível nominal & \multicolumn{1}{c}{$\ell_{p}$} & \multicolumn{1}{c}{$\ell_{B N}$} & \multicolumn{1}{c}{$\ell_{S T}$} \\
\hline $15 \%$ & 23.763 & 16.665 & 18.525 \\
$10 \%$ & 17.661 & 11.356 & 12.998 \\
$5 \%$ & 10.571 & 5.952 & 7.191 \\
$3 \%$ & 7.240 & 3.676 & 4.701 \\
$1 \%$ & 3.214 & 1.303 & 1.928 \\
$0.5 \%$ & 1.923 & 0.683 & 1.116 \\
$0.1 \%$ & 0.585 & 0.146 & 0.333 \\
$0.05 \%$ & 0.343 & 0.075 & 0.197 \\
$0.01 \%$ & 0.098 & 0.016 & 0.055 \\
\hline
\end{tabular}

pela estatística de teste obtida da verossimilhança original. Enquanto a estatística de teste derivada de $\ell_{B N}(\phi)$ tem média e variância iguais a 1.082 e 2.320 , tais valores, relativos ao uso de $\ell_{p}(\phi)$, são 1.468 e 4.126, bem maiores do que os valores assintóticos, 1 e 2 , respectivamente.

Tabela 3.6. Médias e variâncias amostrais de diferentes estatísticas de teste e os valores assintóticos destas, distribuição normal, $n=5$.

\begin{tabular}{ccccc}
\hline momento & $\chi_{1}^{2}$ & $\ell_{p}$ & $\ell_{B N}$ & $\ell_{S T}$ \\
\hline média & 1.000 & 1.468 & 1.082 & 1.192 \\
variância & 2.000 & 4.126 & 2.320 & 2.964 \\
\hline
\end{tabular}

EXEMPLO 2.3 (continuação): distribuição beta com parâmetros $a$ e $b$.

Aqui, é considerado apenas o caso em que $\mu=a$ e $\nu=b$. Estes parâmetros não são ortogonais e quanto maior a diferença entre estes (em módulo), maior a assimetria da distribuição.

Supondo o parâmetro $b$ desconhecido, considere a estimação do parâmetro $a$. As aproximações $\bar{\ell}_{B N}(a)$ e $\breve{\ell}_{B N}(a)$ foram exatas, mas não foi possível determinar $\tilde{\ell}_{B N}(a)$, o mesmo ocorrendo com $\ell_{M T}(a)$ e $\ell_{S T}(a)$.

A seguir são mostrados resultados numéricos relativos a inferência na distribuição beta. O número de réplicas de Monte Carlo foi 500000 e foram consideradas amostras de tamanho $n=10$. Os valores verdadeiros dos parâmetros usados neste experimento foram $a=2 \mathrm{e}$ $b=1$. A Tabela 3.7 contém estatísticas descritivas de diferentes estimadores.

Comparando os resultados dos três estimadores na Tabela 3.7, o melhor desempenho foi do estimador $\widehat{a}_{A C R}$; por exemplo, seu viés e seu erro quadrático médio (EQM) foram -0.174 e 0.520 , respectivamente, enquanto as correspondentes medidas para $\widehat{a}_{B N}\left(\widehat{a}_{p}\right)$ foram 0.590 
Tabela 3.7. Medidas descritivas de diferentes estimadores de $a$, distribuição beta, $n=10$.

\begin{tabular}{cccccccr}
\hline parâmetro & estimador & média & variância & viés & EQM & assimetria & curtose \\
\hline \multirow{4}{*}{$a=2$} & $\widehat{a}_{p}$ & 2.855 & 3.372 & 0.855 & 4.103 & 4.025 & 47.958 \\
& $\widehat{a}_{B N}$ & 2.590 & 2.712 & 0.590 & 3.060 & 4.050 & 48.895 \\
& $\widehat{a}_{A C R}$ & 1.826 & 0.489 & -0.174 & 0.520 & 1.462 & 7.245 \\
\hline
\end{tabular}

e 3.060 (0.855 e 4.103). Isto é, o erro quadrático médio de $\widehat{a}_{B N}$ é quase seis vezes maior do que aquele de $\widehat{a}_{A C R}$. Note também que as estimativas dos coeficientes de assimetria e curtose de $\widehat{a}_{A C R}$ estão mais próximos de 0 e 3 , respectivamente, do que as estimativas dos demais estimadores.

Tabela 3.8. Taxas de rejeição da hipótese nula $\left(\mathcal{H}_{0}: a=\right.$ 2) de diferentes testes da razão de verossimilhanças, distribuição beta, $n=10$.

\begin{tabular}{crrr}
\hline nível nominal & \multicolumn{1}{c}{$\ell_{p}$} & \multicolumn{1}{c}{$\ell_{B N}$} & \multicolumn{1}{c}{$\ell_{A C R}$} \\
\hline $15 \%$ & 18.985 & 15.765 & 14.287 \\
$10 \%$ & 13.370 & 10.625 & 9.609 \\
$5 \%$ & 7.353 & 5.452 & 4.988 \\
$3 \%$ & 4.769 & 3.315 & 3.082 \\
$1 \%$ & 1.845 & 1.146 & 1.105 \\
$0.5 \%$ & 1.030 & 0.578 & 0.581 \\
$0.1 \%$ & 0.254 & 0.125 & 0.130 \\
$0.05 \%$ & 0.144 & 0.061 & 0.069 \\
$0.01 \%$ & 0.036 & 0.014 & 0.015 \\
\hline
\end{tabular}

A Tabela 3.8 apresenta taxas de rejeição sob a hipótese nula de diferentes testes; a hipótese nula sob teste é $\mathcal{H}_{0}: a=2$. Globalmente, o melhor desempenho no teste é aquele derivado de $\ell_{A C R}$, seguido pelo baseado em $\ell_{B N}$; estes dois testes superaram consideravelmente o teste da razão de verossimilhanças original. Por exemplo, para o nível nominal de $10 \%$, as taxas de rejeição da hipótese nula dos testes baseados em $\ell_{p}, \ell_{B N}$ e $\ell_{A C R}$ foram $13.370 \%, 10.625 \%$ and $9.609 \%$, respectivamente.

Tabela 3.9. Médias e variâncias amostrais de diferentes estatísticas de teste e os valores assintóticos destas, distribuição beta, $n=10$.

\begin{tabular}{ccccc}
\hline momento & $\chi_{1}^{2}$ & $\ell_{p}$ & $\ell_{B N}$ & $\ell_{A C R}$ \\
\hline média & 1.000 & 1.203 & 1.039 & 0.974 \\
variância & 2.000 & 2.863 & 2.153 & 2.067 \\
\hline
\end{tabular}

As médias e variâncias das três estatísticas de teste são apresentadas na Tabela 3.9, assim como seus valores assintóticos (1 e 2). A estatística de teste com os primeiros dois momentos mais próximos da média e da variância assintóticas é aquela obtida usando $\ell_{A C R}$, 
seguida pela baseada em $\ell_{B N}$; de acordo com este critério, esses dois testes claramente superam o teste da razão de verossimilhanças original pois as distribuições nulas exatas das respectivas estatísticas de teste são melhor aproximadas pela distribuição assintótica $\chi^{2}$ do que a estatística da razão de verossimilhanças original (perfilada).

A Figura 3.3 apresenta o gráfico das discrepâncias relativas de quantis de diferentes estatísticas de teste versus os correspondentes quantis assintóticos. As discrepâncias relativas de quantis da estatística de teste baseada no logaritmo da verossimilhança perfilada são positivas e relativamente grandes, em torno de 20\%. As discrepâncias relativas de quantis da estatística de teste baseada em $\ell_{B N}(a)$ é sempre positiva, em torno de $4 \%$, e essa mesma medida para a estatística de teste baseada em $\ell_{A C R}(a)$ é negativa para quantis de ordem inferior e positiva para quantis de ordem superior, sendo mais próxima da ordenada zero em torno da abscissa 3.84, que é o valor crítico de $5 \%$ usado em testes.

Figura 3.3. Gráfico das discrepâncias relativas de quantis, $n=10$.

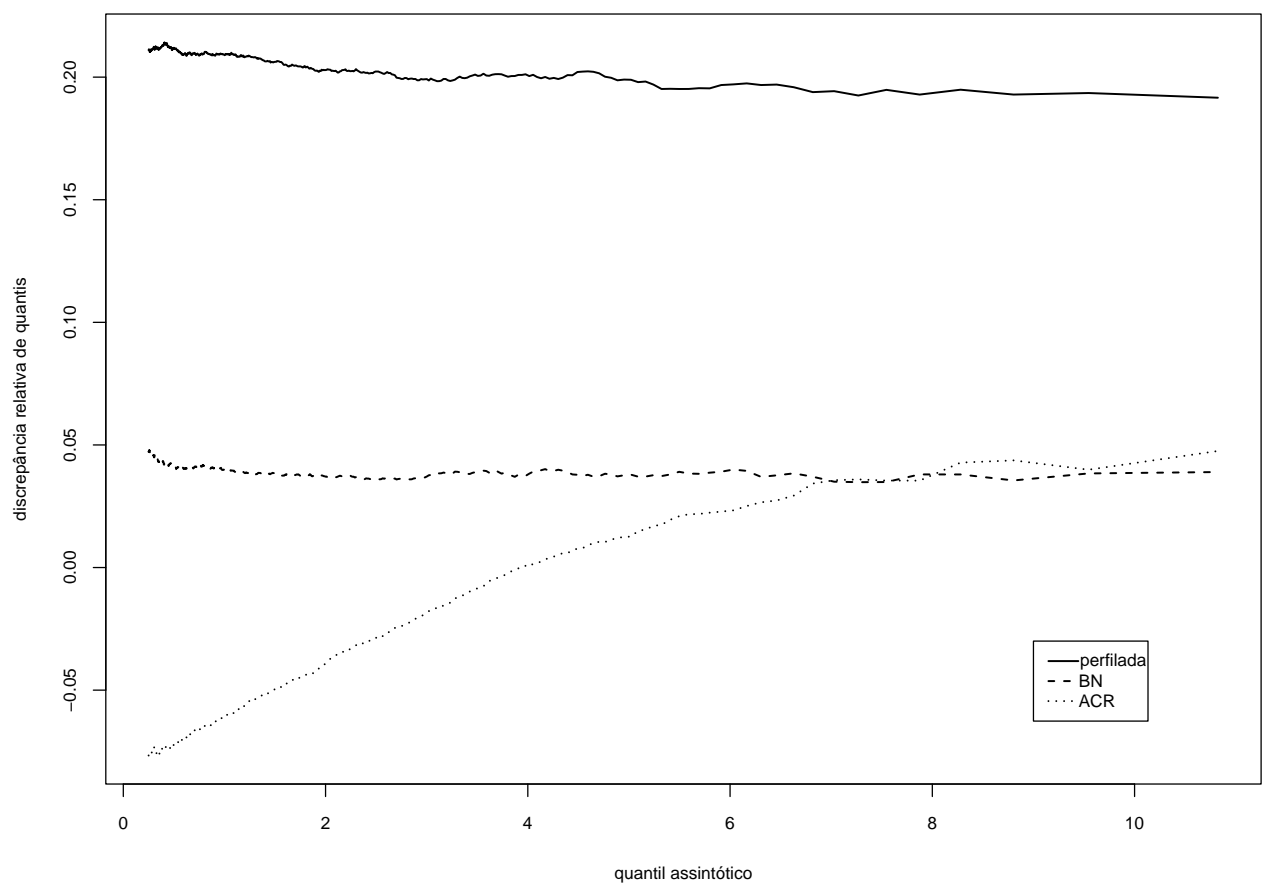

As estatísticas de teste obtidas das verossimilhanças perfiladas modificadas apresentaram média e variância amostrais mais próximas dos valores assintóticos. Este mesmo comportamento foi observado ao comparar os quantis amostrais com os quantis de mesma ordem da distribuição qui-quadrado com um grau de liberdade, conforme o gráfico da Figura 3.3.

EXEMPLO 2.4 (continuação): distribuição gaussiana inversa com média $\gamma$ e coeficiente de variação $\zeta$.

Aqui, é considerado apenas o caso em que $(\mu, \nu)=(\zeta, \gamma)$ e, como nos exemplos anteriores, 
são apresentados resultados numéricos de diferentes estratégias para realização de inferência sobre $\zeta$, considerando o parâmetro de incômodo desconhecido.

Os resultados são baseados em 500000 réplicas de Monte Carlo. O tamanho amostral é $n=10$ e os valores verdadeiros dos parâmetros são $\gamma=5$ e $\zeta=2$.

Note, na Tabela 3.10, que os estimadores de máxima verossimilhança derivados das verossimilhanças perfiladas modificadas são menos viesados do que o estimador original, embora tais estimadores apresentem erros quadráticos médios maiores do que aquele apresentado pelo estimador $\widehat{\zeta}_{p}$. Em valor absoluto, o menor viés é aquele do estimador $\widehat{\zeta}_{C R}$ $(-0.062)$.

Tabela 3.10. Medidas descritivas de diferentes estimadores de $\zeta$, distribuição gaussiana inversa, $n=10$.

\begin{tabular}{cccccccc}
\hline parâmetro & estimador & média & variância & viés & EQM & assimetria & curtose \\
\hline \multirow{5}{*}{$\zeta=2$} & $\widehat{\zeta}_{p}$ & 1.768 & 0.472 & -0.232 & 0.526 & 1.064 & 4.870 \\
& $\widehat{\zeta}_{B N}$ & 1.921 & 0.572 & -0.079 & 0.578 & 1.060 & 4.845 \\
& $\widehat{\widehat{\zeta}}_{B N}$ & 1.871 & 0.512 & -0.129 & 0.529 & 1.072 & 4.907 \\
& $\widehat{\zeta}_{B N}$ & 1.921 & 0.569 & -0.079 & 0.575 & 1.042 & 4.774 \\
& $\widehat{\zeta}_{C R}$ & 1.938 & 0.596 & -0.062 & 0.600 & 1.071 & 4.872 \\
& $\widehat{\zeta}_{S T}$ & 1.916 & 0.568 & -0.084 & 0.575 & 1.058 & 4.841 \\
\hline
\end{tabular}

A Tabela 3.11 apresenta taxas de rejeição sob a hipótese nula de diferentes testes; a hipótese nula sob teste é $\mathcal{H}_{0}: \zeta=2$. Note que as taxas de rejeição sob esta hipótese dos testes baseados nas verossimilhanças perfiladas modificadas estão mais próximas dos níveis nominais do que o teste da razão de verossimilhanças original. Os testes baseados em $\ell_{B N}$ e $\breve{\ell}_{B N}$ tiveram os melhores desempenhos.

Tabela 3.11. Taxas de rejeição da hipótese nula $\left(\mathcal{H}_{0}: \zeta=2\right)$ de diferentes testes da razão de verossimilhanças, distribuição gaussiana inversa, $n=10$.

\begin{tabular}{crrrrrr}
\hline nível nominal & \multicolumn{1}{c}{$\ell_{p}$} & \multicolumn{1}{c}{$\ell_{B N}$} & \multicolumn{1}{c}{$\bar{\ell}_{B N}$} & \multicolumn{1}{c}{$\breve{\ell}_{B N}$} & \multicolumn{1}{c}{$\ell_{C R}$} & \multicolumn{1}{c}{$\ell_{S T}$} \\
\hline $15 \%$ & 19.167 & 14.884 & 14.509 & 14.743 & 13.133 & 15.625 \\
$10 \%$ & 13.485 & 9.924 & 9.272 & 9.848 & 8.548 & 10.522 \\
$5 \%$ & 7.430 & 4.973 & 4.095 & 4.927 & 4.134 & 5.394 \\
$3 \%$ & 4.795 & 2.995 & 2.165 & 2.964 & 2.445 & 3.304 \\
$1 \%$ & 1.850 & 1.025 & 0.506 & 1.013 & 0.804 & 1.228 \\
$0.5 \%$ & 1.019 & 0.519 & 0.207 & 0.512 & 0.403 & 0.689 \\
$0.1 \%$ & 0.256 & 0.107 & 0.028 & 0.105 & 0.081 & 0.175 \\
$0.05 \%$ & 0.145 & 0.054 & 0.014 & 0.053 & 0.041 & 0.096 \\
$0.01 \%$ & 0.037 & 0.012 & 0.003 & 0.012 & 0.010 & 0.024 \\
\hline
\end{tabular}

As médias e variâncias das estatísticas de teste são apresentadas na Tabela 3.12, assim como seus valores assintóticos (1 e 2). Aqui, mais uma vez, são evidenciados os desempenhos 
das estatísticas de teste baseadas em $\ell_{B N}$ e $\breve{\ell}_{B N}$, pois tais estatísticas têm os primeiros dois momentos mais próximos da média e da variância assintóticas. Evidência similar obtém-se da Figura 3.4: as curvas de discrepâncias relativas de quantis destes testes (denotados como "BN" e "aprox. BN-2") estão mais próximas da ordenada zero do que as demais.

Tabela 3.12. Médias e variâncias amostrais de diferentes estatísticas de teste e os valores assintóticos destas, distribuição gaussiana inversa, $n=10$.

\begin{tabular}{cccccccc}
\hline momento & $\chi_{1}^{2}$ & $\ell_{p}$ & $\ell_{B N}$ & $\bar{\ell}_{B N}$ & $\breve{\ell}_{B N}$ & $\ell_{C R}$ & $\ell_{S T}$ \\
\hline média & 1.000 & 1.210 & 0.998 & 0.957 & 0.993 & 0.916 & 1.042 \\
variância & 2.000 & 2.875 & 2.012 & 1.600 & 2.000 & 1.749 & 2.240 \\
\hline
\end{tabular}

Figura 3.4. Gráfico das discrepâncias relativas de quantis, $n=10$.

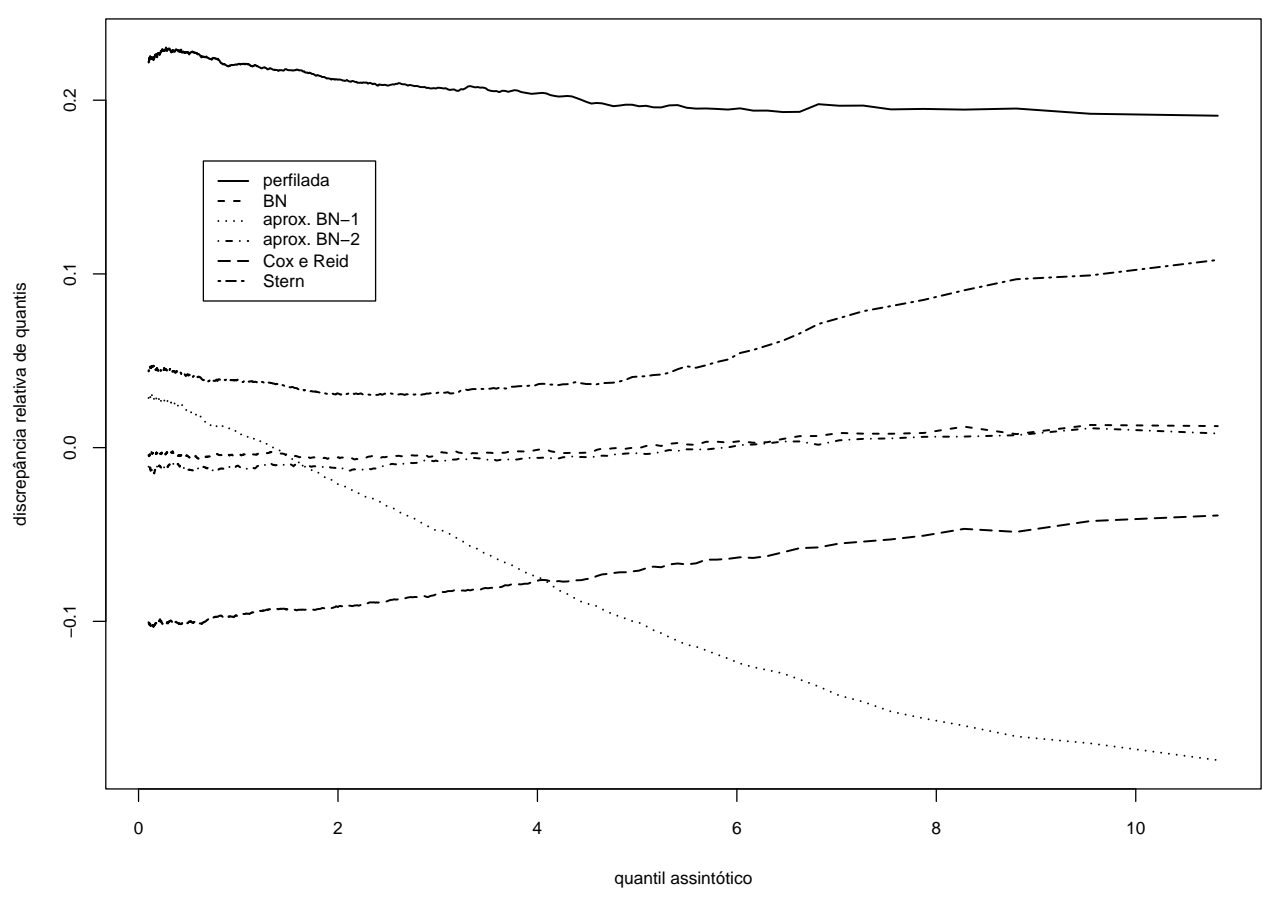

\subsection{CONCLUSÕES}

Inúmeros artigos publicados nas últimas duas décadas tratam de apresentar inferência sobre um subconjunto dos parâmetros que indexam um modelo qualquer, isto é, sobre como realizar inferência na presença de parâmetros de incômodo. Dentro deste contexto, diversas 
modificações da verossimilhança perfilada foram apresentadas no Capítulo 2, acompanhadas de algumas ilustrações em modelos probabilísticos pertencentes à família exponencial biparamétrica. Neste capítulo, foram apresentados resultados numéricos de simulações de Monte Carlo referentes a estes modelos que evidenciam a efetividade de tais modificações. Os resultados indicam que os vieses dos estimadores obtidos das verossimilhanças perfiladas ajustadas são menores do que aquele do estimador de máxima verossimilhança usual (perfilada). Adicionalmente, o tamanho do teste da razão de verossimilhanças original (perfilada) é tipicamente superestimado em pequenas amostras e os testes obtidos das verossimilhanças perfiladas ajustadas mostraram-se mais precisos. Em geral, todos os ajustes considerados produziram inferências mais precisas do que aquelas advindas do uso da verossimilhança perfilada original; nenhum desses ajustes foi uniformemente superior aos demais. O modelo considerado neste capítulo tem apenas um parâmetro de incômodo e, mesmo em tal situação, os ganhos de desempenho do uso de verossimilhanças perfiladas ajustadas em amostras finitas não são negligenciáveis. Assim, está claro que estes ajustes são mesmo mais promissores quando aplicados a situações em que o parâmetro de incômodo é vetorial. 


\section{CAPÍTULO 4 \\ PARÂMETRO DE HETEROGENEIDADE DA $\mathcal{G}_{A}^{0}(\alpha, \gamma, \mathcal{L})$ : TESTES UNICAUDAIS E ESTIMAÇÃO}

\subsection{INTRODUÇÃO}

A modelagem de fenômenos aleatórios confunde-se com a própria conceituação de Estatística, o que tem sido responsável por sua penetração em inúmeras áreas do conhecimento humano. A inferência estatística paramétrica lida com a construção de modelos paramétricos a serem ajustados a dados empíricos. A aplicação das técnicas deste ramo da Estatística proporcionou grandes avanços na área de processamento de imagens de radar, que acarretaram impactos nos diversos campos de estudos das ciências ambientais.

Entre os vários sistemas de radar, o tipo SAR (Synthetic Aperture Radar) tem evoluído e ocupado posição de destaque ao longo dos últimos 50 anos. A missão Apollo, que culminou com a chegada do homem à Lua em 1969, utilizou radares SAR para mapear a superfície lunar. As agências espaciais têm lançado vários satélites transportando estes radares, por exemplo: SEASAT (1978), SIR-A (1981), SIR-B (1984) e SIR-C (1994) da NASA, ERS-1 (1991) e ERS-2 da Agência Espacial Européia, JERS-1 (1992) da Agência Espacial Japonesa e Radarsat-1 (1995) da Agência Espacial Canadense. Existem restrições para dispor um radar no espaço, e, por isto, os sistemas orbitais são simples comparativamente aos aerotransportados, que utilizam diversas combinações de freqüência e polarização para o sinal eletromagnético. Isto permite uma maior extração de informações da área imageada. Entre os sistemas aerotransportados, destaca-se NASA/JPL AirSAR, desenvolvido pelo Laboratório de Propulsão a Jato (JPL-Jet Propulsion Laboratory) da NASA e utilizado para pesquisas ambientais.

O sistema SAR compõe a imagem de uma área a partir de sub-áreas imaginariamente delimitadas, denominadas células de resolução. A dimensão destas células é calibrada no radar e depende de vários parâmetros do sistema, como potência e freqüência do sinal eletromagnético. Dentro de cada célula de resolução existem alvos, cada alvo contribuindo para o retroespalhamento (reflexão) final do sinal eletromagnético daquela célula.

Para compor a imagem de uma região, os sistemas SAR utilizam dois atributos do campo elétrico do sinal de retorno: a amplitude $(A)$ e a fase $(\phi)$. Por isto, este processo de composição é dito coerente. Esses sistemas medem o par $(A \cos \phi, A \sin \phi)$. A amplitude está associada às características morfológicas e dielétricas do objeto, enquanto a fase associa-se à distância entre o objeto e o sensor do radar.

Ao processar as informações contidas no sinal de retorno a fim de compor a imagem de uma região, faz-se necessária a escolha de um critério para diferenciar as células de 
resolução. Tal diferenciação é habitualmente baseada nos valores assumidos por uma das grandezas físicas do sinal de retorno: $A \cos \phi, A \sin \phi, \phi, A, I$ ou $\log I$, onde $I=A^{2}$ denota a intensidade do sinal de retorno. Esta escolha da grandeza física determina o formato no qual a imagem será composta. Existem ainda sistemas chamados polarimétricos, aptos a lidar com o sinal completo, mas sua disseminação é limitada atualmente.

Uma imagem SAR é constituída por milhões de pixels, que idealmente representam as manifestações dos sinais de retorno das células de resolução através de diversos níveis de cinza. A proposta da abordagem estatística é compor essa imagem a partir de realizações independentes de uma variável aleatória, denotada por $z$.

Dependendo da área sujeita à monitoração do radar, as características físicas dos objetos, que são determinantes sobre as propriedades físicas do sinal de retorno de cada célula de resolução, podem variar bastante. As regiões imageadas são classificadas em três tipos: homogêneo, heterogêneo e extremamente heterogêneo. Para alguns sensores de radar e sob certas condições, esses tipos de regiões podem ser exemplificados, respectivamente, por uma área coberta de pasto, por uma região de floresta e por uma área urbana.

Para modelar regiões constituídas de diferentes naturezas de elementos e com a finalidade de ter um certo controle sobre essa diversidade a ponto de estabelecer critérios para distinguir as células de resolução, desejam-se distribuições polivalentes, ou seja, aquelas capazes de modelar dados provenientes de qualquer tipo de região, independentemente do seu grau de heterogeneidade. Até 1997 não existia uma distribuição com tal propriedade, além disso, as distribuições até então utilizadas não apresentavam bons resultados na análise e na modelagem de imagens de áreas urbanas, por exemplo.

Além de preencher a lacuna de uma distribuição para o sinal de retorno de regiões extremamente heterogêneas, a aplicação da distribuição $\mathcal{G}_{A}^{0}(\alpha, \gamma, \mathcal{L})$ proposta por Frery et al. (1997a) a dados reais atestou sua capacidade para modelar satisfatoriamente o sinal de retorno no formato amplitude de regiões heterogêneas e homogêneas. Este capítulo considera tal distribuição e, aqui, $z \sim \mathcal{G}_{A}^{0}(\alpha, \gamma, \mathcal{L})$.

O parâmetro $\alpha$ (parâmetro de interesse) é sempre negativo e possui interpretação importante: quanto maior o valor deste parâmetro, maior a heterogeneidade da área imageada. Entretanto, condiciona-se este fato à constância de alguns aspectos do sistema, como potência e freqüência do sinal emitido pelo radar nas diversas regiões. Desse modo, para valores de $\alpha$ entre -5 e 0 , associam-se regiões extremamente heterogêneas, entre -10 e -5 , associam-se regiões heterogêneas e valores menores que -10 correspondem a regiões homogêneas, segundo Frery et al. (1997a). Esta 'polivalência' da distribuição $\mathcal{G}_{A}^{0}$ é muito importante, pois estabelece um critério para a tipificação da região imageada.

O parâmetro $\gamma$ (parâmetro de perturbação) é sempre positivo e sua raiz quadrada é chamada de parâmetro de escala da família de distribuições $\mathcal{G}_{A}^{0}(\alpha, \gamma, \mathcal{L})$.

$\mathrm{O}$ parâmetro $\mathcal{L}$ representa o número de vezes que o radar SAR imageia uma região (number of looks), pode ser controlado no processo de geração da imagem e, portanto, é considerado conhecido, embora existam trabalhos na área de sensoriamento remoto onde é 
tratada sua estimação. $\mathcal{L} \geq 1$, geralmente sendo usados os valores 1,3 e 8 .

Uma parte deste capítulo consiste na estimação do parâmetro de heterogeneidade $\alpha$ através das maximizações da verossimilhança original $\ell$, da aproximação para a verossimilhança perfilada modificada proposta por Barndorff-Nielsen (1983), denotada por $\tilde{\ell}_{B N}$ (Seção 2.11.4), e também da verossimilhança perfilada ajustada proposta por Cox e Reid (1987, 1989), $\ell_{C R}$. Resultados numéricos comparando tais estimativas são apresentados. Diferentemente do método computacional de inferência estatística denominado bootstrap, utilizado em Cribari-Neto et al. (2002) para correção das estimativas de máxima verossimilhança do parâmetro $\alpha$, espera-se que as modificações sobre a verossimilhança perfilada produzam estimadores com vieses, em módulo, menores do que o valor absoluto do viés apresentado pelo estimador original. Pelas técnicas de bootstrap (Efron, 1979, 1990, 1993), as estimativas do viés deste estimador são obtidas numericamente e são subtraídas das respectivas estimativas de máxima verossimilhança. Assim, obtêm-se estimativas corrigidas até segunda ordem.

Neste capítulo também são realizados testes unicaudais sobre $\alpha$, utilizando as raízes

quadradas sinalizadas das estatísticas da razão de verossimilhanças obtidas de $\ell, \ell_{C R}$ e $\tilde{\ell}_{B N}$. Este enfoque é considerado por Sartori et al. (1999) para o ajuste de Barndorff-Nielsen (1983, 1994). Eles mostraram que as propriedades de tal estatística são freqüentemente superiores àquelas da estatística da razão de verossimilhanças sinalizada usual. A partir dos intervalos de valores de $\alpha$ característicos de cada tipo de região (extremamente heterogênea, heterogênea e homogênea), são realizados dois testes de hipóteses. O objetivo é comparar os desempenhos das verossimilhanças perfiladas a partir das distorções de tamanho dos testes associados e do poder destes.

\subsection{FUNÇÕES DE VEROSSIMILHANÇA PERFILADA}

\subsubsection{INTRODUÇÃO}

Se $z$ é uma variável aleatória com distribuição $\mathcal{G}_{A}^{0}(\alpha, \gamma, \mathcal{L})$, então sua função densidade de probabilidade é dada por

$$
p(z ; \alpha, \gamma, \mathcal{L})=p(z)=\frac{2 \mathcal{L}^{\mathcal{L}} \Gamma(\mathcal{L}-\alpha) z^{2 \mathcal{L}-1}}{\gamma^{\alpha} \Gamma(\mathcal{L}) \Gamma(-\alpha)\left(\gamma+\mathcal{L} z^{2}\right)^{\mathcal{L}-\alpha}}, \quad z \geq 0
$$

sua função de distribuição sendo dada por

$$
F(z)=\Upsilon_{2 \mathcal{L},-2 \alpha}\left(-\alpha z^{2} / \gamma\right)
$$

onde $\Upsilon_{2 \mathcal{L},-2 \alpha}(\cdot)$ é a função de distribuição de uma variável aleatória com distribuição $\mathcal{F}$ de Snedecor com graus de liberdade $2 \mathcal{L}$ e $-2 \alpha$. Esta relação entre as duas funções de distribuição facilita a realização de simulações computacionais. 
Essa distribuição não pertence à família exponencial biparamétrica e os estimadores de máxima verossimilhança não são estatísticas suficientes e minimais do par $(\alpha, \gamma)$. O momento de ordem $r$ dessa distribuição é expresso por

$$
E\left\{z^{r}\right\}=\left(\frac{\gamma}{\mathcal{L}}\right)^{r / 2} \frac{\Gamma(-\alpha-r / 2) \Gamma(\mathcal{L}+r / 2)}{\Gamma(-\alpha) \Gamma(\mathcal{L})} .
$$

A Figura 4.1 mostra as curvas de três funções densidades de probabilidades de variáveis com distribuições $\mathcal{G}_{A}^{0}(\alpha, \gamma, \mathcal{L})$, ou seja, curvas para diferentes ternas $(\alpha, \gamma, \mathcal{L})$. Estas funções estão normalizadas de tal forma que o valor esperado de $z$ seja 1 (um). São considerados valores do parâmetro $\alpha$ relativos aos três tipos de regiões: extremamente heterogêneo $(-1)$, heterogêneo $(-8)$ e homogêneo $(-15)$.

Figura 4.1. Funções densidades de probabilidades de variáveis com distribuições $\mathcal{G}_{A}^{0}(-1,0.405,1)$ (sólida), $\mathcal{G}_{A}^{0}(-8,7.882,3)$ (tracejada) e $\mathcal{G}_{A}^{0}(-15,14.704,8)$ (pontilhada).

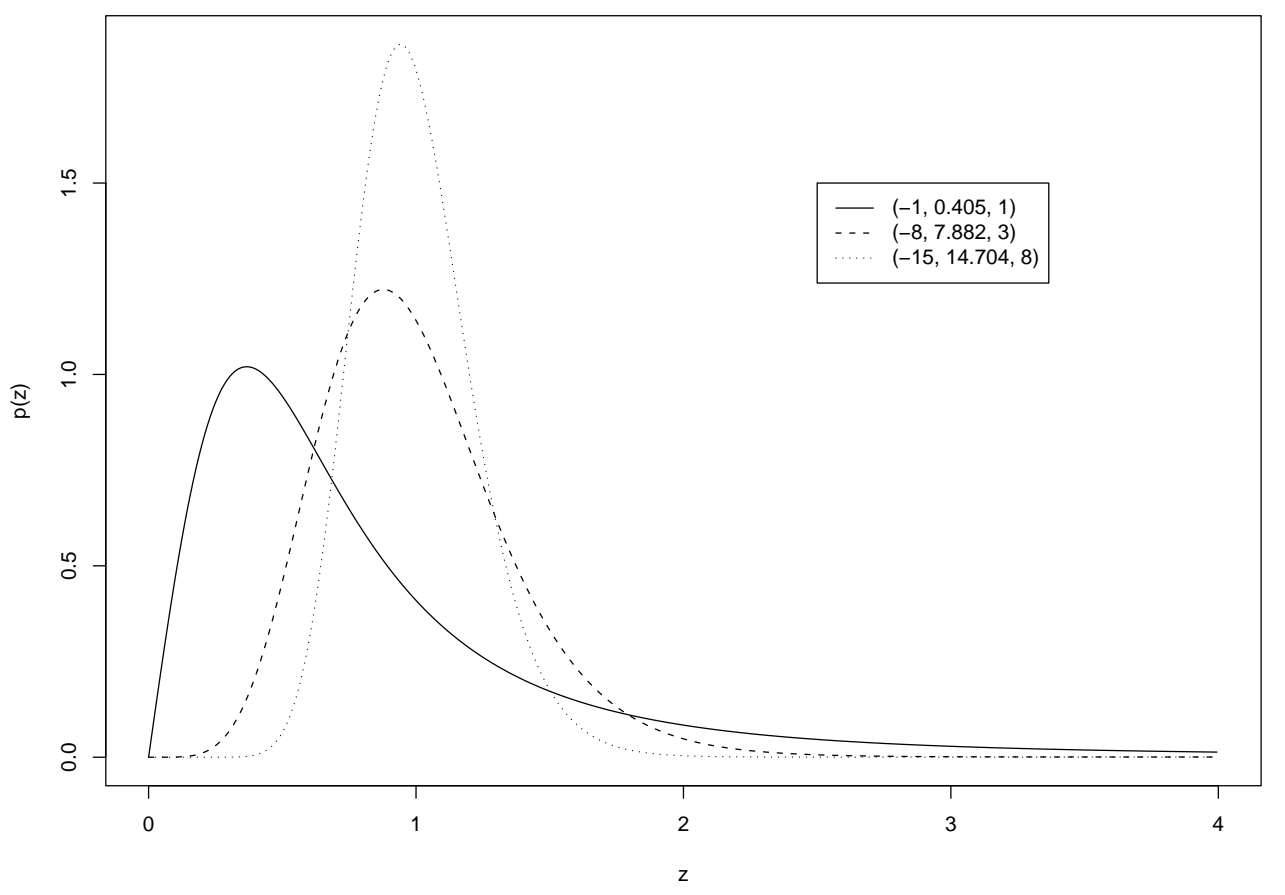

O logaritmo da função de verossimilhança associada a $n$ observações independentes e identicamente distribuídas $\left(z_{1}, \ldots, z_{n}\right)$ de uma variável aleatória com distribuição $\mathcal{G}_{A}^{0}(\alpha, \gamma, \mathcal{L})$ é dado por

$$
\ell(\alpha, \gamma)=n \log \Gamma(\mathcal{L}-\alpha)-n \alpha \log \gamma-n \log \Gamma(-\alpha)-(\mathcal{L}-\alpha) \sum_{i=1}^{n} \log \left(\gamma+\mathcal{L} z_{i}^{2}\right)
$$

Aqui, são consideradas apenas parcelas que dependem dos parâmetros $\alpha$ e $\gamma$. Derivando esta função parcialmente com relação aos parâmetros $\alpha$ e $\gamma$, os estimadores de máxima 
verossimilhança, $\widehat{\alpha}$ e $\widehat{\gamma}$, são a solução simultânea das duas equações a seguir, onde $\psi(\cdot)$ denota a função digama:

$$
n[\psi(-\widehat{\alpha})-\psi(\mathcal{L}-\widehat{\alpha})]+\sum_{i=1}^{n} \log \left(\frac{\widehat{\gamma}+\mathcal{L} z_{i}^{2}}{\widehat{\gamma}}\right)=0 \quad \text { e } \quad-\frac{n \widehat{\alpha}}{\widehat{\gamma}}-(\mathcal{L}-\widehat{\alpha}) \sum_{i=1}^{n} \frac{1}{\widehat{\gamma}+\mathcal{L} z_{i}^{2}}=0 .
$$

Estas equações são não-lineares. Em casos como este, o vetor solução $\widehat{\theta}=(\widehat{\alpha}, \widehat{\gamma})$ do sistema pode ser obtido por técnicas iterativas, como os métodos de Newton-Raphson, escore de Fisher e quase-Newton.

O logaritmo da função de verossimilhança perfilada é expresso por

$$
\ell_{p}(\alpha)=n \log \Gamma(\mathcal{L}-\alpha)-n \alpha \log \widehat{\gamma}_{\alpha}-n \log \Gamma(-\alpha)-(\mathcal{L}-\alpha) \sum_{i=1}^{n} \log \left(\widehat{\gamma}_{\alpha}+\mathcal{L} z_{i}^{2}\right),
$$

onde $\widehat{\gamma}_{\alpha}$ é a raiz da equação

$$
-\frac{n \alpha}{\widehat{\gamma}_{\alpha}}-(\mathcal{L}-\alpha) \sum_{i=1}^{n} \frac{1}{\widehat{\gamma}_{\alpha}+\mathcal{L} z_{i}^{2}}=0 .
$$

\subsubsection{APROXIMAÇÕES PARA A VEROSSIMILHANÇA PROPOSTA POR BARNDORFF-NIELSEN}

Da seção anterior, sabe-se que $\widehat{\gamma}_{\alpha}$ não possui forma fechada. Assim, usando as definições da Seção 2.11, aqui são apresentadas as expressões de três aproximações para $\ell_{B N}(\alpha)$ como função do $\operatorname{par}\left(\alpha, \widehat{\gamma}_{\alpha}\right)$. Tais funções não necessitam da especificação de uma estatística ancilar ou da determinação de uma parametrização ortogonal. São dadas por:

(i)

$$
\begin{gathered}
\bar{\ell}_{B N}(\alpha)=\ell_{p}(\alpha)+\frac{1}{2} \log j_{\gamma \gamma}\left(\alpha, \widehat{\gamma}_{\alpha}\right)-\log \left\{\frac{\widehat{\alpha}}{\widehat{\gamma}}\left[H\left(1, \mathcal{L} ; 1+\widehat{\alpha} ; \widehat{\gamma}_{\alpha}\right)-H\left(1, \mathcal{L} ; \widehat{\alpha} ; \frac{\widehat{\gamma}}{\widehat{\gamma}_{\alpha}}\right)\right]\right. \\
\left.+\left(\frac{\widehat{\alpha}}{\widehat{\gamma}}-\frac{\mathcal{L}-\widehat{\alpha}}{\widehat{\gamma}_{\alpha}-\widehat{\gamma}}\right) \frac{\Gamma(\mathcal{L}-\widehat{\alpha})}{\Gamma(\mathcal{L}) \Gamma(-\widehat{\alpha})}\left(1-\frac{\widehat{\gamma}}{\widehat{\gamma}_{\alpha}}\right)^{\widehat{\alpha}-\mathcal{L}}\left(\frac{\widehat{\gamma}_{\alpha}}{\widehat{\gamma}}\right)^{\widehat{\alpha}} \pi \csc (\widehat{\alpha} \pi)\right\}-\log \left(\frac{\mathcal{L}-\alpha}{\widehat{\gamma}_{\alpha}}\right)
\end{gathered}
$$

(ii)

$$
\breve{\ell}_{B N}(\alpha)=\ell_{p}(\alpha)+\frac{1}{2} \log j_{\gamma \gamma}\left(\alpha, \widehat{\gamma}_{\alpha}\right)-\log \left\{-\frac{n \alpha \widehat{\alpha}}{\widehat{\gamma} \widehat{\gamma}_{\alpha}}+\sum_{i=1}^{n}\left[\frac{(\mathcal{L}-\alpha)(\mathcal{L}-\widehat{\alpha})}{\left(\widehat{\gamma}_{\alpha}+\mathcal{L} z_{i}^{2}\right)\left(\widehat{\gamma}+\mathcal{L} z_{i}^{2}\right)}\right]\right\}
$$

$$
\tilde{\ell}_{B N}(\alpha)=\ell_{p}(\alpha)+\frac{1}{2} \log j_{\gamma \gamma}\left(\alpha, \widehat{\gamma}_{\alpha}\right)-\log (\mathcal{L}-\alpha)-\log \left[\sum_{i=1}^{n}\left(\frac{z_{i}}{\widehat{\gamma}_{\alpha}+\mathcal{L} z_{i}^{2}}\right)^{2}\right],
$$


onde

$$
j_{\gamma \gamma}\left(\alpha, \widehat{\gamma}_{\alpha}\right)=-\frac{n \alpha}{\widehat{\gamma}_{\alpha}^{2}}-(\mathcal{L}-\alpha) \sum_{i=1}^{n} \frac{1}{\left(\widehat{\gamma}_{\alpha}+\mathcal{L} z_{i}^{2}\right)^{2}}
$$

$\mathrm{e}$

$$
H(a, b ; c ; t)=\frac{\Gamma(c)}{\Gamma(a) \Gamma(b)} \sum_{k=0}^{\infty} \frac{\Gamma(a+k) \Gamma(b+k) t^{k}}{\Gamma(c+k) k !}
$$

é a função hipergeométrica.

Embora sejam mostradas três aproximações para $\ell_{B N}(\alpha)$, devido a dificuldades numéricas, apenas a terceira, $\tilde{\ell}_{B N}(\alpha)$, foi usada para a realização das simulações computacionais deste capítulo.

\subsubsection{VEROSSIMILHANÇA PROPOSTA POR COX E REID}

Cox e Reid (1989) discutiram a possibilidade de encontrar uma parametrização ortogonal $(\mu, \lambda)$ sob a qual a diferença entre $\widehat{\lambda}_{\mu}$ e $\widehat{\lambda}$ (respectivamente, estimador de máxima verossimilhança restrito e irrestrito do parâmetro de incômodo) fosse $O_{p}\left(n^{-3 / 2}\right)$, ao invés de $O_{p}\left(n^{-1}\right)$. Em tal caso, a verossimilhança perfilada ajustada proposta por Cox e Reid (1987) seria equivalente àquela proposta por Barndorff-Nielsen (1983) a menos de um erro de ordem $O_{p}\left(n^{-3 / 2}\right)$

e, assim, $\ell_{C R}$ teria viés da informação de ordem $O\left(n^{-1}\right)$ (DiCiccio et al., 1996). Embora Cox e Reid (1989) tenham mostrado a impossibilidade de obter tal parametrização ortogonal em geral, eles forneceram um critério para escolha entre parametrizações ortogonais. Utilizando esse critério, aqui foi obtida uma versão para $\ell_{C R}$ da qual as estimativas de máxima verossimilhança do parâmetro de heterogeneidade mostraram-se bem menos viesadas do que as estimativas obtidas da verossimilhança original. Este critério também foi utilizado por Yang e Xie (2003) a fim de obter estimativas menos viesadas para o parâmetro de forma da distribuição de Weibull, conforme os resultados apresentados no Capítulo 5 desta tese.

Pela equação (4) de Cox e Reid (1989), supondo $\mu$ (parâmetro de interesse) e $\lambda$ (parâmetro de incômodo) ortogonais, a diferença $\widehat{\lambda}_{\mu}-\widehat{\lambda}$, pode ser expressa por

$$
n\left(\widehat{\lambda}_{\mu}-\widehat{\lambda}\right)=-\frac{Z_{11}\left(\mu_{0}, \lambda_{0}\right)}{i_{02}\left(\mu_{0}, \lambda_{0}\right)} \delta_{\mu}-\frac{1}{2} \frac{i_{21}\left(\mu_{0}, \lambda_{0}\right)}{i_{02}\left(\mu_{0}, \lambda_{0}\right)} \delta_{\mu}^{2}+O_{p}\left(n^{-1 / 2}\right),
$$

em que $\left(\mu_{0}, \lambda_{0}\right)$ são os valores verdadeiros dos parâmetros, $n$ é o tamanho da amostra aleatória observada, $\delta_{\mu}$ é fixado, $i_{r s}=E\left\{n^{-1} \partial^{r+s} \ell(\mu, \lambda) / \partial \mu^{r} \partial \lambda^{s}\right\}$ e $Z_{r s}$ é uma variável aleatória limitada de média zero.

A partir da equação (5) de Cox e Reid (1989), a diferença $\widehat{\lambda}_{\mu}^{*}-\widehat{\lambda}^{*}$, onde $\lambda^{*}=h(\lambda)$ é uma transformação não-linear de $\lambda$, pode ser expressa como

$$
n\left(\widehat{\lambda}_{\mu}^{*}-\widehat{\lambda}^{*}\right)=-\frac{Z_{11}^{*}\left(\mu_{0}, \lambda_{0}\right)}{i_{02}^{*}\left(\mu_{0}, \lambda_{0}\right)} \delta_{\mu}-\left.\frac{1}{2} \frac{i_{21}\left(\mu_{0}, \lambda_{0}\right)}{i_{02}\left(\mu_{0}, \lambda_{0}\right)}\left(\frac{\mathrm{d} \lambda^{*}}{\mathrm{~d} \lambda}\right)\right|_{\lambda_{0}} \delta_{\mu}^{2}+O_{p}\left(n^{-1 / 2}\right) .
$$


Em geral, a primeira parcela desta expansão não é nula. Caso em que tal termo é zero ocorre quando $\mu$ e $\lambda$ são parâmetros canônicos de uma família exponencial $(2,2)$. O coeficiente de $\delta_{\mu}^{2}$ é nulo se, e somente se, $i_{21}\left(\mu_{0}, \lambda_{0}\right)=0$, uma condição que independe da transformação $h(\lambda)=\lambda^{*}$. Note que, embora de forma limitada, a dependência de $\widehat{\lambda}_{\mu}$ em relação a $\mu$ é reduzida pela transformação $h(\lambda)=\lambda^{*}$ ao fazer o coeficiente de $\delta_{\mu}^{2}$ independente de $\lambda$ (lembre que $\lambda=\lambda(\mu, \nu)$, onde $(\mu, \nu)$ é a parametrização original não-ortogonal). Neste caso, a variação quadrática de $\hat{\lambda}_{\mu}^{*}$ com $\mu$ torna-se tão livre dos efeitos do parâmetro de incômodo quanto possível. Esta escolha é produzida para cada $\mu_{0}$ fixado ao requerer que

$$
\frac{\mathrm{d} \lambda^{*}}{\mathrm{~d} \lambda}=c \frac{i_{02}\left(\mu_{0}, \lambda\right)}{i_{21}\left(\mu_{0}, \lambda\right)}
$$

ou seja,

$$
\lambda^{*}=c \int^{\lambda} \frac{i_{02}\left(\mu_{0}, \zeta\right)}{i_{21}\left(\mu_{0}, \zeta\right)} \mathrm{d} \zeta
$$

em que $c$ é qualquer constante conveniente. Ou seja, inicialmente é derivada uma verossimilhança perfilada ajustada segundo Cox e Reid (1987) (Seção 2.5) e, reparametrizando o parâmetro de incômodo conforme Cox e Reid (1989), é obtida uma nova verossimilhança perfilada modificada.

Também é possível escrever as equações definindo $\lambda^{*}=h(\lambda)$ em termos de uma parametrização não-ortogonal $(\mu, \nu)$. Neste caso, é necessário resolver duas equações, a saber:

$$
i_{02} \frac{\partial \nu(\mu, \lambda)}{\partial \mu}=-i_{11}
$$

e

$$
i_{02} \frac{\partial \nu(\mu, \lambda)}{\partial \lambda}=c\left\{i_{21}+2 i_{12} \frac{\partial \nu(\mu, \lambda)}{\partial \mu}+i_{03}\left(\frac{\partial \nu(\mu, \lambda)}{\partial \mu}\right)^{2}-i_{02} \frac{\partial^{2} \nu(\mu, \lambda)}{\partial \mu^{2}}\right\}
$$

Aqui, por simplicidade, omitiu-se o argumento $(\mu, \nu)$ dos termos $i_{r s}(\mu, \nu)$, definidos por $i_{r s}=E\left\{n^{-1} \partial^{r+s} \ell(\mu, \nu) / \partial \mu^{r} \partial \nu^{s}\right\}$.

Os parâmetros $\alpha$ (interesse) e $\gamma$ (incômodo) que indexam a distribuição $\mathcal{G}_{A}^{0}$ não são ortogonais. Neste caso, as equações diferenciais parciais (4.1) são expressas por

$$
\frac{\mathcal{L} \alpha}{\gamma^{2}(\mathcal{L}-\alpha+1)} \frac{\partial \gamma(\alpha, \lambda)}{\partial \alpha}=\frac{\mathcal{L}}{\gamma(\mathcal{L}-\alpha)}
$$

$\mathrm{e}$

$$
\begin{aligned}
\frac{\mathcal{L} \alpha}{\gamma^{2}(\mathcal{L}-\alpha+1)} \frac{\partial \gamma(\alpha, \lambda)}{\partial \lambda} & =c\left\{\frac{2\left(\mathcal{L}^{2}-2 \alpha \mathcal{L}+\mathcal{L}\right)}{\gamma^{2}(\mathcal{L}-\alpha+1)(\mathcal{L}-\alpha)} \frac{\partial \gamma(\alpha, \lambda)}{\partial \alpha}-\frac{\mathcal{L} \alpha}{\gamma^{2}(\mathcal{L}-\alpha+1)} \frac{\partial^{2} \gamma(\alpha, \lambda)}{\partial \alpha^{2}}\right. \\
-\frac{2 \alpha}{\gamma^{3}} & {\left.\left[1-\frac{(1-\alpha)(2-\alpha)}{(\mathcal{L}-\alpha+1)(\mathcal{L}-\alpha+2)}\right]\left[\frac{\partial \gamma(\alpha, \lambda)}{\partial \alpha}\right]^{2}\right\} . }
\end{aligned}
$$


Aqui, $i_{21}(\alpha, \gamma)=0$.

Da primeira destas equações, vem

$$
\gamma(\alpha, \lambda)=-\alpha\left(\frac{\alpha}{\alpha-\mathcal{L}}\right)^{1 / \mathcal{L}} \exp [h(\lambda)]
$$

Assim,

$$
\begin{gathered}
\frac{\partial \gamma}{\partial \lambda}=-\alpha\left(\frac{\alpha}{\alpha-\mathcal{L}}\right)^{1 / \mathcal{L}} \exp [h(\lambda)] h^{\prime}(\lambda), \\
\frac{\partial \gamma}{\partial \alpha}=-\frac{\mathcal{L}-\alpha+1}{\mathcal{L}-\alpha}\left(\frac{\alpha}{\alpha-\mathcal{L}}\right)^{1 / \mathcal{L}} \exp [h(\lambda)]
\end{gathered}
$$

$\mathrm{e}$

$$
\frac{\partial^{2} \gamma}{\partial \alpha^{2}}=-\frac{\mathcal{L}+1}{\alpha(\alpha-\mathcal{L})^{2}}\left(\frac{\alpha}{\alpha-\mathcal{L}}\right)^{1 / \mathcal{L}} \exp [h(\lambda)],
$$

em que $h^{\prime}(\lambda)=\mathrm{d} h(\lambda) / \mathrm{d} \lambda$. Substituindo estas derivadas na segunda das equações (4.1), vem

$$
h^{\prime}(\lambda)=\frac{c}{(\mathcal{L}-\alpha)^{2} \alpha^{2}}\left[\frac{2(\alpha-1)}{\mathcal{L}-\alpha+2}-(1+3 \mathcal{L})\right] .
$$

Logo,

$$
h(\lambda)=\frac{c}{(\mathcal{L}-\alpha)^{2} \alpha^{2}}\left[\frac{2(\alpha-1)}{\mathcal{L}-\alpha+2}-(1+3 \mathcal{L})\right] \lambda .
$$

Dessa forma,

$$
\gamma(\alpha, \lambda)=-\alpha\left(\frac{\alpha}{\alpha-\mathcal{L}}\right)^{1 / \mathcal{L}} \exp \left\{\frac{c}{(\mathcal{L}-\alpha)^{2} \alpha^{2}}\left[\frac{2(\alpha-1)}{\mathcal{L}-\alpha+2}-(1+3 \mathcal{L})\right] \lambda\right\}
$$

Substituindo $\gamma=\gamma(\alpha, \lambda)$ na verossimilhança original $\ell(\alpha, \gamma)$, obtém-se $\ell(\alpha, \lambda)$, a saber:

$$
\begin{gathered}
\ell(\alpha, \lambda)=n \log \Gamma(\mathcal{L}-\alpha)-n \alpha \log (-\alpha)-n \log \Gamma(-\alpha)-\frac{n \alpha}{\mathcal{L}} \log \left(\frac{\alpha}{\alpha-\mathcal{L}}\right) \\
-\frac{n c}{\alpha(\mathcal{L}-\alpha)^{2}}\left[\frac{2(\alpha-1)}{\mathcal{L}-\alpha+2}-(1+3 \mathcal{L})\right] \lambda \\
-(\mathcal{L}-\alpha) \sum_{i=1}^{n} \log \left\{-\alpha\left(\frac{\alpha}{\alpha-\mathcal{L}}\right)^{1 / \mathcal{L}} \exp \left[\frac{c}{(\mathcal{L}-\alpha)^{2} \alpha^{2}}\left(\frac{2(\alpha-1)}{\mathcal{L}-\alpha+2}-(1+3 \mathcal{L})\right) \lambda\right]+\mathcal{L} z_{i}^{2}\right\} .
\end{gathered}
$$

Como foi afirmado anteriormente, $c$ é qualquer constante conveniente independente de $\lambda$. Assim, uma parametrização ortogonal ótima é obtida ao fazer

$$
c=\alpha^{2}(\mathcal{L}-\alpha)^{K}\left[\frac{2(\alpha-1)}{\mathcal{L}-\alpha+2}-(1+3 \mathcal{L})\right]^{-1},
$$


onde $K>1$ é uma constante inteira determinada empiricamente. Por conseguinte, $h(\lambda)=$ $\lambda(\mathcal{L}-\alpha)^{K-2}$ e

$$
\begin{gathered}
\ell(\alpha, \lambda)=n \log \Gamma(\mathcal{L}-\alpha)-n \alpha \log (-\alpha)-n \log \Gamma(-\alpha)-\frac{n \alpha}{\mathcal{L}} \log \left(\frac{\alpha}{\alpha-\mathcal{L}}\right)-n \alpha \lambda(\mathcal{L}-\alpha)^{K-2} \\
-(\mathcal{L}-\alpha) \sum_{i=1}^{n} \log \left\{-\alpha\left(\frac{\alpha}{\alpha-\mathcal{L}}\right)^{1 / \mathcal{L}} \exp \left[\lambda(\mathcal{L}-\alpha)^{K-2}\right]+\mathcal{L} z_{i}^{2}\right\} .
\end{gathered}
$$

Fixando o parâmetro de interesse $(\alpha)$, o estimador de máxima verossimilhança restrito do parâmetro de perturbação $\left(\widehat{\lambda}_{\alpha}\right)$ satisfaz

$$
\begin{aligned}
\frac{\partial \ell\left(\alpha, \widehat{\lambda}_{\alpha}\right)}{\partial \lambda} & =-n \alpha(\mathcal{L}-\alpha)^{K-2}+\alpha\left(\frac{\alpha}{\alpha-\mathcal{L}}\right)^{1 / \mathcal{L}}(\mathcal{L}-\alpha)^{K-1} \exp \left[\widehat{\lambda}_{\alpha}(\mathcal{L}-\alpha)^{K-2}\right] \times \\
& \times \sum_{i=1}^{n}\left\{-\alpha\left(\frac{\alpha}{\alpha-\mathcal{L}}\right)^{1 / \mathcal{L}} \exp \left[\widehat{\lambda}_{\alpha}(\mathcal{L}-\alpha)^{K-2}\right]+\mathcal{L} z_{i}^{2}\right\}^{-1}=0 .
\end{aligned}
$$

Note que tal estimador não tem forma fechada.

A informação observada relativa ao parâmetro $\lambda$ é dada por

$$
\begin{gathered}
j_{\lambda \lambda}(\alpha, \lambda)=-\alpha \mathcal{L}\left(\frac{\alpha}{\alpha-\mathcal{L}}\right)^{1 / \mathcal{L}}(\mathcal{L}-\alpha)^{2 K-3} \exp \left[\lambda(\mathcal{L}-\alpha)^{K-2}\right] \times \\
\times \sum_{i=1}^{n} z_{i}^{2}\left\{-\alpha\left(\frac{\alpha}{\alpha-\mathcal{L}}\right)^{1 / \mathcal{L}} \exp \left[\lambda(\mathcal{L}-\alpha)^{K-2}\right]+\mathcal{L} z_{i}^{2}\right\}^{-2} .
\end{gathered}
$$

Portanto,

$$
\ell_{C R}(\alpha)=\ell\left(\alpha, \widehat{\lambda}_{\alpha}\right)-\frac{1}{2} \log j_{\lambda \lambda}\left(\alpha, \widehat{\lambda}_{\alpha}\right)
$$

\subsection{RESULTADOS NUMÉRICOS}

\subsubsection{INTRODUÇÃO}

Uma imagem SAR é constituída por milhões de pixels, que idealmente representam a manifestação do sinal de retorno de cada célula de resolução através de diversos níveis de cinza. As técnicas de filtragem (análise) de imagens podem ser utéis para vários fins, desde a restauração de efeitos indesejados (borramento por movimento do observador e/ou do alvo) até a recuperação de feições mascaradas pelo sistema imageador. Ver Jain (1989) para várias taxonomias e exemplos. 
Em todos os casos, a definição do tamanho da janela a ser empregado é essencial. Quanto maior, mais informação disponível, porém maior também a possibilidade de serem utilizados dados oriundos de mais de uma classe (categoria) de elementos presentes na região imageada. É por esta razão que é desejável a utilização da menor quantidade possível de dados no processo de filtragem ou, em outras palavras, de janelas pequenas.

É costumeiro o uso de janelas quadradas, sendo a menor possível a de tamanho 3 pixels $\times 3$ pixels. Uma janela desta dimensão, isto é, que emprega nove observações, pode ser pequena demais para permitir inferência com certo nível de precisão. Em várias aplicações, o uso de janelas de tamanhos $7 \times 7,9 \times 9$ ou $11 \times 11$ tem conduzido a bons resultados (Frery et al., 1997b).

Dessa forma, nas simulações computacionais deste capítulo, foram geradas amostras de tamanhos 25, 49, 81 e 121, ou seja, simularam-se janelas de dimensões $5 \times 5,7 \times 7$, $9 \times 9$ e $11 \times 11$. Um programa escrito na linguagem de programação Ox gerou 10000 amostras de Monte Carlo da distribuição $\mathcal{G}_{A}^{0}(\alpha, \gamma, \mathcal{L})$ com o par ordenado $(\alpha, \mathcal{L})$ assumindo os seguintes valores: $(-1,1),(-1,3),(-5,3),(-5,8),(-8,3),(-8,8),(-10,3),(-10,8)$, $(-15,3),(-15,8)$. Entre os valores adotados para $\alpha$, alguns são característicos dos três tipos de regiões: extremamente heterogêneo $(-1)$, heterogêneo $(-8)$ e homogêneo $(-15)$. $\alpha=-5$ e $\alpha=-10$ representam valores de fronteira entre estas regiões. $\mathcal{L}=1$ representa a situação mais adversa possível para composição de uma imagem. O parâmetro $\gamma$ assumiu os valores resultantes da substituição de cada par $(\alpha, \mathcal{L})$ na expressão

$$
\gamma=\mathcal{L}\left(\frac{\Gamma(-\alpha) \Gamma(\mathcal{L})}{\Gamma(-\alpha-1 / 2) \Gamma(\mathcal{L}+1 / 2)}\right)^{2}
$$

Este procedimento garantiu que o primeiro momento da distribuição tivesse valor unitário.

A seguir são apresentados resultados numéricos referentes à estimação pontual do parâmetro de heterogeneidade $\alpha$. São considerados os estimadores de máxima verossimilhança derivados de $\ell, \tilde{\ell}_{B N}$ e $\ell_{C R}$. As maximizações dessas funções foram realizadas utilizando o algoritmo de otimização alternada proposto por Frery et al. (2004) e descrito no Apêndice $\mathrm{B}$ desta tese. Os resultados são apresentados de forma análoga ao Capítulo 3 e, diferentemente desse capítulo, foram calculados os vieses relativos dos estimadores (v.r.): $100 \times$ (viés / parâmetro)\%. Assim, quanto à organização dos resultados da estimação pontual do parâmetro de heterogeneidade, os seguintes valores são apresentados em tabelas: valor verdadeiro, tamanho amostral $(n), \mathcal{L}$ (number of looks), média, estimativas do viés, da variância e do erro quadrático médio (EQM), viés relativo (v.r.) e estimativas dos coeficientes de assimetria e de curtose. Para denotar os estimadores de máxima verossimilhança, foi utilizada a notação definida no capítulo anterior e resumida na Tabela 3.1.

Além disso, foram realizados testes unicaudais sobre $\alpha$, utilizando as raízes quadradas sinalizadas das estatísticas da razão de verossimilhanças:

$$
\operatorname{sinal}(\widehat{\alpha}-\alpha) \sqrt{R V}, \quad \operatorname{sinal}\left(\widehat{\tilde{\alpha}}_{B N}-\alpha\right) \sqrt{R V_{B N}} \quad \text { e } \operatorname{sinal}\left(\widehat{\alpha}_{C R}-\alpha\right) \sqrt{R V_{C R}},
$$


onde $R V, \tilde{R V}{ }_{B N}$ e $R V_{C R}$ denotam as estatísticas da razão de verossimilhanças baseadas na verossimilhança original e nas versões ajustadas da verossimilhança perfilada denotadas por $\tilde{\ell}_{B N}$ e $\ell_{C R}$, respectivamente.

A partir dos intervalos de valores de $\alpha$ característicos de cada tipo de região, foram realizados dois testes de hipóteses:

(i) regiões heterogênea e homogênea $\times$ região extremamente heterogênea

$$
\mathcal{H}_{0}: \alpha \leq-5 \quad \text { contra } \quad \mathcal{H}_{1}:-5<\alpha<0
$$

(ii) região homogênea $\times$ regiões heterogênea e extremamente heterogênea

$$
\mathcal{H}_{0}: \alpha \leq-10 \quad \text { contra } \quad \mathcal{H}_{1}:-10<\alpha<0 .
$$

Aqui o objetivo foi comparar os desempenhos das verossimilhanças perfiladas a partir das distorções das taxas de rejeição dos testes sob a hipótese nula com relação aos valores nominais (tamanho), assim como a partir das taxas de rejeição dos mesmos, dado que $\mathcal{H}_{0}$ é falsa (poder). As distribuições assintóticas das estatísticas de teste é a normal padrão. Em situações práticas, estes testes podem ser utéis para um analista que precisa distinguir numa imagem, por exemplo, onde termina uma área urbana e inicia uma região de mata (teste (i)) ou de pastagem (teste (ii)).

Analogamente aos gráficos de discrepâncias relativas de quantis versus quantis da distribuição $\chi_{1}^{2}$ apresentados no capítulo anterior, foram construídos gráficos considerando os quantis da distribuição normal padrão. Entretanto, devido à indeterminação na mediana (zero), denotando o quantil amostral de ordem $q$ do conjunto de valores da estatística de teste por $\pm \sqrt{R V}(q)$, no caso do teste baseado na verossimilhança original, e o correspondente quantil da distribuição normal padrão por $N(0,1)(q)$, a discrepância considerada foi definida como

$$
\pm \sqrt{R V}(q)-N(0,1)(q)
$$

Quanto à organização dos resultados dos testes de hipóteses sobre o parâmetro de interesse, os seguintes valores são apresentados em tabelas: taxa de rejeição observada sob a hipótese nula, considerando níveis nominais de $10 \%$ e 5\%; média e variância amostrais das estatísticas de teste, bem como os valores assintóticos destes momentos; taxa de rejeição do teste, dado que $\mathcal{H}_{0}$ é falsa, considerando nível de significância de $5 \%$.

Aqui cabe uma observação importante: os resultados relativos a $\ell_{C R}(\alpha)$ foram obtidos ao fazer $K=2.5$ e $K=4$ nessa função, pois, de forma empírica, comparativamente a outros valores de $K$, tais valores produziram bons resultados numéricos, tanto na estimação de $\alpha$ quanto nos resultados obtidos pelas estatísticas de teste.

\subsubsection{ESTIMAÇÃO PONTUAL}

Esta seção apresenta resultados da estimação pontual do parâmetro $\alpha$ obtidos a partir de simulações de Monte Carlo. Para cada para $(n, \mathcal{L})$, foram geradas pouco mais que 10000 
(dez mil) amostras, pois, em menos de 1\% dessas, o algoritmo de estimação não apresentou convergência. Assim, garantiu-se resultados baseados, efetivamente, num conjunto de 10000 amostras.

A situação inicial, $\alpha=-1$, representa a simulação de observações da amplitude do sinal de retorno proveniente de uma região extremamente heterogênea, por exemplo, uma área urbana. O acompanhamento do processo de expansão das cidades é de grande importância para o planejamento urbano e a composição de imagens para estas regiões só foi viabilizada, com resultados satisfatórios, através da proposta da distribuição $\mathcal{G}_{A}^{0}(\alpha, \gamma, \mathcal{L})$ por Frery et al. (1997).

A Tabela 4.1 apresenta estatísticas descritivas de diferentes estimadores do parâmetro de heterogeneidade $\alpha$. Entre estes, $\widehat{\alpha}_{C R, K=4}$, obtido a partir da maximização de $\ell_{C R}(\alpha)$ para $K=4$, apresentou desempenho consideravelmente superior aos demais, tanto em termos de viés quanto em termos de erro quadrático médio (EQM).

Tabela 4.1. Medidas descritivas de diferentes estimadores de $\alpha$, distribuição $\mathcal{G}_{A}^{0}(\alpha, \gamma, \mathcal{L}), \alpha=-1$.

\begin{tabular}{ccccccccc}
\hline$(n, \mathcal{L})$ & estimador & média & variância & viés & EQM & v.r. $(\%)$ & assimetria & curtose \\
\hline \multirow{3}{*}{$(49,1)$} & $\widehat{\alpha}$ & -1.203 & 1.380 & -0.203 & 1.421 & 20.349 & -40.724 & 2467.124 \\
& $\widehat{\widetilde{\alpha}}_{B N}$ & -1.156 & 0.431 & -0.156 & 0.455 & 15.556 & -12.049 & 303.737 \\
& $\widehat{\alpha}_{C R, K=4}$ & -1.010 & 0.097 & -0.010 & 0.097 & 0.973 & -1.803 & 8.835 \\
& $\widehat{\alpha}_{C R, K=2.5}$ & -1.105 & 0.193 & -0.105 & 0.204 & 10.471 & -3.344 & 24.822 \\
\hline \multirow{2}{*}{$(25,3)$} & $\widehat{\alpha}$ & -1.198 & 0.358 & -0.198 & 0.397 & 19.768 & -6.553 & 96.423 \\
& $\widehat{\tilde{\alpha}}_{B N}$ & -1.151 & 0.285 & -0.151 & 0.308 & 15.116 & -5.268 & 66.652 \\
& $\widehat{\alpha}_{C R, K=4}$ & -1.066 & 0.145 & -0.066 & 0.150 & 6.643 & -2.120 & 12.085 \\
& $\widehat{\alpha}_{C R, K=2.5}$ & -1.126 & 0.220 & -0.126 & 0.236 & 12.627 & -3.422 & 29.257 \\
\hline
\end{tabular}

Para uma janela de tamanho $7 \times 7(n=49)$ e número de visadas $(\mathcal{L})$ igual a um, situação mais adversa possível para produção de uma imagem em termos deste parâmetro, o viés relativo do estimador $\widehat{\alpha}_{C R, K=4}(0.973 \%)$ foi aproximadamente vinte vezes menor do que aquele apresentado pelo estimador de máxima verossimilhança original $\widehat{\alpha}$ (20.349\%). As estimativas dos erros quadráticos médios desses estimadores foram iguais a 0.097 e 1.421, respectivamente, ou seja, o valor apresentado por $\widehat{\alpha}_{C R, K=4}$ foi aproximadamente 14 vezes menor do que aquele apresentado por $\widehat{\alpha}$. As estimativas dos coeficientes de assimetria $(-1.803)$ e de curtose (8.835) da distribuição do estimador $\widehat{\alpha}_{C R, K=4}$ foram as mais próximas dos valores assintóticos, 0 e 3, respectivamente, enquanto as estimativas relativas a $\widehat{\alpha}$ apresentaram-se muito distantes: -40.724 e 2467.124 , respectivamente.

Reduzindo à metade o tamanho amostral, considere agora uma janela de tamanho $5 \times 5$ $(n=25)$ e número de visadas $(\mathcal{L})$ igual a três. Neste caso, o viés relativo do estimador $\widehat{\alpha}_{C R, K=4}(6.643 \%)$ foi aproximadamente três vezes menor do que aquele apresentado pelo estimador de máxima verossimilhança original $\widehat{\alpha}(19.768 \%)$, enquanto a estimativa de sua variância foi aproximadamente metade daquela apresentada por $\widehat{\alpha}$ : 0.145 e 0.358 , respectivamente. Mais uma vez, as estimativas dos coeficientes de assimetria e de curtose da 
distribuição de $\widehat{\alpha}_{C R, K=4}(-2.120$ e 12.085) foram as mais próximas dos valores assintóticos e consideravelmente inferiores, em módulo, às estimativas associadas a $\widehat{\alpha}$ : -6.553 e 96.423 , respectivamente.

Agora, considere o caso em que $\alpha=-5$, um valor do parâmetro de heterogeneidade no limiar de regiões classificadas como heterogêneas e extremamente heterogêneas, segundo a escala de heterogeneidade definida a partir de valores assumidos por tal parâmetro. A Tabela 4.2 apresenta estatísticas descritivas de diferentes estimadores de $\alpha$. Novamente, entre os estimadores, $\widehat{\alpha}_{C R, K=4}$ apresentou desempenho consideravelmente superior aos demais, tanto em termos de viés quanto em termos de erro quadrático médio (EQM).

Tabela 4.2. Medidas descritivas de diferentes estimadores de $\alpha$, distribuição $\mathcal{G}_{A}^{0}(\alpha, \gamma, \mathcal{L}), \alpha=-5$.

\begin{tabular}{ccrrrrrrr}
\hline$(n, \mathcal{L})$ & estimador & média & variância & \multicolumn{1}{c}{ viés } & EQM & v.r.(\%) & assimetria & curtose \\
\hline \multirow{3}{*}{$(25,8)$} & $\widehat{\alpha}$ & -8.440 & 426.668 & -3.440 & 438.502 & 68.802 & -17.992 & 415.553 \\
& $\widehat{\tilde{\alpha}}_{B N}$ & -7.998 & 424.218 & -2.998 & 433.203 & 59.950 & -18.215 & 422.298 \\
& $\widehat{\alpha}_{C R, K=4}$ & -5.100 & 4.909 & -0.100 & 4.919 & 2.001 & -1.873 & 8.536 \\
& $\widehat{\alpha}_{C R, K=2.5}$ & -6.326 & 101.089 & -1.326 & 102.849 & 26.530 & -40.517 & 2087.235 \\
\hline \multirow{2}{*}{$(81,3)$} & $\widehat{\alpha}$ & -6.921 & 162.281 & -1.921 & 165.970 & 38.415 & -23.567 & 755.522 \\
& $\widehat{\widetilde{\alpha}}_{B N}$ & -6.815 & 162.472 & -1.815 & 165.766 & 36.295 & -23.566 & 755.209 \\
& $\widehat{\alpha}_{C R, K=4}$ & -4.763 & 2.590 & 0.237 & 2.647 & -4.749 & -1.777 & 8.989 \\
& $\widehat{\alpha}_{C R, K=2.5}$ & -5.663 & 9.200 & -0.663 & 9.639 & 13.251 & -4.813 & 50.625 \\
\hline
\end{tabular}

Por exemplo, para $(n, \mathcal{L})=(81,3)$, o erro quadrático médio de $\widehat{\alpha}_{C R, K=4}$ foi igual a 2.647, sessenta e duas vezes menor do que o valor apresentado pelo estimador original, 165.970. Comparando os módulos dos vieses relativos desses estimadores, o valor apresentado por este último (38.415\%) é oito vezes maior do que a estimativa associada ao estimador $\widehat{\alpha}_{C R, K=4}, 4.749 \%$. Vale destacar também que, entre os estimadores, os coeficientes de assimetria $(-1.777)$ e de curtose (8.989) deste apresentaram-se mais próximos aos valores assintóticos, 0 e 3, respectivamente.

A Floresta Amazônica, que cobre grande parte da região norte do Brasil, é um típico exemplo de uma região heterogênea. Nesta área, a diversidade de textura das copas das árvores não é tão grande quanto as diferenças observadas numa cidade e nem tão pequena quanto aquela apresentada pelos campos da região sul do Brasil (região dos Pampas gaúchos).

Valores do parâmetro de heterogeneidade entre - 5 e -10 são característicos de áreas heterogêneas. A Tabela 4.3 apresenta resultados da estimação pontual do parâmetro $\alpha$ quando seu verdadeiro valor é igual a -8 . Mais uma vez, o melhor desempenho foi do estimador $\widehat{\alpha}_{C R, K=4}$. Por exemplo, para $(n, \mathcal{L})=(49,8)$, as estimativas do erro quadrático médio (EQM) e do viés relativo (v.r.) deste foram, respectivamente, iguais a 7.997 e $-2.148 \%$, seguido pelo estimador $\widehat{\alpha}_{C R, K=2.5}$, cujas estimativas foram iguais a 64.838 e $15.687 \%$. Note também que os coeficientes de assimetria (-1.661) e de curtose (7.812) da distribuição de $\widehat{\alpha}_{C R, K=4}$ mostraram-se mais próximos aos valores assintóticos. 
Tabela 4.3. Medidas descritivas de diferentes estimadores de $\alpha$, distribuição $\mathcal{G}_{A}^{0}(\alpha, \gamma, \mathcal{L}), \alpha=-8$.

\begin{tabular}{ccrrrrrrr}
\hline$(n, \mathcal{L})$ & estimador & \multicolumn{1}{c}{ média } & variância & \multicolumn{1}{c}{ viés } & \multicolumn{1}{c}{ EQM } & v.r.(\%) & assimetria & curtose \\
\hline & $\widehat{\alpha}$ & -10.606 & 126.190 & -2.606 & 132.983 & 32.580 & -16.621 & 494.639 \\
$(49,8)$ & $\widehat{\tilde{\alpha}}_{B N}$ & -10.324 & 126.096 & -2.324 & 131.496 & 29.049 & -16.714 & 497.405 \\
& $\widehat{\alpha}_{C R, K=4}$ & -7.828 & 7.968 & 0.172 & 7.997 & -2.148 & -1.661 & 7.812 \\
& $\widehat{\alpha}_{C R, K=2.5}$ & -9.255 & 63.263 & -1.255 & 64.838 & 15.687 & -32.205 & 1700.708 \\
\hline \multirow{2}{*}{$(121,3)$} & $\widehat{\alpha}$ & -11.792 & 318.949 & -3.792 & 333.328 & 47.400 & -12.154 & 217.989 \\
& $\widehat{\tilde{\alpha}}_{B N}$ & -11.648 & 319.535 & -3.648 & 332.840 & 45.594 & -12.151 & 217.739 \\
& $\widehat{\alpha}_{C R, K=4}$ & -7.195 & 6.063 & 0.805 & 6.711 & -10.065 & -2.754 & 41.184 \\
& $\widehat{\alpha}_{C R, K=2.5}$ & -9.471 & 137.024 & -1.471 & 139.189 & 18.389 & -21.475 & 654.171 \\
\hline
\end{tabular}

Agora considere $\alpha=-10$, um valor do parâmetro de heterogeneidade no limiar de regiões classificadas como heterogêneas e homogêneas. A Tabela 4.4 apresenta resultados da estimação pontual de $\alpha$. Aqui também o melhor desempenho foi do estimador $\widehat{\alpha}_{C R, K=4}$. Na Tabela 4.4 , para $(n, \mathcal{L})=(121,3)$, as estimativas das variâncias de $\widehat{\alpha}, \widehat{\tilde{\alpha}}_{B N}, \widehat{\alpha}_{C R, K=2.5}$, foram, respectivamente, iguais a 565.638, 566.338 e 229.064, muito superiores à estimativa apresentada por $\widehat{\alpha}_{C R, K=4}, 8.398$.

Tabela 4.4. Medidas descritivas de diferentes estimadores de $\alpha$, distribuição $\mathcal{G}_{A}^{0}(\alpha, \gamma, \mathcal{L}), \alpha=-10$.

\begin{tabular}{ccccccccc}
\hline$(n, \mathcal{L})$ & estimador & \multicolumn{1}{c}{ média } & variância & \multicolumn{1}{c}{ viés } & EQM & v.r.(\%) & assimetria & curtose \\
\hline \multirow{3}{*}{$(81,8)$} & $\widehat{\alpha}$ & -12.112 & 93.041 & -2.112 & 97.502 & 21.122 & -18.525 & 565.235 \\
& $\widehat{\widetilde{\alpha}}_{B N}$ & -11.856 & 91.807 & -1.856 & 95.253 & 18.562 & -18.942 & 582.709 \\
& $\widehat{\alpha}_{C R, K=4}$ & -9.742 & 10.278 & 0.258 & 10.345 & -2.584 & -1.710 & 8.472 \\
& $\widehat{\alpha}_{C R, K=2.5}$ & -11.021 & 24.471 & -1.021 & 25.513 & 10.209 & -3.686 & 32.524 \\
\hline \multirow{2}{*}{$(121,3)$} & $\widehat{\alpha}$ & -16.601 & 565.638 & -6.601 & 609.214 & 66.012 & -6.731 & 66.176 \\
& $\widehat{\widetilde{\alpha}}_{B N}$ & -16.584 & 566.338 & -6.584 & 609.686 & 65.839 & -6.725 & 66.102 \\
& $\widehat{\alpha}_{C R, K=4}$ & -8.422 & 8.398 & 1.578 & 10.886 & -15.775 & -1.218 & 5.002 \\
& $\widehat{\alpha}_{C R, K=2.5}$ & -12.390 & 229.064 & -2.390 & 234.774 & 23.895 & -11.351 & 190.176 \\
\hline
\end{tabular}

Tamanha diferença não foi observada nas estimativas dos vieses. Enquanto os estimadores $\widehat{\alpha}, \widehat{\tilde{\alpha}}_{B N}, \widehat{\alpha}_{C R, K=2.5}$ apresentaram vieses, em módulo, iguais a 6.601, 6.584 e 2.390, o estimador $\widehat{\alpha}_{C R, K=4}$ apresentou viés igual a 1.578 .

A Tabela 4.5 apresenta medidas descritivas de diferentes estimadores de $\alpha$ quando seu valor verdadeiro é igual a -15 . Como mencionado anteriormente, tal valor é característico de uma área classificada como homogênea; a região de fronteira entre Brasil e Uruguai é um exemplo típico, são os Pampas gaúchos.

Para $(n, \mathcal{L})=(81,8)$, note que os vieses relativos dos estimadores $\widehat{\alpha}, \widehat{\tilde{\alpha}}_{B N}, \widehat{\alpha}_{C R, K=4}$ e $\widehat{\alpha}_{C R, K=2.5}$ foram, em módulo, respectivamente, iguais a $41.515 \%, 40.759 \%, 8.907 \%$ e $17.413 \%$. Nesta mesma ordem, considerando as estimativas dos erros quadráticos médios, têm-se 739.104, 739.982, 27.969 e 286.727. Observe que o estimador com desempenho mais próximo ao do estimador $\widehat{\alpha}_{C R, K=4}$ tem erro quadrático médio dez vezes maior. Considerando 
os valores assintóticos dos coeficientes de assimetria e curtose de estimadores de máxima verossimilhança como valores verdadeiros, as estimativas apresentadas por $\widehat{\alpha}_{C R, K=4}$ foram as menos viesadas, 3.142 e 43.471, em módulo. Para $(n, \mathcal{L})=(121,3)$, o estimador $\widehat{\alpha}_{C R, K=2.5}$ apresentou o menor viés relativo em valor absoluto, $3.762 \%$, entretanto o estimador $\widehat{\alpha}_{C R, K=4}$ apresentou menor erro quadrático médio, 32.670.

Tabela 4.5. Medidas descritivas de diferentes estimadores de $\alpha$, distribuição $\mathcal{G}_{A}^{0}(\alpha, \gamma, \mathcal{L}), \alpha=-15$.

\begin{tabular}{cccrrrrrr}
\hline$(n, \mathcal{L})$ & estimador & \multicolumn{1}{c}{ média } & variância & \multicolumn{1}{c}{ viés } & \multicolumn{1}{c}{ EQM } & v.r.(\%) & assimetria & curtose \\
\hline \multirow{3}{*}{$(81,8)$} & $\widehat{\alpha}$ & -21.227 & 700.326 & -6.227 & 739.104 & 41.515 & -10.996 & 178.476 \\
& $\widehat{\widetilde{\alpha}}_{B N}$ & -21.114 & 702.603 & -6.114 & 739.982 & 40.759 & -10.960 & 177.639 \\
& $\widehat{\alpha}_{C R, K=4}$ & -13.664 & 26.184 & 1.336 & 27.969 & -8.907 & -3.142 & 43.471 \\
& $\widehat{\alpha}_{C R, K=2.5}$ & -17.612 & 279.904 & -2.612 & 286.727 & 17.413 & -18.027 & 526.996 \\
\hline \multirow{2}{*}{$(121,3)$} & $\widehat{\alpha}$ & -32.439 & 2543.590 & -17.439 & 2847.704 & 116.259 & -3.867 & 19.710 \\
& $\widehat{\tilde{\alpha}}_{B N}$ & -32.238 & 2551.771 & -17.238 & 2848.905 & 114.917 & -3.861 & 19.657 \\
& $\widehat{\alpha}_{C R, K=4}$ & -10.252 & 10.128 & 4.748 & 32.670 & -31.652 & -0.674 & 2.930 \\
& $\widehat{\alpha}_{C R, K=2.5}$ & -15.564 & 72.390 & -0.564 & 72.709 & 3.762 & -1.477 & 5.059 \\
\hline
\end{tabular}

Observando os resultados das cinco tabelas apresentadas nesta seção, os estimadores com melhores desempenhos foram aqueles obtidos do ajuste sobre a verossimilhança perfilada proposto por Cox e Reid (1987, 1989), que requer a ortogonalidade entre os parâmetros de interesse e de incômodo, acarretando a independência assintótica dos estimadores de máxima verossimilhança destes. Os comportamentos dos estimadores derivados da verossimilhança original e do ajuste sobre a verossimilhança perfilada denotado por $\tilde{\ell}_{B N}$ foram bastante similares.

\subsubsection{TESTES UNICAUDAIS}

Esta seção apresenta resultados relativos aos testes de hipóteses realizados sobre o parâmetro $\alpha$ e obtidos a partir de simulações de Monte Carlo. Taxas de rejeição dos testes sob a hipótese nula e quantis amostrais das distribuições nulas das estatísticas de teste foram calculados a partir de pouco mais que 10000 (dez mil) amostras para cada par $(n, \mathcal{L})$, como mencionado anteriormente. Taxas de rejeição dos testes, dado que $\mathcal{H}_{0}$ é falsa, foram calculadas a partir de pouco mais que 5000 amostras de Monte Carlo, pois, em menos de 1\% dessas, o algoritmo não apresentou convergência. Assim, neste caso, garantiu-se resultados baseados, efetivamente, em 5000 amostras.

(i) regiões heterogênea e homogênea $\times$ região extremamente heterogênea

$$
\mathcal{H}_{0}: \alpha \leq-5 \quad \text { contra } \quad \mathcal{H}_{1}:-5<\alpha<0
$$

A Tabela 4.6 apresenta taxas de rejeição da hipótese nula de diferentes testes da razão 
Tabela 4.6. Taxas de rejeição da hipótese nula de diferentes testes da razão de verossimilhanças sinalizada, distribuição $\mathcal{G}_{A}^{0}(\alpha, \gamma, \mathcal{L}), \alpha=-5$.

\begin{tabular}{cccccc}
\hline$(n, \mathcal{L})$ & nível nominal & $\ell$ & $\tilde{\ell}_{B N}$ & $\ell_{C R, K=4}$ & $\ell_{C R, K=2.5}$ \\
\hline \multirow{2}{*}{$(25,8)$} & $10 \%$ & 6.513 & 8.037 & 12.725 & 8.925 \\
& $5 \%$ & 3.150 & 3.925 & 6.400 & 4.375 \\
\hline \multirow{2}{*}{$(81,3)$} & $10 \%$ & 7.920 & 9.080 & 16.320 & 10.500 \\
& $5 \%$ & 3.880 & 4.570 & 8.770 & 5.500 \\
\hline
\end{tabular}

de verossimilhanças sinalizada, considerando dois níveis de significância, $5 \%$ e $10 \%$. O valor verdadeiro do parâmetro $\alpha$ é -5 e são considerados dois pares $(n, \mathcal{L}):(25,8)$ e $(81,3)$.

Observe na Tabela 4.6 que os testes baseados em $\tilde{\ell}_{B N}$ e $\ell_{C R, K=2.5}$ apresentaram taxas de rejeição mais próximas aos níveis nominais; por exemplo, para um nível de significância de $10 \%$ e par $(n, \mathcal{L})=(81,3)$, os valores relativos aos testes baseados em $\ell, \tilde{\ell}_{B N}, \ell_{C R, K=4} \mathrm{e}$ $\ell_{C R, K=2.5}$ foram iguais a $7.920 \%, 9.080 \%, 16.320 \%$ e $10.500 \%$, respectivamente. Vale lembrar que $\alpha=-5$ é um valor do parâmetro de heterogeneidade no limiar de regiões classificadas como heterogêneas e extremamente heterogêneas. Note que o teste baseado em $\ell_{C R, K=4}$ foi bastante liberal, rejeitando a hipótese nula além do esperado.

Tabela 4.7. Médias e variâncias amostrais de diferentes estatísticas de teste e os valores assintóticos destas, distribuição $\mathcal{G}_{A}^{0}(\alpha, \gamma, \mathcal{L}), \alpha=-5$.

\begin{tabular}{ccccccc}
\hline$(n, \mathcal{L})$ & momento & $N(0,1)$ & \multicolumn{1}{c}{$\ell$} & $\tilde{\ell}_{B N}$ & $\ell_{C R, K=4}$ & $\ell_{C R, K=2.5}$ \\
\hline \multirow{2}{*}{$(25,8)$} & média & 0 & -0.245 & -0.113 & 0.237 & -0.019 \\
& variância & 1 & 1.035 & 1.014 & 0.829 & 0.953 \\
\hline \multirow{2}{*}{$(81,3)$} & média & 0 & -0.143 & -0.069 & 0.385 & 0.052 \\
& variância & 1 & 1.017 & 1.007 & 0.825 & 0.946 \\
\hline
\end{tabular}

A Tabela 4.7 apresenta médias e variâncias amostrais das diferentes estatísticas de teste e os valores assintóticos destas. Note que a estatística baseada em $\ell_{C R, K=4}$ apresentou momentos empíricos bem distantes dos valores assintóticos; por exemplo, para $(n, \mathcal{L})=$ $(81,3)$, a média e a variância foram, respectivamente, iguais a 0.385 e 0.825 . As estatísticas de teste baseadas em $\tilde{\ell}_{B N}$ e $\ell_{C R, K=2.5}$ apresentaram momentos amostrais mais próximos aos valores assintóticos. Ainda para $(n, \mathcal{L})=(81,3)$, para estes testes, as médias foram iguais a -0.069 e 0.052 , enquanto as variâncias, 1.007 e 0.946, respectivamente.

Os gráficos das Figuras 4.2 e 4.3 apresentam as curvas de discrepâncias entre os quantis amostrais das estatísticas de teste e os respectivos quantis assintóticos versus os quantis da normal padrão (quantis assintóticos). Quanto mais próxima da ordenada nula as curvas estiverem, melhor será a aproximação da distribuição nula da estatística de teste pela distribuição $\mathcal{N}(0,1)$. Para $(n, \mathcal{L})=(25,8)$, o gráfico da Figura 4.2 apresenta tais curvas. Por este gráfico, a melhor aproximação pela normal padrão ocorre para a distribuição nula da estatística de teste baseada em $\ell_{C R, K=2.5}$, embora os resultados das Tabelas 4.6 e 4.7 não indiquem claramente isto. No gráfico da Figura $4.3,(n, \mathcal{L})=(81,3)$, vale destacar a melhor 
Figura 4.2. Gráfico das discrepâncias de quantis, distribuição $\mathcal{G}_{A}^{0}, \alpha=-5,(n, \mathcal{L})=(25,8)$.

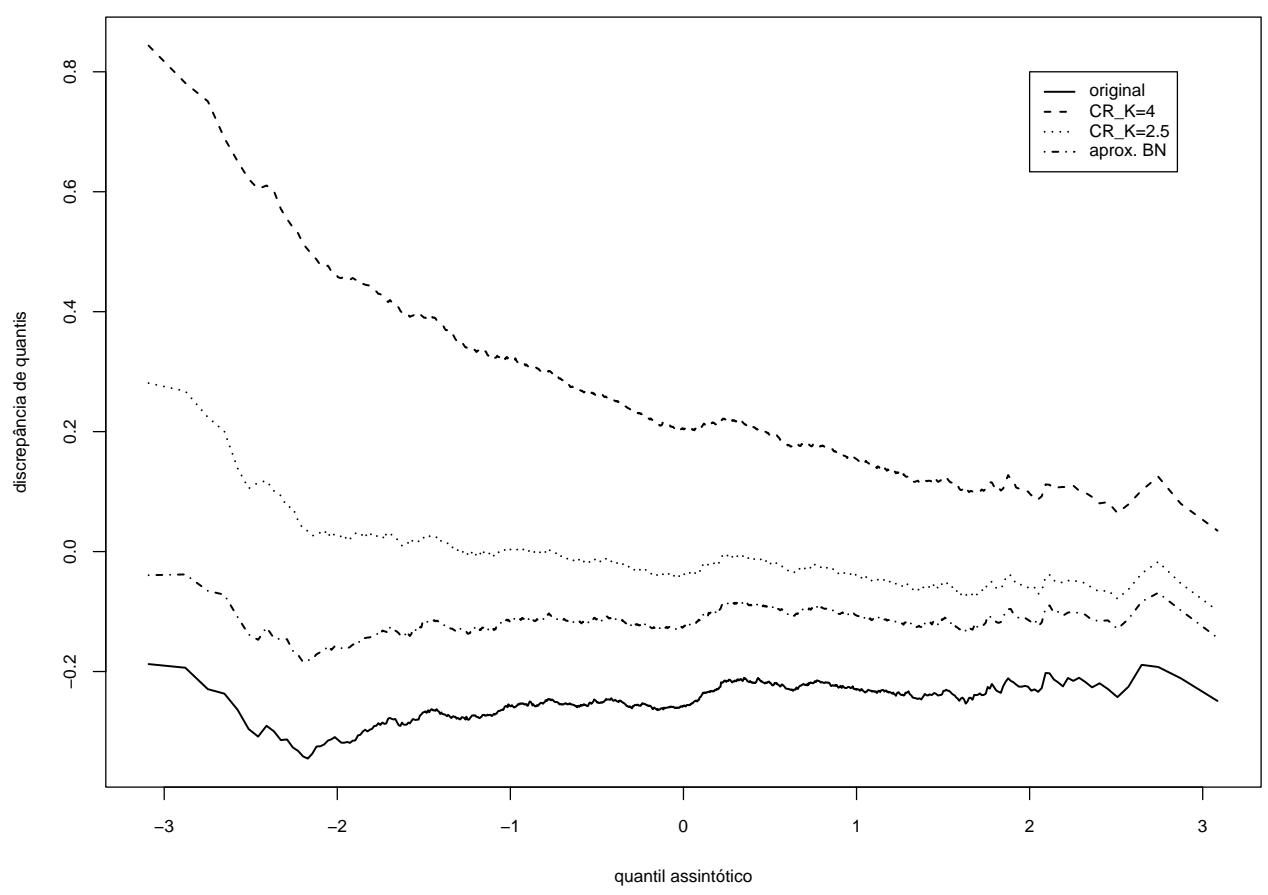

Figura 4.3. Gráfico das discrepâncias de quantis, distribuição $\mathcal{G}_{A}^{0}, \alpha=-5,(n, \mathcal{L})=(81,3)$.

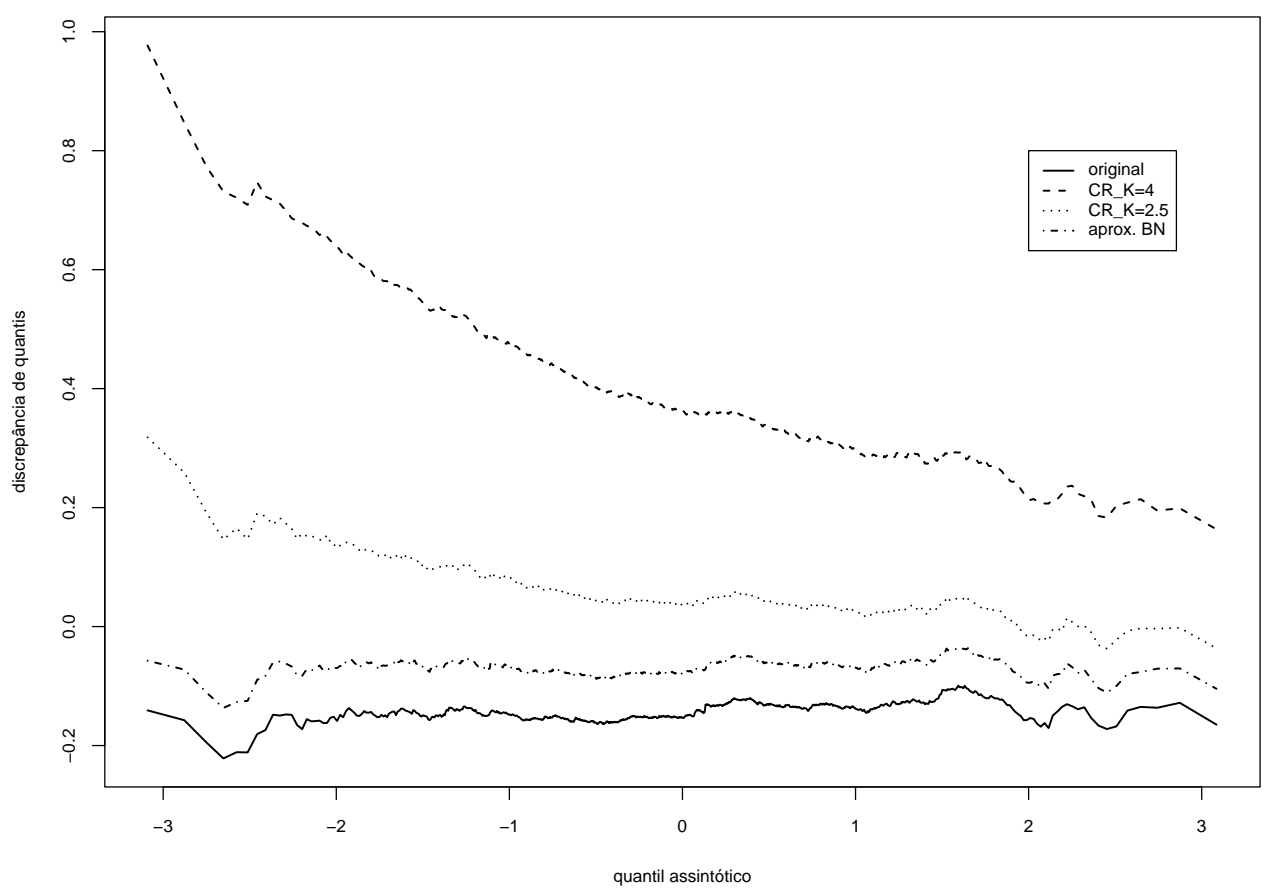

aproximação da distribuição da estatística baseada em $\tilde{\ell}_{B N}$.

Em ambos os gráficos, note a pobre aproximação da distribuição nula da estatística de 
teste baseada em $\ell_{C R, K=4}$ pela distribuição $\mathcal{N}(0,1)$, embora a maximização dessa verossimilhança tenha fornecido as melhores estimativas pontuais, tanto em termos de menor viés quanto em termos de menor variância. Lembre que o viés da função escore tem forte influência sobre o viés da estimativa de máxima verossimilhança, que é o zero da função de estimação definida pela função escore. A nulidade do viés de uma função de estimação é uma condição necessária para que o zero desta, ou seja, o estimador obtido desta função, seja consistente. Por outro lado, a nulidade do viés da informação de Fisher garante boas propriedades distribucionais assintóticas para testes baseados em tal função de estimação, como, por exemplo, melhor aproximação pela distribuição qui-quadrado para testes da razão de verossimilhanças, de Wald e escore de Rao (McCullagh e Tibshirani, 1990).

Tabela 4.8. Taxas de rejeição da hipótese nula de diferentes testes da razão de verossimilhanças sinalizada, nível de significância de $5 \%$, distribuição $\mathcal{G}_{A}^{0}(\alpha, \gamma, \mathcal{L}), \alpha=-5$.

\begin{tabular}{ccrrr}
\hline$(n, \mathcal{L})$ & \multicolumn{1}{c}{$\alpha$} & \multicolumn{1}{c}{$\ell$} & \multicolumn{1}{c}{$\tilde{\ell}_{B N}$} & $\ell_{C R, K=2.5}$ \\
\hline & -4.9 & 3.160 & 3.940 & 4.420 \\
& -4.5 & 5.540 & 6.680 & 7.580 \\
& -4.0 & 9.840 & 12.080 & 13.380 \\
$(25,8)$ & -3.5 & 19.300 & 22.060 & 23.740 \\
& -3.0 & 33.660 & 37.500 & 39.420 \\
& -2.5 & 53.620 & 57.740 & 59.520 \\
& -2.0 & 77.060 & 79.620 & 80.920 \\
& -1.0 & 99.660 & 99.680 & 99.680 \\
\hline & -4.9 & 4.540 & 5.280 & 6.160 \\
& -4.5 & 7.580 & 8.420 & 9.540 \\
& -4.0 & 14.560 & 15.980 & 17.700 \\
$(81,3)$ & -3.5 & 27.560 & 29.860 & 32.600 \\
& -3.0 & 48.980 & 51.340 & 54.400 \\
& -2.5 & 74.320 & 75.640 & 77.760 \\
& -2.0 & 93.780 & 94.580 & 95.200 \\
& -1.0 & 100.000 & 100.000 & 100.000 \\
\hline
\end{tabular}

A Tabela 4.8 apresenta taxas de rejeição da hipótese nula, dado que esta é falsa, para diferentes testes da razão de verossimilhanças sinalizadas, mais precisamente, testes baseados em $\ell, \tilde{\ell}_{B N}$ e $\ell_{C R, K=2.5}$. Foi considerado nível de significância de $5 \%$.

Para $(n, \mathcal{L})=(25,8)$ e esse nível de significância, as taxas de rejeição dos testes baseados em $\ell, \tilde{\ell}_{B N}$ e $\ell_{C R, K=2.5}$ sob a hipótese nula foram iguais a $3.150 \%, 3.925 \%$ e $4.375 \%$ (Tabela 4.6), respectivamente. Pela Tabela 4.8 , dado que $\mathcal{H}_{0}$ é falsa, note que as taxas associadas a $\ell_{C R, K=2.5}$ são sempre maiores do que as demais, seguidas pelas taxas do teste baseado em $\tilde{\ell}_{B N}$. Ou seja, os dois testes construídos a partir de ajustes sobre a verossimilhança perfilada são, aparentemente, mais poderosos que o teste da razão de verossimilhanças sinalizada original e, entre estes, aquele baseado em $\ell_{C R, K=2.5}$ é mais poderoso. Por exemplo, para $\alpha=$ 
-2.5, as taxas de rejeição dos testes obtidos de $\ell, \tilde{\ell}_{B N}$ e $\ell_{C R, K=2.5}$ foram, respectivamente, iguais a $53.620 \%, 57.740 \%$ e $59.520 \%$. Conclusões semelhantes podem ser obtidas para $(n, \mathcal{L})=(81,3)$. Por exemplo, para $\alpha=-3.5$, as taxas de rejeição dos testes obtidos de $\ell$, $\tilde{\ell}_{B N}$ e $\ell_{C R, K=2.5}$ foram, respectivamente, iguais a $27.560 \%, 29.860 \%$ e $32.600 \%$. Aqui, para o nível de significância de $5 \%$, sob a hipótese nula, as taxas de rejeição dos testes baseados em $\ell, \tilde{\ell}_{B N}$ e $\ell_{C R, K=2.5}$ foram iguais a $3.880 \%, 4.570 \%$ e $5.500 \%$ (Tabela 4.6 ), respectivamente.

(ii) região homogênea $\times$ regiões heterogênea e extremamente heterogênea

$$
\mathcal{H}_{0}: \alpha \leq-10 \quad \text { contra } \quad \mathcal{H}_{1}:-10<\alpha<0
$$

A Tabela 4.9 apresenta taxas de rejeição da hipótese nula de diferentes testes da razão de verossimilhanças. Aqui $\alpha=-10$, um valor do parâmetro de heterogeneidade no limiar de regiões classificadas como heterogêneas e homogêneas. São considerados dois pares $(n, \mathcal{L})$ : $(81,8)$ e $(121,3)$. Considerando nível nominal de $10 \%$, para $(n, \mathcal{L})=(81,8)$, as taxas de rejeição apresentadas pelos testes baseados em $\ell, \tilde{\ell}_{B N}, \ell_{C R, K=4}$ e $\ell_{C R, K=2.5}$ foram iguais a $8.060 \%, 9.410 \%, 15.300 \%$ e $10.770 \%$, enquanto para $(n, \mathcal{L})=(121,3)$, tais valores foram iguais a $8.660 \%, 9.640 \%, 23.400 \%$ e $12.050 \%$, respectivamente. O teste com taxas de rejeição mais próximas aos níveis nominais é aquele baseado em $\tilde{\ell}_{B N}$.

Tabela 4.9. Taxas de rejeição da hipótese nula de diferentes testes da razão de verossimilhanças sinalizada, distribuição $\mathcal{G}_{A}^{0}(\alpha, \gamma, \mathcal{L}), \alpha=-10$.

\begin{tabular}{cccccc}
\hline$(n, \mathcal{L})$ & nível nominal & $\ell$ & $\tilde{\ell}_{B N}$ & $\ell_{C R, K=4}$ & $\ell_{C R, K=2.5}$ \\
\hline \multirow{2}{*}{$(81,8)$} & $10 \%$ & 8.060 & 9.410 & 15.300 & 10.770 \\
& $5 \%$ & 4.070 & 4.650 & 7.950 & 5.250 \\
\hline \multirow{2}{*}{$(121,3)$} & $10 \%$ & 8.660 & 9.640 & 23.400 & 12.050 \\
& $5 \%$ & 4.350 & 4.960 & 12.770 & 6.450 \\
\hline
\end{tabular}

A Tabela 4.10 apresenta médias e variâncias amostrais das diferentes estatísticas de teste e os valores assintóticos destas. A estatística de teste com momentos empíricos mais próximos aos valores assintóticos é aquela baseada em $\tilde{\ell}_{B N}$; por exemplo, para $(n, \mathcal{L})=(121,3)$, a média e a variância empíricas foram iguais a -0.054 e 1.022, contra -0.118 e 1.026 daquela baseada na verossimilhança original, respectivamente.

Tabela 4.10. Médias e variâncias amostrais de diferentes estatísticas de teste e os valores assintóticos destas, distribuição $\mathcal{G}_{A}^{0}(\alpha, \gamma, \mathcal{L}), \alpha=-10$.

\begin{tabular}{cccrrrc}
\hline \multirow{2}{*}{$(n, \mathcal{L})$} & momento & $N(0,1)$ & \multicolumn{1}{c}{$\ell$} & \multicolumn{1}{c}{$\tilde{\ell}_{B N}$} & $\ell_{C R, K=4}$ & $\ell_{C R, K=2.5}$ \\
\hline \multirow{2}{*}{$(81,8)$} & média & 0 & -0.125 & -0.050 & 0.320 & 0.047 \\
& variância & 1 & 1.031 & 1.025 & 0.885 & 0.982 \\
\hline \multirow{2}{*}{$(121,3)$} & média & 0 & -0.118 & -0.054 & 0.647 & 0.147 \\
& variância & 1 & 1.026 & 1.022 & 0.742 & 0.905 \\
\hline
\end{tabular}


Figura 4.4. Gráfico das discrepâncias de quantis, distribuição $\mathcal{G}_{A}^{0}, \alpha=-10,(n, \mathcal{L})=(81,8)$.

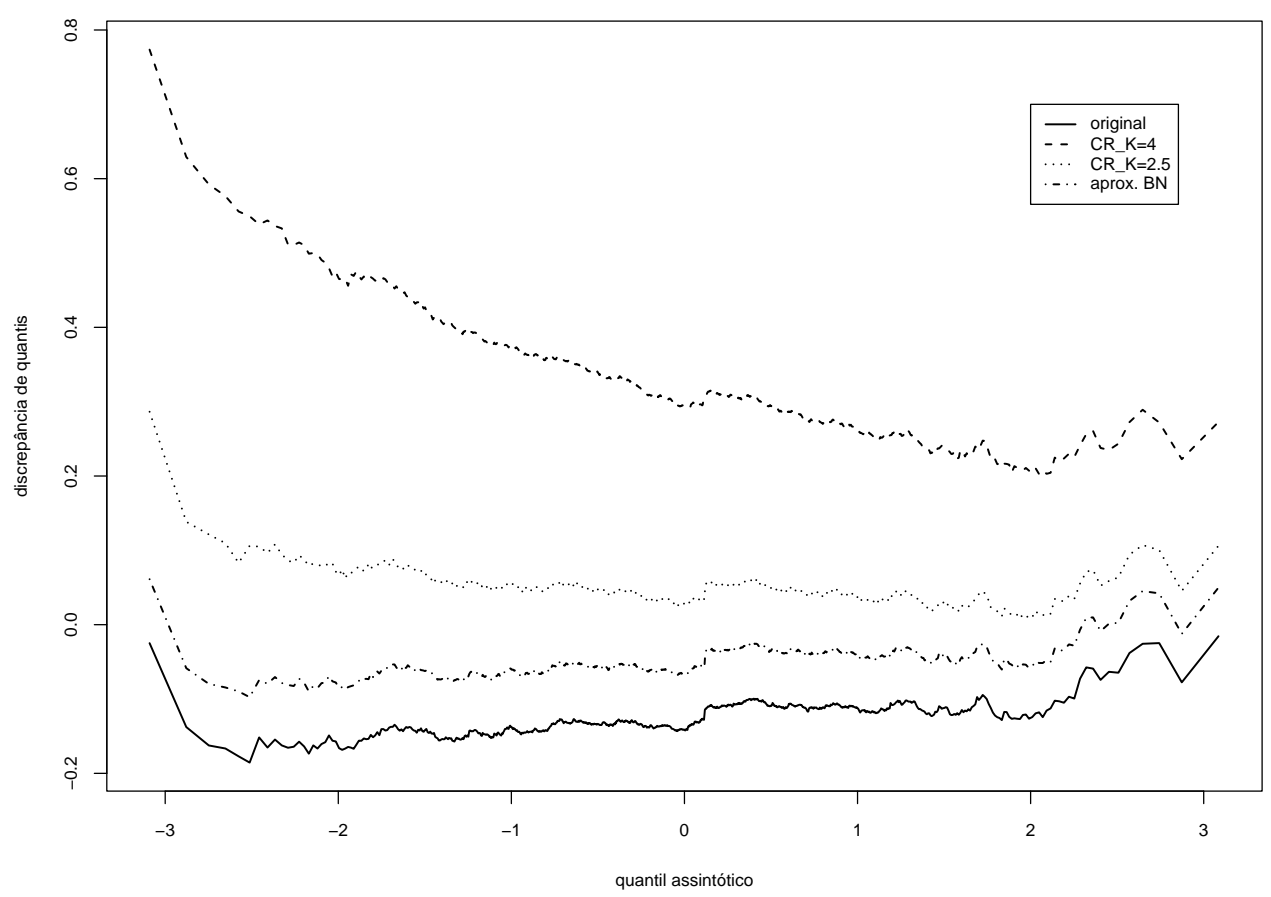

Figura 4.5. Gráfico das discrepâncias de quantis, distribuição $\mathcal{G}_{A}^{0}, \alpha=-10,(n, \mathcal{L})=(121,3)$.

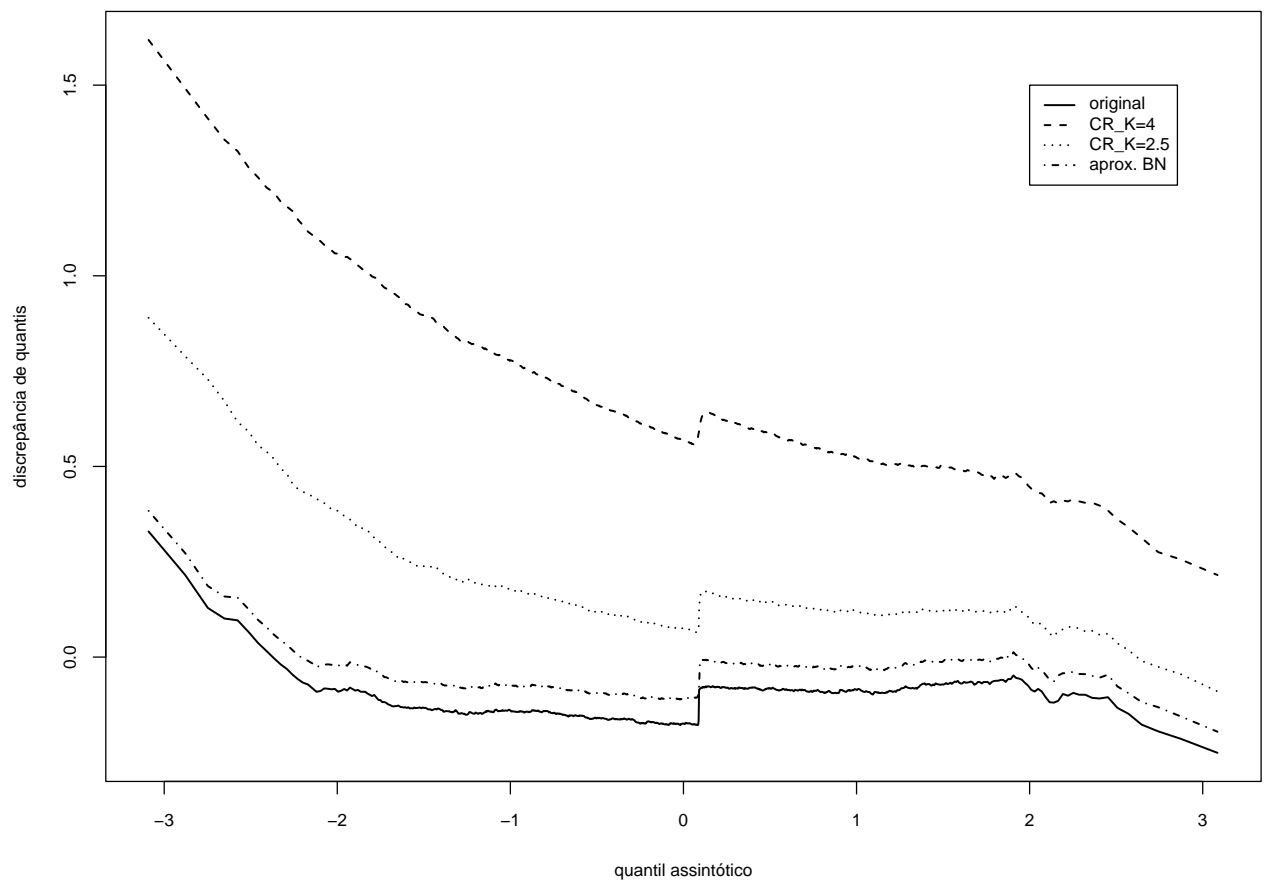

Os gráficos das Figuras 4.4 e 4.5 apresentam as curvas de discrepâncias entre os quantis amostrais das estatísticas de teste e os respectivos quantis assintóticos versus os quantis da normal padrão (quantis assintóticos). Para $(n, \mathcal{L})=(81,8)$, Figura 4.4 , as curvas relativas 
aos ajustes sobre a verossimilhança perfilada denotadas por $\tilde{\ell}_{B N}$ e $\ell_{C R, K=2.5}$ apresentaram-se mais próximas à ordenada nula. Para $(n, \mathcal{L})=(121,3)$, a estatística de teste cuja distribuição nula foi melhor aproximada pela distribuição $\mathcal{N}(0,1)$ foi aquela baseada em $\tilde{\ell}_{B N}$, seguida pela estatística obtida da verossimilhança original, conforme Figura 4.5.

No gráfico da Figura 4.5, note a mudança de patamar das curvas próximo ao quantil zero. Nos gráficos anteriores, tal mudança não foi tão acentuada quanto aqui.

Tabela 4.11. Taxas de rejeição da hipótese nula de diferentes testes da razão de verossimilhanças sinalizada, nível de significância de $5 \%$, distribuição $\mathcal{G}_{A}^{0}(\alpha, \gamma, \mathcal{L}), \alpha=-10$.

\begin{tabular}{crrrr}
\hline$(n, \mathcal{L})$ & \multicolumn{1}{c}{$\alpha$} & \multicolumn{1}{c}{$\ell$} & \multicolumn{1}{c}{$\tilde{\ell}_{B N}$} & $\ell_{C R, K=2.5}$ \\
\hline & -9.5 & 5.780 & 6.680 & 7.500 \\
& -9.0 & 8.260 & 9.300 & 10.460 \\
$(81,8)$ & -8.0 & 15.760 & 17.380 & 19.140 \\
& -7.0 & 31.440 & 33.920 & 36.000 \\
& -6.0 & 52.980 & 55.680 & 58.260 \\
& -5.0 & 77.940 & 80.160 & 81.700 \\
& -3.0 & 99.880 & 99.880 & 99.920 \\
\hline$(121,3)$ & -9.5 & 5.680 & 6.560 & 8.020 \\
& -9.0 & 7.240 & 7.820 & 9.380 \\
& -8.0 & 11.520 & 12.480 & 15.460 \\
& -7.0 & 20.720 & 22.240 & 26.100 \\
& -6.0 & 35.440 & 37.480 & 41.840 \\
& -5.0 & 57.760 & 59.660 & 63.900 \\
\hline
\end{tabular}

A Tabela 4.11 apresenta taxas de rejeição da hipótese nula, dado que esta é falsa, para diferentes testes da razão de verossimilhanças sinalizadas, mais precisamente, testes baseados em $\ell, \tilde{\ell}_{B N}$ e $\ell_{C R, K=2.5}$. Foi considerado nível de significância de $5 \%$.

Para $(n, \mathcal{L})=(81,8)$ e esse nível de significância, as taxas de rejeição dos testes baseados em $\ell, \tilde{\ell}_{B N}$ e $\ell_{C R, K=2.5}$ sob a hipótese nula foram iguais a $4.070 \%, 4.650 \%$ e $5.250 \%$ (Tabela 4.9), respectivamente. Pela Tabela 4.11 , dado que $\mathcal{H}_{0}$ é falsa, note que as taxas associadas a $\ell_{C R, K=2.5}$ são sempre maiores do que as demais, seguidas pelos valores do teste baseado em $\tilde{\ell}_{B N}$. Ou seja, os dois testes construídos a partir de ajustes sobre a verossimilhança perfilada são, aparentemente, mais poderosos que o teste da razão de verossimilhanças sinalizada original e, entre estes, aquele baseado em $\ell_{C R, K=2.5}$ é mais poderoso. Por exemplo, para $\alpha=-6$, as taxas de rejeição dos testes obtidos de $\ell, \tilde{\ell}_{B N}$ e $\ell_{C R, K=2.5}$ foram, respectivamente, iguais a $52.980 \%, 55.680 \%$ e $58.260 \%$. Conclusões semelhantes podem ser obtidas para $(n, \mathcal{L})=(121,3)$. Por exemplo, para $\alpha=-7$, as taxas de rejeição dos testes obtidos de $\ell$, $\tilde{\ell}_{B N}$ e $\ell_{C R, K=2.5}$ foram, respectivamente, iguais a $20.720 \%, 22.240 \%$ e $26.100 \%$. Aqui, para o nível de significância de $5 \%$, sob a hipótese nula, as taxas de rejeição dos testes baseados em 
$\ell, \tilde{\ell}_{B N}$ e $\ell_{C R, K=2.5}$ foram iguais a $4.350 \%, 4.960 \%$ e $6.450 \%$ (Tabela 4.9 ), respectivamente. Note que essa última taxa está bem acima do nível nominal.

Considerando os resultados das tabelas e dos gráficos desta seção, quanto aos tamanhos dos testes, observa-se que aqueles baseados em $\ell$ e $\tilde{\ell}_{B N}$ são conservadores, rejeitando a hipótese nula aquém do esperado, enquanto os testes baseados em $\ell_{C R, K=2.5}$ e $\ell_{C R, K=4}$ são liberais, em geral. Quanto a aproximação da distribuição nula da estatística de teste pela normal reduzida, os melhores resultados foram apresentados pelas estatísticas calculadas a partir de $\tilde{\ell}_{B N}$ e $\ell_{C R, K=2.5}$. Por último, quanto ao poder dos testes, nota-se que aqueles baseados em ajustes sobre a verossimilhança perfilada, $\tilde{\ell}_{B N}$ e $\ell_{C R, K=2.5}$, rejeitam mais a hipótese nula quando esta é falsa. Portanto, entre os quatro testes da razão de verossimilhanças sinalizadas, aquele obtido de $\tilde{\ell}_{B N}$ apresentou melhor desempenho.

\subsection{CONCLUSÕES}

Ao longo da Seção 4.3, foram apresentados resultados numéricos relativos à estimação pontual e a testes de hipóteses sobre o parâmetro de heterogeneidade da distribuição $\mathcal{G}_{A}^{0}(\alpha, \gamma, \mathcal{L})$, utilizada para modelar o sinal de retorno no formato amplitude de regiões imageadas por radares do tipo SAR. Para isto, foram usados os estimadores de máxima verossimilhança derivados da verossimilhança original e de versões ajustadas da verossimilhança perfilada, mais precisamente, $\tilde{\ell}_{B N}$ (ver Seção 2.11.4) e $\ell_{C R}$ (ver Seções 2.5 e 4.2.3).

De acordo com os resultados mostrados na Seção 4.3.2, os estimadores com melhores desempenhos foram aqueles obtidos do ajuste sobre a verossimilhança perfilada proposto por Cox e Reid $(1987,1989)$, que requer a ortogonalidade entre os parâmetros de interesse e de incômodo, acarretando a independência assintótica dos estimadores de máxima verossimilhança destes. Tanto o valor absoluto do viés quanto o erro quadrático médio dos estimadores $\widehat{\alpha}_{C R, K=2.5}$ e $\widehat{\alpha}_{C R, K=4}$ foram sempre menores que aqueles relativos aos estimadores $\widehat{\alpha}$ e $\widehat{\tilde{\alpha}}_{B N}$. Os resultados apresentados por estes foram bastante similares.

Diferentemente do método computacional de inferência estatística denominado bootstrap, utilizado em Cribari-Neto et al. (2002) para correção das estimativas de máxima verossimilhança do parâmetro $\alpha$, espera-se que as modificações sobre a verossimilhança perfilada produzam estimadores com vieses, em módulo, menores do que o valor absoluto do viés apresentado pelo estimador original. Pelas técnicas de bootstrap (Efron, 1979, 1990, 1993), as estimativas do viés deste estimador são obtidas numericamente e são subtraídas das respectivas estimativas de máxima verossimilhança. Assim, obtêm-se estimativas corrigidas até segunda ordem.

Utilizando tais técnicas, para $\alpha=-1$ e $(n, \mathcal{L})=(49,1)$, segundo Cribari-Neto et al. (2002, p. 816, Table 2), o estimador corrigido com menor viés em valor absoluto foi aquele denotado por $\bar{\alpha}_{1}$, cuja estimativa para esta quantidade foi de 0.033 , enquanto que, 
em termos de erro quadrático médio, o melhor estimador foi aquele denotado por $\bar{\alpha}_{2}$, com estimativa para esta quantidade igual a 0.186. Tais resultados foram baseados em 5000 amostras de Monte Carlo, a partir das quais eram geradas 2000 réplicas bootstrap para correções das estimativas de máxima verossimilhança.

De acordo com a Tabela 4.1, apresentada na Seção 4.3.2, o módulo da estimativa do viés de $\widehat{\alpha}_{C R, K=4}$ foi de 0.010, enquanto a estimativa do erro quadrático médio deste estimador foi de 0.097 , ambos inferiores aos valores relativos a $\bar{\alpha}_{1}$ e $\bar{\alpha}_{2}$, respectivamente.

A Seção 4.3.3 contém resultados relativos a testes unicaudais sobre o parâmetro $\alpha$. O objetivo dessa seção foi comparar os diferentes testes da razão de verossimilhanças sinalizadas a partir do poder e das distorções de tamanho dos mesmos. Para isto, foram apresentadas taxas de rejeição da hipótese nula para níveis nominais de 5\% e 10\% sob ambas as hiṕoteses (nula e alternativa), além de gráficos de discrepâncias entre quantis amostrais e quantis assintóticos das estatísticas de teste.

Entre os testes da razão de verossimilhanças sinalizadas, aquele baseado na aproximação para a verossimilhança perfilada modificada proposta por Barndorff-Nielsen (1983) e denotado por $\tilde{\ell}_{B N}$ apresentou, de forma consistente, taxas de rejeição sob $\mathcal{H}_{0}$ próximas aos níveis nominais e quantis amostrais próximos aos quantis assintóticos, seguido pelo teste baseado em $\ell_{C R, K=2.5}$. Dado que a hipótese nula é falsa, estes dois testes apresentaram taxas de rejeição maiores que o teste original $(\ell)$.

Portanto, para a estimação pontual do parâmetro de heterogeneidade da distribuição $\mathcal{G}_{A}^{0}(\alpha, \gamma, \mathcal{L})$, este trabalho sugere o uso do estimador $\widehat{\alpha}_{C R, K=4}$, enquanto que, para a realização de testes unicaudais sobre $\alpha$, este trabalho sugere a utilização do teste baseado em $\tilde{\ell}_{B N}$. Estes testes são importantes para identificar o tipo de região imageada: extremamente heterogêneo, heterogêneo e homogêneo. 


\section{CAPÍTULO 5 \\ ESTIMADORES DE MÁXIMA VEROSSIMILHANÇA E TESTES DA RAZÃO DE VEROSSIMILHANÇAS NO MODELO DE WEIBULL}

\subsection{INTRODUÇÃO}

Nas indústrias, a estimação do tempo de vida médio de um produto ou o cálculo da probabilidade deste durar um certo período de tempo são bastante comuns, pois são características muito importantes na avaliação da qualidade do produto pelos consumidores. Na área médica, por exemplo, em estudos de câncer, o tempo entre o diagnóstico da doença e o falecimento do paciente ou o tempo entre a remissão (paciente livre de sintomas) e a recorrência da doença (recidiva) são importantes para a avaliação da evolução desta.

Quando a compreensão de um fenômeno envolve a observação do tempo até a ocorrência de um evento de interesse ou o tempo entre dois eventos de interesse, nos dois casos, chamado tempo de falha, a Estatística responde aos questionamentos através das técnicas desenvolvidas em análise de sobrevivência (termo usado na área médica) e em confiabilidade (termo usado na área industrial), pois, comumente, a presença de censura nos dados impossibilita o uso das técnicas estatísticas clássicas, como análise de regressão e planejamento de experimentos. Uma vasta literatura trata deste assunto, vale mencionar Lawless (1982), Cox e Oakes (1984), Collet (1994), Klein e Moeschberger (1997), Meeker e Escobar (1998).

Dado censurado corresponde à observação parcial do tempo de falha, mais precisamente, decorre do fato da não observação do tempo até a ocorrência do evento de interesse por alguma razão. Nesta situação, sabe-se apenas que o tempo é superior àquele observado ou que a falha ocorreu num certo intervalo de tempo. Mesmo assim, a observação censurada fornece informação e sua omissão na análise estatística dos dados implicaria em conclusões viciadas.

O tempo de falha é uma variável aleatória não-negativa e, usualmente, é caracterizado por duas funções: função de sobrevivência e função de taxa de falha (ou de risco). A função de sobrevivência é definida como a probabilidade de uma observação não falhar até um certo tempo $t$, ou seja, por exemplo, a probabilidade de um paciente com câncer sobreviver a $t$ meses. A função de taxa de falha é definida, basicamente, como a probabilidade de que a falha ocorra num dado intervalo de tempo; contextualizando, dado que o paciente sobreviveu a 6 meses, essa função forneceria a probabilidade dele sobreviver ao sétimo mês. Matematicamente, as funções de sobrevivência e de taxa de falha instantânea são expressas, respectivamente, por

$$
S(t)=\operatorname{Pr}(y \geq t) \quad \text { e } \quad h(t)=\lim _{\Delta t \rightarrow 0} \frac{\operatorname{Pr}(t \leq y<t+\Delta t \mid y \geq t)}{\Delta t} .
$$


Os modelos paramétricos consistem em distribuições de probabilidade para o tempo de falha e são usados freqüentemente na área industrial. Por causa da sua flexibilidade em modelar taxas de falhas crescente, decrescente e constante, a distribuição de Weibull é largamente utilizada em confiabilidade. Como a inclinação da função de taxa de falha para essa distribuição é determinada pelo valor do seu parâmetro de forma, a estimação deste é de particular interesse.

Se $y$ é uma variável aleatória com distribuição de Weibull, denotada por $W B(\alpha, \beta)$, então sua função densidade de probabilidade é dada por

$$
p(y ; \alpha, \beta)=\left(\frac{\beta}{\alpha}\right)\left(\frac{y}{\alpha}\right)^{\beta-1} \exp \left\{-\left(\frac{y}{\alpha}\right)^{\beta}\right\}, \quad \alpha, \beta, y>0,
$$

em que $\beta$ é o parâmetro de forma (configuração) e $\alpha$ é o parâmetro de escala. Aqui,

$$
E\{y\}=\alpha \Gamma(1+1 / \beta) \quad \text { e } \quad \operatorname{Var}\{y\}=\alpha^{2}\left[\Gamma(1+2 / \beta)-\Gamma(1+1 / \beta)^{2}\right] .
$$

As funções de sobrevivência e de taxa de falha são expressas, respectivamente, por

$$
S(y ; \alpha, \beta)=\exp \left[-\left(\frac{y}{\alpha}\right)^{\beta}\right] \quad \text { e } \quad h(y)=\frac{\beta}{\alpha}\left(\frac{y}{\alpha}\right)^{\beta-1} .
$$

Essa distribuição não pertence à família exponencial biparamétrica e os estimadores de máxima verossimilhança não são estatísticas suficientes e minimais do par $(\alpha, \beta)$ (ver Lawless, 1982, Capítulo 4).

O estimador de máxima verossimilhança do parâmetro $\beta$ pode ser bastante viesado. Resultados numéricos obtidos por Yang e Xie (2003) mostram que o estimador derivado da maximização da verossimilhança perfilada modificada proposta por Cox e Reid (1987, 1989) é mais eficiente do que o estimador original e é aproximadamente não viesado, apresentando viés relativo estimado menor do que $1 \%$ na maioria das situações (com ou sem censura). No trabalho de Yang e Xie (2003), observa-se também que quanto maior a proporção de observações censuradas ou quanto menor o tamanho da amostra, mais eficiente é o estimador derivado de tal verossimilhança relativamente ao estimador de máxima verossimilhança original. Os resultados foram obtidos para observações independentes e identicamente distribuídas.

Analogamente a esse trabalho, uma parte deste capítulo consiste na estimação do parâmetro de forma da distribuição de Weibull através das maximizações das aproximações para a verossimilhança perfilada proposta por Barndorff-Nielsen (Seção 2.11), denotadas por $\bar{\ell}_{B N}$, $\breve{\ell}_{B N}$ e $\tilde{\ell}_{B N}$, que não necessitam da especificação de uma estatística ancilar e nem da obtenção de uma parametrização ortogonal entre os parâmetros de interesse e de incômodo. Resultados numéricos comparando tais estimativas àquelas obtidas da maximização de $\ell_{C R}$ derivada por Yang e Xie (2003) são apresentados. Além disso, diferentemente de tal trabalho, são realizados testes de hipóteses sobre $\beta$, usando as estatísticas da razão de verossimilhanças obtidas de tais aproximações e de $\ell_{C R}$. Mais uma vez, o objetivo é comparar os desempenhos das verossimilhanças perfiladas modificadas a partir das distorções de tamanho dos 
testes associados. Aqui também são constestados os resultados obtidos por simulações de Monte Carlo em Yang e Xie (2003), mais precisamente, é provado que os vieses relativos dos estimadores do parâmetro de forma $\beta$ são constantes, diferentemente dos resultados numéricos observados em tal trabalho. Exemplos numéricos com conjuntos de dados reais são também apresentados.

Numa segunda parte, as observações da variável y são apenas independentes, não são identicamente distribuídas. Neste caso, supõe-se um modelo de regressão, ou seja, é estudado e simulado o caso em que se modela o parâmetro $\alpha$ através de algumas covariáveis, diferentemente do trabalho de Yang e Xie (2003). O objetivo dessa parte é observar o desempenho das verossimilhanças perfiladas modificadas na presença de mais de um parâmetro de incômodo. Aqui também são realizados testes da razão de verossimilhanças sobre os coeficientes da regressão, e, para isso, são consideradas as estatísticas de teste baseadas na verossimilhança original e na aproximação $\tilde{\ell}_{B N}$.

Os resultados envolvem dados completos (sem censura) e dados censurados e são apresentados de forma similar aos capítulos anteriores.

\subsection{TESTE DE HIPÓTESE E ESTIMAÇÃO DO PARÂMETRO DE FORMA}

\subsubsection{OBSERVAÇÕES INDEPENDENTES E IDENTICAMENTE DISTRIBUÍDAS}

\subsubsection{DADOS COMPLETOS}

Considerando inicialmente dados não-censurados, sejam $y_{1}, \ldots, y_{n}$ observações independentes e identicamente distribuídas de uma variável aleatória com distribuição de Weibull. O logaritmo da função de verossimilhança do par $(\alpha, \beta)$ é dado por

$$
\ell(\alpha, \beta)=n \log \left(\frac{\beta}{\alpha}\right)+(\beta-1) \sum_{j=1}^{n} \log \left(\frac{y_{j}}{\alpha}\right)-\sum_{j=1}^{n}\left(\frac{y_{j}}{\alpha}\right)^{\beta} .
$$

Fixando o parâmetro de interesse $\beta$, o estimador de máxima verossimilhança restrito de $\alpha$ é

$$
\widehat{\alpha}_{\beta}=\left(\frac{1}{n} \sum_{j=1}^{n} y_{j}^{\beta}\right)^{1 / \beta} .
$$

Substituindo o parâmetro de incômodo $\alpha$ por este estimador em $\ell(\alpha, \beta)$ vem

$$
\ell_{p}(\beta)=n \log \beta-n \log \sum_{j=1}^{n} y_{j}^{\beta}+\beta \sum_{j=1}^{n} \log y_{j},
$$


cuja primeira derivada com respeito a $\beta$ é

$$
u_{p}(\beta)=\frac{n}{\beta}-\frac{n \sum_{j=1}^{n} y_{j}^{\beta} \log y_{j}}{\sum_{j=1}^{n} y_{j}^{\beta}}+\sum_{j=1}^{n} \log y_{j}
$$

A informação observada $j_{\alpha \alpha}(\alpha, \beta)$ avaliada em $\left(\widehat{\alpha}_{\beta}, \beta\right)$ é expressa por

$$
j_{\alpha \alpha}\left(\widehat{\alpha}_{\beta}, \beta\right)=\beta^{2}\left(\sum_{j=1}^{n} y_{j}^{\beta}\right)^{-2 / \beta} n^{1+2 / \beta}
$$

As aproximações para $\ell_{B N}(\beta)$ são baseadas em aproximações para a derivada do espaço amostral $\ell_{\alpha ; \widehat{\alpha}}(\alpha, \beta ; \widehat{\alpha}, \widehat{\beta}, a)$ conforme descrito na Seção 2.11, em que a é uma estatística ancilar que garante a suficiência e a minimalidade da terna $(\widehat{\alpha}, \widehat{\beta}, a)$.

Aqui, a primeira dessas aproximações é baseada em covariâncias de derivadas do logaritmo da função de verossimilhança (ver Seção 2.11.2), a saber:

$$
\bar{\ell}_{B N}(\beta)=\ell_{p}(\beta)+\frac{1}{2} \log j_{\alpha \alpha}\left(\widehat{\alpha}_{\beta}, \beta\right)-\log I_{\alpha}\left(\widehat{\alpha}_{\beta}, \beta ; \widehat{\alpha}, \widehat{\beta}\right)
$$

em que

$$
I_{\alpha}\left(\alpha, \beta ; \alpha_{0}, \beta_{0}\right)=E_{\left(\alpha_{0}, \beta_{0}\right)}\left\{\ell_{\alpha}(\alpha, \beta) \ell_{\alpha}\left(\alpha_{0}, \beta_{0}\right)\right\} .
$$

Calculando $I_{\alpha}\left(\widehat{\alpha}_{\beta}, \beta ; \widehat{\alpha}, \widehat{\beta}\right)$, vem

$$
I_{\alpha}\left(\widehat{\alpha}_{\beta}, \beta ; \widehat{\alpha}, \widehat{\beta}\right)=\frac{n \beta^{2} \widehat{\alpha}^{\beta-1}}{\widehat{\alpha}_{\beta}^{\beta+1}} \Gamma\left(\frac{\beta}{\widehat{\beta}}+1\right) .
$$

Logo,

$$
\bar{\ell}_{B N}(\beta)=(n-1) \log \beta-\beta\left(\log \widehat{\alpha}-\sum_{j=1}^{n} \log y_{j}\right)-\log \Gamma\left(\frac{\beta}{\widehat{\beta}}+1\right)-(n-1) \log \sum_{j=1}^{n} y_{j}^{\beta},
$$

onde $\widehat{\alpha}$ e $\widehat{\beta}$ são os estimadores de máxima verossimilhança e não têm formas fechadas. Dessa maneira,

$$
\bar{u}_{B N}(\beta)=\frac{n-1}{\beta}-\log \widehat{\alpha}+\sum_{j=1}^{n} \log y_{j}-\frac{1}{\widehat{\beta}} \psi\left(\frac{\beta}{\widehat{\beta}}+1\right)-\frac{(n-1) \sum_{j=1}^{n} y_{j}^{\beta} \log y_{j}}{\sum_{j=1}^{n} y_{j}^{\beta}} .
$$

A segunda das aproximações é baseada em covariâncias empíricas de derivadas do logaritmo da função de verossimilhança (ver Seção 2.11.3):

$$
\breve{\ell}_{B N}(\beta)=\ell_{p}(\beta)+\frac{1}{2} \log j_{\alpha \alpha}\left(\widehat{\alpha}_{\beta}, \beta\right)-\log \breve{I}_{\alpha}\left(\widehat{\alpha}_{\beta}, \beta ; \widehat{\alpha}, \widehat{\beta}\right)
$$


em que

$$
\breve{I}_{\alpha}\left(\alpha, \beta ; \alpha_{0}, \beta_{0}\right)=\sum_{j=1}^{n} \ell_{\alpha}^{(j)}(\alpha, \beta) \ell_{\alpha}^{(j)}\left(\alpha_{0}, \beta_{0}\right)
$$

Assim,

$$
\breve{I}_{\alpha}\left(\widehat{\alpha}_{\beta}, \beta ; \widehat{\alpha}, \widehat{\beta}\right)=\frac{\beta}{\widehat{\alpha}_{\beta}}\left(\frac{\widehat{\beta}}{\widehat{\alpha}^{\widehat{\beta}+1}} \frac{\sum_{j=1}^{n} y_{j}^{\beta+\widehat{\beta}}}{\widehat{\alpha}_{\beta}^{\beta}}-\frac{n \widehat{\beta}}{\widehat{\alpha}}\right)
$$

e, conseqüentemente,

$$
\breve{\ell}_{B N}(\beta)=n \log \beta-(n-1) \log \sum_{j=1}^{n} y_{j}^{\beta}+\beta \sum_{j=1}^{n} \log y_{j}-\log \left[\sum_{j=1}^{n} y_{j}^{\beta}\left(y_{j}^{\widehat{\beta}}-\widehat{\alpha}^{\widehat{\beta}}\right)\right] \text {. }
$$

Aqui,

$$
\breve{u}_{B N}(\beta)=\frac{n}{\beta}+\sum_{j=1}^{n} \log y_{j}-\frac{(n-1) \sum_{j=1}^{n} y_{j}^{\beta} \log y_{j}}{\sum_{j=1}^{n} y_{j}^{\beta}}-\frac{\sum_{j=1}^{n}\left(y_{j}^{\widehat{\beta}}-\widehat{\alpha}^{\widehat{\beta}}\right) y_{j}^{\beta} \log y_{j}}{\sum_{j=1}^{n}\left(y_{j}^{\widehat{\beta}}-\widehat{\alpha}^{\beta}\right) y_{j}^{\beta}} .
$$

A última das aproximações de $\ell_{B N}(\beta)$ é baseada numa estatística aproximadamente ancilar (ver Seção 2.11.4) e é expressa por

$$
\tilde{\ell}_{B N}(\beta)=\ell_{p}(\beta)+\frac{1}{2} \log j_{\alpha \alpha}\left(\widehat{\alpha}_{\beta}, \beta\right)-\log \left[\ell_{\alpha ; y}\left(\widehat{\alpha}_{\beta}, \beta\right) \widehat{V}_{\alpha}\right]
$$

onde os $j$-ésimos componentes dos vetores $\ell_{\alpha ; y}\left(\widehat{\alpha}_{\beta}, \beta\right)$ (vetor-linha) e $\widehat{V}_{\alpha}$ (vetor-coluna) são, respectivamente,

$$
\frac{\beta^{2} y_{j}^{\beta-1}}{\widehat{\alpha}_{\beta}^{\beta+1}} \text { e } \quad \frac{y_{j}}{\widehat{\alpha}} \text {. }
$$

Assim,

$$
\ell_{\alpha ; y}\left(\widehat{\alpha}_{\beta}, \beta\right) \widehat{V}_{\alpha}=\frac{\beta^{2} n^{1+1 / \beta}}{\widehat{\alpha}\left(\sum_{j=1}^{n} y_{j}^{\beta}\right)^{1 / \beta}}
$$

e, portanto,

$$
\tilde{\ell}_{B N}(\beta)=(n-1) \log \beta-n \log \sum_{j=1}^{n} y_{j}^{\beta}+\beta \sum_{j=1}^{n} \log y_{j} .
$$

A função escore perfilada modificada é dada por

$$
\tilde{u}_{B N}(\beta)=\frac{n-1}{\beta}-\frac{n \sum_{j=1}^{n} y_{j}^{\beta} \log y_{j}}{\sum_{j=1}^{n} y_{j}^{\beta}}+\sum_{j=1}^{n} \log y_{j} .
$$


Sob a parametrização ortogonal $(\lambda, \beta)$, em que $\lambda=\log (\alpha) / \beta+(1-\gamma) / \beta^{2}$, Yang e Xie (2003) obtiveram

$$
\ell_{C R}(\beta)=\ell_{p}(\beta)-2 \log \beta=(n-2) \log \beta-n \log \sum_{j=1}^{n} y_{j}^{\beta}+\beta \sum_{j=1}^{n} \log y_{j}
$$

$\mathrm{e}$

$$
u_{C R}(\beta)=u_{p}(\beta)-\frac{2}{\beta}=\frac{n-2}{\beta}-\frac{n \sum_{j=1}^{n} y_{j}^{\beta} \log y_{j}}{\sum_{j=1}^{n} y_{j}^{\beta}}+\sum_{j=1}^{n} \log y_{j} .
$$

Aqui, $\gamma=0.577215 \ldots$ é a constante de Euler.

Observe que os estimadores de máxima verossimilhança associados às funções de verossimilhanças perfiladas não têm formas fechadas. Assim, estimativas para $\beta$ são obtidas numericamente e alguns resultados são apresentados na Seção 5.2.1.3.

\subsubsection{DADOS CENSURADOS}

Geralmente, pesquisas na área médica que envolvem uma resposta temporal são prospectivas e de longa duração e, por isso, ao final, dificilmente são observados os tempos de falhas de todos os indivíduos. Assim, quando o estudo termina após a ocorrência do evento de interesse em um número pré-determinado destes, as demais observações são censuradas. Este caso define a censura do tipo II.

Sejam $y_{(1)}, \ldots, y_{(r)}$ as $r$ menores observações numa amostra aleatória de tamanho $n$ de uma variável com distribuição $W B(\alpha, \beta)$, isto é, dados com presença de censura do tipo II. Neste caso, a função de verossimilhança é expressa por

$$
L(\alpha, \beta)=\prod_{j=1}^{r} p\left(y_{(j)} ; \alpha, \beta\right) \prod_{j=r+1}^{n} S\left(y_{(r)} ; \alpha, \beta\right)=\left[S\left(y_{(r)} ; \alpha, \beta\right)\right]^{n-r} \prod_{j=1}^{r} p\left(y_{(j)} ; \alpha, \beta\right) .
$$

Dessa forma,

$$
\ell(\alpha, \beta)=\log L(\alpha, \beta)=r \log \beta-\beta r \log \alpha+(\beta-1) \sum_{j=1}^{r} \log y_{(j)}-\frac{1}{\alpha^{\beta}} \sum_{j=1}^{r} y_{(j)}^{\beta}-\frac{(n-r) y_{(r)}^{\beta}}{\alpha^{\beta}} .
$$

O estimador de máxima verossimilhança restrito de $\alpha$ é

$$
\widehat{\alpha}_{\beta}=\left[\frac{1}{r}\left(\sum_{j=1}^{r} y_{(j)}^{\beta}+(n-r) y_{(r)}^{\beta}\right)\right]^{1 / \beta} .
$$

Note que se não há censura nos dados $(r=n)$, então este estimador coincide com aquele obtido na seção anterior. 
Portanto, pela definição de verossimilhança perfilada, tem-se

$$
\ell_{p}(\beta)=\ell\left(\widehat{\alpha}_{\beta}, \beta\right)=r \log \beta-r \log \left(\sum_{j=1}^{r} y_{(j)}^{\beta}+(n-r) y_{(r)}^{\beta}\right)+\beta \sum_{j=1}^{r} \log y_{(j)},
$$

cuja primeira derivada relativa ao parâmetro $\beta$ define

$$
u_{p}(\beta)=\frac{r}{\beta}-r \frac{\sum_{j=1}^{r} y_{(j)}^{\beta} \log y_{(j)}+(n-r) y_{(r)}^{\beta} \log y_{(r)}}{\sum_{j=1}^{r} y_{(j)}^{\beta}+(n-r) y_{(r)}^{\beta}}+\sum_{j=1}^{r} \log y_{(j)} .
$$

Aqui,

$$
j_{\alpha \alpha}\left(\widehat{\alpha}_{\beta}, \beta\right)=\beta^{2}\left(\sum_{j=1}^{r} y_{(j)}^{\beta}+(n-r) y_{(r)}^{\beta}\right)^{-2 / \beta} r^{1+2 / \beta} .
$$

Não foi possível a determinação da aproximação $\bar{\ell}_{B N}(\beta)$ para dados com presença de censura tipo II, pois sua obtenção requer cálculos de esperanças matemáticas de potências de estatísticas de ordem impossíveis de serem finalizados analiticamente. Vale lembrar que esta pseudo-verossimilhança tem o mesmo erro de aproximação de $\breve{\ell}_{B N}$. Analogamente à seção anterior, duas aproximações para $\ell_{B N}(\beta)$ e suas respectivas funções escore são dadas por

(i)

$$
\begin{gathered}
\breve{\ell}_{B N}(\beta)=r \log \beta+\beta \sum_{j=1}^{r} \log y_{(j)}-(r-1) \log \left[\sum_{j=1}^{r} y_{(j)}^{\beta}+(n-r) y_{(r)}^{\beta}\right] \\
-\log \left[r\left(\sum_{j=1}^{r} y_{(j)}^{\beta+\widehat{\beta}}+(n-r) y_{(r)}^{\beta+\widehat{\beta}}\right)+(n-r)^{2} y_{(r)}^{\beta+\widehat{\beta}}-\left(\sum_{j=1}^{r} y_{(j)}^{\widehat{\beta}}\right)\left(\sum_{j=1}^{r} y_{(j)}^{\beta}\right)\right]
\end{gathered}
$$

$\mathrm{e}$

$$
\begin{gathered}
\breve{u}_{B N}(\beta)=\frac{r}{\beta}+\sum_{j=1}^{r} \log y_{(j)}-(r-1) \frac{\sum_{j=1}^{r} y_{(j)}^{\beta} \log y_{(j)}+(n-r) y_{(r)}^{\beta} \log y_{(r)}}{\sum_{j=1}^{r} y_{(j)}^{\beta}+(n-r) y_{(r)}^{\beta}} \\
-\frac{r\left(\sum_{j=1}^{r} y_{(j)}^{\beta+\widehat{\beta}} \log y_{(j)}+(n-r) y_{(r)}^{\beta+\widehat{\beta}} \log y_{(r)}\right)}{r\left(\sum_{j=1}^{r} y_{(j)}^{\beta+\widehat{\beta}}+(n-r) y_{(r)}^{\beta+\widehat{\beta}}\right)+(n-r)^{2} y_{(r)}^{\beta+\widehat{\beta}}-\left(\sum_{j=1}^{r} y_{(j)}^{\widehat{\beta}}\right)\left(\sum_{j=1}^{r} y_{(j)}^{\beta}\right)} \\
-\frac{(n-r)^{2} y_{(r)}^{\beta+\widehat{\beta}} \log y_{(r)}-\left(\sum_{j=1}^{r} y_{(j)}^{\widehat{\beta}}\right)\left(\sum_{j=1}^{r} y_{(j)}^{\beta} \log y_{(j)}\right)}{r\left(\sum_{j=1}^{r} y_{(j)}^{\beta+\widehat{\beta}}+(n-r) y_{(r)}^{\beta+\widehat{\beta}}\right)+(n-r)^{2} y_{(r)}^{\beta+\widehat{\beta}}-\left(\sum_{j=1}^{r} y_{(j)}^{\widehat{\beta}}\right)\left(\sum_{j=1}^{r} y_{(j)}^{\beta}\right)},
\end{gathered}
$$


onde $\widehat{\beta}$ é o estimador de máxima verossimilhança obtido de dados com presença de censura tipo II e não tem forma fechada;

(ii)

$$
\tilde{\ell}_{B N}(\beta)=(r-1)\left[\log \beta-\log \left(\sum_{j=1}^{r} y_{(j)}^{\beta}+(n-r) y_{(r)}^{\beta}\right)\right]+\beta \sum_{j=1}^{r} \log y_{(j)}-\log \sum_{j=1}^{r} y_{(j)}^{\beta}
$$

$\mathrm{e}$

$$
\begin{aligned}
\tilde{u}_{B N}(\beta)=(r-1) & {\left[\frac{1}{\beta}-\frac{\sum_{j=1}^{r} y_{(j)}^{\beta} \log y_{(j)}+(n-r) y_{(r)}^{\beta} \log y_{(r)}}{\sum_{j=1}^{r} y_{(j)}^{\beta}+(n-r) y_{(r)}^{\beta}}\right] } \\
& +\sum_{j=1}^{r} \log y_{(j)}-\frac{\sum_{j=1}^{r} y_{(j)}^{\beta} \log y_{(j)}}{\sum_{j=1}^{r} y_{(j)}^{\beta}} .
\end{aligned}
$$

Sob a mesma parametrização ortogonal obtida no caso de dados completos, Yang e Xie (2003) encontraram

$$
\ell_{C R}(\beta)=\ell_{p}(\beta)-2 \log \beta=(r-2) \log \beta-r \log \left[\sum_{j=1}^{r} y_{(j)}^{\beta}+(n-r) y_{(r)}^{\beta}\right]+\beta \sum_{j=1}^{r} \log y_{(j)}
$$

$\mathrm{e}$

$$
u_{C R}(\beta)=u_{p}(\beta)-\frac{2}{\beta}=\frac{r-2}{\beta}-r \frac{\sum_{j=1}^{r} y_{(j)}^{\beta} \log y_{(j)}+(n-r) y_{(r)}^{\beta} \log y_{(r)}}{\sum_{j=1}^{r} y_{(j)}^{\beta}+(n-r) y_{(r)}^{\beta}}+\sum_{j=1}^{r} \log y_{(j)} .
$$

Considere agora dados com presença de censura do tipo I, mais precisamente, $y_{j}=$ $\min \left(t_{j}, c\right)$, onde $t_{j} \sim W B(\alpha, \beta)(j=1, \ldots, n)$ e $c$ é uma constante positiva fixada, representando o tempo de censura. Tipicamente, este tipo de censura ocorre em estudos clínicos que são concluídos após um período pré-estabelecido de tempo. Nesta situação, os dados podem ser convenientemente representados por $n$ pares de variáveis aleatórias $\left(y_{j}, \delta_{j}\right)$, onde

$$
y_{j}=\min \left(t_{j}, c\right) \quad \text { e } \quad \delta_{j}=\left\{\begin{array}{l}
1, t_{j} \leq c \\
0, t_{j}>c
\end{array}\right.
$$

A função densidade de probabilidade conjunta do par $\left(y_{j}, \delta_{j}\right)$ é

$$
p\left(y_{j} ; \alpha, \beta\right)^{\delta_{j}} S(c ; \alpha, \beta)^{1-\delta_{j}}
$$

em que $p(\cdot ; \alpha, \beta)$ e $S(\cdot ; \alpha, \beta)$ denotam, respectivamente, a função densidade e a função de sobrevivência associadas à distribuição de Weibull $W B(\alpha, \beta)$. 
Considere uma amostra aleatória de $n$ pares $\left(y_{j}, \delta_{j}\right), j=1, \ldots, n$. A função de verossimilhança é dada por

$$
L(\alpha, \beta)=\prod_{j=1}^{n} p\left(y_{j} ; \alpha, \beta\right)^{\delta_{j}} S(c ; \alpha, \beta)^{1-\delta_{j}}
$$

Dessa forma,

$$
L(\alpha, \beta)=\left(\frac{\beta}{\alpha}\right)^{r} \exp \left[-\sum_{j=1}^{n}\left(\frac{y_{j}}{\alpha}\right)^{\beta}\right] \prod_{j \in \bar{C}}\left(\frac{y_{j}}{\alpha}\right)^{\beta-1}
$$

onde $\bar{C}$ denota o conjunto de índices das observações não-censuradas (falhas) e $r$ é o número total destas. No caso de dados com censura tipo II, $r$ é fixado, enquanto, aqui, $r$ é aleatório. Conseqüentemente, o logaritmo dessa função é expresso por

$$
\ell(\alpha, \beta)=r \log \beta-r \log \alpha-\sum_{j=1}^{n}\left(\frac{y_{j}}{\alpha}\right)^{\beta}+(\beta-1) \sum_{j \in \bar{C}} \log \left(\frac{y_{j}}{\alpha}\right)
$$

em que $r=\sum \delta_{j}$ é o número observado de falhas.

Fixando o parâmetro de interesse $\beta$, o estimador de máxima verossimilhança restrito de $\alpha$ é

$$
\widehat{\alpha}_{\beta}=\left(\frac{1}{r} \sum_{j=1}^{n} y_{j}^{\beta}\right)^{1 / \beta}
$$

Usando a definição de verossimilhança perfilada tem-se que

$$
\ell_{p}(\beta)=r \log \beta-r \log \sum_{j=1}^{n} y_{j}^{\beta}+\beta \sum_{j \in \bar{C}} \log y_{j},
$$

cuja primeira derivada com relação a $\beta$ define a função escore perfilada, a saber:

$$
u_{p}(\beta)=\frac{r}{\beta}-\frac{r \sum_{j=1}^{n} y_{j}^{\beta} \log y_{j}}{\sum_{j=1}^{n} y_{j}^{\beta}}+\sum_{j \in \bar{C}} \log y_{j} .
$$

Aqui,

$$
j_{\alpha \alpha}\left(\widehat{\alpha}_{\beta}, \beta\right)=\beta^{2}\left(\sum_{j=1}^{n} y_{j}^{\beta}\right)^{-2 / \beta} r^{1+2 / \beta} .
$$

Pelo mesmo motivo do caso de dados com presença de censura tipo II, aqui também não foi possível a determinação da pseudo-verossimilhança $\bar{\ell}_{B N}(\beta)$. Duas aproximações para $\ell_{B N}(\beta)$ e suas respectivas derivadas são dadas por

$$
\breve{\ell}_{B N}(\beta)=r \log \beta+\beta \sum_{j \in \bar{C}} \log y_{j}-(r-1) \log \sum_{j=1}^{n} y_{j}^{\beta}
$$




$$
-\log \left[r\left(\sum_{j \in \bar{C}} y_{j}^{\beta+\widehat{\beta}}+(n-r) c^{\beta+\widehat{\beta}}\right)+(n-r)^{2} c^{\beta+\widehat{\beta}}-\left(\sum_{j \in \bar{C}} y_{j}^{\widehat{\beta}}\right)\left(\sum_{j \in \bar{C}} y_{j}^{\beta}\right)\right]
$$

$\mathrm{e}$

$$
\begin{gathered}
\breve{u}_{B N}(\beta)=\frac{r}{\beta}+\sum_{j \in \bar{C}} \log y_{j}-(r-1) \frac{\sum_{j=1}^{n} y_{j}^{\beta} \log y_{j}}{\sum_{j=1}^{n} y_{j}^{\beta}} \\
-\frac{r\left(\sum_{j \in \bar{C}} y_{j}^{\beta+\widehat{\beta}} \log y_{j}+(n-r) c^{\beta+\widehat{\beta}} \log c\right)}{r\left(\sum_{j \in \bar{C}} y_{j}^{\beta+\widehat{\beta}}+(n-r) c^{\beta+\widehat{\beta}}\right)+(n-r)^{2} c^{\beta+\widehat{\beta}}-\left(\sum_{j \in \bar{C}} y_{j}^{\widehat{\beta}}\right)\left(\sum_{j \in \bar{C}} y_{j}^{\beta}\right)} \\
-\frac{(n-r)^{2} c^{\beta+\widehat{\beta}} \log c-\left(\sum_{j \in \bar{C}} y_{j}^{\widehat{\beta}}\right)\left(\sum_{j \in \bar{C}} y_{j}^{\beta} \log y_{j}\right)}{r\left(\sum_{j \in \bar{C}} y_{j}^{\beta+\widehat{\beta}}+(n-r) c^{\beta+\widehat{\beta}}\right)+(n-r)^{2} c^{\beta+\widehat{\beta}}-\left(\sum_{j \in \bar{C}} y_{j}^{\beta}\right)\left(\sum_{j \in \bar{C}} y_{j}^{\beta}\right)},
\end{gathered}
$$

onde $\widehat{\beta}$ é o estimador de máxima verossimilhança obtido de dados com presença de censura tipo I e não tem forma fechada;

(ii)

$$
\tilde{\ell}_{B N}(\beta)=(r-1)\left[\log \beta-\log \sum_{j=1}^{n} y_{j}^{\beta}\right]+\beta \sum_{j \in \bar{C}} \log y_{j}-\log \sum_{j \in \bar{C}} y_{j}^{\beta}
$$

$\mathrm{e}$

$$
\tilde{u}_{B N}(\beta)=(r-1)\left[\frac{1}{\beta}-\frac{\sum_{j=1}^{n} y_{j}^{\beta} \log y_{j}}{\sum_{j=1}^{n} y_{j}^{\beta}}\right]+\sum_{j \in \bar{C}} \log y_{j}-\frac{\sum_{j \in \bar{C}} y_{j}^{\beta} \log y_{j}}{\sum_{j \in \bar{C}} y_{j}^{\beta}} .
$$

Sob uma nova parametrização ortogonal $(\lambda, \beta)$, em que $\lambda=\log (\alpha)+(1-\gamma) / \beta$, Yang e Xie (2003) obtiveram

$$
\ell_{C R}(\beta)=\ell_{p}(\beta)-\log \beta=(r-1) \log \beta-r \log \sum_{j=1}^{n} y_{j}^{\beta}+\beta \sum_{j \in \bar{C}} \log y_{j}
$$

e

$$
u_{C R}(\beta)=u_{p}(\beta)-\frac{1}{\beta}=\frac{r-1}{\beta}-\frac{r \sum_{j=1}^{n} y_{j}^{\beta} \log y_{j}}{\sum_{j=1}^{n} y_{j}^{\beta}}+\sum_{j \in \bar{C}} \log y_{j} .
$$

Aqui, $\gamma=0.577215 \ldots$ é a constante de Euler. 
Também com dados censurados, os estimadores de máxima verossimilhança associados às funções de verossimilhanças perfiladas não têm formas fechadas. Assim, estimativas para $\beta$ são obtidas numericamente e alguns resultados são apresentados na próxima seção.

O ajuste de Cox e Reid $(1987,1989)$ requer um parâmetro de perturbação ortogonal ao parâmetro de interesse. A obtenção desta ortogonalidade é baseada na resolução de uma equação diferencial parcial cujas parcelas são elementos da matriz de informação de Fisher. Devido à impossibilidade do cálculo de esperanças matemáticas de potências de estatísticas de ordem na construção da matriz de informação de Fisher no caso de dados censurados, vale ressaltar que as duas parametrizações ortogonais usadas (censura tipo I e censura tipo II) foram obtidas a partir da informação de Fisher do caso de dados completos, segundo Yang e Xie (2003).

\subsubsection{RESULTADOS NUMÉRICOS}

Esta seção apresenta resultados numéricos oriundos de simulações de Monte Carlo. O parâmetro de interesse é o parâmetro de forma da distribuição de Weibull e os resultados são apresentados de forma análoga ao Capítulo 3, considerando dados completos e dados censurados. Diferentemente daquele capítulo, aqui são apresentados os vieses relativos dos estimadores (v.r.): $100 \times$ (viés / parâmetro)\%. Todos os resultados são baseados em 100000 (cem mil) réplicas de Monte Carlo. Para identificar os estimadores e os testes da razão de verossimilhanças, é usada a notação da Tabela 3.1.

Foram geradas amostras de tamanhos $n=30$ e $n=20$. No caso de dados com censura do tipo II, foram simuladas as seguintes situações para o par $(n, r):(30,20)$ e $(30,10),(20$, $15)$ e $(20,10)$. No caso de censura tipo I, foram consideradas amostras com $25 \%$ e $50 \%$ de censura. O parâmetro $\alpha$ foi fixado em 100 e dois diferentes valores para $\beta$ foram usados: 1 (distribuição exponencial) e 5, representando, respectivamente, funções de taxa de falha constante e crescente.

Aqui cabe uma importante observação relativa às distribuições dos estimadores de máxima verossimilhança considerados: as razões (estimador de $\beta$ ) / $\beta$ são quantidades pivotais com respeito ao par $(\alpha, \beta)$. Este resultado vale tanto para dados completos quanto para dados censurados e está demonstrado no Apêndice D deste texto. Conseqüentemente, os vieses relativos e os coeficientes de assimetria e de curtose dos estimadores não dependem dos valores verdadeiros dos parâmetros $\alpha$ e $\beta$, variam apenas com o tamanho da amostra e com a proporção de censura observada.

Além da estimação do parâmetro de forma $\beta$, foram realizados testes da razão de verossimilhanças sobre este. Aqui o objetivo foi comparar os desempenhos dos ajustes sobre a verossimilhança perfilada a partir das distorções dos tamanhos dos testes com relação aos níveis nominais. Usando o fato da razão (estimador de $\beta$ ) $/ \beta$ ser uma quantidade pivotal, pode-se mostrar que a probabilidade do erro tipo I não depende de $\alpha$ e nem do valor de $\beta$ 
fixado na hipótese nula em cada um dos testes, varia apenas com o tamanho amostral e com o nível de censura, ou seja, sob a hipótese nula, as estatísticas de teste dependem apenas de quantidades pivotais e ancilares para $(\alpha, \beta)$. Estes resultados também são demonstrados no Apêndice D.

As afirmações nos dois parágrafos precedentes representam uma contestação ao trabalho de Yang e Xie (2003), no qual foram simuladas situações para diferentes valores do parâmetro $\beta$. Por exemplo, considerando dados com censura tipo II e $(n, r)=(30,15)$, para $\beta=3$, o viés relativo estimado de $\widehat{\beta}_{C R}$ foi igual a -0.17 , enquanto para $\beta=8,0.66$. Fato mais grave do que essas estimativas serem diferentes é a oposição dos seus sinais, totalmente em desacordo com o resultado apresentado no Apêndice D. Considerando dados com censura tipo I, $n=20$ e nível de censura de $25 \%$, para $\beta=0.5$, o viés relativo estimado de $\widehat{\beta}_{C R}$ foi de $1.01 \mathrm{e}$, para $\beta=2$, 1.52. Resultados de simulação relativos a testes da razão de verossimilhanças não foram apresentados em tal trabalho.

Assim, os resultados numéricos apresentados aqui se diferenciam pelos tamanhos amostrais e níveis de censura considerados, não pelos valores verdadeiros dos parâmetros $\alpha \mathrm{e}$ $\beta$.

Tabela 5.1. Medidas descritivas de diferentes estimadores de $\beta$, distribuição de Weibull, dados completos, $\beta=5$.

\begin{tabular}{|c|c|c|c|c|c|c|c|c|}
\hline$n$ & estimador & média & variância & viés & EQM & v.r. $(\%)$ & assimetria & curtose \\
\hline \multirow{5}{*}{20} & $\widehat{\beta}_{p}$ & 5.376 & 1.049 & 0.376 & 1.191 & 7.529 & 0.928 & 4.708 \\
\hline & $\widehat{\bar{\beta}}_{B N}$ & 5.207 & 0.983 & 0.207 & 1.025 & 4.136 & 0.922 & 4.694 \\
\hline & $\widehat{\breve{\beta}}_{B N}$ & 5.229 & 0.993 & 0.229 & 1.046 & 4.587 & 0.930 & 4.710 \\
\hline & $\widehat{\tilde{\beta}}_{B N}$ & 5.208 & 0.983 & 0.208 & 1.026 & 4.152 & 0.924 & 4.699 \\
\hline & $\widehat{\beta}_{C R}$ & 5.036 & 0.919 & 0.036 & 0.920 & 0.726 & 0.920 & 4.689 \\
\hline \multirow{5}{*}{30} & $\widehat{\beta}_{p}$ & 5.243 & 0.624 & 0.243 & 0.683 & 4.862 & 0.703 & 3.989 \\
\hline & $\widehat{\bar{\beta}}_{B N}$ & 5.134 & 0.598 & 0.134 & 0.616 & 2.680 & 0.699 & 3.981 \\
\hline & $\widehat{\widehat{\beta}}_{B N}$ & 5.145 & 0.601 & 0.145 & 0.622 & 2.898 & 0.704 & 3.992 \\
\hline & $\widehat{\widetilde{\beta}}_{B N}$ & 5.134 & 0.598 & 0.134 & 0.616 & 2.688 & 0.700 & 3.984 \\
\hline & $\widehat{\beta}_{C R}$ & 5.025 & 0.572 & 0.025 & 0.573 & 0.493 & 0.698 & 3.979 \\
\hline
\end{tabular}

Considerando dados completos, a Tabela 5.1 contém estatísticas descritivas de diferentes estimadores. O valor verdadeiro do parâmetro $\beta$ é 5 . Para $n=30$ e baseando-se no viés relativo, o melhor desempenho foi do estimador $\widehat{\beta}_{C R}(0.493 \%)$, seguido pelos estimadores $\widehat{\bar{\beta}}_{B N}(2.680 \%), \widehat{\widetilde{\beta}}_{B N}(2.688 \%), \widehat{\breve{\beta}}_{B N}(2.898 \%)$ e, por último, $\widehat{\beta}_{p}(4.862 \%)$. Note que o viés relativo de $\widehat{\beta}_{p}$ é quase dez vezes maior do que o respectivo viés de $\widehat{\beta}_{C R}$. Reduzindo o tamanho amostral para $n=20$, as medidas se deterioram, mas essa relação de ordem baseada no viés relativo mantém-se.

Para $n=30$, os coeficientes de assimetria amostrais das distribuições dos estimadores $\widehat{\beta}_{p}$, 
$\widehat{\bar{\beta}}_{B N}, \widehat{\breve{\beta}}_{B N}, \widehat{\tilde{\beta}}_{B N}$ e $\widehat{\beta}_{C R}$ foram, respectivamente, iguais a $0.703,0.699,0.704,0.700$ e 0.698 , enquanto as correspondentes estimativas dos coeficientes de curtose foram 3.989, 3.982, 3.992, 3.984 e 3.979. Estimadores de máxima verossimilhança têm distribuição assintótica normal e, portanto, essas estimativas estão próximas dos valores assintóticos, 0 e 3, respectivamente.

As diferenças entre as estimativas das variâncias não foram tão acentuadas quanto àquelas observadas no viés. Por exemplo, para $n=20$, os valores apresentados por $\widehat{\beta}_{p}$, $\widehat{\bar{\beta}}_{B N}, \widehat{\breve{\beta}}_{B N}, \widehat{\widetilde{\beta}}_{B N}$ e $\widehat{\beta}_{C R}$ foram, respectivamente, iguais a $1.049,0.983,0.993,0.983$ e 0.919 . Como as potências de ordem dois dos vieses dos estimadores foram de pequeno valor, segundo o erro quadrático médio (EQM), os estimadores apresentaram desempenhos semelhantes.

Tabela 5.2. Taxas de rejeição da hipótese nula de diferentes testes da razão de verossimilhanças, $\mathcal{H}_{0}: \beta=1$, distribuição de Weibull, dados completos.

\begin{tabular}{|c|c|c|c|c|c|c|}
\hline$n$ & nível nominal & $\ell_{p}$ & $\bar{\ell}_{B N}$ & $\breve{\ell}_{B N}$ & $\tilde{\ell}_{B N}$ & $\ell_{C R}$ \\
\hline \multirow{9}{*}{20} & $15 \%$ & 16.575 & 15.003 & 14.949 & 14.987 & 14.747 \\
\hline & $10 \%$ & 11.353 & 10.122 & 10.115 & 10.105 & 9.800 \\
\hline & $5 \%$ & 6.020 & 5.113 & 5.105 & 5.090 & 4.937 \\
\hline & $3 \%$ & 3.751 & 3.116 & 3.104 & 3.107 & 2.996 \\
\hline & $1 \%$ & 1.390 & 1.058 & 1.040 & 1.047 & 0.997 \\
\hline & $0.5 \%$ & 0.758 & 0.542 & 0.558 & 0.539 & 0.459 \\
\hline & $0.1 \%$ & 0.164 & 0.099 & 0.097 & 0.097 & 0.087 \\
\hline & $0.05 \%$ & 0.078 & 0.049 & 0.048 & 0.048 & 0.048 \\
\hline & $0.01 \%$ & 0.019 & 0.009 & 0.011 & 0.009 & 0.009 \\
\hline \multirow{9}{*}{30} & $15 \%$ & 16.305 & 15.124 & 15.144 & 15.127 & 14.891 \\
\hline & $10 \%$ & 10.903 & 10.070 & 10.096 & 10.072 & 9.893 \\
\hline & $5 \%$ & 5.538 & 4.971 & 4.953 & 4.965 & 4.889 \\
\hline & $3 \%$ & 3.358 & 2.953 & 2.956 & 2.941 & 2.870 \\
\hline & $1 \%$ & 1.178 & 0.990 & 0.976 & 0.991 & 0.925 \\
\hline & $0.5 \%$ & 0.610 & 0.472 & 0.468 & 0.474 & 0.454 \\
\hline & $0.1 \%$ & 0.133 & 0.100 & 0.102 & 0.100 & 0.095 \\
\hline & $0.05 \%$ & 0.082 & 0.059 & 0.060 & 0.060 & 0.049 \\
\hline & $0.01 \%$ & 0.019 & 0.013 & 0.014 & 0.013 & 0.015 \\
\hline
\end{tabular}

A Tabela 5.2 apresenta taxas de rejeição sob a hipótese nula de diferentes testes da razão de verossimilhanças com vários níveis de significância. Embora essas taxas não dependam da hipótese considerada, vale mencionar que $\mathcal{H}_{0}: \beta=1$. Para $n=30$ e nível de significância de $10 \%$, as taxas de rejeição dos testes baseados em $\ell_{p}, \bar{\ell}_{B N}, \breve{\ell}_{B N}, \tilde{\ell}_{B N}$ e $\ell_{C R}$ foram, respectivamente, iguais a $10.903 \%, 10.070 \%, 10.096 \%, 10.072 \%$ e $9.893 \%$. Todas as verossimilhanças perfiladas ajustadas produziram inferências mais precisas relativamente aos resultados obtidos pelo teste da razão de verossimilhanças original e, aqui, não cabe qualquer destaque particular entre os desempenhos dessas.

Na Tabela 5.3 são mostradas a média e a variância empíricas de diferentes estatísticas de 
teste. Observe que as estatísticas baseadas nas verossimilhanças perfiladas ajustadas tiveram os dois primeiros momentos mais próximos dos valores assintóticos comparativamente aos valores apresentados pela estatística de teste obtida da verossimilhança original, $\ell_{p}$. Por exemplo, para $n=20$, a estatística de teste baseada em $\tilde{\ell}_{B N}$ apresentou média e variância iguais a 1.004 e 2.036 , respectivamente, e a estatística baseada em $\ell_{p}, 1.083$ e 2.370 .

Tabela 5.3. Médias e variâncias amostrais de diferentes estatísticas de teste e os valores assintóticos destas, $\mathcal{H}_{0}: \beta=1$, distribuição de Weibull, dados completos.

\begin{tabular}{cccccccc}
\hline \multirow{2}{*}{$n$} & momento & $\chi_{1}^{2}$ & $\ell_{p}$ & $\bar{\ell}_{B N}$ & $\breve{\ell}_{B N}$ & $\tilde{\ell}_{B N}$ & $\ell_{C R}$ \\
\hline \multirow{2}{*}{20} & média & 1.000 & 1.083 & 1.005 & 1.004 & 1.004 & 0.990 \\
& variância & 2.000 & 2.370 & 2.042 & 2.038 & 2.036 & 1.972 \\
\hline \multirow{2}{*}{30} & média & 1.000 & 1.055 & 1.003 & 1.002 & 1.002 & 0.993 \\
& variância & 2.000 & 2.203 & 1.993 & 1.992 & 1.991 & 1.953 \\
\hline
\end{tabular}

Figura 5.1. Gráfico das discrepâncias relativas de quantis, distribuição de Weibull, $\mathcal{H}_{0}: \beta=1$, dados completos, $n=20$.

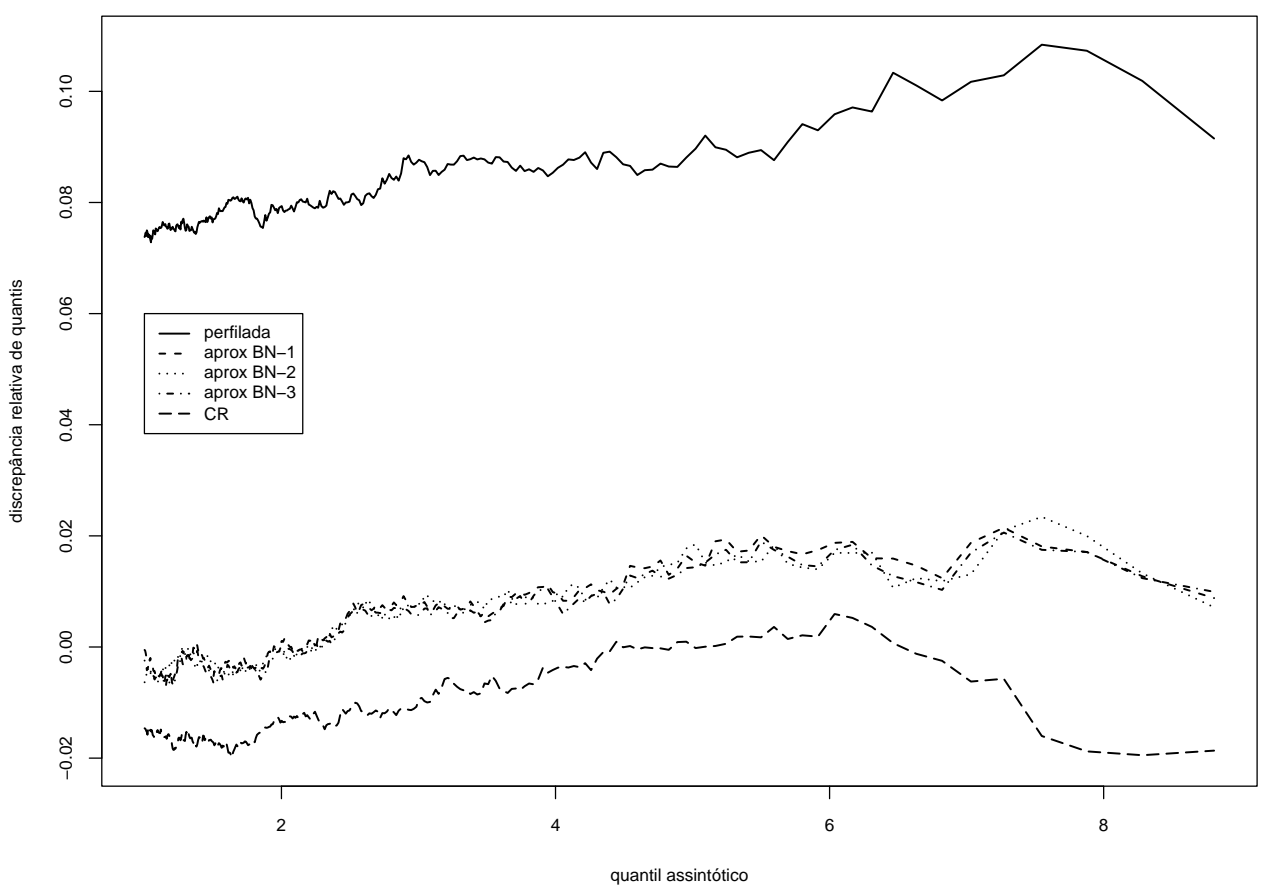

A Figura 5.1 apresenta o gráfico das discrepâncias relativas de quantis versus os correspondentes quantis assintóticos para $n=20$. Quanto mais próxima da ordenada zero a curva estiver, melhor a aproximação da distribuição da estatística de teste por sua distribuição assintótica qui-quadrado. Note que as curvas de discrepâncias de quantis das estatísticas de teste baseadas em $\bar{\ell}_{B N}$ (aprox BN-1), $\breve{\ell}_{B N}$ (aprox BN-2) e $\tilde{\ell}_{B N}$ (aprox BN-3) apresentaram comportamentos semelhantes, posicionadas entre as ordenada zero e 0.02 (2\%), diferentemente da curva associada à verossimilhança perfilada original, posicionada entre as ordenadas 
$0.08(8 \%)$ e $0.10(10 \%)$. Portanto, as estatísticas de teste baseadas nas verossimilhanças perfiladas ajustadas apresentaram distribuições nulas melhor aproximadas pela distribuição $\chi_{1}^{2}$. Isto também é observado na Figura 5.2, que apresenta gráfico similar para $n=30$.

Figura 5.2. Gráfico das discrepâncias relativas de quantis, distribuição de Weibull, $\mathcal{H}_{0}: \beta=1$, dados completos, $n=30$.

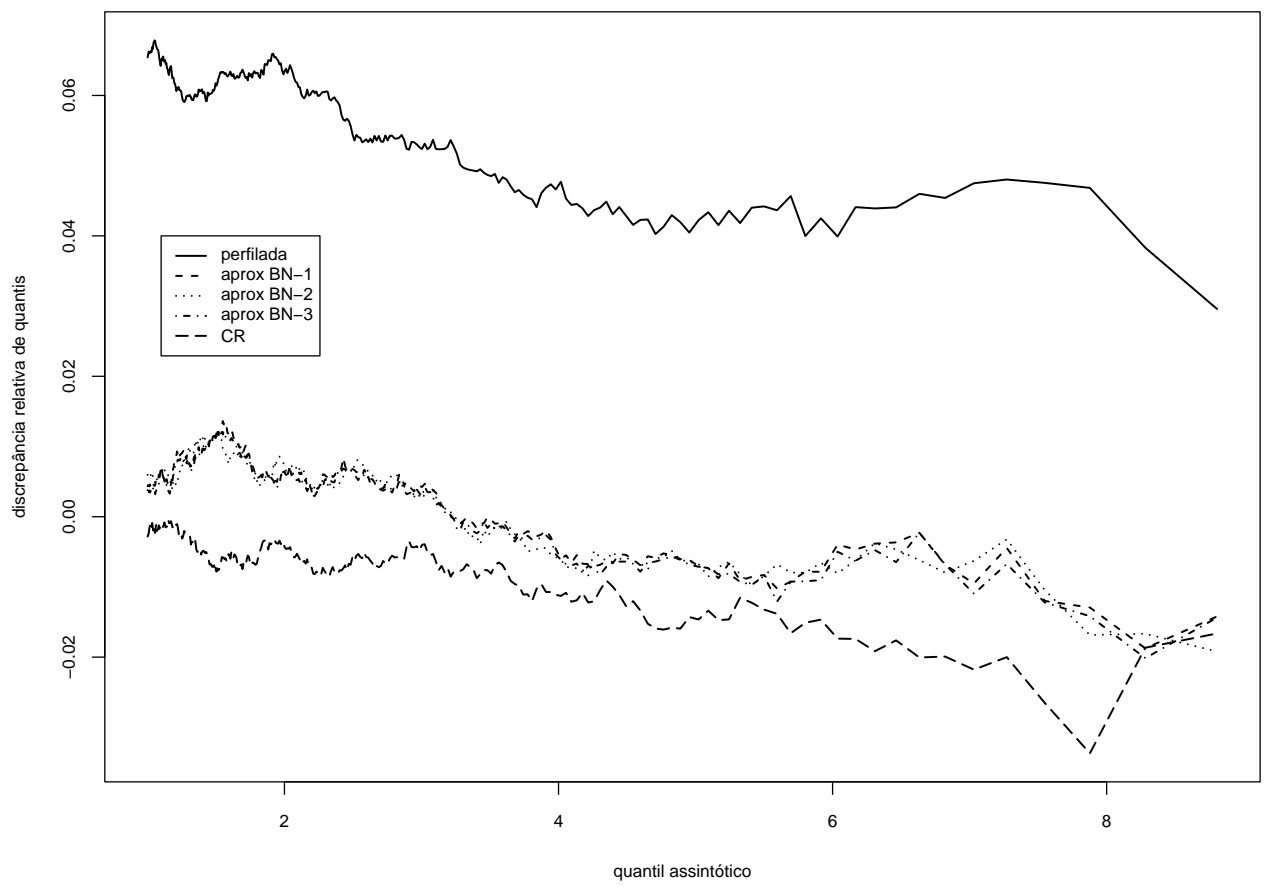

Quando um estudo clínico termina após a ocorrência do evento de interesse em um número pré-determinado de indivíduos, as demais observações são ditas censuradas. Este caso define a censura do tipo II e resultados numéricos são apresentados a seguir.

A Tabela 5.4 contém estatísticas descritivas de diferentes estimadores para diferentes níveis de censura $(n, r): 25 \%(20,15), 33.33 \%(30,20), 50 \%(20,10)$ e $66.66 \%(30,10)$. Vale mencionar que $\beta=5$.

Comparativamente à situação anterior (dados completos), a presença de censura evidenciou ainda mais o melhor desempenho do estimador $\widehat{\beta}_{C R}$, tanto em termo de menor erro quadrático médio, quanto em termo de menor viés relativo, que, surpreendentemente, diminuiu. Por outro lado, relativamente a essas duas medidas, a presença de censura deteriorou os resultados dos demais estimadores, reduzindo, por exemplo, a diferença entre os desempenhos de $\widehat{\beta}_{p}$ e $\widehat{\breve{\beta}}_{B N}$, ambos superados por $\widehat{\widetilde{\beta}}_{B N}$.

Para $(n, r)=(30,20)$, o viés relativo e o erro quadrático médio do estimador $\widehat{\beta}_{p}$ foram iguais a $9.091 \%$ e 1.583, respectivamente, enquanto, para $\widehat{\breve{\beta}}_{B N}, 7.863 \%$ e 1.501 , e, para $\widehat{\widetilde{\beta}}_{B N}$, $6.088 \%$ e 1.394 . Note que o viés relativo de $\widehat{\beta}_{p}$ é quase trinta vezes maior do que o respectivo viés de $\widehat{\beta}_{C R}(0.308 \%)$, o menor deles. Para um nível de censura de $50 \%,(n, r)=(20,10)$, o 
Tabela 5.4. Medidas descritivas de diferentes estimadores de $\beta$, distribuição de Weibull, dados censurados tipo II, $\beta=5$.

\begin{tabular}{ccccccccc}
\hline$(n, r)$ & estimador & média & variância & viés & EQM & v.r.(\%) & assimetria & curtose \\
\hline \multirow{3}{*}{$(20,15)$} & $\widehat{\beta}_{p}$ & 5.610 & 1.985 & 0.610 & 2.358 & 12.204 & 1.146 & 5.658 \\
& $\widehat{\beta}_{B N}$ & 5.510 & 1.916 & 0.510 & 2.176 & 10.201 & 1.147 & 5.660 \\
& $\widehat{\tilde{\beta}}_{B N}$ & 5.398 & 1.835 & 0.398 & 1.993 & 7.951 & 1.143 & 5.646 \\
& $\widehat{\beta}_{C R}$ & 5.030 & 1.593 & 0.030 & 1.594 & 0.600 & 1.143 & 5.644 \\
\hline \multirow{3}{*}{$(30,20)$} & $\widehat{\beta}_{p}$ & 5.455 & 1.377 & 0.455 & 1.583 & 9.091 & 0.931 & 4.718 \\
& $\widehat{\beta}_{B N}$ & 5.393 & 1.346 & 0.393 & 1.501 & 7.863 & 0.931 & 4.718 \\
& $\widehat{\tilde{\beta}}_{B N}$ & 5.304 & 1.301 & 0.304 & 1.394 & 6.088 & 0.929 & 4.716 \\
& $\widehat{\beta}_{C R}$ & 5.015 & 1.164 & 0.015 & 1.164 & 0.308 & 0.929 & 4.716 \\
\hline \multirow{3}{*}{$(20,10)$} & $\widehat{\beta}_{p}$ & 6.050 & 4.278 & 1.050 & 5.380 & 21.000 & 1.636 & 9.273 \\
& $\widehat{\beta}_{B N}$ & 5.962 & 4.155 & 0.962 & 5.081 & 19.248 & 1.636 & 9.263 \\
& $\widehat{\tilde{\beta}}_{B N}$ & 5.707 & 3.806 & 0.707 & 4.306 & 14.133 & 1.638 & 9.308 \\
& $\widehat{\beta}_{C R}$ & 5.002 & 2.923 & 0.002 & 2.923 & 0.037 & 1.636 & 9.280 \\
\hline \multirow{3}{*}{$(30,10)$} & $\widehat{\beta}_{p}$ & 6.132 & 4.778 & 1.132 & 6.060 & 22.643 & 1.622 & 8.240 \\
& $\widehat{\aleph}_{B N}$ & 6.078 & 4.695 & 1.078 & 5.857 & 21.560 & 1.622 & 8.242 \\
& $\widehat{\tilde{\beta}}_{B N}$ & 5.789 & 4.260 & 0.789 & 4.882 & 15.774 & 1.619 & 8.215 \\
& $\widehat{\beta}_{C R}$ & 5.010 & 3.189 & 0.010 & 3.189 & 0.202 & 1.620 & 8.223 \\
\hline
\end{tabular}

viés relativo do estimador original é quase seiscentas vezes maior: $21 \% / 0.037 \%$. O aumento da proporção de censura na amostra foi acompanhado pela redução do viés de $\widehat{\beta}_{C R}$.

Observe na Tabela 5.4 que as diferenças entre as estimativas das variâncias não são tão acentuadas quanto àquelas observadas entre os vieses. Em geral, há aumento dessas estimativas com o aumento da proporção de dados censurados na amostra. Por exemplo, para $(n, r)=(20,15)$, as estimativas das variâncias de $\widehat{\beta}_{p}, \widehat{\breve{\beta}}_{B N}, \widehat{\widetilde{\beta}}_{B N}$ e $\widehat{\beta}_{C R}$ foram iguais a $1.985,1.916,1.835$ e 1.593 , enquanto, para $(n, r)=(30,10), 4.778,4.695,4.260$ e 3.189, respectivamente. Note que a variância de $\widehat{\beta}_{C R}$ praticamente dobrou. Isto reflete-se no comportamento das estimativas dos erros quadráticos médios.

Sobre as estimativas dos coeficientes de assimetria e de curtose, assim como constatado nos valores apresentados para as variâncias, à medida que a proporção de censura aumenta, tais estimativas distanciam-se ainda mais dos valores assintóticos associados a um estimador de máxima verossimilhança, 0 e 3, respectivamente. Note que, embora não seja a combinação com menor proporção de censura, para $(n, r)=(30,20)$, tais estimativas estiveram mais próximas dos valores assintóticos, respectivamente: 0.931 e $4.718\left(\widehat{\beta}_{p}, \widehat{\breve{\beta}}_{B N}\right), 0.929$ e $4.716\left(\widehat{\tilde{\beta}}_{B N}, \widehat{\beta}_{C R}\right)$. Além disso, as variâncias e os vieses dos estimadores neste caso foram menores do que as estimativas apresentadas no caso $(n, r)=(20,15)$, que tem uma proporção de censura menor, $25 \%$. Talvez o melhor resultado destas medidas de desempenho 
dos estimadores deva-se ao fato da maior quantidade de tempos de falhas observada em $(n, r)=(30,20)$, que apresenta uma proporção de censura de $33.33 \%$.

Tabela 5.5. Taxas de rejeição da hipótese nula de diferentes testes da razão de verossimilhanças, $\mathcal{H}_{0}: \beta=1$, distribuição de Weibull, dados censurados tipo II.

\begin{tabular}{|c|c|c|c|c|c|c|c|c|}
\hline \multicolumn{5}{|c|}{$(n, r)=(30,20)$} & \multicolumn{4}{|c|}{$(n, r)=(20,15)$} \\
\hline nível nominal & $\ell_{p}$ & $\breve{\ell}_{B N}$ & $\tilde{\ell}_{B N}$ & $\ell_{C R}$ & $\ell_{p}$ & $\breve{\ell}_{B N}$ & $\tilde{\ell}_{B N}$ & $\ell_{C R}$ \\
\hline $15 \%$ & 16.876 & 16.162 & 15.226 & 14.909 & 17.649 & 16.463 & 15.385 & 14.894 \\
\hline $10 \%$ & 11.596 & 10.959 & 10.207 & 9.951 & 12.259 & 11.279 & 10.348 & 9.971 \\
\hline $5 \%$ & 6.018 & 5.620 & 5.181 & 5.024 & 6.500 & 5.845 & 5.248 & 5.040 \\
\hline $3 \%$ & 3.805 & 3.544 & 3.148 & 3.001 & 4.153 & 3.699 & 3.193 & 3.030 \\
\hline $1 \%$ & 1.369 & 1.232 & 1.052 & 0.995 & 1.591 & 1.352 & 1.097 & 1.019 \\
\hline $0.5 \%$ & 0.718 & 0.632 & 0.515 & 0.483 & 0.831 & 0.699 & 0.567 & 0.510 \\
\hline $0.1 \%$ & 0.144 & 0.125 & 0.104 & 0.105 & 0.193 & 0.155 & 0.116 & 0.110 \\
\hline $0.05 \%$ & 0.084 & 0.065 & 0.048 & 0.052 & 0.097 & 0.083 & 0.062 & 0.060 \\
\hline \multirow[t]{2}{*}{$0.01 \%$} & 0.018 & 0.015 & 0.006 & 0.006 & 0.023 & 0.020 & 0.013 & 0.018 \\
\hline & \multicolumn{4}{|c|}{$(n, r)=(30,10)$} & \multicolumn{4}{|c|}{$(n, r)=(20,10)$} \\
\hline nível nominal & $\ell_{p}$ & $\breve{\ell}_{B N}$ & $\tilde{\ell}_{B N}$ & $\ell_{C R}$ & $\ell_{p}$ & $\breve{\ell}_{B N}$ & $\tilde{\ell}_{B N}$ & $\ell_{C R}$ \\
\hline $15 \%$ & 19.345 & 18.774 & 16.066 & 15.124 & 18.697 & 17.843 & 15.493 & 14.771 \\
\hline $10 \%$ & 13.654 & 13.226 & 10.935 & 10.172 & 13.110 & 12.352 & 10.361 & 9.937 \\
\hline $5 \%$ & 7.582 & 7.244 & 5.569 & 5.147 & 7.199 & 6.662 & 5.260 & 4.932 \\
\hline $3 \%$ & 4.834 & 4.615 & 3.375 & 3.160 & 4.572 & 4.215 & 3.196 & 2.933 \\
\hline $1 \%$ & 1.873 & 1.774 & 1.191 & 1.041 & 1.763 & 1.563 & 1.054 & 0.984 \\
\hline $0.5 \%$ & 1.048 & 0.963 & 0.587 & 0.515 & 0.934 & 0.822 & 0.529 & 0.514 \\
\hline $0.1 \%$ & 0.252 & 0.232 & 0.108 & 0.095 & 0.224 & 0.187 & 0.110 & 0.111 \\
\hline $0.05 \%$ & 0.125 & 0.112 & 0.051 & 0.042 & 0.134 & 0.111 & 0.050 & 0.049 \\
\hline $0.01 \%$ & 0.030 & 0.025 & 0.012 & 0.008 & 0.027 & 0.021 & 0.014 & 0.008 \\
\hline
\end{tabular}

A Tabela 5.5 apresenta taxas de rejeição sob a hipótese nula de diferentes testes da razão de verossimilhanças com vários níveis de significância. Embora a hipótese nula considerada não exerça influência sobre tais taxas, aqui $\mathcal{H}_{0}: \beta=1$. O teste da razão de verossimilhanças baseado na verossimilhança perfilada ajustada proposta por Cox e Reid $(1987,1989)$ foi aquele que apresentou taxas de rejeição mais próximas dos níveis nominais, seguido pelo teste baseado em $\tilde{\ell}_{B N}$. Por exemplo, para $(n, r)=(30,20)$ e nível de significância de $5 \%$, as taxas de rejeição dos testes baseados em $\ell_{p}, \breve{\ell}_{B N}, \tilde{\ell}_{B N}$ e $\ell_{C R}$ foram iguais a $6.018 \%, 5.620 \%$, $5.181 \%$ e $5.024 \%$, enquanto, para $(n, r)=(20,10)$ e nível de significância de $0.5 \%, 0.934 \%$, $0.822 \%, 0.529 \%$ e $0.514 \%$, respectivamente.

As médias e variâncias das estatísticas de teste são apresentadas na Tabela 5.6, assim como seus valores assintóticos (1 e 2, respectivamente). Entre as estatísticas, aquela com os momentos empíricos mais próximos dos valores assintóticos foi a baseada em $\ell_{C R}$, seguida pela obtida de $\tilde{\ell}_{B N}$. Por exemplo, para $(n, r)=(30,10)$, ou seja, $66.66 \%$ de dados censurados na amostra, as estimativas da média e da variância das estatísticas foram iguais a: 1.218 e 
Tabela 5.6. Médias e variâncias amostrais de diferentes estatísticas de teste e os valores assintóticos destas, $\mathcal{H}_{0}: \beta=1$, distribuição de Weibull, dados censurados tipo II.

\begin{tabular}{cccccccccc}
\hline & \multicolumn{9}{c}{$(n, r)=(30,20)$} \\
\hline momento & $\chi_{1}^{2}$ & $\ell_{p}$ & $\breve{\ell}_{B N}$ & $\tilde{\ell}_{B N}$ & $\ell_{C R}$ & $\ell_{p}$ & $\breve{\ell}_{B N}$ & $\tilde{\ell}_{B N}$ & $\ell_{C R}$ \\
\hline média & 1 & 1.096 & 1.062 & 1.016 & 1.001 & 1.132 & 1.077 & 1.023 & 1.002 \\
variância & 2 & 2.364 & 2.227 & 2.042 & 1.992 & 2.560 & 2.334 & 2.105 & 2.026 \\
\hline \multicolumn{1}{c}{$(n, r)=(30,10)$} & & & $(n, r)=(20,10)$ & \\
\hline momento & $\chi_{1}^{2}$ & $\ell_{p}$ & $\breve{\ell}_{B N}$ & $\tilde{\ell}_{B N}$ & $\ell_{C R}$ & $\ell_{p}$ & $\breve{\ell}_{B N}$ & $\tilde{\ell}_{B N}$ & $\ell_{C R}$ \\
\hline média & 1 & 1.218 & 1.189 & 1.049 & 1.012 & 1.187 & 1.142 & 1.023 & 0.996 \\
variância & 2 & 2.890 & 2.772 & 2.171 & 2.031 & 2.760 & 2.576 & 2.079 & 1.993 \\
\hline
\end{tabular}

Figura 5.3. Gráfico das discrepâncias relativas de quantis, distribuição de Weibull, $\mathcal{H}_{0}: \beta=1$, dados censurados tipo II, $(n, r)=(20,15)$.

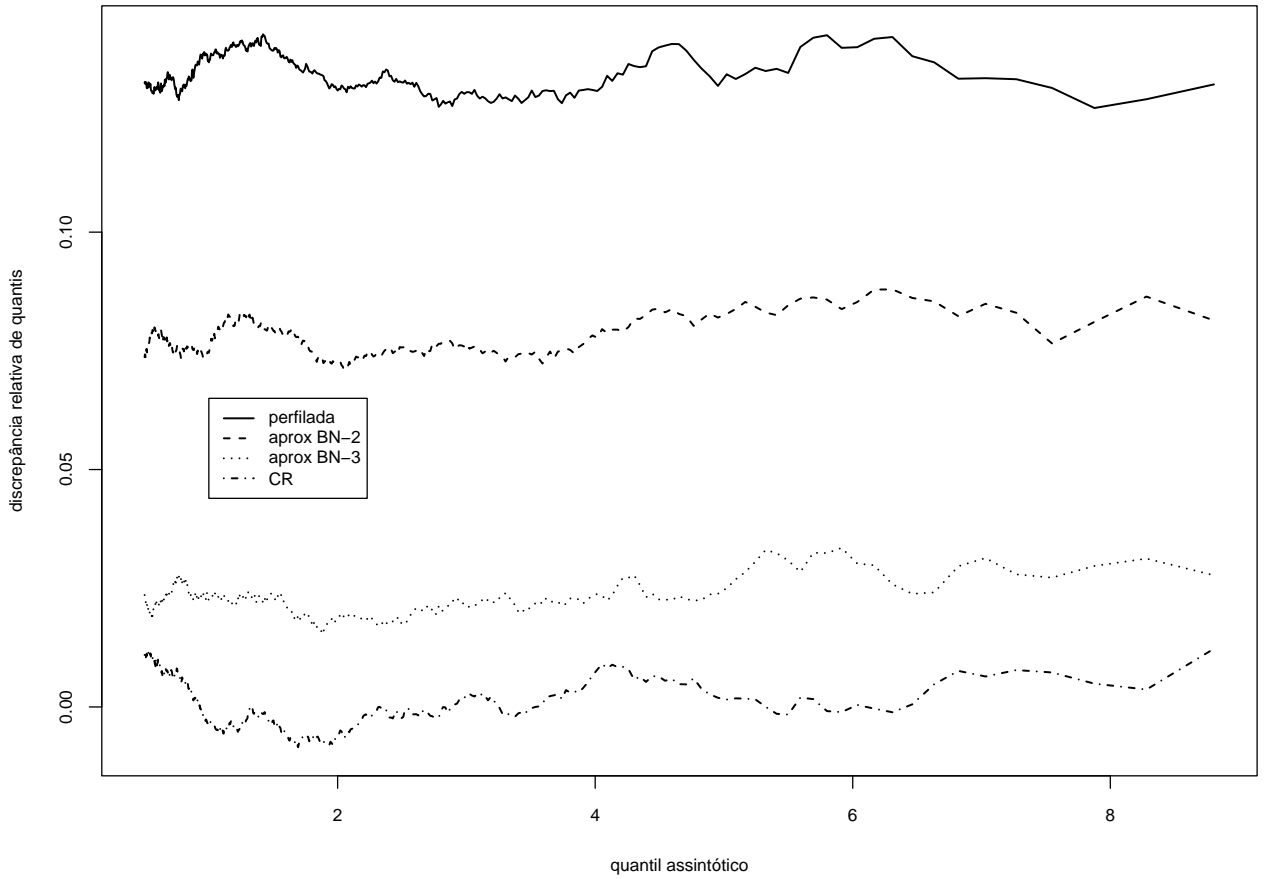

$2.890\left(\ell_{p}\right), 1.189$ e $2.772\left(\breve{\ell}_{B N}\right), 1.049$ e $2.171\left(\tilde{\ell}_{B N}\right), 1.012$ e $2.031\left(\ell_{C R}\right)$, respectivamente.

As Figuras 5.3, 5.4, 5.5 e 5.6 apresentam os gráficos das discrepâncias relativas de quantis versus os correspondentes quantis assintóticos das estatísticas de teste para cada uma das combinações $(n, r)$ consideradas: $(20,15),(30,20),(20,10)$ e $(30,10)$, respectivamente. Todos os testes mostraram-se liberais, rejeitando a hipótese nula além do valor nominal (Tabela 5.5). Um reflexo disto é o posicionamento das curvas de discrepâncias de quantis na região positiva do eixo das ordenadas, conforme as quatro figuras. Note que as curvas das estatísticas de teste baseadas em $\ell_{C R}$ e $\tilde{\ell}_{B N}$ (aprox BN-3) estão mais próximas da ordenada zero e, portanto, as respectivas distribuições nulas são melhor aproximadas pela distribuição assintótica $\chi_{1}^{2}$. Por outro lado, à medida que a proporção de censura aumenta, os pata- 
Figura 5.4. Gráfico das discrepâncias relativas de quantis, distribuição de Weibull, $\mathcal{H}_{0}: \beta=1$, dados censurados tipo II, $(n, r)=(30,20)$.

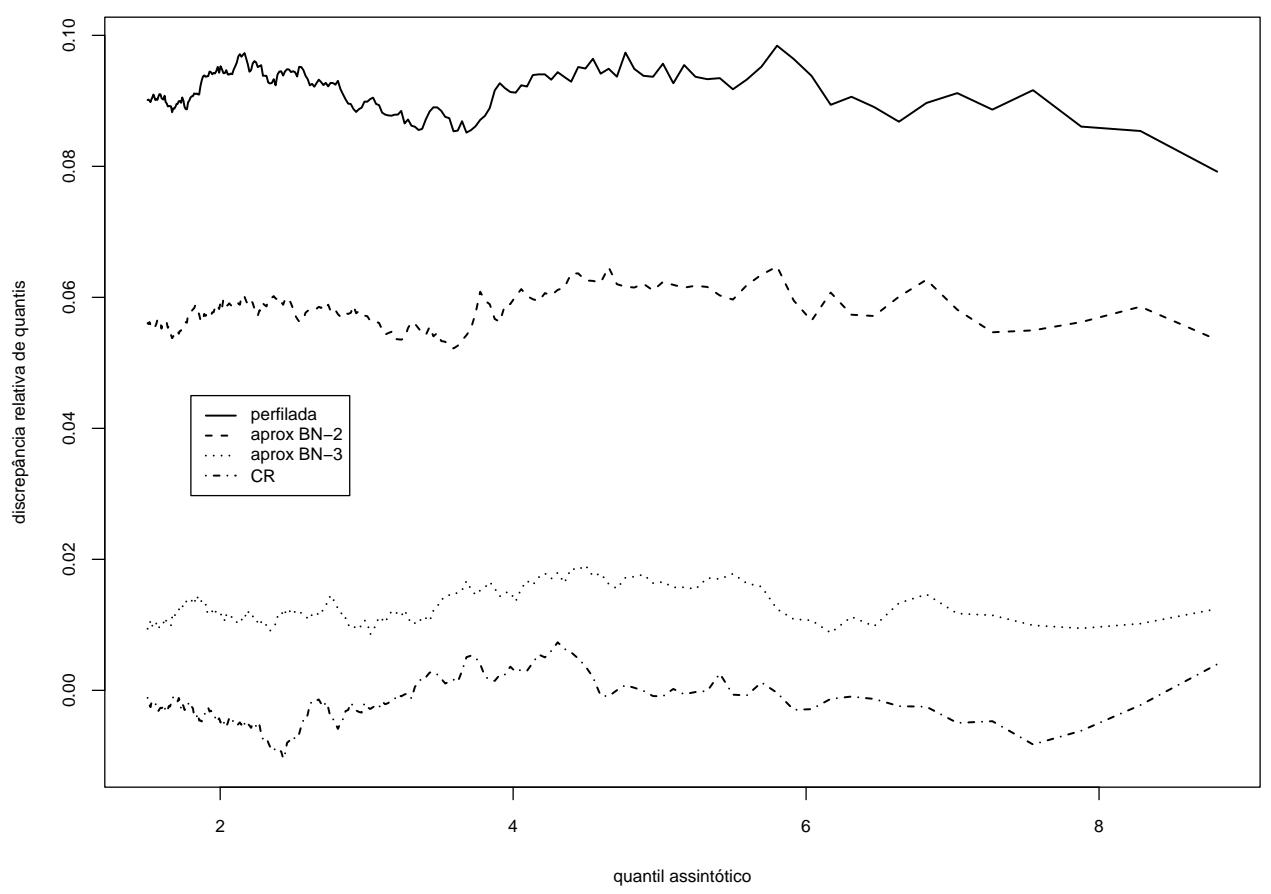

Figura 5.5. Gráfico das discrepâncias relativas de quantis, distribuição de Weibull, $\mathcal{H}_{0}: \beta=1$, dados censurados tipo II, $(n, r)=(20,10)$.

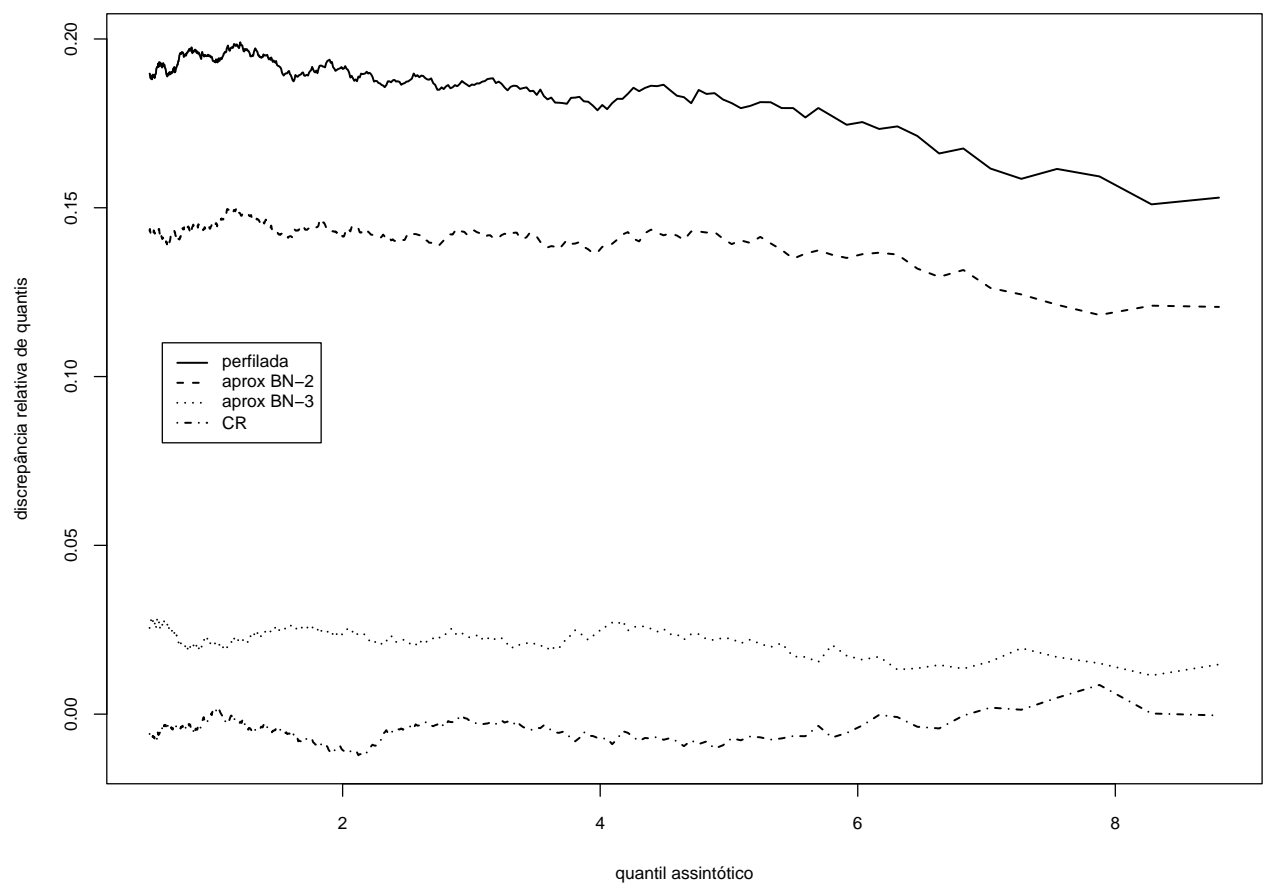

mares das curvas associadas a $\ell_{p}$ e a $\breve{\ell}_{B N}$ (aprox BN-2) claramente deslocam-se para cima, 
Figura 5.6. Gráfico das discrepâncias relativas de quantis, distribuição de Weibull, $\mathcal{H}_{0}: \beta=1$, dados censurados tipo II, $(n, r)=(30,10)$.

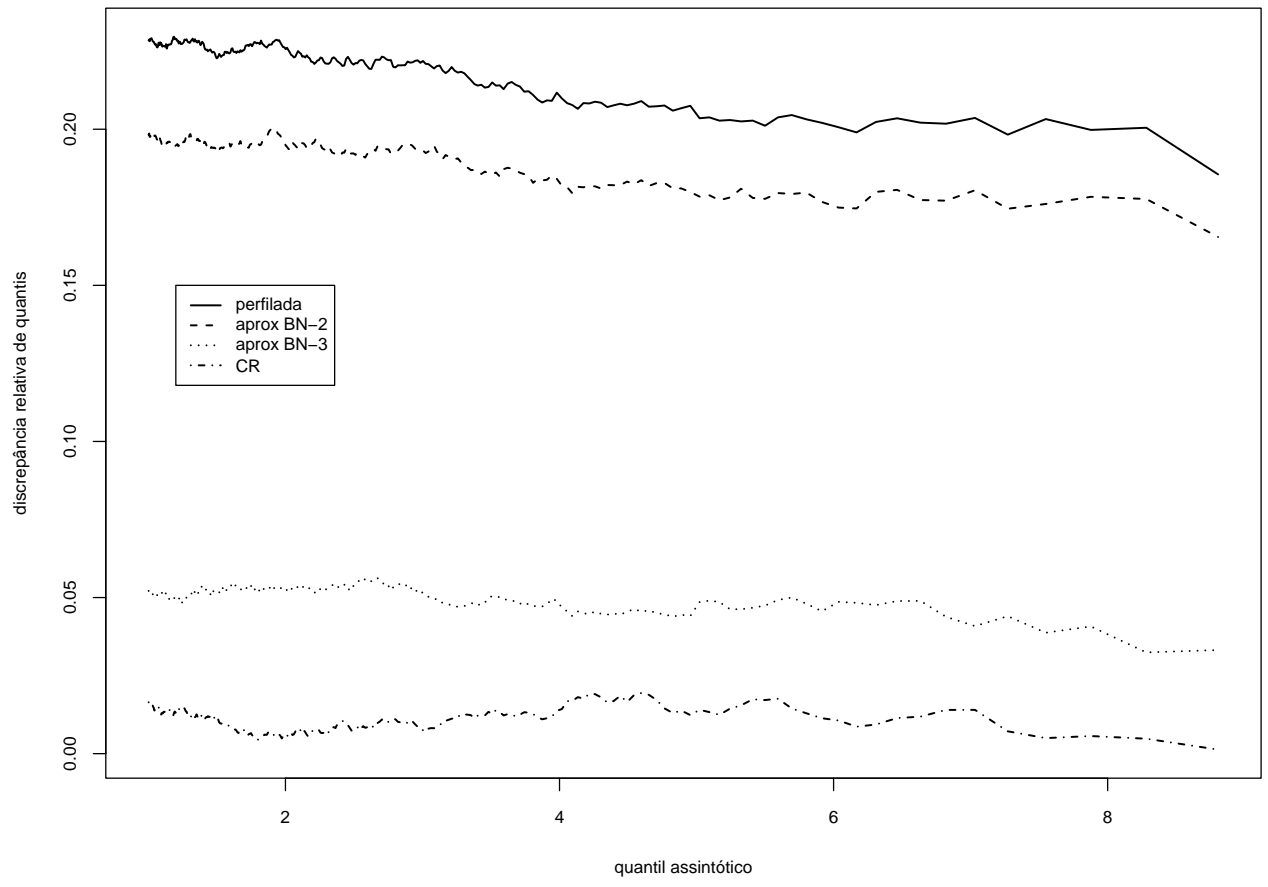

distanciando-se da ordenada zero.

A partir dos resultados das Tabelas 5.5 e 5.6 e dos gráficos das Figuras 5.3, 5.4, 5.5 e 5.6, o teste baseado em $\ell_{C R}$ supera os demais testes da razão de verossimilhanças.

Agora considere dados com censura tipo I. Tipicamente, este tipo de censura ocorre em estudos clínicos que são concluídos após um período pré-estabelecido de tempo.

Diferentemente dos dados com censura tipo II, não é possível fixar o número de falhas numa amostra Monte Carlo, ou seja, a proporção de observações censuradas. Aqui se faz o seguinte: supondo uma proporção (probabilidade) de censura desejada $\left(p_{0}\right)$, calcula-se, usando a função distribuição do tempo de falha, o tempo de censura $(c)$ necessário para que tal proporção seja observada, mais precisamente,

$$
\operatorname{Pr}(y>c)=p_{0} \quad \Longrightarrow \quad c=\alpha\left(-\log p_{0}\right)^{1 / \beta}
$$

Como as amostras são de tamanhos finitos, em geral, tal proporção não é observada em cada uma das amostras, mas o valor médio das proporções de censura é calculado e comparado com a proporção de censura desejada. Aqui foram considerados dois valores para $p_{0}$ : $25 \%$ e $50 \%$. Os valores médios das proporções de censura observados foram iguais a $25.001 \%$ e $49.998 \%$, muito próximos aos valores nominais.

A Tabela 5.7 apresenta estatísticas descritivas de diferentes estimadores de $\beta$. Mais uma vez, o valor verdadeiro do parâmetro de interesse foi 5. Assim como ocorreu com os resultados obtidos a partir de dados censurados do tipo II, a medida que mais diferencia 
Tabela 5.7. Medidas descritivas de diferentes estimadores de $\beta$, distribuição de Weibull, dados censurados tipo I, $\beta=5$.

\begin{tabular}{|c|c|c|c|c|c|c|c|c|}
\hline \multicolumn{9}{|c|}{$n=20$} \\
\hline$p_{0}$ & estimador & média & variância & viés & EQM & v.r. $(\%)$ & assimetria & curtose \\
\hline \multirow{4}{*}{$25 \%$} & $\widehat{\beta}_{p}$ & 5.340 & 1.625 & 0.340 & 1.740 & 6.792 & 0.914 & 4.707 \\
\hline & $\breve{\beta}_{B N}$ & 5.236 & 1.556 & 0.236 & 1.612 & 4.722 & 0.916 & 4.715 \\
\hline & $\tilde{\beta}_{B N}$ & 5.155 & 1.526 & 0.155 & 1.550 & 3.097 & 0.907 & 4.674 \\
\hline & $\widehat{\beta}_{C R}$ & 5.065 & 1.498 & 0.065 & 1.502 & 1.301 & 0.899 & 4.643 \\
\hline \multirow{4}{*}{$50 \%$} & $\widehat{\beta}_{p}$ & 5.527 & 3.314 & 0.527 & 3.592 & 10.536 & 1.746 & 11.596 \\
\hline & $\breve{\beta}_{B N}$ & 5.438 & 3.209 & 0.438 & 3.400 & 8.753 & 1.752 & 11.671 \\
\hline & $\tilde{\beta}_{B N}$ & 5.245 & 2.977 & 0.245 & 3.037 & 4.902 & 1.635 & 10.206 \\
\hline & $\widehat{\beta}_{C R}$ & 5.024 & 2.736 & 0.024 & 2.737 & 0.481 & 1.515 & 9.024 \\
\hline \multicolumn{9}{|c|}{$n=30$} \\
\hline$p_{0}$ & estimador & média & variância & viés & EQM & v.r. $(\%)$ & assimetria & curtose \\
\hline \multirow{4}{*}{$25 \%$} & & 5.220 & 0.994 & 0.220 & 1.043 & 4.397 & 0.698 & 3.983 \\
\hline & $\breve{\beta}_{B N}$ & 5.153 & 0.966 & 0.153 & 0.990 & 3.060 & 0.698 & 3.986 \\
\hline & $\tilde{\beta}_{B N}$ & 5.099 & 0.954 & 0.099 & 0.964 & 1.979 & 0.694 & 3.973 \\
\hline & $\widehat{\beta}_{C R}$ & 5.042 & 0.943 & 0.042 & 0.944 & 0.830 & 0.690 & 3.959 \\
\hline \multirow{4}{*}{$50 \%$} & $\widehat{\beta}_{p}$ & 5.336 & 1.867 & 0.336 & 1.979 & 6.713 & 1.167 & 6.889 \\
\hline & $\breve{\beta}_{B N}$ & 5.279 & 1.827 & 0.279 & 1.904 & 5.576 & 1.169 & 6.904 \\
\hline & $\tilde{\beta}_{B N}$ & 5.154 & 1.747 & 0.154 & 1.771 & 3.083 & 1.133 & 6.540 \\
\hline & $\widehat{\beta}_{C R}$ & 5.020 & 1.666 & 0.020 & 1.666 & 0.395 & 1.089 & 6.080 \\
\hline
\end{tabular}

os estimadores é o viés. Dessa forma, segundo esta medida, $\widehat{\beta}_{C R}$ foi o estimador que apresentou melhor desempenho. Por exemplo, para $n=20$ e $p_{0}=25 \%$, os vieses relativos dos estimadores $\widehat{\beta}_{p}, \widehat{\breve{\beta}}_{B N}, \widehat{\tilde{\beta}}_{B N}$ e $\widehat{\beta}_{C R}$ foram, respectivamente, iguais a $6.792 \%, 4.722 \%, 3.097 \%$ e $1.301 \%$. Observe que a discrepância de desempenho entre $\widehat{\beta}_{C R}$ e os demais estimadores não foi tão acentuada como aquela vista no caso de dados com presença de censura do tipo II, $(n, r)=(20,15)$. Note também que o aumento da proporção de censura na amostra acarretou aumento de viés dos estimadores, exceto de $\widehat{\beta}_{C R}$, que reduziu. Para $n=20$ e $p_{0}=50 \%$, o viés relativo deste estimador foi igual a $0.481 \%$.

A Tabela 5.8 apresenta taxas de rejeição sob a hipótese nula de diferentes testes da razão de verossimilhanças. Aqui $\mathcal{H}_{0}: \beta=1$. Diferentemente dos resultados relativos a amostras com presença de censura do tipo II, o teste da razão de verossimilhanças baseado em $\ell_{p}$ apresentou taxas de rejeição mais próximas aos níveis nominais. Assim, restou uma pequena margem para melhoria destas taxas através de ajustes em $\ell_{p}$. Por exemplo, para $\left(n, p_{0}\right)=(30,25 \%)$ e nível de significância de $1 \%$, os testes da razão de verossimilhanças baseados em $\ell_{p}, \breve{\ell}_{B N}, \tilde{\ell}_{B N}$ e $\ell_{C R}$ apresentaram taxas de rejeição da hipótese nula iguais a $1.097 \%, 1.004 \%, 0.960 \%$ e $1.037 \%$, respectivamente. Em alguns casos, a taxa apresentada 
Tabela 5.8. Taxas de rejeição da hipótese nula de diferentes testes da razão de verossimilhanças, $\mathcal{H}_{0}: \beta=1$, distribuição de Weibull, dados censurados tipo I.

\begin{tabular}{|c|c|c|c|c|c|c|c|c|}
\hline \multirow[b]{2}{*}{ nível nominal } & \multicolumn{4}{|c|}{$\left(n, p_{0}\right)=(30,25 \%)$} & \multicolumn{4}{|c|}{$\left(n, p_{0}\right)=(20,25 \%)$} \\
\hline & $\ell_{p}$ & $\breve{\ell}_{B N}$ & $\tilde{\ell}_{B N}$ & $\ell_{C R}$ & $\ell_{p}$ & $\breve{\ell}_{B N}$ & $\tilde{\ell}_{B N}$ & $\ell_{C R}$ \\
\hline $15 \%$ & 15.444 & 14.975 & 14.824 & 15.173 & 15.687 & 14.867 & 14.603 & 15.170 \\
\hline $10 \%$ & 10.438 & 9.973 & 9.810 & 10.072 & 10.465 & 9.899 & 9.684 & 10.254 \\
\hline $5 \%$ & 5.283 & 4.998 & 4.941 & 5.103 & 5.499 & 5.069 & 4.892 & 5.146 \\
\hline $3 \%$ & 3.208 & 2.983 & 2.936 & 3.066 & 3.366 & 3.049 & 2.944 & 3.156 \\
\hline $1 \%$ & 1.097 & 1.004 & 0.960 & 1.037 & 1.226 & 1.073 & 1.033 & 1.104 \\
\hline $0.5 \%$ & 0.566 & 0.504 & 0.485 & 0.516 & 0.646 & 0.567 & 0.528 & 0.589 \\
\hline $0.1 \%$ & 0.127 & 0.119 & 0.111 & 0.125 & 0.145 & 0.110 & 0.095 & 0.128 \\
\hline $0.05 \%$ & 0.062 & 0.057 & 0.054 & 0.061 & 0.072 & 0.055 & 0.053 & 0.057 \\
\hline \multirow[t]{2}{*}{$0.01 \%$} & 0.009 & 0.008 & 0.008 & 0.011 & 0.013 & 0.013 & 0.013 & 0.015 \\
\hline & \multicolumn{4}{|c|}{$\left(n, p_{0}\right)=(30,50 \%)$} & \multicolumn{4}{|c|}{$\left(n, p_{0}\right)=(20,50 \%)$} \\
\hline nível nominal & $\ell_{p}$ & $\breve{\ell}_{B N}$ & $\tilde{\ell}_{B N}$ & $\ell_{C R}$ & $\ell_{p}$ & $\breve{\ell}_{B N}$ & $\tilde{\ell}_{B N}$ & $\ell_{C R}$ \\
\hline $15 \%$ & 15.042 & 14.700 & 14.225 & 14.876 & 15.087 & 14.534 & 13.658 & 14.716 \\
\hline $10 \%$ & 10.050 & 9.787 & 9.367 & 9.878 & 10.071 & 9.686 & 8.919 & 9.732 \\
\hline $5 \%$ & 4.997 & 4.815 & 4.534 & 4.912 & 5.066 & 4.830 & 4.422 & 4.883 \\
\hline $3 \%$ & 3.017 & 2.905 & 2.731 & 2.940 & 3.087 & 2.903 & 2.573 & 2.969 \\
\hline $1 \%$ & 1.034 & 0.995 & 0.896 & 0.985 & 1.043 & 0.974 & 0.849 & 1.045 \\
\hline $0.5 \%$ & 0.525 & 0.476 & 0.415 & 0.483 & 0.558 & 0.507 & 0.430 & 0.504 \\
\hline $0.1 \%$ & 0.106 & 0.099 & 0.083 & 0.095 & 0.109 & 0.089 & 0.075 & 0.099 \\
\hline $0.05 \%$ & 0.053 & 0.046 & 0.037 & 0.042 & 0.061 & 0.047 & 0.036 & 0.045 \\
\hline $0.01 \%$ & 0.008 & 0.005 & 0.002 & 0.004 & 0.016 & 0.012 & 0.009 & 0.014 \\
\hline
\end{tabular}

pelo teste baseado em $\ell_{p}$ foi até mais próxima ao nível nominal do que as demais; por exemplo, para $\left(n, p_{0}\right)=(20,50 \%)$ e nível nominal de $10 \%$, tal taxa de rejeição foi igual a $10.071 \%$, enquanto os testes baseados nas versões ajustadas de $\ell_{p}$ apresentaram valores iguais a $9.686 \%\left(\breve{\ell}_{B N}\right), 8.919 \%\left(\tilde{\ell}_{B N}\right)$ e $9.732 \%\left(\ell_{C R}\right)$. Portanto, as melhorias advindas dos testes baseados em $\breve{\ell}_{B N}, \tilde{\ell}_{B N}$ e $\ell_{C R}$ são inexpressivas, não cabendo qualquer destaque entre estes.

Na Tabela 5.9, analogamente ao ocorrido com as taxas de rejeição da hipótese nula, os momentos da estatística de teste baseada na verossimilhança perfilada original não diferiram muito dos seus valores assintóticos. Por exemplo, para $\left(n, p_{0}\right)=(30,25 \%)$, a média e a variância da estatítica de teste baseada em $\ell_{p}$ foram iguais a 1.028 e 2.108, enquanto as estimativas de tais momentos relativos ao uso de $\breve{\ell}_{B N}$ foram 1.003 e 2.007 , respectivamente. Para $\left(n, p_{0}\right)=(30,50 \%)$, a média e a variância amostrais da estatística obtida de $\ell_{p}$ foram as mais próximas de 1 (um) e 2 (dois): 1.003 e 2.006, respectivamente.

As Figuras 5.7, 5.8, 5.9 e 5.10 apresentam os gráficos das discrepâncias relativas de quantis versus os correspondentes quantis assintóticos para cada uma das combinações $\left(n, p_{0}\right)$ consideradas: $(20,25 \%),(30,25 \%),(20,50 \%)$ e $(30,50 \%)$, respectivamente. Lembre que quanto mais próxima da ordenada zero a curva estiver, melhor a aproximação assintótica 
Tabela 5.9. Médias e variâncias amostrais de diferentes estatísticas de teste e os valores assintóticos destas, $\mathcal{H}_{0}: \beta=1$, distribuição de Weibull, dados censurados tipo I.

\begin{tabular}{cccccccccc}
\hline & \multicolumn{4}{c}{$\left(n, p_{0}\right)=(30,25 \%)$} & \multicolumn{4}{c}{$\left(n, p_{0}\right)=(20,25 \%)$} \\
\hline momento & $\chi_{1}^{2}$ & $\ell_{p}$ & $\breve{\ell}_{B N}$ & $\tilde{\ell}_{B N}$ & $\ell_{C R}$ & $\ell_{p}$ & $\breve{\ell}_{B N}$ & $\tilde{\ell}_{B N}$ & $\ell_{C R}$ \\
\hline média & 1 & 1.028 & 1.003 & 0.995 & 1.012 & 1.042 & 1.003 & 0.989 & 1.017 \\
variância & 2 & 2.108 & 2.007 & 1.973 & 2.042 & 2.206 & 2.043 & 1.989 & 2.097 \\
\hline \multicolumn{1}{c}{$\left(n, p_{0}\right)=(30,50 \%)$} & & \multicolumn{3}{c}{$\left(n, p_{0}\right)=(20,50 \%)$} \\
\hline momento & $\chi_{1}^{2}$ & $\ell_{p}$ & $\breve{\ell}_{B N}$ & $\tilde{\ell}_{B N}$ & $\ell_{C R}$ & $\ell_{p}$ & $\breve{\ell}_{B N}$ & $\tilde{\ell}_{B N}$ & $\ell_{C R}$ \\
\hline média & 1 & 1.003 & 0.989 & 0.964 & 0.995 & 1.005 & 0.982 & 0.944 & 0.994 \\
variância & 2 & 2.006 & 1.948 & 1.854 & 1.973 & 2.042 & 1.955 & 1.806 & 1.997 \\
\hline
\end{tabular}

Figura 5.7. Gráfico das discrepâncias relativas de quantis, distribuição de Weibull, $\mathcal{H}_{0}: \beta=1$, dados censurados tipo I, $n=20,25 \%$ de censura.

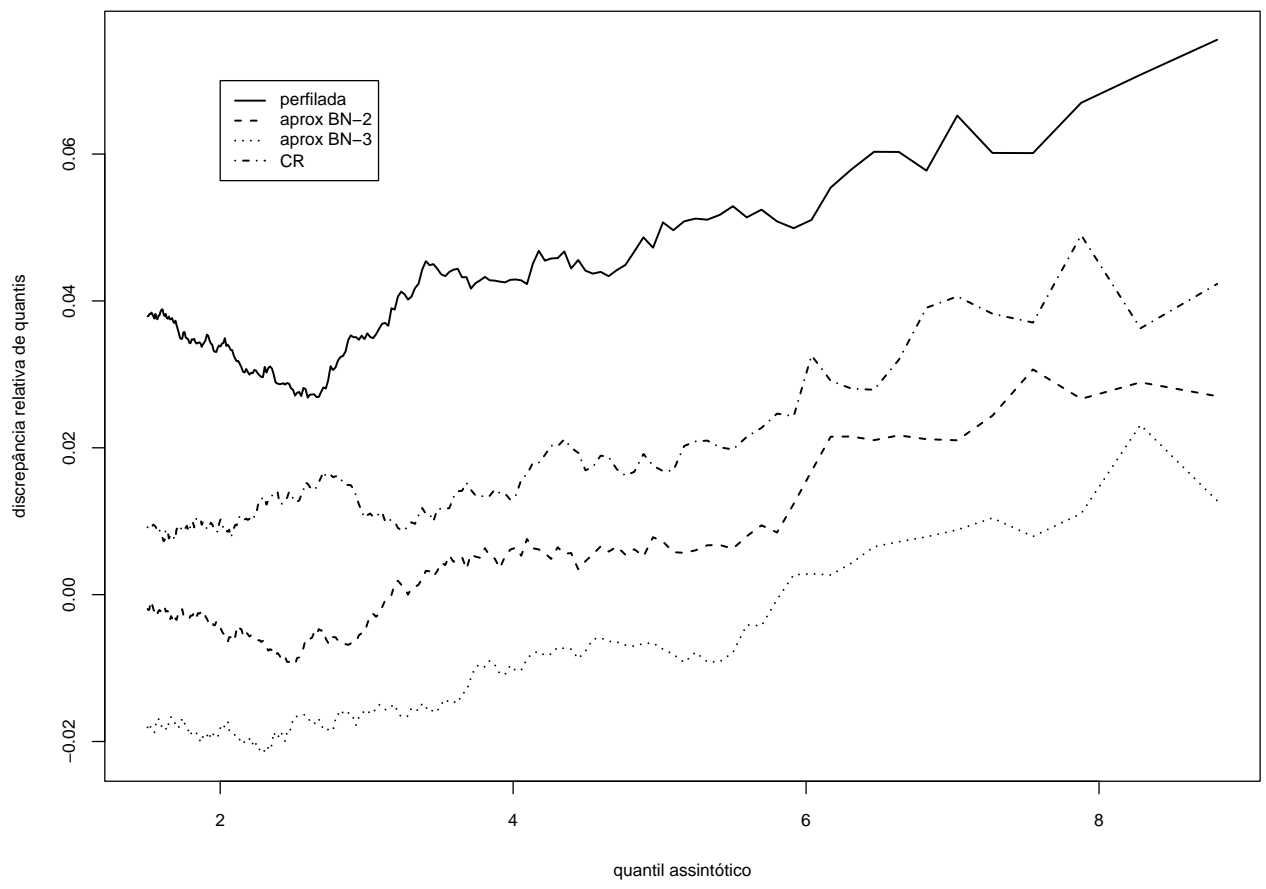

utilizada no teste.

Para $p_{0}=25 \%$, Figuras 5.7 e 5.8, a curva de discrepâncias de quantis da estatística de teste baseada em $\breve{\ell}_{B N}$ (aprox BN-2) é a mais próxima da ordenada zero, posicionada entre as curvas baseadas em $\tilde{\ell}_{B N}$ (aprox BN-3) e $\ell_{C R}$, que estão abaixo e acima dessa ordenada, respectivamente. Para $\left(n, p_{0}\right)=(30,25 \%)$, as três curvas estão praticamente dispostas na faixa de $-1 \%$ a $+1 \%$ do eixo vertical, ou seja, os quantis amostrais das distribuições dessas estatísticas diferem dos quantis assintóticos em no máximo 1\% dos respectivos valores destes.

Para $p_{0}=50 \%$, Figuras 5.9 e 5.10, entre as curvas de discrepâncias de quantis, aquela baseada em $\ell_{p}$ é a mais próxima da ordenada zero. Com exceção desta, todas as curvas estão 
Figura 5.8. Gráfico das discrepâncias relativas de quantis, distribuição de Weibull, $\mathcal{H}_{0}: \beta=1$, dados censurados tipo I, $n=30,25 \%$ de censura.

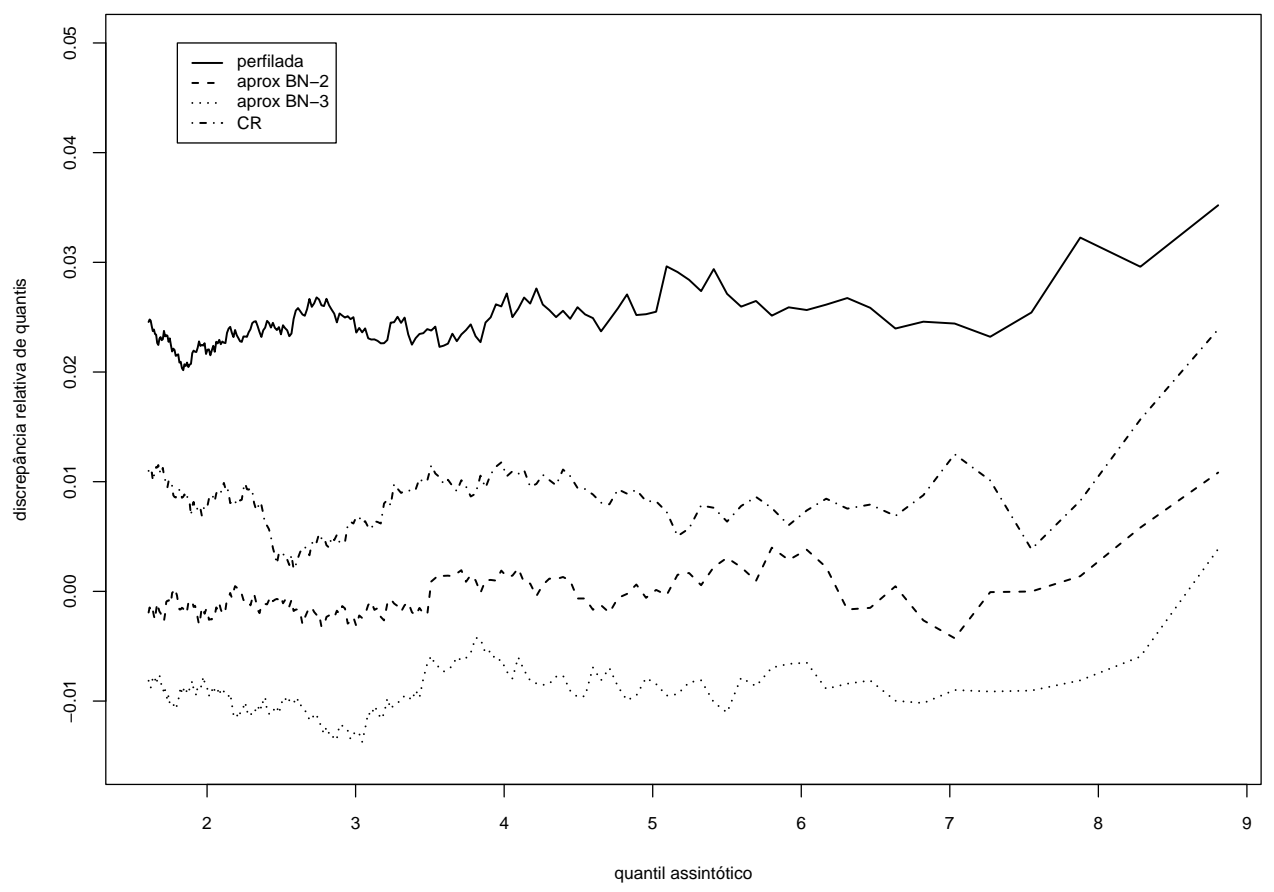

Figura 5.9. Gráfico das discrepâncias relativas de quantis, distribuição de Weibull, $\mathcal{H}_{0}: \beta=1$, dados censurados tipo I, $n=20,50 \%$ de censura.

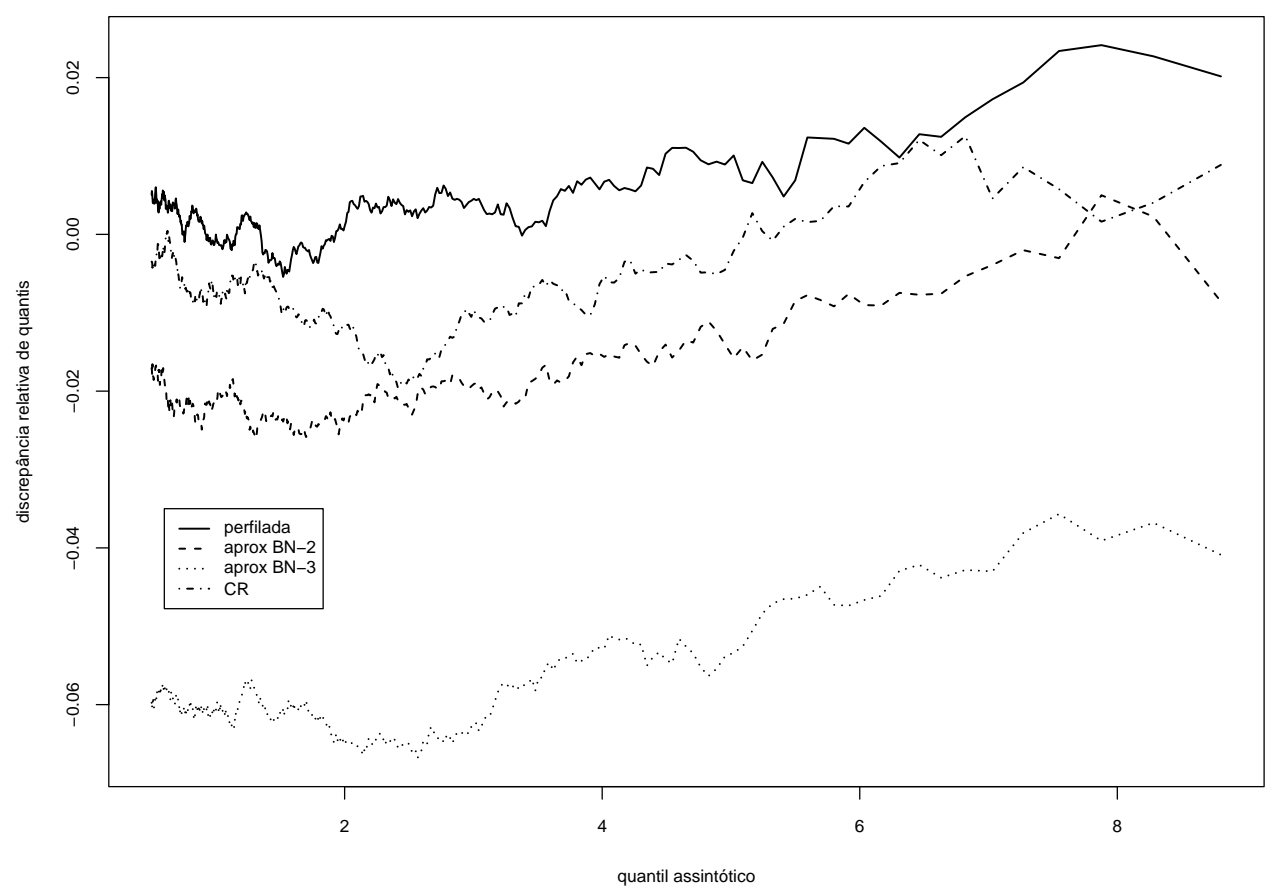

posicionadas abaixo da ordenada zero na maior parte de suas extensões, indicando que os 
Figura 5.10. Gráfico das discrepâncias relativas de quantis, distribuição de Weibull, $\mathcal{H}_{0}: \beta=1$, dados censurados tipo I, $n=30,50 \%$ de censura.

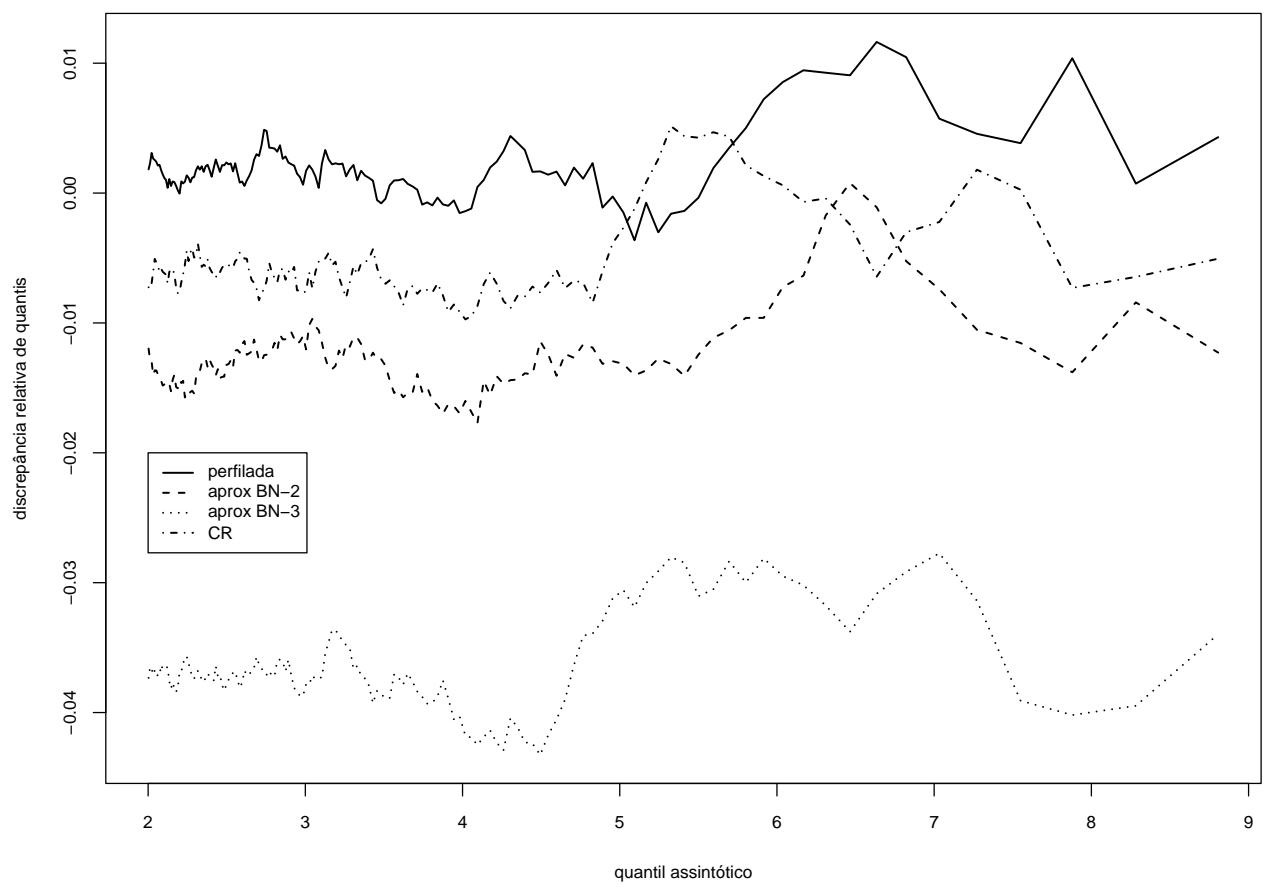

quantis amostrais das estatísticas de teste subestimaram os respectivos quantis assintóticos, conforme resultados da Tabela 5.8.

\subsubsection{EXEMPLOS NUMÉRICOS}

Considere agora dois conjuntos de dados bem conhecidos obtidos de Lawless (1982). Em ambos os casos, assume-se que os dados são independentes e seguem uma distribuição de Weibull. É realizada inferência sobre o parâmetro de forma $\beta$ usando a verossimilhança perfilada e suas versões modificadas derivadas nesta seção.

Os dados a seguir são tempos entre falhas sucessivas de equipamentos de ar-condicionado de aeronaves Boeing 720 (Proschan, 1963; Lawless, 1982, p. 134): 74, 57, 48, 29, 502, 12, $70,21,29,386,59,27,153,26$ and 326. Aqui, $n=15$ e não há censura. A hipótese nula de interesse é $\mathcal{H}_{0}: \beta=1$. As estatísticas da razão de verossimilhanças baseadas em $\ell, \bar{\ell}_{B N}, \breve{\ell}_{B N}$, $\tilde{\ell}_{B N}$ e $\ell_{C R}$ são, respectivamente, 0.409, 0.658, 0.655, 0.682 e 1.033. No nível de significância de $10 \%$, nenhum dos testes rejeita a hipótese nula. Isto é, todos os cinco testes apontam para a mesma conclusão: o modelo exponencial não pode ser rejeitado como o mecanismo gerador de dados. As estimativas de máxima verossimilhança obtidas das maximizações da verossimilhança perfilada e das verossimilhanças perfiladas modificadas são $\widehat{\beta}=0.888$, $\widehat{\bar{\beta}}_{B N}=0.858, \widehat{\breve{\beta}}_{B N}=0.857, \widehat{\tilde{\beta}}_{B N}=0.856$ e $\widehat{\beta}_{C R}=0.823$.

O segundo exemplo numérico, baseado em Mann e Fertig (1973) — ver também Law- 
less (1982, p. 86) - , consiste de tempos de falhas de componentes de aeronaves sujeitos a um teste de vida útil. Os dados são censurados do tipo II: 13 componentes foram colocados sob teste até que o décimo falhasse. Os tempos de falhas (em horas) observados são 0.22, $0.50,0.88,1.00,1.32,1.33,1.54,1.76,2.50,3.00$. Aqui, as estatísticas da razão de verossimilhanças para o teste de $\mathcal{H}_{0}: \beta=1$ obtidas de $\ell, \breve{\ell}_{B N}, \tilde{\ell}_{B N}$ e $\ell_{C R}$ são $1.435,1.160,0.960$ e 0.354 , respectivamente. Entre essas, independentemente da estatística escolhida para o teste, a hipótese nula não é rejeitada nos níveis de significância usuais, isto é, o modelo exponencial não pode ser rejeitado. Considere, contudo, a hipótese nula $\mathcal{H}_{0}: \beta=2$. Os testes baseados em $\ell, \breve{\ell}_{B N}$ e $\tilde{\ell}_{B N}$ não rejeitam $\mathcal{H}_{0}$ no nível de significância de $10 \%$, diferentemente do teste baseado na verossimilhança perfilada modificada proposta por Cox e Reid (1987), $\ell_{C R}$. Este teste é o único que distingue entre $\beta=1$ and $\beta=2$. As estimativas para $\beta$ obtidas ao maximizar a verossimilhança perfilada e suas versões modificadas são $\widehat{\beta}=1.417$, $\widehat{\breve{\beta}}_{B N}=1.374, \widehat{\tilde{\beta}}_{B N}=1.343$ e $\widehat{\beta}_{C R}=1.204$.

\subsubsection{CONCLUSÕES}

O estimador de máxima verossimilhança do parâmetro de forma da distribuição de Weibull é bastante viesado. De acordo com os resultados numéricos apresentados na Seção 5.2.1.3, o estimador com melhor desempenho foi $\widehat{\beta}_{C R}$, tanto em termos de menor valor absoluto do viés quanto em termos de menor erro quadrático médio. Este estimador é obtido da maximização de uma verossimilhança perfilada modificada que requer, para seu cálculo, a ortogonalidade entre os parâmetros de interesse e de incômodo, ou seja, a independência assintótica dos estimadores de máxima verossimilhança destes parâmetros, diferentemente das demais verossimilhanças consideradas $\left(\ell_{p}, \bar{\ell}_{B N}, \breve{\ell}_{B N}, \tilde{\ell}_{B N}\right)$, que não exigem tal parametrização.

Embora seja destacado o desempenho do estimador $\widehat{\beta}_{C R}$, vale ressaltar que as simulações de Monte Carlo mostraram que os estimadores obtidos das demais verossimilhanças perfiladas ajustadas apresentaram menores vieses e menores erros quadráticos médios comparativamente ao estimador de máxima verossimilhança usual $\left(\widehat{\beta}_{p}\right)$. Entretanto esta diferença de desempenho diminuiu à medida que aumentou a proporção de dados censurados na amostra, diferentemente do estimador $\widehat{\beta}_{C R}$, que se mostrou insensível a tal mudança.

Considerando dados completos, os testes baseados nas verossimilhanças perfiladas ajustadas apresentaram desempenhos semelhantes, com as taxas de rejeição da hipótese nula bem próximas dos valores nominais, enquanto o teste da razão de verossimilhanças usual (perfilada) mostrou-se mais liberal. Para dados com presença de censura do tipo II, os testes

baseados em $\bar{\ell}_{B N}, \breve{\ell}_{B N}$ e $\tilde{\ell}_{B N}$ deterioraram-se, mas ainda mostraram-se mais confiáveis do que o teste baseado em $\ell_{p}$. A presença de dados censurados não deteriorou o desempenho da estatística de teste obtida de $\ell_{C R}$. A média e a variância empíricas desta estatística permaneceram próximas aos valores assintóticos e os testes baseados nesta verossimilhança continuaram apresentando taxas de rejeição próximas dos níveis nominais. Entretanto, para 
dados com presença de censura do tipo I, o desempenho da estatítica de teste baseada em $\ell_{p}$ foi bastante satisfatório, tornando inexpressivas as melhorias das taxas de rejeição da hipótese nula e das estimativas dos momentos advindas de modificações sobre $\ell_{p}$.

Portanto, em geral, considerando observações independentes e identicamente distribuídas, entre as verossimilhanças perfiladas utilizadas neste capítulo para realizar inferências sobre o parâmetro de forma da distribuição de Weibull, aquela que produziu o melhor estimador de máxima verossimilhança e cuja distribuição nula da estatística de teste é melhor aproximada pela distribuição assintótica $\chi_{1}^{2}$ foi a verossimilhança perfilada ajustada proposta por Cox e Reid $(1987,1989), \ell_{C R}$.

\subsubsection{OBSERVAÇÕES INDEPENDENTES}

\subsubsection{INTRODUÇÃO}

Esta seção trata da extensão do modelo considerado anteriormente através da suposição de um modelo de regressão. Diferentemente do trabalho de Yang e Xie (2003), aqui é estudado e simulado o caso em que se modela o parâmetro $\alpha$ através de algumas covariáveis. $\mathrm{O}$ objetivo é observar o desempenho das verossimilhanças perfiladas modificadas na presença de mais de um parâmetro de incômodo sem a suposição de variáveis aleatórias identicamente distribuídas, mas mantendo a independência entre estas.

Assim, seja $t \sim W B(\alpha(\mathbf{x}), \beta)$, onde $\mathbf{x}=\left(x_{1}, x_{2}, \ldots, x_{p}\right)$ é uma coleção de covariáveis. Logo, $\log t=y \sim V E(\eta(\mathbf{x}), \sigma)$, ou seja, $y$ tem distribuição valor extremo com parâmetros de locação $\eta(\mathbf{x})$ e de escala $\sigma$, mais precisamente,

$$
p(y ; \eta(\mathbf{x}), \sigma)=\frac{1}{\sigma} \exp \left\{\frac{y-\eta(\mathbf{x})}{\sigma}-\exp \left(\frac{y-\eta(\mathbf{x})}{\sigma}\right)\right\},
$$

em que $-\infty<y<\infty, \eta(\mathbf{x})=\log \alpha(\mathbf{x})$ e $\sigma=\beta^{-1}$. Usualmente, $\eta(\mathbf{x})=\mathbf{x} \phi$, onde $\phi=$ $\left(\phi_{1}, \phi_{2}, \ldots, \phi_{p}\right)^{\top}$.

Os resultados são obtidos de forma análoga à seção anterior, entretanto, entre as aproximações para $\ell_{B N}$, apenas aquela baseada numa estatística ancilar $\left(\tilde{\ell}_{B N}\right)$ é considerada, pois, juntamente com $\ell_{C R}$, apresentaram os melhores resultados no caso de observações independentes e identicamente distribuídas. São considerados dados completos e dados censurados. Resultados numéricos relativos a estimação e a testes de hipóteses sobre $\sigma^{-1}$, parâmetro de forma da distribuição de Weibull, são apresentados no final desta seção.

\subsubsection{DADOS COMPLETOS}

Considerando inicialmente dados não-censurados, sejam $y_{1}, \ldots, y_{n}$ observações independentes de variáveis aleatórias com distribuições valores extremos: $y_{j} \sim V E\left(\eta\left(\mathbf{x}_{j}\right), \sigma\right)$, onde 
$j=1, \ldots, n$. O logaritmo da função de verossimilhança do vetor paramétrico $(\sigma, \phi)$ é dado por

$$
\ell(\sigma, \phi)=-n \log \sigma+\sum_{j=1}^{n} \frac{y_{j}-\mathbf{x}_{j} \phi}{\sigma}-\sum_{j=1}^{n} \exp \left(\frac{y_{j}-\mathbf{x}_{j} \phi}{\sigma}\right)
$$

em que $\mathbf{x}_{j}=\left(x_{j 1}, x_{j 2}, \ldots, x_{j p}\right)$ para $j=1, \ldots, n$ e $x_{j 1}=1, \forall j$. Fazendo $z_{j}=\left(y_{j}-\mathbf{x}_{j} \phi\right) / \sigma$, o gradiente dessa função é expresso por

$$
\frac{\partial \ell(\sigma, \phi)}{\partial \sigma}=-\frac{n}{\sigma}-\frac{1}{\sigma} \sum_{j=1}^{n} z_{j}+\frac{1}{\sigma} \sum_{j=1}^{n} z_{j} \exp \left(z_{j}\right)
$$

$\mathrm{e}$

$$
\frac{\partial \ell(\sigma, \phi)}{\partial \phi_{s}}=-\frac{1}{\sigma} \sum_{j=1}^{n} x_{j s}+\frac{1}{\sigma} \sum_{j=1}^{n} x_{j s} \exp \left(z_{j}\right)
$$

onde $s=1, \ldots, p$.

Observe que, fixando o parâmetro de interesse $\sigma\left(\beta^{-1}\right)$, o estimador de máxima verossimilhança restrito do vetor paramétrico $\phi$ não tem forma fechada. Conseqüentemente, não é possível obter explicitamente $\ell_{p}(\sigma)$. Derivando pela segunda vez $\ell(\sigma, \phi)$, vem

$$
\begin{gathered}
\frac{\partial^{2} \ell(\sigma, \phi)}{\partial \phi_{t} \partial \phi_{s}}=-\frac{1}{\sigma^{2}} \sum_{j=1}^{n} x_{j s} x_{j t} \exp \left(z_{j}\right) \\
\frac{\partial^{2} \ell(\sigma, \phi)}{\partial \sigma^{2}}=\frac{n}{\sigma^{2}}+\frac{2}{\sigma^{2}} \sum_{j=1}^{n} z_{j}-\frac{2}{\sigma^{2}} \sum_{j=1}^{n} z_{j} \exp \left(z_{j}\right)-\frac{1}{\sigma^{2}} \sum_{j=1}^{n} z_{j}^{2} \exp \left(z_{j}\right)
\end{gathered}
$$

$\mathrm{e}$

$$
\frac{\partial^{2} \ell(\sigma, \phi)}{\partial \sigma \partial \phi_{s}}=\frac{\partial^{2} \ell(\sigma, \phi)}{\partial \phi_{s} \partial \sigma}=\frac{1}{\sigma^{2}} \sum_{j=1}^{n} x_{j s}-\frac{1}{\sigma^{2}} \sum_{j=1}^{n} x_{j s} \exp \left(z_{j}\right)-\frac{1}{\sigma^{2}} \sum_{j=1}^{n} x_{j s} z_{j} \exp \left(z_{j}\right),
$$

em que $s, t=1, \ldots, p$. Note que $z_{j}(j=1, \ldots, n)$ tem distribuição valor extremo padrão $\left(z_{j} \sim V E(0,1)\right): p\left(z_{j}\right)=\exp \left(z_{j}-\exp \left(z_{j}\right)\right),-\infty<z_{j}<\infty$.

A fim de obter a matriz de informação de Fisher, é necessário calcular as esperanças matemáticas dessas derivadas de ordem dois, para isto, usam-se os seguintes resultados (Lawless, 1982, Capítulo 6):

(i) $E\left\{z_{j}\right\}=-\gamma$,

(ii) $E\left\{\exp \left(z_{j}\right)\right\}=1$,

(iii) $E\left\{z_{j} \exp \left(z_{j}\right)\right\}=1-\gamma$,

(iv) $E\left\{z_{j}^{2} \exp \left(z_{j}\right)\right\}=\frac{\pi^{2}}{6}+\gamma^{2}-2 \gamma$.

Estas esperanças estão relacionadas com a função gama e suas derivadas, ver Seção 1.3 .2 e Apêndice B em Lawless (1982). $\gamma$ é a constante de Euler, aproximadamente igual a 0.5772. 
Conseqüentemente, a matriz de informação de Fisher é dada por

$$
I(\sigma, \phi)=\frac{1}{\sigma^{2}}\left(\begin{array}{cc}
X^{\top} X & \mathbf{m} \\
\mathbf{m}^{\top} & n\left[\pi^{2} / 6+(1-\gamma)^{2}\right]
\end{array}\right),
$$

onde $X$ é uma matriz $n \times p$ com linhas iguais a $\mathbf{x}_{j}(j=1, \ldots, n)$ e $\mathbf{m}$ é um vetor $p \times 1$ com $t$-ésimo componente igual a $(1-\gamma) \sum_{j=1}^{n} x_{j t}$. Os elementos desta matriz são necessários para a obtenção do ajuste de Cox e Reid $(1987,1989)$.

As aproximações para $\ell_{B N}(\sigma)$ são baseadas em aproximações para a derivada do espaço amostral $\ell_{\phi ; \widehat{\phi}}(\sigma, \phi ; \widehat{\sigma}, \widehat{\phi}, a)$ conforme a Seção 2.11, em que a é uma estatística ancilar que garante a suficiência e a minimalidade da terna $(\widehat{\sigma}, \widehat{\phi}, a)$. Aqui, $\widehat{\sigma}$ e $\widehat{\phi}$ são estimadores de máxima verossimilhança de $\sigma$ e $\phi$ respectivamente.

A aproximação para $\ell_{B N}(\sigma)$ considerada nesta seção é aquela baseada numa estatística aproximadamente ancilar (ver Seção 2.11.4), expressa por

$$
\tilde{\ell}_{B N}(\sigma)=\ell_{p}(\sigma)+\frac{1}{2} \log \left|j_{\phi \phi}\left(\sigma, \widehat{\phi}_{\sigma}\right)\right|-\log \left|\ell_{\phi ; y}\left(\sigma, \widehat{\phi}_{\sigma}\right) \widehat{V}_{\phi}\right| \text {. }
$$

Como $\widehat{\phi}_{\sigma}$ não tem forma fechada, $\tilde{\ell}_{B N}(\sigma)$ é igual à soma

$$
\ell(\sigma, \phi)+\frac{1}{2} \log \left|j_{\phi \phi}(\sigma, \phi)\right|-\log \left|\ell_{\phi ; y}(\sigma, \phi) \widehat{V}_{\phi}\right|
$$

sujeita às restrições da forma

$$
\frac{\partial \ell(\sigma, \phi)}{\partial \phi_{s}}=-\frac{1}{\sigma} \sum_{j=1}^{n} x_{j s}+\frac{1}{\sigma} \sum_{j=1}^{n} x_{j s} \exp \left(z_{j}\right)=0,
$$

onde $s=1, \ldots, p$. Aqui, $j_{\phi \phi}(\sigma, \phi)$ é a matriz de informação observada relativa ao vetor $\phi$, ou seja, é uma matriz $p \times p$ com elemento $(s, t)$ dado por

$$
j_{\phi \phi}^{(s, t)}(\sigma, \phi)=\frac{1}{\sigma^{2}} \sum_{j=1}^{n} x_{j s} x_{j t} \exp \left(z_{j}\right) .
$$

Numa forma matricial,

$$
j_{\phi \phi}(\sigma, \phi)=\frac{1}{\sigma^{2}} X^{\top} Z X
$$

onde $Z=\operatorname{diag}\left(\exp \left(z_{1}\right), \ldots, \exp \left(z_{n}\right)\right)$, isto é, $Z$ é uma matriz quadrada de ordem $n$ com elemento $(i, j)$ definido por

$$
Z^{(i, j)}=\exp \left(z_{j}\right) \text { se } i=j \quad \text { e } \quad Z^{(i, j)}=0 \text { se } i \neq j
$$

Derivando os componentes $\partial \ell(\sigma, \phi) / \partial \phi_{s}$ do vetor escore $(s=1, \ldots, p)$ com relação ao vetor de observações $y=\left(y_{1}, \ldots, y_{n}\right)$, obtém-se $\ell_{\phi ; y}(\sigma, \phi)$, uma matriz $p \times n$ com elemento $(s, j)$ expresso por

$$
\ell_{\phi ; y}^{(s, j)}(\sigma, \phi)=\frac{1}{\sigma^{2}} x_{j s} \exp \left(z_{j}\right)
$$


Numa forma matricial,

$$
\ell_{\phi ; y}(\sigma, \phi)=\frac{1}{\sigma^{2}} X^{\top} Z
$$

Por fim, seguindo a definição para $\widehat{V}_{\phi}$ dada na Seção 2.11.4, vem

$$
\widehat{V}_{\phi}=X
$$

Portanto, para pares $(\sigma, \phi)$ satisfazendo as restrições

$$
\frac{\partial \ell(\sigma, \phi)}{\partial \phi_{s}}=0
$$

onde $s=1, \ldots, p$, a função $\tilde{\ell}_{B N}(\sigma)$ é dada por

$$
\ell(\sigma, \phi)+p \log \sigma-\frac{1}{2} \log \left|X^{\top} Z X\right| .
$$

Considerando observações independentes e identicamente distribuídas (Seção 5.2.1) de uma variável com distribuição de Weibull $W B(\alpha, \beta)$, a fim de obter $\ell_{C R}(\beta)$, o ajuste de Cox e Reid $(1987,1989)$ para a verossimilhança perfilada, Yang e Xie $(2003)$ propuseram a parametrização ortogonal $\left(\beta, \lambda^{*}\right)$, onde $\lambda^{*}=\log (\lambda) / \beta$. Aqui, $\lambda$ é um parâmetro ortogonal a $\beta$, obtido segundo Cox e Reid (1987), sendo dado por

$$
\lambda=\alpha \exp \left(\frac{1-\gamma}{\beta}\right)
$$

enquanto $\lambda^{*}$ é uma reparametrização deste, obtida segundo o enfoque de Cox e Reid (1989):

$$
\lambda^{*}=\frac{\log (\alpha)}{\beta}+\frac{1-\gamma}{\beta^{2}} .
$$

Partindo da parametrização ortogonal $\left(\beta, \lambda^{*}\right)$, os resultados apresentados pelo estimador de máxima verossimilhança e pelo teste da razão de verossimilhanças derivados de $\ell_{C R}(\beta)$ foram ótimos, conforme a Seção 5.2.1.3 e o trabalho de Yang e Xie (2003).

Assim, reproduzindo tal parametrização ortogonal ótima a partir do par $(\sigma, \phi)$ que indexa a distribuição valor extremo, em que $\sigma=\beta^{-1}$ e $\log \alpha\left(\mathbf{x}_{j}\right)=\eta\left(\mathbf{x}_{j}\right)=\mathbf{x}_{j} \phi(j=1, \ldots, n)$, obtém-se o seguinte vetor paramétrico de dimensão $p$,

$$
\lambda^{o}=\sigma\left[\phi+(1-\gamma) \sigma\left(X^{\top} X\right)^{-1} X^{\top} \mathbb{1}_{n}\right]
$$

onde $\mathbb{1}_{n}$ é um vetor $n \times 1$ de uns. Ou seja, a fim de obter $\ell_{C R}(\sigma)$, indexa-se a distribuição valor extremo por $\left(\sigma, \lambda^{o}\right)$, onde $\sigma$ (parâmetro de interesse) é ortogonal ao vetor $\lambda^{o}$ (parâmetro de incômodo). Esta extensão não foi derivada em Yang e Xie (2003) e é descrita com maiores detalhes a seguir.

Para obter $\lambda=\left(\lambda_{1}, \ldots, \lambda_{p}\right)^{\top}$ ortogonal ao parâmetro $\sigma$, é necessário resolver a equação diferencial parcial

$$
i_{\phi \sigma}+i_{\phi \phi} \frac{\partial \phi(\sigma, \lambda)}{\partial \sigma}=0
$$


onde $i_{\phi \sigma}$ e $i_{\phi \phi}$ são os blocos superiores direito (diagonal secundária) e esquerdo (diagonal principal) da matriz de informação de Fisher $I(\sigma, \phi)$, respectivamente. Assim,

$$
\frac{1}{\sigma^{2}} \mathbf{m}+\frac{1}{\sigma^{2}} X^{\top} X \frac{\partial \phi(\sigma, \lambda)}{\partial \sigma}=0
$$

ou ainda,

$$
(1-\gamma) X^{\top} \mathbb{1}_{n}+X^{\top} X \frac{\partial \phi(\sigma, \lambda)}{\partial \sigma}=0
$$

Logo,

$$
\frac{\partial \phi(\sigma, \lambda)}{\partial \sigma}=-(1-\gamma)\left(X^{\top} X\right)^{-1} X^{\top} \mathbb{1}_{n}
$$

e uma solução dessa equação é

$$
\phi=-(1-\gamma) \sigma\left(X^{\top} X\right)^{-1} X^{\top} 1_{n}+\log \lambda .
$$

Seguindo a sugestão de Yang e Xie (2003), baseada na proposta de Cox e Reid (1989), é usada a parametrização ortogonal $\left(\sigma, \lambda^{o}\right)$ para obtenção de $\ell_{C R}(\sigma)$, onde $\lambda^{o}=\sigma \log \lambda$, justificando, dessa forma, a expressão apresentada inicialmente.

Portanto, o logaritmo da função de verossimilhança de $\left(\sigma, \lambda^{o}\right)$ é dado por

$$
\ell^{\curvearrowright}\left(\sigma, \lambda^{o}\right)=-n \log \sigma+\frac{1}{\sigma} \sum_{j=1}^{n} y_{j}-\frac{1}{\sigma^{2}} \sum_{j=1}^{n} \mathbf{x}_{j} \lambda^{o}-\sum_{j=1}^{n} \exp \left(q_{j}\right)
$$

onde

$$
q_{j}=\frac{y_{j}}{\sigma}-\frac{1}{\sigma^{2}} \mathbf{x}_{j} \lambda^{o}+(1-\gamma) \mathbf{x}_{j}\left(X^{\top} X\right)^{-1} X^{\top} 1_{n}
$$

para $j=1, \ldots, n$.

Para $s=1, \ldots, p$, os componentes do vetor escore relativos a $\lambda^{o}$ são expressos por

$$
\frac{\partial \ell^{\diamond}\left(\sigma, \lambda^{o}\right)}{\partial \lambda_{s}^{o}}=-\frac{1}{\sigma^{2}}\left[\sum_{j=1}^{n} x_{j s}-\sum_{j=1}^{n} x_{j s} \exp \left(q_{j}\right)\right] .
$$

Note que não é possível obter $\ell_{p}^{\diamond}(\sigma)$ de forma explícita, pois $\widehat{\lambda_{\sigma}^{o}}$ não tem forma fechada.

O ajuste de Cox e Reid (1987) para a verossimilhança perfilada requer a informação observada relativa ao parâmetro de incômodo $\lambda^{o}$ :

$$
-\frac{\partial^{2} \ell^{\diamond}\left(\sigma, \lambda^{o}\right)}{\partial \lambda_{t}^{o} \lambda_{s}^{o}}=\frac{1}{\sigma^{4}} \sum_{j=1}^{n} x_{j s} x_{j t} \exp \left(q_{j}\right)
$$

onde $s, t=1, \ldots, p$. Numa forma matricial,

$$
j_{\lambda^{o} \lambda^{o}}^{\diamond}\left(\sigma, \lambda^{o}\right)=\frac{1}{\sigma^{4}} X^{\top} Q X,
$$


onde $Q=\operatorname{diag}\left(\exp \left(q_{1}\right), \ldots, \exp \left(q_{n}\right)\right)$, analogamente à matriz $Z$ definida anteriormente.

Portanto, para pares $\left(\sigma, \lambda^{o}\right)$ satisfazendo as restrições

$$
\frac{\partial \ell^{\diamond}\left(\sigma, \lambda^{o}\right)}{\partial \lambda_{s}^{o}}=0
$$

onde $s=1, \ldots, p$, a função $\ell_{C R}(\sigma)$ é dada por

$$
\ell^{\diamond}\left(\sigma, \lambda^{o}\right)+2 p \log \sigma-\frac{1}{2} \log \left|X^{\top} Q X\right| .
$$

\subsubsection{DADOS CENSURADOS}

Dadas $n$ observações independentes $y_{j}$, em que $y_{j} \sim V E\left(\eta\left(\mathbf{x}_{j}\right), \sigma\right)$ para $j=1, \ldots, n$, sejam $C$ e $\bar{C}$ os conjuntos de índices para os quais $y_{j}$ é o logaritmo de um tempo de censura ou de um tempo de falha, respectivamente. No caso de dados censurados, a função de verossimilhança é dada por

$$
L(\sigma, \phi)=\prod_{j \in \bar{C}} \frac{1}{\sigma} \exp \left[\frac{y_{j}-\mathbf{x}_{j} \phi}{\sigma}-\exp \left(\frac{y_{j}-\mathbf{x}_{j} \phi}{\sigma}\right)\right] \prod_{j \in C} \exp \left[-\exp \left(\frac{y_{j}-\mathbf{x}_{j} \phi}{\sigma}\right)\right]
$$

e, assim, seu logaritmo é expresso por

$$
\ell(\sigma, \phi)=-r \log \sigma+\sum_{j \in \bar{C}} \frac{y_{j}-\mathbf{x}_{j} \phi}{\sigma}-\sum_{j=1}^{n} \exp \left(\frac{y_{j}-\mathbf{x}_{j} \phi}{\sigma}\right),
$$

onde $r$ é o número observado de tempos de falhas. No caso de dados com censura tipo II, $r$ é fixado, mas, para dados com censura tipo I, é aleatório.

Da mesma forma que para dados completos, seja $z_{j}=\left(y_{j}-\mathbf{x}_{j} \phi\right) / \sigma$. Os componentes do vetor escore são dados por

$$
\frac{\partial \ell(\sigma, \phi)}{\partial \sigma}=-\frac{r}{\sigma}-\frac{1}{\sigma} \sum_{j \in \bar{C}} z_{j}+\frac{1}{\sigma} \sum_{j=1}^{n} z_{j} \exp \left(z_{j}\right)
$$

$\mathrm{e}$

$$
\frac{\partial \ell(\sigma, \phi)}{\partial \phi_{s}}=-\frac{1}{\sigma} \sum_{j \in \bar{C}} x_{j s}+\frac{1}{\sigma} \sum_{j=1}^{n} x_{j s} \exp \left(z_{j}\right)
$$

onde $s=1, \ldots, p$. Observe que, fixando o parâmetro de interesse $\sigma\left(\beta^{-1}\right)$, o estimador de máxima verossimilhança restrito do vetor paramétrico $\phi$ não tem forma fechada. Conseqüentemente, não é possível obter explicitamente $\ell_{p}(\sigma)$. Derivando pela segunda vez $\ell(\sigma, \phi)$, vem

$$
\frac{\partial^{2} \ell(\sigma, \phi)}{\partial \phi_{t} \partial \phi_{s}}=-\frac{1}{\sigma^{2}} \sum_{j=1}^{n} x_{j s} x_{j t} \exp \left(z_{j}\right)
$$




$$
\frac{\partial^{2} \ell(\sigma, \phi)}{\partial \sigma^{2}}=\frac{r}{\sigma^{2}}+\frac{2}{\sigma^{2}} \sum_{j \in \bar{C}} z_{j}-\frac{2}{\sigma^{2}} \sum_{j=1}^{n} z_{j} \exp \left(z_{j}\right)-\frac{1}{\sigma^{2}} \sum_{j=1}^{n} z_{j}^{2} \exp \left(z_{j}\right)
$$

$\mathrm{e}$

$$
\frac{\partial^{2} \ell(\sigma, \phi)}{\partial \sigma \partial \phi_{s}}=\frac{\partial^{2} \ell(\sigma, \phi)}{\partial \phi_{s} \partial \sigma}=\frac{1}{\sigma^{2}} \sum_{j \in \bar{C}} x_{j s}-\frac{1}{\sigma^{2}} \sum_{j=1}^{n} x_{j s} \exp \left(z_{j}\right)-\frac{1}{\sigma^{2}} \sum_{j=1}^{n} x_{j s} z_{j} \exp \left(z_{j}\right),
$$

em que $s, t=1, \ldots, p$.

Como $\widehat{\phi}_{\sigma}$ não tem forma fechada, $\tilde{\ell}_{B N}(\sigma)$ é igual a

$$
\ell(\sigma, \phi)+\frac{1}{2} \log \left|j_{\phi \phi}(\sigma, \phi)\right|-\log \left|\ell_{\phi ; y}(\sigma, \phi) \widehat{V}_{\phi}\right|
$$

sujeita às restrições da forma

$$
\frac{\partial \ell(\sigma, \phi)}{\partial \phi_{s}}=-\frac{1}{\sigma} \sum_{j \in \bar{C}} x_{j s}+\frac{1}{\sigma} \sum_{j=1}^{n} x_{j s} \exp \left(z_{j}\right)=0
$$

onde $s=1, \ldots, p$.

O elemento $(s, t)$ da matriz de informação observada relativa ao vetor $\phi$ é dado por

$$
j_{\phi \phi}^{(s, t)}(\sigma, \phi)=\frac{1}{\sigma^{2}} \sum_{j=1}^{n} x_{j s} x_{j t} \exp \left(z_{j}\right),
$$

em que $s, t=1, \ldots, p$. Numa forma matricial,

$$
j_{\phi \phi}(\sigma, \phi)=\frac{1}{\sigma^{2}} X^{\top} Z X
$$

onde $Z=\operatorname{diag}\left(\exp \left(z_{1}\right), \ldots, \exp \left(z_{n}\right)\right)$. Embora essa expressão seja igual àquela para dados completos, lembre que existem observações censuradas na amostra.

Derivando os componentes $\partial \ell(\sigma, \phi) / \partial \phi_{s}$ do vetor escore $(s=1, \ldots, p)$ com relação ao vetor de observações $y=\left(y_{1}, \ldots, y_{n}\right)$, obtém-se $\ell_{\phi ; y}(\sigma, \phi)$, cujo elemento $(s, j)$ é dado por

$$
\ell_{\phi ; y}^{(s, j)}(\sigma, \phi)=\frac{1}{\sigma^{2}} x_{j s} \exp \left(z_{j}\right)
$$

Numa forma matricial,

$$
\ell_{\phi ; y}(\sigma, \phi)=\frac{1}{\sigma^{2}} X^{\top} Z
$$

A distribuição da variável que modela o tempo de censura não depende de $\phi$. Logo, seguindo a definição para $\widehat{V}_{\phi}$ dada na Seção 2.11.4, vem

$$
\widehat{V}_{\phi}=\left(v_{1}^{\top}, \ldots, v_{n}^{\top}\right)^{\top},
$$

onde

$$
j \in \bar{C} \Longrightarrow v_{j}=\mathbf{x}_{j} \quad \text { e } \quad j \in C \Longrightarrow v_{j}=0_{1 \times p}
$$


Portanto, para pares $(\sigma, \phi)$ satisfazendo as restrições

$$
\frac{\partial \ell(\sigma, \phi)}{\partial \phi_{s}}=0
$$

onde $s=1, \ldots, p$, a função $\tilde{\ell}_{B N}(\sigma)$ é dada por

$$
\ell(\sigma, \phi)+p \log \sigma+\frac{1}{2} \log \left|X^{\top} Z X\right|-\log \left|X^{\top} Z \widehat{V}_{\phi}\right|
$$

Para observações independentes e identicamente distribuídas, Yang e Xie (2003) usaram, no caso de dados censurados, a fim de derivar $\ell_{C R}(\beta)$, duas parametrizações ortogonais obtidas a partir da informação de Fisher relativa à verossimilhança para dados completos. Mais precisamente, para dados com censura do tipo II, eles indexaram a distribuição de Weibull com a mesma parametrização do caso de dados completos $\left(\beta, \lambda^{*}\right)=(\beta, \log \lambda / \beta)$, enquanto, para dados com censura do tipo I, a parametrização proposta, baseada nos resultados de Cox e Reid (1989), foi $(\beta, \log \lambda)$. Aqui, $\lambda=\alpha \exp \{(1-\gamma) / \beta\}$.

Portanto, reproduzindo tais parametrizações ortogonais ótimas a partir do par $(\sigma, \phi)$ que indexa a distribuição valor extremo, em que $\sigma=\beta^{-1}$ e $\log \alpha\left(\mathbf{x}_{j}\right)=\eta\left(\mathbf{x}_{j}\right)=\mathbf{x}_{j} \phi$ $(j=1, \ldots, n)$, o logaritmo da função de verossimilhança para $\left(\sigma, \lambda^{o}\right)$, no caso de dados censurados do tipo II, é dado por

$$
\ell^{*}\left(\sigma, \lambda^{o}\right)=-r \log \sigma+\frac{1}{\sigma} \sum_{j \in \bar{C}} y_{j}-\frac{1}{\sigma^{2}} \sum_{j \in \bar{C}} \mathbf{x}_{j} \lambda^{o}-\sum_{j=1}^{n} \exp \left(q_{j}\right),
$$

onde

$$
q_{j}=\frac{y_{j}}{\sigma}-\frac{1}{\sigma^{2}} \mathbf{x}_{j} \lambda^{o}+(1-\gamma) \mathbf{x}_{j}\left(X^{\top} X\right)^{-1} X^{\top} \mathbf{1}_{n}
$$

para $j=1, \ldots, n$. Ou seja, a fim de obter $\ell_{C R}(\sigma)$, indexa-se a distribuição valor extremo por $\left(\sigma, \lambda^{o}\right)$, onde $\sigma$ (parâmetro de interesse) é ortogonal ao vetor $\lambda^{o}$ (conjunto de parâmetros de incômodo), definido anteriormente.

Para $s=1, \ldots, p$, os componentes do vetor escore relativos a $\lambda^{o}$ são expressos por

$$
\frac{\partial \ell^{*}\left(\sigma, \lambda^{o}\right)}{\partial \lambda_{s}^{o}}=-\frac{1}{\sigma^{2}}\left[\sum_{j \in \bar{C}} x_{j s}-\sum_{j=1}^{n} x_{j s} \exp \left(q_{j}\right)\right] .
$$

Note que não é possível obter $\ell_{p}^{*}(\sigma)$ de forma explícita, pois $\widehat{\lambda_{\sigma}^{o}}$ não tem forma fechada.

O ajuste de Cox e Reid (1987) para a verossimilhança perfilada requer a informação observada relativa ao parâmetro de incômodo $\lambda^{o}$ :

$$
-\frac{\partial^{2} \ell^{*}\left(\sigma, \lambda^{o}\right)}{\partial \lambda_{t}^{o} \lambda_{s}^{o}}=\frac{1}{\sigma^{4}} \sum_{j=1}^{n} x_{j s} x_{j t} \exp \left(q_{j}\right)
$$


onde $s, t=1, \ldots, p$. Numa forma matricial,

$$
j_{\lambda^{o} \lambda^{o}}^{*}\left(\sigma, \lambda^{o}\right)=\frac{1}{\sigma^{4}} X^{\top} Q X
$$

onde $Q=\operatorname{diag}\left(\exp \left(q_{1}\right), \ldots, \exp \left(q_{n}\right)\right)$, analogamente à matriz $Z$ definida anteriormente. Mais uma vez, embora essa expressão seja igual àquela para dados completos, lembre que existem observações censuradas (tipo II) na amostra.

Portanto, para pares $\left(\sigma, \lambda^{o}\right)$ satisfazendo as restrições

$$
\frac{\partial \ell^{*}\left(\sigma, \lambda^{o}\right)}{\partial \lambda_{s}^{o}}=0
$$

onde $s=1, \ldots, p$, a função $\ell_{C R}(\sigma)$ é dada por

$$
\ell^{*}\left(\sigma, \lambda^{o}\right)+2 p \log \sigma-\frac{1}{2} \log \left|X^{\top} Q X\right| .
$$

Agora, para dados censurados do tipo I, seguindo Yang e Xie (2003), a parametrização ortogonal ótima é definida por $\left(\sigma, \lambda^{\dagger}\right)=\left(\sigma, \lambda^{o} / \sigma\right)=(\sigma, \log \lambda)$. Assim, o logaritmo da função de verossimilhança é dado por

$$
\ell^{\star}\left(\sigma, \lambda^{\dagger}\right)=-r \log \sigma+\frac{1}{\sigma} \sum_{j \in \bar{C}} y_{j}-\frac{1}{\sigma} \sum_{j \in \bar{C}} \mathbf{x}_{j} \lambda^{\dagger}-\sum_{j=1}^{n} \exp \left(w_{j}\right),
$$

onde

$$
w_{j}=\frac{y_{j}}{\sigma}-\frac{1}{\sigma} \mathbf{x}_{j} \lambda^{\dagger}+(1-\gamma) \mathbf{x}_{j}\left(X^{\top} X\right)^{-1} X^{\top} \mathbb{1}_{n}
$$

para $j=1, \ldots, n$. Ou seja, a fim de obter $\ell_{C R}(\sigma)$, indexa-se a distribuição valor extremo por $\left(\sigma, \lambda^{\dagger}\right.$ ), onde $\sigma$ (parâmetro de interesse) é ortogonal ao vetor $\lambda^{\dagger}=\lambda^{o} / \sigma=\log \lambda$ (parâmetro de incômodo).

Para $s=1, \ldots, p$, os componentes do vetor escore relativos a $\lambda^{\dagger}$ são expressos por

$$
\frac{\partial \ell^{\star}\left(\sigma, \lambda^{\dagger}\right)}{\partial \lambda_{s}^{\dagger}}=-\frac{1}{\sigma}\left[\sum_{j \in \bar{C}} x_{j s}-\sum_{j=1}^{n} x_{j s} \exp \left(w_{j}\right)\right] .
$$

Mais uma vez, observe que não é possível obter $\ell_{p}^{\star}(\sigma)$ de forma explícita, pois $\widehat{\lambda_{\sigma}^{\dagger}}$ não tem forma fechada.

O ajuste de Cox e Reid (1987) para a verossimilhança perfilada requer a informação observada relativa ao parâmetro de incômodo $\lambda^{\dagger}$ :

$$
-\frac{\partial^{2} \ell^{\star}\left(\sigma, \lambda^{\dagger}\right)}{\partial \lambda_{t}^{\dagger} \lambda_{s}^{\dagger}}=\frac{1}{\sigma^{2}} \sum_{j=1}^{n} x_{j s} x_{j t} \exp \left(w_{j}\right)
$$


onde $s, t=1, \ldots, p$. Numa forma matricial,

$$
j_{\lambda^{\dagger} \lambda^{\dagger}}^{\star}\left(\sigma, \lambda^{\dagger}\right)=\frac{1}{\sigma^{2}} X^{\top} W X
$$

em que $W=\operatorname{diag}\left(\exp \left(w_{1}\right), \ldots, \exp \left(w_{n}\right)\right)$, analogamente à matriz $Z$ definida anteriormente.

Portanto, para pares $\left(\sigma, \lambda^{\dagger}\right)$ satisfazendo as restrições

$$
\frac{\partial \ell^{\star}\left(\sigma, \lambda^{\dagger}\right)}{\partial \lambda_{s}^{\dagger}}=0,
$$

onde $s=1, \ldots, p$, a função $\ell_{C R}(\sigma)$ é calculada por

$$
\ell^{\star}\left(\sigma, \lambda^{\dagger}\right)+p \log \sigma-\frac{1}{2} \log \left|X^{\top} W X\right| .
$$

Tanto para dados completos como para dados censurados, os estimadores de máxima verossimilhança de $\sigma$ não têm formas fechadas. Assim, estimativas são obtidas numericamente e alguns resultados são apresentados na próxima seção para $\beta=\sigma^{-1}$, o parâmetro de forma da distribuição de Weibull, baseados no princípio da invariância dos estimadores de máxima verossimilhança. Além disso, são mostrados resultados relativos a testes da razão de verossimilhanças sobre este parâmetro.

\subsubsection{RESULTADOS NUMÉRICOS}

Esta seção apresenta resultados numéricos oriundos de simulações de Monte Carlo. Embora as funções de verossimilhanças derivadas tenham $\sigma$ como argumento, o parâmetro de interesse é $\sigma^{-1}$, o parâmetro de configuração da distribuição de Weibull. Maximizando tais funções, pelo princípio da invariância dos estimadores de máxima verossimilhança, são obtidas estimativas para $\beta$. Os resultados são apresentados de forma análoga à Seção 5.2.1.3, considerando dados completos e dados censurados, e são baseados em 10000 (dez mil) réplicas de Monte Carlo. Foram geradas amostras de tamanhos 30 e 20 com os mesmos níveis de censura do caso de observações independentes e identicamente distribuídas. Assim, no caso de dados com censura do tipo II, foram simuladas as seguintes situações para o par $(n, r)$ : $(30,20)$ e $(30,10),(20,15)$ e $(20,10)$. No caso de censura tipo I, para $n=20$, foram consideradas amostras com $25 \%$ e $50 \%$ de censura.

Os resultados numéricos foram obtidos para uma e três covariadas, ou seja, dois e quatro parâmetros de incômodo $(p=2,4)$, pois o modelo de regressão proposto tem intercepto $\phi_{1}$ $\left(x_{1}=1\right)$. Os valores verdadeiros dos parâmetros de incômodo são todos iguais a um, ou seja, $\phi_{i}=1 \forall i$. No vetor de covariadas $\mathbf{x}=\left(1, x_{2}, \ldots, x_{p}\right)$, os componentes $x_{2}, \ldots, x_{p}$ são realizações independentes de uma variável aleatória uniforme $U(0,1)$.

Aqui cabe uma importante observação relativa às distribuições dos estimadores de máxima verossimilhança considerados: para dados completos, as razões (estimador de $\sigma$ ) / $\sigma$ 
são quantidades pivotais com respeito aos parâmetros da distribuição valor extremo. Conseqüentemente, os vieses relativos e os coeficientes de assimetria e de curtose dos estimadores não dependem dos valores verdadeiros dos parâmetros $\sigma$ e $\phi$, depedem apenas do tamanho amostral $n$ e da matriz de especificação do modelo $X$. Para os estimadores $\widehat{\sigma}$ e $\widehat{\tilde{\sigma}}_{B N}$, este resultado também é válido no caso de dados com presença de censura do tipo II, o que não ocorre com o estimador $\widehat{\sigma}_{C R}$. Alguns desses resultados estão demonstrados no Apêndice D deste texto, assim como o motivo pelo qual as razões (estimador de $\sigma$ ) / $\sigma$ não são quantidades pivotais no caso de dados com presença de censura do tipo I.

Por isto, no caso de dados completos, os resultados numéricos apresentados são referentes a simulações em que $\sigma=1 / 5$, ou seja, $\beta=5$ (função de taxa de falha crescente), bem como no caso de dados com presença de censura do tipo II. Além disso, para este tipo de censura, como a razão $\widehat{\sigma}_{C R} / \sigma$ não é uma quantidade pivotal para $(\sigma, \phi)$, são apresentados resultados em que $\sigma=1$ e $\sigma=2$, ou seja, $\beta=1$ (função de taxa de falha constante) e $\beta=1 / 2$ (função de taxa de falha decrescente), respectivamente. Para dados censurados do tipo I, foram obtidos resultados para os três valores do parâmetro $\sigma$ aqui considerados: 2, 1 e 1/5.

Além da estimação do parâmetro $\sigma\left(=\beta^{-1}\right)$, foram realizados testes da razão de verossimilhanças sobre este. Aqui o objetivo foi comparar os desempenhos dos ajustes sobre a verossimilhança perfilada a partir das distorções dos tamanhos dos testes com relação aos níveis nominais na presença de mais de um parâmetro de incômodo. Usando o fato da razão (estimador de $\sigma$ ) / $\sigma$ ser uma quantidade pivotal, pode-se mostrar, para dados completos, que a probabilidade do erro tipo I não depende de $\phi$ e nem do valor de $\sigma$ fixado na hipótese nula, varia apenas com o tamanho amostral $n$ e com a matriz de especificação do modelo $X$, ou seja, neste caso, as estatísticas de teste dependem apenas de quantidades pivotais e ancilares para $(\sigma, \phi)$. Este resultado é demonstrado no Apêndice $\mathrm{D}$ e não vale para dados censurados. Para este caso, foram realizados testes de hipóteses considerando funções de taxas de falhas decrescente, constante e crescente:

$$
\mathcal{H}_{0}: \beta=0.5, \quad \mathcal{H}_{0}: \beta=1 \quad \text { e } \quad \mathcal{H}_{0}: \beta=5
$$

Considerando dados completos, a Tabela 5.10 contém estatísticas descritivas de diferentes estimadores. Na presença de dois parâmetros de incômodo, o estimador $\widehat{\beta}_{C R}$ apresentou, em módulo, o menor viés relativo: $3.043 \%(n=20)$ e $1.896 \%(n=30)$. Para estes tamanhos amostrais, as estimativas das variâncias de $\widehat{\beta}_{C R}$ foram as menores, iguais a 0.912 e 0.572 , respectivamente. Os coeficientes de assimetria e curtose apresentaram valores próximos aos assintóticos. Por exemplo, para $n=20$, tais coeficientes relativos à distribuição de $\widehat{\beta}_{C R}$ foram iguais a 0.905 e 4.529, respectivamente. Com quatro parâmetros de incômodo, em termos de viés, o melhor desempenho foi do estimador $\widetilde{\widetilde{\beta}}_{B N}$, que apresentou vieses relativos, para $n=20$ e $n=30$, iguais a $5.023 \%$ e $2.901 \%$. Embora este estimador tenha apresentado menor viés, $\widehat{\beta}_{C R}$ apresentou menores estimativas de erro quadrático médio (EQM) nos dois tamanhos amostrais considerados: $1.254(n=20)$ e $0.692(n=30)$. O aumento da quantidade de parâmetros de incômodo acarretou aumento das estimativas dos vieses dos 
Tabela 5.10. Medidas descritivas de diferentes estimadores de $\beta=\sigma^{-1}$, distribuição valor extremo, dados completos, $\beta=5$.

\begin{tabular}{|c|c|c|c|c|c|c|c|c|}
\hline \multicolumn{9}{|c|}{$\eta(\mathbf{x})=\phi_{1}+\phi_{2} x_{2}$} \\
\hline$n$ & estimador & média & variância & viés & EQM & v.r. $(\%)$ & assimetria & curtose \\
\hline \multirow{3}{*}{20} & $\widehat{\beta}$ & 5.569 & 1.210 & 0.569 & 1.534 & 11.380 & 0.918 & 4.555 \\
\hline & $\tilde{\beta}_{B N}$ & 5.217 & 1.057 & 0.217 & 1.104 & 4.333 & 0.911 & 4.538 \\
\hline & $\widehat{\beta}_{C R}$ & 4.848 & 0.912 & -0.152 & 0.935 & -3.043 & 0.905 & 4.529 \\
\hline \multirow{3}{*}{30} & $\widehat{\beta}$ & 5.359 & 0.684 & 0.359 & 0.812 & 7.174 & 0.733 & 4.075 \\
\hline & $\tilde{\tilde{\beta}}_{B N}$ & 5.136 & 0.627 & 0.136 & 0.645 & 2.712 & 0.727 & 4.053 \\
\hline & $\widehat{\beta}_{C R}$ & 4.905 & 0.572 & -0.095 & 0.581 & -1.896 & 0.720 & 4.032 \\
\hline \multicolumn{9}{|c|}{$\eta(\mathbf{x})=\phi_{1}+\phi_{2} x_{2}+\phi_{3} x_{3}+\phi_{4} x_{4}$} \\
\hline$n$ & estimador & média & variância & viés & EQM & v.r. (\%) & assimetria & curtose \\
\hline \multirow{3}{*}{20} & $\widehat{\beta}$ & 6.024 & 1.667 & 1.024 & 2.716 & 20.484 & 1.041 & 5.155 \\
\hline & $\tilde{\tilde{\beta}}_{B N}$ & 5.251 & 1.259 & 0.251 & 1.322 & 5.023 & 1.032 & 5.118 \\
\hline & $\widehat{\beta}_{C R}$ & 4.389 & 0.881 & -0.611 & 1.254 & -12.214 & 1.018 & 5.054 \\
\hline \multirow{3}{*}{30} & $\widehat{\beta}$ & 5.618 & 0.824 & 0.618 & 1.206 & 12.364 & 0.769 & 4.184 \\
\hline & $\tilde{\tilde{\beta}}_{B N}$ & 5.145 & 0.689 & 0.145 & 0.710 & 2.901 & 0.756 & 4.137 \\
\hline & $\widehat{\beta}_{C R}$ & 4.635 & 0.559 & -0.365 & 0.692 & -7.291 & 0.744 & 4.097 \\
\hline
\end{tabular}

Tabela 5.11. Taxas de rejeição da hipótese nula de diferentes testes da razão de verossimilhanças, $\mathcal{H}_{0}: \beta=\sigma^{-1}=5$, distribuição valor extremo, dados completos.

\begin{tabular}{rrrrrrrr}
\hline & \multicolumn{3}{c}{$\eta(\mathbf{x})=\phi_{1}+\phi_{2} x_{2}$} & \multicolumn{3}{c}{$\eta(\mathbf{x})=\phi_{1}+\phi_{2} x_{2}+\phi_{3} x_{3}+\phi_{4} x_{4}$} \\
\hline \multicolumn{1}{r}{$n$} & nível nominal & \multicolumn{1}{c}{$\ell$} & \multicolumn{1}{c}{$\tilde{\ell}_{B N}$} & \multicolumn{1}{c}{$\ell_{C R}$} & \multicolumn{1}{c}{$\ell$} & \multicolumn{1}{c}{$\tilde{\ell}_{B N}$} & \multicolumn{1}{c}{$\ell_{C R}$} \\
\hline \multirow{4}{*}{20} & $15 \%$ & 19.750 & 15.470 & 16.050 & 29.120 & 15.500 & 24.610 \\
& $10 \%$ & 14.170 & 10.420 & 10.900 & 22.300 & 10.410 & 18.610 \\
& $5 \%$ & 7.910 & 5.210 & 5.780 & 14.280 & 5.350 & 10.630 \\
& $1 \%$ & 2.050 & 0.880 & 1.090 & 4.850 & 1.090 & 3.040 \\
& $0.1 \%$ & 0.250 & 0.070 & 0.150 & 0.980 & 0.100 & 0.340 \\
& $0.05 \%$ & 0.140 & 0.040 & 0.060 & 0.700 & 0.050 & 0.210 \\
\hline \multirow{4}{*}{30} & $15 \%$ & 18.280 & 15.240 & 15.750 & 24.360 & 14.860 & 20.510 \\
& $10 \%$ & 12.610 & 10.330 & 10.690 & 17.870 & 9.980 & 14.510 \\
& $5 \%$ & 6.770 & 5.120 & 5.420 & 10.440 & 5.050 & 8.040 \\
& $1 \%$ & 1.530 & 0.860 & 0.980 & 3.180 & 0.900 & 2.100 \\
& $0.1 \%$ & 0.180 & 0.110 & 0.110 & 0.500 & 0.130 & 0.260 \\
& $0.05 \%$ & 0.100 & 0.080 & 0.060 & 0.310 & 0.080 & 0.120 \\
\hline
\end{tabular}

estimadores. Note que os dois estimadores derivados das maximizações de versões ajustadas de $\ell_{p}$ apresentaram desempenhos bem superiores ao estimador de máxima verossimilhança original. O sinal negativo do viés do estimador $\widehat{\beta}_{C R}$ indica que este subestimou o verdadeiro valor do parâmetro $\beta$.

A Tabela 5.11 apresenta taxas de rejeição sob a hipótese nula de diferentes testes da razão 
de verossimilhanças com vários níveis de significância. Aqui $\mathcal{H}_{0}: \beta=5$, mas vale lembrar que tais taxas não dependem da hipótese nula considerada. Para quatro parâmetros de incômodo e $n=30$, considere, por exemplo, o nível de significância de $10 \%$. As taxas de rejeição dos testes baseados em $\ell, \tilde{\ell}_{B N}$ e $\ell_{C R}$ foram, respectivamente, iguais a $17.870 \%, 9.980 \%$ e $14.510 \%$. Para dois parâmetros de incômodo, $n=20$ e nível nominal de $15 \%$, tais taxas foram $19.750 \%$, $15.470 \%$ e $16.050 \%$, respectivamente. Aqui as duas verossimilhanças perfiladas ajustadas produziram inferências mais precisas relativamente aos resultados obtidos pelo teste da razão de verossimilhanças original, mas vale destacar o desempenho do teste baseado em $\tilde{\ell}_{B N}$, que apresentou taxas mais próximas aos níveis nominais. O desempenho da estatística de teste baseada em $\ell_{C R}$ deteriora-se bastante com o aumento da presença de parâmetros de incômodo.

As médias e variâncias das estatísticas de teste são apresentadas na Tabela 5.12, assim como seus valores assintóticos (1 e 2, respectivamente). Para quatro parâmetros de incômodo e $n=30$, as estatísticas de teste baseadas em $\ell, \tilde{\ell}_{B N}$ e $\ell_{C R}$ apresentaram média e variância amostrais, respectivamente, iguais a 1.476 e 4.031, 0.999 e 1.987, 1.277 e 3.019. A estatística baseada em $\tilde{\ell}_{B N}$ foi aquela que apresentou momentos empíricos mais próximos aos assintóticos.

Tabela 5.12. Médias e variâncias amostrais de diferentes estatísticas de teste e os valores assintóticos destas, $\mathcal{H}_{0}: \beta=\sigma^{-1}=5$, distribuição valor extremo, dados completos.

\begin{tabular}{ccccccccc}
\hline & & \multicolumn{3}{c}{$\eta(\mathbf{x})=\phi_{1}+\phi_{2} x_{2}$} & \multicolumn{2}{c}{$\eta(\mathbf{x})=\phi_{1}+\phi_{2} x_{2}+\phi_{3} x_{3}+\phi_{4} x_{4}$} \\
\hline \multirow{2}{*}{$n$} & momento & $\chi_{1}^{2}$ & $\ell$ & $\tilde{\ell}_{B N}$ & $\ell_{C R}$ & $\ell$ & $\tilde{\ell}_{B N}$ & $\ell_{C R}$ \\
\hline \multirow{2}{*}{20} & média & 1 & 1.238 & 1.010 & 1.058 & 1.780 & 1.019 & 1.485 \\
& variância & 2 & 2.967 & 1.996 & 2.169 & 5.519 & 2.071 & 3.745 \\
\hline \multirow{2}{*}{30} & média & 1 & 1.148 & 1.002 & 1.031 & 1.476 & 0.999 & 1.277 \\
& variância & 2 & 2.598 & 1.982 & 2.085 & 4.031 & 1.987 & 3.019 \\
\hline
\end{tabular}

Os desempenhos das estatísticas de teste nas Tabelas 5.11 e 5.12 são reflexos das aproximações das distribuições destas pela distribuição assintótica qui-quadrado com um grau de liberdade. As qualidades dessas aproximações são melhor avaliadas através das discrepâncias relativas de quantis, conforme as Figuras 5.11 e 5.12.

Para o caso de dois parâmetros de perturbação, a Figura 5.11 apresenta o gráfico das curvas de discrepâncias relativas de quantis versus os correspondentes quantis assintóticos. Quanto mais próxima da ordenada zero a curva estiver, melhor a aproximação da distribuição da estatística de teste pela distribuição qui-quadrado com um grau de liberdade. Assim, as estatísticas baseadas em $\ell_{C R}(\mathrm{CR})$ e $\tilde{\ell}_{B N}$ (aprox BN) têm distribuições nulas melhor aproximadas pela distribuição $\chi_{1}^{2}$ do que aquela derivada da verossimilhança original $\ell$, com destaque para a segunda. Para o caso de quatro parâmetros de perturbação, é apresentada a Figura 5.12. Note os deslocamentos das curvas associadas a $\ell$ e $\ell_{C R}$ para patamares mais distantes da ordenada nula, enquanto a curva relativa a $\tilde{\ell}_{B N}($ aprox $\mathrm{BN})$ permanece próxima a esta. 
Figura 5.11. Gráfico das discrepâncias relativas de quantis, distribuição valor extremo, $\eta(\mathbf{x})=\phi_{1}+\phi_{2} x_{2}, \mathcal{H}_{0}: \beta=\sigma^{-1}=5$, dados completos, $n=20$.

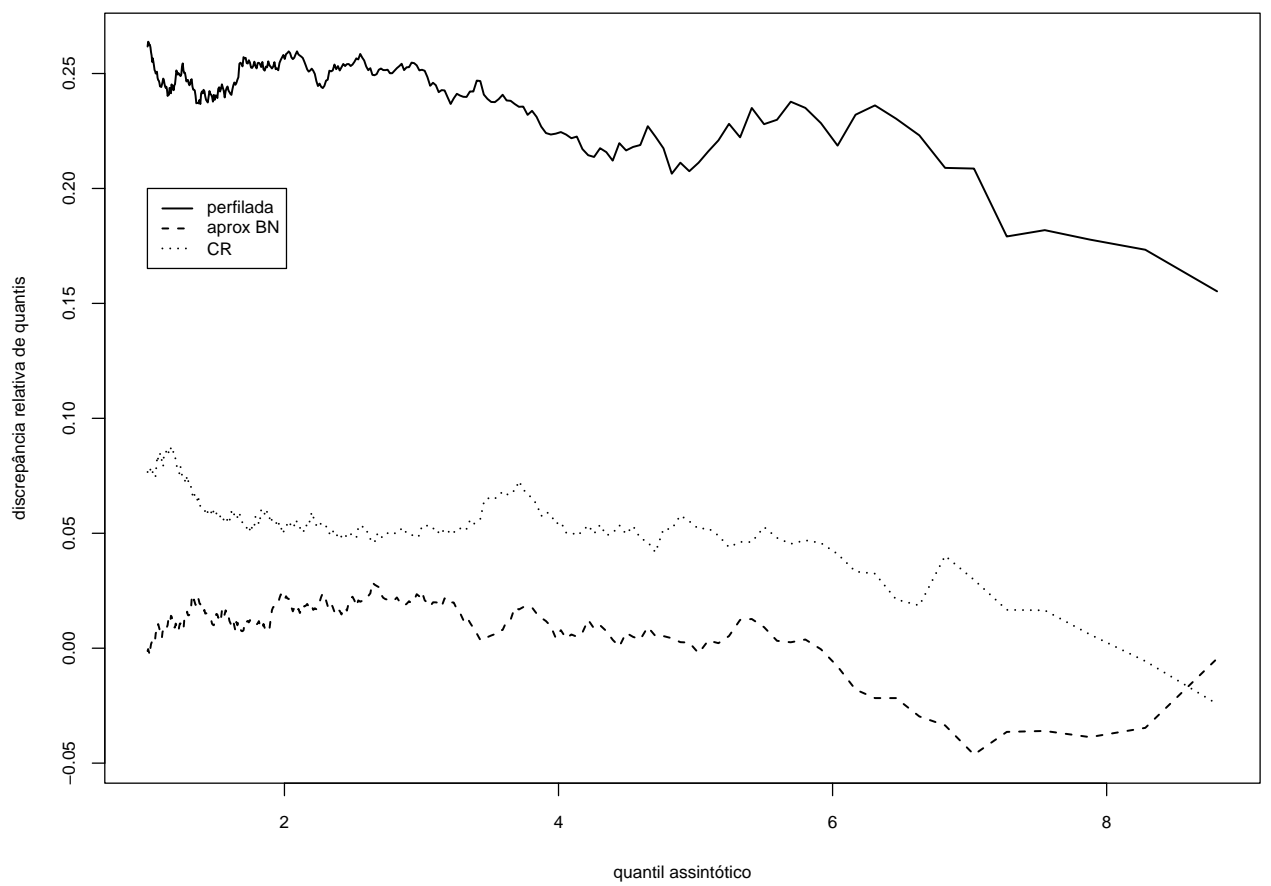

Figura 5.12. Gráfico das discrepâncias relativas de quantis, distribuição valor extremo, $\eta(\mathbf{x})=\phi_{1}+\phi_{2} x_{2}+\phi_{3} x_{3}+\phi_{4} x_{4}, \mathcal{H}_{0}: \beta=\sigma^{-1}=5$, dados completos, $n=20$.

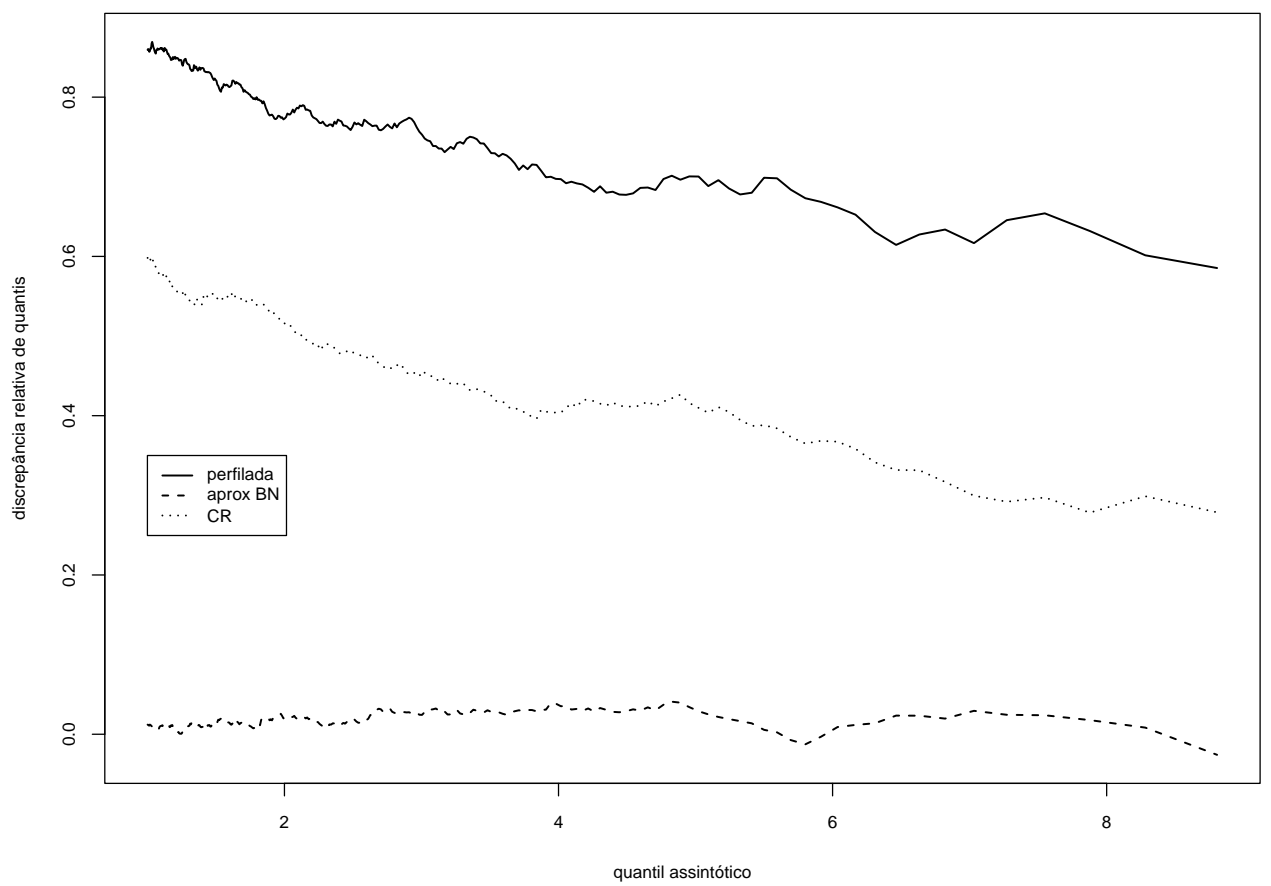

Agora considere dados com presença de censura do tipo II. A Tabela 5.13 contém es- 
Tabela 5.13. Medidas descritivas de diferentes estimadores de $\beta=\sigma^{-1}$, distribuição valor extremo, dados censurados tipo II, $\beta=5$.

\begin{tabular}{|c|c|c|c|c|c|c|c|c|}
\hline \multicolumn{9}{|c|}{$\eta(\mathbf{x})=\phi_{1}+\phi_{2} x_{2}$} \\
\hline$(n, r)$ & estimador & média & variância & viés & EQM & v.r.(\%) & assimetria & curtose \\
\hline \multirow{3}{*}{$(20,15)$} & $\widehat{\beta}$ & 5.756 & 1.824 & 0.756 & 2.395 & 15.121 & 1.003 & 4.767 \\
\hline & $\tilde{\tilde{\beta}}_{B N}$ & 5.289 & 1.522 & 0.289 & 1.606 & 5.786 & 0.986 & 4.716 \\
\hline & $\widehat{\beta}_{C R}$ & 4.753 & 1.290 & -0.247 & 1.352 & -4.945 & 0.996 & 4.782 \\
\hline \multirow{3}{*}{$(30,20)$} & $\widehat{\beta}$ & 5.545 & 1.249 & 0.545 & 1.546 & 10.906 & 0.862 & 4.355 \\
\hline & $\tilde{\beta}_{B N}$ & 5.218 & 1.098 & 0.218 & 1.146 & 4.369 & 0.854 & 4.342 \\
\hline & $\widehat{\beta}_{C R}$ & 4.811 & 0.972 & -0.189 & 1.008 & -3.789 & 0.865 & 4.379 \\
\hline \multirow{3}{*}{$(20,10)$} & $\widehat{\beta}$ & 6.196 & 3.880 & 1.196 & 5.311 & 23.920 & 1.562 & 8.901 \\
\hline & $\tilde{\tilde{\beta}}_{B N}$ & 5.445 & 2.949 & 0.445 & 3.148 & 8.910 & 1.519 & 8.473 \\
\hline & $\widehat{\beta}_{C R}$ & 4.399 & 2.119 & -0.601 & 2.480 & -12.023 & 1.577 & 8.920 \\
\hline \multirow{3}{*}{$(30,10)$} & $\widehat{\beta}$ & 6.193 & 4.461 & 1.193 & 5.884 & 23.855 & 1.739 & 9.705 \\
\hline & $\tilde{\beta}_{B N}$ & 5.490 & 3.496 & 0.490 & 3.735 & 9.792 & 1.744 & 9.871 \\
\hline & $\widehat{\beta}_{C R}$ & 4.186 & 2.137 & -0.814 & 2.801 & -16.286 & 1.715 & 9.356 \\
\hline \multicolumn{9}{|c|}{$\eta(\mathbf{x})=\phi_{1}+\phi_{2} x_{2}+\phi_{3} x_{3}+\phi_{4} x_{4}$} \\
\hline$(n, r)$ & estimador & média & variância & viés & EQM & v.r. $(\%)$ & assimetria & curtose \\
\hline \multirow{3}{*}{$(20,15)$} & $\widehat{\beta}$ & 6.299 & 2.617 & 1.299 & 4.304 & 25.972 & 1.244 & 5.940 \\
\hline & $\tilde{\tilde{\beta}}_{B N}$ & 5.247 & 1.793 & 0.247 & 1.854 & 4.933 & 1.214 & 5.821 \\
\hline & $\widehat{\beta}_{C R}$ & 3.993 & 1.117 & -1.007 & 2.131 & -20.142 & 1.187 & 5.706 \\
\hline \multirow{3}{*}{$(30,20)$} & $\widehat{\beta}$ & 5.833 & 1.447 & 0.833 & 2.140 & 16.656 & 0.946 & 4.641 \\
\hline & $\tilde{\beta}_{B N}$ & 5.130 & 1.109 & 0.130 & 1.126 & 2.597 & 0.926 & 4.592 \\
\hline & $\widehat{\beta}_{C R}$ & 4.295 & 0.836 & -0.705 & 1.334 & -14.101 & 0.923 & 4.605 \\
\hline \multirow{3}{*}{$(20,10)$} & $\widehat{\beta}$ & 7.014 & 6.250 & 2.014 & 10.306 & 40.279 & 1.780 & 9.805 \\
\hline & $\tilde{\beta}_{B N}$ & 5.245 & 3.381 & 0.245 & 3.441 & 4.909 & 1.710 & 9.258 \\
\hline & $\widehat{\beta}_{C R}$ & 2.735 & 1.313 & -2.265 & 6.442 & -45.294 & 1.721 & 8.852 \\
\hline \multirow{3}{*}{$(30,10)$} & $\widehat{\beta}$ & 6.731 & 5.588 & 1.731 & 8.585 & 34.622 & 1.818 & 10.242 \\
\hline & $\tilde{\beta}_{B N}$ & 5.129 & 3.160 & 0.129 & 3.177 & 2.580 & 1.722 & 9.704 \\
\hline & $\widehat{\beta}_{C R}$ & 2.558 & 1.240 & -2.442 & 7.205 & -48.845 & 1.855 & 10.363 \\
\hline
\end{tabular}

tatísticas descritivas de diferentes estimadores para vários níveis de censura, ou seja, para vários pares $(n, r)$, onde $r$ é o número de falhas. Como esperado, com a presença de censura nos dados, percebe-se, através das medidas de avaliação da qualidade de estimadores, como EQM e viés, que, em geral, as estimativas deterioram-se. Lembre que as estimativas dos vieses relativos e dos coeficientes de assimetria e de curtose dos estimadores $\widehat{\beta}$ e $\widehat{\widetilde{\beta}}_{B N}$ dependem apenas do par $(n, r)$ e da matriz de especificação do modelo. A única coluna comum às duas matrizes consideradas $(p=2,4)$, é aquela correspondente ao intercepto, coluna $n \times 1$ de uns.

De acordo com a Tabela 5.13, comparando os estimadores em termos de viés, aquele que apresentou menores valores foi $\widehat{\tilde{\beta}}_{B N}$. Exceto pelas duas situações representadas por $(n, r)=$ 
$(20,15)$ e $(n, r)=(30,20)$ e parte sistemática do modelo dada por $\eta(\mathbf{x})=\phi_{1}+\phi_{2} x_{2}$, em que o estimador $\widehat{\beta}_{C R}$ apresentou vieses relativos, em módulo, iguais a $4.945 \%$ e $3.789 \%$, contra $5.786 \%$ e $4.369 \%$ de $\widehat{\widetilde{\beta}}_{B N}$, em todos os demais casos, o viés apresentado por este foi inferior. Por exemplo, para o modelo com quatro parâmetros de incômodo e $(n, r)=(30,10)$, os vieses relativos apresentados pelos estimadores $\widehat{\beta}, \widehat{\tilde{\beta}}_{B N}$ e $\widehat{\beta}_{C R}$ foram, em módulo, respectivamente, iguais a $34.622 \%, 2.580 \%$ e $48.845 \%$. Note a deterioração das estimativas produzidas por $\widehat{\beta}_{C R}$ com os aumentos da presença de censura nos dados e da quantidade de regressores. Para as situações apresentadas, este estimador sempre subestimou o verdadeiro valor de $\beta$. Comparando os estimadores em termos de erro quadrático médio (EQM), observa-se que o estimador $\widehat{\beta}_{C R}$ é melhor que os demais quando a parte sistemática do modelo é dada por $\eta(\mathbf{x})=\phi_{1}+\phi_{2} x_{2}$, enquanto $\widehat{\tilde{\beta}}_{B N}$ é o melhor estimador na presença de quatro parâmetros de incômodo. Na primeira situação, para $(n, r)=(20,10)$, as estimativas do EQM associadas a $\widehat{\beta}, \widehat{\tilde{\beta}}_{B N}$ e $\widehat{\beta}_{C R}$ foram, respectivamente, iguais a $5.311,3.148$ e 2.480 . Na segunda situação, $\eta(\mathbf{x})=\phi_{1}+\phi_{2} x_{2}+\phi_{3} x_{3}+\phi_{4} x_{4}$, para $(n, r)=(30,10)$, as estimativas do EQM associadas a $\widehat{\beta}, \widehat{\tilde{\beta}}_{B N}$ e $\widehat{\beta}_{C R}$ foram, respectivamente, iguais a $8.585,3.177$ e 7.205 .

Tabela 5.14. Medidas descritivas do estimador $\widehat{\beta}_{C R}$, distribuição valor extremo, $\eta(\mathbf{x})=$ $\phi_{1}+\phi_{2} x_{2}$, dados censurados tipo II.

\begin{tabular}{cccccccc}
\hline \multicolumn{7}{c}{$\beta=0.5$} \\
\hline$(n, r)$ & média & variância & viés & EQM & v.r. $(\%)$ & assimetria & curtose \\
\hline$(20,15)$ & 0.457 & 0.014 & -0.043 & 0.016 & -8.671 & 1.172 & 5.663 \\
$(30,20)$ & 0.465 & 0.010 & -0.035 & 0.011 & -7.075 & 0.892 & 4.459 \\
$(20,10)$ & 0.406 & 0.021 & -0.094 & 0.030 & -18.739 & 1.993 & 13.364 \\
$(30,10)$ & 0.396 & 0.021 & -0.104 & 0.032 & -20.898 & 1.809 & 10.500 \\
\hline & & & & & & \\
$(n, r)$ & média & variância & viés & EQM & v.r. $(\%)$ & assimetria & curtose \\
\hline$(20,15)$ & 0.916 & 0.055 & -0.084 & 0.062 & -8.391 & 1.145 & 5.539 \\
$(30,20)$ & 0.931 & 0.041 & -0.069 & 0.046 & -6.870 & 0.919 & 4.608 \\
$(20,10)$ & 0.814 & 0.084 & -0.186 & 0.119 & -18.559 & 1.990 & 12.706 \\
$(30,10)$ & 0.792 & 0.083 & -0.208 & 0.126 & -20.758 & 1.946 & 12.153 \\
\hline
\end{tabular}

Como mencionado anteriormente, para dados censurados, a razão $\widehat{\sigma}_{C R} / \sigma$ não é uma quantidade pivotal para $(\sigma, \phi)$. Por conseguinte, as estimativas dos vieses relativos e dos coeficientes de assimetria e de curtose de $\widehat{\beta}_{C R}$ variam com $\beta$. Para $\beta=0.5$ e $\beta=1$, a Tabela 5.14 contém estatísticas descritivas deste estimador para diferentes níveis de censura. Note que as estimativas são mais viesadas do que aquelas referentes a $\beta=5$. Além disso, em geral, as estimativas dos coeficientes de assimetria e de curtose estão mais distantes dos valores assintóticos, 0 e 3 , respectivamente.

A Tabela 5.15 apresenta taxas de rejeição sob a hipótese nula $\left(\mathcal{H}_{0}: \beta=5\right)$ de diferentes testes da razão de verossimilhanças com vários níveis de significância. Seja com dois ou com quatro parâmetros de incômodo, o teste baseado em $\tilde{\ell}_{B N}$ foi aquele que apresentou taxas 
Tabela 5.15. Taxas de rejeição da hipótese nula de diferentes testes da razão de verossimilhanças, $\mathcal{H}_{0}: \beta=\sigma^{-1}=5$, distribuição valor extremo, dados censurados tipo II.

\begin{tabular}{rcrrrrrr}
\hline & & \multicolumn{3}{c}{$\eta(\mathbf{x})=\phi_{1}+\phi_{2} x_{2}$} & \multicolumn{1}{c}{$\eta(\mathbf{x})=\phi_{1}+\phi_{2} x_{2}+\phi_{3} x_{3}+\phi_{4} x_{4}$} \\
\hline$(n, r)$ & nível nominal & \multicolumn{1}{c}{$\ell$} & \multicolumn{1}{c}{$\tilde{\ell}_{B N}$} & \multicolumn{1}{c}{$\ell_{C R}$} & \multicolumn{1}{c}{$\ell$} & \multicolumn{1}{c}{$\tilde{\ell}_{B N}$} & \multicolumn{1}{c}{$\ell_{C R}$} \\
\hline & $15 \%$ & 20.760 & 14.700 & 16.620 & 30.470 & 14.530 & 33.240 \\
& $10 \%$ & 15.050 & 9.800 & 11.610 & 23.620 & 9.790 & 25.520 \\
$(20,15)$ & $5 \%$ & 8.350 & 4.720 & 6.120 & 15.480 & 5.120 & 15.900 \\
& $1 \%$ & 2.070 & 0.860 & 1.470 & 5.730 & 1.020 & 4.960 \\
& $0.1 \%$ & 0.290 & 0.040 & 0.150 & 1.220 & 0.130 & 0.880 \\
& $0.05 \%$ & 0.190 & 0.040 & 0.060 & 0.760 & 0.040 & 0.520 \\
\hline \multirow{5}{*}{$(30,20)$} & $15 \%$ & 19.410 & 15.080 & 17.270 & 25.460 & 14.910 & 28.220 \\
& $10 \%$ & 14.130 & 10.230 & 11.590 & 19.170 & 9.850 & 21.510 \\
& $5 \%$ & 7.950 & 5.030 & 6.090 & 11.750 & 4.980 & 13.190 \\
& $1 \%$ & 1.860 & 0.980 & 1.490 & 3.760 & 1.000 & 3.790 \\
& $0.1 \%$ & 0.270 & 0.100 & 0.140 & 0.740 & 0.090 & 0.620 \\
& $0.05 \%$ & 0.160 & 0.060 & 0.050 & 0.440 & 0.040 & 0.450 \\
\hline \multirow{6}{*}{$(20,10)$} & $15 \%$ & 22.340 & 13.870 & 20.890 & 34.560 & 14.160 & 57.040 \\
& $10 \%$ & 16.330 & 8.980 & 14.860 & 27.720 & 9.420 & 48.700 \\
& $5 \%$ & 8.970 & 4.540 & 8.440 & 18.790 & 4.470 & 35.680 \\
& $1 \%$ & 2.520 & 0.760 & 2.210 & 7.340 & 0.950 & 16.060 \\
& $0.1 \%$ & 0.290 & 0.070 & 0.260 & 1.840 & 0.090 & 3.910 \\
& $0.05 \%$ & 0.180 & 0.070 & 0.140 & 1.210 & 0.060 & 2.490 \\
\hline & $15 \%$ & 20.710 & 14.560 & 24.160 & 31.010 & 13.840 & 62.600 \\
& $10 \%$ & 14.960 & 9.630 & 17.680 & 24.040 & 9.200 & 54.340 \\
& $5 \%$ & 8.850 & 4.390 & 10.020 & 15.280 & 4.400 & 41.670 \\
& $1 \%$ & 2.380 & 0.840 & 2.890 & 5.530 & 0.890 & 20.570 \\
& $0.1 \%$ & 0.380 & 0.100 & 0.320 & 1.340 & 0.080 & 5.720 \\
& $0.05 \%$ & 0.200 & 0.070 & 0.200 & 0.810 & 0.040 & 3.780 \\
\hline
\end{tabular}

de rejeição mais próximas aos níveis nominais. Por exemplo, considerando o modelo com três regressores e $(n, r)=(20,15)$, para o nível de significância de $1 \%$, os testes baseados em $\ell, \tilde{\ell}_{B N}$ e $\ell_{C R}$ apresentaram taxas de rejeição, respectivamente, iguais a $5.730 \%, 1.020 \%$ e $4.960 \%$. Com um regressor, $(n, r)=(30,10)$ e nível nominal de $5 \%$, as taxas de rejeição foram, respectivamente, iguais a $8.850 \%, 4.390 \%$ e $10.020 \%$. Note na tabela que os desempenhos dos testes baseados em $\ell_{C R}$ e na verossimilhança original deterioram-se bastante com os aumentos da quantidade de parâmetros de incômodo e de presença de censura nos dados.

As médias e variâncias empíricas das estatísticas de teste são apresentadas na Tabela 5.16. Considerando o modelo com uma covariada e $(n, r)=(30,20)$, as médias e variâncias das estatísticas baseadas em $\ell, \tilde{\ell}_{B N}$ e $\ell_{C R}$ foram, respectivamente, iguais a 1.224 e 2.923 , 1.010 e $2.014,1.107$ e 2.396. Note que os valores associados à aproximação $\tilde{\ell}_{B N}$ apresentaramse mais próximos aos valores assintóticos. Considerando três regressores e $(n, r)=(20,10)$, tais momentos amostrais foram, respectivamente, iguais a 2.149 e 7.573, 0.965 e 1.886, 3.530 
Tabela 5.16. Médias e variâncias amostrais de diferentes estatísticas de teste e os valores assintóticos destas, $\mathcal{H}_{0}: \beta=\sigma^{-1}=5$, distribuição valor extremo, dados censurados tipo II.

\begin{tabular}{ccccccccc}
\hline & \multicolumn{4}{c}{$\eta(\mathbf{x})=\phi_{1}+\phi_{2} x_{2}$} & \multicolumn{2}{c}{$\eta(\mathbf{x})=\phi_{1}+\phi_{2} x_{2}+\phi_{3} x_{3}+\phi_{4} x_{4}$} \\
\hline \multirow{2}{*}{$(n, r)$} & momento & $\chi_{1}^{2}$ & $\ell$ & $\tilde{\ell}_{B N}$ & $\ell_{C R}$ & $\ell$ & $\tilde{\ell}_{B N}$ & $\ell_{C R}$ \\
\hline \multirow{2}{*}{$(20,15)$} & média & 1 & 1.282 & 0.985 & 1.102 & 1.879 & 0.988 & 1.926 \\
& variância & 2 & 3.109 & 1.872 & 2.340 & 6.146 & 1.998 & 5.456 \\
\hline \multirow{2}{*}{$(30,20)$} & média & 1 & 1.224 & 1.010 & 1.107 & 1.566 & 0.993 & 1.686 \\
& variância & 2 & 2.923 & 2.014 & 2.396 & 4.580 & 1.968 & 4.593 \\
\hline \multirow{2}{*}{$(20,10)$} & média & 1 & 1.364 & 0.955 & 1.292 & 2.149 & 0.965 & 3.530 \\
& variância & 2 & 3.491 & 1.792 & 3.093 & 7.573 & 1.886 & 11.417 \\
\hline \multirow{2}{*}{$(30,10)$} & média & 1 & 1.305 & 0.957 & 1.451 & 1.892 & 0.963 & 4.032 \\
& variância & 2 & 3.394 & 1.867 & 3.633 & 6.347 & 1.865 & 13.359 \\
\hline
\end{tabular}

e 11.417.

Os resultados das Tabelas 5.15 e 5.16 são reflexos da qualidade das aproximações das distribuições das estatísticas de teste pela distribuição assintótica qui-quadrado com um grau de liberdade. Tais aproximações podem ser melhor avaliadas através das discrepâncias relativas entre os quantis empíricos e os quantis assintóticos dessas estatísticas. As Figuras $5.13,5.14,5.15$ e 5.16 apresentam as curvas de discrepâncias relativas associadas às três estatísticas de teste consideradas para $(n, r)=(20,15)$ e $(n, r)=(20,10)$ e parte sistemática do modelo constituída por um e três regressores.

Para o caso de dois parâmetros de perturbação e $(n, r)=(20,15)$, a Figura 5.13 apresenta o gráfico das curvas de discrepâncias relativas de quantis versus os correspondentes quantis assintóticos. Como afirmado anteriormente, quanto mais próxima da ordenada zero a curva estiver, melhor a aproximação da distribuição nula da estatística de teste pela distribuição qui-quadrado. Assim, aquela baseada em $\tilde{\ell}_{B N}$ (aprox BN) tem a distribuição melhor aproximada pela distribuição $\chi_{1}^{2}$. Observe que as diferenças entre os quantis amostrais e os quantis assintóticos da estatística baseada em $\ell_{C R}(\mathrm{CR})$ é, aproximadamente, igual a $10 \%$. Aumentando o nível de censura para $50 \%,(n, r)=(20,10)$, note na Figura 5.14 os deslocamentos das curvas relativas às estatísticas baseadas em $\ell$ e $\ell_{C R}$ para patamares mais distantes da ordenada nula e a aproximação destas. A curva associada a $\tilde{\ell}_{B N}$ permaneceu próxima a esta ordenada.

Para o caso de quatro parâmetros de incômodo, as Figuras 5.15 e 5.16 apresentam os gráficos das curvas de discrepâncias relativas de quantis versus os correspondentes quantis assintóticos para $(n, r)=(20,15)$ e $(n, r)=(20,10)$, respectivamente. Note que, entre as estatísticas de teste, mais uma vez, aquela cuja distribuição nula é melhor aproximada pela distribuição $\chi_{1}^{2}$ também é a baseada em $\tilde{\ell}_{B N}$ (aprox BN). Observe as deteriorações das aproximações das distribuições nulas das estatísticas de teste baseadas em $\ell$ e $\ell_{C R}$ pela distribuição qui-quadrado com o aumento da proporção de censura na amostra. No gráfico da Figura 5.16, tal aproximação relativa a estatística baseada em $\ell_{C R}$ é bastante pobre.

Usando o fato da razão (estimador de $\sigma$ ) / $\sigma$ ser uma quantidade pivotal, pode-se mostrar, 
Figura 5.13. Gráfico das discrepâncias relativas de quantis, distribuição valor extremo, $\eta(\mathbf{x})=\phi_{1}+\phi_{2} x_{2}, \mathcal{H}_{0}: \beta=\sigma^{-1}=5$, dados censurados tipo II, $(n, r)=(20,15)$.

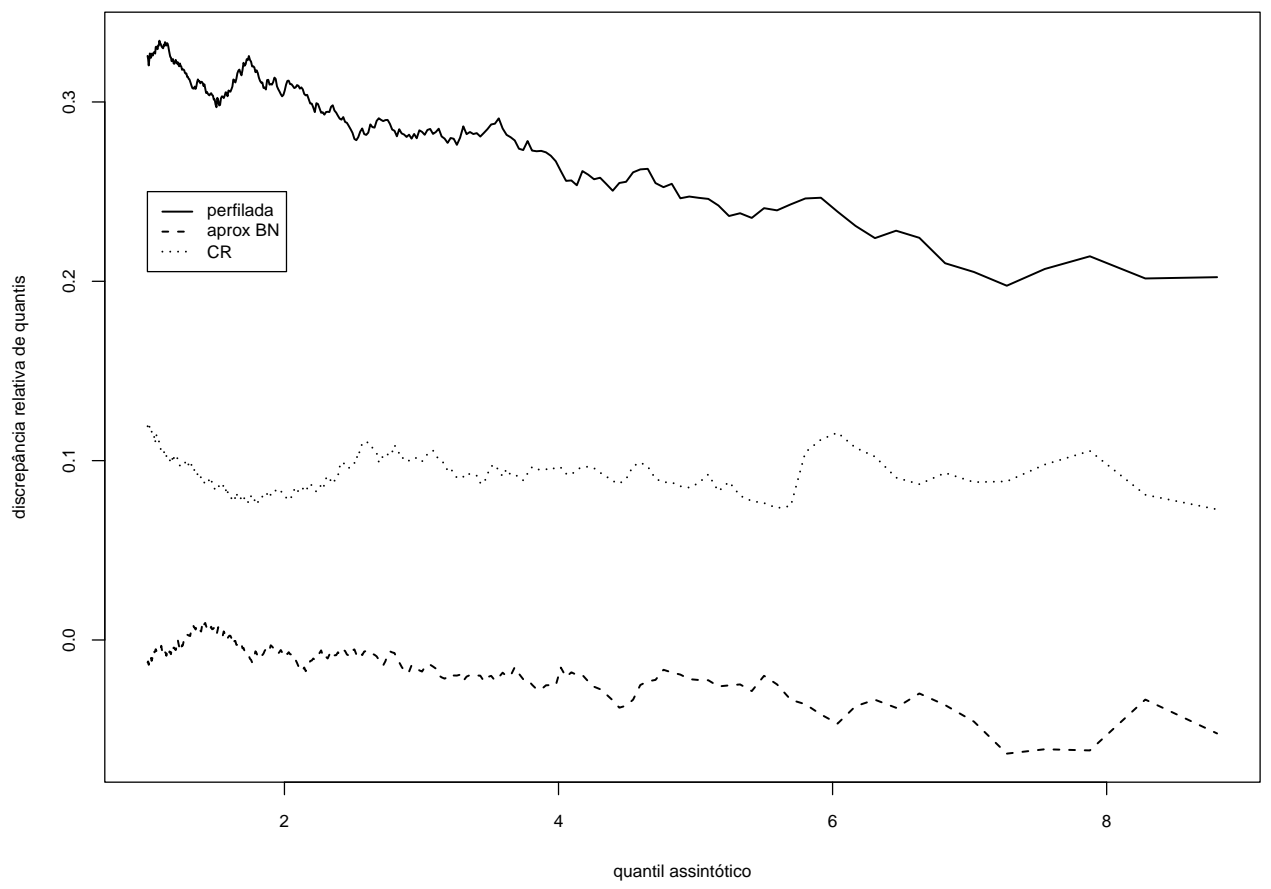

Figura 5.14. Gráfico das discrepâncias relativas de quantis, distribuição valor extremo, $\eta(\mathbf{x})=\phi_{1}+\phi_{2} x_{2}, \mathcal{H}_{0}: \beta=\sigma^{-1}=5$, dados censurados tipo II, $(n, r)=(20,10)$.

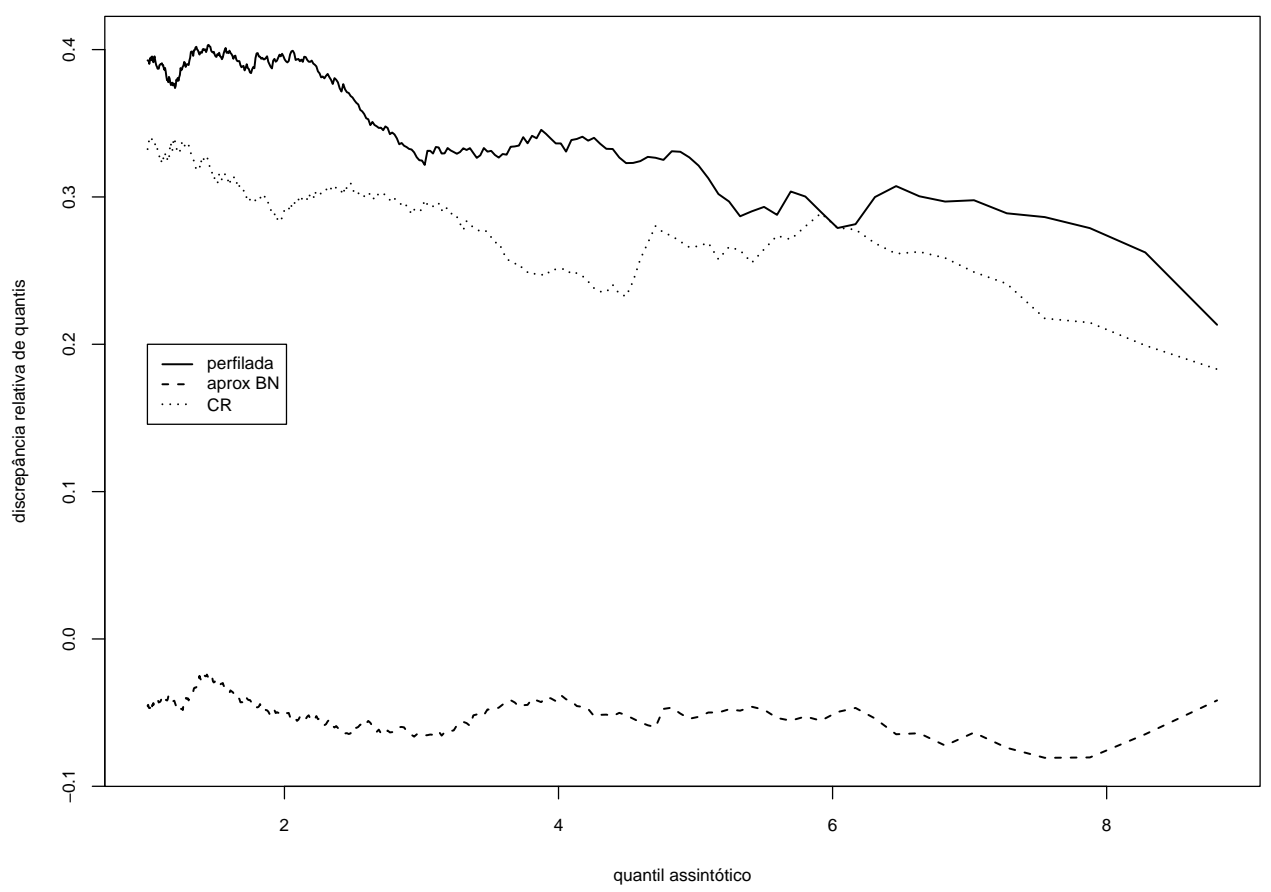

para dados completos, que a probabilidade do erro tipo I não depende de $\phi$ e nem do valor 
Figura 5.15. Gráfico das discrepâncias relativas de quantis, distribuição valor extremo, $\eta(\mathbf{x})=\phi_{1}+\phi_{2} x_{2}+\phi_{3} x_{3}+\phi_{4} x_{4}, \mathcal{H}_{0}: \beta=\sigma^{-1}=5$, dados censurados tipo II, $(n, r)=(20,15)$.

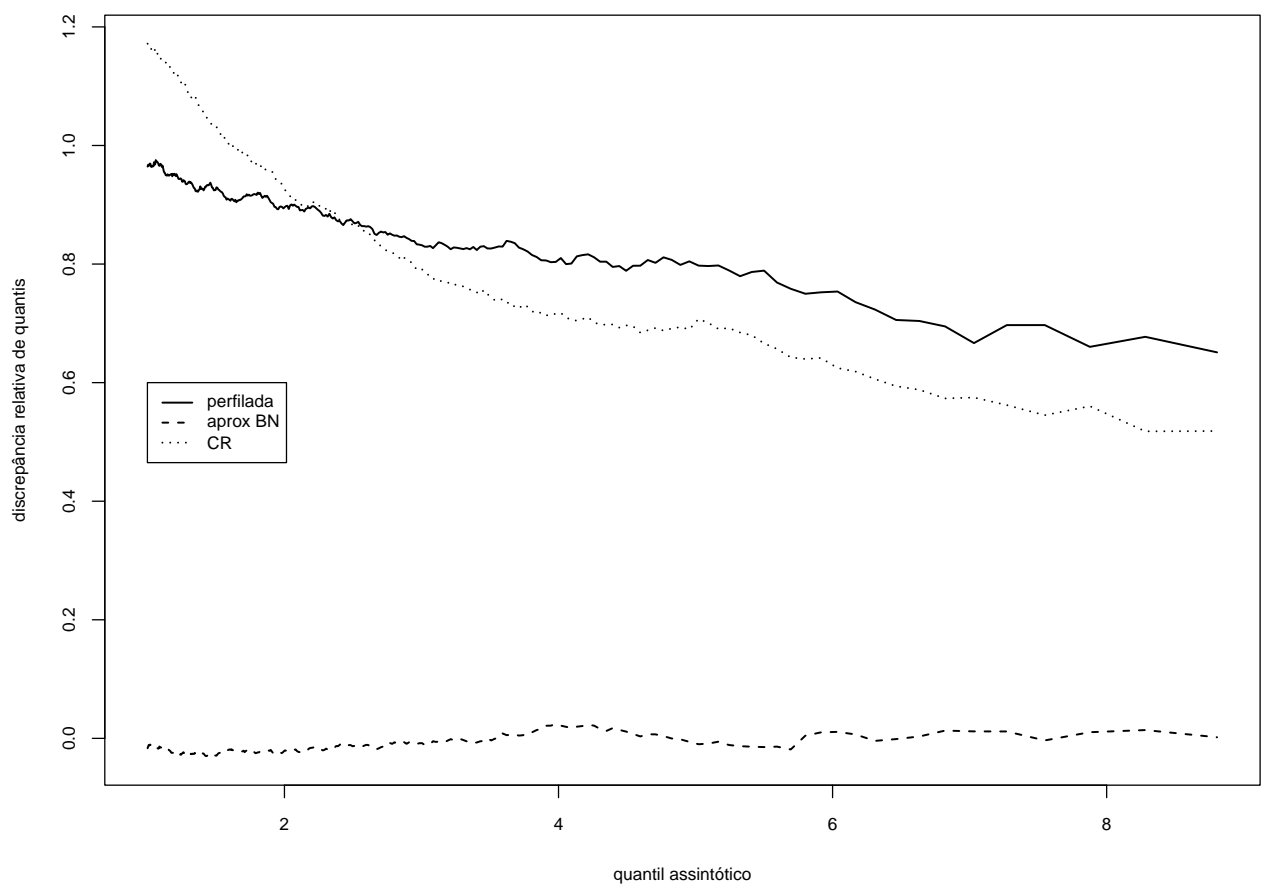

Figura 5.16. Gráfico das discrepâncias relativas de quantis, distribuição valor extremo, $\eta(\mathbf{x})=\phi_{1}+\phi_{2} x_{2}+\phi_{3} x_{3}+\phi_{4} x_{4}, \mathcal{H}_{0}: \beta=\sigma^{-1}=5$, dados censurados tipo II, $(n, r)=(20,10)$.

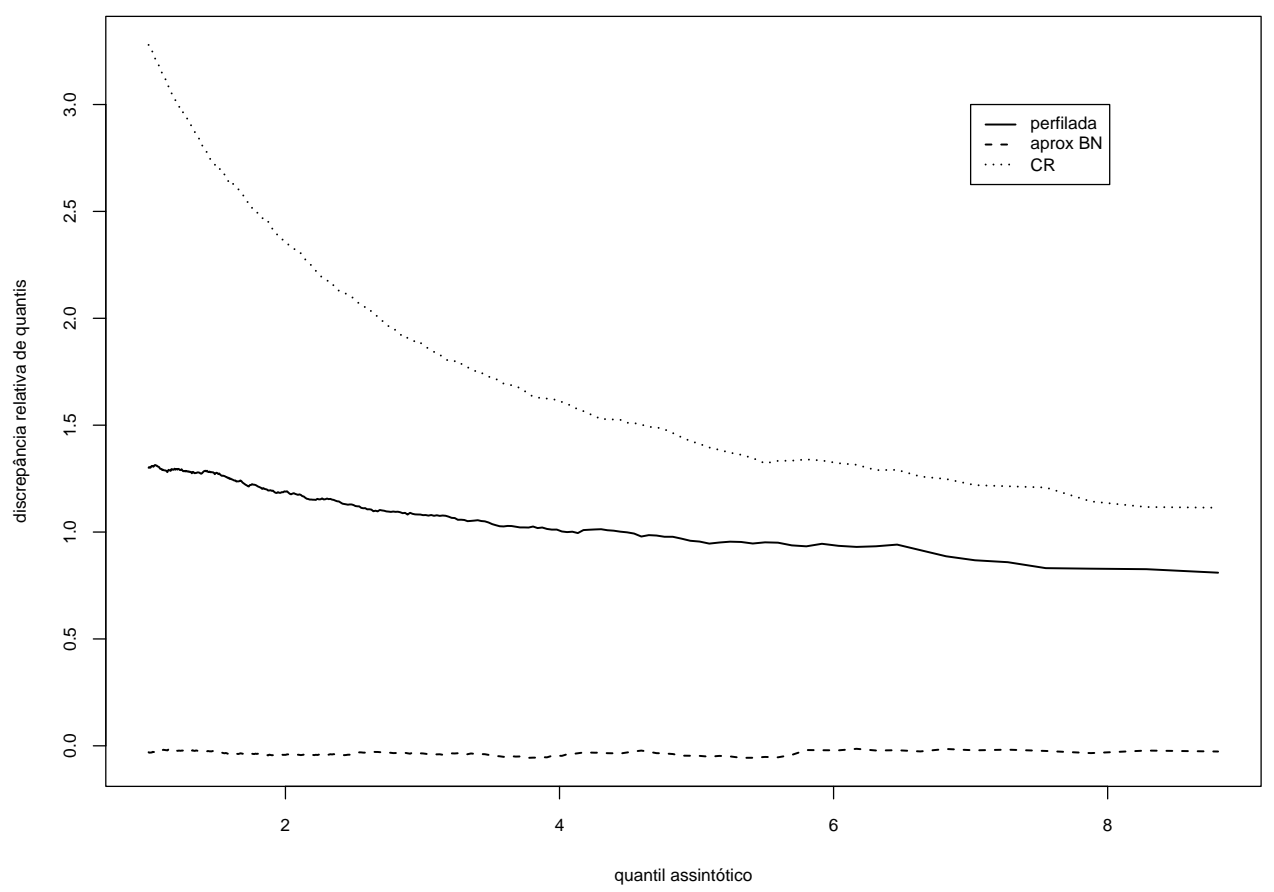

de $\sigma$ fixado na hipótese nula, varia apenas com o tamanho amostral $n$ e com a matriz de 
especificação do modelo $X$. Este resultado não vale para dados censurados. Para este caso, além dos testes da razão de verossimilhanças em que $\mathcal{H}_{0}: \beta=5$, também foram realizados testes em que $\mathcal{H}_{0}: \beta=0.5$ e $\mathcal{H}_{0}: \beta=1$. Resultados numéricos são apresentados a seguir de forma resumida para dados censurados do tipo II.

Tabela 5.17. Taxas de rejeição da hipótese nula de diferentes testes da razão de verossimilhanças, distribuição valor extremo, $\eta(\mathbf{x})=\phi_{1}+\phi_{2} x_{2}$, dados censurados tipo II.

\begin{tabular}{|c|c|c|c|c|c|c|c|}
\hline & & \multicolumn{3}{|c|}{$\mathcal{H}_{0}: \beta=0.5$} & \multicolumn{3}{|c|}{$\mathcal{H}_{0}: \beta=1$} \\
\hline$(n, r)$ & nível nominal & $\ell$ & $\tilde{\ell}_{B N}$ & $\ell_{C R}$ & $\ell$ & $\tilde{\ell}_{B N}$ & $\ell_{C R}$ \\
\hline \multirow{6}{*}{$(20,15)$} & $15 \%$ & 19.590 & 14.700 & 20.000 & 19.550 & 14.780 & 19.790 \\
\hline & $10 \%$ & 14.020 & 9.870 & 14.190 & 13.920 & 9.710 & 13.930 \\
\hline & $5 \%$ & 7.740 & 5.070 & 7.660 & 7.730 & 4.940 & 7.430 \\
\hline & $1 \%$ & 2.160 & 0.960 & 1.840 & 2.090 & 0.990 & 1.810 \\
\hline & $0.1 \%$ & 0.250 & 0.070 & 0.250 & 0.270 & 0.060 & 0.250 \\
\hline & $0.05 \%$ & 0.130 & 0.060 & 0.150 & 0.150 & 0.020 & 0.150 \\
\hline \multirow{6}{*}{$(30,20)$} & $15 \%$ & 18.670 & 14.760 & 19.280 & 18.490 & 15.120 & 19.220 \\
\hline & $10 \%$ & 12.930 & 10.190 & 13.140 & 12.960 & 9.940 & 13.300 \\
\hline & $5 \%$ & 6.860 & 4.970 & 6.850 & 7.080 & 5.130 & 7.160 \\
\hline & $1 \%$ & 1.580 & 0.870 & 1.770 & 1.660 & 0.930 & 1.860 \\
\hline & $0.1 \%$ & 0.170 & 0.100 & 0.240 & 0.150 & 0.150 & 0.270 \\
\hline & $0.05 \%$ & 0.120 & 0.060 & 0.170 & 0.090 & 0.070 & 0.160 \\
\hline \multirow{6}{*}{$(20,10)$} & $15 \%$ & 20.450 & 14.500 & 26.100 & 20.660 & 14.140 & 25.810 \\
\hline & $10 \%$ & 14.790 & 9.660 & 19.150 & 14.600 & 9.220 & 19.080 \\
\hline & $5 \%$ & 8.560 & 4.800 & 11.070 & 8.050 & 4.610 & 11.370 \\
\hline & $1 \%$ & 2.280 & 0.930 & 3.350 & 2.250 & 0.930 & 3.100 \\
\hline & $0.1 \%$ & 0.330 & 0.100 & 0.440 & 0.400 & 0.130 & 0.450 \\
\hline & $0.05 \%$ & 0.170 & 0.070 & 0.250 & 0.270 & 0.080 & 0.270 \\
\hline \multirow{6}{*}{$(30,10)$} & $15 \%$ & 20.030 & 14.750 & 27.860 & 20.210 & 14.170 & 27.310 \\
\hline & $10 \%$ & 14.640 & 9.560 & 21.010 & 14.720 & 9.520 & 20.460 \\
\hline & $5 \%$ & 8.630 & 4.520 & 12.520 & 8.260 & 4.410 & 12.620 \\
\hline & $1 \%$ & 2.000 & 0.970 & 3.470 & 1.990 & 0.890 & 3.500 \\
\hline & $0.1 \%$ & 0.420 & 0.090 & 0.560 & 0.430 & 0.120 & 0.520 \\
\hline & $0.05 \%$ & 0.240 & 0.040 & 0.320 & 0.240 & 0.050 & 0.280 \\
\hline
\end{tabular}

Considerando o modelo de regressão com uma covariada, a Tabela 5.17 apresenta taxas de rejeição sob as hipóteses $\mathcal{H}_{0}: \beta=0.5$ e $\mathcal{H}_{0}: \beta=1$ de diferentes testes da razão de verossimilhanças com vários níveis de significância. Como observado anteriormente, o teste baseado em $\tilde{\ell}_{B N}$ foi aquele que apresentou taxas de rejeição mais próximas aos níveis nominais. Os testes baseados em $\ell_{C R}$ e na verossimilhança original foram bastante liberais. As taxas não diferiram tanto daquelas apresentadas no teste da hipótese $\mathcal{H}_{0}: \beta=5$.

As médias e variâncias empíricas das estatísticas de teste são apresentadas na Tabela 5.18. Note que os valores associados a $\tilde{\ell}_{B N}$ apresentaram-se mais próximos aos valores assintóticos. 
Tabela 5.18. Médias e variâncias amostrais de diferentes estatísticas de teste e os valores assintóticos destas, distribuição valor extremo, $\eta(\mathbf{x})=\phi_{1}+\phi_{2} x_{2}$, dados censurados tipo II.

\begin{tabular}{ccccccccc}
\hline & & \multicolumn{3}{c}{$\mathcal{H}_{0}: \beta=0.5$} & \multicolumn{3}{c}{$\mathcal{H}_{0}: \beta=1$} \\
\hline \multirow{2}{*}{$(n, r)$} & momento & $\chi_{1}^{2}$ & $\ell$ & $\tilde{\ell}_{B N}$ & $\ell_{C R}$ & $\ell$ & $\tilde{\ell}_{B N}$ & $\ell_{C R}$ \\
\hline \multirow{2}{*}{$(20,15)$} & média & 1 & 1.237 & 1.002 & 1.239 & 1.237 & 0.996 & 1.223 \\
& variância & 2 & 3.022 & 1.989 & 2.869 & 2.997 & 1.961 & 2.804 \\
\hline \multirow{2}{*}{$(30,20)$} & média & 1 & 1.164 & 1.004 & 1.209 & 1.174 & 1.011 & 1.209 \\
& variância & 2 & 2.596 & 1.956 & 2.797 & 2.671 & 1.998 & 2.796 \\
\hline \multirow{2}{*}{$(20,10)$} & média & 1 & 1.291 & 0.977 & 1.552 & 1.285 & 0.972 & 1.542 \\
& variância & 2 & 3.349 & 1.965 & 4.030 & 3.356 & 1.956 & 3.983 \\
\hline \multirow{2}{*}{$(30,10)$} & média & 1 & 1.280 & 0.978 & 1.645 & 1.271 & 0.968 & 1.627 \\
& variância & 2 & 3.205 & 1.922 & 4.378 & 3.223 & 1.907 & 4.304 \\
\hline
\end{tabular}

Os resultados das Tabelas 5.17 e 5.18 sugerem que a distribuição nula da estatística de teste baseada nesta função é aquela que melhor aproxima-se da distribuição $\chi_{1}^{2}$.

Considerando observações independentes e identicamente distribuídas, o ajuste proposto por Cox e Reid $(1987,1989)$ para a verossimilhança perfilada e usado por Yang e Xie (2003) para derivar $\ell_{C R}$ apresentou ótimos resultados, tanto em termos de estimação de $\beta$ quanto em termos de testes de hipóteses sobre tal parâmetro, conforme a Seção 5.2.1.3. Naquele caso, o parâmetro de perturbação era unidimensional e a ortogonalidade entre este e o parâmetro de interesse, necessária para obter tal ajuste, foi obtida com dados completos e usada também para derivar $\ell_{C R}$ no caso de dados censurados.

Nesta seção, as observações não são identicamente distribuídas, pois é considerado um modelo de regressão para $\alpha$, o parâmetro de escala da distribuição de Weibull. Assim, o parâmetro de incômodo não é mais unidimensional. Derivando de forma análoga a parametrização ortogonal proposta por Yang e Xie (2003), observa-se que os resultados relativos a $\ell_{C R}$ deterioram-se à medida que aumentam o número de parâmetros de incômodo e a presença de censura nos dados. Possivelmente, este mal desempenho deve-se ao fato da utilização de uma parametrização que não é ortogonal para dados censurados, pois lembre que Yang e Xie (2003) propuseram parametrizações ortogonais sob dados completos para um parâmetro de incômodo. Assim, ao considerar dois ou mais parâmetros de perturbação, o desempenho de tal ajuste sobre a verossimilhança perfilada não foi o mesmo. Vale lembrar que o ajuste de Cox e Reid $(1987,1989)$ não é invariante sob reparametrizações que preservam o parâmetro de interesse (ver Seção 2.5). Além disso, em geral, para este ajuste, o viés da informação é de ordem $O(1)$, prejudicando seu desempenho em testes assintóticos, como razão de verossimilhanças, Wald e escore de Rao. Por outro lado, vale destacar o ótimo desempenho da aproximação $\tilde{\ell}_{B N}$, que não requer uma estatística ancilar para seu cálculo, diferentemente de $\ell_{B N}$, e nem a ortogonalidade entre os parâmetros de interesse e de perturbação.

Agora considere dados com presença de censura do tipo I. Diferentemente dos dados com censura do tipo II, não é possível fixar o número de falhas numa amostra Monte Carlo, ou seja, a proporção de observações censuradas. 
No caso de observações independentes e identicamente distribuídas, supondo uma proporção (probabilidade) de censura desejada $\left(p_{0}\right)$, foi calculado, usando a função distribuição do tempo de falha, o tempo de censura $(c)$ necessário para que tal proporção fosse observada,

$$
c=\alpha\left(-\log p_{0}\right)^{1 / \beta},
$$

onde $\alpha$ e $\beta$ são os parâmetros da distribuição de Weibull.

No caso de observações independentes, não necessariamente identicamente distribuídas, a derivação desse tempo de censura de forma análoga não é possível. Assim, empiricamente, baseado em resultados de simulações, usou-se a seguinte expressão para $c$,

$$
c=\left[\frac{1}{n} \sum_{j=1}^{n} \alpha\left(\mathbf{x}_{j}\right)\right]\left(-\log p_{0}\right)^{1 / \beta} .
$$

Note que esta expressão, no caso de observações independentes e identicamente distribuídas, é igual àquela proposta anteriormente. Mais uma vez, como as amostras são de tamanhos finitos, em geral, tal proporção não é observada em cada uma das amostras, mas o valor médio das proporções de censura é calculado e comparado com a proporção de censura desejada.

Aqui, para $n=20$, são considerados dois níveis de censura $\left(p_{0}\right)$ : $25 \%$ e $50 \%$. Vale lembrar que os resultados são baseados em 10000 (dez mil) réplicas de Monte Carlo.

Diferentemente dos casos de dados completos e dados com presença de censura do tipo II, aqui as razões (estimador de $\sigma$ )/ $\sigma$ não são quantidades pivotais. Assim, os vieses relativos e os coeficientes de assimetria e de curtose dos estimadores dependem dos valores dos parâmetros, assim como as taxas de rejeição dos testes da razão de verossimilhanças.

Considerando inicialmente $25 \%$ de censura, para o modelo com uma covariada e $\beta=0.5$, o percentual de censura médio observado foi de $24.51 \%$, enquanto para $\beta=1$ e $\beta=5$, tais percentuais foram de $24.20 \%$ e $29.82 \%$, respectivamente. Os percentuais para o modelo com três regressores foram iguais a $23.44 \%, 23.10 \%$ e $30.23 \%$, respectivamente.

A Tabela 5.19 contém estatísticas descritivas de diferentes estimadores de $\beta$ para $p_{0}=$ $25 \%$. Observe que, para os dois modelos, os estimadores $\widehat{\tilde{\beta}}_{B N}$ e $\widehat{\beta}_{C R}$ apresentaram desempenhos superiores ao estimador $\widehat{\beta}$, tanto em termos de viés, quanto em termos de erro quadrático médio (EQM). Entre os dois, considerando o erro quadrático médio como medida de comparação, os resultados foram muito próximos, não cabendo qualquer destaque. Entretanto, observando o módulo das estimativas dos vieses relativos destes, na presença de dois parâmetros de incômodo, o estimador $\widehat{\beta}_{C R}$ apresenta valores menores para esta medida, enquanto na presença de quatro parâmetros de perturbação, para $\beta=0.5$ e $\beta=1$, os melhores resultados são apresentados por $\widehat{\tilde{\beta}}_{B N}$. Por exemplo, para $\beta=1$ e uma covariada, os módulos dos vieses relativos de $\widehat{\beta}, \widehat{\tilde{\beta}}_{B N}$ e $\widehat{\beta}_{C R}$ foram, respectivamente, iguais a $9.977 \%$, $2.438 \%$ e $0.860 \%$. Para o modelo com três covariadas, tais valores foram iguais a $18.851 \%$, $1.240 \%$ e $3.696 \%$, respectivamente. Os resultados relativos aos coeficientes de assimetria e 
Tabela 5.19. Medidas descritivas de diferentes estimadores de $\beta=\sigma^{-1}$, distribuição valor extremo, dados censurados tipo I, $n=20,25 \%$ de censura.

\begin{tabular}{|c|c|c|c|c|c|c|c|c|}
\hline \multicolumn{9}{|c|}{$\eta(\mathbf{x})=\phi_{1}+\phi_{2} x_{2}$} \\
\hline parâmetro & estimador & média & variância & viés & EQM & v.r.(\%) & assimetria & curtose \\
\hline \multirow{3}{*}{$\beta=0.5$} & $\widehat{\beta}$ & 0.549 & 0.018 & 0.049 & 0.020 & 9.772 & 0.973 & 4.917 \\
\hline & $\tilde{\tilde{\beta}}_{B N}$ & 0.511 & 0.016 & 0.011 & 0.016 & 2.285 & 0.969 & 4.917 \\
\hline & $\widehat{\beta}_{C R}$ & 0.494 & 0.015 & -0.006 & 0.015 & -1.243 & 0.950 & 4.821 \\
\hline \multirow{3}{*}{$\beta=1$} & $\widehat{\beta}$ & 1.100 & 0.070 & 0.100 & 0.080 & 9.977 & 0.911 & 4.546 \\
\hline & $\tilde{\tilde{\beta}}_{B N}$ & 1.024 & 0.062 & 0.024 & 0.062 & 2.438 & 0.915 & 4.600 \\
\hline & $\widehat{\beta}_{C R}$ & 0.991 & 0.060 & -0.009 & 0.060 & -0.860 & 0.893 & 4.488 \\
\hline \multirow{3}{*}{$\beta=5$} & $\widehat{\beta}$ & 5.646 & 1.922 & 0.646 & 2.339 & 12.911 & 1.043 & 5.063 \\
\hline & $\tilde{\tilde{\beta}}_{B N}$ & 5.171 & 1.589 & 0.171 & 1.618 & 3.411 & 1.000 & 4.911 \\
\hline & $\widehat{\beta}_{C R}$ & 5.138 & 1.640 & 0.138 & 1.659 & 2.764 & 1.014 & 4.938 \\
\hline \multicolumn{9}{|c|}{$\eta(\mathbf{x})=\phi_{1}+\phi_{2} x_{2}+\phi_{3} x_{3}+\phi_{4} x_{4}$} \\
\hline parâmetro & estimador & média & variância & viés & EQM & v.r. $(\%)$ & assimetria & curtose \\
\hline \multirow{3}{*}{$\beta=0.5$} & $\beta$ & 0.590 & 0.022 & 0.090 & 0.030 & 17.906 & 1.084 & 5.441 \\
\hline & $\widehat{\tilde{\beta}}_{B N}$ & 0.504 & 0.017 & 0.004 & 0.017 & 0.882 & 1.034 & 5.204 \\
\hline & $\widehat{\beta}_{C R}$ & 0.475 & 0.016 & -0.025 & 0.017 & -4.971 & 1.024 & 5.201 \\
\hline \multirow{3}{*}{$\beta=1$} & $\widehat{\beta}$ & 1.189 & 0.091 & 0.189 & 0.127 & 18.851 & 1.114 & 5.592 \\
\hline & $\tilde{\tilde{\beta}}_{B N}$ & 1.012 & 0.067 & 0.012 & 0.067 & 1.240 & 1.071 & 5.392 \\
\hline & $\widehat{\beta}_{C R}$ & 0.963 & 0.066 & -0.037 & 0.067 & -3.696 & 1.070 & 5.420 \\
\hline \multirow{3}{*}{$\beta=5$} & $\widehat{\beta}$ & 6.259 & 2.895 & 1.259 & 4.479 & 25.172 & 1.299 & 6.165 \\
\hline & $\tilde{\tilde{\beta}}_{B N}$ & 5.147 & 1.919 & 0.147 & 1.940 & 2.945 & 1.243 & 6.012 \\
\hline & $\widehat{\beta}_{C R}$ & 5.078 & 1.952 & 0.078 & 1.958 & 1.553 & 1.217 & 5.836 \\
\hline
\end{tabular}

de curtose dos estimadores para as várias situações são similares e razoavelmente viesados, se considerarmos os valores assintóticos, 0 e 3, respectivamente, como verdadeiros.

A Tabela 5.20 apresenta taxas de rejeição sob a hipótese nula de diferentes testes da razão de verossimilhanças com distintos níveis de significância. As médias e variâncias empíricas das estatísticas de teste são apresentadas na Tabela 5.21.

Entre as estatísticas de teste, a distribuição nula daquela baseada em $\tilde{\ell}_{B N}$ foi a melhor aproximada pela distribuição $\chi_{1}^{2}$. Isto pode ser comprovado pela proximidade entre as taxas de rejeição das hipóteses nulas e os níveis nominais (Tabela 5.20) e pela pequena diferença entre os momentos amostrais dessa estatística e os momentos assintóticos (Tabela 5.21). Aqui os valores tabelados dependeram da hipótese nula considerada.

Para o modelo com três covariadas, sob $\mathcal{H}_{0}: \beta=1$, as taxas de rejeição apresentadas pelos testes da razão de verossimilhanças baseados em $\ell, \tilde{\ell}_{B N}$ e $\ell_{C R}$ foram iguais a $17.490 \%$, $9.570 \%$ e $12.560 \%$, respectivamente, para o nível nominal de $10 \%$. Os correspondentes momentos empíricos das estatísticas de teste foram iguais a: 1.479, 0.981, 1.160 (média) e 4.242, 1.920, 2.589 (variância). Para o modelo com uma covariada, ainda sob $\mathcal{H}_{0}: \beta=1$, as taxas de rejeição foram iguais a 12.010\%, 9.490\% e 10.760\%, enquanto os momentos empíricos das 
Tabela 5.20. Taxas de rejeição da hipótese nula de diferentes testes da razão de verossimilhanças, distribuição valor extremo, $\beta=\sigma^{-1}$, dados censurados tipo I, $n=20,25 \%$ de censura.

\begin{tabular}{|c|c|c|c|c|c|c|c|}
\hline \multirow[b]{2}{*}{$\mathcal{H}_{0}:$} & \multirow[b]{2}{*}{ nível nominal } & \multicolumn{3}{|c|}{$\eta(\mathbf{x})=\phi_{1}+\phi_{2} x_{2}$} & \multicolumn{3}{|c|}{$\eta(\mathbf{x})=\phi_{1}+\phi_{2} x_{2}+\phi_{3} x_{3}+\phi_{4} x_{4}$} \\
\hline & & $\ell$ & $\tilde{\ell}_{B N}$ & $\ell_{C R}$ & $\ell$ & $\tilde{\ell}_{B N}$ & $\ell_{C R}$ \\
\hline \multirow{6}{*}{$\beta=0.5$} & $15 \%$ & 17.180 & 14.410 & 16.210 & 22.980 & 14.300 & 18.740 \\
\hline & $10 \%$ & 11.550 & 9.470 & 11.010 & 16.750 & 9.250 & 13.110 \\
\hline & $5 \%$ & 6.250 & 4.810 & 5.830 & 9.810 & 4.590 & 7.150 \\
\hline & $1 \%$ & 1.690 & 0.940 & 1.230 & 2.830 & 0.960 & 1.740 \\
\hline & $0.1 \%$ & 0.170 & 0.090 & 0.160 & 0.570 & 0.100 & 0.140 \\
\hline & $0.05 \%$ & 0.100 & 0.040 & 0.090 & 0.350 & 0.040 & 0.090 \\
\hline \multirow{6}{*}{$\beta=1$} & $15 \%$ & 17.490 & 14.510 & 16.380 & 23.900 & 14.480 & 18.490 \\
\hline & $10 \%$ & 12.010 & 9.490 & 10.760 & 17.490 & 9.570 & 12.560 \\
\hline & $5 \%$ & 6.470 & 4.870 & 5.570 & 10.710 & 4.920 & 6.790 \\
\hline & $1 \%$ & 1.630 & 0.920 & 1.180 & 3.180 & 0.900 & 1.590 \\
\hline & $0.1 \%$ & 0.210 & 0.070 & 0.140 & 0.630 & 0.070 & 0.130 \\
\hline & $0.05 \%$ & 0.090 & 0.030 & 0.060 & 0.360 & 0.040 & 0.070 \\
\hline \multirow{6}{*}{$\beta=5$} & $15 \%$ & 19.170 & 14.690 & 15.790 & 28.340 & 15.010 & 16.460 \\
\hline & $10 \%$ & 13.560 & 10.070 & 10.780 & 21.750 & 9.950 & 11.130 \\
\hline & $5 \%$ & 7.650 & 4.640 & 5.100 & 14.070 & 5.010 & 5.630 \\
\hline & $1 \%$ & 1.730 & 0.960 & 1.090 & 5.110 & 1.020 & 1.280 \\
\hline & $0.1 \%$ & 0.230 & 0.080 & 0.110 & 0.940 & 0.100 & 0.110 \\
\hline & $0.05 \%$ & 0.170 & 0.040 & 0.040 & 0.660 & 0.060 & 0.070 \\
\hline
\end{tabular}

Tabela 5.21. Médias e variâncias amostrais de diferentes estatísticas de teste e os valores assintóticos destas, distribuição valor extremo, $\beta=\sigma^{-1}$, dados censurados tipo I, $n=20,25 \%$ de censura.

\begin{tabular}{ccccccccc}
\hline & & \multicolumn{4}{c}{$\eta(\mathbf{x})=\phi_{1}+\phi_{2} x_{2}$} & \multicolumn{2}{c}{$\eta(\mathbf{x})=\phi_{1}+\phi_{2} x_{2}+\phi_{3} x_{3}+\phi_{4} x_{4}$} \\
\hline $\mathcal{H}_{0}:$ & momento & $\chi_{1}^{2}$ & $\ell$ & $\tilde{\ell}_{B N}$ & $\ell_{C R}$ & $\ell$ & $\tilde{\ell}_{B N}$ & $\ell_{C R}$ \\
\hline \multirow{2}{*}{$\beta=0.5$} & média & 1 & 1.122 & 0.989 & 1.072 & 1.418 & 0.967 & 1.181 \\
& variância & 2 & 2.557 & 1.955 & 2.270 & 3.928 & 1.883 & 2.672 \\
\hline \multirow{2}{*}{$\beta=1$} & média & 1 & 1.126 & 0.986 & 1.063 & 1.479 & 0.981 & 1.160 \\
& variância & 2 & 2.527 & 1.933 & 2.239 & 4.242 & 1.920 & 2.589 \\
\hline \multirow{2}{*}{$\beta=5$} & média & 1 & 1.206 & 0.988 & 1.032 & 1.762 & 0.991 & 1.062 \\
& variância & 2 & 2.833 & 1.923 & 2.106 & 5.599 & 1.979 & 2.238 \\
\hline
\end{tabular}

estatísticas de teste: 1.126, 0.986 e 1.063 (média) e 2.527, 1.933 e 2.239 (variância), respectivamente. Observe a deterioração da aproximação para a distribuição $\chi_{1}^{2}$ apresentada pela estatística baseada na verossimilhança original $\ell$.

As Figuras 5.17 e 5.18 apresentam os gráficos das curvas de discrepâncias relativas de quantis versus os correspondentes quantis assintóticos das estatísticas de teste. Refletindo os resultados tabelados, tanto para o modelo com uma covariada (Figura 5.17) quanto para o modelo com três covariadas (Figura 5.18), a estatística de teste baseada em $\tilde{\ell}_{B N}$ (aprox $\mathrm{BN})$ tem distribuição melhor aproximada pela $\chi_{1}^{2}$, comparativamente às demais. 
Figura 5.17. Gráfico das discrepâncias relativas de quantis, distribuição valor extremo, $\eta(\mathbf{x})=\phi_{1}+\phi_{2} x_{2}, \mathcal{H}_{0}: \beta=\sigma^{-1}=1$, dados censurados tipo I, $25 \%$ de censura.

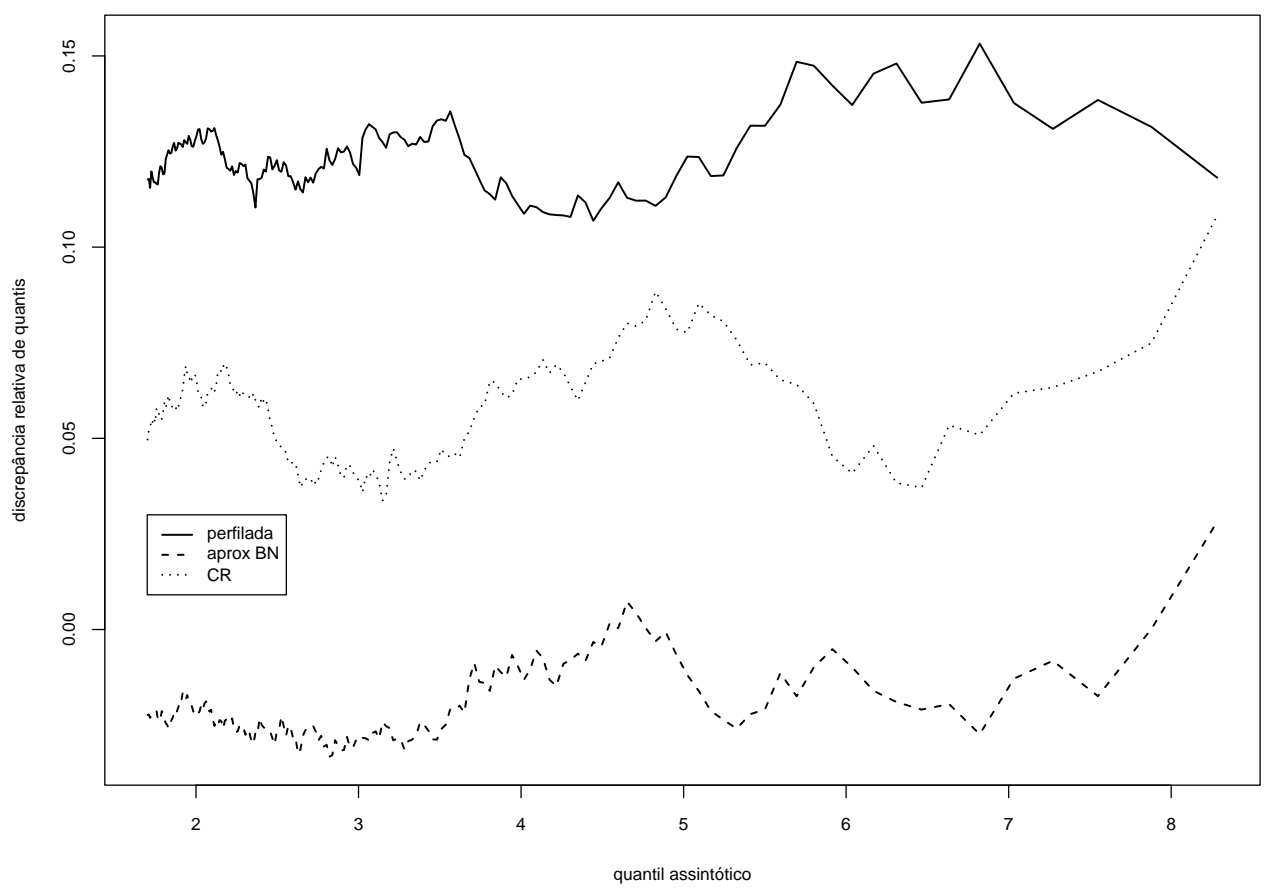

Figura 5.18. Gráfico das discrepâncias relativas de quantis, distribuição valor extremo, $\eta(\mathbf{x})=\phi_{1}+\phi_{2} x_{2}+\phi_{3} x_{3}+\phi_{4} x_{4}, \mathcal{H}_{0}: \beta=\sigma^{-1}=1$, dados censurados tipo I, $25 \%$ de censura.

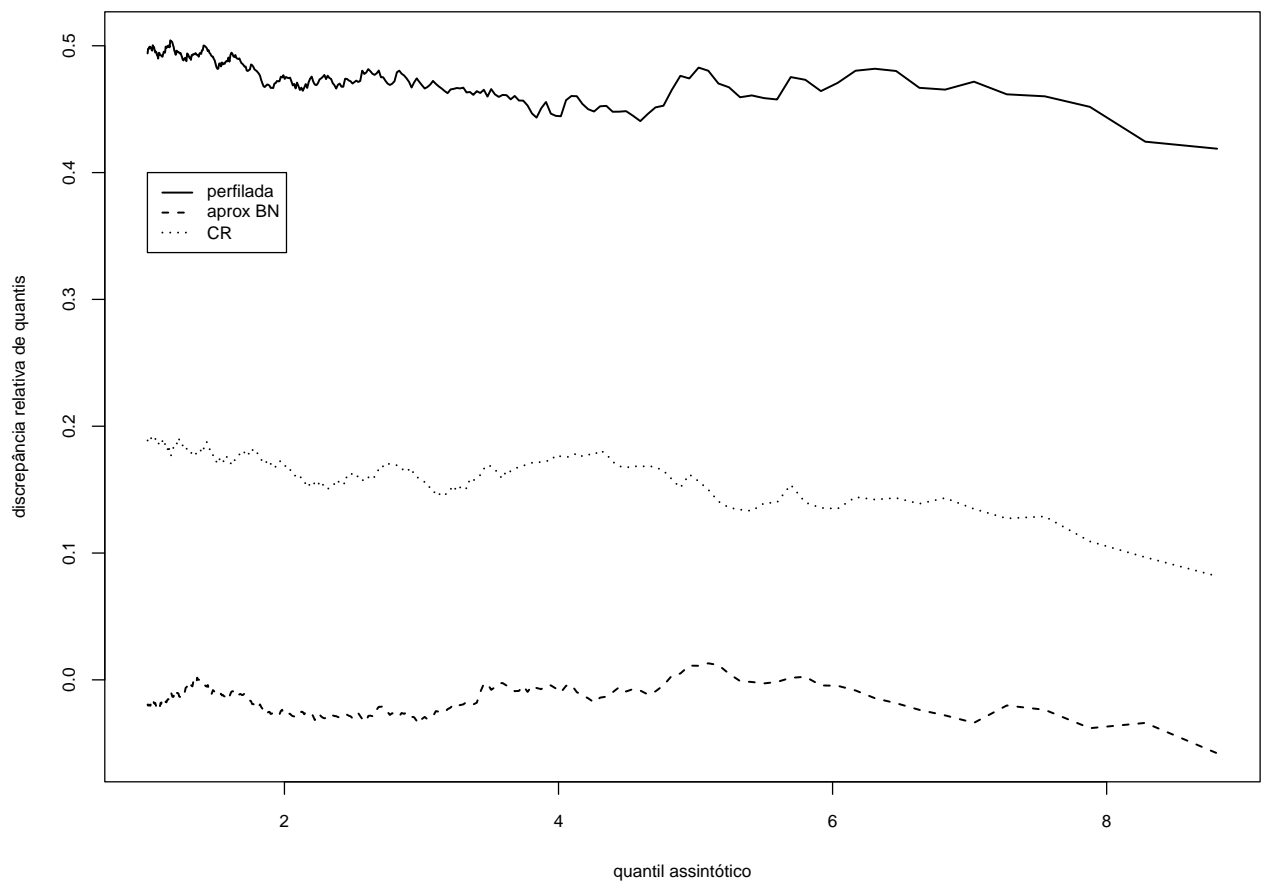


Tabela 5.22. Medidas descritivas de diferentes estimadores de $\beta=\sigma^{-1}$, distribuição valor extremo, dados censurados tipo I, $n=20,50 \%$ de censura.

\begin{tabular}{|c|c|c|c|c|c|c|c|c|}
\hline \multicolumn{9}{|c|}{$\eta(\mathbf{x})=\phi_{1}+\phi_{2} x_{2}$} \\
\hline parâmetro & estimador & média & variância & viés & EQM & v.r.(\%) & assimetria & curtose \\
\hline \multirow{3}{*}{$\beta=0.5$} & $\widehat{\beta}$ & 0.567 & 0.038 & 0.067 & 0.043 & 13.489 & 2.283 & 19.336 \\
\hline & $\tilde{\tilde{\beta}}_{B N}$ & 0.513 & 0.031 & 0.013 & 0.031 & 2.660 & 2.010 & 15.017 \\
\hline & $\widehat{\beta}_{C R}$ & 0.468 & 0.026 & -0.032 & 0.027 & -6.380 & 1.794 & 13.251 \\
\hline \multirow{3}{*}{$\beta=1$} & $\widehat{\beta}$ & 1.133 & 0.138 & 0.133 & 0.155 & 13.261 & 1.637 & 8.919 \\
\hline & $\tilde{\tilde{\beta}}_{B N}$ & 1.026 & 0.114 & 0.026 & 0.115 & 2.622 & 1.561 & 8.484 \\
\hline & $\widehat{\beta}_{C R}$ & 0.942 & 0.099 & -0.058 & 0.103 & -5.798 & 1.376 & 7.227 \\
\hline \multirow{3}{*}{$\beta=5$} & $\widehat{\beta}$ & 5.750 & 2.586 & 0.750 & 3.149 & 15.009 & 1.335 & 7.918 \\
\hline & $\widetilde{\tilde{\beta}}_{B N}$ & 5.167 & 2.054 & 0.167 & 2.082 & 3.341 & 1.260 & 7.386 \\
\hline & $\widehat{\beta}_{C R}$ & 5.094 & 2.135 & 0.094 & 2.144 & 1.889 & 1.286 & 7.466 \\
\hline \multicolumn{9}{|c|}{$\eta(\mathbf{x})=\phi_{1}+\phi_{2} x_{2}+\phi_{3} x_{3}+\phi_{4} x_{4}$} \\
\hline parâmetro & estimador & média & variância & viés & EQM & v.r. $(\%)$ & assimetria & curtose \\
\hline \multirow{3}{*}{$\beta=0.5$} & $\widehat{Q}$ & 0.607 & 0.044 & 0.107 & 0.055 & 21.303 & 2.036 & 14.206 \\
\hline & $\tilde{\tilde{\beta}}_{B N}$ & 0.490 & 0.030 & -0.010 & 0.030 & -2.010 & 2.343 & 23.653 \\
\hline & $\widehat{\beta}_{C R}$ & 0.407 & 0.023 & -0.093 & 0.032 & -18.664 & 1.244 & 6.933 \\
\hline \multirow{3}{*}{$\beta=1$} & $\widehat{\beta}$ & 1.217 & 0.159 & 0.217 & 0.206 & 21.703 & 1.630 & 8.713 \\
\hline & $\widehat{\tilde{\beta}}_{B N}$ & 0.985 & 0.107 & -0.015 & 0.107 & -1.470 & 1.564 & 9.085 \\
\hline & $\widehat{\beta}_{C R}$ & 0.848 & 0.091 & -0.152 & 0.114 & -15.158 & 1.266 & 6.653 \\
\hline \multirow{3}{*}{$\beta=5$} & $\widehat{\beta}$ & 6.471 & 4.260 & 1.471 & 6.425 & 29.428 & 2.162 & 15.907 \\
\hline & $\tilde{\beta}_{B N}$ & 5.113 & 2.495 & 0.113 & 2.508 & 2.269 & 1.765 & 11.124 \\
\hline & $\widehat{\beta}_{C R}$ & 5.003 & 2.562 & 0.003 & 2.562 & 0.054 & 1.782 & 11.438 \\
\hline
\end{tabular}

Aumentando o percentual de censura tipo I para 50\%, as Tabelas 5.22, 5.23 e 5.24 apresentam, respectivamente: estatísticas descritivas de diferentes estimadores; taxas de rejeição sob a hipótese nula de diferentes testes da razão de verossimilhanças com vários níveis de significância; médias e variâncias das estatísticas de teste, assim como seus valores assintóticos.

Para o modelo com uma covariada e $\beta=0.5$, o percentual de censura médio observado foi de $49.22 \%$, enquanto para $\beta=1$ e $\beta=5$, tais percentuais foram de $48.16 \%$ e $42.15 \%$, respectivamente. Os percentuais para o modelo com três regressores foram iguais a $47.68 \%$, $45.18 \%$ e $40.33 \%$, respectivamente.

O aumento da presença de censura nos dados acarreta as deteriorações das medidas de desempenho dos estimadores e das aproximações das distribuições das estatísticas da razão de verossimilhanças pela distribuição assintótica qui-quadrado. Note nas tabelas que as diferenças de desempenhos dos estimadores e das estatísticas de teste não são tão acentuadas quanto àquelas observadas no caso de dados com presença de censura do tipo II.

De acordo com os resultados da Tabela 5.22, para $\beta=0.5$ e $\beta=1$, o estimador $\widehat{\widetilde{\beta}}_{B N}$ apresentou, em módulo, vieses relativos consideravelmente inferiores aos demais. Para $\beta=5$, 
Tabela 5.23. Taxas de rejeição da hipótese nula de diferentes testes da razão de verossimilhanças, distribuição valor extremo, $\beta=\sigma^{-1}$, dados censurados tipo I, $n=20,50 \%$ de censura.

\begin{tabular}{|c|c|c|c|c|c|c|c|}
\hline \multirow[b]{2}{*}{$\mathcal{H}_{0}:$} & \multirow[b]{2}{*}{ nível nominal } & \multicolumn{3}{|c|}{$\eta(\mathbf{x})=\phi_{1}+\phi_{2} x_{2}$} & \multicolumn{3}{|c|}{$\eta(\mathbf{x})=\phi_{1}+\phi_{2} x_{2}+\phi_{3} x_{3}+\phi_{4} x_{4}$} \\
\hline & & $\ell$ & $\tilde{\ell}_{B N}$ & $\ell_{C R}$ & $\ell$ & $\tilde{\ell}_{B N}$ & $\ell_{C R}$ \\
\hline \multirow{6}{*}{$\beta=0.5$} & $15 \%$ & 16.920 & 13.760 & 18.150 & 20.940 & 12.950 & 27.310 \\
\hline & $10 \%$ & 11.520 & 9.200 & 12.450 & 15.200 & 8.560 & 20.690 \\
\hline & $5 \%$ & 6.140 & 4.390 & 6.660 & 8.660 & 4.370 & 12.470 \\
\hline & $1 \%$ & 1.330 & 0.790 & 1.750 & 2.300 & 0.920 & 4.090 \\
\hline & $0.1 \%$ & 0.240 & 0.080 & 0.210 & 0.370 & 0.090 & 0.790 \\
\hline & $0.05 \%$ & 0.170 & 0.040 & 0.110 & 0.260 & 0.050 & 0.450 \\
\hline \multirow{6}{*}{$\beta=1$} & $15 \%$ & 16.900 & 13.910 & 17.730 & 21.490 & 13.370 & 24.510 \\
\hline & $10 \%$ & 11.450 & 8.820 & 12.330 & 15.740 & 9.100 & 18.140 \\
\hline & $5 \%$ & 5.930 & 4.520 & 6.640 & 9.130 & 4.340 & 11.020 \\
\hline & $1 \%$ & 1.370 & 0.800 & 1.600 & 2.670 & 0.830 & 3.340 \\
\hline & $0.1 \%$ & 0.250 & 0.090 & 0.130 & 0.430 & 0.070 & 0.490 \\
\hline & $0.05 \%$ & 0.130 & 0.030 & 0.060 & 0.230 & 0.050 & 0.280 \\
\hline \multirow{6}{*}{$\beta=5$} & $15 \%$ & 18.820 & 14.050 & 15.540 & 29.360 & 14.510 & 17.130 \\
\hline & $10 \%$ & 13.120 & 8.950 & 10.230 & 22.680 & 9.750 & 11.630 \\
\hline & $5 \%$ & 7.300 & 4.480 & 5.440 & 14.650 & 4.800 & 6.140 \\
\hline & $1 \%$ & 1.730 & 0.860 & 1.170 & 5.230 & 1.030 & 1.430 \\
\hline & $0.1 \%$ & 0.270 & 0.070 & 0.110 & 1.110 & 0.100 & 0.130 \\
\hline & $0.05 \%$ & 0.160 & 0.050 & 0.060 & 0.800 & 0.050 & 0.080 \\
\hline
\end{tabular}

por este critério, o melhor desempenho foi do estimador $\widehat{\beta}_{C R}$.

Quanto aos testes da razão de verossimilhanças, baseando-se nos resultados das Tabelas 5.23 e 5.24, aquele cuja distribuição nula da estatística de teste melhor se aproximou da quiquadrado foi o baseado em $\tilde{\ell}_{B N}$, pois as taxas de rejeição e os momentos amostrais apresentaram-se, respectivamente, mais próximos dos níveis nominais e dos valores assintóticos destes.

Tabela 5.24. Médias e variâncias amostrais de diferentes estatísticas de teste e os valores assintóticos destas, distribuição valor extremo, $\beta=\sigma^{-1}$, dados censurados tipo I, $n=20,50 \%$ de censura.

\begin{tabular}{ccccccccc}
\hline & & \multicolumn{3}{c}{$\eta(\mathbf{x})=\phi_{1}+\phi_{2} x_{2}$} & \multicolumn{2}{c}{$\eta(\mathbf{x})=\phi_{1}+\phi_{2} x_{2}+\phi_{3} x_{3}+\phi_{4} x_{4}$} \\
\hline $\mathcal{H}_{0}:$ & momento & $\chi_{1}^{2}$ & $\ell$ & $\tilde{\ell}_{B N}$ & $\ell_{C R}$ & $\ell$ & $\tilde{\ell}_{B N}$ & $\ell_{C R}$ \\
\hline \multirow{2}{*}{$\beta=0.5$} & média & 1 & 1.091 & 0.953 & 1.161 & 1.297 & 0.922 & 1.661 \\
& variância & 2 & 2.472 & 1.841 & 2.654 & 3.406 & 1.797 & 4.807 \\
\hline \multirow{2}{*}{$\beta=1$} & média & 1 & 1.088 & 0.947 & 1.141 & 1.344 & 0.929 & 1.512 \\
& variância & 2 & 2.429 & 1.813 & 2.555 & 3.620 & 1.796 & 4.076 \\
\hline \multirow{2}{*}{$\beta=5$} & média & 1 & 1.198 & 0.962 & 1.038 & 1.816 & 0.987 & 1.101 \\
& variância & 2 & 2.804 & 1.836 & 2.132 & 6.040 & 1.990 & 2.448 \\
\hline
\end{tabular}

As Figuras 5.19 e 5.20 apresentam os gráficos das curvas de discrepâncias relativas de quantis versus os correspondentes quantis assintóticos das estatísticas de teste. Refletindo os resultados tabelados, tanto para o modelo com uma covariada (Figura 5.19) quanto para 
Figura 5.19. Gráfico das discrepâncias relativas de quantis, distribuição valor extremo, $\eta(\mathbf{x})=$ $\phi_{1}+\phi_{2} x_{2}, \mathcal{H}_{0}: \beta=\sigma^{-1}=1$, dados censurados tipo I, $50 \%$ de censura.

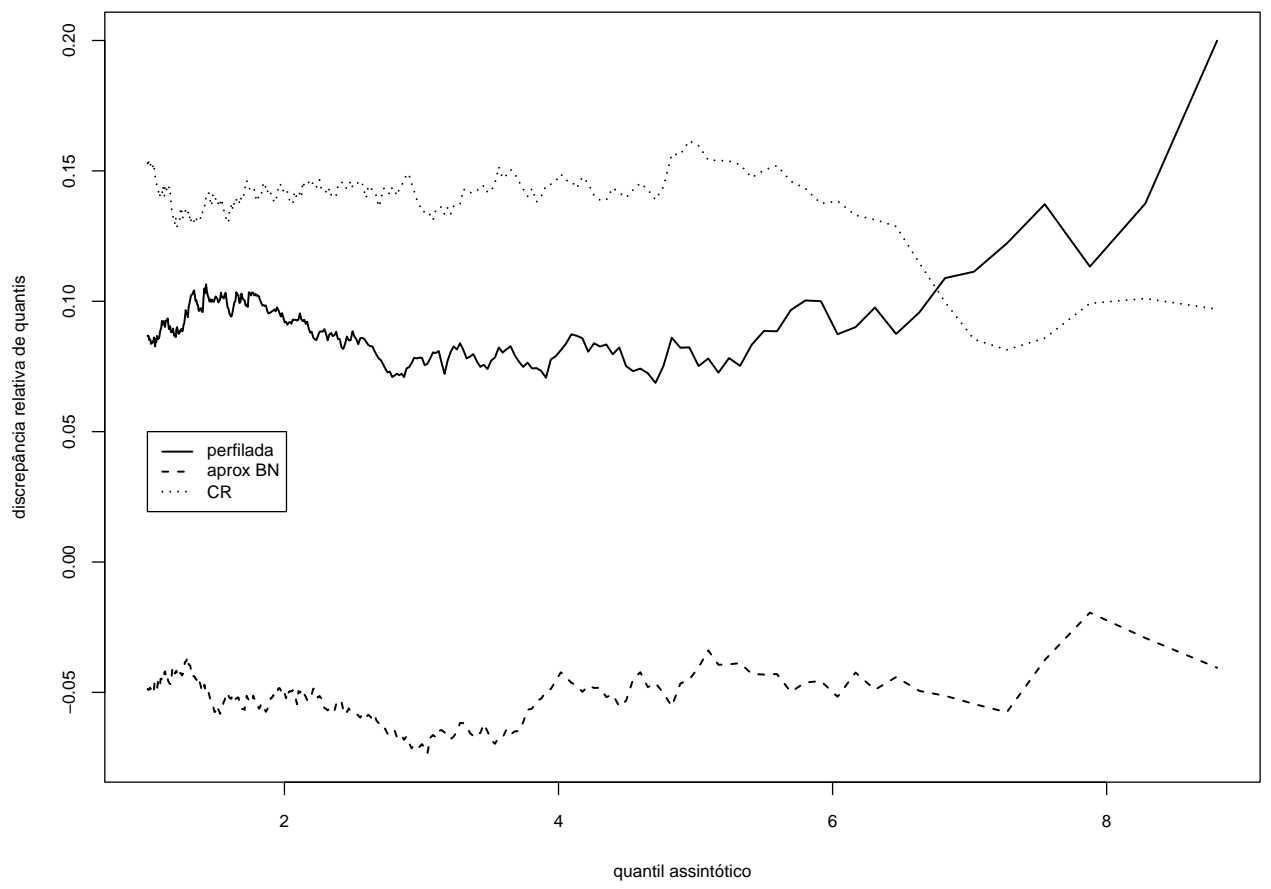

Figura 5.20. Gráfico das discrepâncias relativas de quantis, distribuição valor extremo, $\eta(\mathbf{x})=$ $\phi_{1}+\phi_{2} x_{2}+\phi_{3} x_{3}+\phi_{4} x_{4}, \mathcal{H}_{0}: \beta=\sigma^{-1}=1$, dados censurados tipo I, $50 \%$ de censura.

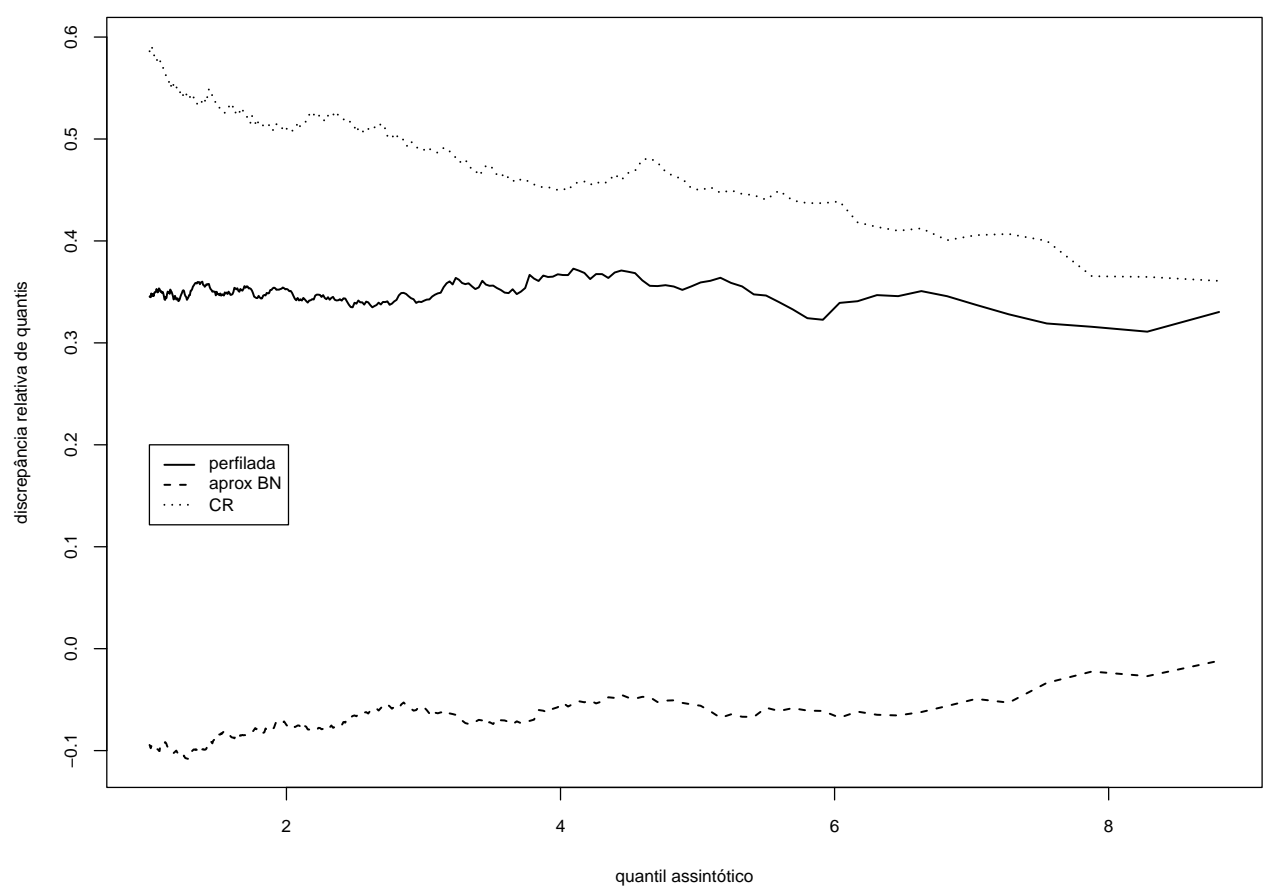

o modelo com três covariadas (Figura 5.20), a estatística de teste baseada em $\tilde{\ell}_{B N}$ (aprox 
BN) tem distribuição melhor aproximada pela $\chi_{1}^{2}$.

\subsubsection{CONCLUSÕES}

O estimador de máxima verossimilhança do parâmetro de forma $\left(\beta=\sigma^{-1}\right)$ da distribuição de Weibull é bastante viesado. Na presença de mais de um parâmetro de incômodo, o estimador que apresentou, de forma consistente, resultados bastante satisfatórios foi $\widehat{\tilde{\beta}}_{B N}$. O desempenho do estimador $\widehat{\beta}_{C R}$ deteriorou-se gradativamente com os aumentos da presença de censura nos dados e da quantidade de parâmetros de perturbação, possivelmente causado pela não ortogonalidade dos parâmetros de interesse e de incômodo. Lembre que tal parametrização foi obtida a partir da verossimilhança de uma amostra sem censura e representa uma adaptação daquela derivada no caso de observações independentes e identicamente distribuídas. Em algumas situações, o estimador de máxima verossimilhança original apresentou, em módulo, viés inferior àquele estimado para $\widehat{\beta}_{C R}$.

Entre os testes da razão de verossimilhanças, aqueles baseados na verossimilhança original $\ell$ e na versão ajustada da verossimilhança perfilada denotada por $\ell_{C R}$ foram sempre liberais, rejeitando a hipótese nula além do esperado (nível nominal). Entre estes, vale mencionar que o teste original apresentou, em algumas situações, taxas de rejeição mais próximas aos níveis nominais que o teste corrigido. Por outro lado, as taxas de rejeição do teste baseado em $\tilde{\ell}_{B N}$ sempre foram próximas aos níveis nominais.

Portanto, em geral, considerando observações independentes, mas não identicamente distribuídas, entre as verossimilhanças utilizadas neste capítulo para realizar inferências sobre o parâmetro de forma da distribuição de Weibull, aquela que produziu o melhor estimador de máxima verossimilhança e cuja distribuição nula da estatística de teste é melhor aproximada pela distribuição assintótica qui-quadrado foi a verossimilhança perfilada ajustada denotada por $\tilde{\ell}_{B N}$ (ver Seção 2.11.4).

\subsection{TESTES SOBRE OS COEFICIENTES DA REGRESSÃO}

\subsubsection{INTRODUÇÃO}

Da mesma forma que foram realizados testes da razão de verossimilhanças sobre $\beta=\sigma^{-1}$, podem ser realizados testes sobre os coeficientes da regressão $\phi=\left(\phi_{1}, \ldots, \phi_{p}\right)^{\top}$.

O ajuste para a verossimilhança perfilada proposto por Cox e Reid $(1987)\left(\ell_{C R}\right)$ requer a ortogonalidade entre os parâmetros de interesse e de incômodo. Devido à dificuldade em se derivar tal parametrização quando o parâmetro de interesse é parte do vetor $\phi$, aqui são apresentados apenas resultados de testes da razão de verossimilhanças baseados na verossi- 
milhança original e na aproximação para o ajuste de Barndorff-Nielsen denotada por $\tilde{\ell}_{B N}$, que apresentou resultados bastante satisfatórios na seção anterior. São considerados dados completos e dados censurados.

Dessa forma, fazendo $\phi=\left(\phi^{(1)^{\top}}, \phi^{(2)^{\top}}\right)^{\top}$, onde $\phi^{(1)}$ é um vetor $k \times 1(k<p)$, hipóteses da forma $\mathcal{H}_{0}: \phi^{(1)}=\phi_{0}^{(1)}$ podem ser avaliadas usando o teste da razão de verossimilhanças:

$$
R V=2\left\{\ell\left(\widehat{\sigma}, \widehat{\phi^{(1)}}, \widehat{\phi^{(2)}}\right)-\ell\left(\widehat{\sigma_{0}}, \phi_{0}^{(1)}, \widehat{\phi_{0}^{(2)}}\right)\right\}
$$

onde $\left(\widehat{\sigma}, \widehat{\phi^{(1)}}, \widehat{\phi^{(2)}}\right)$ é a estimativa de máxima verossimilhança do vetor $(\sigma, \phi), \widehat{\sigma_{0}}$ e $\widehat{\phi_{0}^{(2)}}$ são as estimativas de máxima verossimilhança de $\sigma$ e de $\phi^{(2)}$ sob a hipótese nula, respectivamente. A hipótese alternativa é $\mathcal{H}_{1}: \phi^{(1)} \neq \phi_{0}^{(1)}$. Lembre que, assintoticamente, $R V \sim \chi_{k}^{2}$. Aqui,

$$
\ell(\sigma, \phi)=-r \log \sigma+\sum_{j \in \bar{C}} \frac{y_{j}-\mathbf{x}_{j} \phi}{\sigma}-\sum_{j=1}^{n} \exp \left(\frac{y_{j}-\mathbf{x}_{j} \phi}{\sigma}\right)
$$

é a verossimilhança original, onde $r$ é o número observado de tempos de falhas. No caso de dados completos, $r=n$.

Nesta seção, segundo notação usada ao longo do Capítulo $2, \mu=\phi^{(1)}$ (interesse) e $\nu=$ $\left(\sigma, \phi^{(2)}\right)$ (incômodo). A aproximação para $\ell_{B N}$ baseada numa estatística aproximadamente ancilar é dada por (ver Seção 2.11.4)

$$
\tilde{\ell}_{B N}(\mu)=\ell_{p}(\mu)+\frac{1}{2} \log \left|j_{\nu \nu}\left(\mu, \widehat{\nu}_{\mu}\right)\right|-\log \left|\ell_{\nu ; y}\left(\mu, \widehat{\nu}_{\mu}\right) \widehat{V}_{\nu}\right|
$$

A fim de obter $\widehat{\nu}_{\mu}$, sejam

$$
\frac{\partial \ell(\sigma, \phi)}{\partial \sigma}=-\frac{r}{\sigma}-\frac{1}{\sigma} \sum_{j \in \bar{C}}\left(\frac{y_{j}-\mathbf{x}_{j} \phi}{\sigma}\right)+\frac{1}{\sigma} \sum_{j=1}^{n}\left(\frac{y_{j}-\mathbf{x}_{j} \phi}{\sigma}\right) \exp \left(\frac{y_{j}-\mathbf{x}_{j} \phi}{\sigma}\right)
$$

$\mathrm{e}$

$$
\frac{\partial \ell(\sigma, \phi)}{\partial \phi_{t}^{(2)}}=-\frac{1}{\sigma} \sum_{j \in \bar{C}} x_{j t}+\frac{1}{\sigma} \sum_{j=1}^{n} x_{j t} \exp \left(\frac{y_{j}-\mathbf{x}_{j} \phi}{\sigma}\right)
$$

onde $\phi_{t}^{(2)}$ é o $t$-ésimo componente do vetor $\phi^{(2)}$, que tem dimensão $(p-k) \times 1$. Para $j=1, \ldots, n$, seja $\eta\left(\mathbf{x}_{j}\right)=\mathbf{x}_{j} \phi=\mathbf{x}_{j}^{(1)} \phi^{(1)}+\mathbf{x}_{j}^{(2)} \phi^{(2)}$. Assim, o conjunto das derivadas relativas aos componentes de $\phi^{(2)}$ é dado pelo vetor

$$
\frac{\partial \ell(\sigma, \phi)}{\partial \phi^{(2)}}=-\frac{1}{\sigma} \sum_{j \in \bar{C}} \mathbf{x}_{j}^{(2)^{\top}}+\frac{1}{\sigma} \sum_{j=1}^{n} \exp \left(\frac{y_{j}-\mathbf{x}_{j} \phi}{\sigma}\right) \mathbf{x}_{j}^{(2)^{\top}}
$$

Igualando tais derivadas a zero, $\widehat{\sigma}_{\phi^{(1)}}$ e $\widehat{\phi^{(2)}}{ }_{\phi^{(1)}}$ são as raízes do sistema de $(p-k)+1$ equações. Note que estas quantidades não têm formas fechadas e, por conseqüência, não é 
possível expressar $\ell_{p}\left(\phi^{(1)}\right)$ como função apenas de $\phi^{(1)}$. Assim, a verossimilhança perfilada original é igual a $\ell=\ell(\sigma, \phi)$ sujeita às $(p-k)+1$ restrições definidas por

$$
\frac{\partial \ell}{\partial \sigma}=0 \quad \text { e } \quad \frac{\partial \ell}{\partial \phi^{(2)}}=0_{(p-k) \times 1}
$$

A aproximação $\tilde{\ell}_{B N}(\mu)$ depende da matriz de informação observada relativa aos parâmetros de incômodo. As derivadas de ordem dois da função $\ell(\sigma, \phi)$ são dadas por

$$
\begin{gathered}
\frac{\partial^{2} \ell}{\partial \sigma^{2}}=\frac{r}{\sigma^{2}}+\frac{2}{\sigma^{2}} \sum_{j \in \bar{C}}\left(\frac{y_{j}-\mathbf{x}_{j} \phi}{\sigma}\right)-\frac{2}{\sigma^{2}} \sum_{j=1}^{n}\left(\frac{y_{j}-\mathbf{x}_{j} \phi}{\sigma}\right) \exp \left(\frac{y_{j}-\mathbf{x}_{j} \phi}{\sigma}\right) \\
-\frac{1}{\sigma^{2}} \sum_{j=1}^{n}\left(\frac{y_{j}-\mathbf{x}_{j} \phi}{\sigma}\right)^{2} \exp \left(\frac{y_{j}-\mathbf{x}_{j} \phi}{\sigma}\right) \\
\frac{\partial^{2} \ell}{\partial \phi^{(2)^{\top}} \partial \phi^{(2)}}=-\frac{1}{\sigma^{2}} \sum_{j=1}^{n} \exp \left(\frac{y_{j}-\mathbf{x}_{j} \phi}{\sigma}\right) \mathbf{x}_{j}^{(2)^{\top}} \mathbf{x}_{j}^{(2)}
\end{gathered}
$$

e

$$
\begin{gathered}
\frac{\partial^{2} \ell}{\partial \sigma \partial \phi^{(2)}}=\frac{1}{\sigma^{2}} \sum_{j \in \bar{C}} \mathbf{x}_{j}^{(2)^{\top}}-\frac{1}{\sigma^{2}} \sum_{j=1}^{n} \exp \left(\frac{y_{j}-\mathbf{x}_{j} \phi}{\sigma}\right) \mathbf{x}_{j}^{(2)^{\top}} \\
-\frac{1}{\sigma^{2}} \sum_{j=1}^{n}\left(\frac{y_{j}-\mathbf{x}_{j} \phi}{\sigma}\right) \exp \left(\frac{y_{j}-\mathbf{x}_{j} \phi}{\sigma}\right) \mathbf{x}_{j}^{(2)^{\top}}
\end{gathered}
$$

Desse modo,

$$
j_{\nu \nu}(\sigma, \phi)=\left(\begin{array}{cc}
-\partial^{2} \ell / \partial \sigma^{2} & -\left(\partial \ell / \partial \sigma \partial \phi^{(2)}\right)^{\top} \\
-\partial \ell / \partial \sigma \partial \phi^{(2)} & -\partial \ell / \partial \phi^{(2)^{\top}} \partial \phi^{(2)}
\end{array}\right)
$$

onde $\nu=\left(\sigma, \phi^{(2)}\right) . j_{\nu \nu}\left(\mu, \widehat{\nu}_{\mu}\right)$ é igual a $j_{\nu \nu}(\sigma, \phi)$ sujeita às $(p-k)+1$ restrições definidas anteriormente.

Por último, a expressão da função $\tilde{\ell}_{B N}(\mu)$ depende de $\ell_{\nu ; y}\left(\mu, \widehat{\nu}_{\mu}\right)$ e de $\widehat{V}_{\nu}$, definidos na Seção 2.11.4. $\ell_{\nu ; y}\left(\mu, \widehat{\nu}_{\mu}\right)$ é uma matriz $(p-k+1) \times n$ com $j$-ésima coluna dada por

$$
\left(\begin{array}{c}
-\frac{1}{\sigma^{2}} I_{(j \in \bar{C})}+\frac{1}{\sigma^{2}} \exp \left(\frac{y_{j}-\mathbf{x}_{j} \phi}{\sigma}\right)+\frac{1}{\sigma^{2}}\left(\frac{y_{j}-\mathbf{x}_{j} \phi}{\sigma}\right) \exp \left(\frac{y_{j}-\mathbf{x}_{j} \phi}{\sigma}\right) \\
\frac{1}{\sigma^{2}} \exp \left(\frac{y_{j}-\mathbf{x}_{j} \phi}{\sigma}\right) \mathbf{x}_{j}^{(2)^{\top}}
\end{array}\right)
$$

sujeita às $(p-k)+1$ restrições citadas anteriormente. Aqui $\mathbb{I}_{(j \in \bar{C})}=1$ se $y_{j}$ for uma observação não-censurada, ou seja, se $j \in \bar{C}$. Caso contrário, esta função será nula. $\widehat{V}_{\nu}$ é uma matriz $n \times(p-k+1)$ com $i$-ésima linha dada por

$$
\left(\left[\frac{y_{i}-\mathbf{x}_{i} \widehat{\phi}}{\widehat{\sigma}}\right] \quad \mathbf{x}_{i}^{(2)}\right),
$$


onde $\widehat{\sigma}$ e $\widehat{\phi}$ são as estimativas de máxima verossimilhança de $\sigma$ e $\phi$, respectivamente.

Usando esses resultados e propriedades de determinantes de matrizes constituídas por blocos, para pares $(\sigma, \phi)$ satisfazendo as restrições

$$
\frac{\partial \ell(\sigma, \phi)}{\partial \sigma}=0 \quad \text { e } \quad \frac{\partial \ell(\sigma, \phi)}{\partial \phi^{(2)}}=0_{(p-k) \times 1}
$$

após manipulações algébricas, a função $\tilde{\ell}_{B N}\left(\phi^{(1)}\right)=\tilde{\ell}_{B N}(\sigma, \phi)$ é dada por

$$
\begin{gathered}
\ell(\sigma, \phi)+(p-k+1) \log \sigma-\frac{1}{2} \log \left|\sum_{j=1}^{n} \exp \left(z_{j}\right) \mathbf{x}_{j}^{(2)^{\top}} \mathbf{x}_{j}^{(2)}\right|+\frac{1}{2} \log \left\{-r-2 \sum_{j \in \bar{C}} z_{j}+2 \sum_{j=1}^{n} z_{j} \exp \left(z_{j}\right)\right. \\
+\sum_{j=1}^{n} z_{j}^{2} \exp \left(z_{j}\right)-\left[-\sum_{j \in \bar{C}} \mathbf{x}_{j}^{(2)}+\sum_{j=1}^{n} \exp \left(z_{j}\right) \mathbf{x}_{j}^{(2)}+\sum_{j=1}^{n} z_{j} \exp \left(z_{j}\right) \mathbf{x}_{j}^{(2)}\right]\left[\sum_{j=1}^{n} \exp \left(z_{j}\right) \mathbf{x}_{j}^{(2)^{\top}} \mathbf{x}_{j}^{(2)}\right]^{-1} \\
\left.\times\left[-\sum_{j \in \bar{C}} \mathbf{x}_{j}^{(2)^{\top}}+\sum_{j=1}^{n} \exp \left(z_{j}\right) \mathbf{x}_{j}^{(2)^{\top}}+\sum_{j=1}^{n} z_{j} \exp \left(z_{j}\right) \mathbf{x}_{j}^{(2)}\right]\right\} \\
-\log \left\{\sum_{j=1}^{n}\left[-I_{(j \in \bar{C})}+\left(1+z_{j}\right) \exp \left(z_{j}\right)\right] \widehat{z}_{j}-\left[\sum_{j=1}^{n}\left[-I_{(j \in \bar{C})}+\left(1+z_{j}\right) \exp \left(z_{j}\right)\right] \mathbf{x}_{j}^{(2)}\right]\right. \\
\left.\times\left[\sum_{j=1}^{n} \exp \left(z_{j}\right) \mathbf{x}_{j}^{(2)} \mathbf{x}_{j}^{(2)}\right]^{-1}\left[\sum_{j=1}^{n} \exp \left(z_{j}\right) \widehat{z}_{j} \mathbf{x}_{j}^{(2)}\right]\right\}
\end{gathered}
$$

onde

$$
z_{j}=\frac{y_{j}-\mathbf{x}_{j} \phi}{\sigma} \quad \text { e } \quad \widehat{z}_{j}=\frac{y_{j}-\mathbf{x}_{j} \widehat{\phi}}{\widehat{\sigma}}
$$

para $j=1, \ldots, n$.

Assim, a estatística da razão de verossimilhanças baseada em $\tilde{\ell}_{B N}\left(\phi^{(1)}\right)=\tilde{\ell}_{B N}(\sigma, \phi)=$ $\tilde{\ell}_{B N}\left(\sigma, \phi^{(1)}, \phi^{(2)}\right)$ é definida por

$$
R V=2\left\{\tilde{\ell}_{B N}\left(\widehat{\sigma}_{B N}, \widehat{\phi}_{B N}^{(1)}, \widehat{\phi^{(2)}}{ }_{B N}\right)-\tilde{\ell}_{B N}\left(\widehat{\sigma}_{0 B N}, \phi_{0}^{(1)}, \widehat{\phi_{0}^{(2)}}{ }_{B N}\right)\right\},
$$

onde $\tilde{\ell}_{B N}\left(\widehat{\sigma}_{B N}, \widehat{\phi^{(1)}}{ }_{B N}, \widehat{\phi^{(2)}} B N\right)$ é o valor máximo da função $\tilde{\ell}_{B N}\left(\sigma, \phi^{(1)}, \phi^{(2)}\right)$ sujeita às restrições

$$
\frac{\partial \ell(\sigma, \phi)}{\partial \sigma}=0 \quad \text { e } \quad \frac{\partial \ell(\sigma, \phi)}{\partial \phi^{(2)}}=0_{(p-k) \times 1}
$$

enquanto $\tilde{\ell}_{B N}\left(\widehat{\sigma}_{0 B N}, \phi_{0}^{(1)}, \widehat{\phi_{0}^{(2)}}{ }_{B N}\right)$ é o valor máximo de $\tilde{\ell}_{B N}\left(\sigma, \phi_{0}^{(1)}, \phi^{(2)}\right)$ sujeita às restrições

$$
\frac{\partial \ell\left(\sigma, \phi_{0}^{(1)}, \phi^{(2)}\right)}{\partial \sigma}=0 \quad \text { e } \quad \frac{\partial \ell\left(\sigma, \phi_{0}^{(1)}, \phi^{(2)}\right)}{\partial \phi^{(2)}}=0_{(p-k) \times 1} .
$$




\subsubsection{RESULTADOS NUMÉRICOS}

Esta seção apresenta resultados numéricos relativos a testes da razão de verossimilhanças realizados sobre o vetor $\phi$, que constitui a parte sistemática do modelo de regressão de Weibull. Esses resultados são baseados em 10000 (dez mil) amostras de Monte Carlo.

Para dados completos, $\beta=\sigma^{-1}=1$, assim como todos os componentes do vetor $\phi$, $\phi_{i}=1 \forall i$. Nestes casos, a probabilidade do erro tipo I não depende de $\sigma$ e nem do valor fixado para os componentes do vetor $\phi$ na hipótese nula, conforme demonstrado no Apêndice D. No caso de dados com presença de censura, são considerados três valores para $\sigma: 2,1 \mathrm{e}$ 0.2 (ou seja, $\beta=0.5,1$ e 5 , respectivamente). Aqui, em alguns casos, $\phi_{i}=1 \forall i$, em outros, $\phi_{i}=3 \forall i$.

Da mesma forma da seção anterior, aqui são considerados dois modelos de regressão, cujas partes sistemáticas são dadas por

$$
\eta(\mathbf{x})=\phi_{1}+\phi_{2} x_{2}
$$

$\mathrm{e}$

$$
\eta(\mathbf{x})=\phi_{1}+\phi_{2} x_{2}+\phi_{3} x_{3}+\phi_{4} x_{4}
$$

Considerando o modelo com uma covariada, os valores atribuídos a $x_{2}$ são realizações independentes de uma variável aleatória com distribuição uniforme contínua $U(0,100)$. Os resultados são baseados numa amostra de tamanho $n=20$ e, para dados censurados, são considerados dois níveis de censura: $25 \%$ e $50 \%$. No estudo de poder dos testes realizado ao final desta seção, os valores atribuídos a $x_{2}$ são realizações independentes de uma variável aleatória com distribuição uniforme contínua $U(0,1)$. Diferentemente dos demais resultados, estes são baseados em 5000 (cinco mil) amostras de Monte Carlo.

Para o modelo com três covariadas, em algumas situações, $x_{2}, x_{3}$ e $x_{4}$ são realizações independentes de uma variável aleatória $t$-Student com um grau de liberdade (distribuição de Cauchy), em outras, $x_{2}, x_{3}$ e $x_{4}$ são realizações independentes de uma variável uniforme contínua $U(0,100)$. Aqui $n=50$ e, para dados censurados, são considerados dois níveis de censura: $30 \%$ e $50 \%$.

Para o modelo com uma covariada e dados completos, $n=20$, a Tabela 5.25 apresenta taxas de rejeição sob a hipótese nula de diferentes testes da razão de verossimilhanças com distintos níveis de significância. As médias e variâncias empíricas das respectivas estatísticas de teste são apresentadas na Tabela 5.26. Por estas tabelas, note que as taxas de rejeição dos testes baseados em $\tilde{\ell}_{B N}$ estão mais próximas aos níveis nominais, assim como os momentos empíricos das estatísticas de teste baseadas nesta função estão mais próximos aos valores assintóticos. Isto são indicadores da boa aproximação da distribuição nula da estatística de teste obtida de $\tilde{\ell}_{B N}$ pela distribuição assintótica qui-quadrado.

Por exemplo, para o nível de significância de $5 \%$, sob a hipótese nula, os testes baseados em $\tilde{\ell}_{B N}$ apresentaram taxas de rejeição iguais a $5.070 \%\left(\mathcal{H}_{0}: \phi_{2}=1\right)$ e $5.090 \%\left(\mathcal{H}_{0}: \phi_{1}=\right.$ $\phi_{2}=1$ ) contra $7.150 \%$ e $6.950 \%$, respectivamente, dos testes baseados na verossimilhança 
original. Para $\mathcal{H}_{0}: \phi_{1}=\phi_{2}=1$, a média e a variância amostrais da estatística de teste obtida de $\tilde{\ell}_{B N}$ foram iguais a 1.997 e 4.120, respectivamente, contra 2.240 e 5.135 da estatística derivada de $\ell$.

Tabela 5.25. Taxas de rejeição da hipótese nula de diferentes testes da razão de verossimilhanças, $\sigma=\beta^{-1}=1$, distribuição valor extremo, dados completos, $n=20$.

\begin{tabular}{lrrrr}
\hline \multirow{2}{*}{ nível nominal } & \multicolumn{4}{c}{$\eta(\mathbf{x})=\phi_{1}+\phi_{2} x_{2}$} \\
\cline { 2 - 5 } & \multicolumn{1}{c}{$\mathcal{H}_{0}: \phi_{2}=1$} & \multicolumn{1}{c}{$\mathcal{H}_{0}: \phi_{1}=\phi_{2}=1$} \\
\cline { 2 - 5 } & \multicolumn{1}{c}{$\tilde{\ell}_{B N}$} & \multicolumn{1}{c}{$\ell$} & $\tilde{\ell}_{B N}$ \\
\hline $15 \%$ & 17.900 & 14.150 & 18.630 & 15.150 \\
$10 \%$ & 12.430 & 9.650 & 13.130 & 10.030 \\
$5 \%$ & 7.150 & 5.070 & 6.950 & 5.090 \\
$1 \%$ & 1.860 & 1.000 & 1.680 & 1.160 \\
$0.1 \%$ & 0.260 & 0.130 & 0.300 & 0.130 \\
$0.05 \%$ & 0.150 & 0.020 & 0.130 & 0.070 \\
\hline
\end{tabular}

Tabela 5.26. Médias e variâncias amostrais de diferentes estatísticas de teste e os valores assintóticos destas, $\beta=\sigma^{-1}=1$, distribuição valor extremo, dados completos, $n=20$.

\begin{tabular}{ccccccc}
\hline \multirow{2}{*}{ momento } & \multicolumn{4}{c}{$\eta(\mathbf{x})=\phi_{1}+\phi_{2} x_{2}$} \\
\cline { 2 - 7 } & $\chi_{1}^{2}$ & $\ell$ & $\tilde{\ell}_{B N}: \phi_{2}=1$ & $\chi_{2}^{2}$ & $\ell$ & $\tilde{\ell}_{B N}$ \\
\cline { 2 - 7 } & 1 & 1.170 & 0.983 & 2 & 2.240 & 1.997 \\
média & 2 & 2.782 & 1.989 & 4 & 5.135 & 4.120 \\
variância & 2 &
\end{tabular}

Os gráficos das Figuras 5.21 e 5.22 apresentam as curvas de discrepâncias relativas de quantis das estatísticas de teste baseadas na verossimilhança original $\ell$ e no ajuste sobre a verossimilhança perfilada denotado por $\tilde{\ell}_{B N}$. Estes gráficos são relativos aos testes sobre o vetor $\phi$ no modelo com uma covariada, considerando dados completos $(n=20)$. Observe que os posicionamentos das curvas associadas a $\tilde{\ell}_{B N}$, sempre próximas à ordenada nula, atestam os bons desempenhos dos testes baseados em tal função.

Para o modelo com três covariadas e dados completos, $n=50$, a Tabela 5.27 apresenta taxas de rejeição sob a hipótese nula de diferentes testes da razão de verossimilhanças com distintos níveis de significância. As médias e variâncias empíricas das estatísticas de teste são apresentadas na Tabela 5.28.

Observe que as taxas de rejeição dos testes baseados em $\tilde{\ell}_{B N}$ estão mais próximas aos níveis nominais comparativamente às taxas associadas aos testes da razão de verossimilhanças originais $(\ell)$. Entretanto, diferentemente da situação anterior, os desempenhos daqueles testes deterioram-se com o aumento do número de parâmetros testados. Por exemplo, considerando as hipóteses $\mathcal{H}_{0}: \phi_{4}=1, \mathcal{H}_{0}: \phi_{3}=\phi_{4}=1$ e $\mathcal{H}_{0}: \phi_{2}=\phi_{3}=\phi_{4}=1$, para o nível de significância de $15 \%$, as taxas de rejeição dos testes baseados em $\tilde{\ell}_{B N}$ foram iguais a 
Figura 5.21. Gráfico das discrepâncias relativas de quantis, distribuição valor extremo, $\eta(\mathbf{x})=\phi_{1}+\phi_{2} x_{2}, \beta=\sigma^{-1}=1, \mathcal{H}_{0}: \phi_{2}=1$, dados completos, $n=20$.

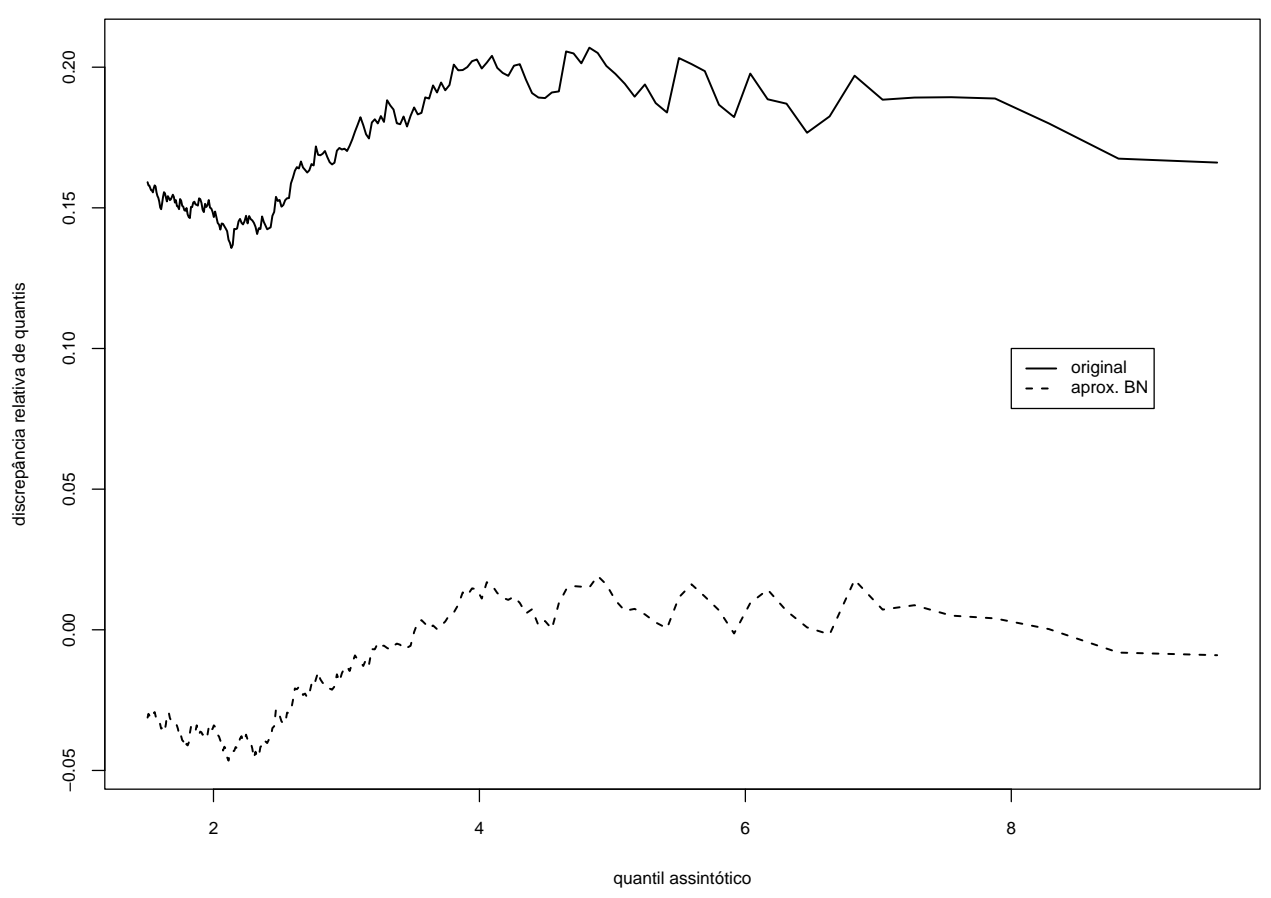

Figura 5.22. Gráfico das discrepâncias relativas de quantis, distribuição valor extremo, $\eta(\mathbf{x})=\phi_{1}+\phi_{2} x_{2}, \beta=\sigma^{-1}=1, \mathcal{H}_{0}: \phi_{1}=\phi_{2}=1$, dados completos, $n=20$.

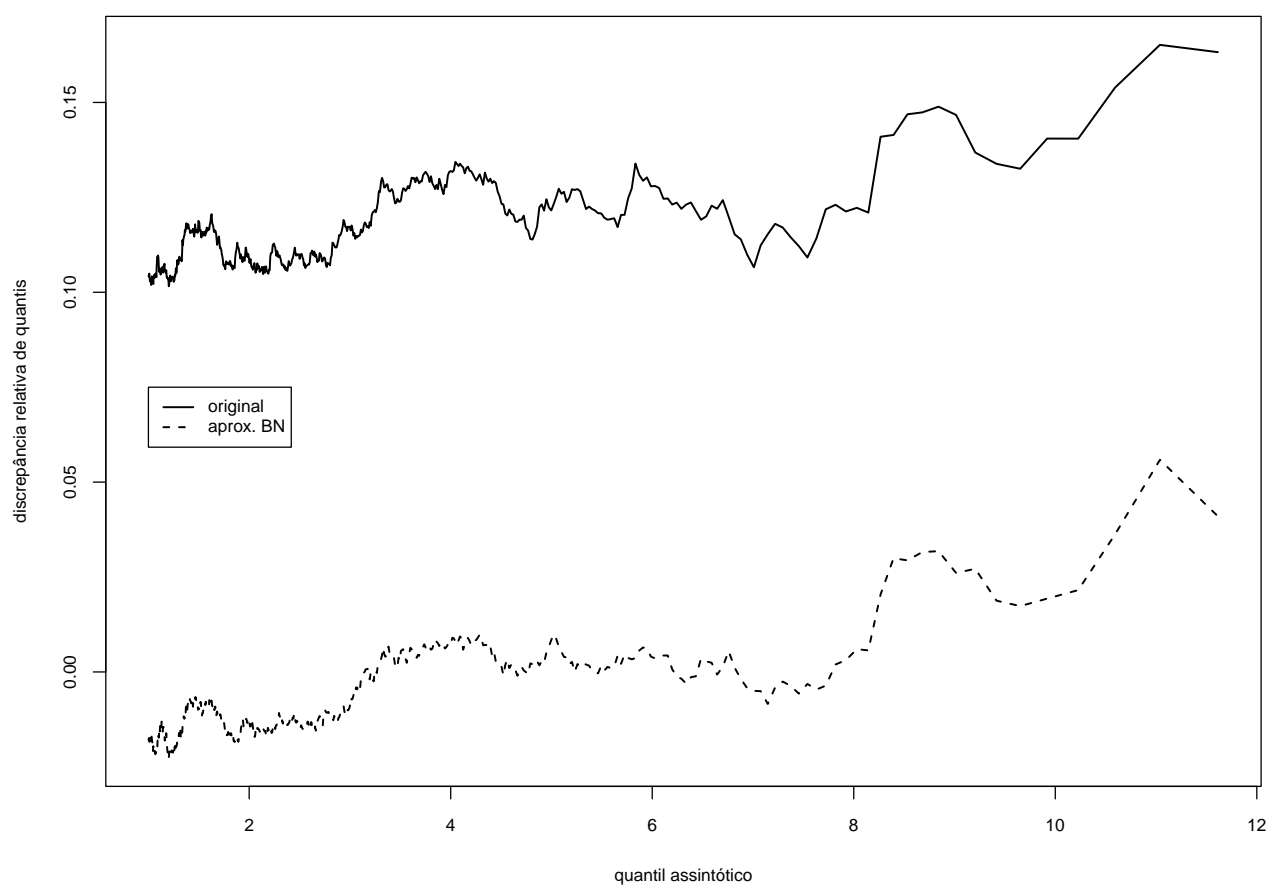

$15.020 \%, 16.770 \%$ e $17.510 \%$, respectivamente. Aqui as covariáveis $x_{2}, x_{3}$ e $x_{4}$ são realizações 
Tabela 5.27. Taxas de rejeição da hipótese nula de diferentes testes da razão de verossimilhanças, $\sigma=\beta^{-1}=1$, distribuição valor extremo, dados completos, $n=50$.

\begin{tabular}{crrrrrr}
\hline & \multicolumn{6}{c}{$\eta(\mathbf{x})=\phi_{1}+\phi_{2} x_{2}+\phi_{3} x_{3}+\phi_{4} x_{4}$} \\
\cline { 2 - 7 } nível nominal & \multicolumn{1}{c}{$\mathcal{H}_{0}: \phi_{4}=1$} & \multicolumn{1}{c}{$\mathcal{H}_{0}: \phi_{3}=\phi_{4}=1$} & \multicolumn{1}{c}{$\mathcal{H}_{0}: \phi_{2}=\phi_{3}=\phi_{4}=1$} \\
\cline { 2 - 7 } & \multicolumn{1}{c}{$\ell$} & \multicolumn{1}{c}{$\tilde{\ell}_{B N}$} & \multicolumn{1}{c}{$\ell$} & \multicolumn{1}{c}{$\tilde{\ell}_{B N}$} & $\ell$ & $\tilde{\ell}_{B N}$ \\
\hline $15 \%$ & 17.460 & 15.020 & 19.440 & 16.770 & 20.030 & 17.510 \\
$10 \%$ & 12.130 & 10.240 & 13.730 & 11.530 & 14.120 & 11.970 \\
$5 \%$ & 6.790 & 5.380 & 7.570 & 5.940 & 7.830 & 6.180 \\
$1 \%$ & 1.600 & 1.060 & 2.000 & 1.290 & 1.790 & 1.350 \\
$0.1 \%$ & 0.200 & 0.120 & 0.210 & 0.140 & 0.230 & 0.130 \\
$0.05 \%$ & 0.110 & 0.040 & 0.120 & 0.070 & 0.120 & 0.100 \\
\hline
\end{tabular}

Tabela 5.28. Médias e variâncias amostrais de diferentes estatísticas de teste e os valores assintóticos destas, $\beta=\sigma^{-1}=1$, distribuição valor extremo, dados completos, $n=50$.

\begin{tabular}{cccccccccc}
\hline & \multicolumn{8}{c}{$\eta(\mathbf{x})=\phi_{1}+\phi_{2} x_{2}+\phi_{3} x_{3}+\phi_{4} x_{4}$} \\
\cline { 2 - 10 } momento & \multicolumn{3}{c}{$\mathcal{H}_{0}: \phi_{4}=1$} & \multicolumn{1}{c}{$\mathcal{H}_{0}: \phi_{3}=\phi_{4}=1$} & \multicolumn{1}{c}{$\mathcal{H}_{0}: \phi_{2}=\phi_{3}=\phi_{4}=1$} \\
\cline { 2 - 11 } & $\chi_{1}^{2}$ & $\ell$ & $\tilde{\ell}_{B N}$ & $\chi_{2}^{2}$ & $\ell$ & $\tilde{\ell}_{B N}$ & $\chi_{3}^{2}$ & $\ell$ & $\tilde{\ell}_{B N}$ \\
\hline média & 1 & 1.137 & 1.020 & 2 & 2.329 & 2.137 & 3 & 3.437 & 3.223 \\
variância & 2 & 2.636 & 2.124 & 4 & 5.336 & 4.516 & 6 & 7.668 & 6.779 \\
\hline
\end{tabular}

independentes de uma variável aleatória com distribuição de Cauchy.

Os desempenhos dos testes segundo os resultados das Tabelas 5.27 e 5.28 são reflexos das qualidades das aproximações das distribuições nulas das estatísticas de teste pelas distribuições qui-quadrado. Assim como as taxas de rejeição dos testes baseados em $\tilde{\ell}_{B N}$ deterioraram-se com o aumento da quantidade de parâmetros de perturbação, os momentos empíricos das correspondentes estatísticas de teste distanciaram-se dos valores assintóticos.

Considerando o modelo com três covariadas, os gráficos das Figuras 5.23, 5.24 e 5.25 apresentam as curvas de discrepências relativas de quantis das estatísticas de teste baseadas na verossimilhança original $\ell$ e no ajuste sobre a verossimilhança perfilada denotado por $\tilde{\ell}_{B N}$. Com o aumento do número de parâmetros testados, observe os afastamentos das curvas com relação à ordenada nula, concordando com os resultados apresentados nas Tabelas $5.27 \mathrm{e}$ 5.28. Mesmo com tal deterioração, o desempenho do teste baseado em $\tilde{\ell}_{B N}$ foi melhor que o teste original.

Considerando agora o modelo com uma covariada, $\beta=\sigma^{-1}=1$ e dados censurados do tipo II, a Tabela 5.29 apresenta taxas de rejeição sob a hipótese nula de diferentes testes da razão de verossimilhanças com distintos níveis de significância. As médias e variâncias empíricas das estatísticas de teste são apresentadas na Tabela 5.30.

$\mathrm{Na}$ Tabela 5.29, para os dois níveis de censura considerados, note que os testes baseados em $\tilde{\ell}_{B N}$ apresentaram taxas mais próximas aos níveis nominais. Por exemplo, para $(n, r)=$ $(20,10), \mathcal{H}_{0}: \phi_{1}=\phi_{2}=1$ e nível de significância de $0.1 \%$, tal teste apresentou taxa 
Figura 5.23. Gráfico das discrepâncias relativas de quantis, distribuição valor extremo, $\eta(\mathbf{x})=\phi_{1}+\phi_{2} x_{2}+\phi_{3} x_{3}+\phi_{4} x_{4}, \beta=\sigma^{-1}=1, \mathcal{H}_{0}: \phi_{4}=1$, dados completos, $n=50$.

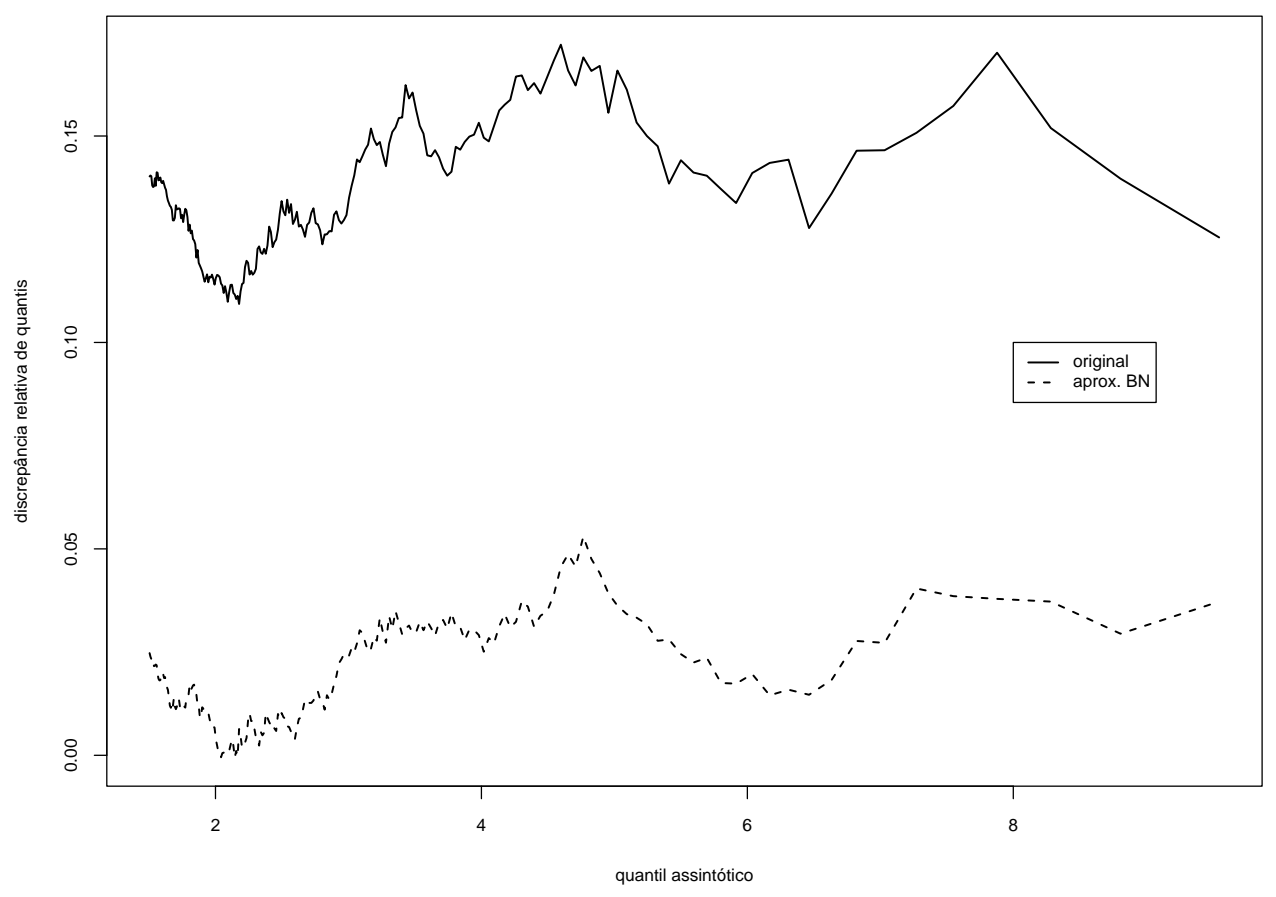

Figura 5.24. Gráfico das discrepâncias relativas de quantis, distribuição valor extremo, $\eta(\mathbf{x})=\phi_{1}+\phi_{2} x_{2}+\phi_{3} x_{3}+\phi_{4} x_{4}, \beta=\sigma^{-1}=1, \mathcal{H}_{0}: \phi_{3}=\phi_{4}=1$, dados completos, $n=50$.

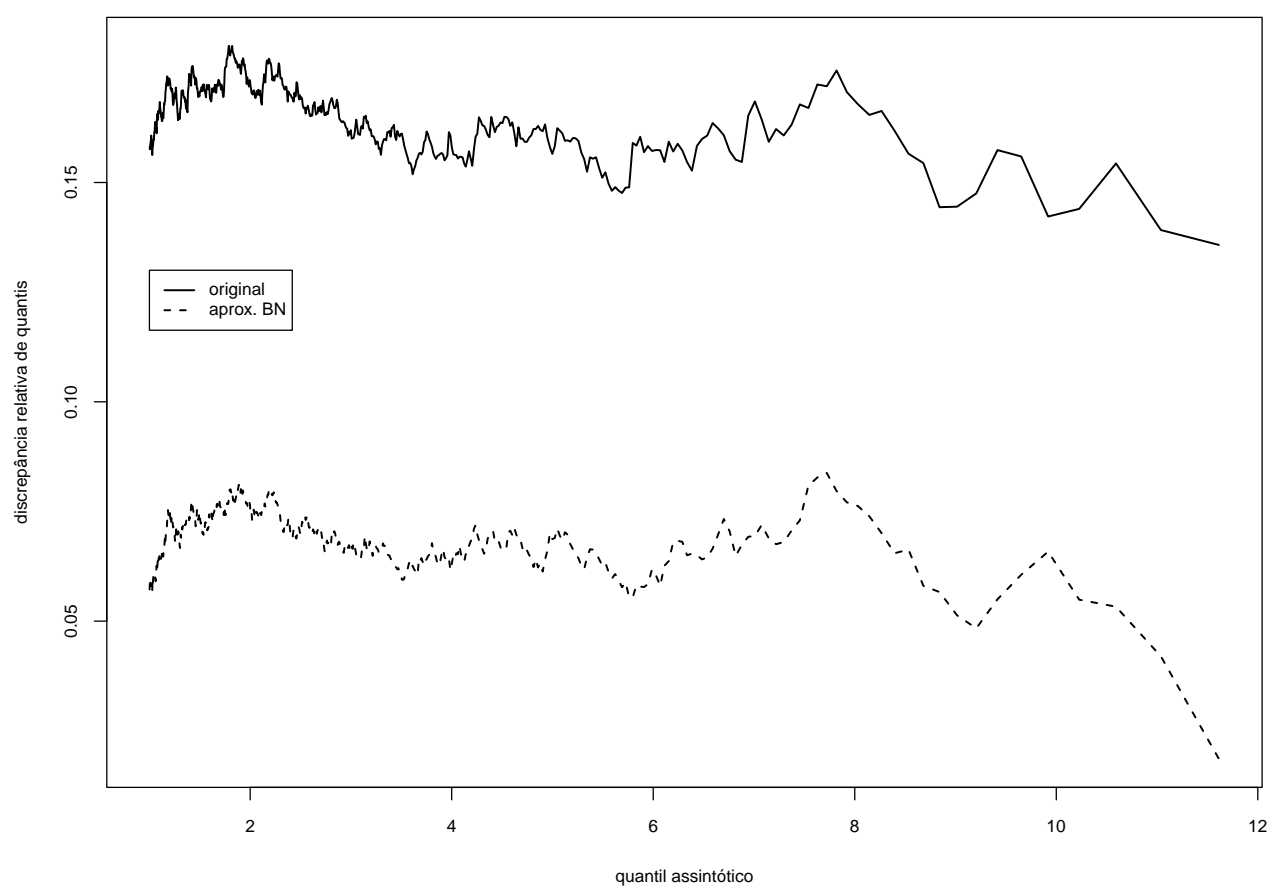

igual ao nível nominal, enquanto o teste original apresentou taxa igual a 0.390\%. Para esta 
Figura 5.25. Gráfico das discrepâncias relativas de quantis, distribuição valor extremo, $\eta(\mathbf{x})=\phi_{1}+\phi_{2} x_{2}+\phi_{3} x_{3}+\phi_{4} x_{4}, \beta=\sigma^{-1}=1, \mathcal{H}_{0}: \phi_{2}=\phi_{3}=\phi_{4}=1$, dados completos, $n=50$.

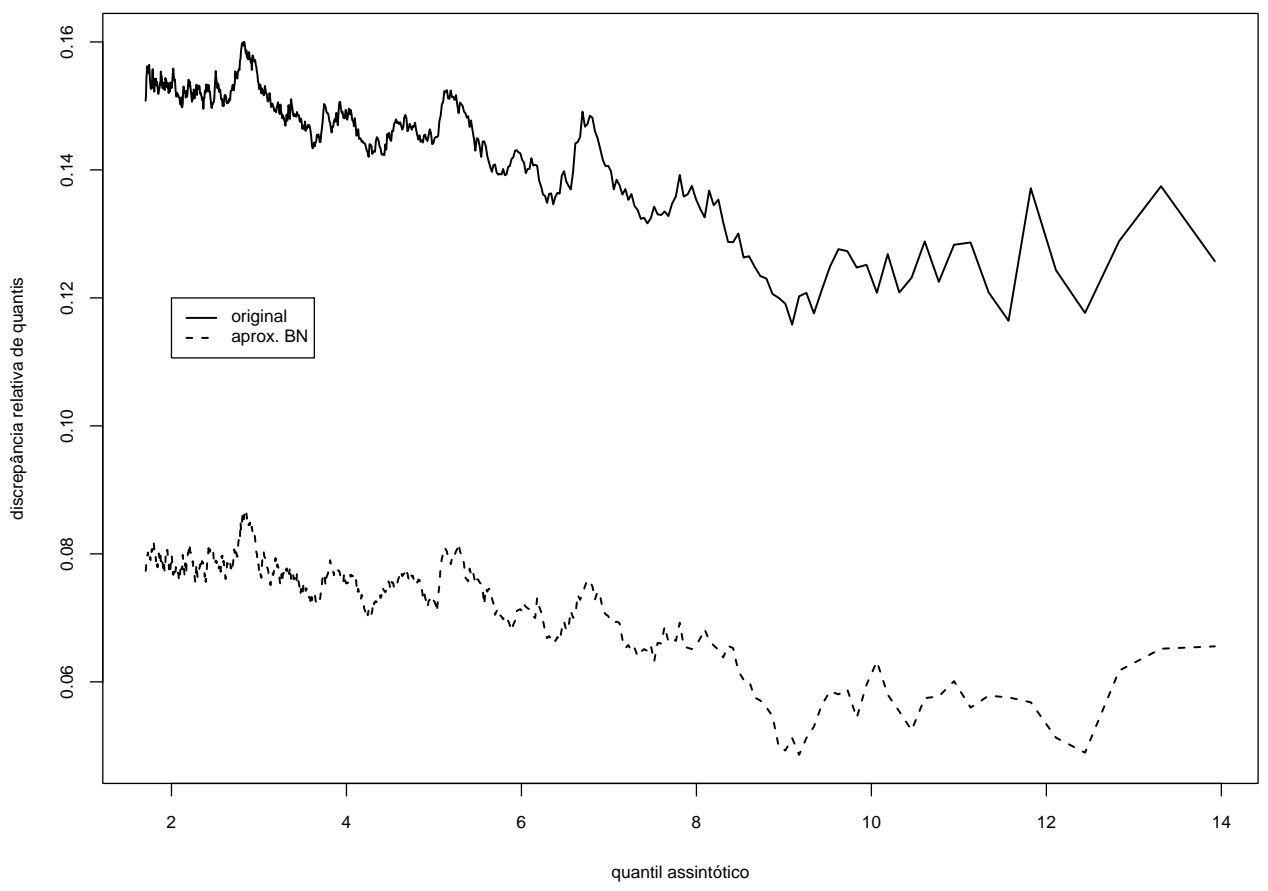

Tabela 5.29. Taxas de rejeição da hipótese nula de diferentes testes da razão de verossimilhanças, $\sigma=\beta^{-1}=1$, distribuição valor extremo, dados censurados tipo II.

\begin{tabular}{rcrrrr}
\hline & & \multicolumn{4}{c}{$\eta(\mathbf{x})=\phi_{1}+\phi_{2} x_{2}$} \\
\cline { 3 - 6 }$(n, r)$ & nível nominal & \multicolumn{2}{c}{$\mathcal{H}_{0}: \phi_{2}=1$} & \multicolumn{1}{c}{$\mathcal{H}_{0}: \phi_{1}=\phi_{2}=1$} \\
\cline { 3 - 6 } & & \multicolumn{1}{c}{$\ell$} & \multicolumn{1}{c}{$\tilde{\ell}_{B N}$} & \multicolumn{1}{c}{$\ell$} & $\tilde{\ell}_{B N}$ \\
\hline \multirow{3}{*}{$(20,15)$} & $15 \%$ & 19.530 & 14.380 & 19.080 & 14.660 \\
& $10 \%$ & 13.780 & 9.590 & 13.570 & 9.970 \\
& $1 \%$ & 7.460 & 4.470 & 7.360 & 4.840 \\
& $0.1 \%$ & 1.850 & 0.810 & 1.840 & 0.860 \\
& $0.05 \%$ & 0.250 & 0.050 & 0.260 & 0.100 \\
$(20,10)$ & $15 \%$ & 21.480 & 13.870 & 22.030 & 15.360 \\
& $10 \%$ & 15.530 & 9.060 & 16.170 & 10.570 \\
& $5 \%$ & 9.020 & 4.420 & 9.650 & 5.470 \\
& $1 \%$ & 2.420 & 0.700 & 2.690 & 1.140 \\
& $0.1 \%$ & 0.330 & 0.020 & 0.390 & 0.100 \\
& $0.05 \%$ & 0.220 & 0.020 & 0.210 & 0.040 \\
\hline
\end{tabular}

mesma situação, a média e a variância amostrais da estatística de teste obtida do ajuste sobre a verossimilhança perfilada são iguais a 2.029 e 4.173, respectivamente, contra 2.534 e 
6.319 da verossimilhança original. Pela Tabela 5.30, os momentos empíricos das estatísticas baseadas em $\tilde{\ell}_{B N}$ apresentaram-se mais próximos aos valores assintóticos. Observe também que os testes baseados em $\tilde{\ell}_{B N}$ não se deterioraram com a presença de censura nos dados, diferentemente do teste original $(\ell)$.

Tabela 5.30. Médias e variâncias amostrais de diferentes estatísticas de teste e os valores assintóticos destas, $\beta=\sigma^{-1}=1$, distribuição valor extremo, dados censurados tipo II.

\begin{tabular}{cccccccc}
\hline \multirow{2}{*}{$(n, r)$} & \multicolumn{6}{c}{$\eta(\mathbf{x})=\phi_{1}+\phi_{2} x_{2}$} \\
\cline { 3 - 8 } & momento & \multicolumn{3}{c}{$\mathcal{H}_{0}: \phi_{2}=1$} & \multicolumn{4}{c}{$\mathcal{H}_{0}: \phi_{1}=\phi_{2}=1$} \\
\cline { 3 - 8 } & & $\chi_{1}^{2}$ & $\ell$ & $\tilde{\ell}_{B N}$ & $\chi_{2}^{2}$ & $\ell$ & $\tilde{\ell}_{B N}$ \\
\hline \multirow{2}{*}{$(20,15)$} & média & 1 & 1.220 & 0.969 & 2 & 2.308 & 1.983 \\
& variância & 2 & 2.831 & 1.815 & 4 & 5.181 & 3.881 \\
\hline \multirow{2}{*}{$(20,10)$} & média & 1 & 1.331 & 0.941 & 2 & 2.534 & 2.029 \\
& variância & 2 & 3.380 & 1.742 & 4 & 6.319 & 4.173 \\
\hline
\end{tabular}

Ainda sobre o modelo com uma covariada, $\beta=\sigma^{-1}=1$ e dados censurados do tipo II, os gráficos das Figuras 5.26 e 5.27 apresentam as curvas de discrepências relativas de quantis das estatísticas de teste baseadas na verossimilhança original $\ell$ e no ajuste sobre a verossimilhança perfilada denotado por $\tilde{\ell}_{B N}$, cujas curvas, por estarem mais próximas à ordenada nula, ratificam os resultados das Tabelas 5.29 e 5.30, como as melhores aproximações das distribuições nulas das estatísticas de teste pela distribuição qui-quadrado.

Figura 5.26. Gráfico das discrepâncias relativas de quantis, distribuição valor extremo, $\eta(\mathbf{x})=\phi_{1}+\phi_{2} x_{2}, \beta=\sigma^{-1}=1, \mathcal{H}_{0}: \phi_{2}=1$, dados censurados tipo II, $(n, r)=(20,10)$.

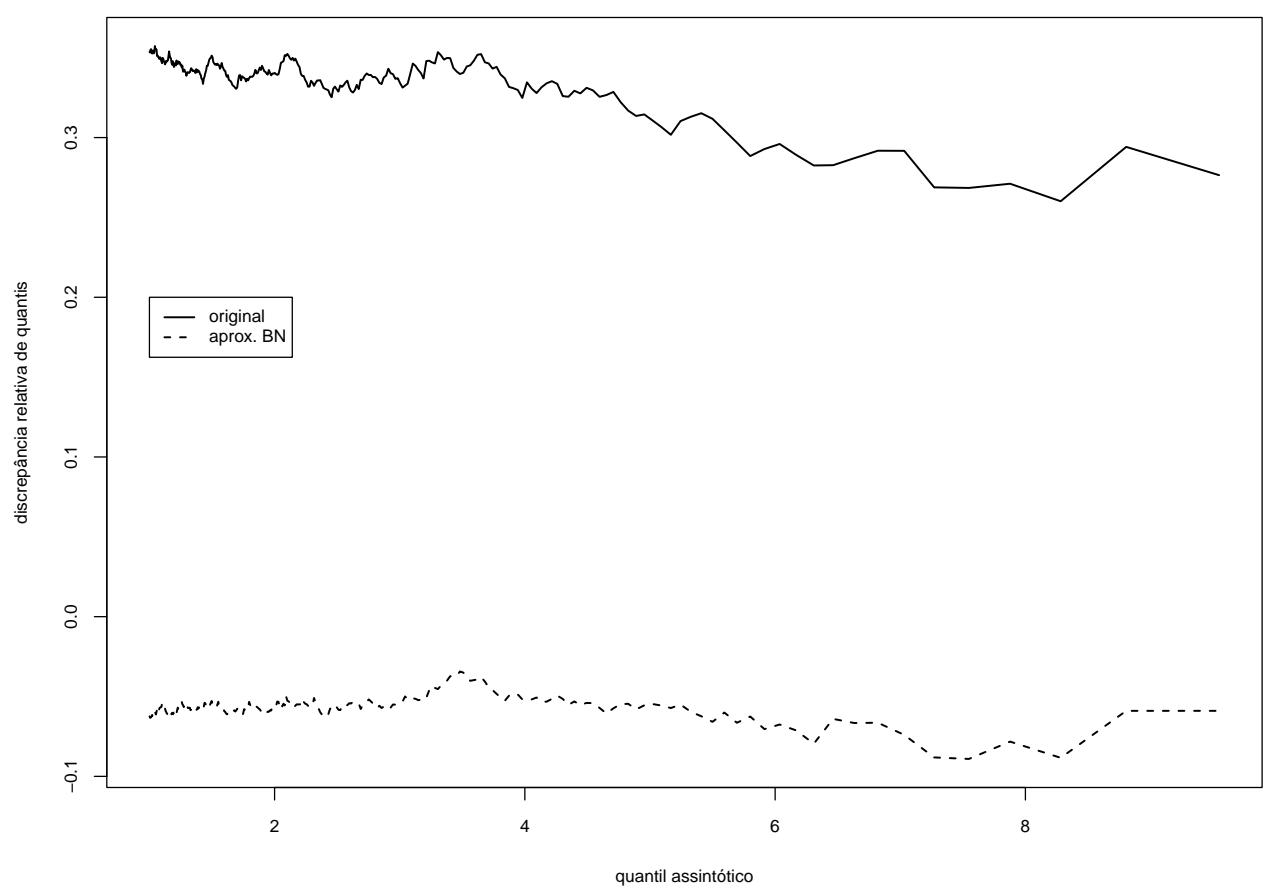


Figura 5.27. Gráfico das discrepâncias relativas de quantis, distribuição valor extremo, $\eta(\mathbf{x})=\phi_{1}+\phi_{2} x_{2}, \beta=\sigma^{-1}=1, \mathcal{H}_{0}: \phi_{1}=\phi_{2}=1$, dados censurados tipo II, $(n, r)=(20,10)$.

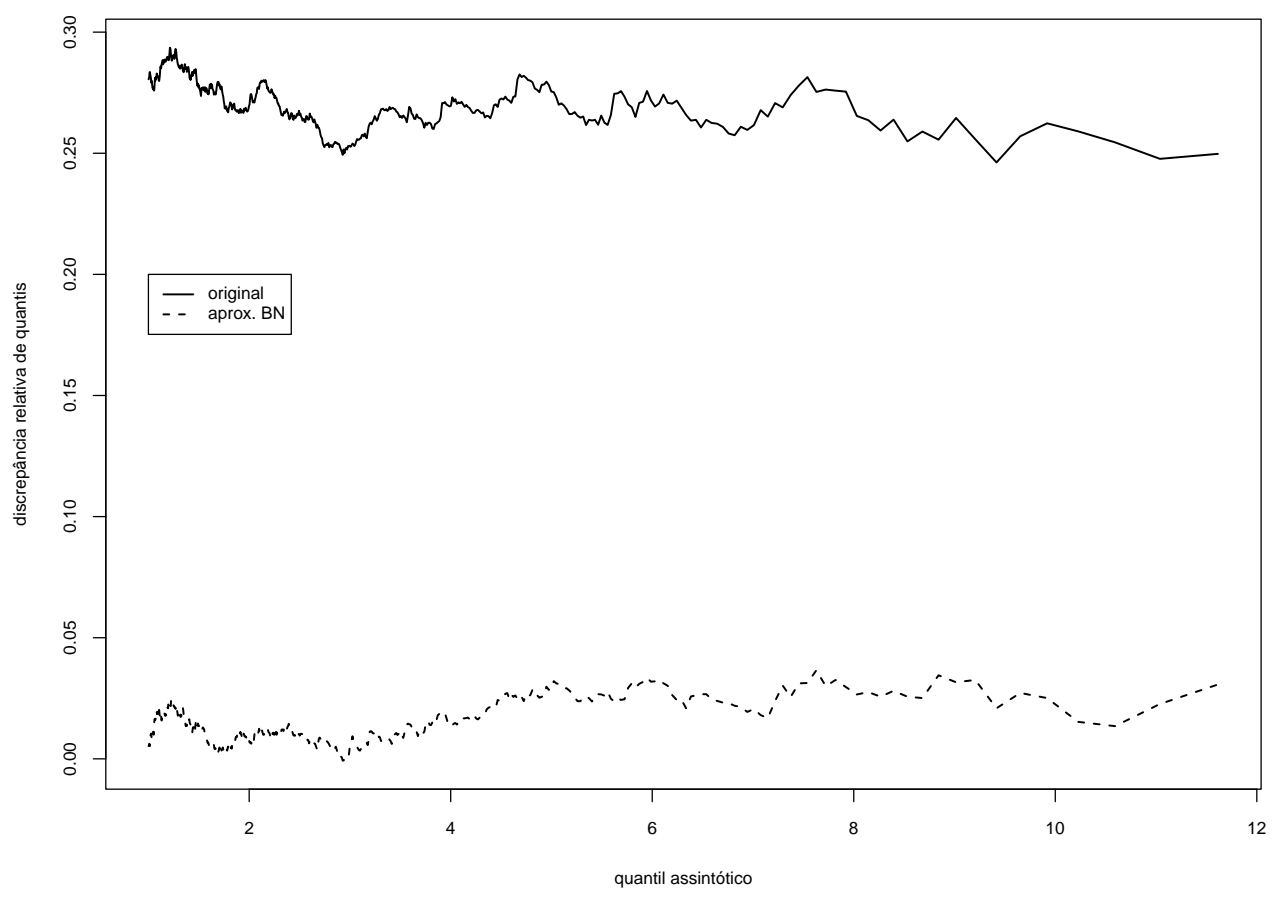

Tabela 5.31. Taxas de rejeição da hipótese nula de diferentes testes da razão de verossimilhanças, distribuição valor extremo, $\eta(\mathbf{x})=\phi_{1}+\phi_{2} x_{2}$, dados censurados tipo II.

\begin{tabular}{lcrrrr}
\hline & & \multicolumn{4}{c}{$\mathcal{H}_{0}: \phi_{1}=\phi_{2}=3$} \\
\cline { 3 - 6 }$(n, r)$ & nível nominal & \multicolumn{2}{c}{$\beta=0.5$} & \multicolumn{2}{c}{$\beta=5$} \\
\cline { 3 - 6 } & & \multicolumn{1}{c}{$\ell$} & $\tilde{\ell}_{B N}$ & \multicolumn{1}{c}{$\ell$} \\
\hline & $15 \%$ & 18.900 & 14.700 & 18.830 & 14.820 \\
& $10 \%$ & 13.640 & 9.930 & 13.550 & 9.910 \\
$(20,15)$ & $5 \%$ & 7.410 & 4.780 & 7.580 & 4.870 \\
& $1 \%$ & 1.860 & 0.890 & 1.790 & 0.930 \\
& $0.1 \%$ & 0.280 & 0.080 & 0.260 & 0.060 \\
& $0.05 \%$ & 0.120 & 0.030 & 0.120 & 0.030 \\
\hline & $15 \%$ & 22.520 & 15.640 & 23.000 & 15.630 \\
& $10 \%$ & 16.500 & 10.650 & 16.850 & 10.580 \\
& $5 \%$ & 9.700 & 5.530 & 9.850 & 5.370 \\
& $1 \%$ & 2.730 & 1.080 & 2.810 & 1.050 \\
& $0.1 \%$ & 0.410 & 0.100 & 0.430 & 0.100 \\
& $0.05 \%$ & 0.230 & 0.040 & 0.230 & 0.030 \\
\hline
\end{tabular}

Resultados para $\beta=0.5$ e $\beta=5$ são apresentados nas Tabelas 5.31 e 5.32 . Os resultados dos testes para diferentes níveis de censura são semelhantes àqueles apresentados nas Tabelas 
5.29 e 5.30. Mais uma vez, os testes baseados em $\tilde{\ell}_{B N}$ foram melhores do que os testes originais e não se deterioraram tanto com a presença de censura nos dados. Aqui $\phi_{i}=3, \forall i$.

Tabela 5.32. Médias e variâncias amostrais de diferentes estatísticas de teste e os valores assintóticos destas, distribuição valor extremo, $\eta(\mathbf{x})=\phi_{1}+\phi_{2} x_{2}$, dados censurados tipo II.

\begin{tabular}{cccccccc}
\hline \multirow{2}{*}{$(n, r)$} & & \multicolumn{4}{c}{$\mathcal{H}_{0}: \phi_{1}=\phi_{2}=3$} \\
\cline { 4 - 7 } & momento & $\chi_{2}^{2}$ & \multicolumn{2}{c}{$\beta=0.5$} & \multicolumn{2}{c}{$\beta=5$} \\
\cline { 4 - 7 } & & & $\ell$ & $\tilde{\ell}_{B N}$ & $\ell$ & $\tilde{\ell}_{B N}$ \\
\hline \multirow{2}{*}{$(20,15)$} & média & 2 & 2.309 & 1.985 & 2.312 & 1.981 \\
& variância & 4 & 5.197 & 3.903 & 5.266 & 3.928 \\
\hline \multirow{2}{*}{$(20,10)$} & média & 2 & 2.544 & 2.034 & 2.566 & 2.028 \\
& variância & 4 & 6.375 & 4.202 & 6.489 & 4.190 \\
\hline
\end{tabular}

Considerando o modelo com três covariadas, $\beta=\sigma^{-1}=1$ e dados censurados do tipo II, a Tabela 5.33 apresenta taxas de rejeição sob a hipótese nula de diferentes testes da razão de verossimilhanças com distintos níveis de significância. As médias e variâncias empíricas das estatísticas de teste são apresentadas na Tabela 5.34. Os resultados são baseados numa matriz de especificação $X$ cujos elementos são realizações independentes de uma variável com distribuição de Cauchy.

Tabela 5.33. Taxas de rejeição da hipótese nula de diferentes testes da razão de verossimilhanças, $\sigma=\beta^{-1}=1$, distribuição valor extremo, dados censurados tipo II.

\begin{tabular}{|c|c|c|c|c|c|c|c|}
\hline \multirow{3}{*}{$(n, r)$} & \multirow{3}{*}{ nível nominal } & \multicolumn{6}{|c|}{$\eta(\mathbf{x})=\phi_{1}+\phi_{2} x_{2}+\phi_{3} x_{3}+\phi_{4} x_{4}$} \\
\hline & & \multicolumn{2}{|c|}{$\mathcal{H}_{0}: \phi_{4}=1$} & \multicolumn{2}{|c|}{$\mathcal{H}_{0}: \phi_{3}=\phi_{4}=1$} & \multicolumn{2}{|c|}{$\mathcal{H}_{0}: \phi_{2}=\phi_{3}=\phi_{4}=1$} \\
\hline & & $\ell$ & $\tilde{\ell}_{B N}$ & $\ell$ & $\tilde{\ell}_{B N}$ & $\ell$ & $\tilde{\ell}_{B N}$ \\
\hline \multirow{6}{*}{$(50,35)$} & $15 \%$ & 17.950 & 14.860 & 21.080 & 17.610 & 21.540 & 18.380 \\
\hline & $10 \%$ & 12.630 & 10.220 & 15.120 & 12.050 & 15.330 & 12.780 \\
\hline & $5 \%$ & 7.010 & 5.200 & 8.250 & 6.280 & 8.580 & 6.530 \\
\hline & $1 \%$ & 1.770 & 1.090 & 2.080 & 1.290 & 2.120 & 1.500 \\
\hline & $0.1 \%$ & 0.270 & 0.100 & 0.290 & 0.120 & 0.250 & 0.130 \\
\hline & $0.05 \%$ & 0.120 & 0.070 & 0.130 & 0.060 & 0.140 & 0.070 \\
\hline \multirow{6}{*}{$(50,25)$} & $15 \%$ & 19.130 & 14.260 & 22.330 & 17.290 & 22.810 & 18.800 \\
\hline & $10 \%$ & 13.400 & 9.530 & 16.080 & 11.930 & 16.520 & 12.750 \\
\hline & $5 \%$ & 7.470 & 4.710 & 9.100 & 6.360 & 9.250 & 6.790 \\
\hline & $1 \%$ & 2.000 & 1.010 & 2.270 & 1.180 & 2.440 & 1.530 \\
\hline & $0.1 \%$ & 0.290 & 0.130 & 0.330 & 0.150 & 0.360 & 0.180 \\
\hline & $0.05 \%$ & 0.200 & 0.050 & 0.200 & 0.060 & 0.200 & 0.090 \\
\hline
\end{tabular}

Assim como ocorreu com os resultados obtidos de dados completos, observe que as taxas de rejeição dos testes baseados em $\tilde{\ell}_{B N}$ estão mais próximas aos níveis nominais comparativamente às taxas associadas aos testes da razão de verossimilhanças originais $(\ell)$, entretanto, os desempenhos daqueles testes deterioram-se com o aumento do número de parâmetros 
testados. Por exemplo, considerando as hipóteses $\mathcal{H}_{0}: \phi_{4}=1, \mathcal{H}_{0}: \phi_{3}=\phi_{4}=1 \mathrm{e}$ $\mathcal{H}_{0}: \phi_{2}=\phi_{3}=\phi_{4}=1$, para o nível de significância de $10 \%$ e $(n, r)=(50,25)$, as taxas de rejeição dos testes baseados em $\tilde{\ell}_{B N}$ foram iguais a $9.530 \%, 11.930 \%$ e $12.750 \%$, respectivamente.

Além disso, acompanhando as deteriorações das taxas de rejeição dos testes baseados em $\tilde{\ell}_{B N}$ sob a hipótese nula com o aumento do número de parâmetros testados, os momentos amostrais das respectivas estatísticas de teste distanciaram-se dos valores assintóticos. Por exemplo, considerando a mesma ordem das hipóteses mencionadas anteriormente e o mesmo nível de censura, as médias e variâncias empíricas de tais estatísticas foram iguais, respectivamente, a 0.982 e 1.967, 2.153 e 4.539, 3.307 e 7.191 (Tabela 5.34).

Tabela 5.34. Médias e variâncias amostrais de diferentes estatísticas de teste e os valores assintóticos destas, $\beta=\sigma^{-1}=1$, distribuição valor extremo, dados censurados tipo II.

\begin{tabular}{|c|c|c|c|c|c|c|c|c|c|c|}
\hline \multirow{3}{*}{$(n, r)$} & \multirow{3}{*}{ momento } & \multicolumn{9}{|c|}{$\eta(\mathbf{x})=\phi_{1}+\phi_{2} x_{2}+\phi_{3} x_{3}+\phi_{4} x_{4}$} \\
\hline & & \multicolumn{3}{|c|}{$\mathcal{H}_{0}: \phi_{4}=1$} & \multicolumn{3}{|c|}{$\mathcal{H}_{0}: \phi_{3}=\phi_{4}=1$} & \multicolumn{3}{|c|}{$\mathcal{H}_{0}: \phi_{2}=\phi_{3}=\phi_{4}=1$} \\
\hline & & $\chi_{1}^{2}$ & $\ell$ & $\tilde{\ell}_{B N}$ & $\chi_{2}^{2}$ & $\ell$ & $\tilde{\ell}_{B N}$ & $\chi_{3}^{2}$ & $\ell$ & $\tilde{\ell}_{B N}$ \\
\hline \multirow{2}{*}{$(50,35)$} & média & 1 & 1.159 & 0.997 & 2 & 2.418 & 2.158 & 3 & 3.558 & 3.283 \\
\hline & variância & 2 & 2.795 & 2.060 & 4 & 5.635 & 4.546 & 6 & 8.168 & 7.042 \\
\hline \multirow{2}{*}{$(50,25)$} & média & 1 & 1.218 & 0.982 & 2 & 2.519 & 2.153 & 3 & 3.685 & 3.307 \\
\hline & variância & 2 & 3.031 & 1.967 & 4 & 6.053 & 4.539 & 6 & 8.719 & 7.191 \\
\hline
\end{tabular}

Tabela 5.35. Taxas de rejeição da hipótese nula de diferentes testes da razão de verossimilhanças, $\sigma=\beta^{-1}=1$, distribuição valor extremo, dados censurados tipo II, $x_{2}, x_{3}, x_{4} \sim U(0,100)$.

\begin{tabular}{|c|c|c|c|c|c|c|c|}
\hline \multirow{3}{*}{$(n, r)$} & \multirow{3}{*}{ nível nominal } & \multicolumn{6}{|c|}{$\eta(\mathbf{x})=\phi_{1}+\phi_{2} x_{2}+\phi_{3} x_{3}+\phi_{4} x_{4}$} \\
\hline & & \multicolumn{2}{|c|}{$\mathcal{H}_{0}: \phi_{4}=1$} & \multicolumn{2}{|c|}{$\mathcal{H}_{0}: \phi_{3}=\phi_{4}=1$} & \multicolumn{2}{|c|}{$\mathcal{H}_{0}: \phi_{2}=\phi_{3}=\phi_{4}=1$} \\
\hline & & $\ell$ & $\tilde{\ell}_{B N}$ & $\ell$ & $\tilde{\ell}_{B N}$ & $\ell$ & $\tilde{\ell}_{B N}$ \\
\hline \multirow{6}{*}{$(50,35)$} & $15 \%$ & 18.320 & 14.670 & 19.300 & 15.010 & 19.160 & 15.330 \\
\hline & $10 \%$ & 12.650 & 9.550 & 13.160 & 9.780 & 13.270 & 10.400 \\
\hline & $5 \%$ & 6.900 & 4.740 & 7.020 & 4.890 & 7.230 & 5.460 \\
\hline & $1 \%$ & 1.570 & 0.870 & 1.750 & 0.950 & 1.540 & 0.990 \\
\hline & $0.1 \%$ & 0.190 & 0.080 & 0.220 & 0.070 & 0.170 & 0.100 \\
\hline & $0.05 \%$ & 0.080 & 0.060 & 0.120 & 0.040 & 0.100 & 0.010 \\
\hline
\end{tabular}

A utilização de observações de uma variável com distribuição de Cauchy na matriz de especificação do modelo $X$ tem, por objetivo, gerar um conjunto de dados com a presença de pontos de alavanca (ou de alto leverage), os quais têm comportamentos atípicos com relação aos demais pontos num ajuste de regressão, pois as variâncias dos valores ajustados dos pontos próximos àqueles são maiores do que as variâncias dos valores ajustados correspondentes aos demais. Para o modelo com três covariadas e $(n, r)=(50,35)$, as Tabelas 5.35 e 5.36 apresentam resultados numéricos considerando realizações independentes de uma variável uniforme contínua $U(0,100)$ para os elementos da matriz X. Note que as aproximações das distribuições nulas das estatísticas de teste baseadas em $\tilde{\ell}_{B N}$ pelas distribuições 
qui-quadrado não se deterioram tanto com o aumento do número de parâmetros testados, pois as taxas de rejeição da hipótese nula dos respectivos testes estão próximas aos níveis nominais, bem como os momentos amostrais, dos valores assintóticos.

Tabela 5.36. Médias e variâncias amostrais de diferentes estatísticas de teste e os valores assintóticos destas, $\beta=\sigma^{-1}=1$, distribuição valor extremo, dados censurados tipo II, $x_{2}, x_{3}, x_{4} \sim U(0,100)$.

\begin{tabular}{ccccccccccc}
\hline & \multicolumn{8}{c}{$\eta(\mathbf{x})=\phi_{1}+\phi_{2} x_{2}+\phi_{3} x_{3}+\phi_{4} x_{4}$} \\
\cline { 3 - 11 }$(n, r)$ & momento & \multicolumn{3}{c}{$\mathcal{H}_{0}: \phi_{4}=1$} & \multicolumn{3}{c}{$\mathcal{H}_{0}: \phi_{3}=\phi_{4}=1$} & \multicolumn{2}{c}{$\mathcal{H}_{0}: \phi_{2}=\phi_{3}=\phi_{4}=1$} \\
\cline { 3 - 12 } & & $\chi_{1}^{2}$ & $\ell$ & $\tilde{\ell}_{B N}$ & $\chi_{2}^{2}$ & $\ell$ & $\tilde{\ell}_{B N}$ & $\chi_{3}^{2}$ & $\ell$ & $\tilde{\ell}_{B N}$ \\
\hline \multirow{2}{*}{$(50,35)$} & média & 1 & 1.163 & 0.990 & 2 & 2.276 & 1.988 & 3 & 3.361 & 3.034 \\
& variância & 2 & 2.618 & 1.884 & 4 & 5.099 & 3.902 & 6 & 7.301 & 6.045 \\
\hline
\end{tabular}

Os gráficos das Figuras 5.28, 5.29 e 5.30 apresentam as curvas de discrepâncias relativas de quantis das estatísticas de teste baseadas na verossimilhança original $\ell$ e no ajuste sobre a verossimilhança perfilada denotado por $\tilde{\ell}_{B N}$ para dados censurados tipo II, $(n, r)=(50,25)$. Aqui $x_{2}, x_{3}$ e $x_{4}$ são realizações independentes de uma variável com distribuição de Cauchy. Na Figura 5.28, $\mathcal{H}_{0}: \phi_{4}=1$, enquanto na Figura 5.29, $\mathcal{H}_{0}: \phi_{3}=\phi_{4}=1$. Na Figura 5.30, $\mathcal{H}_{0}: \phi_{2}=\phi_{3}=\phi_{4}=1$. Assim os quantis assintóticos são obtidos de variáveis qui-quadrado com um, dois e três graus de liberdade. Note que à medida que aumenta o número de parâmetros testados, as curvas deslocam-se para cima, distanciando-se da ordenada nula. Ou seja, as aproximações das distribuições nulas das estatísticas de teste pela qui-quadrado deterioram-se.

Figura 5.28. Gráfico das discrepâncias relativas de quantis, distribuição valor extremo, $\eta(\mathbf{x})=\phi_{1}+\phi_{2} x_{2}+\phi_{3} x_{3}+\phi_{4} x_{4}, \beta=\sigma^{-1}=1, \mathcal{H}_{0}: \phi_{4}=1$, dados censurados tipo II, $(n, r)=(50,25)$.

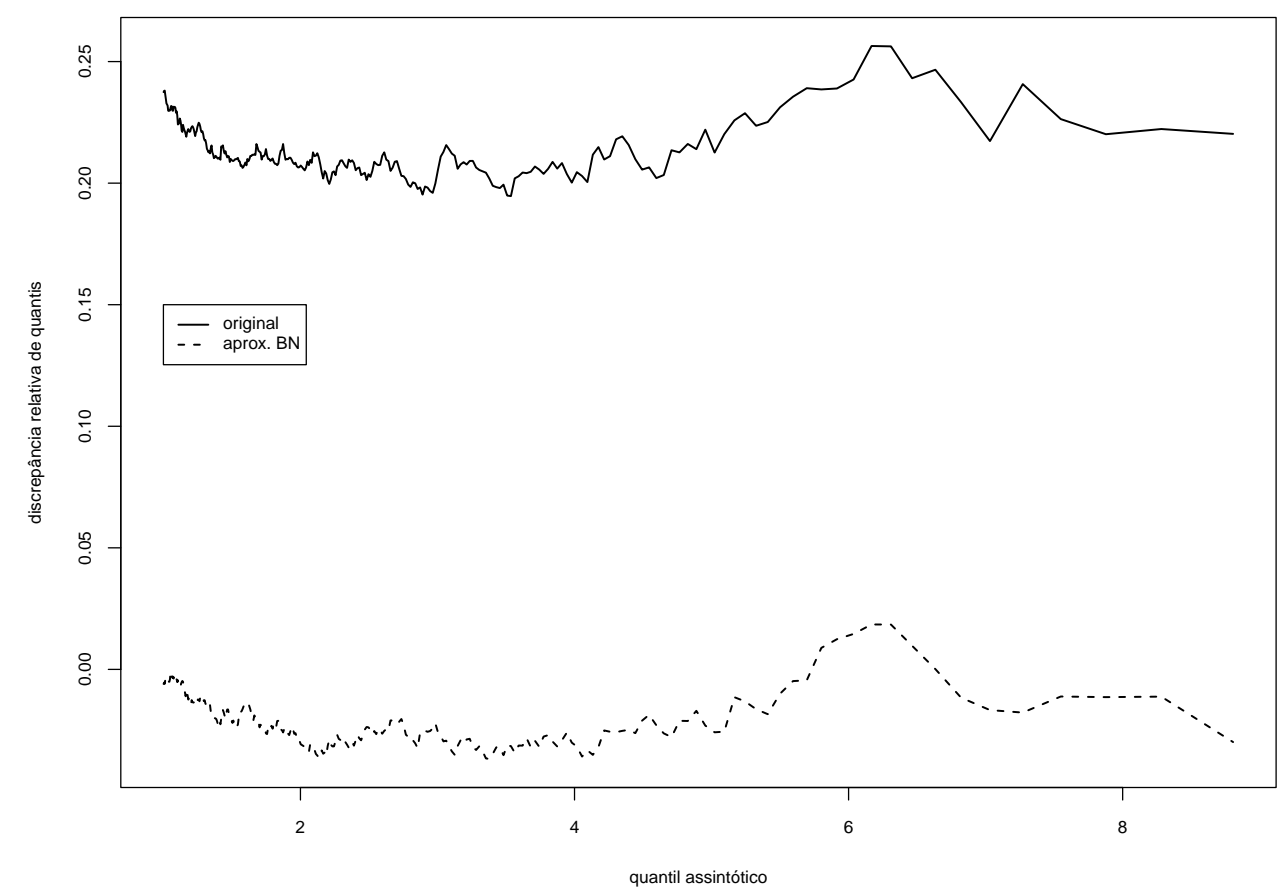


Figura 5.29. Gráfico das discrepâncias relativas de quantis, distribuição valor extremo, $\eta(\mathbf{x})=\phi_{1}+\phi_{2} x_{2}+\phi_{3} x_{3}+\phi_{4} x_{4}, \beta=\sigma^{-1}=1, \mathcal{H}_{0}: \phi_{3}=\phi_{4}=1$, dados censurados tipo II, $(n, r)=(50,25)$.

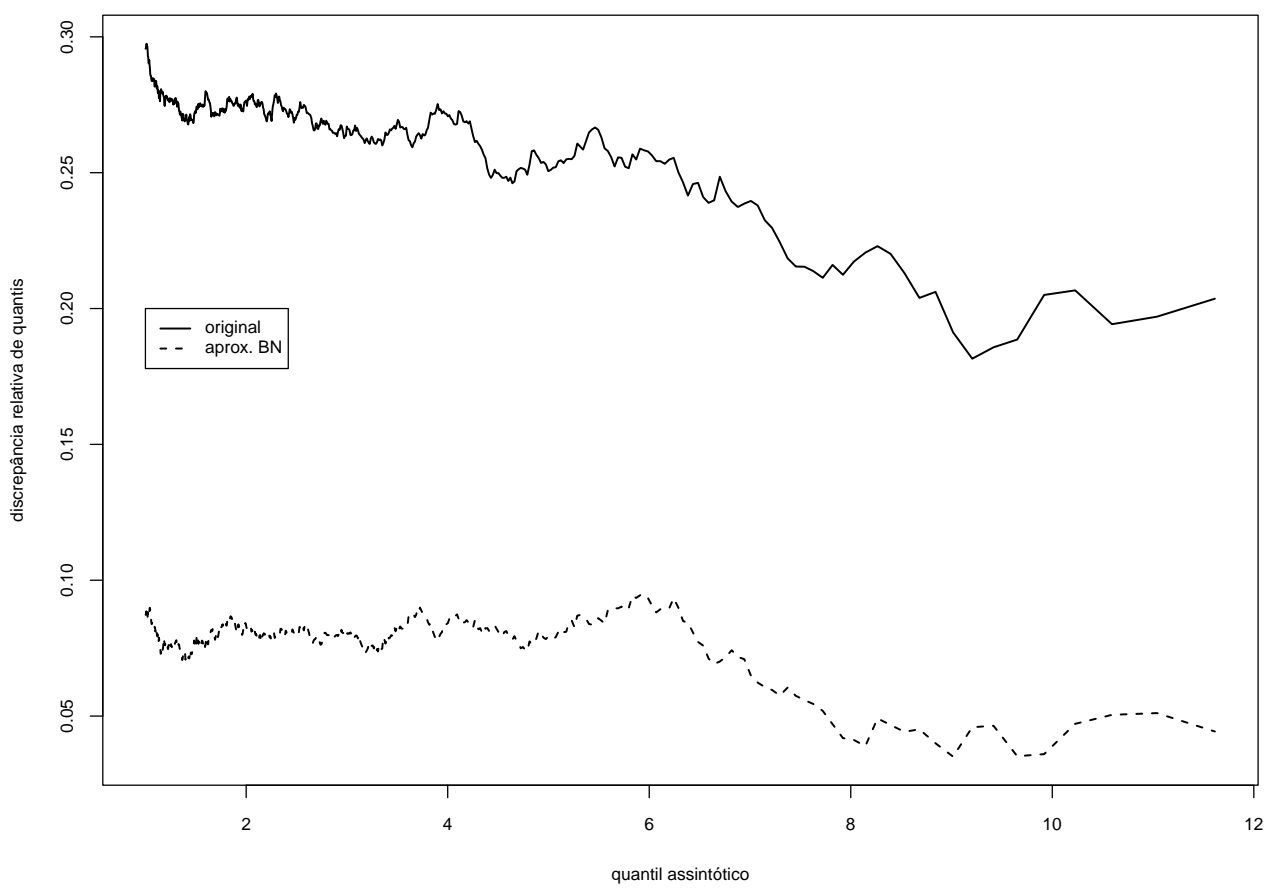

Figura 5.30. Gráfico das discrepâncias relativas de quantis, distribuição valor extremo, $\eta(\mathbf{x})=\phi_{1}+\phi_{2} x_{2}+\phi_{3} x_{3}+\phi_{4} x_{4}, \beta=\sigma^{-1}=1, \mathcal{H}_{0}: \phi_{2}=\phi_{3}=\phi_{4}=1$, dados censurados tipo II, $(n, r)=(50,25)$.

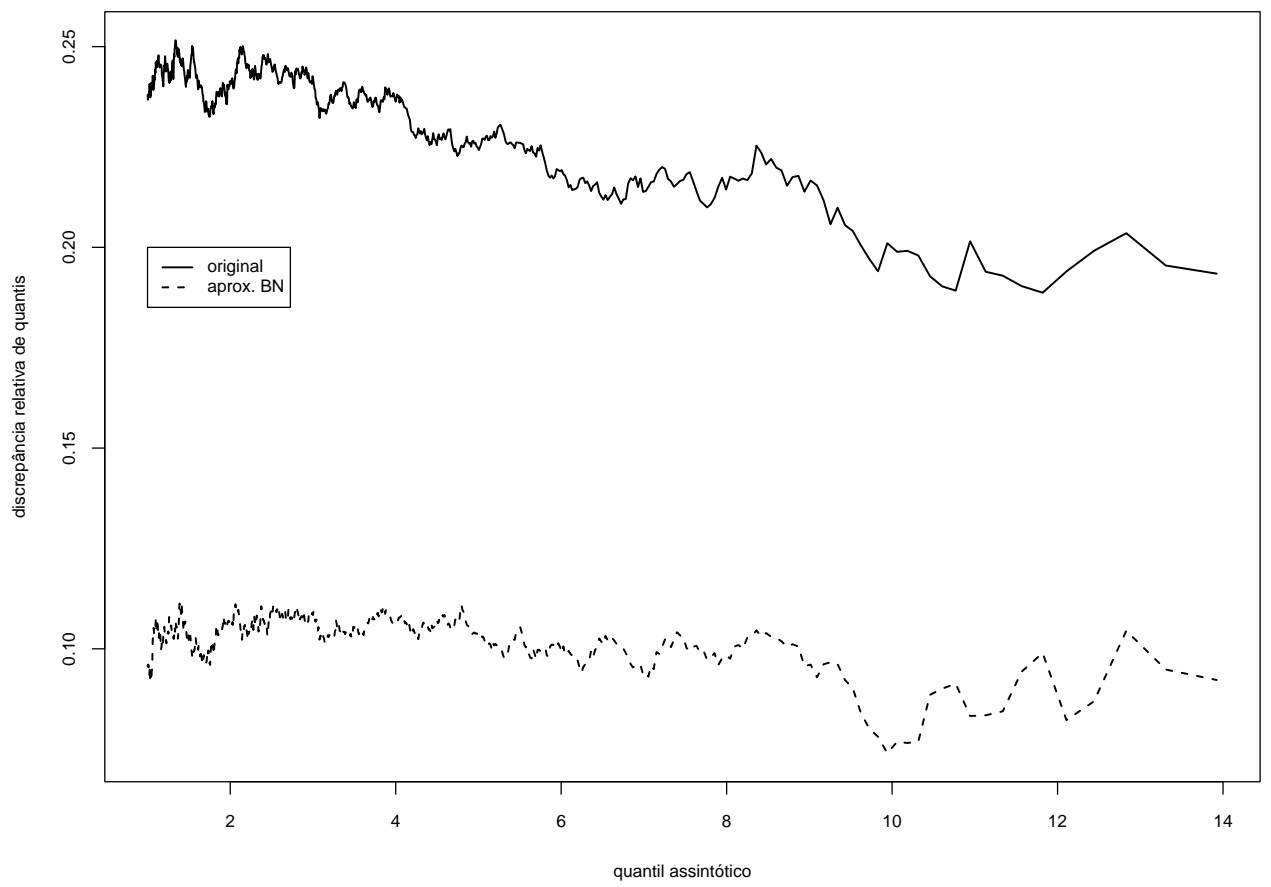


Ainda sobre o modelo com três covariadas, censura tipo II e $(n, r)=(50,35)$, resultados numéricos para $\beta=0.5$ e $\beta=5$ são apresentados nas Tabelas 5.37 e 5.38. Para $\beta=0.5$, os valores das covariadas $x_{2}, x_{3}$ e $x_{4}$ são realizações independentes de uma variável aleatória com distribuição de Cauchy, enquanto para $\beta=5$, são realizações de uma variável aleatória com distribuição uniforme contínua $U(0,100)$. Aqui $\phi_{i}=3, \forall i$.

Nas Tabelas 5.37 e 5.38, para $\beta=0.5$, note as deteriorações das estimativas dos tamanhos dos testes baseados em $\tilde{\ell}_{B N}$ com o aumento do número de parâmetros testados, bem como os afastamentos dos momentos amostrais das respectivas estatísticas de testes dos seus valores assintóticos. Isto não ocorre quando $\beta=5$. Nos dois casos, os testes da razão de verossimilhanças originais são sempre liberais, rejeitando as hipóteses nulas além do esperado. As médias e as variâncias empíricas das estatísticas de testes baseadas em $\ell$ estão bem afastadas dos seus correspondentes valores assintóticos. Os resultados são semelhantes aos casos em que $\beta=\sigma^{-1}=1$. Em todos os casos, os testes baseados em $\tilde{\ell}_{B N}$ apresentaram desempenhos superiores aos testes originais.

Tabela 5.37. Taxas de rejeição da hipótese nula de diferentes testes da razão de verossimilhanças, distribuição valor extremo, dados censurados tipo II, $(n, r)=(50,35)$.

\begin{tabular}{|c|c|c|c|c|c|c|c|}
\hline \multirow{3}{*}{$\beta$} & \multirow{3}{*}{ nível nominal } & \multicolumn{6}{|c|}{$\eta(\mathbf{x})=\phi_{1}+\phi_{2} x_{2}+\phi_{3} x_{3}+\phi_{4} x_{4}$} \\
\hline & & \multicolumn{2}{|c|}{$\mathcal{H}_{0}: \phi_{4}=3$} & \multicolumn{2}{|c|}{$\mathcal{H}_{0}: \phi_{3}=\phi_{4}=3$} & \multicolumn{2}{|c|}{$\mathcal{H}_{0}: \phi_{2}=\phi_{3}=\phi_{4}=3$} \\
\hline & & $\ell$ & $\tilde{\ell}_{B N}$ & $\ell$ & $\tilde{\ell}_{B N}$ & $\ell$ & $\tilde{\ell}_{B N}$ \\
\hline \multirow{6}{*}{0.5} & $15 \%$ & 18.160 & 15.000 & 21.360 & 17.740 & 21.840 & 18.700 \\
\hline & $10 \%$ & 12.640 & 9.880 & 15.100 & 12.080 & 15.060 & 12.620 \\
\hline & $5 \%$ & 7.440 & 5.140 & 8.340 & 6.260 & 8.580 & 6.780 \\
\hline & $1 \%$ & 1.920 & 1.160 & 2.140 & 1.300 & 2.220 & 1.580 \\
\hline & $0.1 \%$ & 0.380 & 0.140 & 0.280 & 0.120 & 0.340 & 0.200 \\
\hline & $0.05 \%$ & 0.160 & 0.040 & 0.160 & 0.100 & 0.160 & 0.100 \\
\hline \multirow{6}{*}{5} & $15 \%$ & 19.100 & 15.640 & 19.480 & 15.160 & 19.360 & 15.040 \\
\hline & $10 \%$ & 13.420 & 10.220 & 13.500 & 9.620 & 12.980 & 10.040 \\
\hline & $5 \%$ & 7.320 & 5.160 & 7.100 & 4.860 & 7.500 & 5.520 \\
\hline & $1 \%$ & 1.880 & 1.060 & 1.920 & 0.920 & 1.720 & 1.200 \\
\hline & $0.1 \%$ & 0.220 & 0.120 & 0.240 & 0.100 & 0.160 & 0.120 \\
\hline & $0.05 \%$ & 0.140 & 0.080 & 0.140 & 0.040 & 0.120 & 0.040 \\
\hline
\end{tabular}

Tabela 5.38. Médias e variâncias amostrais de diferentes estatísticas de teste e os valores assintóticos destas, distribuição valor extremo, dados censurados tipo II, $(n, r)=(50,35)$.

\begin{tabular}{|c|c|c|c|c|c|c|c|c|c|c|}
\hline \multirow{3}{*}{$\beta$} & \multirow{3}{*}{ momento } & \multicolumn{9}{|c|}{$\eta(\mathbf{x})=\phi_{1}+\phi_{2} x_{2}+\phi_{3} x_{3}+\phi_{4} x_{4}$} \\
\hline & & \multicolumn{3}{|c|}{$\mathcal{H}_{0}: \phi_{4}=3$} & \multicolumn{3}{|c|}{$\mathcal{H}_{0}: \phi_{3}=\phi_{4}=3$} & \multicolumn{3}{|c|}{$\mathcal{H}_{0}: \phi_{2}=\phi_{3}=\phi_{4}=3$} \\
\hline & & $\chi_{1}^{2}$ & $\ell$ & $\tilde{\ell}_{B N}$ & $\chi_{2}^{2}$ & $\ell$ & $\tilde{\ell}_{B N}$ & $\chi_{3}^{2}$ & $\ell$ & $\tilde{\ell}_{B N}$ \\
\hline \multirow{2}{*}{0.5} & média & 1 & 1.179 & 1.010 & 2 & 2.435 & 2.168 & 3 & 3.581 & 3.301 \\
\hline & variância & 2 & 2.915 & 2.127 & 4 & 5.741 & 4.596 & 6 & 8.302 & 7.126 \\
\hline \multirow{2}{*}{5} & média & 1 & 1.226 & 1.073 & 2 & 2.308 & 2.010 & 3 & 3.385 & 3.049 \\
\hline & variância & 2 & 2.803 & 1.994 & 4 & 5.196 & 3.973 & 6 & 7.418 & 6.139 \\
\hline
\end{tabular}


Agora considere dados com presença de censura do tipo I. Diferentemente dos dados com censura do tipo II, não é possível fixar o número de falhas numa amostra Monte Carlo, ou seja, a proporção de observações censuradas.

No caso de observações independentes e identicamente distribuídas (Seção 5.2.1.3), supondo uma proporção (probabilidade) de censura desejada $\left(p_{0}\right)$, foi calculado, usando a função distribuição do tempo de falha, o tempo de censura $(c)$ necessário para que tal proporção fosse observada,

$$
c=\alpha\left(-\log p_{0}\right)^{1 / \beta},
$$

onde $\alpha$ e $\beta$ são os parâmetros da distribuição de Weibull.

No caso de observações independentes, não necessariamente identicamente distribuídas, a derivação desse tempo de censura de forma análoga não é possível. Assim, empiricamente, baseado em resultados de simulações, foi usada a seguinte expressão para $c$,

$$
c=\alpha_{\left[1-p_{0}\right]}\left(-\log p_{0}\right)^{1 / \beta},
$$

onde $\alpha_{\left[1-p_{0}\right]}$ denota o quantil amostral de ordem $\left(1-p_{0}\right)$ do conjunto $\left\{\alpha\left(\mathbf{x}_{1}\right), \ldots, \alpha\left(\mathbf{x}_{n}\right)\right\}$. Note que esta expressão, no caso de observações independentes e identicamente distribuídas, é igual àquela proposta anteriormente. Mais uma vez, como as amostras são de tamanhos finitos, em geral, tal proporção não é observada em cada uma das amostras, mas o valor médio das proporções de censura é calculado e comparado com a proporção de censura desejada.

Aqui, tanto no modelo com uma covariada quanto no modelo com três covariadas, os valores destas são realizações independentes de uma variável aleatória com distribuição uniforme contínua $U(0,100)$. No primeiro caso, $\eta(\mathbf{x})=\phi_{1}+\phi_{2} x_{2}$, para $n=20$, são considerados dois níveis de censura: $25 \%$ e $50 \%$. No segundo caso, $\eta(\mathbf{x})=\phi_{1}+\phi_{2} x_{2}+\phi_{3} x_{3}+\phi_{4} x_{4}$, para $n=50$, foram simuladas duas proporções de censura: $p_{0}=30 \%$ e $p_{0}=50 \%$. Vale lembrar que os resultados são baseados em 10000 (dez mil) réplicas de Monte Carlo.

Considerando inicialmente o modelo com uma covariada e $p_{0}=25 \%$, as proporções de censura médias observadas foram iguais a: $22.78 \%(\beta=0.5), 23.11 \%(\beta=1)$ e $24.76 \%$ $(\beta=5)$. A Tabela 5.39 apresenta taxas de rejeição sob a hipótese nula de diferentes testes da razão de verossimilhanças com distintos níveis de significância. As médias e variâncias empíricas das estatísticas de teste são apresentadas na Tabela 5.40.

Entre as estatísticas de teste, as distribuições nulas daquelas baseadas em $\tilde{\ell}_{B N}$ foram melhor aproximadas pelas distribuições qui-quadrado. Isto pode ser comprovado pelas proximidades entre as taxas de rejeição das hipóteses nulas e os níveis nominais (Tabela 5.39) e pelas pequenas diferenças entre os momentos amostrais dessas estatísticas e os momentos assintóticos (Tabela 5.40). Aqui os valores tabelados dependeram da hipótese nula considerada.

Por exemplo, para $\beta=0.5$ e nível nominal de $10 \%$, os testes baseados em $\tilde{\ell}_{B N}$ e na verossimilhança original $\ell$ apresentaram taxas de rejeição de $\mathcal{H}_{0}: \phi_{2}=1$ iguais a $9.580 \%$ e $13.630 \%$, respectivamente, enquanto tais taxas relativas à rejeição de $\mathcal{H}_{0}: \phi_{1}=\phi_{2}=1$ 
foram iguais a $8.460 \%$ e $13.200 \%$, respectivamente. Note que, em geral, os testes baseados no ajuste sobre a verossimilhança perfilada são conservadores. Pela Tabela 5.40 , para $\beta=5$ e sob $\mathcal{H}_{0}: \phi_{2}=1$, as médias e as variâncias das estatísticas de teste foram: 1.218 e 2.857 $(\ell), 0.992$ e $1.930\left(\tilde{\ell}_{B N}\right)$, respectivamente.

Tabela 5.39. Taxas de rejeição da hipótese nula de diferentes testes da razão de verossimilhanças, distribuição valor extremo, dados censurados tipo I, $n=20,25 \%$ de censura.

\begin{tabular}{|c|c|c|c|c|c|}
\hline \multirow{3}{*}{$\beta$} & \multirow{3}{*}{ nível nominal } & \multicolumn{4}{|c|}{$\eta(\mathbf{x})=\phi_{1}+\phi_{2} x_{2}$} \\
\hline & & \multicolumn{2}{|c|}{$\mathcal{H}_{0}: \phi_{2}=1$} & \multicolumn{2}{|c|}{$\mathcal{H}_{0}: \phi_{1}=\phi_{2}=1$} \\
\hline & & $\ell$ & $\tilde{\ell}_{B N}$ & $\ell$ & $\tilde{\ell}_{B N}$ \\
\hline \multirow{6}{*}{0.5} & $15 \%$ & 19.220 & 15.000 & 18.890 & 12.910 \\
\hline & $10 \%$ & 13.630 & 9.580 & 13.200 & 8.460 \\
\hline & $5 \%$ & 7.370 & 4.760 & 7.500 & 4.260 \\
\hline & $1 \%$ & 1.870 & 0.960 & 1.680 & 0.730 \\
\hline & $0.1 \%$ & 0.240 & 0.070 & 0.230 & 0.060 \\
\hline & $0.05 \%$ & 0.090 & 0.020 & 0.140 & 0.040 \\
\hline \multirow{6}{*}{1} & $15 \%$ & 19.400 & 14.960 & 18.710 & 12.770 \\
\hline & $10 \%$ & 13.590 & 9.600 & 13.020 & 8.440 \\
\hline & $5 \%$ & 7.420 & 4.790 & 7.540 & 4.230 \\
\hline & $1 \%$ & 1.830 & 0.910 & 1.710 & 0.650 \\
\hline & $0.1 \%$ & 0.220 & 0.070 & 0.250 & 0.060 \\
\hline & $0.05 \%$ & 0.090 & 0.030 & 0.130 & 0.040 \\
\hline \multirow{6}{*}{5} & $15 \%$ & 19.240 & 14.680 & 18.960 & 13.660 \\
\hline & $10 \%$ & 13.710 & 10.070 & 13.590 & 8.870 \\
\hline & $5 \%$ & 7.590 & 4.800 & 7.500 & 4.280 \\
\hline & $1 \%$ & 1.900 & 1.030 & 1.820 & 0.750 \\
\hline & $0.1 \%$ & 0.270 & 0.060 & 0.250 & 0.050 \\
\hline & $0.05 \%$ & 0.100 & 0.010 & 0.120 & 0.030 \\
\hline
\end{tabular}

Tabela 5.40. Médias e variâncias amostrais de diferentes estatísticas de teste e os valores assintóticos destas, distribuição valor extremo, dados censurados tipo I, $n=20,25 \%$ de censura.

\begin{tabular}{cccccccc}
\hline & & \multicolumn{5}{c}{$\eta(\mathbf{x})=\phi_{1}+\phi_{2} x_{2}$} \\
\cline { 3 - 8 }$\beta$ & momento & \multicolumn{3}{c}{$\mathcal{H}_{0}: \phi_{2}=1$} & \multicolumn{3}{c}{$\mathcal{H}_{0}: \phi_{1}=\phi_{2}=1$} \\
\cline { 3 - 8 } & & $\chi_{1}^{2}$ & $\ell$ & $\tilde{\ell}_{B N}$ & $\chi_{2}^{2}$ & $\ell$ & $\tilde{\ell}_{B N}$ \\
\hline \multirow{2}{*}{0.5} & média & 1 & 1.208 & 0.984 & 2 & 2.283 & 1.848 \\
& variância & 2 & 2.844 & 1.920 & 4 & 5.220 & 3.484 \\
\hline \multirow{2}{*}{1} & média & 1 & 1.208 & 0.986 & 2 & 2.287 & 1.854 \\
& variância & 2 & 2.823 & 1.911 & 4 & 5.172 & 3.464 \\
\hline \multirow{2}{*}{5} & média & 1 & 1.218 & 0.992 & 2 & 2.308 & 1.893 \\
& variância & 2 & 2.857 & 1.930 & 4 & 5.237 & 3.611 \\
\hline
\end{tabular}


Aumentando o percentual de censura para $50 \%$ e considerando também $\phi_{i}=3$, $\forall i$, a Tabela 5.41 apresenta taxas de rejeição sob a hipótese nula de diferentes testes da razão de verossimilhanças com distintos níveis de significância. As médias e variâncias empíricas das estatísticas de teste são apresentadas na Tabela 5.42. Sob a hipótese $\mathcal{H}_{0}: \phi_{1}=\phi_{2}=3$, os percentuais médios de censura observados foram iguais a $48.07 \%(\beta=0.5), 48.59 \%(\beta=1)$ e $49.93 \%(\beta=5)$. Sob a hipótese $\mathcal{H}_{0}: \phi_{1}=\phi_{2}=1$, tais percentuais foram, respectivamente, iguais a $48.90 \%, 48.85 \%$ e $49.74 \%$.

Tabela 5.41. Taxas de rejeição da hipótese nula de diferentes testes da razão de verossimilhanças, distribuição valor extremo, dados censurados tipo I, $n=20,50 \%$ de censura.

\begin{tabular}{|c|c|c|c|c|c|}
\hline \multirow{3}{*}{$\beta$} & \multirow{3}{*}{ nível nominal } & \multicolumn{4}{|c|}{$\eta(\mathbf{x})=\phi_{1}+\phi_{2} x_{2}$} \\
\hline & & \multicolumn{2}{|c|}{$\mathcal{H}_{0}: \phi_{1}=\phi_{2}=1$} & \multicolumn{2}{|c|}{$\mathcal{H}_{0}: \phi_{1}=\phi_{2}=3$} \\
\hline & & $\ell$ & $\tilde{\ell}_{B N}$ & $\ell$ & $\tilde{\ell}_{B N}$ \\
\hline \multirow{6}{*}{0.5} & $15 \%$ & 21.420 & 12.020 & 21.560 & 13.150 \\
\hline & $10 \%$ & 15.030 & 7.590 & 15.740 & 8.580 \\
\hline & $5 \%$ & 8.460 & 3.400 & 9.110 & 4.050 \\
\hline & $1 \%$ & 1.990 & 0.470 & 2.390 & 0.690 \\
\hline & $0.1 \%$ & 0.240 & 0.020 & 0.310 & 0.040 \\
\hline & $0.05 \%$ & 0.130 & 0.010 & 0.150 & 0.030 \\
\hline \multirow{6}{*}{1} & $15 \%$ & 21.730 & 12.340 & 21.750 & 13.000 \\
\hline & $10 \%$ & 15.420 & 7.820 & 15.750 & 8.430 \\
\hline & $5 \%$ & 8.670 & 3.500 & 9.020 & 3.930 \\
\hline & $1 \%$ & 2.190 & 0.590 & 2.360 & 0.690 \\
\hline & $0.1 \%$ & 0.270 & 0.030 & 0.300 & 0.040 \\
\hline & $0.05 \%$ & 0.140 & 0.030 & 0.160 & 0.020 \\
\hline \multirow{6}{*}{5} & $15 \%$ & 22.720 & 13.550 & 22.820 & 13.570 \\
\hline & $10 \%$ & 16.570 & 9.150 & 16.700 & 9.230 \\
\hline & $5 \%$ & 9.620 & 4.520 & 9.710 & 4.390 \\
\hline & $1 \%$ & 2.730 & 0.720 & 2.710 & 0.690 \\
\hline & $0.1 \%$ & 0.360 & 0.040 & 0.350 & 0.030 \\
\hline & $0.05 \%$ & 0.200 & 0.010 & 0.170 & 0.000 \\
\hline
\end{tabular}

Pelos resultados da Tabela 5.41, os testes da razão de verossimilhanças baseados nas verossimilhanças originais tornam-se ainda mais liberais, enquanto os testes da razão de verossimilhanças baseados em $\tilde{\ell}_{B N}$ caracterizam-se pelo seu conservadorismo, rejeitando as hipóteses nulas aquém do esperado, e apresentam os melhores desempenhos. Note que as taxas de rejeição dependem da hipótese nula considerada e variam com o valor do parâmetro $\beta=\sigma^{-1}$. Esta mesma observação vale para as estimativas dos momentos das estatísticas de teste, conforme os resultados apresentados na Tabela 5.42. Observe que as estimativas associadas a $\tilde{\ell}_{B N}$ estão mais próximas aos valores assintóticos. 
Por exemplo, sob $\mathcal{H}_{0}: \phi_{1}=\phi_{2}=3$, para $\beta=5$ e nível nominal de $5 \%$, as taxas de rejeição dos testes baseados na verossimilhança original e em $\tilde{\ell}_{B N}$ foram, respectivamente, iguais a $9.710 \%$ e $4.390 \%$. Aqui as médias e variâncias amostrais das estatísticas de teste foram: 2.553 e $6.326(\ell), 1.882$ e $3.589\left(\tilde{\ell}_{B N}\right)$, respectivamente.

Tabela 5.42. Médias e variâncias amostrais de diferentes estatísticas de teste e os valores assintóticos destas, distribuição valor extremo, dados censurados tipo I, $n=20,50 \%$ de censura.

\begin{tabular}{ccccccc}
\hline \multirow{2}{*}{$\beta$} & \multirow{2}{*}{ momento } & $\chi_{2}^{2}$ & \multicolumn{4}{c}{$\eta(\mathbf{x})=\phi_{1}+\phi_{2} x_{2}$} \\
\cline { 4 - 7 } & & & $\mathcal{H}_{0}: \phi_{1}=\phi_{2}=1$ & $\mathcal{H}_{0}: \phi_{1}=\phi_{2}=3$ \\
\cline { 3 - 7 } & & & $\tilde{\ell}_{B N}$ & $\ell$ & $\tilde{\ell}_{B N}$ \\
\hline \multirow{2}{*}{0.5} & média & 2 & 2.420 & 1.769 & 2.478 & 1.844 \\
& variância & 4 & 5.565 & 3.093 & 5.989 & 3.478 \\
\hline \multirow{2}{*}{1} & média & 2 & 2.466 & 1.805 & 2.489 & 1.846 \\
& variância & 4 & 5.749 & 3.228 & 5.983 & 3.407 \\
\hline \multirow{2}{*}{5} & média & 2 & 2.548 & 1.886 & 2.553 & 1.882 \\
& variância & 4 & 6.337 & 3.620 & 6.326 & 3.589 \\
\hline
\end{tabular}

Figura 5.31. Gráfico das discrepâncias relativas de quantis, distribuição valor extremo, $\eta(\mathbf{x})=\phi_{1}+\phi_{2} x_{2}, \beta=\sigma^{-1}=1, \mathcal{H}_{0}: \phi_{2}=1$, dados censurados tipo I, $p_{0}=25 \%$.

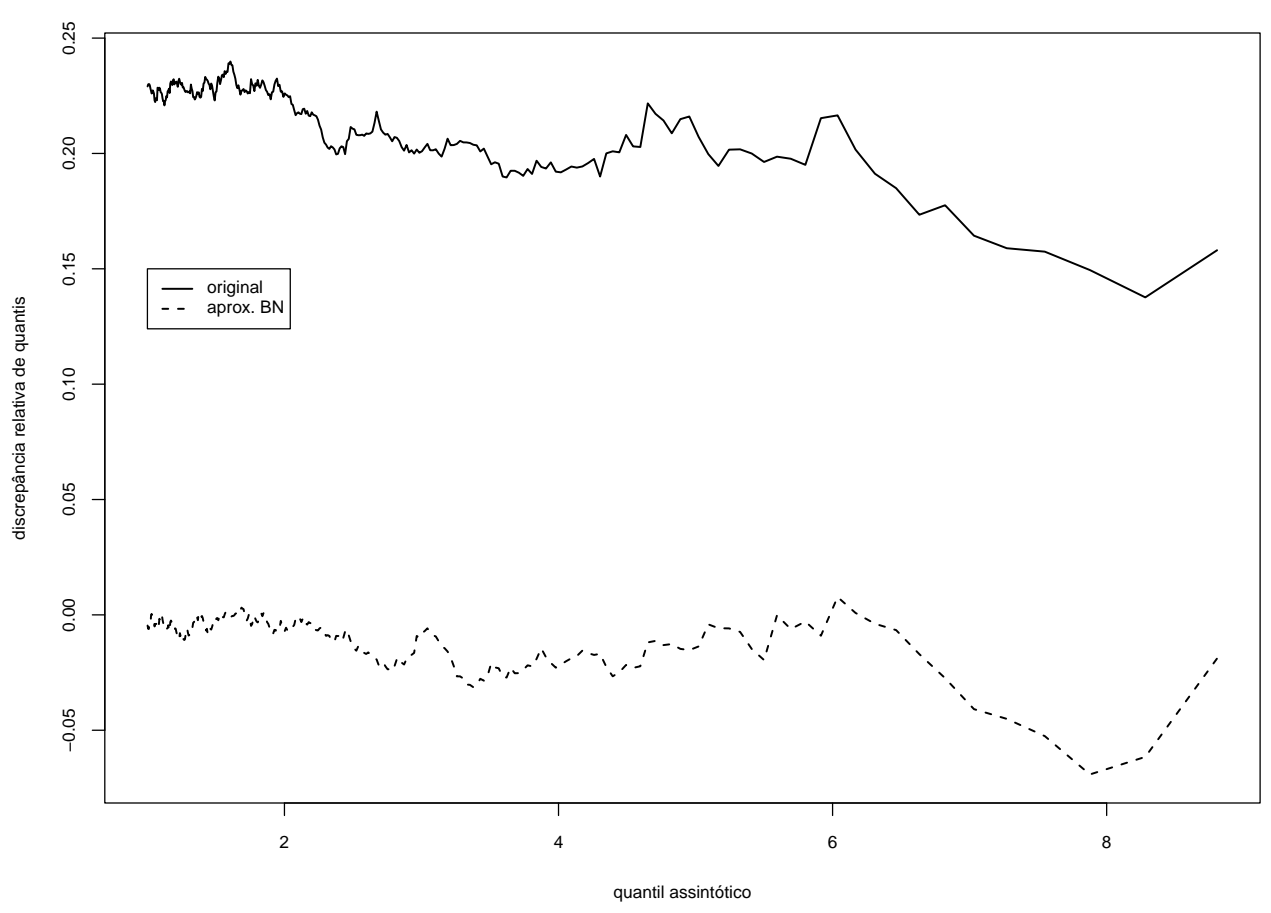

Os gráficos das Figuras 5.31 e 5.32 apresentam as curvas de discrepâncias relativas de quantis das estatísticas de teste baseadas na verossimilhança original $\ell$ e no ajuste sobre a verossimilhança perfilada denotado por $\tilde{\ell}_{B N}$ para dados censurados tipo I, $p_{0}=25 \%$. Na Figura 5.31, $\mathcal{H}_{0}: \phi_{2}=1$, enquanto na Figura 5.32, $\mathcal{H}_{0}: \phi_{1}=\phi_{2}=1$. Assim os quantis 
assintóticos são obtidos de variáveis qui-quadrado com um e dois graus de liberdade. Note que à medida que aumenta o número de parâmetros testados, as curvas deslocam-se para baixo.

Figura 5.32. Gráfico das discrepâncias relativas de quantis, distribuição valor extremo, $\eta(\mathbf{x})=\phi_{1}+\phi_{2} x_{2}, \beta=\sigma^{-1}=1, \mathcal{H}_{0}: \phi_{1}=\phi_{2}=1$, dados censurados tipo I, $p_{0}=25 \%$.

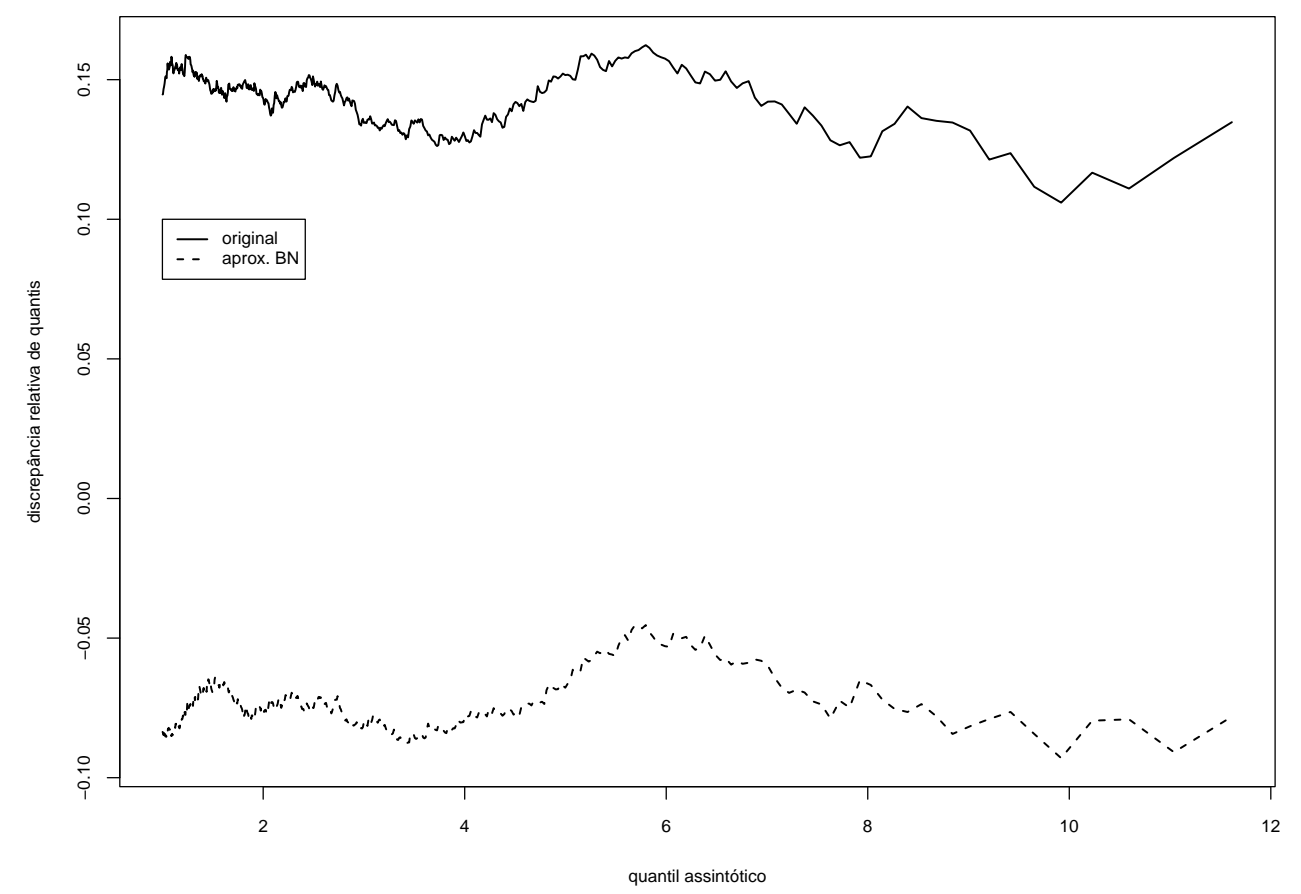

Considerando agora o modelo com três covariadas e $p_{0}=30 \%$, a Tabela 5.43 apresenta taxas de rejeição sob a hipótese nula de diferentes testes da razão de verossimilhanças com distintos níveis de significância. As médias e variâncias empíricas das estatísticas de teste são apresentadas na Tabela 5.44. Aqui $x_{2}, x_{3}$ e $x_{4}$ são realizações independentes de uma variável aleatória uniforme contínua $U(0,100)$, sendo assim este modelo uma extensão daquele apresentado anteriormente através da inclusão de duas covariáveis. Dessa forma, os comportamentos dos testes baseados nas verossimilhanças originais e em $\tilde{\ell}_{B N}$ são semelhantes à situação anterior e, mais uma vez, vale destacar destacar o ótimo desempenho deste. Para $\beta=0.5$, a censura média observada nas amostras foi de $29.30 \%$, enquanto para $\beta=1$, $29.32 \%$. Para $\beta=5$, tal percentual foi de $29.70 \%$.

Os gráficos das Figuras 5.33, 5.34 e 5.35 apresentam as curvas de discrepâncias relativas de quantis das estatísticas de teste baseadas na verossimilhança original $\ell$ e no ajuste sobre a verossimilhança perfilada denotado por $\tilde{\ell}_{B N}$ para dados censurados tipo I, $p_{0}=30 \%$. Na Figura 5.33, $\mathcal{H}_{0}: \phi_{4}=1$, enquanto na Figura 5.34, $\mathcal{H}_{0}: \phi_{3}=\phi_{4}=1$. Na Figura 5.35, $\mathcal{H}_{0}: \phi_{2}=\phi_{3}=\phi_{4}=1$. Assim os quantis assintóticos são obtidos de variáveis qui-quadrado com um, dois e três graus de liberdade. Nas três figuras, a curva associada ao teste baseado em $\tilde{\ell}_{B N}$ está mais próxima a ordenada nula. 
Tabela 5.43. Taxas de rejeição da hipótese nula de diferentes testes da razão de verossimilhanças, distribuição valor extremo, dados censurados tipo I, $n=50,30 \%$ de censura.

\begin{tabular}{|c|c|c|c|c|c|c|c|}
\hline \multirow{3}{*}{$\beta$} & \multirow{3}{*}{ nível nominal } & \multicolumn{6}{|c|}{$\eta(\mathbf{x})=\phi_{1}+\phi_{2} x_{2}+\phi_{3} x_{3}+\phi_{4} x_{4}$} \\
\hline & & \multicolumn{2}{|c|}{$\mathcal{H}_{0}: \phi_{4}=1$} & \multicolumn{2}{|c|}{$\mathcal{H}_{0}: \phi_{3}=\phi_{4}=1$} & \multicolumn{2}{|c|}{$\mathcal{H}_{0}: \phi_{2}=\phi_{3}=\phi_{4}=1$} \\
\hline & & $\ell$ & $\tilde{\ell}_{B N}$ & $\ell$ & $\tilde{\ell}_{B N}$ & $\ell$ & $\tilde{\ell}_{B N}$ \\
\hline \multirow{6}{*}{0.5} & $15 \%$ & 17.910 & 14.670 & 18.500 & 14.770 & 18.740 & 15.690 \\
\hline & $10 \%$ & 12.460 & 9.710 & 12.750 & 9.770 & 13.020 & 10.540 \\
\hline & $5 \%$ & 6.790 & 4.690 & 7.020 & 4.960 & 7.130 & 5.560 \\
\hline & $1 \%$ & 1.630 & 0.930 & 1.640 & 0.970 & 1.580 & 1.010 \\
\hline & $0.1 \%$ & 0.140 & 0.060 & 0.170 & 0.050 & 0.130 & 0.080 \\
\hline & $0.05 \%$ & 0.070 & 0.030 & 0.060 & 0.030 & 0.060 & 0.010 \\
\hline \multirow{6}{*}{1} & $15 \%$ & 18.030 & 14.650 & 18.800 & 14.880 & 18.870 & 15.470 \\
\hline & $10 \%$ & 12.720 & 9.700 & 12.870 & 9.780 & 13.060 & 10.350 \\
\hline & $5 \%$ & 6.830 & 4.630 & 6.900 & 4.860 & 7.160 & 5.420 \\
\hline & $1 \%$ & 1.610 & 0.910 & 1.660 & 1.000 & 1.630 & 1.000 \\
\hline & $0.1 \%$ & 0.170 & 0.070 & 0.160 & 0.060 & 0.170 & 0.070 \\
\hline & $0.05 \%$ & 0.070 & 0.050 & 0.070 & 0.030 & 0.070 & 0.020 \\
\hline \multirow{6}{*}{5} & $15 \%$ & 18.260 & 14.640 & 19.280 & 14.920 & 19.170 & 15.530 \\
\hline & $10 \%$ & 12.700 & 9.730 & 13.280 & 9.850 & 13.200 & 10.410 \\
\hline & $5 \%$ & 6.870 & 4.840 & 7.060 & 4.880 & 7.180 & 5.390 \\
\hline & $1 \%$ & 1.570 & 0.940 & 1.740 & 0.990 & 1.560 & 0.930 \\
\hline & $0.1 \%$ & 0.170 & 0.070 & 0.220 & 0.050 & 0.130 & 0.060 \\
\hline & $0.05 \%$ & 0.070 & 0.050 & 0.080 & 0.030 & 0.070 & 0.030 \\
\hline
\end{tabular}

Tabela 5.44. Médias e variâncias amostrais de diferentes estatísticas de teste e os valores assintóticos destas, distribuição valor extremo, dados censurados tipo I, $n=50,30 \%$ de censura.

\begin{tabular}{|c|c|c|c|c|c|c|c|c|c|c|}
\hline \multirow{3}{*}{$\beta$} & \multirow{3}{*}{ momento } & \multicolumn{9}{|c|}{$\eta(\mathbf{x})=\phi_{1}+\phi_{2} x_{2}+\phi_{3} x_{3}+\phi_{4} x_{4}$} \\
\hline & & \multicolumn{3}{|c|}{$\mathcal{H}_{0}: \phi_{4}=1$} & \multicolumn{3}{|c|}{$\mathcal{H}_{0}: \phi_{3}=\phi_{4}=1$} & \multicolumn{3}{|c|}{$\mathcal{H}_{0}: \phi_{2}=\phi_{3}=\phi_{4}=1$} \\
\hline & & $\chi_{1}^{2}$ & $\ell$ & $\tilde{\ell}_{B N}$ & $\chi_{2}^{2}$ & $\ell$ & $\tilde{\ell}_{B N}$ & $\chi_{3}^{2}$ & $\ell$ & $\tilde{\ell}_{B N}$ \\
\hline \multirow{2}{*}{0.5} & média & 1 & 1.157 & 0.990 & 2 & 2.247 & 1.988 & 3 & 3.336 & 3.066 \\
\hline & variância & 2 & 2.558 & 1.886 & 4 & 4.942 & 3.895 & 6 & 7.200 & 6.114 \\
\hline \multirow{2}{*}{1} & média & 1 & 1.161 & 0.988 & 2 & 2.254 & 1.984 & 3 & 3.340 & 3.056 \\
\hline & variância & 2 & 2.584 & 1.886 & 4 & 4.978 & 3.887 & 6 & 7.223 & 6.086 \\
\hline \multirow{2}{*}{5} & média & 1 & 1.163 & 0.991 & 2 & 2.286 & 1.997 & 3 & 3.350 & 3.050 \\
\hline & variância & 2 & 2.600 & 1.900 & 4 & 5.064 & 3.915 & 6 & 7.204 & 6.067 \\
\hline
\end{tabular}

Aumentando o percentual de censura para $p_{0}=50 \%$, a Tabela 5.45 apresenta taxas de rejeição sob a hipótese nula de diferentes testes da razão de verossimilhanças com distintos níveis de significância. As médias e variâncias empíricas das estatísticas de teste são apresentadas na Tabela 5.46. Aqui é considerada apenas a hipótese $\mathcal{H}_{0}: \phi_{2}=\phi_{3}=\phi_{4}=1$, cujas taxas de rejeição dos testes têm se apresentado mais distantes dos níveis nominais. 
Figura 5.33. Gráfico das discrepâncias relativas de quantis, distribuição valor extremo, $\eta(\mathbf{x})=\phi_{1}+\phi_{2} x_{2}+\phi_{3} x_{3}+\phi_{4} x_{4}, \beta=\sigma^{-1}=1, \mathcal{H}_{0}: \phi_{4}=1$, dados censurados tipo I, $p_{0}=30 \%$.

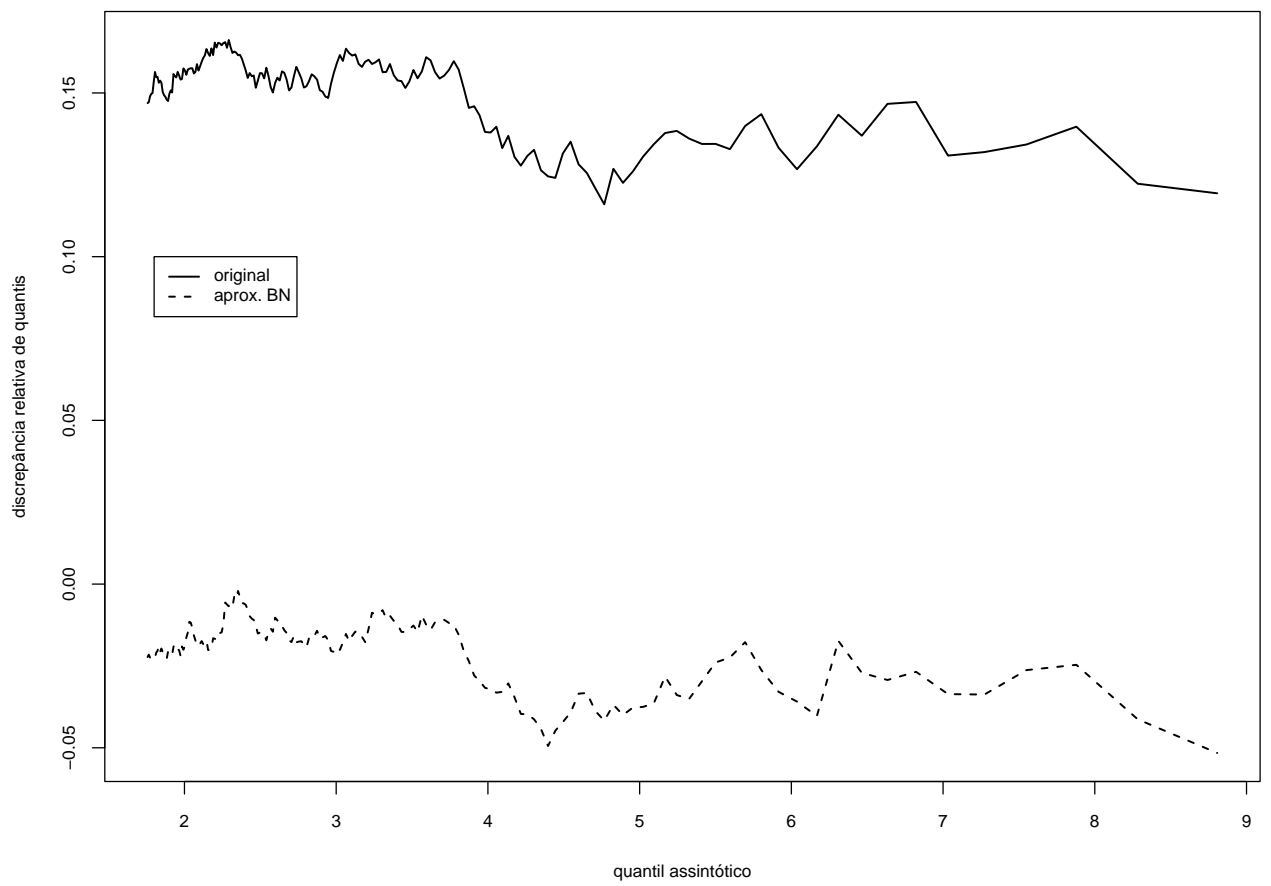

Figura 5.34. Gráfico das discrepâncias relativas de quantis, distribuição valor extremo, $\eta(\mathbf{x})=\phi_{1}+\phi_{2} x_{2}+\phi_{3} x_{3}+\phi_{4} x_{4}, \beta=\sigma^{-1}=1, \mathcal{H}_{0}: \phi_{3}=\phi_{4}=1$, dados censurados tipo I, $p_{0}=30 \%$.

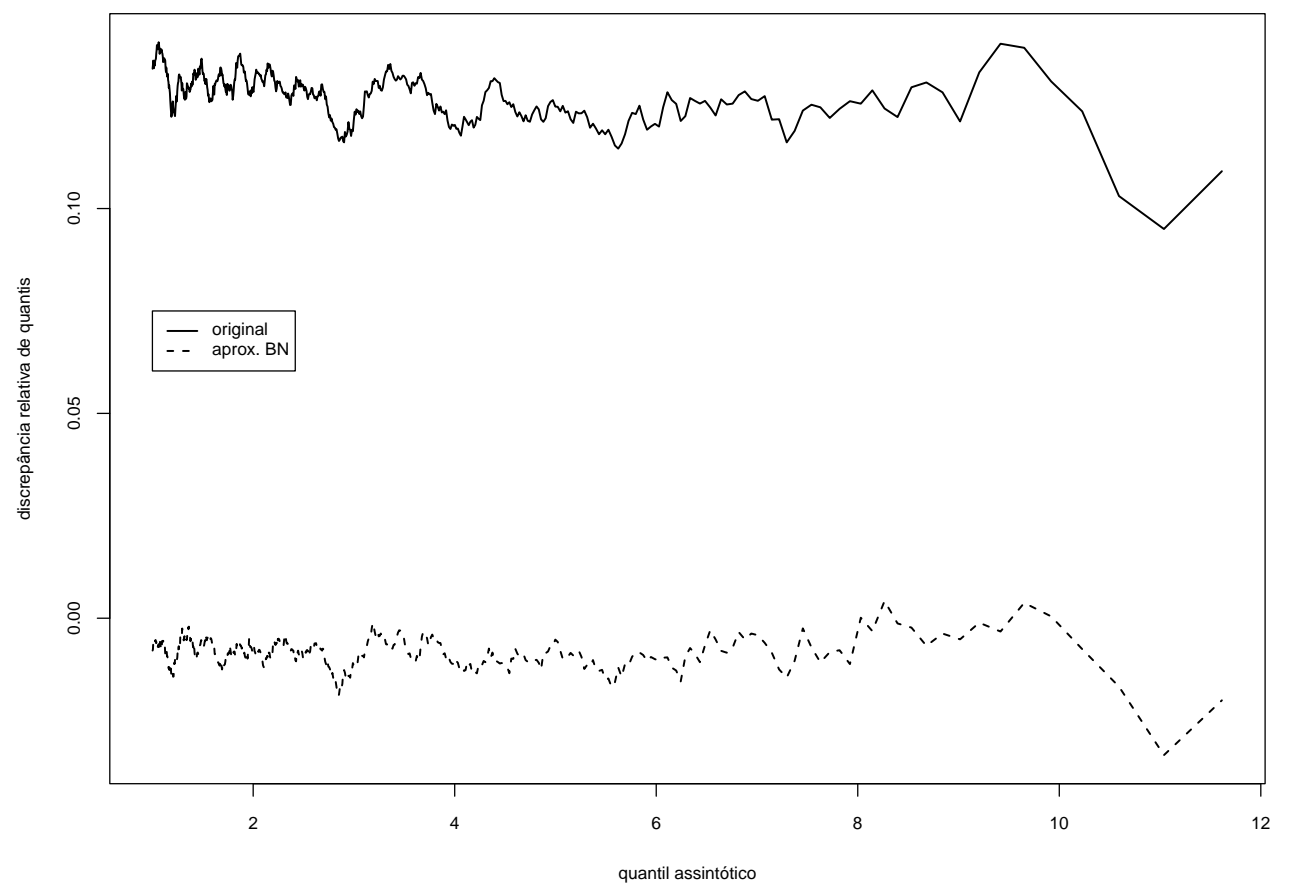

Para $\beta=0.5$, a censura média observada nas amostras foi de $49.06 \%$, enquanto para $\beta=1$, 
Figura 5.35. Gráfico das discrepâncias relativas de quantis, distribuição valor extremo, $\eta(\mathbf{x})=\phi_{1}+\phi_{2} x_{2}+\phi_{3} x_{3}+\phi_{4} x_{4}, \beta=\sigma^{-1}=1, \mathcal{H}_{0}: \phi_{2}=\phi_{3}=\phi_{4}=1$, dados censurados tipo I, $p_{0}=30 \%$.

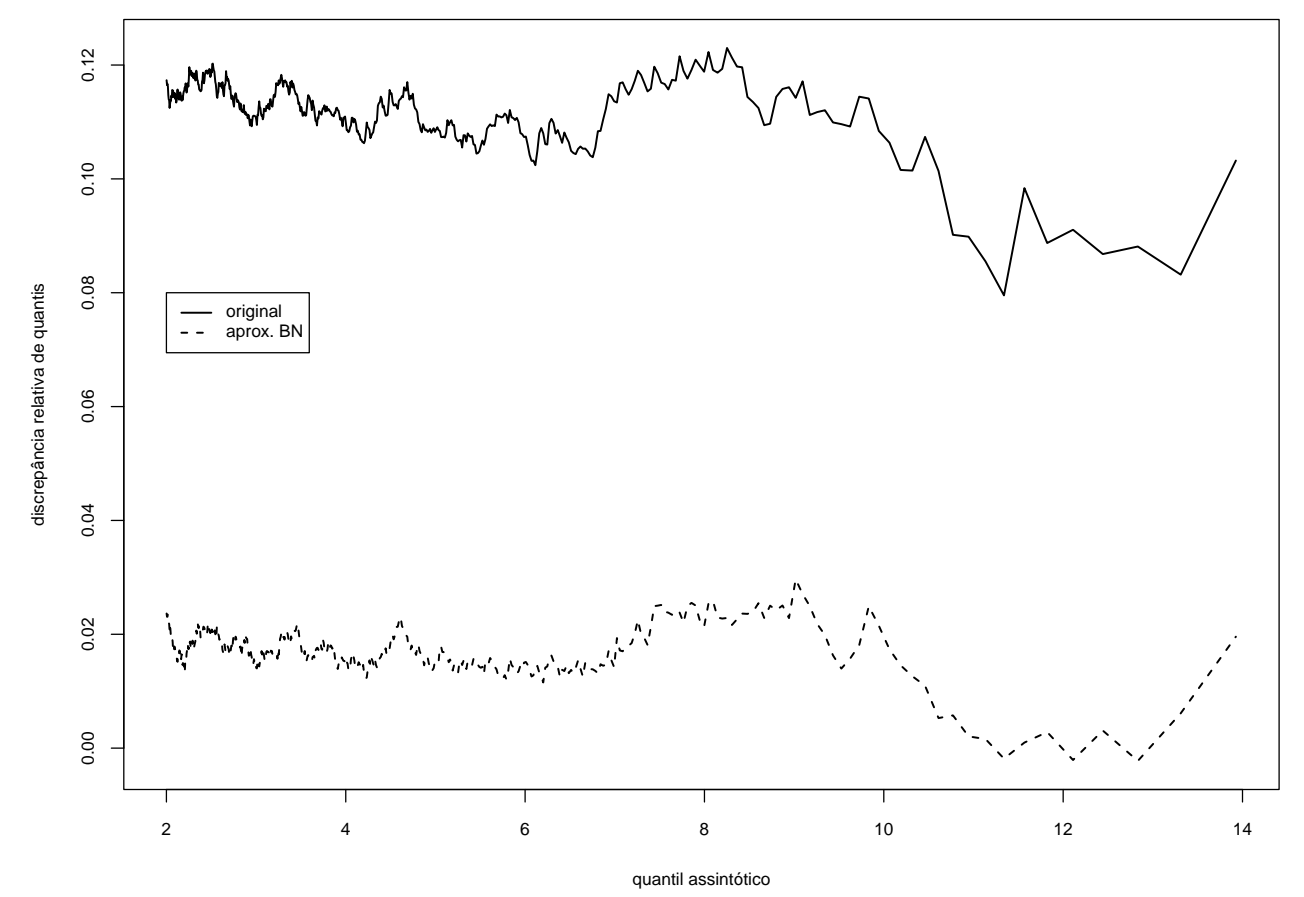

Tabela 5.45. Taxas de rejeição da hipótese nula de diferentes testes da razão de verossimilhanças, distribuição valor extremo, $\eta(\mathbf{x})=\phi_{1}+\phi_{2} x_{2}+\phi_{3} x_{3}+\phi_{4} x_{4}$, dados censurados tipo I, $n=50,50 \%$ de censura.

\begin{tabular}{crrrrrr}
\hline & \multicolumn{9}{c}{$\mathcal{H}_{0}: \phi_{2}=\phi_{3}=\phi_{4}=1$} \\
\cline { 2 - 7 } nível nominal & \multicolumn{2}{c}{$\beta=0.5$} & \multicolumn{2}{c}{$\beta=1$} & \multicolumn{2}{c}{$\beta=5$} \\
\cline { 2 - 7 } & \multicolumn{1}{c}{$\ell$} & \multicolumn{1}{c}{$\ell$} & \multicolumn{1}{c}{$\tilde{\ell}_{B N}$} & \multicolumn{1}{c}{$\ell$} & $\tilde{\ell}_{B N}$ \\
\hline $15 \%$ & 20.260 & 16.280 & 20.390 & 16.490 & 20.960 & 16.030 \\
$10 \%$ & 14.410 & 11.260 & 14.840 & 11.350 & 14.840 & 10.880 \\
$5 \%$ & 8.110 & 5.790 & 8.450 & 5.720 & 8.310 & 5.590 \\
$1 \%$ & 2.100 & 1.230 & 2.050 & 1.170 & 2.160 & 1.040 \\
$0.1 \%$ & 0.240 & 0.110 & 0.250 & 0.120 & 0.250 & 0.110 \\
$0.05 \%$ & 0.130 & 0.070 & 0.140 & 0.050 & 0.130 & 0.060 \\
\hline
\end{tabular}

49.24\%. Para $\beta=5$, tal percentual foi de $49.84 \%$. De acordo com os resultados da Tabela 5.45 , ambos os testes são liberais, mas aquele baseado em $\tilde{\ell}_{B N}$ apresenta taxas mais próximas aos níveis nominais, assim como os momentos amostrais das suas estatísticas de teste estão mais próximos dos valores assintóticos (Tabela 5.46).

Embora os testes da razão de verossimilhanças baseados na verossimilhança original $\ell$ tenham se mostrado bastante liberais ao longo desta seção, a seguir são mostrados alguns resultados relativos ao poder dos testes. Aqui os valores atribuídos a $x_{2}$ são realizações 
Tabela 5.46. Médias e variâncias amostrais de diferentes estatísticas de teste e os valores assintóticos destas, distribuição valor extremo, $\eta(\mathbf{x})=\phi_{1}+\phi_{2} x_{2}+\phi_{3} x_{3}+\phi_{4} x_{4}$, dados censurados tipo I, $n=50,50 \%$ de censura.

\begin{tabular}{cccccccc}
\hline \multirow{2}{*}{ momento } & & \multicolumn{6}{c}{$\mathcal{H}_{0}: \phi_{2}=\phi_{3}=\phi_{4}=1$} \\
\cline { 3 - 8 } & $\chi_{3}^{2}$ & \multicolumn{2}{c}{$\beta=0.5$} & \multicolumn{2}{c}{$\beta=1$} & \multicolumn{2}{c}{$\beta=5$} \\
\cline { 3 - 8 } & & $\ell$ & $\tilde{\ell}_{B N}$ & $\ell$ & $\tilde{\ell}_{B N}$ & $\ell$ & $\tilde{\ell}_{B N}$ \\
\hline média & 3 & 3.489 & 3.136 & 3.510 & 3.124 & 3.526 & 3.106 \\
variância & 6 & 7.950 & 6.486 & 7.994 & 6.411 & 8.020 & 6.304 \\
\hline
\end{tabular}

de uma variável aleatória uniforme contínua $U(0,1)$. Os resultados são baseados em 5000 amostras de Monte Carlo, $\beta=\sigma^{-1}=1$ e o nível de significância é igual a $5 \%$.

$\mathrm{Na}$ Tabela 5.47, mais precisamente, na coluna censura, é usada a seguinte notação: "0" denota dados completos; "r $=15$ " denota dados censurados tipo II, $(n, r)=(20,15)$; " $p_{0}=25 \% "$ denota dados censurados tipo I com nível de censura nominal igual a $25 \%$. De acordo com esta tabela, os testes da razão de verossimilhanças baseados em $\ell$ rejeitam mais a hipótese nula quando esta é falsa. Aliás, até mesmo quando esta é verdadeira, conforme os resultados apresentados ao longo da seção. Por exemplo, para dados censurados do tipo II $\left(r=15\right.$ na tabela) e $\phi_{1}=\phi_{2}=3.0$, o teste original rejeitou a hipótese nula $55.560 \%$ das vezes, enquanto o teste baseado em $\tilde{\ell}_{B N}$ apresentou taxa de rejeição igual a $47.480 \%$. Aqui vale ressaltar também a perda de poder dos testes com a presença de censura nos dados. Por exemplo, enquanto para dados completos os testes baseados em $\ell$ e $\tilde{\ell}_{B N}$ rejeitaram a hipótese nula $26.700 \%$ e $20.520 \%$ quando $\phi_{1}=\phi_{2}=2.0$, para dados censurados tipo II, os percentuais foram iguais a $19.080 \%$ e $14.780 \%$, respectivamente.

Tabela 5.47. Taxas de rejeição da hipótese nula de diferentes testes da razão de verossimilhanças, distribuição valor extremo, $n=20$.

\begin{tabular}{crrr}
\hline \multirow{2}{*}{ censura } & $\phi_{i}$ & \multicolumn{2}{c}{$\eta(\mathbf{x})=\phi_{1}+\phi_{2} x_{2}, \mathcal{H}_{0}: \phi_{2}=1$} \\
\cline { 3 - 4 } & & \multicolumn{1}{c}{$\ell$} & $\tilde{\ell}_{B N}$ \\
\hline \multirow{3}{*}{0} & 1.1 & 7.160 & 5.220 \\
& 2.0 & 26.700 & 20.520 \\
& 3.0 & 70.320 & 63.760 \\
$r=15$ & 5.0 & 99.200 & 98.900 \\
\hline \multirow{3}{*}{$p_{0}=25 \%$} & 1.1 & 7.780 & 4.500 \\
& 2.0 & 19.080 & 14.780 \\
& 3.0 & 55.560 & 47.480 \\
& 5.0 & 96.650 & 94.900 \\
\hline & 1.1 & 7.440 & 4.800 \\
& 2.0 & 22.080 & 16.320 \\
\hline
\end{tabular}




\subsubsection{CONCLUSÕES}

Baseando-se nos resultados numéricos apresentados ao longo desta seção referentes ao tamanho e ao poder dos testes da razão de verossimilhanças construídos a partir da verossimilhança original $\ell$ e da versão ajustada da verossimilhança perfilada denotada por $\tilde{\ell}_{B N}$, este trabalho sugere a utilização do segundo teste para realizar inferências sobre $\phi$, vetor paramétrico que constitui a parte sistemática do modelo de regressão de Weibull.

Os testes baseados em $\tilde{\ell}_{B N}$ apresentaram taxas de rejeição sob a hipótese nula mais próximas aos níveis nominais. Os testes baseados em $\ell$ rejeitaram demais, mesmo quando $\mathcal{H}_{0}$ era verdadeira, sendo bastante liberais nestes casos. 


\section{APÊNDICE A}

\section{DETALHES COMPUTACIONAIS}

\section{A.1. GERADORES DE NÚMEROS ALEATÓRIOS}

A linguagem de programação 0x dispõe de três geradores de números aleatórios denominados:

- "PM" - Park e Miller modificado, de período aproximadamente $2^{32}$ e que exige uma semente,

- "GM" - George Marsaglia, de período aproximadamente $2^{60}$ e que exige duas sementes,

- “LE" - Pierre L'Ecuyer, de período aproximadamente $2^{113}$ e que exige quatro sementes.

Maiores detalhes sobre estes geradores podem ser encontrados em Doornik (2001). O gerador utilizado nas simulações foi o segundo, "GM" - George Marsaglia. Este gerador passa em testes rigorosos de aleatoriedade (como, e.g., a bateria de testes "Die Hard" desenvolvida por George Marsaglia) e possui uma eficiente implementação em C (e, por conseqüência, em 0x). O código fonte em C dos três geradores é dado em Doornik (1999, Appendix A5).

\section{A.2. COMANDOS DE MAXIMIZAÇÃO}

Quando os estimadores de máxima verossimilhança derivados das várias verossimilhanças perfiladas exemplificadas no Capítulo 2 não possuíam formas fechadas ou quando não foi possível obter explicitamente a verossimilhança perfilada, seja essa modificada ou não, devido a $\widehat{\nu}_{\mu}$ também não possuir forma fechada, foram utilizados os seguintes comandos da linguagem de programação 0x para obter as estimativas dos parâmetros de interesse: MaxBFGS e MaxSQP, respectivamente. Estes pertencem ao pacote de maximização da versão 3.30 da linguagem de programação 0x, que também disponibiliza comandos para diferenciação numérica. Diferentemente de MaxBFGS, MaxSQP maximiza funções com restrições nas variáveis (parâmetros).

O comando MaxBFGS maximiza funções usando o método quase-Newton desenvolvido por Broyden, Fletcher, Goldfarb e Shanno (BFGS) com a possibilidade de escolha de condições e parâmetros da maximização, como a utilização de derivadas analíticas ou numéricas da função a ser maximizada, os critérios de convergência, o número máximo de iterações, etc.. As simulações computacionais realizadas utilizaram derivadas analíticas a fim de se obter maior precisão e eficiência. Maiores detalhes sobre o comando MaxBFGS podem ser encontrados 
em Doornik (2001). Para uma discussão do método BFGS e sua implementação em C, ver Press et al. (1992, pp. 425-430). A idéia central deste método e a razão da denominação quase-Newton são explicadas a seguir.

Numa vizinhança de um ponto $v(k)$ (escalar ou vetor), em que $k$ indica a iteração do processo, pois este método de maximização é numérico, uma função qualquer $f$ pode ser aproximada por sua série de Taylor até segunda ordem como

$$
f(v)=f(v(k))+(v-v(k))^{\top} \nabla f(v(k))+\frac{1}{2}(v-v(k))^{\top} A(v(k))(v-v(k)),
$$

onde $\nabla f(v(k))$ é o gradiente de $f$ avaliado em $v(k), A(v(k))$ é a verdadeira matriz hessiana avaliada em $v(k)$ e $v$ pertence a uma vizinhança de $v(k)$. Desta expressão obtém-se

$$
\nabla f(v)=\nabla f(v(k))+A(v(k))(v-v(k)) .
$$

No método de Newton, faz-se $\nabla f(v)=0$ para determinar o próximo ponto da iteração:

$$
0=\nabla f(v(k))+A(v(k))(v-v(k)) \Rightarrow v=v(k)-A^{-1}(v(k)) \nabla f(v(k)),
$$

onde $v$ é uma aproximação para o ponto de máximo da função $f$.

Assim, aproximações para o ponto de máximo desta função são obtidas através da lei de recorrência

$$
v(k+1)=v(k)-A^{-1}(v(k)) \nabla f(v(k))
$$

ou, generalizando,

$$
v(k+1)=v(k)-s(k) A^{-1}(v(k)) \nabla f(v(k)),
$$

onde $s(k)$ é um escalar determinado por um procedimento de busca linear a partir de $v(k) \mathrm{e}$ na direção de $-A^{-1}(v(k)) \nabla f(v(k))$ de tal forma que $f(v(k))$ cresça ao longo desta direção. $\mathrm{O}$ valor de $f$ aumenta com a maior taxa possível quando os pontos $v(k)$ movem-se numa direção definida pelo gradiente em $v(k)$. Assim, o aumento da função $f$ é caracterizado pela desigualdade

$$
\nabla^{\top} f(v(k))(v-v(k))>0 \Rightarrow-(v-v(k))^{\top} A(v(k))(v-v(k))>0,
$$

e, portanto, $A$ deve ser negativa-definida $\forall k$.

Em geral, longe de um ponto de máximo, não há garantia que a matriz hessiana $A$ seja negativa-definida. Logo, o incremento $-s(k) A^{-1}(v(k)) \nabla f(v(k))$ pode mover o ponto $v(k)$ para um ponto $v(k+1)$ onde o valor da função é menor. Desta forma, a idéia deste método quase-Newton é construir iterativamente uma boa aproximação para $-A^{-1}$, isto é, construir uma seqüência de matrizes simétricas e positivas-definidas $Q(k)$ tal que

$$
\lim _{k \rightarrow \infty} Q(k)=-A^{-1}
$$

Daí vem a denominação 'quase' em quase-Newton, pois não se usa a verdadeira matriz hessiana de $f$ e sim, uma aproximação. 
Este método inicia com uma aproximação para a matriz $-A^{-1}$ que seja positiva-definida e simétrica, usualmente a matriz identidade de mesma ordem, e constrói as aproximações $Q(k)$ 's de tal forma que sejam positivas-definidas e simétricas. Dessa forma, mesmo longe do máximo, isto garante que os pontos se movem em direção crescente. A lei de recorrência desta seqüência de matrizes é expressa por

$$
\begin{gathered}
Q(k+1)=Q(k)+\frac{(v(k+1)-v(k)) \odot(v(k+1)-v(k))}{(v(k+1)-v(k))^{\top}(\nabla f(v(k+1))-\nabla f(v(k)))} \\
-\frac{[Q(k)(\nabla f(v(k+1))-\nabla f(v(k)))] \odot[Q(k)(\nabla f(v(k+1))-\nabla f(v(k)))]}{(\nabla f(v(k+1))-\nabla f(v(k)))^{\top} Q(k)(\nabla f(v(k+1))-\nabla f(v(k)))} \\
+(\nabla f(v(k+1))-\nabla f(v(k)))^{\top} Q(k)(\nabla f(v(k+1))-\nabla f(v(k))) U \odot U,
\end{gathered}
$$

onde $U$ é o vetor-coluna definido por

$$
\begin{gathered}
U=\frac{v(k+1)-v(k)}{(v(k+1)-v(k))^{\top}(\nabla f(v(k+1))-\nabla f(v(k)))} \\
-\frac{Q(k)(\nabla f(v(k+1))-\nabla f(v(k)))}{(\nabla f(v(k+1))-\nabla f(v(k)))^{\top} Q(k)(\nabla f(v(k+1))-\nabla f(v(k)))}
\end{gathered}
$$

e $\odot$ denota o produto direto ('outer product') de dois vetores, cujo resultado para $x \odot w$ é uma matriz de elemento $(i, j)$ dado por $x_{i} w_{j}$.

Portanto, o ponto de máximo da função $f$, segundo o método BFGS, é obtido iterativamente através da expressão

$$
v(k+1)=v(k)+s(k) Q(v(k)) \nabla f(v(k)) .
$$

Como já foi mencionado anteriormente, o comando MaxBFGS não maximiza funções cujas variáveis (parâmetros) estejam sujeitas a restrições. Particularmente, considere o Exemplo 2.3 (distribuição gama com parâmetros $\omega$ e $\vartheta$ ), apresentado no Capítulo 2 (seção 2.4). Neste exemplo, quando $\vartheta$ é o parâmetro de interesse, o logaritmo da verossimilhança perfilada é expresso em função da estimativa de máxima verossimilhança restrita $\widehat{\omega}_{\vartheta}$, que não possui forma fechada, mas é raiz de uma equação conhecida. Mais precisamente,

$$
\ell_{p}(\vartheta)=l\left(\widehat{\omega}_{\vartheta}, \vartheta\right)=n \widehat{\omega}_{\vartheta} \log \vartheta+\widehat{\omega}_{\vartheta} \sum_{j=1}^{n} \log y_{j}-\vartheta \sum_{j=1}^{n} y_{j}-n \log \Gamma\left(\widehat{\omega}_{\vartheta}\right)
$$

em que $\widehat{\omega}_{\vartheta}$ satisfaz a equação

$$
n \log \vartheta+\sum_{j=1}^{n} \log y_{j}-n \psi\left(\widehat{\omega}_{\vartheta}\right)=0 .
$$

Observe que não é possível usar o comando MaxBFGS para maximizar $\ell_{p}(\vartheta)$, pois, devido ao desconhecimento da expressão de $\widehat{\omega}_{\vartheta}$, tal verossimilhança não está explicitamente 
(unicamente) definida em função do parâmetro (variável) $\vartheta$. Aqui, a estimativa de máxima verossimilhança perfilada $\widehat{\vartheta}_{p}$ vem como solução da maximização da função

$$
l(\omega, \vartheta)=n \omega \log \vartheta+\omega \sum_{j=1}^{n} \log y_{j}-\vartheta \sum_{j=1}^{n} y_{j}-n \log \Gamma(\omega)
$$

sujeita à restrição de igualdade

$$
n \log \vartheta+\sum_{j=1}^{n} \log y_{j}-n \psi(\omega)=0 .
$$

Em problemas deste tipo, a linguagem de programção $0 \mathrm{x}$ disponibiliza no seu pacote de maximização o comando MaxSQP.

Este comando maximiza funções não-lineares sujeitas a restrições não-lineares usando uma técnica de programação quadrática sequencial, que, basicamente, consiste em gerar passos (iterações) resolvendo subproblemas quadráticos. É um dos métodos mais eficazes para este tipo de otimização. Para uma discussão do método SQP (Sequential Quadratic Programming), ver Nocedal e Wright (1999). O algoritmo deste método implementado em Ox é similar ao Algoritmo 18.7 em Nocedal e Wright (1999). Maiores detalhes sobre o comando MaxSQP podem ser encontrados em: http://www.nuff.ox.ac.uk/Users/Doornik/doc/ox/index.html. 


\section{APÊNDICE B}

\section{OTIMIZAÇÃO ALTERNADA}

Este apêndice consiste na apresentação do algoritmo implementado na linguagem de programação Ox e utilizado para as simulações computacionais do Capítulo 4 - Parâmetro de heterogeneidade da $\mathcal{G}_{A}^{0}(\alpha, \gamma, \mathcal{L})$ : testes unicaudais e estimação.

Em algumas situações, a solução da equação de verossimilhança,

$$
\frac{\partial \ell(\theta)}{\partial \theta}=0
$$

pode ser obtida explicitamente. Em situações mais complicadas, a solução dessa equação é obtida por procedimentos numéricos, como os métodos de Newton-Raphson e escoring de Fisher.

Problemas numéricos surgem quando a função de verossimilhança não é côncava o suficiente para permitir otimização direta. Para estes casos, aqui é apresentado um algoritmo de otimização alternada proposto por Frery et al. (2004). Embora a discussão e os resultados estejam concentrados na distribuição $\mathcal{G}_{A}^{0}(\alpha, \gamma, \mathcal{L})$, a proposta pode ser aplicada a outras situações onde há ausência de convergência dos algoritmos de otimização usuais.

Após um estudo comparativo de várias rotinas de maximização, como os métodos de Newton-Raphson, BFGS (proposto por Broyden, Fletcher, Goldfarb e Shanno), simplex downhill e algoritmo de Broyden, usando como função objetivo o logaritmo da função de verossimilhança do par $(\alpha, \gamma)$, Frery et al. perceberam que a otimização simultânea não era suficientemente confiável. Os resultados mostraram que, em regiões de concavidade pouco acentuada, nas quais os algoritmos de otimização simultânea eram pouco eficazes, as superfícies poderiam ser divididas para produzir funções melhor comportadas.

Assim, uma análise de funções marginais, ou seja, uma decomposição da função $\ell(\alpha, \gamma)$ em partes dependentes de cada um dos parâmetros foi conduzida e analisada para uma variedade de situações. Os bons resultados provenientes deste procedimento motivaram a proposta de um algoritmo alternado que consistiu em escrever duas equações a partir do logaritmo da função de verossimilhança do par $(\alpha, \gamma)$ : uma dependendo de $\alpha$, dado $\gamma$ fixado, e outra dependendo de $\gamma$, dado $\alpha$ fixado. Mais precisamante, o conjunto de equações foi definido, respectivamente, por

$$
\ell_{1}\left(\alpha ; \gamma(j), z_{1}, \ldots, z_{n}\right)=\log \left[\frac{\Gamma(\mathcal{L}-\alpha)}{\gamma(j)^{\alpha} \Gamma(-\alpha)}\right]+\frac{\alpha}{n} \sum_{i=1}^{n} \log \left(\gamma(j)+\mathcal{L} z_{i}^{2}\right)
$$

e

$$
\ell_{2}\left(\gamma ; \alpha(j), z_{1}, \ldots, z_{n}\right)=-\alpha(j) \log \gamma-\frac{\mathcal{L}-\alpha(j)}{n} \sum_{i=1}^{n} \log \left(\gamma+\mathcal{L} z_{i}^{2}\right)
$$


onde $\gamma(j)$ e $\alpha(j)$ são valores fixados dos parâmetros.

Dessa forma, dado um valor inicial para $\gamma, \gamma(0)$, maximiza-se a primeira equação com relação ao parâmetro $\alpha$ e obtém-se $\alpha(0)$. Depois, usando este valor inicial obtido para $\alpha$, maximiza-se a segunda equação com relação a $\gamma$, obtendo $\gamma(1)$. Logo, definido um critério de parada para este algoritmo, são obtidas estimativas de máxima verossimilhança para os parâmetros iterativamente.

O algoritmo proposto por Frery et al. (2004) é descrito de forma mais detalhada abaixo.

\section{Otimização alternada para estimação de parâmetros}

Leia o conjunto dos dados de entrada $\mathbf{z}=\left(z_{i}\right)_{1 \leq i \leq n}$, fixe a menor variação entre iterações consecutivas para executar o passo 3 (tipicamente $\delta=10^{-4}$ ) e o número máximo de iterações (tipicamente $M=1000)$. Então:

1. calcule uma estimativa inicial de $\gamma$, por exemplo

$$
\gamma(0)=\mathcal{L}\left(m_{1} \frac{\Gamma(\mathcal{L})}{\Gamma(\mathcal{L}+1 / 2)}\right)^{2}
$$

onde $m_{1}=n^{-1} \sum_{i=1}^{n} z_{i}$ é o primeiro momento amostral;

2. defina os valores necessários para executar o passo (3c) pela primeira vez por $\delta=10^{-3}$ e $\alpha(0)=-10^{6}$ e inicialize o contador $j=1$;

3. enquanto $\epsilon \geq \delta$ e $j \leq M$, faça

(a) encontre $\alpha(j)=\arg \max _{\alpha \in \mathbb{R}^{-}} \ell_{1}(\alpha ; \gamma(j-1), \mathbf{z})$;

(b) encontre $\gamma(j)=\arg \max _{\gamma \in \mathbb{R}^{+}} \ell_{2}(\gamma ; \alpha(j), \mathbf{z})$;

(c) calcule

$$
\epsilon=\left|\frac{\alpha(j)-\alpha(j-1)}{\alpha(j)}\right|+\left|\frac{\gamma(j)-\gamma(j-1)}{\gamma(j)}\right|,
$$

o valor absoluto da variação relativa entre iterações consecutivas,

(d) atualize o contador $j \leftarrow j+1$;

4. se $\epsilon>\delta$ retorne uma mensagem de erro, senão retorne a estimativa $(\alpha(j-1), \gamma(j-1))$ e uma mensagem de sucesso.

Uma extensão imediata para este algoritmo foi implementada no programa utilizado nas simulações computacionais do Capítulo 4 a fim de comportar algumas restrições sobre os parâmetros. Mais precisamente, as etapas de maximização das funções marginais relativas aos logaritmos de funções de verossimilhanças perfiladas modificadas foram realizadas sob restrições de igualdades envolvendo os parâmetros. 


\section{APÊNDICE C}

\section{PROGRAMA COMPUTACIONAL}

\section{C.1. INTRODUÇÃO}

Este apêndice consiste na apresentação do programa implementado na linguagem de programação Ox e utilizado para as simulações referentes ao EXEMPLO 2.4 - DISTRIBUIÇÃO GAUSSIANA INVERSA.

Tal programa fornece medidas amostrais de posição e de dispersão dos estimadores de máxima verossimilhança derivados das verossimilhanças perfiladas consideradas nesse exemplo. Também são obtidas taxas de rejeição sob a hipótese nula de testes sobre o parâmetro de interesse, usando as estatísticas da razão de verossimilhanças baseadas nas verossimilhanças perfiladas, bem como média e variância amostrais e assintóticas destas. Além disso, são calculadas as discrepâncias relativas entre os quantis amostrais e os quantis assintóticos dessas estatísticas a fim de construir gráficos da forma: discrepância relativa versus quantil assintótico.

Sobre a organização dos resultados da estimação pontual do parâmetro de interesse, os seguintes valores são apresentados em tabelas: valor verdadeiro, média, viés, estimativa da variância, estimativa do erro quadrático médio (EQM) e estimativas dos coeficientes de assimetria e de curtose.

Quanto à organização dos resultados do teste de hipótese sobre o parâmetro de interesse,

os seguintes valores são apresentados em tabelas: taxa de rejeição observada sob a hipótese nula, considerando níveis nominais de $15 \%, 10 \%, 5 \%, 3 \%, 1 \%, 0.5 \%, 0.1 \%, 0.05 \%$ e 0.01\%;

média e variância amostrais das estatísticas de teste, bem como os valores assintóticos destes momentos. 


\section{C.2. EXEMPLO 2.4: DISTRIBUIÇÃO GAUSSIANA INVERSA}

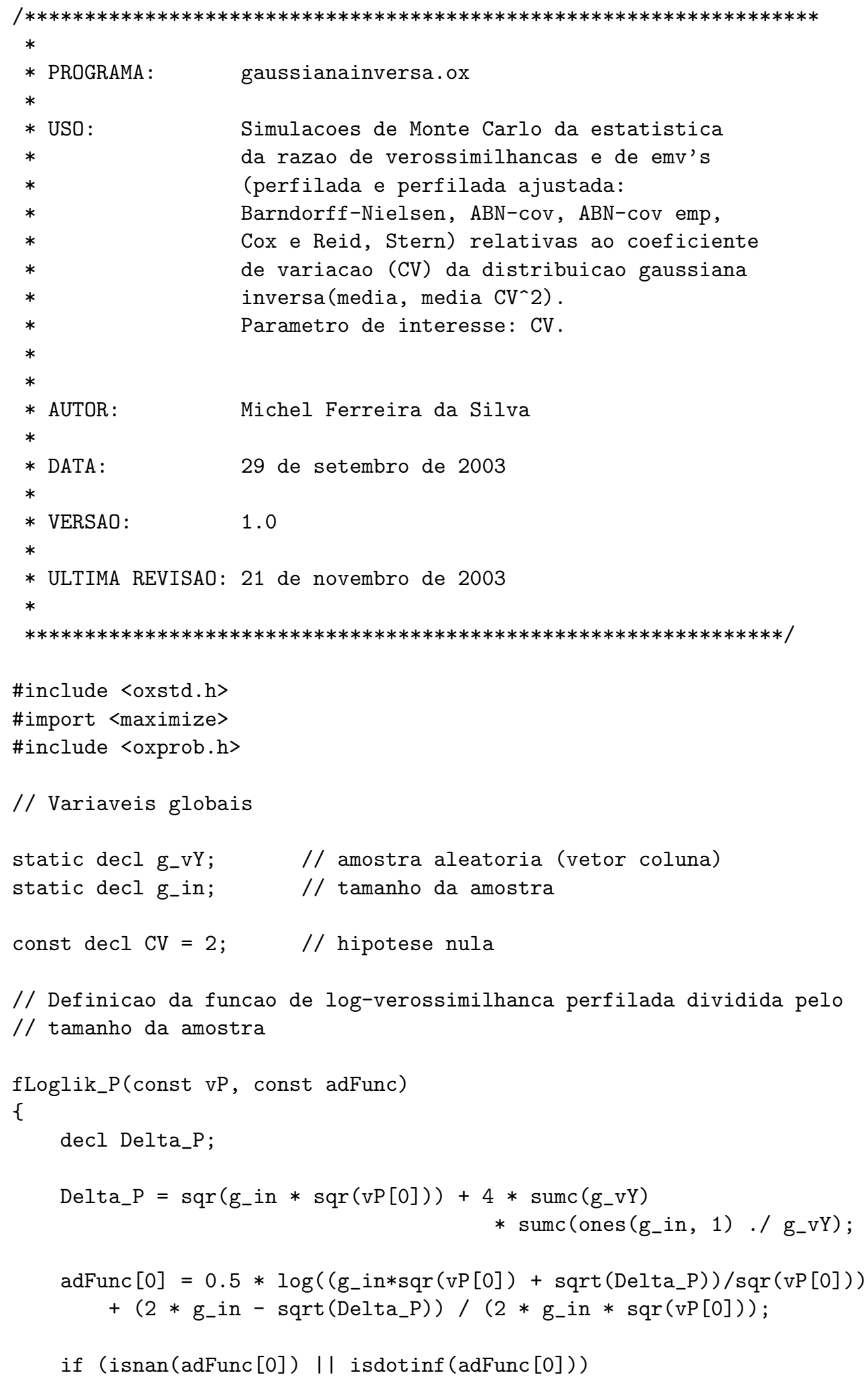




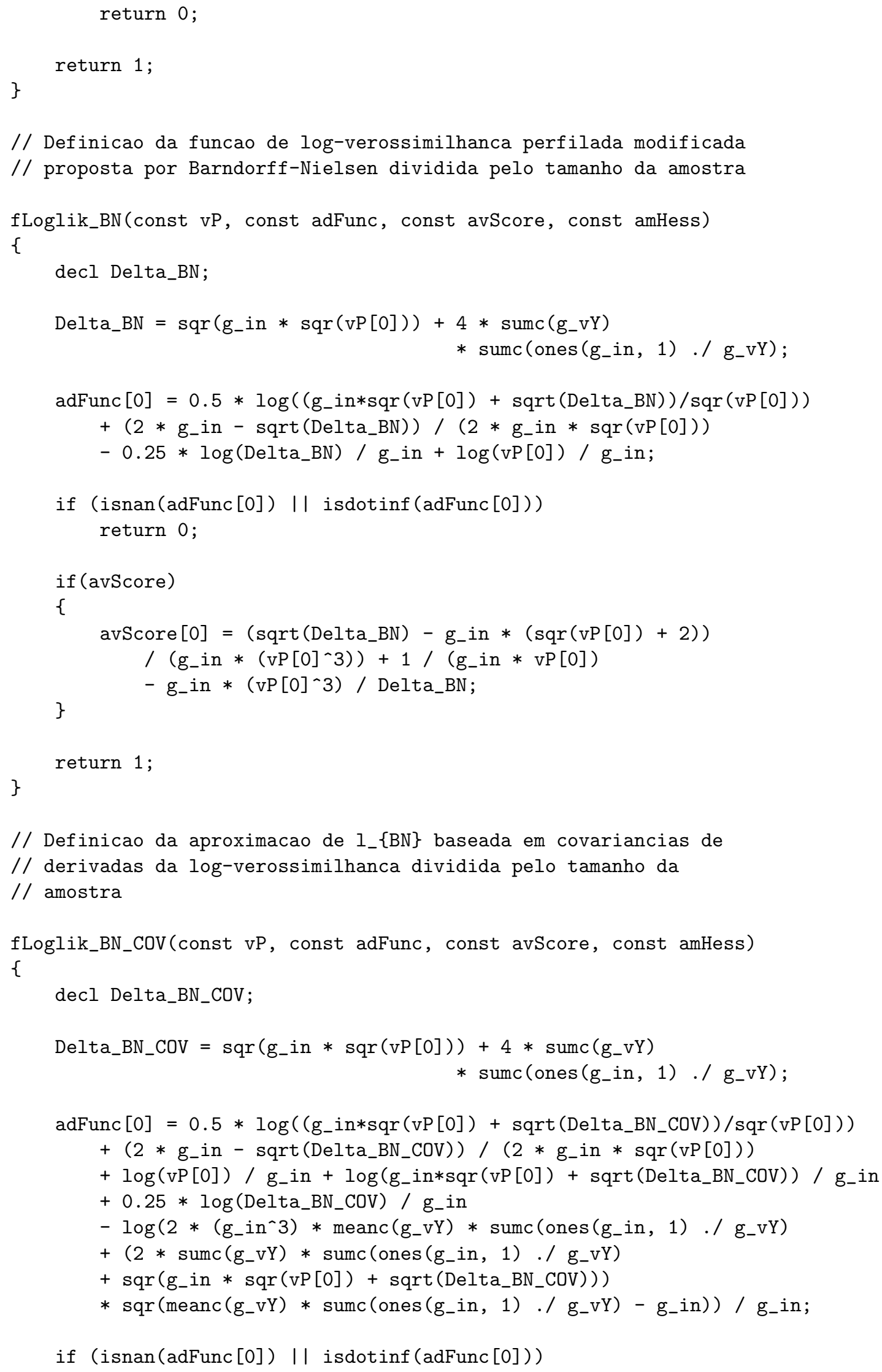




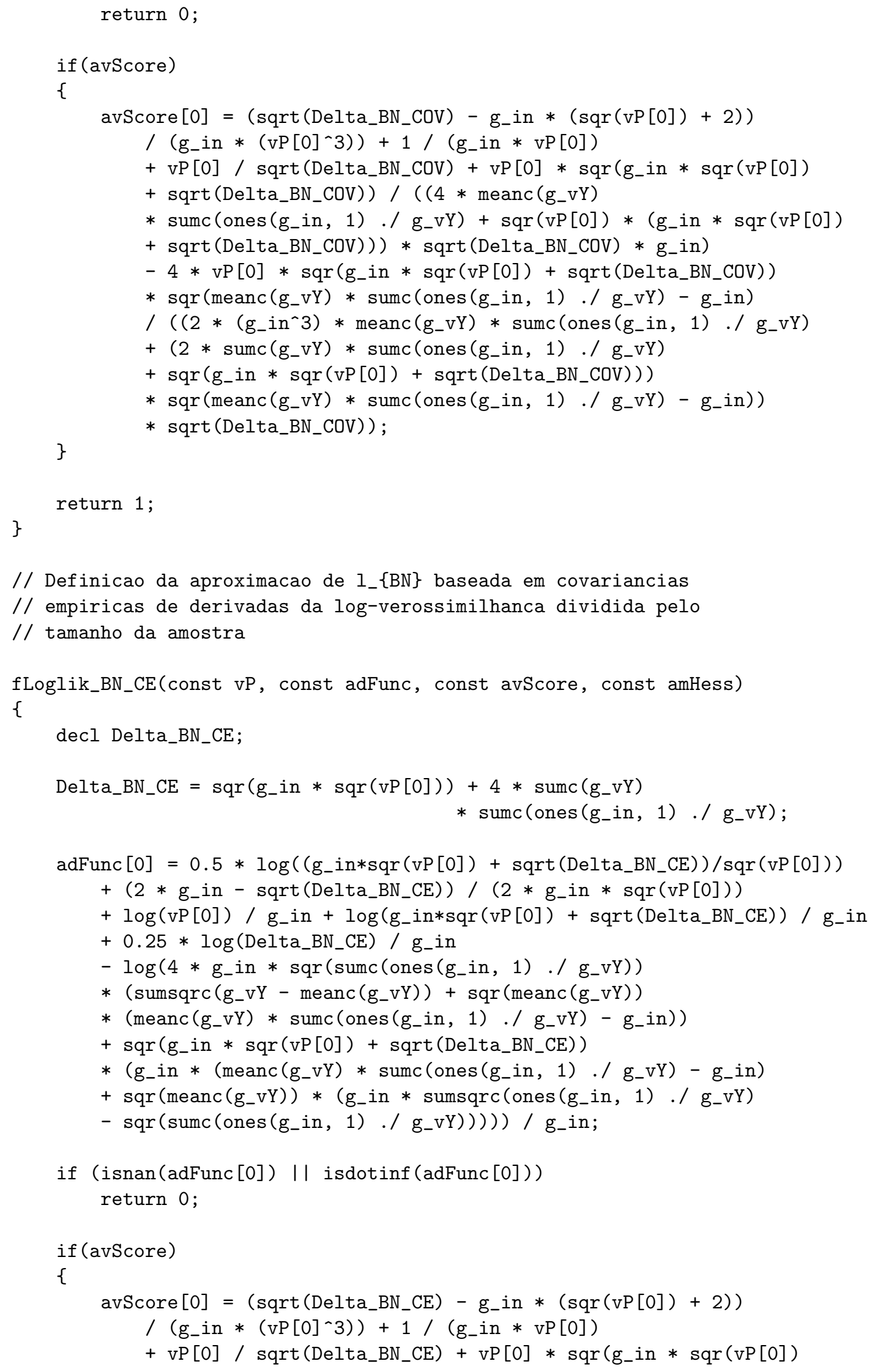




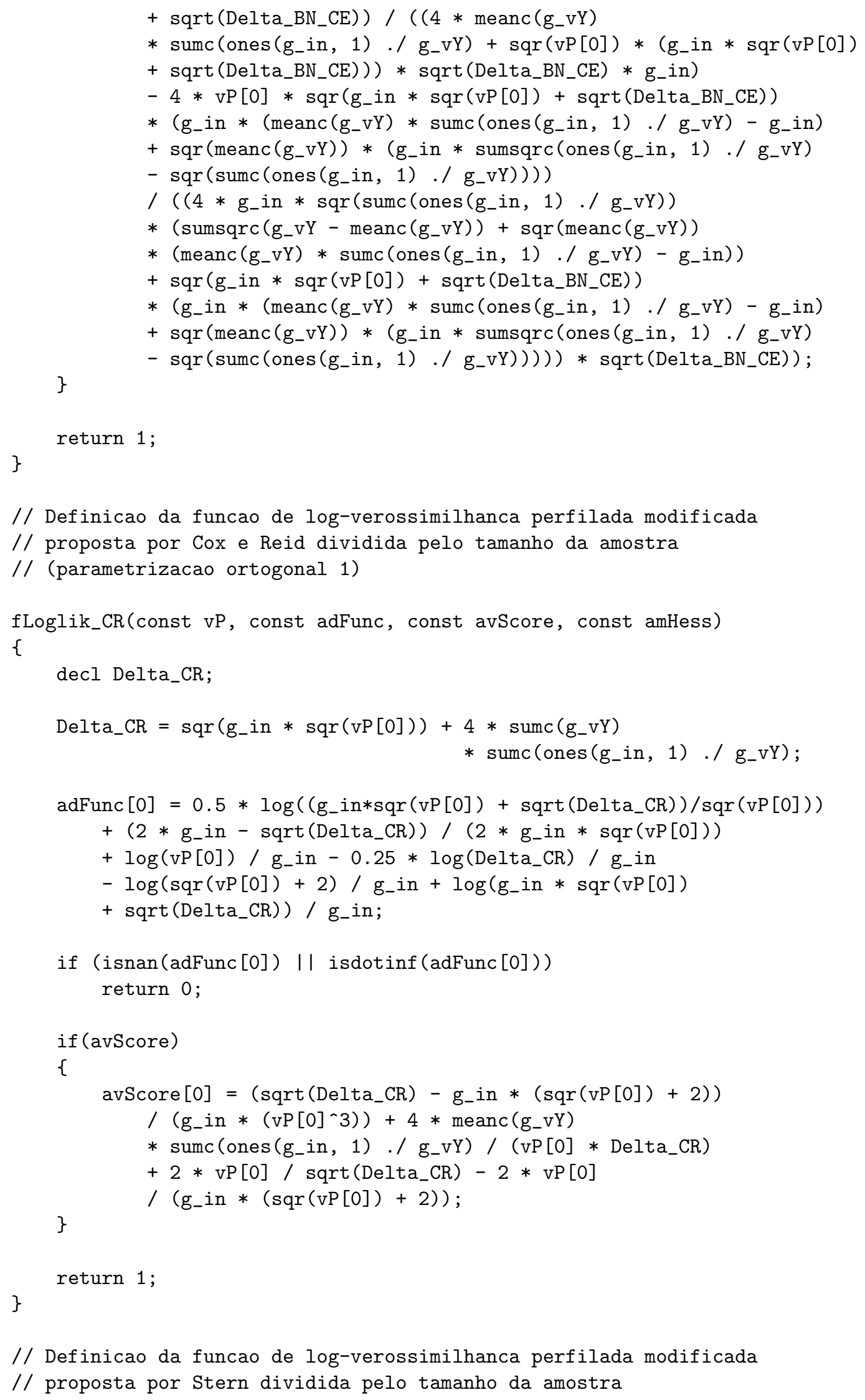


// (parametrizacao ortogonal 1)

fLoglik_ST(const vP, const adFunc, const avScore, const amHess)

\{

decl Delta_ST;

Delta_ST $=\operatorname{sqr}\left(g_{-}\right.$in $\left.* \operatorname{sqr}(v P[0])\right)+4 * \operatorname{sumc}\left(g_{-} v Y\right)$

* sumc(ones (g_in, 1) ./ g_vY);

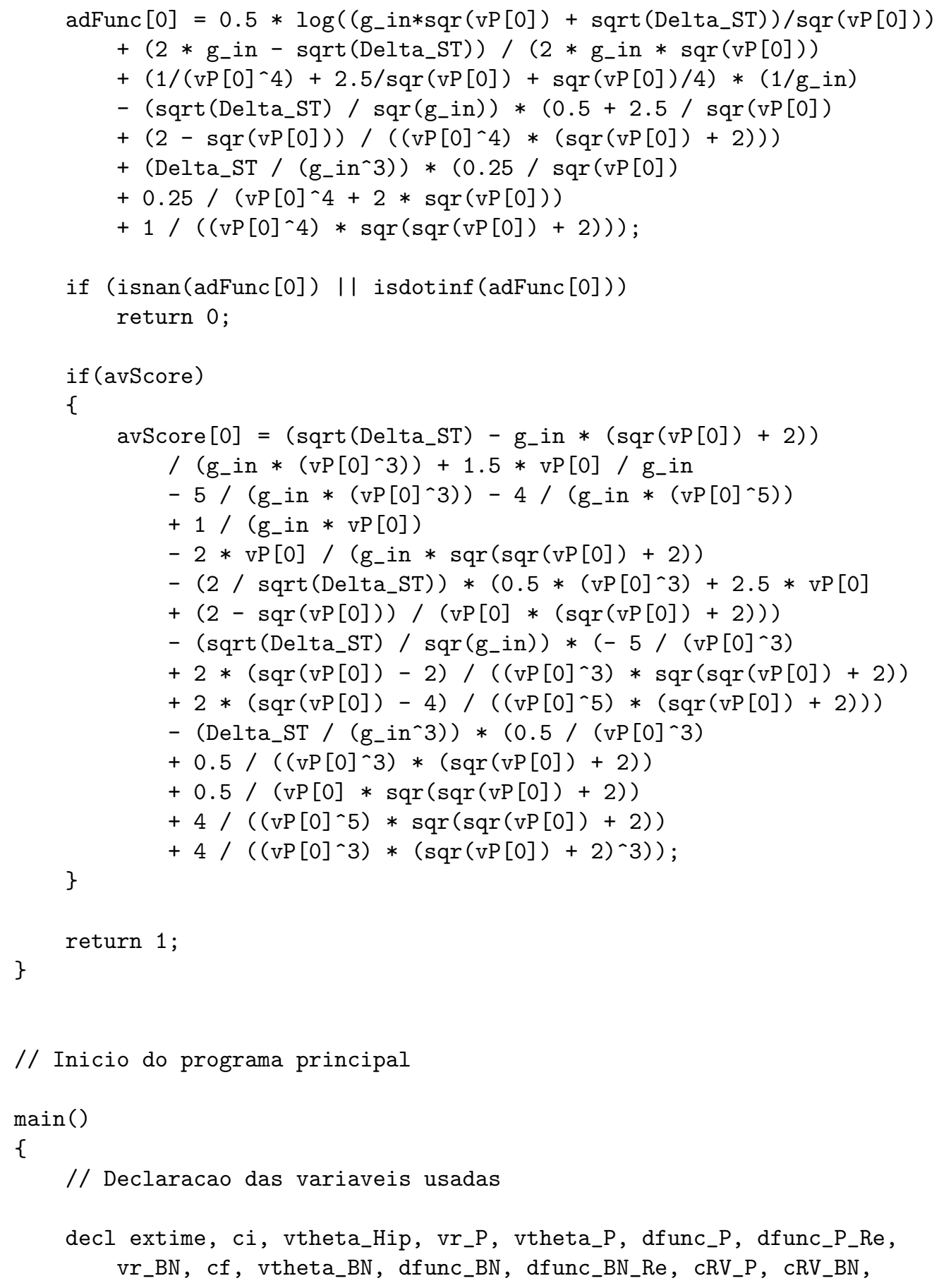




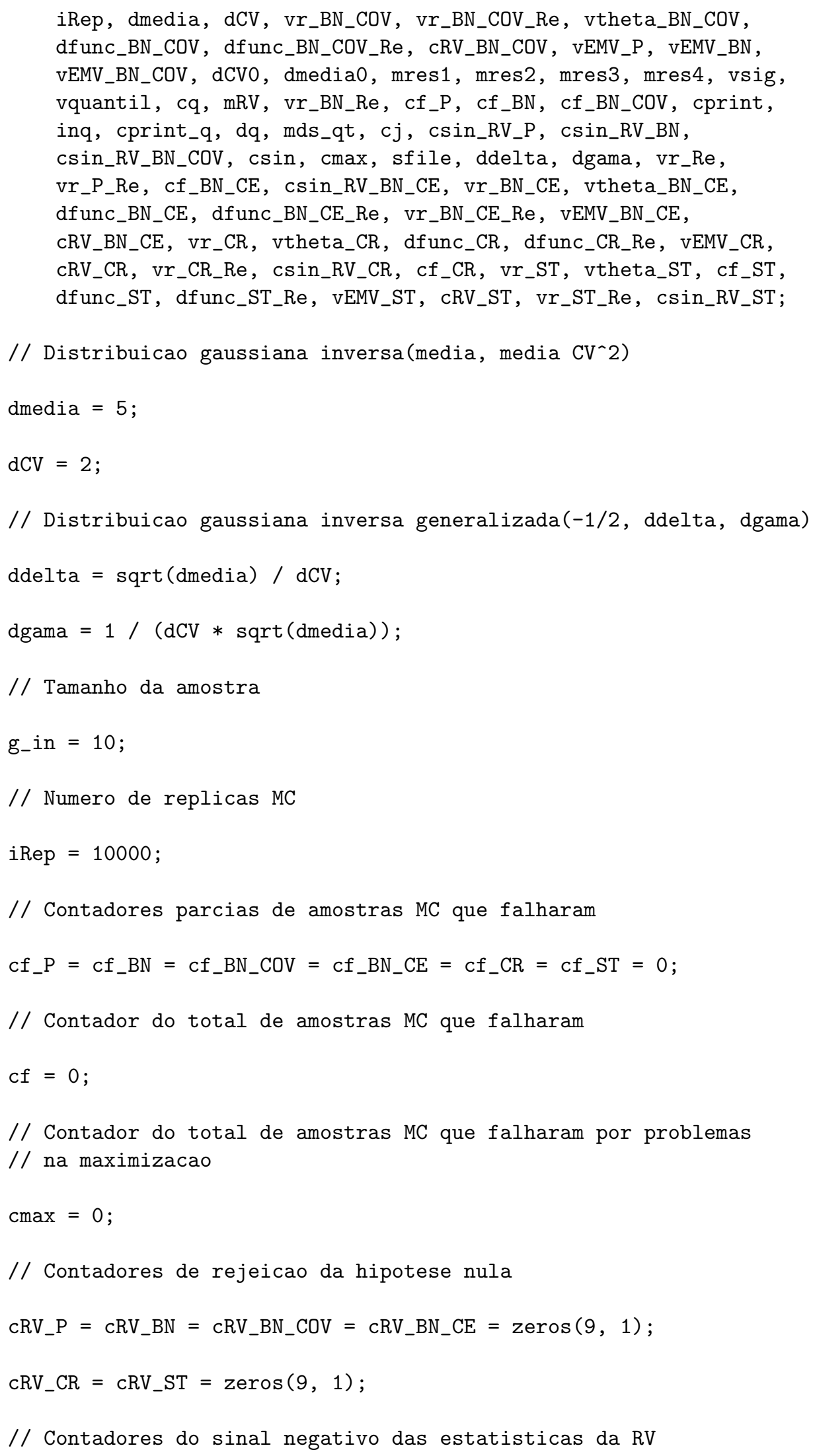




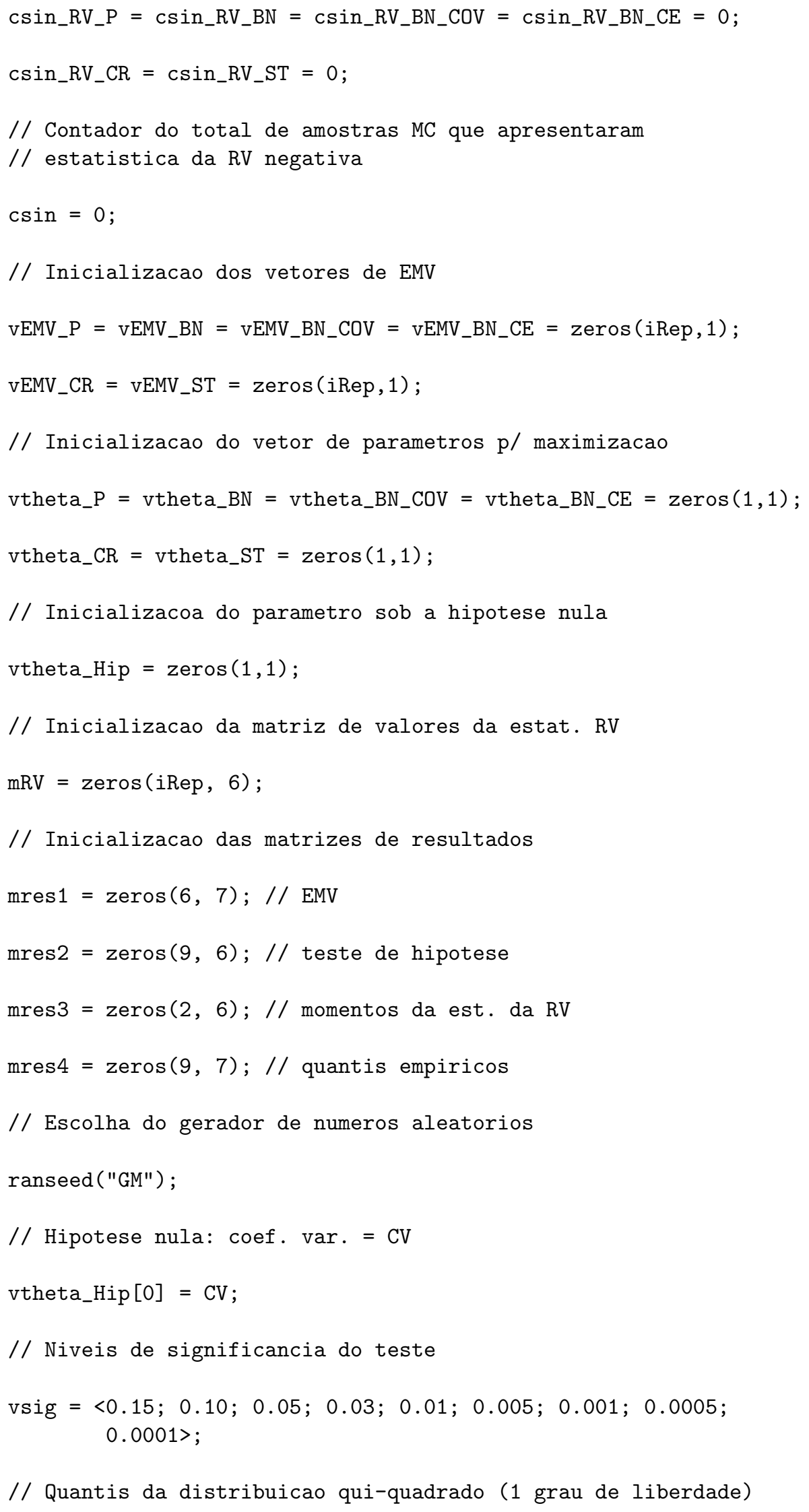




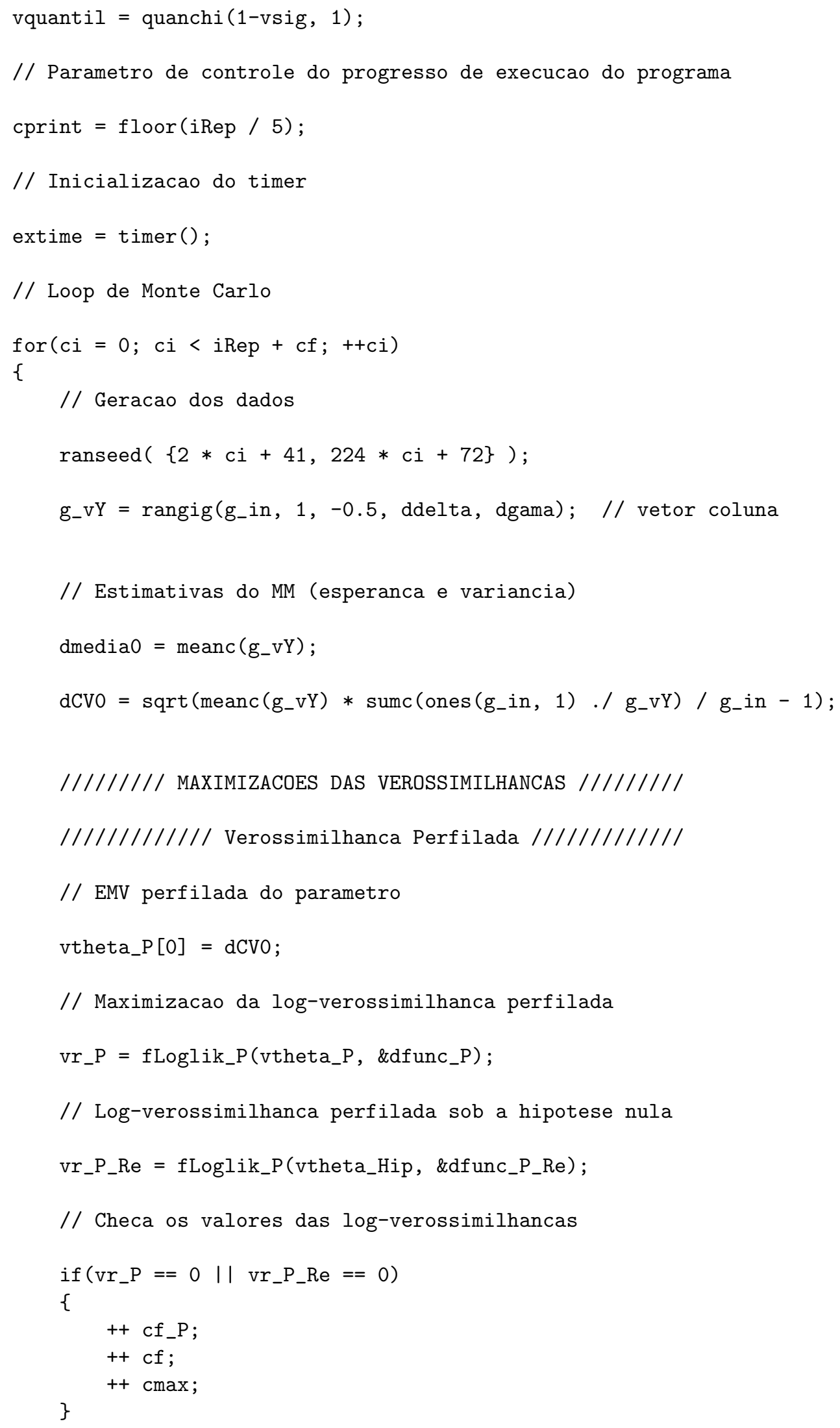




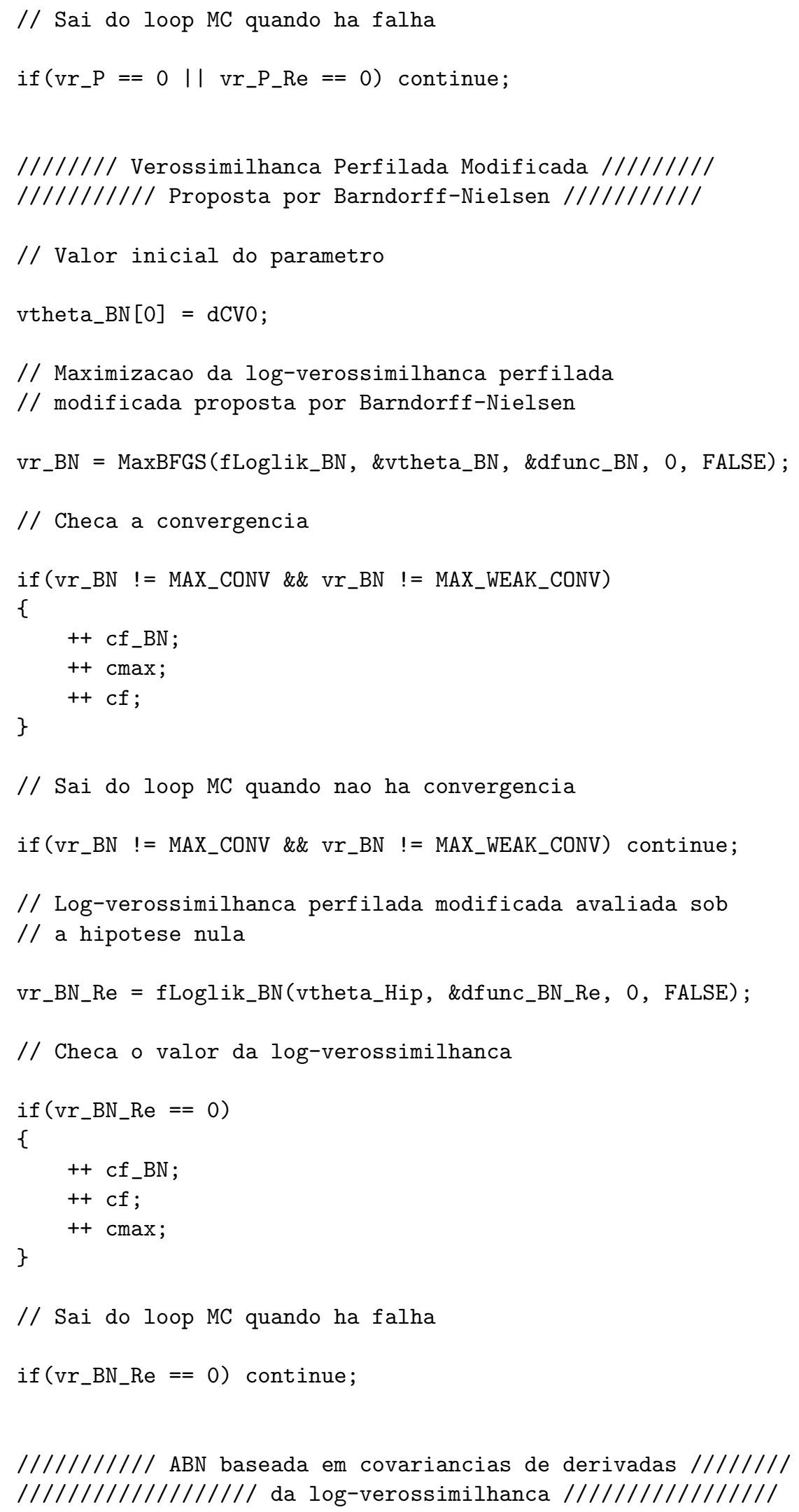




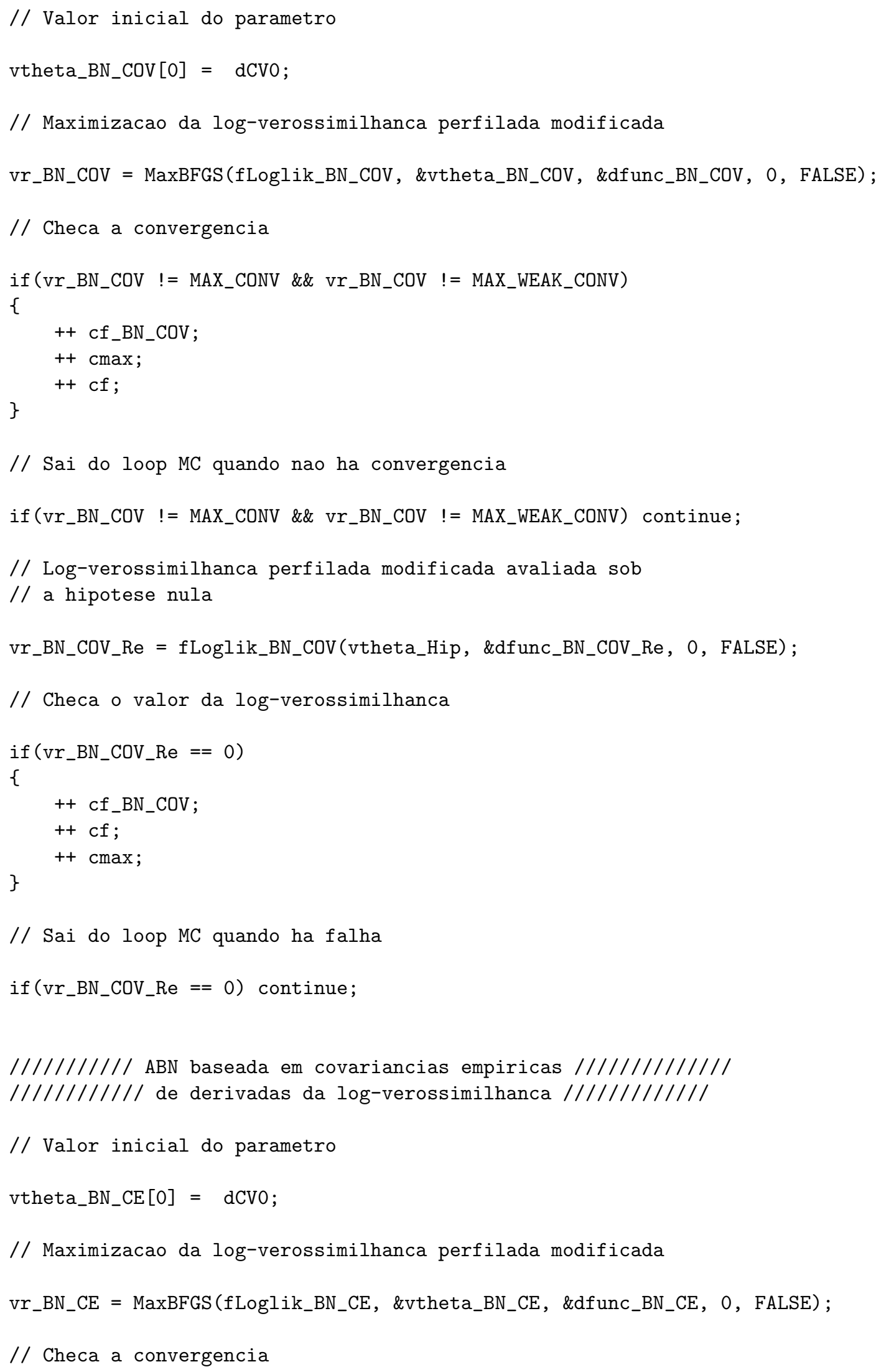




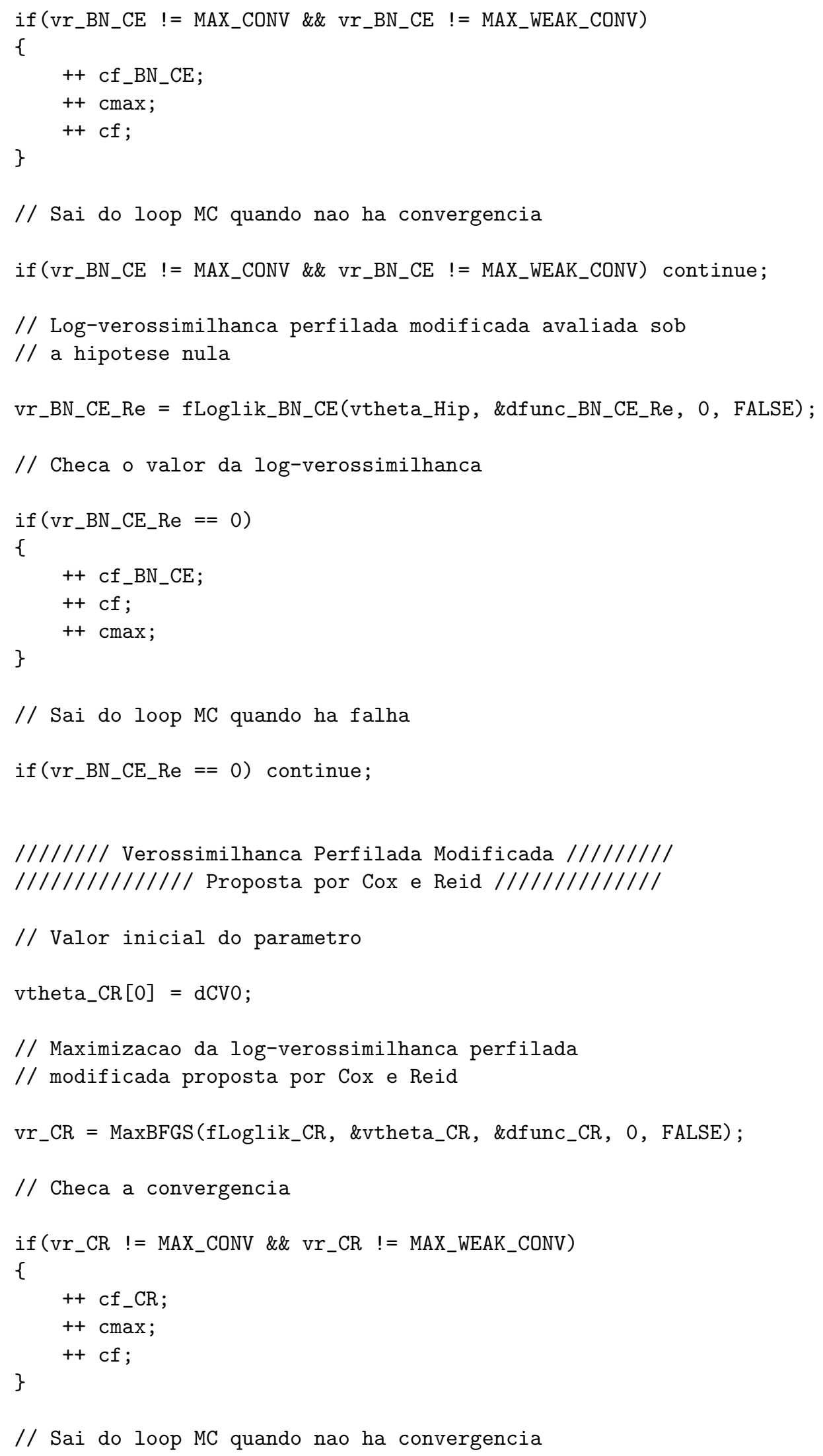




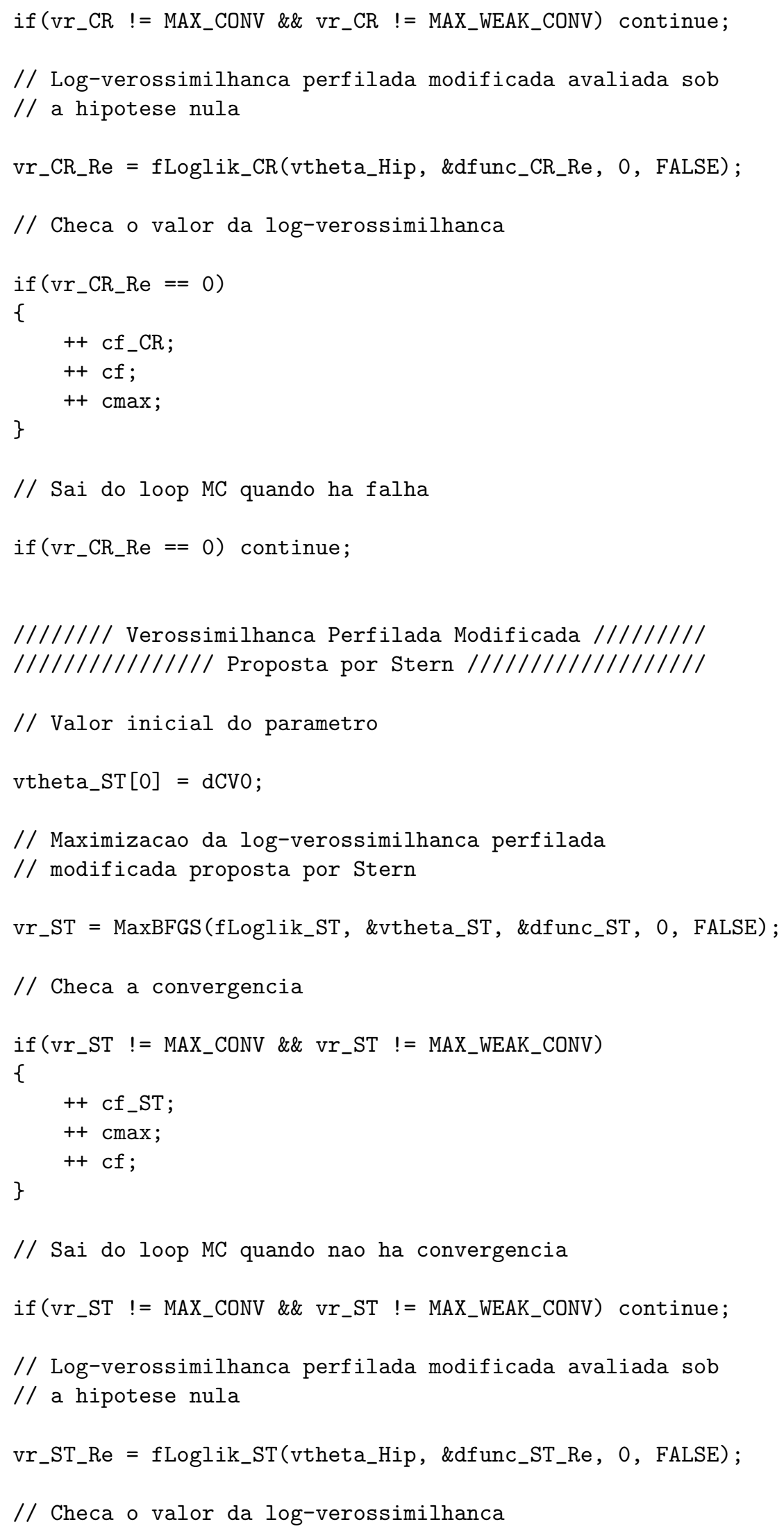




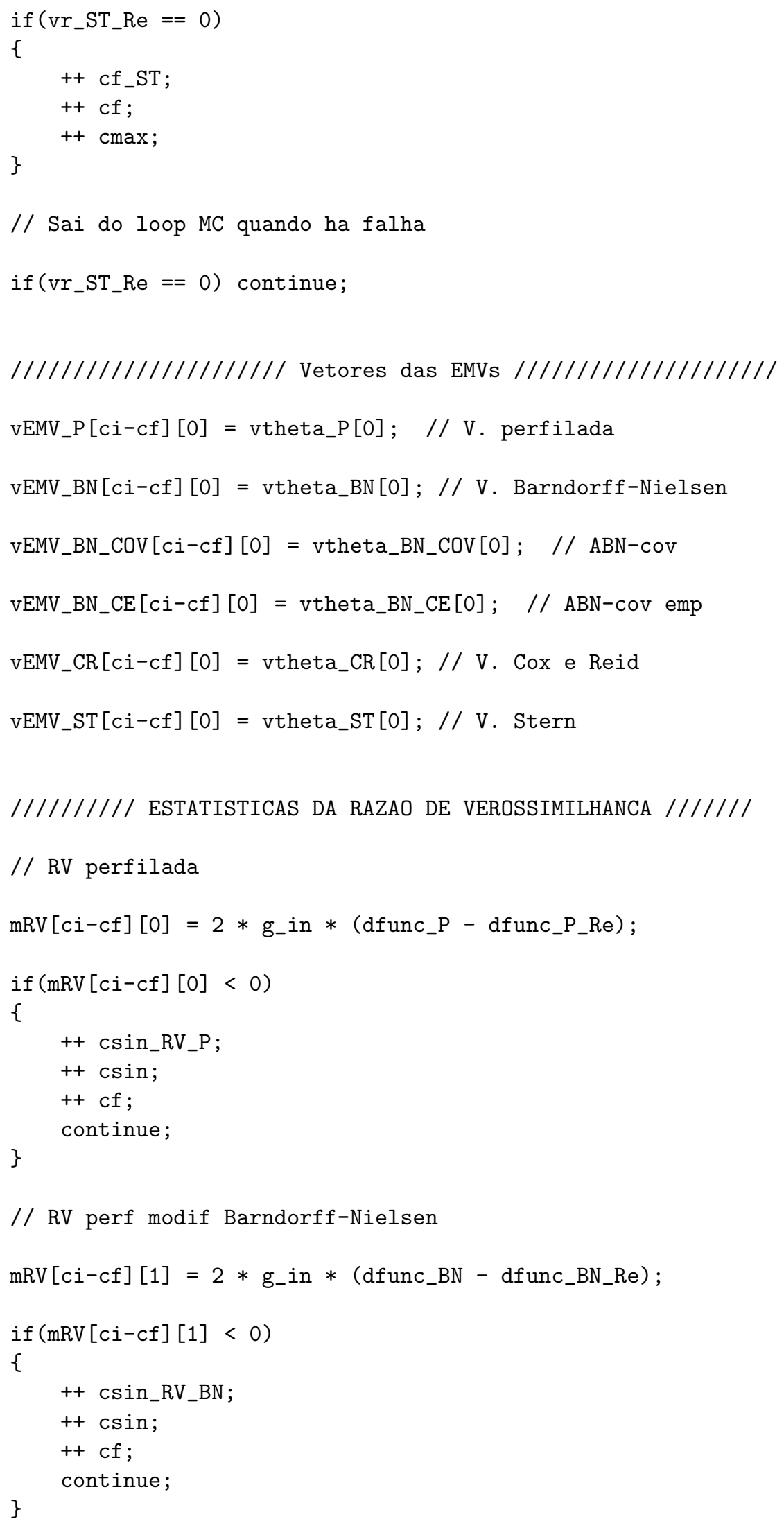




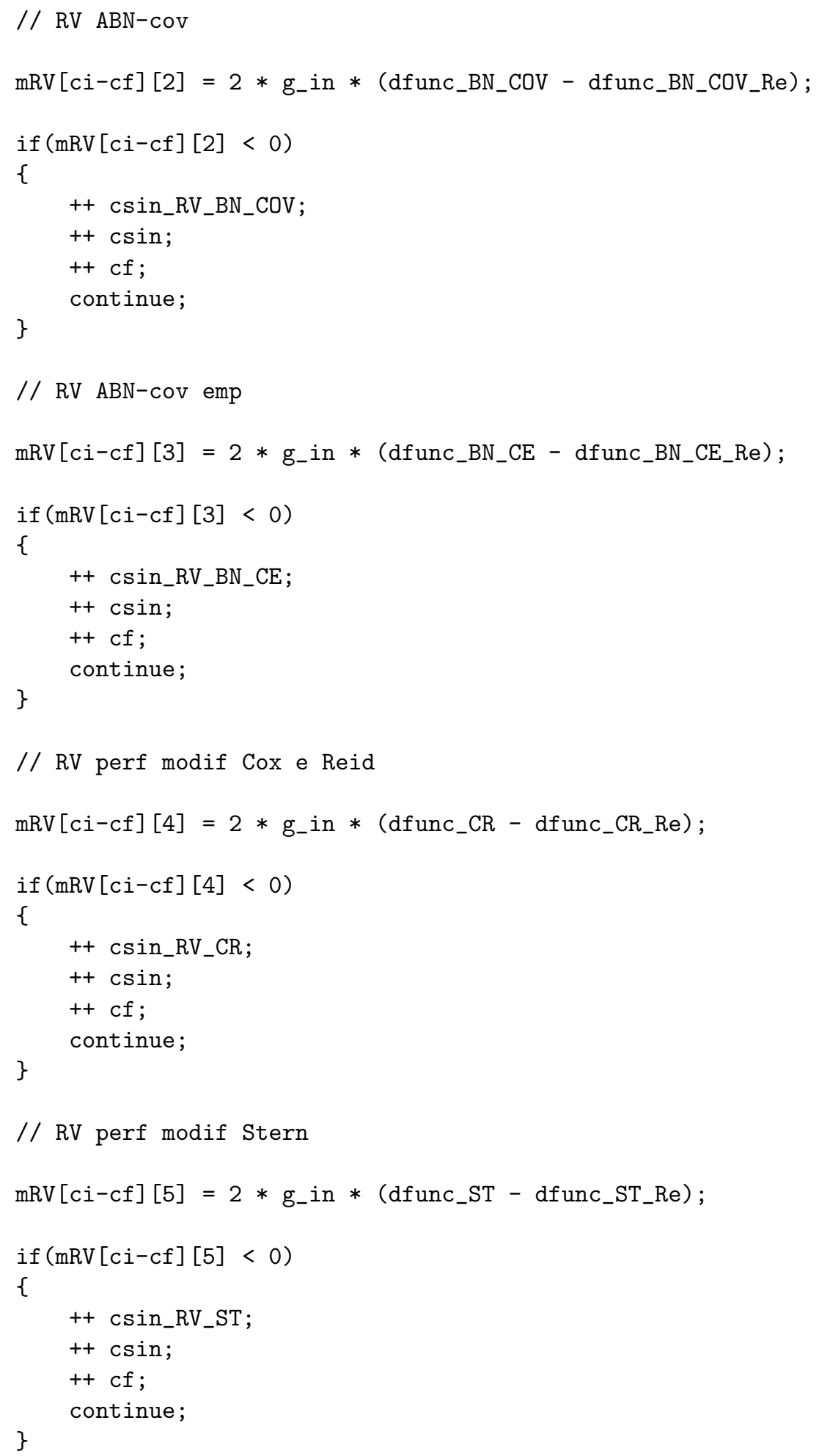




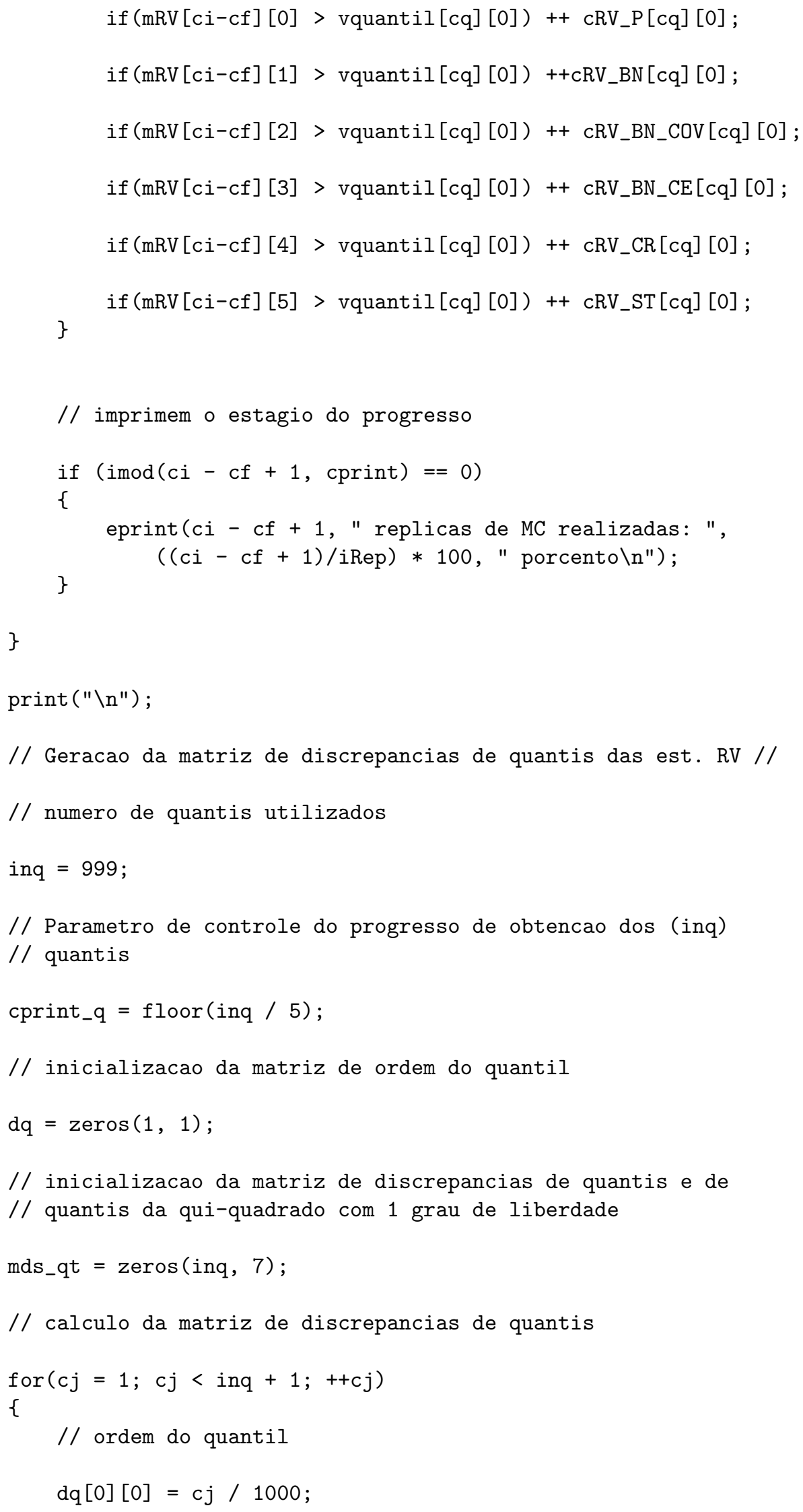




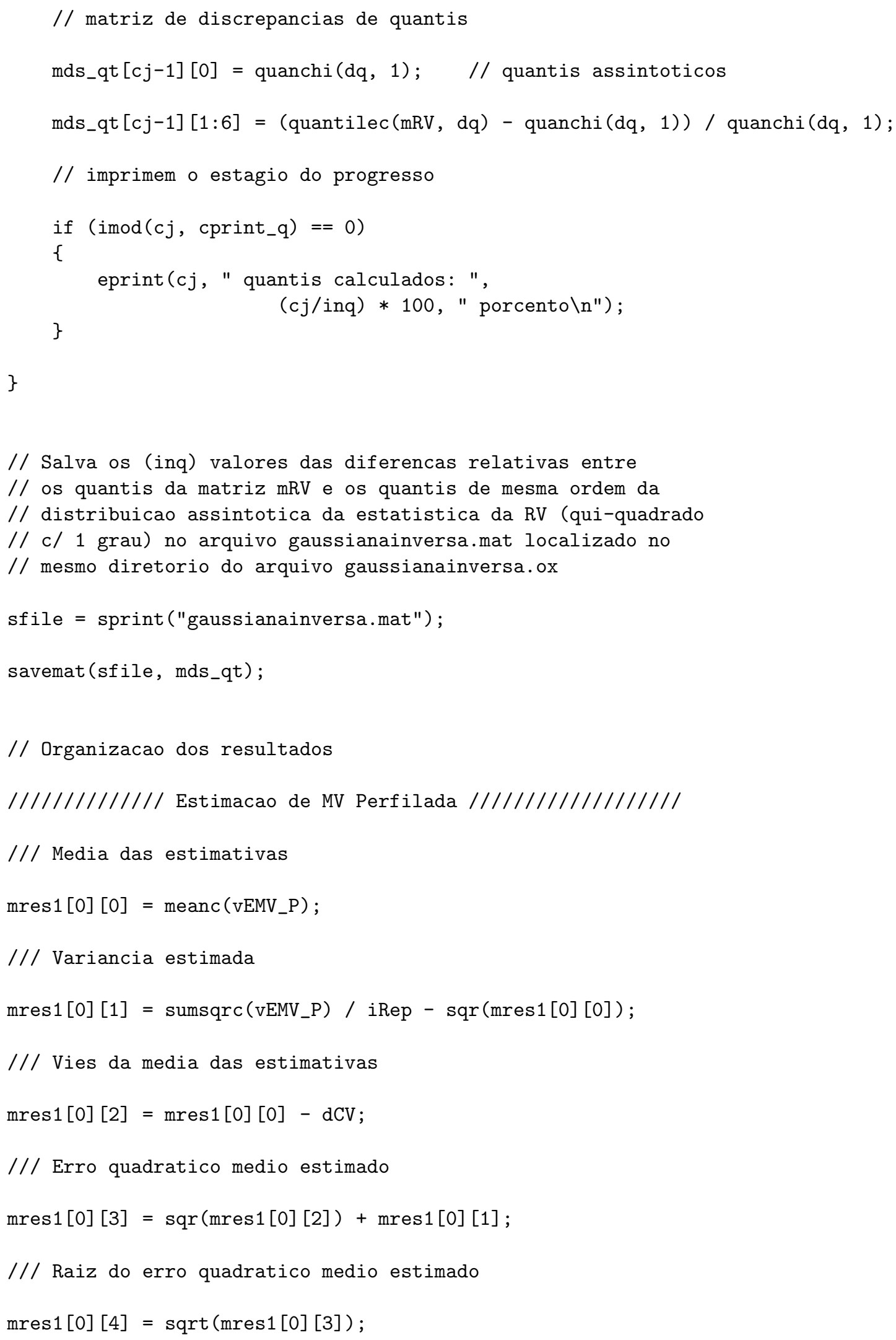


/// Coeficiente de assimetria

$\operatorname{mres} 1[0][5]=\operatorname{sumc}\left(\left(\right.\right.$ standardize $\left.\left(v E M V \_P\right)\right) \cdot$ - 3) / iRep;

/// Coeficiente de curtose

$\operatorname{mres} 1[0][6]=\operatorname{sumc}\left(\left(\right.\right.$ standardize $\left.\left(v E M V \_P\right)\right) \cdot$ - 4) / iRep;

////// Estimacao de MV perf modif Barndorff-Nielsen ///////

/// Media das estimativas

$\operatorname{mres} 1[1][0]=\operatorname{meanc}\left(\mathrm{vEMV} \_\mathrm{BN}\right)$;

/// Variancia estimada

$\operatorname{mres1}[1][1]=\operatorname{sumsqrc}\left(v E M V \_B N\right) / i \operatorname{Rep}-\operatorname{sqr}(\operatorname{mres} 1[1][0])$;

/// Vies da media das estimativas

$\operatorname{mres} 1[1][2]=\operatorname{mres} 1[1][0]-\mathrm{dCV}$;

/// Erro quadratico medio estimado

$\operatorname{mres} 1[1][3]=\operatorname{sqr}(\operatorname{mres} 1[1][2])+\operatorname{mres} 1[1][1]$;

/// Raiz do erro quadratico medio estimado

$\operatorname{mres1}[1][4]=\operatorname{sqrt}(\operatorname{mres} 1[1][3]) ;$

/// Coeficiente de assimetria

$\operatorname{mres} 1[1][5]=\operatorname{sumc}\left(\left(\operatorname{standardize}\left(\mathrm{vEMV} \_\mathrm{BN}\right)\right) \cdot \leadsto 3\right) / \mathrm{iRep}$;

/// Coeficiente de curtose

$\operatorname{mres} 1[1][6]=\operatorname{sumc}\left(\left(\operatorname{standardize}\left(\mathrm{vEMV} \_B N\right)\right) \cdot\right.$ 4) / iRep;

$/ / / / / / / / / / / / /$ Estimacao de MV perf modif ABN-cov $/ / / / / / / / / / / /$

/// Media das estimativas

$\operatorname{mres} 1[2][0]=\operatorname{meanc}($ vEMV_BN_COV) ;

/// Variancia estimada

$\operatorname{mres1}[2][1]=\operatorname{sumsqrc}\left(v E M V \_B N \_C O V\right) / \operatorname{iRep}-\operatorname{sqr}(\operatorname{mres1}[2][0])$;

/// Vies da media das estimativas

$\operatorname{mres1}[2][2]=\operatorname{mres} 1[2][0]-\mathrm{dCV}$; 
/// Erro quadratico medio estimado

$\operatorname{mres1}[2][3]=\operatorname{sqr}(\operatorname{mres} 1[2][2])+\operatorname{mres} 1[2][1]$;

/// Raiz do erro quadratico medio estimado

$\operatorname{mres} 1[2][4]=\operatorname{sqrt}(\operatorname{mres} 1[2][3]) ;$

/// Coeficiente de assimetria

$\operatorname{mres} 1[2][5]=\operatorname{sumc}((\operatorname{standardize}($ vEMV_BN_COV $)) \cdot$ - 3$) /$ iRep;

/// Coeficiente de curtose

$\operatorname{mres1}[2][6]=\operatorname{sumc}\left(\left(\operatorname{standardize}\left(v E M V \_B N \_C O V\right)\right) \cdot \wedge 4\right) /$ iRep;

$/ / / / / / / / / / / / / /$ Estimacao de MV perf modif ABN-cov emp $/ / / / / / / / / / / /$

/// Media das estimativas

$\operatorname{mres} 1[3][0]=\operatorname{meanc}\left(\mathrm{vEMV} \_\right.$BN_CE) ;

/// Variancia estimada

$\operatorname{mres1}[3][1]=\operatorname{sumsqrc}\left(v E M V \_B N \_C E\right) / i \operatorname{Rep}-\operatorname{sqr}(\operatorname{mres} 1[3][0])$;

/// Vies da media das estimativas

$\operatorname{mres1}[3][2]=\operatorname{mres} 1[3][0]-\mathrm{dCV}$;

/// Erro quadratico medio estimado

$\operatorname{mres1}[3][3]=\operatorname{sqr}(\operatorname{mres} 1[3][2])+\operatorname{mres} 1[3][1]$;

/// Raiz do erro quadratico medio estimado

$\operatorname{mres1}[3][4]=\operatorname{sqrt}(\operatorname{mres} 1[3][3])$;

/// Coeficiente de assimetria

$\operatorname{mres} 1[3][5]=\operatorname{sumc}\left(\left(\right.\right.$ standardize $\left.\left(v E M V_{-B N} \_C E\right)\right) \cdot$ - 3) / iRep;

/// Coeficiente de curtose

$\operatorname{mres} 1[3][6]=\operatorname{sumc}\left(\left(\right.\right.$ standardize $\left.\left.\left(v E M V \_B N \_C E\right)\right) \cdot \wedge 4\right) /$ iRep;

$/ / / / / / / / / / /$ Estimacao de MV perf modif Cox e Reid ///////////

/// Media das estimativas 
$\operatorname{mres} 1[4][0]=\operatorname{meanc}\left(\mathrm{vEMV}_{-} \mathrm{CR}\right)$;

/// Variancia estimada

$\operatorname{mres1}[4][1]=\operatorname{sumsqrc}\left(\mathrm{vEMV} \_\mathrm{CR}\right) / \mathrm{iRep}-\operatorname{sqr}(\operatorname{mres} 1[4][0]) ;$

/// Vies da media das estimativas

$\operatorname{mres} 1[4][2]=\operatorname{mres} 1[4][0]-\mathrm{dCV}$;

/// Erro quadratico medio estimado

$\operatorname{mres} 1[4][3]=\operatorname{sqr}(\operatorname{mres} 1[4][2])+\operatorname{mres} 1[4][1]$;

/// Raiz do erro quadratico medio estimado

$\operatorname{mres} 1[4][4]=\operatorname{sqrt}(\operatorname{mres} 1[4][3]) ;$

/// Coeficiente de assimetria

$\operatorname{mres} 1[4][5]=\operatorname{sumc}\left(\left(\operatorname{standardize}\left(v E M V \_C R\right)\right) \cdot\right.$ - 3) / iRep;

/// Coeficiente de curtose

$\operatorname{mres} 1[4][6]=\operatorname{sumc}\left(\left(\operatorname{standardize}\left(\mathrm{vEMV} \_\mathrm{CR}\right)\right) \cdot\right.$ - 4) / iRep;

$/ / / / / / / / / / /$ Estimacao de MV perf modif Stern $/ / / / / / / / / / /$

/// Media das estimativas

$\operatorname{mres} 1[5][0]=\operatorname{meanc}\left(\mathrm{vEMV} \_\mathrm{ST}\right)$;

/// Variancia estimada

$\operatorname{mres1}[5][1]=\operatorname{sumsqrc}\left(v E M V \_S T\right) / i \operatorname{Rep}-\operatorname{sqr}(\operatorname{mres} 1[5][0])$;

/// Vies da media das estimativas

$\operatorname{mres1}[5][2]=\operatorname{mres} 1[5][0]-\mathrm{dCV}$;

/// Erro quadratico medio estimado

$\operatorname{mres} 1[5][3]=\operatorname{sqr}(\operatorname{mres} 1[5][2])+\operatorname{mres} 1[5][1]$;

/// Raiz do erro quadratico medio estimado

$\operatorname{mres1}[5][4]=\operatorname{sqrt}(\operatorname{mres} 1[5][3]) ;$

/// Coeficiente de assimetria

$\operatorname{mres} 1[5][5]=\operatorname{sumc}((\operatorname{standardize}($ vEMV_ST $)) \cdot \wedge 3) /$ iRep; 


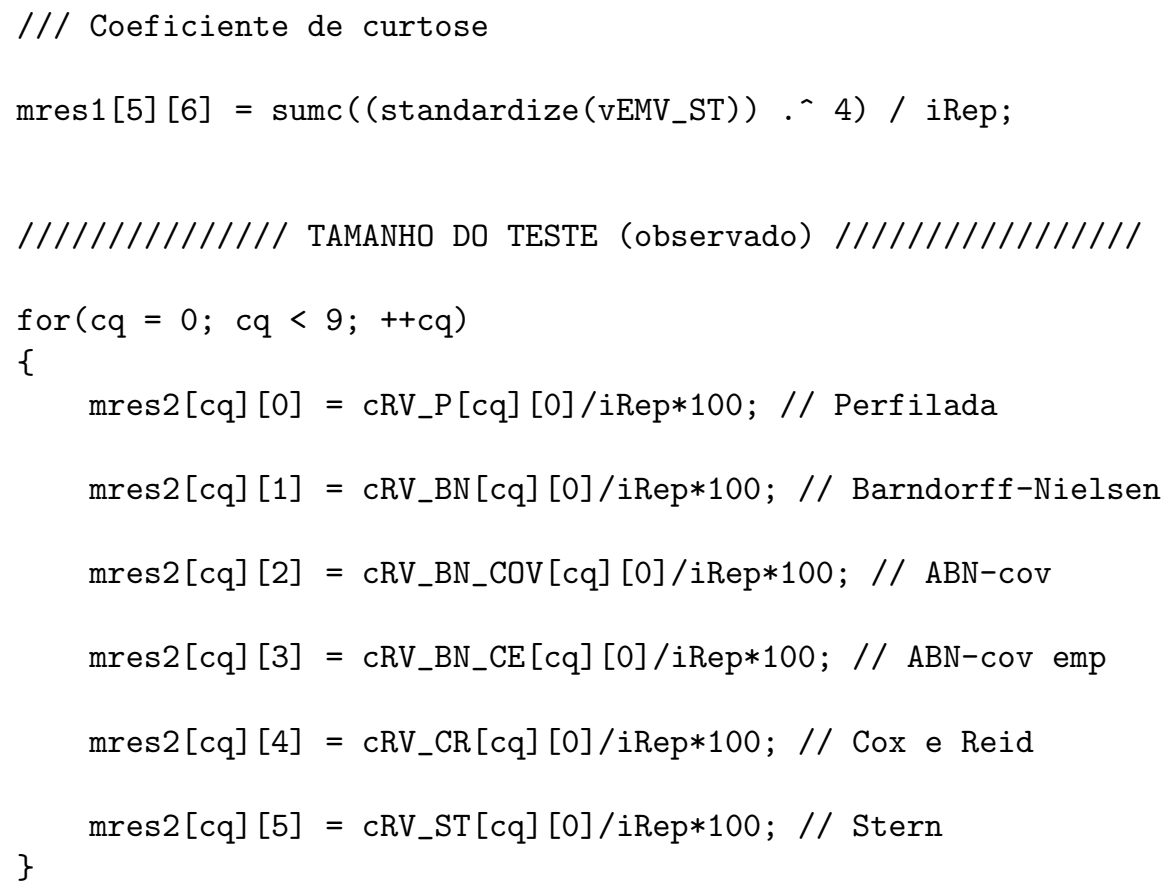

/////////////// MOMENTOS DA ESTATISTICA DA RV ////////////////

// media

$\operatorname{mres} 3[0][]=\operatorname{meanc}(\mathrm{mRV})$;

// variancia

$\operatorname{mres} 3[1][]=\operatorname{varc}(\mathrm{mRV})$;

////////// QUANTIS AMOSTRAIS DA ESTATISTICA DA RV /////////

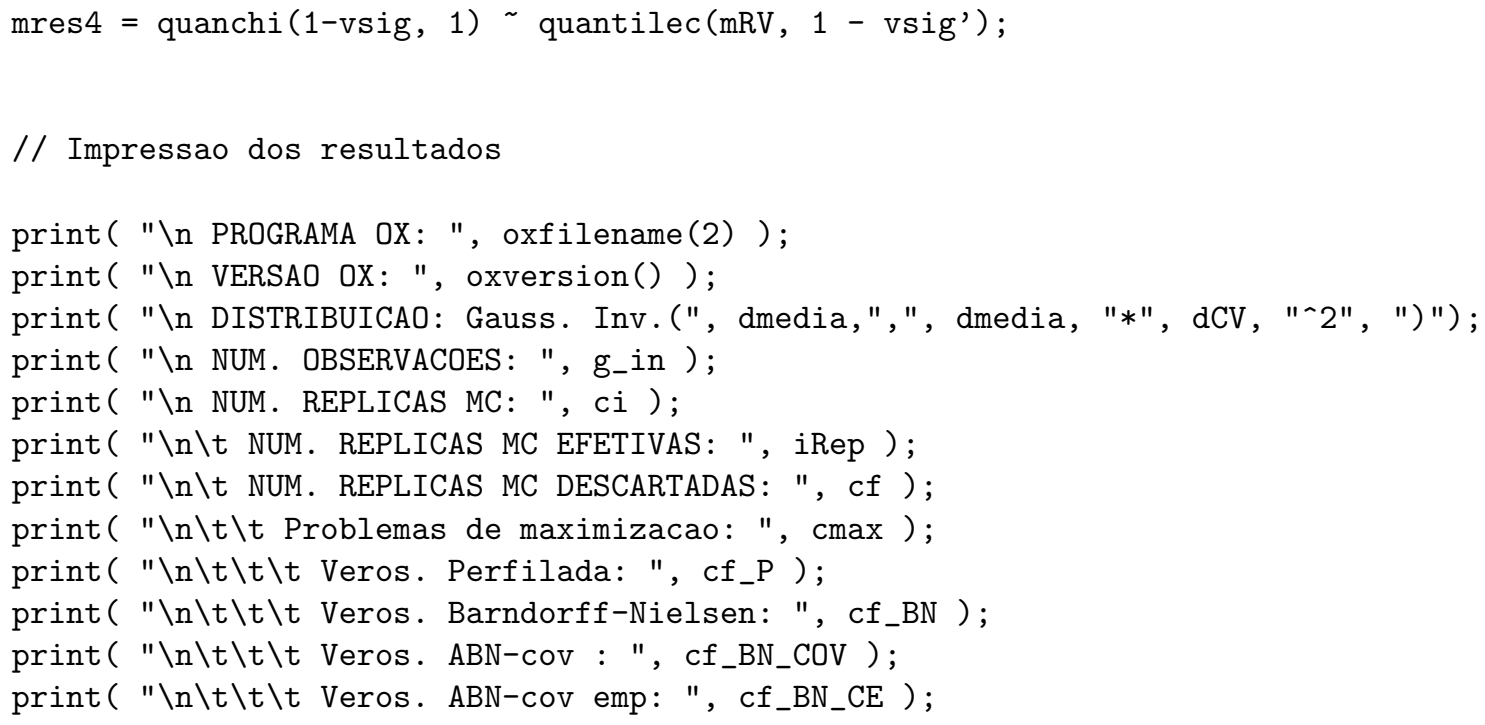




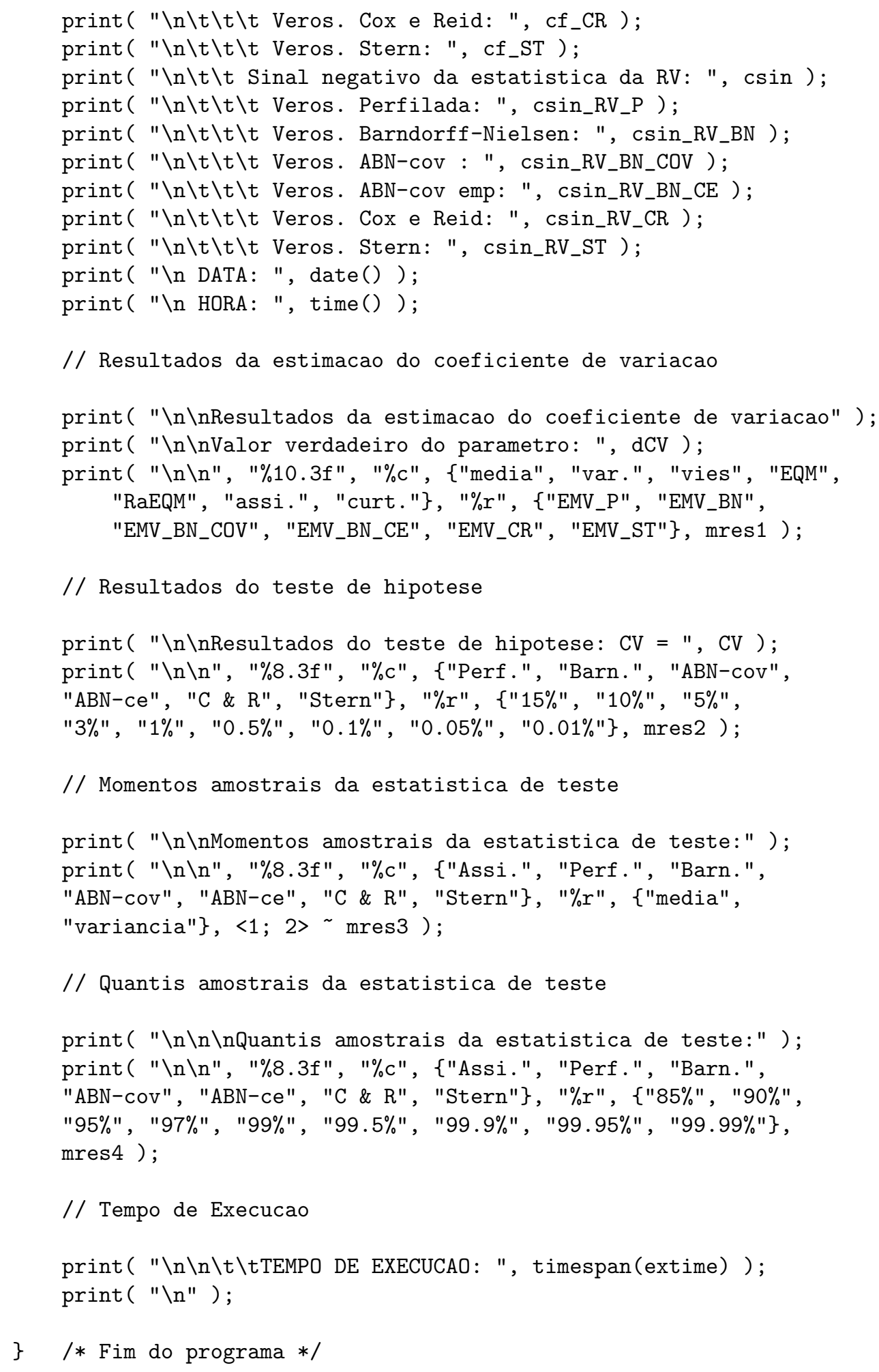




\title{
APÊNDICE D
}

\section{ESTIMADORES DE MÁXIMA VEROSSIMILHANÇA E ESTATÍSTICAS DA RAZÃO DE VEROSSIMILHANÇAS NO MODELO DE WEIBULL: RESULTADOS DISTRIBUCIONAIS}

\author{
D.1. OBSERVAÇÕES INDEPENDENTES E \\ IDENTICAMENTE DISTRIBUÍDAS
}

\section{D.1.1. INTRODUÇÃO}

O Capítulo 5 deste texto trata da estimação e de teste de hipótese sobre o parâmetro de forma da distribuição de Weibull, modelo probabilístico largamente utilizado em confiabilidade. Considerando observações independentes e identicamente distribuídas e todas as verossimilhanças perfiladas, foi mencionado na Seção 5.2.1.3, para dados completos e dados censurados, que as razões ${ }^{\star}$ (e.m.v. de $\beta$ ) / $\beta$ são quantidades pivotais para $(\alpha, \beta)$. Além disso, para testes da razão de verossimilhanças, foi afirmado que a probabilidade do erro tipo I não depende de $\alpha$ e nem do valor fixado para $\beta$ na hipótese nula, depende apenas do tamanho amostral e do nível de censura considerado.

Esses resultados foram obtidos de forma análoga ao trabalho de Thoman et al. (1969), que enunciou e provou os seguintes teoremas envolvendo os estimadores de máxima verossimilhança dos parâmetros $\alpha$ e $\beta$, $\widehat{\alpha}$ e $\widehat{\beta}$, respectivamente, no caso de dados completos:

[1] $\widehat{\beta} / \beta$ é distribuído independentemente de $\alpha$ e $\beta$ e tem a mesma distribuição de $\widehat{\beta}_{11}$, o estimador de máxima verossimilhança de $\beta$ quando $\alpha=\beta=1$.

[2] $\widehat{\beta} \log (\widehat{\alpha} / \alpha)$ é distribuído independentemente de $\alpha$ e $\beta$ e tem a mesma distribuição de $\widehat{\beta}_{11} \log \widehat{\alpha}_{11}$, onde $\widehat{\alpha}_{11}$ é o estimador de máxima verossimilhança de $\alpha$ quando $\alpha=\beta=1$.

Aqui o resultado [1] é mostrado no contexto de verossimilhanças perfiladas, tanto para dados completos quanto para dados censurados. Em seguida, usando esse resultado, é mostrado que a probabilidade do erro tipo I dos testes da razão de verossimilhanças considerados não dependem do par $(\alpha, \beta)$.

Nas demonstrações, é usada a seguinte transformação:

$$
x \sim \mathcal{E} x p(1) \quad \Longrightarrow \quad y=\alpha x^{1 / \beta} \sim W B(\alpha, \beta) .
$$

\footnotetext{
^ e.m.v. = estimador de máxima verossimilhança.
} 


\section{D.1.2. CENSURA TIPO II}

No caso de dados censurados tipo II ( $r$ fixado e $r<n$ ), a função escore relativa ao parâmetro de interesse $\beta$ obtida da função de verossimilhança perfilada é dada por (Seção 5.2.1.2)

$$
u_{p}(\beta)=\frac{r}{\beta}-r \frac{\sum_{j=1}^{r} y_{(j)}^{\beta} \log y_{(j)}+(n-r) y_{(r)}^{\beta} \log y_{(r)}}{\sum_{j=1}^{r} y_{(j)}^{\beta}+(n-r) y_{(r)}^{\beta}}+\sum_{j=1}^{r} \log y_{(j)} .
$$

O e.m.v. de $\beta$ é a raiz da equação $u_{p}(\beta)=0$, portanto satisfaz a igualdade:

$$
\frac{r}{\widehat{\beta}_{p}}-r \frac{\sum_{j=1}^{r} y_{(j)}^{\widehat{\beta}_{p}} \log y_{(j)}+(n-r) y_{(r)}^{\widehat{\beta}_{p}} \log y_{(r)}}{\sum_{j=1}^{r} \widehat{\beta}_{(j)}^{\widehat{\beta}_{p}}+(n-r) y_{(r)}^{\widehat{\beta}_{p}}}+\sum_{j=1}^{r} \log y_{(j)}=0 .
$$

Como $y_{j}=\alpha x_{j}^{1 / \beta}, j=1, \ldots, n$, então $y_{(j)}=\alpha x_{(j)}^{1 / \beta}$ e $\log y_{(j)}=\log \alpha+(1 / \beta) \log x_{(j)}$. Substituindo essas relações na equação anterior, vem

$$
\frac{r}{\widehat{\beta}_{p}}-\frac{r}{\beta} \frac{\sum_{j=1}^{r} x_{(j)}^{\widehat{\beta}_{p} / \beta} \log x_{(j)}+(n-r) x_{(r)}^{\widehat{\beta}_{p} / \beta} \log x_{(r)}}{\sum_{j=1}^{r} x_{(j)}^{\widehat{\beta}_{p} / \beta}+(n-r) x_{(r)}^{\widehat{\beta}_{p} / \beta}}+\frac{1}{\beta} \sum_{j=1}^{r} \log x_{(j)}=0 .
$$

Por último, multiplicando esta equação por $\beta$ em ambos os lados, obtém-se

$$
\frac{r}{\widehat{\beta}_{p} / \beta}-r \frac{\sum_{j=1}^{r} x_{(j)}^{\widehat{\beta}_{p} / \beta} \log x_{(j)}+(n-r) x_{(r)}^{\widehat{\beta}_{p} / \beta} \log x_{(r)}}{\sum_{j=1}^{r} x_{(j)}^{\widehat{\beta}_{p} / \beta}+(n-r) x_{(r)}^{\widehat{\beta}_{p} / \beta}}+\sum_{j=1}^{r} \log x_{(j)}=0 .
$$

Considerando como incógnita desta equação a razão $\widehat{\beta}_{p} / \beta$, observe que a raiz é função apenas das estatísticas de ordem da amostra aleatória de uma variável exponencial padrão $\mathcal{E} x p(1)$, portanto, tal razão é uma quantidade pivotal para $(\alpha, \beta)$. A solução desta equação para $\widehat{\beta}_{p} / \beta$ é a mesma solução de

$$
\frac{r}{\widehat{\beta}_{p}^{11}}-r \frac{\sum_{j=1}^{r} x_{(j)}^{\widehat{\beta}_{p}^{11}} \log x_{(j)}+(n-r) x_{(r)}^{\widehat{\beta}_{p}^{11}} \log x_{(r)}}{\sum_{j=1}^{r} x_{(j)}^{\widehat{\beta}_{p}^{11}}+(n-r) x_{(r)}^{\widehat{\beta}_{p}^{11}}}+\sum_{j=1}^{r} \log x_{(j)}=0
$$

para $\widehat{\beta}_{p}^{11}$, que é o estimador de máxima verossimilhança perfilada de $\beta$ quando $\alpha=\beta=1$. Assim, $\widehat{\beta}_{p}^{11}=\widehat{\beta}_{p} / \beta$, embora $\widehat{\beta}_{p}$ e $\widehat{\beta}_{p}^{11}$ sejam baseados em amostras aleatórias de distribuições distintas. Aqui vale lembrar que $\widehat{\beta}_{p}=\widehat{\beta}$. No caso de dados completos, $r=n$.

Como $\widehat{\beta}_{p}^{11}$ é função de uma amostra aleatória de uma variável exponencial padrão e do par $(r, n)$, quantis $q_{1}$ e $q_{2}$ independentes de $(\alpha, \beta)$ podem ser determinados de tal forma que

$$
\operatorname{Pr}\left(q_{1}<\widehat{\beta}_{p}^{11}<q_{2}\right)=1-\gamma
$$


onde $0<\gamma<1$. Logo, intervalos de confiança para $\beta$ com coeficientes de confiança iguais a $100(1-\gamma) \%$ podem ser construídos com $\alpha$ desconhecido.

A estatística da razão de verossimilhanças é definida por

$$
R V_{p}(\beta)=2\left\{\ell_{p}\left(\widehat{\beta}_{p}\right)-\ell_{p}(\beta)\right\}
$$

Assim, após manipulações algébricas,

$$
\begin{aligned}
R V_{p}(\beta)=2\left\{r \log \left(\widehat{\beta}_{p} / \beta\right)\right. & -r \log \left[\sum_{j=1}^{r} x_{(j)}^{\widehat{\beta}_{p} / \beta}+(n-r) x_{(r)}^{\widehat{\beta}_{p} / \beta}\right]+\left(\frac{\widehat{\beta}_{p}}{\beta}-1\right) \sum_{j=1}^{r} \log x_{(j)} \\
& \left.+r \log \left[\sum_{j=1}^{r} x_{(j)}+(n-r) x_{(r)}\right]\right\} .
\end{aligned}
$$

Note que $\operatorname{Pr}_{(\alpha, \beta)}\left(R V_{p}(\beta) \leq q\right)$ não depende de $\alpha$ e nem do valor fixado para $\beta$ na hipótese nula, depende apenas do tamanho amostral e do nível de censura considerado (valor de $r$ ). Mais uma vez, no caso de dados completos, $r=n$.

Os resultados relativos aos estimadores de máxima verossimilhança derivados das versões ajustadas de $\ell_{p}(\beta)$ são demonstrados de forma análoga, assim como os resultados relacionados aos tamanhos dos testes da razão de verossimilhanças. Portanto, por economia de espaço, a seguir são mostradas as equações de verossimilhança cujas incógnitas são as razões (e.m.v. de $\beta$ ) / $\beta$ e as estatísticas de teste baseadas nas verossimilhanças perfiladas ajustadas.

(i) Ajuste de Cox e Reid: $\widehat{\beta}_{C R} / \beta$ e $R V_{C R}$

Equação de verossimilhança:

$$
\frac{r-2}{\widehat{\beta}_{C R} / \beta}-r \frac{\sum_{j=1}^{r} x_{(j)}^{\widehat{\beta}_{C R} / \beta} \log x_{(j)}+(n-r) x_{(r)}^{\widehat{\beta}_{C R} / \beta} \log x_{(r)}}{\sum_{j=1}^{r} x_{(j)}^{\widehat{\beta}_{C R} / \beta}+(n-r) x_{(r)}^{\widehat{\beta}_{C R} / \beta}}+\sum_{j=1}^{r} \log x_{(j)}=0 .
$$

Estatística da razão de verossimilhanças:

$$
\begin{gathered}
R V_{C R}(\beta)=2\left\{(r-2) \log \left(\widehat{\beta}_{C R} / \beta\right)-r \log \left[\sum_{j=1}^{r} x_{(j)}^{\widehat{\beta}_{C R} / \beta}+(n-r) x_{(r)}^{\widehat{\beta}_{C R} / \beta}\right]\right. \\
\left.+\left(\frac{\widehat{\beta}_{C R}}{\beta}-1\right) \sum_{j=1}^{r} \log x_{(j)}+r \log \left[\sum_{j=1}^{r} x_{(j)}+(n-r) x_{(r)}\right]\right\} .
\end{gathered}
$$


D. EMV E ESTATÍSTICAS DA RAZÃO DE VEROSSIMILHANÇAS NO MODELO DE WEIBULL

(ii) Aproximação para Barndorff-Nielsen (covariância empírica): $\widehat{\breve{\beta}}_{B N} / \beta$ e $\overparen{R V} B N$

Equação de verossimilhança:

$$
\begin{aligned}
& \frac{r}{\widehat{\breve{\beta}}_{B N} / \beta}+\sum_{j=1}^{r} \log x_{(j)}-(r-1) \frac{\sum_{j=1}^{r} x_{(j)}^{\widehat{\breve{\beta}}_{B N} / \beta} \log x_{(j)}+(n-r) x_{(r)}^{\widehat{\breve{\beta}}_{B N} / \beta} \log x_{(r)}}{\sum_{j=1}^{r} x_{(j)}^{\widehat{\breve{\beta}}_{B N} / \beta}+(n-r) x_{(r)}^{\widehat{\breve{\beta}}_{B N} / \beta}} \\
& -\left\{r \sum_{j=1}^{r} x_{(j)}^{\left(\widehat{\widetilde{\beta}}_{B N}+\widehat{\beta}\right) / \beta} \log x_{(j)}+n(n-r) x_{(r)}^{\left(\widehat{\widetilde{\beta}}_{B N}+\widehat{\beta}\right) / \beta} \log x_{(r)}\right. \\
& \left.-\left(\sum_{j=1}^{r} x_{(j)}^{\widehat{\beta} / \beta}\right)\left(\sum_{j=1}^{r} x_{(j)}^{\widehat{\breve{\beta}}_{B N} / \beta} \log x_{(j)}\right)\right\} \\
& /\left\{r \sum_{j=1}^{r} x_{(j)}^{\left(\widehat{\widetilde{\beta}}_{B N}+\widehat{\beta}\right) / \beta}+n(n-r) x_{(r)}^{\left(\widehat{\widetilde{\beta}}_{B N}+\widehat{\beta}\right) / \beta}-\left(\sum_{j=1}^{r} x_{(j)}^{\widehat{\beta} / \beta}\right)\left(\sum_{j=1}^{r} x_{(j)}^{\widehat{\widehat{\beta}}_{B N} / \beta}\right)\right\}=0
\end{aligned}
$$

Estatística da razão de verossimilhanças:

$$
\begin{aligned}
& \breve{R V}{ }_{B N}(\beta)=2\left\{r \log \left(\widehat{\breve{\beta}}_{B N} / \beta\right)-(r-1) \log \left[\sum_{j=1}^{r} x_{(j)}^{\widehat{\breve{\beta}}_{B N} / \beta}+(n-r) x_{(r)}^{\widehat{\breve{\beta}}_{B N} / \beta}\right]\right. \\
& -\log \left[r \sum_{j=1}^{r} x_{(j)}^{\left.\widehat{\widetilde{\beta}}_{B N}+\widehat{\beta}\right) / \beta}+n(n-r) x_{(r)}^{\left.\widehat{\widetilde{\beta}}_{B N}+\widehat{\beta}\right) / \beta}-\left(\sum_{j=1}^{r} x_{(j)}^{\widehat{\beta} / \beta}\right)\left(\sum_{j=1}^{r} x_{(j)}^{\widehat{\widehat{\beta}}_{B N} / \beta}\right)\right] \\
& +\left(\frac{\widehat{\breve{\beta}}_{B N}}{\beta}-1\right) \sum_{j=1}^{r} \log x_{(j)}+(r-1) \log \left[\sum_{j=1}^{r} x_{(j)}+(n-r) x_{(r)}\right] \\
& \left.+\log \left[r \sum_{j=1}^{r} x_{(j)}^{1+\widehat{\beta} / \beta}+n(n-r) x_{(r)}^{1+\widehat{\beta} / \beta}-\left(\sum_{j=1}^{r} x_{(j)}^{\widehat{\beta} / \beta}\right)\left(\sum_{j=1}^{r} x_{(j)}\right)\right]\right\} .
\end{aligned}
$$

Aqui, $\widehat{\beta}$ é o estimador de máxima verossimilhança obtido de dados com presença de censura tipo II e não tem forma fechada. Lembre que $\widehat{\beta}=\widehat{\beta}_{p}$, portanto, $\widehat{\beta} / \beta$ é uma quantidade pivotal para $(\alpha, \beta)$.

(iii) Aproximação para Barndorff-Nielsen (estatística ancilar): $\widehat{\tilde{\beta}}_{B N} / \beta$ e $\tilde{R V} B N$

Equação de verossimilhança:

$$
\frac{r-1}{\widehat{\tilde{\beta}}_{B N} / \beta}-(r-1) \frac{\sum_{j=1}^{r} x_{(j)}^{\widehat{\tilde{\beta}}_{B N} / \beta} \log x_{(j)}+(n-r) x_{(r)}^{\widehat{\tilde{\beta}}_{B N} / \beta} \log x_{(r)}}{\sum_{j=1}^{r} x_{(j)}^{\widehat{\tilde{\beta}}_{B N} / \beta}+(n-r) x_{(r)}^{\widehat{\tilde{\beta}}_{B N} / \beta}}
$$


D. EMV E ESTATÍSTICAS DA RAZÃO DE VEROSSIMILHANÇAS NO MODELO DE WEIBULL

$$
+\sum_{j=1}^{r} \log x_{(j)}-\frac{\sum_{j=1}^{r} x_{(j)}^{\widehat{\tilde{\beta}}_{B N} / \beta} \log x_{(j)}}{\sum_{j=1}^{r} x_{(j)}^{\widehat{\tilde{\beta}}_{B N} / \beta}}=0
$$

Estatística da razão de verossimilhanças:

$$
\begin{gathered}
\tilde{R V_{B N}}(\beta)=2\left\{(r-1) \log \left(\widehat{\tilde{\beta}}_{B N} / \beta\right)-(r-1) \log \left[\sum_{j=1}^{r} x_{(j)}^{\widehat{\tilde{\beta}}_{B N} / \beta}+(n-r) x_{(r)}^{\widehat{\tilde{\beta}}_{B N} / \beta}\right]\right. \\
\left.+(r-1) \log \left[\sum_{j=1}^{r} x_{(j)}+(n-r) x_{(r)}\right]+\left(\frac{\widehat{\tilde{\beta}}_{B N}}{\beta}-1\right) \sum_{j=1}^{r} \log x_{(j)}-\log \sum_{j=1}^{r} x_{(j)}^{\widehat{\tilde{\beta}}_{B N} / \beta}+\log \sum_{j=1}^{r} x_{(j)}\right\} .
\end{gathered}
$$

Nos itens (i), (ii) e (iii), para dados completos, basta fazer $r=n$ nas expressões apresentadas.

(iv) Aproximação para Barndorff-Nielsen (covariâncias): $\widehat{\bar{\beta}}_{B N} / \beta$ e $\overline{R V}_{B N}$ (apenas para dados completos)

Equação de verossimilhança:

$$
\frac{(n-1) \widehat{\beta} / \beta}{\widehat{\bar{\beta}}_{B N} / \beta}-\widehat{\beta} \log \left(\frac{\widehat{\alpha}}{\alpha}\right)+\frac{\widehat{\beta}}{\beta} \sum_{j=1}^{n} \log x_{j}-\psi\left(\frac{\widehat{\bar{\beta}}_{B N} / \beta}{\widehat{\beta} / \beta}+1\right)-\frac{\widehat{\beta}(n-1)}{\beta} \frac{\sum_{j=1}^{n} x_{j}^{\widehat{\bar{\beta}}_{B N} / \beta} \log x_{j}}{\sum_{j=1}^{n} x_{j} \widehat{\bar{\beta}}_{B N} / \beta}=0
$$

Estatística da razão de verossimilhanças:

$$
\begin{aligned}
& \overline{R V}_{B N}(\beta)=2\left\{(n-1) \log \left(\frac{\widehat{\bar{\beta}}_{B N}}{\beta}\right)+\frac{1-\widehat{\bar{\beta}}_{B N} / \beta}{\widehat{\beta} / \beta} \log \left(\frac{\widehat{\alpha}}{\alpha}\right)+\left(\frac{\widehat{\bar{\beta}}_{B N}}{\beta}-1\right) \sum_{j=1}^{n} \log x_{j}\right. \\
& \left.-\log \Gamma\left(\frac{\widehat{\bar{\beta}}_{B N} / \beta}{\widehat{\beta} / \beta}+1\right)+\log \Gamma\left(\frac{1}{\widehat{\beta} / \beta}+1\right)-(n-1) \log \sum_{j=1}^{n} x_{j}^{\widehat{\bar{\beta}}_{B N} / \beta}+(n-1) \log \sum_{j=1}^{n} x_{j}\right\} .
\end{aligned}
$$

Aqui lembre que, pelo trabalho de Thoman et al. (1969), para dados completos, $\widehat{\beta} \log (\widehat{\alpha} / \alpha)$ é distribuído independentemente do par $(\alpha, \beta)$. Além disso, $\widehat{\beta}=\widehat{\beta}_{p}$. 


\section{D.1.3. CENSURA TIPO I}

Considere agora dados com presença de censura do tipo I, mais precisamente, $y_{j}=\min \left(t_{j}, c\right)$, onde $t_{j} \sim W B(\alpha, \beta)(j=1, \ldots, n)$ e $c$ é uma constante positiva fixada, representando o tempo de censura. Nesta situação, os dados podem ser convenientemente representados por $n$ pares de variáveis aleatórias $\left(y_{j}, \delta_{j}\right)$, onde

$$
y_{j}=\min \left(t_{j}, c\right) \quad \text { e } \quad \delta_{j}=\left\{\begin{array}{l}
1, t_{j} \leq c \\
0, t_{j}>c .
\end{array}\right.
$$

Na Seção 5.2.1.3, a fim de simular amostras com proporções de censura $p_{0}$, a constante $c$ foi definida a partir do cálculo da probabilidade $\operatorname{Pr}\left(t_{j}>c\right)=p_{0}$, obtendo-se

$$
c=\alpha\left(-\log p_{0}\right)^{1 / \beta}
$$

Vale lembrar aqui que as observações são independentes e identicamente distribuídas.

Note que $\delta_{j}$ tem distribuição de Bernoulli com probabilidade de sucesso $1-p_{0}$, ou seja, $\delta_{j} \sim \operatorname{Ber}\left(1-p_{0}\right)$. Conseqüentemente, $r=\sum_{j=1}^{n} \delta_{j}$ (número de falhas) é aleatório. Mais precisamente, $r$ tem distribuição binomial com parâmetros $n$ e $1-p_{0}$. Portanto, $r \sim \operatorname{Bin}\left(n, 1-p_{0}\right)$ é ancilar para $(\alpha, \beta)$.

A função escore relativa ao parâmetro de interesse $\beta$ obtida da função de verossimilhança perfilada é dada por (Seção 5.2.1.2)

$$
u_{p}(\beta)=\frac{r}{\beta}-\frac{r \sum_{j=1}^{n} y_{j}^{\beta} \log y_{j}}{\sum_{j=1}^{n} y_{j}^{\beta}}+\sum_{j \in \bar{C}} \log y_{j}
$$

onde $\bar{C}$ denota o conjunto de índices das observações não-censuradas. O e.m.v. de $\beta$ é a raiz da equação $u_{p}(\beta)=0$ e, logo, satisfaz a igualdade

$$
\frac{r}{\widehat{\beta}_{p}}-\frac{r \sum_{j=1}^{n} y_{j}^{\widehat{\beta}_{p}} \log y_{j}}{\sum_{j=1}^{n} \widehat{\beta}_{j}}+\sum_{j \in \bar{C}} \log y_{j}=0
$$

que pode ser re-escrita da forma

$$
\frac{r}{\widehat{\beta}_{p}}-r \frac{\sum_{j \in \bar{C}} t_{j}^{\widehat{\beta}_{p}} \log t_{j}+(n-r) c^{\widehat{\beta}_{p}} \log c}{\sum_{j \in \bar{C}} t_{j}^{\widehat{\beta}_{p}}+(n-r) c^{\widehat{\beta}_{p}}}+\sum_{j \in \bar{C}} \log t_{j}=0 .
$$

Para $j \in \bar{C}, t_{j}=\alpha x_{j}^{1 / \beta}$, onde $x_{j} \sim \mathcal{E} x p(1)$. Além disso, $c=\alpha\left(-\log p_{0}\right)^{1 / \beta}$. Substituindo essas relações na equação acima, vem 


$$
\frac{r}{\widehat{\beta}_{p}}-\frac{r}{\beta} \frac{\sum_{j \in \bar{C}} x_{j}^{\widehat{\beta}_{p} / \beta} \log x_{j}+(n-r)\left(-\log p_{0}\right)^{\widehat{\beta}_{p} / \beta} \log \left(-\log p_{0}\right)}{\sum_{j \in \bar{C}} x_{j}^{\widehat{\beta}_{p} / \beta}+(n-r)\left(-\log p_{0}\right)^{\widehat{\beta}_{p} / \beta}}+\frac{1}{\beta} \sum_{j \in \bar{C}} \log x_{j}=0 .
$$

Por fim, multiplicando ambos os lados por $\beta$, obtém-se

$$
\frac{r}{\widehat{\beta}_{p} / \beta}-r \frac{\sum_{j \in \bar{C}} x_{j}^{\widehat{\beta}_{p} / \beta} \log x_{j}+(n-r)\left(-\log p_{0}\right)^{\widehat{\beta}_{p} / \beta} \log \left(-\log p_{0}\right)}{\sum_{j \in \bar{C}} x_{j}^{\widehat{\beta}_{p} / \beta}+(n-r)\left(-\log p_{0}\right)^{\widehat{\beta}_{p} / \beta}}+\sum_{j \in \bar{C}} \log x_{j}=0 .
$$

Nesta equação, $n$ e $p_{0}$ são fixos e $r$ é ancilar para $(\alpha, \beta)$. Note que

$$
\sum_{j \in \bar{C}} \log x_{j}=\sum_{j=1}^{n} \delta_{j} \log x_{j}, \quad \sum_{j \in \bar{C}} x_{j}^{\widehat{\beta}_{p} / \beta} \log x_{j}=\sum_{j=1}^{n} \delta_{j} x_{j}^{\widehat{\beta}_{p} / \beta} \log x_{j}
$$

$\mathrm{e}$

$$
\sum_{j \in \bar{C}} x_{j}^{\widehat{\beta}_{p} / \beta}=\sum_{j=1}^{n} \delta_{j} x_{j}^{\widehat{\beta}_{p} / \beta}
$$

Portanto, considerando a razão $\left(\widehat{\beta}_{p} / \beta\right)$ como incógnita da equação, a raiz desta depende apenas de quantidades ancilares para $(\alpha, \beta)$. Ou seja, $\left(\widehat{\beta}_{p} / \beta\right)$ é distribuído independentemente desses parâmetros. Assim, o viés relativo de $\widehat{\beta}_{p}$ e os coeficientes de assimetria e de curtose da distribuição deste estimador variam apenas com o tamanho amostral ou com o nível de censura.

A estatística da razão de verossimilhanças é definida por

$$
R V_{p}(\beta)=2\left\{\ell_{p}\left(\widehat{\beta}_{p}\right)-\ell_{p}(\beta)\right\}
$$

Assim, após manipulações algébricas,

$$
\begin{aligned}
& R V_{p}(\beta)=2\left\{r \log \left(\widehat{\beta}_{p} / \beta\right)-r \log \left[\sum_{j \in \bar{C}} \widehat{\beta}_{j} / \beta+(n-r)\left(-\log p_{0}\right)^{\widehat{\beta}_{p} / \beta}\right]\right. \\
& \left.\quad+r \log \left[\sum_{j \in \bar{C}} x_{j}+(n-r)\left(-\log p_{0}\right)\right]+\left(\frac{\widehat{\beta}_{p}}{\beta}-1\right) \sum_{j \in \bar{C}} \log x_{j}\right\} .
\end{aligned}
$$

Note que $\operatorname{Pr}_{(\alpha, \beta)}\left(R V_{p}(\beta) \leq q\right)$ não depende de $\alpha$ e nem do valor fixado para $\beta$ na hipótese nula, depende apenas do tamanho amostral e da proporção de censura considerada (valor de $\left.p_{0}\right)$. 
D. EMV E ESTATÍSTICAS DA RAZÃO DE VEROSSIMILHANÇAS NO MODELO DE WEIBULL

Os resultados relativos aos estimadores de máxima verossimilhança derivados das versões ajustadas de $\ell_{p}(\beta)$ são demonstrados de forma análoga, assim como os resultados relacionados aos tamanhos dos testes da razão de verossimilhanças. Portanto, por economia de espaço, a seguir são mostradas as equações de verossimilhança cujas incógnitas são as razões (e.m.v. de $\beta) / \beta$ e as estatísticas de teste baseadas nas verossimilhanças perfiladas ajustadas.

(i) Ajuste de Cox e Reid: $\widehat{\beta}_{C R} / \beta$ e $R V_{C R}$

Equação de verossimilhança:

$$
\frac{r-1}{\widehat{\beta}_{C R} / \beta}-r \frac{\sum_{j \in \bar{C}} x_{j}^{\widehat{\beta}_{C R} / \beta} \log x_{j}+(n-r)\left(-\log p_{0}\right)^{\widehat{\beta}_{C R} / \beta} \log \left(-\log p_{0}\right)}{\sum_{j \in \bar{C}} x_{j}^{\widehat{\beta}_{C R} / \beta}+(n-r)\left(-\log p_{0}\right)^{\widehat{\beta}_{C R} / \beta}}+\sum_{j \in \bar{C}} \log x_{j}=0 .
$$

Estatística da razão de verossimilhanças:

$$
\begin{aligned}
R V_{C R}(\beta) & =2\left\{(r-1) \log \left(\widehat{\beta}_{C R} / \beta\right)-r \log \left[\sum_{j \in \bar{C}} x_{j}^{\widehat{\beta}_{C R} / \beta}+(n-r)\left(-\log p_{0}\right)^{\widehat{\beta}_{C R} / \beta}\right]\right. \\
& \left.+r \log \left[\sum_{j \in \bar{C}} x_{j}+(n-r)\left(-\log p_{0}\right)\right]+\left(\frac{\widehat{\beta}_{C R}}{\beta}-1\right) \sum_{j \in \bar{C}} \log x_{j}\right\} .
\end{aligned}
$$

(ii) Aproximação para Barndorff-Nielsen (covariância empírica): $\widehat{\breve{\beta}}_{B N} / \beta$ e $\breve{R V} B N$ Equação de verossimilhança:

$$
\begin{aligned}
& \frac{r}{\widehat{\widehat{\beta}}_{B N} / \beta}+\sum_{j \in \bar{C}} \log x_{j}-(r-1) \frac{\sum_{j \in \bar{C}} x_{j}^{\widehat{\widehat{\beta}}_{B N} / \beta} \log x_{j}+(n-r)\left(-\log p_{0}\right)^{\widehat{\beta}_{B N} / \beta} \log \left(-\log p_{0}\right)}{\sum_{j \in \bar{C}} x_{j}^{\widehat{\beta}_{B N} / \beta}+(n-r)\left(-\log p_{0}\right)^{\widehat{\beta}_{B N} / \beta}} \\
& -\left\{r \sum_{j \in \bar{C}} x_{j}^{\left(\widehat{\breve{\beta}}_{B N}+\widehat{\beta}\right) / \beta} \log x_{j}+n(n-r)\left(-\log p_{0}\right)^{\left(\widehat{\breve{\beta}}_{B N}+\widehat{\beta}\right) / \beta} \log \left(-\log p_{0}\right)-\left(\sum_{j \in \bar{C}} x_{j}^{\widehat{\beta} / \beta}\right)\right. \\
& \left.\times\left(\sum_{j \in \bar{C}} x_{j}^{\widehat{\beta}_{B N} / \beta} \log x_{j}\right)\right\} \\
& /\left\{r \sum_{j \in \bar{C}} x_{j}^{\left.\widehat{\widetilde{\beta}}_{B N}+\widehat{\beta}\right) / \beta}+n(n-r)\left(-\log p_{0}\right)^{\left.\widehat{(\breve{\beta}}_{B N}+\widehat{\beta}\right) / \beta}-\left(\sum_{j \in \bar{C}} x_{j}^{\widehat{\beta} / \beta}\right)\left(\sum_{j \in \bar{C}} x_{j}^{\widehat{\breve{\beta}}_{B N} / \beta}\right)\right\}=0 .
\end{aligned}
$$


D. EMV E ESTATÍSTICAS DA RAZÃO DE VEROSSIMILHANÇAS NO MODELO DE WEIBULL

Estatística da razão de verossimilhanças:

$$
\begin{aligned}
& R V_{B N}(\beta)=2\left\{r \log \left(\widehat{\breve{\beta}}_{B N} / \beta\right)-(r-1) \log \left[\sum_{j \in \bar{C}} x_{j}^{\widehat{\breve{\beta}}_{B N} / \beta}+(n-r)\left(-\log p_{0}\right)^{\widehat{\beta}_{B N} / \beta}\right]\right. \\
& -\log \left[r \sum_{j \in \bar{C}} x_{j}^{\left(\widehat{\breve{\beta}}_{B N}+\widehat{\beta}\right) / \beta}+n(n-r)\left(-\log p_{0}\right)^{\left(\widehat{\breve{\beta}}_{B N}+\widehat{\beta}\right) / \beta}-\left(\sum_{j \in \bar{C}} x_{j}^{\widehat{\beta} / \beta}\right)\left(\sum_{j \in \bar{C}} x_{j}^{\widehat{\beta}_{B N} / \beta}\right)\right] \\
& +\left(\frac{\widehat{\vec{\beta}}_{B N}}{\beta}-1\right) \sum_{j \in \bar{C}} \log x_{j}+(r-1) \log \left[\sum_{j \in \bar{C}} x_{j}+(n-r)\left(-\log p_{0}\right)\right] \\
& \left.+\log \left[r \sum_{j \in \bar{C}} x_{j}^{1+\widehat{\beta} / \beta}+n(n-r)\left(-\log p_{0}\right)^{1+\widehat{\beta} / \beta}-\left(\sum_{j \in \bar{C}} x_{j}^{\widehat{\beta} / \beta}\right)\left(\sum_{j \in \bar{C}} x_{j}\right)\right]\right\} .
\end{aligned}
$$

Aqui $\widehat{\beta}$ é o estimador de máxima verossimilhança obtido de dados com presença de censura tipo I e não tem forma fechada. Lembre que $\widehat{\beta}=\widehat{\beta}_{p}$, portanto, $\widehat{\beta} / \beta$ é uma quantidade pivotal para $(\alpha, \beta)$.

(iii) Aproximação para Barndorff-Nielsen (estatística ancilar): $\widehat{\tilde{\beta}}_{B N} / \beta$ e $\tilde{R V} B N$

Equação de verossimilhança:

$$
\begin{gathered}
\frac{r-1}{\widehat{\tilde{\beta}}_{B N} / \beta}-(r-1) \frac{\sum_{j \in \bar{C}} x_{j}^{\widehat{\tilde{\beta}}_{B N} / \beta} \log x_{j}+(n-r)\left(-\log p_{0}\right)^{\widehat{\tilde{\beta}}_{B N} / \beta} \log \left(-\log p_{0}\right)}{\sum_{j \in \bar{C}} x_{j}^{\widehat{\tilde{\beta}}_{B N} / \beta}+(n-r)\left(-\log p_{0}\right)^{\widehat{\tilde{\beta}}_{B N} / \beta}} \\
+\sum_{j \in \bar{C}} \log x_{j}-\frac{\sum_{j \in \bar{C}} x_{j}^{\widehat{\tilde{\beta}}_{B N} / \beta} \log x_{j}}{\sum_{j \in \bar{C}} x_{j}^{\tilde{\beta}_{B N} / \beta}}=0 .
\end{gathered}
$$

Estatística da razão de verossimilhanças:

$$
\begin{aligned}
& \tilde{R V} V_{B N}(\beta)=2\left\{(r-1) \log \left(\widehat{\tilde{\beta}}_{B N} / \beta\right)-(r-1) \log \left[\sum_{j \in \bar{C}} x_{j}^{\widehat{\tilde{\beta}}_{B N} / \beta}+(n-r)\left(-\log p_{0}\right)^{\widehat{\tilde{\beta}}_{B N} / \beta}\right]\right. \\
& \left.+\left(\frac{\widehat{\tilde{\beta}}_{B N}}{\beta}-1\right) \sum_{j \in \bar{C}} \log x_{j}+(r-1) \log \left[\sum_{j \in \bar{C}} x_{j}+(n-r)\left(-\log p_{0}\right)\right]-\log \sum_{j \in \bar{C}} x_{j}^{\widehat{\tilde{\beta}}_{B N} / \beta}+\log \sum_{j \in \bar{C}} x_{j}\right\} .
\end{aligned}
$$




\section{D.2. OBSERVAÇÕES INDEPENDENTES}

\section{D.2.1. INTRODUÇÃO}

Diferentemente da seção anterior, aqui são demonstrados resultados para o caso em que se modela o parâmetro $\alpha$ através de algumas covariáveis, mais precisamente,

$$
t_{j} \sim W B\left(\alpha\left(\mathbf{x}_{j}\right), \beta\right),
$$

onde $\mathbf{x}_{j}=\left(1, x_{j 2}, \ldots, x_{j p}\right)$ é uma coleção de covariáveis com $j=1, \ldots, n$. Por conseguinte, $\log t_{j}=y_{j} \sim V E\left(\eta\left(\mathbf{x}_{j}\right), \sigma\right)$, onde $\sigma=\beta^{-1}$ e $\eta\left(\mathbf{x}_{j}\right)=\log \alpha\left(\mathbf{x}_{j}\right)$. Usualmente,

$$
\eta\left(\mathbf{x}_{j}\right)=\mathbf{x}_{j} \phi
$$

onde $\phi=\left(\phi_{1}, \phi_{2}, \ldots, \phi_{p}\right)^{\top}$. Assim, as observações das variáveis aleatórias não são identicamente distribuídas, são apenas independentes.

Neste contexto, foi mencionado na Seção 5.2.2.4, para os estimadores de máxima verossimilhança de $\sigma$ derivados das funções $\ell$ e $\tilde{\ell}_{B N}$, que as razões (e.m.v. de $\sigma$ ) / $\sigma$ são quantidades pivotais para $(\sigma, \phi)$, tanto para dados completos quanto para dados com presença de censura do tipo II. Para o estimador derivado de $\ell_{C R}$, este resultado apenas é válido no caso de dados completos. Além disso, somente neste caso, as probabilidades dos erros tipo I dos testes da razão de verossimilhanças baseados em tais funções não dependem do par $(\sigma, \phi)$. A seguir, são demonstrados tais resultados, assim como a razão pela qual não valem para dados com presença de censura do tipo I. No caso de dados censurados do tipo II, representam extensões de resultados apresentados em Lawless (1982, Apêndice G) e Verhagen (1961).

Para isto, são exploradas as propriedades de estimadores equivariantes. Suponha que $\tau$ e $\psi$ sejam parâmetros de locação e de escala de uma família de distribuições, respectivamente, e seja $\mathbf{y}=\left(y_{1}, \ldots, y_{n}\right)$ uma amostra aleatória de uma variável distribuída segundo essa família. Estimadores $\widehat{\tau}=\widehat{\tau}(\mathbf{y})$ e $\widehat{\psi}=\widehat{\psi}(\mathbf{y})$ desses parâmetros são ditos equivariantes se

$$
\widehat{\tau}(d \mathbf{y}+k)=d \widehat{\tau}(\mathbf{y})+k \quad \text { e } \quad \widehat{\psi}(d \mathbf{y}+k)=d \widehat{\psi}(\mathbf{y})
$$

onde $d(d>0)$ e $k(-\infty<k<\infty)$ são constantes reais.

A distribuição valor extremo pertence à família de locação-escala e, aqui, seu parâmetro de locação é modelado por algumas covariáveis segundo a seguinte estrutura: $\eta(\mathbf{x})=\mathbf{x} \phi$. O parâmetro de escala é $\sigma$. Neste contexto, dada uma amostra $\mathbf{y}=\left(y_{1}, \ldots, y_{n}\right)$, onde $y_{j} \sim$ $V E\left(\eta\left(\mathbf{x}_{j}\right), \sigma\right)$ para $j=1, \ldots, n$, estimadores para $\phi($ vetor $p \times 1)$ e $\sigma$ são ditos equivariantes se para qualquer vetor de constantes reais $\mathbf{b}($ vetor $p \times 1)$ e qualquer escalar $d(d>0)$ :

$$
\widehat{\phi}(d \mathbf{y}+X \mathbf{b})=\widehat{d \phi}(\mathbf{y})+\mathbf{b} \quad \text { e } \quad \widehat{\sigma}(d \mathbf{y}+X \mathbf{b})=\widehat{d \phi}(\mathbf{y})
$$

onde $X=\left(\mathbf{x}_{1}, \ldots, \mathbf{x}_{n}\right)^{\top}$. 


\section{D.2.2. ESTIMADORES E ESTATÍSTICAS DE TESTES}

No caso de dados censurados, a função de verossimilhança é dada por

$$
L(\sigma, \phi)=\prod_{j \in \bar{C}} \frac{1}{\sigma} \exp \left[\frac{y_{j}-\mathbf{x}_{j} \phi}{\sigma}-\exp \left(\frac{y_{j}-\mathbf{x}_{j} \phi}{\sigma}\right)\right] \prod_{j \in C} \exp \left[-\exp \left(\frac{y_{j}-\mathbf{x}_{j} \phi}{\sigma}\right)\right],
$$

onde $\bar{C}$ denota o conjunto dos índices das observações não-censuradas.

Sejam $\mathbf{y}=\left(y_{1}, \ldots, y_{n}\right)$ observações independentes de variáveis aleatórias com distribuições $y_{j} \sim V E\left(\mathbf{x}_{j} \phi, \sigma\right)(j=1, \ldots, n)$ e $y_{(1)}, \ldots, y_{(r)}$ as $r$ menores observações. Uma amostra de tamanho $n$ definida por $\left(y_{(1)}, \ldots, y_{(r)}, y_{(r)}, \ldots, y_{(r)}\right)$ caracteriza uma amostra com presença de censura do tipo II, onde $r$ é fixado previamente e não depende do par $(\sigma, \phi)$. Note que $r=\# \bar{C}$.

Substituindo o seguinte grupo de transformações na função $L\left(\sigma^{\prime}, \phi^{\prime}\right)=L_{\mathbf{y}^{\prime}}\left(\sigma^{\prime}, \phi^{\prime}\right)$,

$$
y_{j}^{\prime}=d y_{j}+\mathbf{x}_{j} k, \quad \phi^{\prime}=d \phi+k \quad \text { e } \quad \sigma^{\prime}=d \sigma,
$$

em que $d$ é um escalar $(d>0), k$ é um vetor de dimensão $p \times 1$ de constantes reais e $j=1, \ldots, n$, é fácil ver que

$$
L_{\mathbf{y}^{\prime}}\left(\sigma^{\prime}, \phi^{\prime}\right)=\left(\frac{1}{d}\right)^{r} L_{\mathbf{y}}(\sigma, \phi) .
$$

Ou seja,

$$
L_{\mathbf{y}}(\sigma, \phi)=d^{r} L_{\mathbf{y}^{\prime}}\left(\sigma^{\prime}, \phi^{\prime}\right) .
$$

Assim, se $L_{\mathbf{y}}(\sigma, \phi)$ é maximizada em

$$
\widehat{\phi}=\widehat{\phi}\left(y_{(1)}, \ldots, y_{(r)}\right) \text { e } \widehat{\sigma}=\widehat{\sigma}\left(y_{(1)}, \ldots, y_{(r)}\right),
$$

então $L_{\mathbf{y}^{\prime}}(\sigma, \phi)$ é maximizada em

$$
\widehat{\phi}\left(y_{(1)}^{\prime}, \ldots, y_{(r)}^{\prime}\right)=\widehat{d \phi}\left(y_{(1)}, \ldots, y_{(r)}\right)+k \quad \text { e } \widehat{\sigma}\left(y_{(1)}^{\prime}, \ldots, y_{(r)}^{\prime}\right)=d \widehat{\sigma}\left(y_{(1)}, \ldots, y_{(r)}\right) .
$$

Portanto, os estimadores de máxima verossimilhança $\widehat{\phi}$ e $\widehat{\sigma}$ são equivariantes.

Dado $y_{j} \sim V E\left(\mathbf{x}_{j} \phi, \sigma\right)$, então

$$
z_{j}=\frac{y_{j}-\mathbf{x}_{j} \phi}{\sigma} \sim V E(0,1),
$$

para $j=1, \ldots, n$. Assim, $z_{j}$ é uma quantidade pivotal para $(\sigma, \phi)$ e, por conseguinte, a distribuição conjunta das estatísticas de ordem $z_{(1)}, \ldots, z_{(r)}$ também não depende desses parâmetros.

Seja $\mathbf{z}=\left(z_{1}, \ldots, z_{n}\right)$. Note que $\mathbf{z}=\mathbf{y}^{\prime}$ ao fazer $d=1 / \sigma$ e $k=-(1 / \sigma) \phi$. Como $\widehat{\phi}$ e $\widehat{\sigma}$ são equivariantes, então

$$
\widehat{\phi}\left(z_{(1)}, \ldots, z_{(r)}\right)=\frac{1}{\sigma} \widehat{\phi}\left(y_{(1)}, \ldots, y_{(r)}\right)-\frac{1}{\sigma} \phi=\frac{1}{\sigma}\left[\widehat{\phi}\left(y_{(1)}, \ldots, y_{(r)}\right)-\phi\right]
$$


e

$$
\widehat{\sigma}\left(z_{(1)}, \ldots, z_{(r)}\right)=\frac{1}{\sigma} \widehat{\sigma}\left(y_{(1)}, \ldots, y_{(r)}\right)
$$

Logo,

$$
\frac{1}{\sigma}[\widehat{\phi}-\phi] \text { e } \frac{\widehat{\sigma}}{\sigma}
$$

são quantidades pivotais para $(\sigma, \phi)$, pois a distribuição conjunta do vetor $\left(z_{(1)}, \ldots, z_{(r)}\right)$ não depende desses parâmetros. A razão destas quantidades,

$$
\frac{1}{\widehat{\sigma}}[\widehat{\phi}-\phi]
$$

também é uma quantidade pivotal para $(\sigma, \phi)$. Explorando ainda a independência das distribuições dessas quantidades e de $z_{j}(j \in \bar{C})$ com relação ao par $(\sigma, \phi)$, tem-se que

$$
\frac{\sigma}{\widehat{\sigma}}\left[z_{j}-\mathbf{x}_{j} \frac{1}{\sigma}(\widehat{\phi}-\phi)\right]=\frac{\sigma}{\widehat{\sigma}}\left[\frac{y_{j}-\mathbf{x}_{j} \phi}{\sigma}-\mathbf{x}_{j} \frac{1}{\sigma}(\widehat{\phi}-\phi)\right]=\frac{y_{j}-\mathbf{x}_{j} \widehat{\phi}}{\widehat{\sigma}}
$$

é uma estatística ancilar para $(\sigma, \phi)$. No caso de dados completos, $r=\# \bar{C}=n$.

Observe que o fato dessas quantidades serem pivotais é uma conseqüência da equivariância dos estimadores de máxima verossimilhança e do vetor $\left(z_{(1)}, \ldots, z_{(r)}\right)$ ser independentemente distribuído do par $(\sigma, \phi)$. No caso de dados com presença de censura do tipo I, diferentemente da situação em que as observações eram independentes e identicamente distribuídas, tal vetor não é independentemente distribuído do par $(\sigma, \phi)$. Naquela situação, $r \sim \operatorname{Bin}\left(n, 1-p_{0}\right)$, conforme Seção D.1.3.

Aqui $y_{j}=\log \left[\min \left(t_{j}, c\right)\right]$, onde $t_{j} \sim W B\left(\alpha\left(\mathbf{x}_{j}\right), \beta\right)(j=1, \ldots, n)$ e $c$ é uma constante positiva fixada, representando o tempo de censura. Na Seção 5.2.2.4, tal constante foi fixada em

$$
c=\left[\frac{1}{n} \sum_{j=1}^{n} \alpha\left(\mathbf{x}_{j}\right)\right]\left(-\log p_{0}\right)^{1 / \beta}
$$

sendo $p_{0}$ a proporção de censura desejada. Nesta situação, os dados podem ser convenientemente representados por $n$ pares de variáveis aleatórias $\left(y_{j}, \delta_{j}\right)$, onde

$$
y_{j}=\log \left[\min \left(t_{j}, c\right)\right] \quad \text { e } \quad \delta_{j}=\left\{\begin{array}{l}
1, t_{j} \leq c \\
0, t_{j}>c
\end{array}\right.
$$

Note que $r=\sum_{j}^{n} \delta_{j}$. Dessa forma,

$$
\operatorname{Pr}\left(\delta_{j}=1\right)=\operatorname{Pr}\left(t_{j} \leq c\right)=\operatorname{Pr}\left(t_{j} \leq\left[\frac{1}{n} \sum_{j=1}^{n} \alpha\left(\mathbf{x}_{j}\right)\right]\left(-\log p_{0}\right)^{1 / \beta}\right)
$$

Lembre que se $u_{j} \sim \mathcal{E} x p(1)$, então $t_{j}=\alpha\left(\mathbf{x}_{j}\right) u_{j}^{1 / \beta} \sim W B\left(\alpha\left(\mathbf{x}_{j}\right), \beta\right)$ para $j=1, \ldots, n$. Usando esta relação, obtém-se

$$
\operatorname{Pr}\left(\delta_{j}=1\right)=\operatorname{Pr}\left(u_{j} \leq\left[\frac{\sum_{j=1}^{n} \alpha\left(\mathbf{x}_{j}\right)}{n \alpha\left(\mathbf{x}_{j}\right)}\right]^{\beta}\left(-\log p_{0}\right)\right)=1-\exp \left\{\left[\frac{\sum_{j=1}^{n} \alpha\left(\mathbf{x}_{j}\right)}{n \alpha\left(\mathbf{x}_{j}\right)}\right]^{\beta} \log p_{0}\right\} .
$$


Ou seja,

$$
\operatorname{Pr}\left(\delta_{j}=1\right)=1-p_{0}^{\left\{\left[\sum_{j=1}^{n} \alpha\left(\mathbf{x}_{j}\right)\right] /\left[n \alpha\left(\mathbf{x}_{j}\right)\right]\right\}^{\beta}} .
$$

Assim,

$$
\delta_{j} \sim \operatorname{Ber}\left(1-p_{0}^{\left\{\left[\sum_{j=1}^{n} \alpha\left(\mathbf{x}_{j}\right)\right] /\left[n \alpha\left(\mathbf{x}_{j}\right)\right]\right\}^{\beta}}\right)
$$

para $j=1, \ldots, n$. Como $\beta=\sigma^{-1}$ e $\alpha\left(\mathbf{x}_{j}\right)=\exp \left(\mathbf{x}_{j} \phi\right)$, então $r=\sum_{j}^{n} \delta_{j}$ não é ancilar para $(\sigma, \phi)$ e, conseqüentemente, a distribuição conjunta do vetor aleatório $\left(z_{(1)}, \ldots, z_{(r)}\right)$ depende deste par de parâmetros. Portanto, no caso de dados com presença de censura do tipo I e observações independentes, mas não identicamente distribuídas, as estatísticas

$$
\frac{1}{\sigma}[\widehat{\phi}-\phi] \text { e } \frac{\widehat{\sigma}}{\sigma}
$$

não são independentemente distribuídas de $\sigma$ e $\phi$.

Note que

$$
\mathbf{x}_{j}=\mathbf{x}, \forall j \quad \Longrightarrow \quad \delta_{j} \sim \operatorname{Ber}\left(1-p_{0}\right), \forall j
$$

Por conseguinte, $r \sim \operatorname{Bin}\left(n, 1-p_{0}\right)$. Este é o caso de dados com presença de censura do tipo I e observações independentes e identicamente distribuídas.

Voltando ao caso de dados censurados do tipo II, embora $(\widehat{\sigma} / \sigma)$ seja uma quantidade pivotal para $(\sigma, \phi)$, a distribuição dessa razão depende da matriz de especificação do modelo $X$.

Os estimadores de máxima verossimilhança $\widehat{\sigma}$ e $\widehat{\phi}$ são raízes do sistema de equações de verossimilhança (ver Seção 5.2.2.3),

$$
-r-\frac{1}{\widehat{\sigma}} \sum_{j \in \bar{C}}\left(y_{j}-\mathbf{x}_{j} \widehat{\phi}\right)+\frac{1}{\widehat{\sigma}} \sum_{j=1}^{n}\left(y_{j}-\mathbf{x}_{j} \widehat{\phi}\right) \exp \left(\frac{y_{j}-\mathbf{x}_{j} \widehat{\phi}}{\widehat{\sigma}}\right)=0
$$

$\mathrm{e}$

$$
-\frac{1}{\widehat{\sigma}} \sum_{j \in \bar{C}} \mathbf{x}_{j}+\frac{1}{\widehat{\sigma}} \sum_{j=1}^{n} \mathbf{x}_{j} \exp \left(\frac{y_{j}-\mathbf{x}_{j} \widehat{\phi}}{\widehat{\sigma}}\right)=0_{1 \times p}
$$

Multiplicando $(D .2)$ à direita pelo vetor $\widehat{\phi}$ e somando a equação resultante a $(D .1)$, obtém-se

$$
-r-\frac{1}{\widehat{\sigma}} \sum_{j \in \bar{C}} y_{j}+\frac{1}{\widehat{\sigma}} \sum_{j=1}^{n} y_{j} \exp \left(\frac{y_{j}-\mathbf{x}_{j} \widehat{\phi}}{\widehat{\sigma}}\right)=0 .
$$

Como mencionado anteriormente, se $u_{j} \sim \mathcal{E} x p(1)$, então $t_{j}=\alpha\left(\mathbf{x}_{j}\right) u_{j}^{1 / \beta} \sim W B\left(\alpha\left(\mathbf{x}_{j}\right), \beta\right)$ para $j=1, \ldots, n$, onde $\beta=\sigma^{-1}$ e $\alpha\left(\mathbf{x}_{j}\right)=\exp \left(\mathbf{x}_{j} \phi\right)$. Dessa maneira,

$$
y_{j}=\log t_{j}=\mathbf{x}_{j} \phi+\sigma \log u_{j}
$$


D. EMV E ESTATÍSTICAS DA RAZÃO DE VEROSSIMILHANÇAS NO MODELO DE WEIBULL

e, substituindo essa relação na última equação, vem

$$
-r-\frac{1}{\widehat{\sigma}} \sum_{j \in \bar{C}} y_{j}+\frac{1}{\widehat{\sigma}} \sum_{j=1}^{n}\left(\mathbf{x}_{j} \phi+\sigma \log u_{j}\right) \exp \left(\frac{y_{j}-\mathbf{x}_{j} \widehat{\phi}}{\widehat{\sigma}}\right)=0 .
$$

Multiplicando $(D .2)$ à direita pelo vetor $\phi$, obtém-se

$$
\frac{1}{\widehat{\sigma}} \sum_{j \in \bar{C}} \mathbf{x}_{j} \phi=\frac{1}{\widehat{\sigma}} \sum_{j=1}^{n} \mathbf{x}_{j} \phi \exp \left(\frac{y_{j}-\mathbf{x}_{j} \widehat{\phi}}{\widehat{\sigma}}\right) .
$$

Usando este resultado, a equação (D.1) pode ser re-escrita na forma

$$
-r-\frac{1}{\widehat{\sigma} / \sigma} \sum_{j \in \bar{C}} \frac{y_{j}-\mathbf{x}_{j} \phi}{\sigma}+\frac{1}{\widehat{\sigma} / \sigma} \sum_{j=1}^{n} \log \left(u_{j}\right) \exp \left[\frac{\log u_{j}}{\widehat{\sigma} / \sigma}-\mathbf{x}_{j} \frac{1}{\widehat{\sigma}}(\widehat{\phi}-\phi)\right]=0
$$

ou ainda,

$$
-r-\frac{1}{\widehat{\sigma} / \sigma} \sum_{j \in \bar{C}} \log \left(u_{j}\right)+\frac{1}{\widehat{\sigma} / \sigma} \sum_{j=1}^{n} \log \left(u_{j}\right) \exp \left[\frac{\log u_{j}}{\widehat{\sigma} / \sigma}-\mathbf{x}_{j} \frac{1}{\widehat{\sigma}}(\widehat{\phi}-\phi)\right]=0 .
$$

Considerando $(\widehat{\sigma} / \sigma)$ como incógnita desta equação, observe que a raiz depende apenas de estatísticas ancilares e quantidades pivotais para $(\sigma, \phi)$, entretanto depende de $\mathbf{x}_{j}, j=$ $1, \ldots, n$, através do termo $\exp \left[\mathbf{x}_{j}(\widehat{\phi}-\phi) / \widehat{\sigma}\right]$. No caso de dados completos, $r=\# \bar{C}=n$.

A estatística da razão de verossimilhanças é definida por

$$
R V(\sigma)=2\left\{\ell(\widehat{\sigma}, \widehat{\phi})-\ell\left(\sigma, \widehat{\phi}_{\sigma}\right)\right\}
$$

Assim,

$$
\begin{aligned}
R V(\sigma)= & 2\left\{-r \log \left(\frac{\widehat{\sigma}}{\sigma}\right)+\sum_{j \in \bar{C}}\left(\frac{y_{j}-\mathbf{x}_{j} \widehat{\phi}}{\widehat{\sigma}}\right)-\sum_{j=1}^{n} \exp \left(\frac{y_{j}-\mathbf{x}_{j} \widehat{\phi}}{\widehat{\sigma}}\right)\right. \\
& \left.-\sum_{j \in \bar{C}}\left(\frac{y_{j}-\mathbf{x}_{j} \widehat{\phi}_{\sigma}}{\sigma}\right)+\sum_{j=1}^{n} \exp \left(\frac{y_{j}-\mathbf{x}_{j} \widehat{\phi}_{\sigma}}{\sigma}\right)\right\} .
\end{aligned}
$$

Foi demonstrado anteriormente que as parcelas do somatório

$$
\sum_{j \in \bar{C}}\left(\frac{y_{j}-\mathbf{x}_{j} \widehat{\phi}}{\widehat{\sigma}}\right)
$$

são estatísticas ancilares para $(\sigma, \phi)$. Logo, a soma de tais parcelas é uma estatística ancilar também. 
Note da equação $(D .2)$ que

$$
\sum_{j=1}^{n} \exp \left(\frac{y_{j}-\mathbf{x}_{j} \widehat{\phi}}{\widehat{\sigma}}\right)=r
$$

Aqui lembre que $x_{j 1}=1$ para $j=1, \ldots, n$.

No Capítulo 5, a fim de comparar três testes da razão de verossimilhanças a partir das distorções dos tamanhos destes com relação aos valores nominais, são geradas amostras sob a hipótese nula e calculadas as taxas de rejeição dos mesmos. Nesta situação, o valor do parâmetro $\sigma$ é o valor verdadeiro. Assim, $\widehat{\phi}_{\sigma}$ é o estimador de máxima verossimilhança de $\phi$. Portanto, analogamente ao caso em que o par $(\sigma, \phi)$ é desconhecido,

$$
\sum_{j=1}^{n} \exp \left(\frac{y_{j}-\mathbf{x}_{j} \widehat{\phi}_{\sigma}}{\sigma}\right)=r
$$

onde $\widehat{\phi}_{\sigma}$ satisfaz a equação

$$
\frac{\partial \ell\left(\sigma, \widehat{\phi}_{\sigma}\right)}{\partial \phi^{\top}}=-\frac{1}{\sigma} \sum_{j \in \bar{C}} \mathbf{x}_{j}+\frac{1}{\sigma} \sum_{j=1}^{n} \exp \left(\frac{y_{j}-\mathbf{x}_{j} \widehat{\phi}_{\sigma}}{\sigma}\right) \mathbf{x}_{j}=0_{1 \times p},
$$

que pode ser re-escrita na forma,

$$
\sum_{j \in \bar{C}}\left[\exp \left(\frac{y_{j}-\mathbf{x}_{j} \widehat{\phi}_{\sigma}}{\sigma}\right)-1\right] \mathbf{x}_{j}+\sum_{j \notin \bar{C}} \exp \left(\frac{y_{j}-\mathbf{x}_{j} \widehat{\phi}_{\sigma}}{\sigma}\right) \mathbf{x}_{j}=0_{1 \times p} .
$$

Note que, para dados completos,

$$
\sum_{j \in \bar{C}}\left[\exp \left(\frac{y_{j}-\mathbf{x}_{j} \widehat{\phi}_{\sigma}}{\sigma}\right)-1\right] \mathbf{x}_{j}=0_{1 \times p}
$$

e, portanto,

$$
\sum_{j \in \bar{C}}\left(\frac{y_{j}-\mathbf{x}_{j} \widehat{\phi}_{\sigma}}{\sigma}\right)
$$

é independentemente distribuído do par $(\sigma, \phi)$, o que não é observado no caso de dados censurados.

Dessa forma, para dados completos, $\operatorname{Pr}_{(\sigma, \phi)}(R V(\sigma) \leq q)$ não depende de $\phi$ e nem do valor fixado para $\sigma$ na hipótese nula, ou seja, sob esta, observe que a estatística $R V(\sigma)$ depende apenas de quantidades pivotais e estatísticas ancilares para $(\sigma, \phi)$, entretanto, por depender da razão $(\widehat{\sigma} / \sigma)$, sua distribuição depende da matriz de especificação do modelo $X$.

Agora considere a verossimilhança perfilada ajustada denotada por $\tilde{\ell}_{B N}(\sigma)$, que é uma aproximação para $\ell_{B N}(\sigma)$ baseada numa estatística aproximadamente ancilar. Sua expressão é dada pela soma (ver Seção 5.2.2.3)

$$
\ell(\sigma, \phi)+p \log \sigma+\frac{1}{2} \log \left|X^{\top} Z X\right|-\log \left|X^{\top} Z \widehat{V}_{\phi}\right|,
$$


sujeita às restrições da forma

$$
\frac{\partial \ell(\sigma, \phi)}{\partial \phi_{s}}=-\frac{1}{\sigma} \sum_{j \in \bar{C}} x_{j s}+\frac{1}{\sigma} \sum_{j=1}^{n} x_{j s} \exp \left(\frac{y_{j}-\mathbf{x}_{j} \phi}{\sigma}\right)=0
$$

onde $s=1, \ldots, p$. Como $\widehat{\phi}_{\sigma}$ não tem forma fechada, $\tilde{\ell}_{B N}(\sigma)$ é apresentada como função do par $(\sigma, \phi)$, que, por satisfazer às $p$ restrições, garante-se que seja $\left(\sigma, \widehat{\phi}_{\sigma}\right)$. Dessa maneira, aqui $\tilde{\ell}_{B N}(\sigma)=\tilde{\ell}_{B N}(\sigma, \phi)$. Portanto,

$\tilde{\ell}_{B N, \mathbf{y}}(\sigma, \phi)=-(r-p) \log \sigma+\sum_{j \in \bar{C}}\left(\frac{y_{j}-\mathbf{x}_{j} \phi}{\sigma}\right)-\sum_{j=1}^{n} \exp \left(\frac{y_{j}-\mathbf{x}_{j} \phi}{\sigma}\right)+\log \left(\frac{\left|X^{\top} Z X\right|^{1 / 2}}{\left|X^{\top} Z \widehat{V}_{\phi}\right|}\right)$,

onde $Z=\operatorname{diag}\left(\exp \left(z_{1}\right), \ldots, \exp \left(z_{n}\right)\right)$, definido anteriormente.

A propriedade de equivariância dos estimadores de máxima verossimilhança pode ser demonstrada também usando o logaritmo da verossimilhança, pois

$$
L_{\mathbf{y}}(\sigma, \phi)=d^{r} L_{\mathbf{y}^{\prime}}\left(\sigma^{\prime}, \phi^{\prime}\right) \quad \Longleftrightarrow \quad \ell_{\mathbf{y}}(\sigma, \phi)=r \log d+\ell_{\mathbf{y}^{\prime}}\left(\sigma^{\prime}, \phi^{\prime}\right) .
$$

Substituindo o grupo de transformações $\mathbf{y}^{\prime}, \sigma^{\prime}$ e $\phi^{\prime}$, apresentado anteriormente, em $\tilde{\ell}_{B N, \mathbf{y}^{\prime}}\left(\sigma^{\prime}, \phi^{\prime}\right)$, após manipulações algébricas, obtém-se

$$
\tilde{\ell}_{B N, \mathbf{y}}(\sigma, \phi)=(r-p) \log d+\tilde{\ell}_{B N, \mathbf{y}^{\prime}}\left(\sigma^{\prime}, \phi^{\prime}\right) .
$$

Observe que $\partial \ell_{\mathbf{y}}(\sigma, \phi) / \partial \phi_{s}=0 \Longleftrightarrow \partial \ell_{\mathbf{y}^{\prime}}\left(\sigma^{\prime}, \phi^{\prime}\right) / \partial \phi_{s}^{\prime}=0$, onde $s=1, \ldots, p$.

Seja $\widehat{\tilde{\phi}}_{B N}=\widehat{\phi}_{\widehat{\tilde{\sigma}}_{B N}}$ o estimador de máxima verossimilhança de $\phi$ derivado da maximização de $\tilde{\ell}_{B N}(\sigma, \phi)$ sob as $p$ restrições. Logo, se $\tilde{\ell}_{B N, \mathbf{y}}(\sigma, \phi)$ é maximizada em

$$
\widehat{\tilde{\phi}}_{B N}=\widehat{\tilde{\phi}}_{B N}\left(y_{(1)}, \ldots, y_{(r)}\right) \quad \text { e } \quad \widehat{\tilde{\sigma}}_{B N}=\widehat{\tilde{\sigma}}_{B N}\left(y_{(1)}, \ldots, y_{(r)}\right),
$$

então $\tilde{\ell}_{B N, \mathbf{y}^{\prime}}(\sigma, \phi)$ é maximizada em

$$
\widehat{\tilde{\phi}}_{B N}\left(y_{(1)}^{\prime}, \ldots, y_{(r)}^{\prime}\right)=d \widehat{\tilde{\phi}}_{B N}\left(y_{(1)}, \ldots, y_{(r)}\right)+k
$$

$\mathrm{e}$

$$
\widehat{\tilde{\sigma}}_{B N}\left(y_{(1)}^{\prime}, \ldots, y_{(r)}^{\prime}\right)=d \widehat{\tilde{\sigma}}_{B N}\left(y_{(1)}, \ldots, y_{(r)}\right)
$$

Dessa forma, os estimadores de máxima verossimilhança $\widehat{\tilde{\phi}}_{B N}$ e $\widehat{\tilde{\sigma}}_{B N}$ são equivariantes.

De forma similar às demonstrações dos resultados distribucionais de quantidades envolvendo os estimadores de máxima verossimilhança $\widehat{\phi}$ e $\widehat{\sigma}$, pode ser provado que

$$
\frac{1}{\sigma}\left[\widehat{\tilde{\phi}}_{B N}-\phi\right], \quad \frac{\widehat{\tilde{\sigma}}_{B N}}{\sigma}, \quad \frac{1}{\widehat{\tilde{\sigma}}_{B N}}\left[\widehat{\tilde{\phi}}_{B N}-\phi\right] \quad \text { e } \frac{y_{j}-\mathbf{x}_{j} \widehat{\tilde{\phi}}_{B N}}{\widehat{\tilde{\sigma}}_{B N}}
$$


são independentemente distribuídos do par $(\sigma, \phi)$, onde $j \in \bar{C}$. No caso de dados completos, $r=\# \bar{C}=n$. No caso de dados de censurados tipo I, como $r$ é aleatório e sua distribuição depende do par $(\sigma, \phi)$, a distribuição do vetor $\left(z_{(1)}, \ldots, z_{(r)}\right)$ depende desses parâmetros. Conseqüentemente, as quantidades não são pivotais neste caso.

A razão $\widehat{\tilde{\sigma}}_{B N} / \sigma$ é uma quantidade pivotal para $(\sigma, \phi)$ e sua distribuição depende da matriz de especificação do modelo $X$. Da equação de verossimilhança $\partial \tilde{\ell}_{B N}\left(\widehat{\tilde{\sigma}}_{B N}, \widehat{\tilde{\phi}}_{B N}\right) / \partial \sigma=0 \mathrm{e}$ da equação de restrição $\partial \ell\left(\widehat{\tilde{\sigma}}_{B N}, \widehat{\tilde{\phi}}_{B N}\right) / \partial \phi^{\top}=0_{1 \times p}$, após manipulações algébricas, vem

$$
\begin{gathered}
-r+p-\frac{1}{\widehat{\tilde{\sigma}}_{B N}} \sum_{j \in \bar{C}} y_{j}+\frac{1}{\widehat{\tilde{\sigma}}_{B N}} \sum_{j=1}^{n} y_{j} \exp \left(\frac{y_{j}-\mathbf{x}_{j} \widehat{\tilde{\phi}}_{B N}}{\widehat{\tilde{\sigma}}_{B N}}\right) \\
-\frac{1}{2} \operatorname{tr}\left\{\left[X^{\top} Z\left(\widehat{\tilde{\sigma}}_{B N}, \widehat{\tilde{\phi}}_{B N}\right) X\right]^{-1} X^{\top}\left(Z\left(\widehat{\tilde{\sigma}}_{B N}, \widehat{\tilde{\phi}}_{B N}\right) \log Z\left(\widehat{\tilde{\sigma}}_{B N}, \widehat{\tilde{\phi}}_{B N}\right)\right) X\right\} \\
+\operatorname{tr}\left\{\left[X^{\top} Z\left(\widehat{\tilde{\sigma}}_{B N}, \widehat{\tilde{\phi}}_{B N}\right) \widehat{V}_{\phi}\right]^{-1} X^{\top}\left(Z\left(\widehat{\tilde{\sigma}}_{B N}, \widehat{\tilde{\phi}}_{B N}\right) \log Z\left(\widehat{\tilde{\sigma}}_{B N}, \widehat{\tilde{\phi}}_{B N}\right)\right) \widehat{V}_{\phi}\right\}=0,
\end{gathered}
$$

onde

$Z\left(\widehat{\tilde{\sigma}}_{B N}, \widehat{\tilde{\phi}}_{B N}\right)$ é a matriz $Z=\operatorname{diag}\left(\exp \left(z_{1}\right), \ldots, \exp \left(z_{n}\right)\right)$ avaliada em $\left(\widehat{\tilde{\sigma}}_{B N}, \widehat{\tilde{\phi}}_{B N}\right)$,

$\log Z\left(\widehat{\tilde{\sigma}}_{B N}, \widehat{\tilde{\phi}}_{B N}\right)$ é a matriz $\log Z=\operatorname{diag}\left(z_{1}, \ldots, z_{n}\right)$ avaliada em $\left(\widehat{\tilde{\sigma}}_{B N}, \widehat{\tilde{\phi}}_{B N}\right)$ e

$\widehat{V}_{\phi}$ é uma matriz $n \times p$ composta por elementos de $X$.

Aqui foi usada a seguinte propriedade de diferenciação de matrizes, encontrada, por exemplo, em Magnus e Neudecker (1988),

$$
\frac{\partial}{\partial \theta} \log |A(\theta)|=\operatorname{tr}\left\{A(\theta)^{-1} \frac{\partial}{\partial \theta} A(\theta)\right\}
$$

onde $A(\theta)$ é uma matriz quadrada cujos elementos dependem de $\theta$.

Note que as matrizes

$$
\left[X^{\top} Z\left(\widehat{\tilde{\sigma}}_{B N}, \widehat{\tilde{\phi}}_{B N}\right) X\right]^{-1} X^{\top}\left(Z\left(\widehat{\tilde{\sigma}}_{B N}, \widehat{\tilde{\phi}}_{B N}\right) \log Z\left(\widehat{\tilde{\sigma}}_{B N}, \widehat{\tilde{\phi}}_{B N}\right)\right) X
$$

$\mathrm{e}$

$$
\left[X^{\top} Z\left(\widehat{\tilde{\sigma}}_{B N}, \widehat{\tilde{\phi}}_{B N}\right) \widehat{V}_{\phi}\right]^{-1} X^{\top}\left(Z\left(\widehat{\tilde{\sigma}}_{B N}, \widehat{\tilde{\phi}}_{B N}\right) \log Z\left(\widehat{\tilde{\sigma}}_{B N}, \widehat{\tilde{\phi}}_{B N}\right)\right) \widehat{V}_{\phi}
$$

são estatísticas ancilares para $(\sigma, \phi)$ e as distribuições dos seus traços dependem da matriz $X$.

Usando novamente a equação de restrição $\partial \ell\left(\widehat{\tilde{\sigma}}_{B N}, \widehat{\tilde{\phi}}_{B N}\right) / \partial \phi^{\top}=0_{1 \times p}$ e o fato que

$$
y_{j}=\mathbf{x}_{j} \phi+\sigma \log u_{j}
$$

onde $u_{j} \sim \mathcal{E} x p(1), j=1, \ldots, n$, a equação $(D .3)$ torna-se 


$$
\begin{aligned}
-r+ & p-\frac{1}{\hat{\tilde{\sigma}}_{B N} / \sigma} \sum_{j \in \bar{C}} \log u_{j}+\frac{1}{\widehat{\tilde{\sigma}}_{B N} / \sigma} \sum_{j=1}^{n} \log \left(u_{j}\right) \exp \left[\frac{\log u_{j}}{\widehat{\tilde{\sigma}}_{B N} / \sigma}-\mathbf{x}_{j} \frac{1}{\widehat{\tilde{\sigma}}_{B N}}\left(\widehat{\tilde{\phi}}_{B N}-\phi\right)\right] \\
& -\frac{1}{2} \operatorname{tr}\left\{\left[X^{\top} Z\left(\widehat{\tilde{\sigma}}_{B N}, \widehat{\tilde{\phi}}_{B N}\right) X\right]^{-1} X^{\top}\left(Z\left(\widehat{\tilde{\sigma}}_{B N}, \widehat{\tilde{\phi}}_{B N}\right) \log Z\left(\widehat{\tilde{\sigma}}_{B N}, \widehat{\tilde{\phi}}_{B N}\right)\right) X\right\} \\
& +\operatorname{tr}\left\{\left[X^{\top} Z\left(\widehat{\tilde{\sigma}}_{B N}, \widehat{\tilde{\phi}}_{B N}\right) \widehat{V}_{\phi}\right]^{-1} X^{\top}\left(Z\left(\widehat{\tilde{\sigma}}_{B N}, \widehat{\tilde{\phi}}_{B N}\right) \log Z\left(\widehat{\tilde{\sigma}}_{B N}, \widehat{\tilde{\phi}}_{B N}\right)\right) \widehat{V}_{\phi}\right\}=0 .
\end{aligned}
$$

Considerando $\left(\widehat{\tilde{\sigma}}_{B N} / \sigma\right)$ como incógnita desta equação, observe que a raiz depende apenas de estatísticas ancilares e quantidades pivotais para $(\sigma, \phi)$, além da matriz $X$. No caso de dados completos, $r=\# \bar{C}=n$.

A estatística da razão de verossimilhanças é definida por

$$
R V_{B N}(\sigma)=2\left\{\tilde{\ell}_{B N}\left(\widehat{\tilde{\sigma}}_{B N}, \widehat{\tilde{\phi}}_{B N}\right)-\tilde{\ell}_{B N}\left(\sigma, \widehat{\tilde{\phi}}_{B N, \sigma}\right)\right\}
$$

onde $\widehat{\tilde{\phi}}_{B N, \sigma}$ é o estimador de máxima verossimilhança de $\phi$ sob a hipótese nula. Fixando $\sigma$ em $\tilde{\ell}_{B N}(\sigma, \phi)$, tal estimador é obtido maximando esta função sob as restrições

$$
\frac{\partial \ell(\sigma, \phi)}{\partial \phi_{s}}=-\frac{1}{\sigma} \sum_{j \in \bar{C}} x_{j s}+\frac{1}{\sigma} \sum_{j=1}^{n} x_{j s} \exp \left(\frac{y_{j}-\mathbf{x}_{j} \phi}{\sigma}\right)=0
$$

onde $s=1, \ldots, p$. Note que $\widehat{\tilde{\phi}}_{B N, \sigma}$ é diferente de $\widehat{\tilde{\phi}}_{B N}$, pois este é obtido da maximização de $\tilde{\ell}_{B N}(\sigma, \phi)$ sob as $p$ restrições com relação ao par $(\sigma, \phi)$, ou seja, constitui o ponto de máximo global da função sob as restrições, $\left(\widehat{\tilde{\sigma}}_{B N}, \widehat{\tilde{\phi}}_{B N}\right)$.

Feitas estas considerações, a estatística da razão de verossimilhanças é dada por

$$
\begin{aligned}
& R V_{B_{N}}(\sigma)=2\left\{-(r-p) \log \left(\frac{\widehat{\tilde{\sigma}}_{B N}}{\sigma}\right)+\sum_{j \in \bar{C}}\left(\frac{y_{j}-\mathbf{x}_{j} \widehat{\tilde{\phi}}_{B N}}{\widehat{\tilde{\sigma}}_{B N}}\right)-\sum_{j=1}^{n} \exp \left(\frac{y_{j}-\mathbf{x}_{j} \widehat{\tilde{\phi}}_{B N}}{\widehat{\tilde{\sigma}}_{B N}}\right)\right. \\
& \quad+\frac{1}{2} \log \left|X^{\top} Z\left(\widehat{\tilde{\sigma}}_{B N}, \widehat{\tilde{\phi}}_{B N}\right) X\right|-\log \left|X^{\top} Z\left(\widehat{\tilde{\sigma}}_{B N}, \widehat{\tilde{\phi}}_{B N}\right) \widehat{V}_{\phi}\right|-\sum_{j \in \bar{C}}\left(\frac{y_{j}-\mathbf{x}_{j} \widehat{\tilde{\phi}}_{B N, \sigma}}{\sigma}\right) \\
& \left.+\sum_{j=1}^{n} \exp \left(\frac{y_{j}-\mathbf{x}_{j}}{\sigma} \widehat{\tilde{\phi}}_{B N, \sigma}\right)-\frac{1}{2} \log \left|X^{\top} Z\left(\sigma, \widehat{\tilde{\phi}}_{B N, \sigma}\right) X\right|+\log \left|X^{\top} Z\left(\sigma, \widehat{\tilde{\phi}}_{B N, \sigma}\right) \widehat{V}_{\phi}\right|\right\} .
\end{aligned}
$$


D. EMV E ESTATÍSTICAS DA RAZÃO DE VEROSSIMILHANÇAS NO MODELO DE WEIBULL

Conforme demonstração anterior,

$$
\sum_{j \in \bar{C}}\left(\frac{y_{j}-\mathbf{x}_{j} \widehat{\tilde{\phi}}_{B N}}{\widehat{\tilde{\sigma}}_{B N}}\right)
$$

é ancilar para $(\sigma, \phi)$, assim como os determinantes das formas quadráticas

$$
X^{\top} Z\left(\widehat{\tilde{\sigma}}_{B N}, \widehat{\tilde{\phi}}_{B N}\right) X \quad \text { e } \quad X^{\top} Z\left(\widehat{\tilde{\sigma}}_{B N}, \widehat{\tilde{\phi}}_{B N}\right) \widehat{V}_{\phi}
$$

Além disso, entre as $p$ restrições, para $s=1$, vem

$$
\sum_{j=1}^{n} \exp \left(\frac{y_{j}-\mathbf{x}_{j} \widehat{\tilde{\phi}}_{B N}}{\widehat{\tilde{\sigma}}_{B N}}\right)=r
$$

Lembre que $x_{j 1}=1$ para $j=1, \ldots, n$.

Considerando, na hipótese nula, o valor verdadeiro de $\sigma, \widehat{\tilde{\phi}}_{B N, \sigma}$ é o estimador de máxima verossimilhança de $\phi$. Portanto, analogamente ao caso em que o par $(\sigma, \phi)$ é desconhecido,

$$
\sum_{j=1}^{n} \exp \left(\frac{y_{j}-\mathbf{x}_{j} \widehat{\tilde{\phi}}_{B N, \sigma}}{\sigma}\right)=r
$$

Além disso, $\widehat{\tilde{\phi}}_{B N, \sigma}$ satisfaz a equação

$$
\frac{\partial \ell\left(\sigma, \widehat{\tilde{\phi}}_{B N, \sigma}\right)}{\partial \phi^{\top}}=-\frac{1}{\sigma} \sum_{j \in \bar{C}} \mathbf{x}_{j}+\frac{1}{\sigma} \sum_{j=1}^{n} \exp \left(\frac{y_{j}-\mathbf{x}_{j} \widehat{\tilde{\phi}}_{B N, \sigma}}{\sigma}\right) \mathbf{x}_{j}=0_{1 \times p}
$$

que pode ser re-escrita na forma,

$$
\sum_{j \in \bar{C}}\left[\exp \left(\frac{y_{j}-\mathbf{x}_{j} \widehat{\tilde{\phi}}_{B N, \sigma}}{\sigma}\right)-1\right] \mathbf{x}_{j}+\sum_{j \notin \bar{C}} \exp \left(\frac{y_{j}-\mathbf{x}_{j} \widehat{\tilde{\phi}}_{B N, \sigma}}{\sigma}\right) \mathbf{x}_{j}=0_{1 \times p}
$$

Note que, para dados completos,

$$
\sum_{j \in \bar{C}}\left[\exp \left(\frac{y_{j}-\mathbf{x}_{j} \widehat{\tilde{\phi}}_{B N, \sigma}}{\sigma}\right)-1\right] \mathbf{x}_{j}=0_{1 \times p}
$$

e, portanto,

$$
\sum_{j \in \bar{C}}\left(\frac{y_{j}-\mathbf{x}_{j} \widehat{\tilde{\phi}}_{B N, \sigma}}{\sigma}\right)
$$


é independentemente distribuído do par $(\sigma, \phi)$, o que não é observado no caso de dados censurados. Por motivos semelhantes, os determinantes das formas quadráticas

$$
X^{\top} Z\left(\sigma, \widehat{\tilde{\phi}}_{B N, \sigma}\right) X \quad \text { e } \quad X^{\top} Z\left(\sigma, \widehat{\tilde{\phi}}_{B N, \sigma}\right) \widehat{V}_{\phi}
$$

são independentemente distribuídos do par $(\sigma, \phi)$ apenas para dados completos.

Dessa forma, para dados completos, $\operatorname{Pr}_{(\sigma, \phi)}(R V(\sigma) \leq q)$ não depende de $\phi$ e nem do valor fixado para $\sigma$ na hipótese nula, ou seja, sob esta, observe que a estatística $R V(\sigma)$ depende apenas de quantidades pivotais e estatísticas ancilares para $(\sigma, \phi)$, entretanto, sua distribuição depende da matriz de especificação do modelo $X$.

\section{D.3. TESTES SOBRE OS COEFICIENTES DA REGRESSÃO}

\section{D.3.1. INTRODUÇÃO}

Para dados completos, foi demonstrado, na seção anterior, ao testar hipóteses sobre $\sigma$, que a probabilidade do erro tipo I não depende de $\phi$ e nem do valor fixado para tal parâmetro na hipótese nula quando são realizados testes da razão de verossimilhanças baseados em $\ell, \tilde{\ell}_{B N}$

e $\ell_{C R}$ (obtida a partir de uma parametrização ortogonal análoga àquela proposta por Yang e Xie (2003)). Utilizando alguns resultados derivados nessas demonstrações, aqui é provado também que, ao realizar testes da razão de verossimilhanças sobre $\phi$, o tamanho do teste não depende de $\sigma$ e nem do valor fixado para $\phi$ na hipótese nula. Este resultado é válido somente para dados completos, não vale para dados censurados. São considerados apenas os testes baseados na verossimilhança original $\ell$ e na verossimilhança perfilada ajustada $\tilde{\ell}_{B N}$.

\section{D.3.2. ESTATÍSTICAS DE TESTES}

Utilizando notação apresentada na Seção 5.3.1, para dados com presença de censura, o logaritmo da verossimilhança original é dado por

$$
\ell(\sigma, \phi)=-r \log \sigma+\sum_{j \in \bar{C}}\left[\frac{y_{j}-\left(\mathbf{x}_{j}^{(1)} \phi^{(1)}+\mathbf{x}_{j}^{(2)} \phi^{(2)}\right)}{\sigma}\right]-\sum_{j=1}^{n} \exp \left[\frac{y_{j}-\left(\mathbf{x}_{j}^{(1)} \phi^{(1)}+\mathbf{x}_{j}^{(2)} \phi^{(2)}\right)}{\sigma}\right] .
$$

Assim a estatística do teste da razão de verossimilhanças sobre $\phi^{(1)}$ é expressa por 
D. EMV E ESTATÍSTICAS DA RAZÃO DE VEROSSIMILHANÇAS NO MODELO DE WEIBULL

$$
\begin{aligned}
& R V\left(\phi^{(1)}\right)=2\left\{-r \log \widehat{\sigma}+\sum_{j \in \bar{C}}\left[\frac{y_{j}-\mathbf{x}_{j} \widehat{\phi}}{\widehat{\sigma}}\right]-\sum_{j=1}^{n} \exp \left[\frac{y_{j}-\mathbf{x}_{j} \widehat{\phi}}{\widehat{\sigma}}\right]+r \log \widehat{\sigma}_{\phi^{(1)}}\right. \\
& \left.-\sum_{j \in \bar{C}}\left[\frac{y_{j}-\left(\mathbf{x}_{j}^{(1)} \phi^{(1)}+\mathbf{x}_{j}^{(2)} \widehat{\phi}_{\phi^{(2)}}^{(1)}\right)}{\widehat{\sigma}_{\phi^{(1)}}}\right]+\sum_{j=1}^{n} \exp \left[\frac{y_{j}-\left(\mathbf{x}_{j}^{(1)} \phi^{(1)}+\mathbf{x}_{j}^{(2)} \widehat{\phi}_{\phi^{(2)}}^{(1)}\right)}{\widehat{\sigma}_{\phi^{(1)}}}\right]\right\},
\end{aligned}
$$

onde $\widehat{\sigma}_{\phi^{(1)}}$ e $\widehat{\phi^{(2)}} \phi^{(1)}$ são as estimativas de máxima verossimilhança de $\sigma$ e $\phi^{(2)}$ sob a hipótese nula, respectivamente. Essas estimativas são as raízes do sistema de $(p-k)+1$ equações definidas por

$$
\frac{\partial \ell(\sigma, \phi)}{\partial \sigma}=-\frac{r}{\sigma}-\frac{1}{\sigma} \sum_{j \in \bar{C}}\left(\frac{y_{j}-\mathbf{x}_{j} \phi}{\sigma}\right)+\frac{1}{\sigma} \sum_{j=1}^{n}\left(\frac{y_{j}-\mathbf{x}_{j} \phi}{\sigma}\right) \exp \left(\frac{y_{j}-\mathbf{x}_{j} \phi}{\sigma}\right)=0
$$

$\mathrm{e}$

$$
\frac{\partial \ell(\sigma, \phi)}{\partial \phi^{(2)}}=-\frac{1}{\sigma} \sum_{j \in \bar{C}} \mathbf{x}_{j}^{(2)^{\top}}+\frac{1}{\sigma} \sum_{j=1}^{n} \exp \left(\frac{y_{j}-\mathbf{x}_{j} \phi}{\sigma}\right) \mathbf{x}_{j}^{(2)^{\top}}=0_{(p-k) \times 1} .
$$

Ou seja,

$$
\begin{gathered}
-r-\frac{1}{\widehat{\sigma}_{\phi^{(1)}}} \sum_{j \in \bar{C}}\left[y_{j}-\left(\mathbf{x}_{j}^{(1)} \phi^{(1)}+\mathbf{x}_{j}^{(2)} \widehat{\phi}^{(2)}{ }_{\phi^{(1)}}\right)\right] \\
+\frac{1}{\widehat{\sigma}_{\phi^{(1)}}} \sum_{j=1}^{n}\left[y_{j}-\left(\mathbf{x}_{j}^{(1)} \phi^{(1)}+\mathbf{x}_{j}^{(2)} \widehat{\phi}_{\phi^{(2)}}\right)\right] \exp \left[\frac{y_{j}-\left(\mathbf{x}_{j}^{(1)} \phi^{(1)}+\mathbf{x}_{j}^{(2)} \widehat{\phi^{(2)}} \phi^{(1)}\right)}{\widehat{\sigma}_{\phi^{(1)}}}\right]=0
\end{gathered}
$$

$\mathrm{e}$

$$
-\sum_{j \in \bar{C}} \mathbf{x}_{j}^{(2)^{\top}}+\sum_{j=1}^{n} \exp \left[\frac{y_{j}-\left(\mathbf{x}_{j}^{(1)} \phi^{(1)}+\mathbf{x}_{j}^{(2)} \widehat{\phi^{(2)}} \phi^{(1)}\right)}{\widehat{\sigma}_{\phi^{(1)}}}\right] \mathbf{x}_{j}^{(2)}{ }^{\top}=0_{(p-k) \times 1} .
$$

Usando o fato que $y_{j}=\mathbf{x}_{j} \phi+\sigma \log u_{j}=\mathbf{x}_{j}^{(1)} \phi^{(1)}+\mathbf{x}_{j}^{(2)} \phi^{(2)}+\sigma \log u_{j}$, onde $u_{j} \sim \mathcal{E} x p(1)$, $j=1, \ldots, n$, estas equações são expressas por

$$
\begin{aligned}
-r- & \frac{1}{\widehat{\sigma}_{\phi^{(1)}} / \sigma} \sum_{j \in \bar{C}} \log u_{j}+\frac{1}{\widehat{\sigma}_{\phi^{(1)}} / \sigma} \sum_{j=1}^{n} \log \left(u_{j}\right) \exp \left(\frac{\log u_{j}}{\widehat{\sigma}_{\phi^{(1)}} / \sigma}\right) \exp \left[\frac{1}{\widehat{\sigma}_{\phi^{(1)}}} \mathbf{x}_{j}^{(2)}\left(\phi^{(2)}-\widehat{\phi^{(2)}}{ }_{\phi^{(1)}}\right)\right] \\
& +\frac{1}{\widehat{\sigma}_{\phi^{(1)}}} \sum_{j=1}^{n}\left[\mathbf{x}_{j}^{(2)}\left(\phi^{(2)}-\widehat{\phi^{(2)}} \phi^{(1)}\right)\right] \exp \left(\frac{\log u_{j}}{\widehat{\sigma}_{\phi^{(1)}} / \sigma}\right) \exp \left[\frac{1}{\widehat{\sigma}_{\phi^{(1)}}} \mathbf{x}_{j}^{(2)}\left(\phi^{(2)}-\widehat{\phi^{(2)}}{ }_{\phi^{(1)}}\right)\right]
\end{aligned}
$$


D. EMV E ESTATÍSTICAS DA RAZÃO DE VEROSSIMILHANÇAS NO MODELO DE WEIBULL

$$
-\frac{1}{\widehat{\sigma}_{\phi^{(1)}}} \sum_{j \in \bar{C}} \mathbf{x}_{j}^{(2)}\left(\phi^{(2)}-\widehat{\phi^{(2)}} \phi^{(1)}\right)=0
$$

$\mathrm{e}$

$$
-\sum_{j \in \bar{C}} \mathbf{x}_{j}^{(2)^{\top}}+\sum_{j=1}^{n} \exp \left(\frac{\log u_{j}}{\widehat{\sigma}_{\phi^{(1)}} / \sigma}\right) \exp \left[\frac{1}{\widehat{\sigma}_{\phi^{(1)}}} \mathbf{x}_{j}^{(2)}\left(\phi^{(2)}-\widehat{\phi^{(2)}} \phi^{(1)}\right)\right] \mathbf{x}_{j}^{(2)}{ }^{\top}=0_{(p-k) \times 1} .
$$

Multiplicando a equação $(D .5)$ à esquerda por $\left(1 / \widehat{\sigma}_{\phi^{(1)}}\right)\left(\phi^{(2)}-\widehat{\phi^{(2)}}{ }_{\phi^{(1)}}\right)^{\top}$ e usando o resultado para simplificar a equação $(D .4)$, esta torna-se

$-r-\frac{1}{\widehat{\sigma}_{\phi^{(1)}} / \sigma} \sum_{j \in \bar{C}} \log u_{j}+\frac{1}{\widehat{\sigma}_{\phi^{(1)}} / \sigma} \sum_{j=1}^{n} \log \left(u_{j}\right) \exp \left(\frac{\log u_{j}}{\widehat{\sigma}_{\phi^{(1)}} / \sigma}\right) \exp \left[\frac{1}{\widehat{\sigma}_{\phi^{(1)}}} \mathbf{x}_{j}^{(2)}\left(\phi^{(2)}-\widehat{\phi^{(2)}}{ }_{\phi^{(1)}}\right)\right]=0$

Note que a equação $(D .5)$ pode ser re-escrita na forma

$$
\begin{aligned}
& \sum_{j \in \bar{C}}\left\{\exp \left(\frac{\log u_{j}}{\widehat{\sigma}_{\phi^{(1)}} / \sigma}\right) \exp \left[\frac{1}{\widehat{\sigma}_{\phi^{(1)}}} \mathbf{x}_{j}^{(2)}\left(\phi^{(2)}-\widehat{\phi^{(2)}}{ }_{\phi^{(1)}}\right)\right]-1\right\} \mathbf{x}_{j}^{(2)^{\top}} \\
+ & \sum_{j \notin \bar{C}} \exp \left(\frac{\log u_{j}}{\widehat{\sigma}_{\phi^{(1)}} / \sigma}\right) \exp \left[\frac{1}{\widehat{\sigma}_{\phi^{(1)}}} \mathbf{x}_{j}^{(2)}\left(\phi^{(2)}-\widehat{\phi^{(2)}}{ }_{\phi^{(1)}}\right)\right] \mathbf{x}_{j}^{(2)^{\top}}=0_{(p-k) \times 1} .
\end{aligned}
$$

Assim, para dados completos,

$$
\sum_{j \in \bar{C}}\left\{\exp \left(\frac{\log u_{j}}{\widehat{\sigma}_{\phi^{(1)}} / \sigma}\right) \exp \left[\frac{1}{\widehat{\sigma}_{\phi^{(1)}}} \mathbf{x}_{j}^{(2)}\left(\phi^{(2)}-\widehat{\phi^{(2)}}{ }_{\phi^{(1)}}\right)\right]-1\right\} \mathbf{x}_{j}^{(2)^{\top}}=0_{(p-k) \times 1},
$$

e, conseqüentemente, neste caso, pela última versão da equação $(D .4)$, observe que a razão $\widehat{\sigma}_{\phi^{(1)}} / \sigma$ é independentemente distribuída do par $(\sigma, \phi)$, pois é a raiz de uma equação cujos termos são quantidades pivotais e estatísticas ancilares para $(\sigma, \phi)$.

Voltando a estatística de teste, por uma questão de conveniência, esta pode ser re-escrita na forma

$$
\begin{aligned}
& R V\left(\phi^{(1)}\right)=2\left\{-r \log \left(\frac{\widehat{\sigma}}{\sigma}\right)+\sum_{j \in \bar{C}}\left[\frac{y_{j}-\mathbf{x}_{j} \widehat{\phi}}{\widehat{\sigma}}\right]-\sum_{j=1}^{n} \exp \left[\frac{y_{j}-\mathbf{x}_{j} \widehat{\phi}}{\widehat{\sigma}}\right]+r \log \left(\frac{\widehat{\sigma}_{\phi^{(1)}}}{\sigma}\right)\right. \\
& \left.-\sum_{j \in \bar{C}}\left[\frac{y_{j}-\left(\mathbf{x}_{j}^{(1)} \phi^{(1)}+\mathbf{x}_{j}^{(2)} \widehat{\phi^{(2)}} \phi^{(1)}\right)}{\widehat{\sigma}_{\phi^{(1)}}}\right]+\sum_{j=1}^{n} \exp \left[\frac{y_{j}-\left(\mathbf{x}_{j}^{(1)} \phi^{(1)}+\mathbf{x}_{j}^{(2)} \widehat{\phi^{(2)}} \phi^{(1)}\right)}{\widehat{\sigma}_{\phi^{(1)}}}\right]\right\} .
\end{aligned}
$$

Utilizando resultados da seção anterior, lembre que $(\widehat{\sigma} / \sigma)$ é uma quantidade pivotal para $(\sigma, \phi), \sum_{j \in \bar{C}}\left(y_{j}-\mathbf{x}_{j} \widehat{\phi}\right) / \widehat{\sigma}$ é uma estatística ancilar para $(\sigma, \phi)$ e $\sum_{j=1}^{n} \exp \left(y_{j}-\mathbf{x}_{j} \widehat{\phi}\right) / \widehat{\sigma}=r$. 
Na Seção 5.3, a fim de comparar os testes da razão de verossimilhanças baseados em $\ell$ e $\tilde{\ell}_{B N}$ a partir das distorções dos tamanhos destes com relação aos valores nominais, são geradas amostras sob a hipótese nula e calculadas as taxas de rejeição dos mesmos. Nesta situação, os valores dos componentes de $\phi$ sob $\mathcal{H}_{0}$ são os valores verdadeiros. Assim, $\widehat{\sigma}_{\phi(1)}$ e $\widehat{\phi^{(2)}}{ }_{\phi^{(1)}}$ são as estimativas de máxima verossimilhança de $\sigma$ e $\phi^{(2)}$. Portanto, analogamente ao caso em que o par $(\sigma, \phi)$ é desconhecido,

$$
\sum_{j=1}^{n} \exp \left[\frac{y_{j}-\left(\mathbf{x}_{j}^{(1)} \phi^{(1)}+\mathbf{x}_{j}^{(2)} \widehat{\phi^{(2)}} \phi^{(1)}\right)}{\widehat{\sigma}_{\phi^{(1)}}}\right]=r .
$$

Para dados completos, a razão $\left(\widehat{\sigma}_{\phi^{(1)}} / \sigma\right)$ é uma quantidade pivotal para $(\sigma, \phi)$, assim como

$$
\sum_{j \in \bar{C}}\left[\frac{y_{j}-\left(\mathbf{x}_{j}^{(1)} \phi^{(1)}+\mathbf{x}_{j}^{(2)} \widehat{\phi^{(2)}} \phi^{(1)}\right)}{\widehat{\sigma}_{\phi^{(1)}}}\right]
$$

pela equação (D.6). Portanto, neste caso, note que $\operatorname{Pr}_{(\sigma, \phi)}\left(R V\left(\phi^{(1)}\right) \leq q\right)$ não depende do par $\left(\sigma, \phi^{(2)}\right)$ e nem do valor fixado para $\phi^{(1)}$ na hipótese nula, ou seja, sob esta, observe que a estatística $R V\left(\phi^{(1)}\right)$ depende apenas de quantidades pivotais e de estatísticas ancilares para $(\sigma, \phi)$, entretanto, por depender da razão $(\widehat{\sigma} / \sigma)$, sua distribuição depende da matriz de especificação do modelo $X$.

Agora considere o teste da razão de verossimilhanças baseado em $\tilde{\ell}_{B N}\left(\phi^{(1)}\right)$. Para pares $(\sigma, \phi)$ satisfazendo as restrições

$$
\frac{\partial \ell}{\partial \sigma}=0 \quad \text { e } \quad \frac{\partial \ell}{\partial \phi^{(2)}}=0_{(p-k) \times 1},
$$

a função $\tilde{\ell}_{B N}\left(\phi^{(1)}\right)=\tilde{\ell}_{B N}(\sigma, \phi)$ é dada por

$$
\begin{gathered}
\ell(\sigma, \phi)+(p-k+1) \log \sigma-\frac{1}{2} \log \left|\sum_{j=1}^{n} \exp \left(z_{j}\right) \mathbf{x}_{j}^{(2)^{\top}} \mathbf{x}_{j}^{(2)}\right|+\frac{1}{2} \log \left\{-r-2 \sum_{j \in \bar{C}} z_{j}+2 \sum_{j=1}^{n} z_{j} \exp \left(z_{j}\right)\right. \\
+\sum_{j=1}^{n} z_{j}^{2} \exp \left(z_{j}\right)-\left[-\sum_{j \in \bar{C}} \mathbf{x}_{j}^{(2)}+\sum_{j=1}^{n} \exp \left(z_{j}\right) \mathbf{x}_{j}^{(2)}+\sum_{j=1}^{n} z_{j} \exp \left(z_{j}\right) \mathbf{x}_{j}^{(2)}\right]\left[\sum_{j=1}^{n} \exp \left(z_{j}\right) \mathbf{x}_{j}^{(2)} \mathbf{x}_{j}^{(2)}\right]^{-1} \\
\left.\times\left[-\sum_{j \in \bar{C}} \mathbf{x}_{j}^{(2)^{\top}}+\sum_{j=1}^{n} \exp \left(z_{j}\right) \mathbf{x}_{j}^{(2)^{\top}}+\sum_{j=1}^{n} z_{j} \exp \left(z_{j}\right) \mathbf{x}_{j}^{(2)}\right]\right\} \\
-\log \left\{\sum_{j=1}^{n}\left[-I_{(j \in \bar{C})}+\left(1+z_{j}\right) \exp \left(z_{j}\right)\right] \widehat{z}_{j}-\left[\sum_{j=1}^{n}\left[-I_{(j \in \bar{C})}+\left(1+z_{j}\right) \exp \left(z_{j}\right)\right] \mathbf{x}_{j}^{(2)}\right]\right. \\
\left.\times\left[\sum_{j=1}^{n} \exp \left(z_{j}\right) \mathbf{x}_{j}^{(2)} \mathbf{x}_{j}^{(2)}\right]^{-1}\left[\sum_{j=1}^{n} \exp \left(z_{j}\right) \widehat{z}_{j} \mathbf{x}_{j}^{(2)}\right]\right\}
\end{gathered}
$$


onde

$$
z_{j}=\frac{y_{j}-\mathbf{x}_{j} \phi}{\sigma} \quad \text { e } \quad \widehat{z}_{j}=\frac{y_{j}-\mathbf{x}_{j} \widehat{\phi}}{\widehat{\sigma}}
$$

para $j=1, \ldots, n$.

Da mesma forma que foi demonstrado que as parcelas da verossimilhança original $\ell(\sigma, \phi)$ quando maximizadas sob a hipótese nula são independentemente distribuídas do par $(\sigma, \phi)$ no caso de dados completos, pode-se mostrar que as parcelas de $\tilde{\ell}_{B N}\left(\phi^{(1)}\right)=\tilde{\ell}_{B N}(\sigma, \phi)$, sujeitas às restrições

$$
\frac{\partial \ell}{\partial \sigma}=0 \quad \text { e } \quad \frac{\partial \ell}{\partial \phi^{(2)}}=0_{(p-k) \times 1},
$$

quando maximizadas sob as hipóteses nula e alternativa, têm distribuições que não dependem dos parâmetros $\sigma$ e $\phi$. 


\section{REFERÊNCIAS}

[1] Abramowitz, M. e Stegun, I. A. (1965). Handbook of Mathematical Functions. New York: Dover.

[2] Barndorff-Nielsen, O. E. (1980). Conditionality resolutions. Biometrika, 67, 293-310.

[3] Barndorff-Nielsen, O. E. (1983). On a formula for the distribution of the maximum likelihood estimator. Biometrika, 70, 343-365.

[4] Barndorff-Nielsen, O. E. (1994). Adjusted versions of profile likelihood and directed likelihood, and extended likelihood. Journal of the Royal Statistical Society B, 56, 125140.

[5] Barndorff-Nielsen, O. E. (1995). Stable and invariant adjusted profile likelihood and directed likelihood for curved exponential models. Biometrika, 82, 489-499.

[6] Chhikara, R. S. e Folks, J. L. (1989). The Inverse Gaussian Distribution. New York: Marcel Dekker.

[7] Collet, A. (1994). Modelling Survival Data in Medical Research. London: Chapman e Hall.

[8] Cox, D. R. e Oakes, D. (1984). Analysis of Survival Data. London: Chapman e Hall.

[9] Cox, D. R. e Reid, N. (1987). Parameter orthogonality and approximate conditional inference. Journal of the Royal Statistical Society B, 49, 1-39.

[10] Cox, D. R. e Reid, N. (1989). On the stability of maximum-likelihood estimators of orthogonal parameters. The Canadian Journal of Statistics, 17(2), 229-233.

[11] Cox, D. R. e Reid, N. (1992). A note on the difference between profile and modified profile likelihood. Biometrika, 79, 408-411.

[12] Cox, D. R. e Reid, N. (1993). A note on the calculation of adjusted profile likelihood. Journal of the Royal Statistical Society B, 55, 467-471.

[13] Cribari-Neto, F. (1997). Econometric programming environments: GAUSS, Ox and S-PLUS. Journal of Applied Econometrics, 12, 77-89.

[14] Cribari-Neto, F., Frery, A. C. e Silva, M. F. (2002). Improved estimation of clutter properties in speckled imagery. Computational Statistics and Data Analysis, 40, 801824.

[15] Cribari-Neto, F. e Zarkos, S.G. (1999). R: yet another econometric programming environments. Journal of Applied Econometrics, 14, 319-329.

[16] Cribari-Neto, F. e Zarkos, S.G. (2003). Econometric and statistical computing using 0x. Computational Economics, 21, 277-295.

[17] DiCiccio, T. J. e Stern, S. E. (1993). An adjustment to the profile likelihood based on observed information. Technical Report, Department of Statistics, Stanford University. 
[18] DiCiccio, T. J. e Stern, S. E. (1994). Frequentist and Bayesian Bartlett correction of test statistics based on adjusted profile likelihoods. Journal of the Royal Statistical Society $B, 56,397-498$.

[19] DiCiccio, T. J., Martin, M. A., Stern, S. E. e Young, G. A. (1996). Information bias and adjusted profile likelihoods. Journal of the Royal Statistical Society B, 58, 189-203.

[20] Doornik, J.A. (1999). Object-Oriented Matrix Programming Using Ox, 3rd edition. London: Timberlake Consultants.

[21] Doornik, J. A. (2001). Ox 3.0 - An Object-Oriented Matrix Programming Language, 4th edition. London: Timberlake Consultants.

[22] Efron, B. (1979). Bootstrap methods: another look at the jackknife. Annals of Statistics, $7,1-26$.

[23] Efron, B. (1990). More efficient bootstrap computations. Journal of the American Statistical Association, 85, 79-89.

[24] Efron, B. e Tibshirani, R. J. (1993). An Introduction to the Bootstrap. New York: Chapman \& Hall.

[25] Engelhardt, M. (1975). On simple estimation of the parameters of the Weibull or extreme value distribution. Technometrics, 17, 369-374.

[26] Ferrari, S. L. P., Lucambio, F. e Cribari-Neto, F. (2004). Improved profile likelihood inference. Journal of Statistical Planning and Inference, a aparecer.

[27] Ferguson, H., Reid, N. e Cox, D. R. (1991). Estimating equations based on modified profile likelihood. Estimating Functions (ed. V. P. Godambe), 279-293. Oxford: Oxford University Press.

[28] Fergunson, H. (1992). Asymptotic properties of a conditional maximum likelihood estimator. Canadian Journal Statistics, 20, 63-76.

[29] Fraser, D. A. S. e Reid, N. (1995). Ancillaries and third-order significance. Utilitas Mathematica, 47, 33-53.

[30] Fraser, D. A. S. e Reid, N. (1999). Ancillary information for statistical inference. Proceedings of a CRM Symposium on Empirical Bayes and Likelihood Inference (eds. E. Ahmad e N. Reid). New York: Springer-Verlag.

[31] Fraser, D. A. S., Reid, N. e Wu, J. (1999). A simple formula for tail probabilities for frequentist and Bayesian inference. Biometrika, 86, 655-661.

[32] Frery, A. C., Müller, H.--J., Yanasse, C. C. F. e Sant'ana, S. J. S. (1997a). A model for extremely heterogeneous clutter. IEEE Transactions on Geoscience and Remote Sensing, 35, 648-659.

[33] Frery, A. C., Sant'ana, S. J. S., Mascarenhas, N. D. A. e Bustos, O. H. (1997b). Robust inference techniques for speckle noise reduction in 1-look amplitude SAR images. Applied Signal Processing, 4, 61-76. 
[34] Frery, A. C., Cribari-Neto, F. e Souza, M. O. (2004). Analysis of minute features in speckled imagery with maximum likelihood estimation. EURASIP Journal on Applied Signal Processing, 16, 2476-2491.

[35] Harter, H. L. e Moore, A. H. (1968). Maximum likelihood estimation, from doubly censored samples, of the parameters of the first asymptotic distribution of extreme values. Journal of the American Statistical Association, 63, 889-901.

[36] Ihaka, R. e Gentleman, R. (1996). R: a language for data analysis and graphics. Journal of Computational and Graphical Statistics, 5, 299-314.

[37] Jain, A.K. (1989). Fundamentals of Digital Image Processing. Englewood Cliffs: Prentice-Hall.

[38] Klein, J. P. e Moeschberger, M. L. (1997). Survival Analysis Techniques for Censored and Truncated Data. New York: Springer-Verlag.

[39] Knuth, D.E. (1986). The TEXbook. New York: Addison-Wesley.

[40] Lawless, J. F. (1982). Statistical Models and Methods for Lifetime Data. New York: Wiley.

[41] Liang, K.-Y. (1987). Estimating functions and approximate conditional likelihood. Biometrika, 74, 695-702.

[42] Macaskill, G. T. (1993). A note on adjusted profile likelihoods in non-linear regression. Journal of the Royal Statistical Society B, 55, 125-131.

[43] Magnus, J. R. e Neudecker, H. (1988). Matrix Differential Calculus with Applications in Statistics and Econometrics. New York: Wiley.

[44] Mann, N. R. and Fertig, K. W. (1973). Tables for obtaining confidence bounds and tolerance bounds based on best linear invariant estimates of parameters of the extreme value distribution. Technometrics, 15, 87-101.

[45] McCullagh, P. (1987). Tensor Methods in Statistics. London: Chapman e Hall.

[46] McCullagh, P. e Tibishirani, R. (1990). A simple method for the adjustment of profile likelihood. Journal of the Royal Statistical Society B, 52, 325-344.

[47] Meeker, W. Q. e Escobar, L. A. (1998). Statistical Methods for Reliability Data. New York: John Wiley.

[48] Mukerjee, R. (1992). Comparison between the conditional likelihood ratio test and the usual likelihood ratio test. Journal of the Royal Statistical Society B, 54, 184-196.

[49] Nocedal, J. e Wright, S. J. (1999). Numerical Optimization. New York: Springer-Verlag.

[50] Pace, L. e Salvan, A. (1997). Principles of Statistical Inference from a Neo-Fisherian Perspective. Singapore: World Scientific.

[51] Press, W. H., Teukelsky, S. A., Vetterling, W. T. e Flannery, B. P. (1992). Numerical Recipes in C: The Art of Scientific Computing. New York: Cambridge University Press. 
[52] Proschan, F. (1963). Theoretical explanation of observed decreasing failure rate. Technometrics, 5, 375-383.

[53] Ruggiero, M. A. G. e Lopes, V. L. R. (1997). Cálculo numérico: aspectos teóricos e computacionais. São Paulo: Makron Books.

[54] Sartori, N., Bellio, R., Salvan, A. e Pace, L. (1999). The directed modified profile likelihood in models with many parameters. Biometrika, 86, 735-742.

[55] Severini, T. A. (1998a). An approximation to the modified profile likelihood function. Biometrika, 85, 403-411.

[56] Severini, T. A. (1998b). Likelihood functions for the elimination of nuisance parameters. Biometrika, 85, 507-522.

[57] Severini, T. A. (1999). An empirical adjustment to the likelihood ratio statistic. Biometrika, 86, 235-247.

[58] Severini, T. A. (2000a). Likelihood Methods in Statistics. Oxford: Oxford University Press.

[59] Severini, T. A. (2000b). Approximation of sample space derivatives. Technical Report, Department of Statistics, Northwestern University.

[60] Skovgaard, I. M. (1990). On the density of minimum contrast estimators. Annals of Statistics, 18, 779-789.

[61] Skovgaard, I. M. (1996). An explicit large-deviation approximation to one-parameter tests. Bernoulli, 2, 145-165.

[62] Stern, S. E. (1997). A second-order adjustment to the profile likelihood in the case of a multidimensional parameter of interest. Journal of the Royal Statistical Society B, 59, 653-665.

[63] Thoman, D. R., Bain, L. J. e Antle, C. E. (1969). Inferences on the parameters of the Weibull distribution. Technometrics, 11(3), 445-460.

[64] Verhagen, A. M. W. (1961). The estimation of regression and error-scale parameters, when the joint distribution of the errors is of any continuous form and known apart from a scale parameter. Biometrika, 48, 125-132.

[65] Yang, Z. e Xie, M. (2003). Efficient estimation of the Weibull shape parameter based on a modified profile likelihood. Journal of Statistical Computation and Simulation, 73(2), 115-123. 